Journal of

Mechanics of

Materials and Structures

Volume 3, № 4

April 2008

mathematical sciences publishers 


\title{
JOURNAL OF MECHANICS OF MATERIALS AND STRUCTURES
}

http://www.jomms.org

\author{
EDITOR-IN-CHIEF Charles R. Steele
}

Associate Editor Marie-Louise Steele

Division of Mechanics and Computation

Stanford University

Stanford, CA 94305

USA

\section{BOARD OF EDITORS}

D. BIGONI University of Trento, Italy

H. D. Bui École Polytechnique, France

J. P. CARTER University of Sydney, Australia

R. M. Christensen Stanford University, U.S.A.

G. M. L. GLADWELL University of Waterloo, Canada

D. H. Hodges Georgia Institute of Technology, U.S.A.

J. HutChINSON Harvard University, U.S.A.

C. HwU National Cheng Kung University, R.O. China

IWONA JASIUK University of Illinois at Urbana-Champaign

B. L. KARIHALOO University of Wales, U.K.

Y. Y. KIM Seoul National University, Republic of Korea

Z. Mroz Academy of Science, Poland

D. PAMPlonA Universidade Católica do Rio de Janeiro, Brazil

M. B. RUBIN Technion, Haifa, Israel

Y. SHINDO Tohoku University, Japan

A. N. SHUPIKOV Ukrainian Academy of Sciences, Ukraine

T. TARNAI University Budapest, Hungary

F. Y. M. WAN University of California, Irvine, U.S.A.

P. WRIGGERS Universität Hannover, Germany

W. YANG Tsinghua University, P.R. China

F. ZIEGLER Technische Universität Wien, Austria

\section{PRODUCTION}

Paulo Ney de Souza Production Manager

SheIla Newbery Senior Production Editor

SILVIO LEVY Scientific Editor

See inside back cover or http://www.jomms.org for submission guidelines.

Regular subscription rate: $\$ 500$ a year.

Subscriptions, requests for back issues, and changes of address should be sent to Mathematical Sciences Publishers, 798 Evans Hall, Department of Mathematics, University of California, Berkeley, CA 94720-3840. 


\title{
INTERFACIAL CRACK KINKING SUBJECTED TO CONTACT EFFECTS
}

\author{
Baoxiang X. Shan, Assimina A. Pelegri And Yi Pan
}

\begin{abstract}
We investigate the problem of a kinking crack at a bimaterial interface when the two surfaces are in contact near the crack tip. Using a potential function and the dislocation technique, we relate, by a singular integral equation, the stress intensity factors (SIF) at the kinking crack tip to the SIF before crack kinking. We use Gauss-Chebyshev integration formulas to solve this integral equation numerically. We evaluate the kinking angles from a bimaterial interface under conditions of contact using the maximum energy release rate criterion and compare these angles with our experiments and those in the literature. The interfacial crack is demonstrated by simulation and experiments to kink into the more compliant material at an angle of about $80^{\circ}$.
\end{abstract}

\section{Introduction}

Interfacial fracture mechanics has been studied for several decades, and treatments have steadily grown more effective as applications have increased [Sih 1977; Muskhelishvili 1977; Sanford 1997a; 1997b; Hutchinson and Suo 1992]. Using continuum fracture mechanics, there are three candidate criteria for predicting the growth direction for an interfacial crack: maximum loop stress [Wang 1994; Li et al. 2004], maximum energy release rate [Mulville et al. 1978; Sun and Jih 1987], and zero mode II intensity stress factor $\left(K_{I I}=0\right)$ [Sih 1977; Hutchinson and Suo 1992; Sanford 1997b; Banks-Sills and Ashkenazi 2000]. The maximum loop stress criterion was demonstrated to be ineffective and inaccurate [Xie et al. 2005]. The criteria of maximum energy release rate and zero mode II intensity stress factor were shown to predict the same crack propagation direction in most loading conditions [Hutchinson and Suo 1992]. It is thus reasonable to use the maximum energy release rate criterion to predict the direction the crack propagates. Because this rate can be completely expressed in terms of local stress intensity factors [Rice 1988], it is important, in bimaterial systems with complex geometry, to find how the local SIF relates to the loading SIF.

Some have tried using finite element methods (FEM) to predict the kinking angles out of an interface [Leblond and Frelat 2001; Leblond and Frelat 2004]. However, calculating the energy release rate for all possible kinking angles is very tedious and may be inaccurate due to the singular characteristics of stress and strain at a crack tip. With help of a complex variable representation, the dislocation method provides a powerful tool for solving crack problems in both homogeneous solids and nonhomogeneous layered materials [Atkinson 1966; Lo 1978; Hayashi and Nemat-Nasser 1981; Hills et al. 1996]. Therefore, we adopt the dislocation technique here to find the relation between the SIF before and after kinking, under general loading conditions. We then apply the solution to find how cracks kink from a interface between two materials in contact.

Keywords: complex variables, composites, contact effect, interfacial crack, kinking crack, singular integration. 


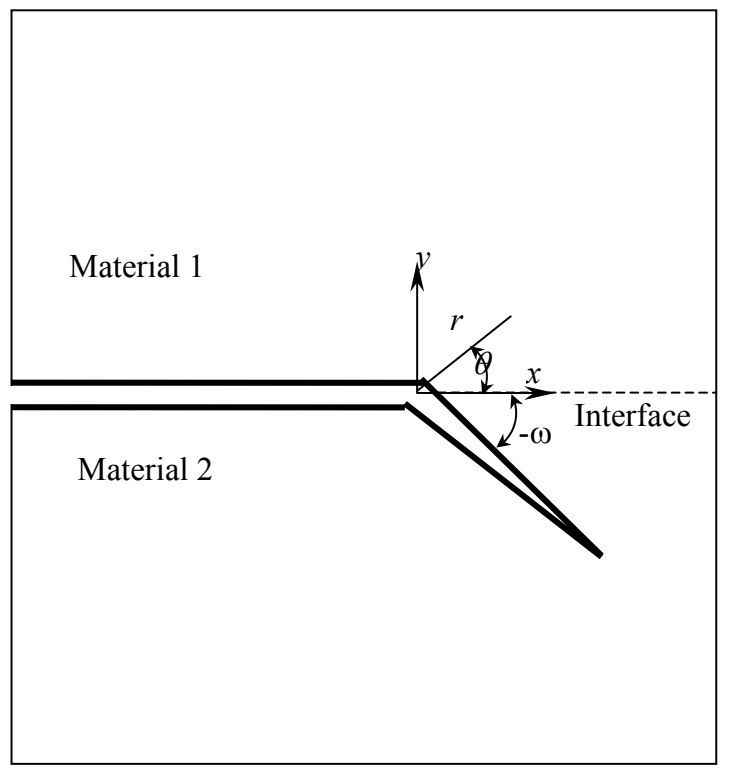

Figure 1. Geometry of kinked crack.

When the surfaces near an interfacial crack tip are in contact, the conditions around the tip locally become pure mode II, at least before the crack kinks [Comninou 1977; Comninou and Dundurs 1979a; 1979b; Fan et al. 1998; Gautesen and Dundurs 1988]. Equivalently, the effect of contact can be included by applying a pure shear loading at infinity or by setting the loading phase angle to $90^{\circ}$ [Leblond and Frelat 2001; 2004].

\section{Problem formulations}

Consider a two-dimensional infinite bimaterial system, with a semiinfinite edge crack on the interface and a significantly smaller kinked crack in the more compliant material labeled 2; see Figure 1. Global loading of this geometry causes a primary semiinfinite interfacial crack to propagate into material 2 . As the linear fracture solution may cause the crack to exhibit an inadmissible flank penetration into the bimaterial interface, the primary semiinfinite interfacial crack before kinking is represented by a contact model [Comninou 1977; Comninou and Dundurs 1979a; 1979b] in which a small contact zone exists around the crack tip; see Figure 2.

In either the linear fracture model or the contact model, the stress intensity factors for the interfacial crack can be expressed in a complex variable [Rice 1988; Hutchinson and Suo 1992]

$$
K=K_{I}+i K_{I I}=|K| e^{i \psi_{0}},
$$

where $i=\sqrt{-1}$, and where $K_{I}$ and $K_{I I}$ are the mode I and II SIF initially applied at infinity. $|K|$ and $\psi_{0}$ are the magnitude and phase angle of the SIF.

When a contact zone occurs around the crack tip, the complex SIF is determined by $K_{I I}$ with a diminished $K_{I}$ [Whitcomb 1981; Sun and Jih 1987]. Due to the contact between the flanks of the 


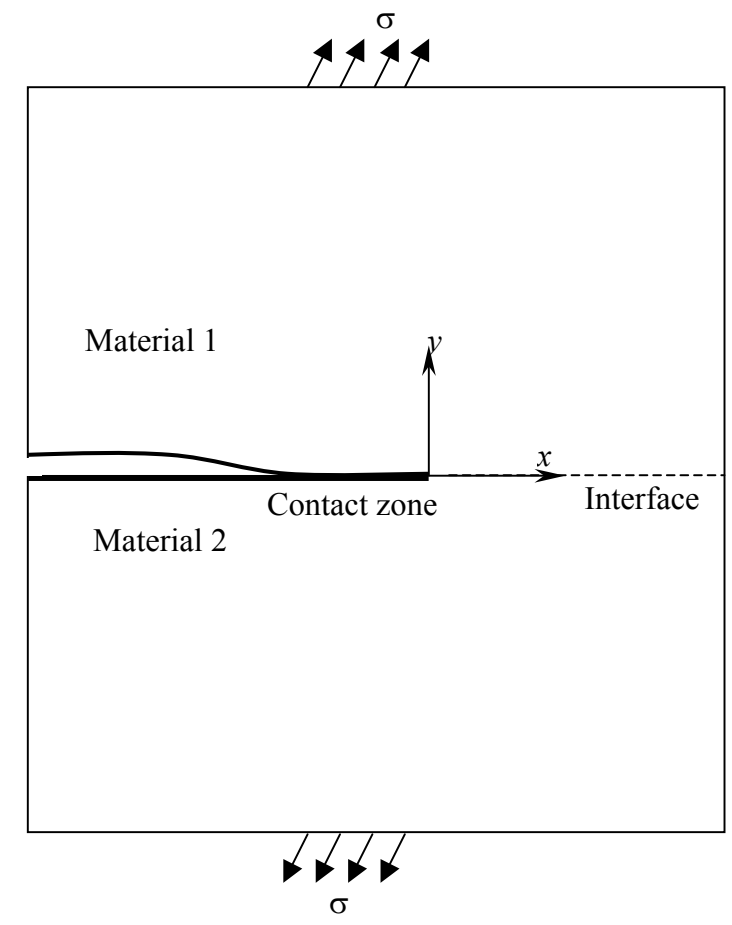

Figure 2. Contact model of primary semiinfinite interfacial crack.

interfacial crack, the mode I SIF disappears, that is, $K_{I}=0$ or $\psi_{0}=90^{\circ}$ [Comninou 1977; Leblond and Frelat 2001; Leblond and Frelat 2004].

After the primary interfacial crack kinks into one side, the stress intensity factor after kinking $K^{\prime}$ can be similarly expressed in a complex variable

$$
K^{\prime}=K_{1}+i K_{2},
$$

where $K_{1}$ and $K_{2}$ are the postkinking mode I and II SIF.

Because the crack tip is confined to a one side after kinking — that is, in a single homogeneous and isotropic medium - the kinked crack problem can be solved by superposition using the basic linear fracture solution for an edge dislocation in material 2 interacting with an initial semiinfinite interfacial crack. As illustrated in Figure 3, the actual kinked crack tip is represented by a virtual dislocation along the crack tip.

The stresses at point $z=t e^{-i \omega}$ caused by a discrete edge dislocation at $z_{0}=\eta e^{-i \omega}$ can be expressed in complex form [Lo 1978; Hayashi and Nemat-Nasser 1981; Wang 1994] as

$$
\sigma_{\theta \theta}(t)+i \sigma_{r \theta}(t)=2 \bar{B} e^{-i \omega}(t-\eta)^{-1}+B H_{1}(t, \eta)+\bar{B} H_{2}(t, \eta),
$$

where

$$
B=\frac{\mu_{2}}{i \pi\left(\kappa_{2}+1\right)} e^{i \theta}\left(u_{r}+i v_{\theta}\right)
$$

is the Burgers vector. The functions $H_{1}$ and $H_{2}$ are specified in Appendix A. 

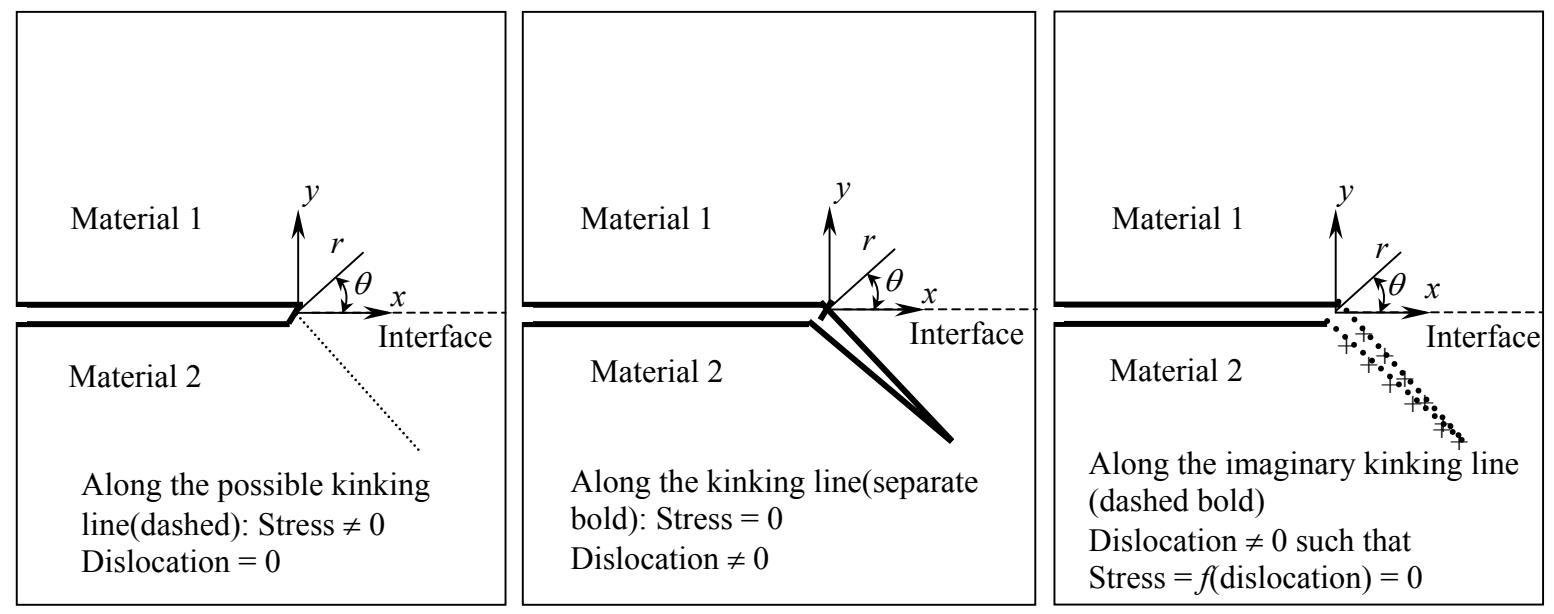

Figure 3. Modeling a kinked crack by dislocation. From left: without kinking; after kinking, in the traditional representation with a crack; after kinking, in the dislocation representation without a crack.

After the kinked crack is represented by a distributed dislocation $B(\eta)$, where $\eta$ is the distance of a discrete dislocation from the initial crack tip, the crack-dislocation relation becomes an integral equation specifying that the net traction is zero on the kinking line (see Figure 3):

$$
2 e^{-i \omega} \int_{0}^{1} \bar{B}(\eta)(t-\eta)^{-1} d \eta+\int_{0}^{1} B(\eta) H_{1}(t, \eta) d \eta+\int_{0}^{1} \bar{B}(\eta) H_{2}(t, \eta) d \eta=-\left(\sigma_{\theta \theta}(t)+i \sigma_{r \theta}(t)\right) .
$$

Because $s=2 \eta-1$ and $s_{0}=2 t-1$, Equation (3) can be equivalently transformed into

$$
2 e^{-i \omega} \int_{-1}^{1} \bar{B}(s)\left(s_{0}-s\right)^{-1} d s+\int_{-1}^{1} B(s) \frac{H_{1}\left(s_{0}, s\right)}{2} d s+\int_{-1}^{1} \bar{B}(s) \frac{H_{2}\left(s_{0}, s\right)}{2} d s=-\left(\sigma_{\theta \theta}\left(s_{0}\right)+i \sigma_{r \theta}\left(s_{0}\right)\right),
$$

where $s$ is new integration variable, $s_{0}$ is the point along the kinking crack from the initial crack tip to the propagated crack tip, and $B(s)$ is the distributed dislocation along the kinking crack.

This integral equation can be solved numerically using a method developed by [Erdogan and Gupta 1972; Sih 1977]. First, the unknown equivalent dislocation function $B(s)$ is factored into a singularity term and a bounded term $P(s)$ [He and Hutchinson 1989]:

$$
B(s)=\left(1-s^{2}\right)^{-1 / 2} P(s) .
$$

Then Equation (4) becomes a set of linear equations

$$
\sum_{i=1}^{n} \frac{1}{n} \bar{P}\left(s_{i}\right)\left[\frac{2 \pi e^{-i \varpi}}{s_{0 k}-s_{i}}+\frac{\pi}{2} H_{2}\left(s_{0 k}, s_{i}\right)\right]+\sum_{i=1}^{n} \frac{1}{n} P\left(s_{i}\right)\left[\frac{\pi}{2} H_{1}\left(s_{0 k}, s_{i}\right)\right]=-\left(\sigma_{\theta \theta}\left(s_{0 k}\right)+i \sigma_{r \theta}\left(s_{0 k}\right)\right),
$$


where $n$ is the number of sampling point and the highest of order for numerical approximation,

$$
s_{i}=\cos \left(\frac{2 i-1}{2 n} \pi\right) \text { for } i=1,2, \ldots, n, \quad \text { and } \quad s_{0 k}=\cos \left(\frac{k}{n} \pi\right) \text { for } k=1,2, \ldots, n-1,
$$

The function $P(s)$ can be expended in Chebyshev polynomials $T_{j}(s)$ of the first kind as

$$
P(s)=\sum_{j=1}^{n} C_{j} T_{j-1}(s) .
$$

Consequently, Equation (5) becomes the set of linear equations

$$
\sum_{j=1}^{n}\left\{C_{j} E_{j}\left(s_{0 k}\right)+\bar{C}_{j} F_{j}\left(s_{0 k}\right)\right\}=-\left(\sigma_{\theta \theta}\left(s_{0 k}\right)+i \sigma_{r \theta}\left(s_{0 k}\right)\right),
$$

where $E_{j}\left(s_{0 k}\right)$ and $F_{j}\left(s_{0 k}\right)$ are the integrated functions that describe the distributed dislocation of stress at the point $s_{0 k}$. They can be written as

$$
\begin{aligned}
& E_{j}\left(s_{0 k}\right)=\int_{-1}^{1} B(s) \frac{H_{1}\left(s_{0 k}, s\right)}{2} d s, \\
& F_{j}\left(s_{0 k}\right)=2 e^{-i \omega} \int_{-1}^{1} \bar{B}(s)\left(s_{0 k}-s\right)^{-1} d s+\int_{-1}^{1} \bar{B}(s) \frac{H_{2}\left(s_{0 k}, s\right)}{2} d s .
\end{aligned}
$$

Because there are $n$ unknowns but only $n-1$ equations in Equation (5), an additional equation needs be introduced. He and Hutchinson [1989] chose

$$
P(-1)=\sum_{j=1}^{n} C_{j} T_{j-1}(-1)=0 .
$$

After the dislocation function is numerically determined from the linear equations, the stress intensity factor after kinking can be calculated, according to [Lo 1978; He and Hutchinson 1989], from

$$
K^{\prime}=K_{1}+i K_{2}=(2 \pi)^{3 / 2} e^{-i \varpi} \lim _{\eta \rightarrow 0}\left\{(1-\eta)^{1 / 2} \bar{B}(\eta)\right\}=(2 \pi)^{3 / 2} e^{-i \varpi} \bar{P}(1) .
$$

This equation relates the SIF after kinking to that before, because as $P(1)$ is connected to the initially applied SIF $K$ through Equation (5).

In plane strain, the initial energy release rate $G$ of the interfacial crack is related to the initial SIF $K$ as [He and Hutchinson 1989]

$$
G_{0}=\left(\frac{1-v_{1}}{\mu_{1}}+\frac{1-v_{2}}{\mu_{2}}\right)\left(\frac{K \bar{K}}{4 \cosh ^{2} \pi \varepsilon}\right),
$$

where $\mu_{1}$ and $\nu_{1}$ are the shear modulus and Poisson's ratio of material 1 , and $\mu_{2}$ and $\nu_{2}$ are the shear modulus and Poisson's ratio of material 2. The material mismatch index $\varepsilon$ is defined as

$$
\varepsilon=\frac{1}{2 \pi} \ln \left(\frac{1-\beta}{1+\beta}\right) .
$$


$\alpha$ and $\beta$ are Dundur's material parameters, defined in plane strain as

$$
\alpha=\frac{\mu_{1}\left(1-v_{2}\right)-\mu_{2}\left(1-v_{1}\right)}{\mu_{1}\left(1-v_{2}\right)+\mu_{2}\left(1-v_{1}\right)} \quad \text { and } \quad \beta=\frac{1}{2} \frac{\mu_{1}\left(1-2 v_{2}\right)-\mu_{2}\left(1-2 v_{1}\right)}{\mu_{1}\left(1-v_{2}\right)+\mu_{2}\left(1-v_{1}\right)} .
$$

As the crack propagates in a homogeneous medium after kinking, the energy release rate $G$ is given by [He and Hutchinson 1989]

$$
G=\frac{1-v_{2}}{2 \mu_{2}}\left(K_{1}^{2}+K_{2}^{2}\right),
$$

where $\mu_{2}$ and $v_{2}$ are the shear modulus and Poisson's ratio of material 2 and where $K_{1}$ and $K_{2}$ are the postkinking SIFs of mode I and II.

We will next evaluate, for different material combinations, the relative energy release rate $G / G_{0}$, and we will predict the kinking angle by the criterion of maximum energy release rate.

\section{Numerical solution and results}

3.1. Validation of program. The numerical simulation is in MATLAB. Before implementing the numerical analysis, we validate the program by comparing our results with [He and Hutchinson 1989, Table 1] for $\alpha=0, \beta=0$ and $\alpha=0.56, \beta=0.12$. In both cases, the kinking angle is set to $45^{\circ}$ and the initial SIF $K$ is set to 1 ; see the results in Table 1 .

Comparing our results with those of [He and Hutchinson 1989], we find that the real parts of $P(1)$ agree very well. The imaginary parts of $P(1)$ are close in value, but ours takes a negative sign. Our calculation is further validated by Cotterell and Rice's equation [1980].

From Table 1, we also see that the values of $P(1)$ differ by less than 0.1 percent in going from $N=40$ to $N=100$. Thus, for the rest of this paper, we will use $N=40$.

3.2. Numerical simulation results. Figure 4 plots how relative energy release rate varies with angle, for different material combinations. In the simulation, the Poisson's ratios of both materials are assumed to be same, and the ratio of material Young's moduli $E_{1} / E_{2}$ are chosen as 1,2, 5, 10 and 100. The figure demonstrates that in each case the relative release energy rate reaches its maximum at some angle.

\begin{tabular}{ccc}
\hline \multicolumn{3}{c}{$\alpha=\beta=0$} \\
$N$ & $P_{R}(1)$ & $P_{m}(1)$ \\
\hline 4 & 0.04923 & -0.02096 \\
8 & 0.04976 & -0.02108 \\
12 & 0.04989 & -0.02107 \\
16 & 0.04994 & -0.02106 \\
20 & 0.04997 & -0.02105 \\
40 & 0.05001 & -0.02102 \\
\hline
\end{tabular}

\begin{tabular}{ccc}
\hline \multicolumn{3}{c}{$\alpha=0.56, \beta=0.12$} \\
$N$ & $P_{R}(1)$ & $P_{m}(1)$ \\
\hline 4 & 0.04149 & -0.01805 \\
8 & 0.04183 & -0.01871 \\
12 & 0.04193 & -0.01879 \\
20 & 0.04199 & -0.01883 \\
40 & 0.04201 & -0.01885 \\
100 & 0.04202 & -0.01885 \\
\hline
\end{tabular}

Table 1. Calculation of $P(1)$ for $\omega=45^{\circ}$ and $K=1$. 


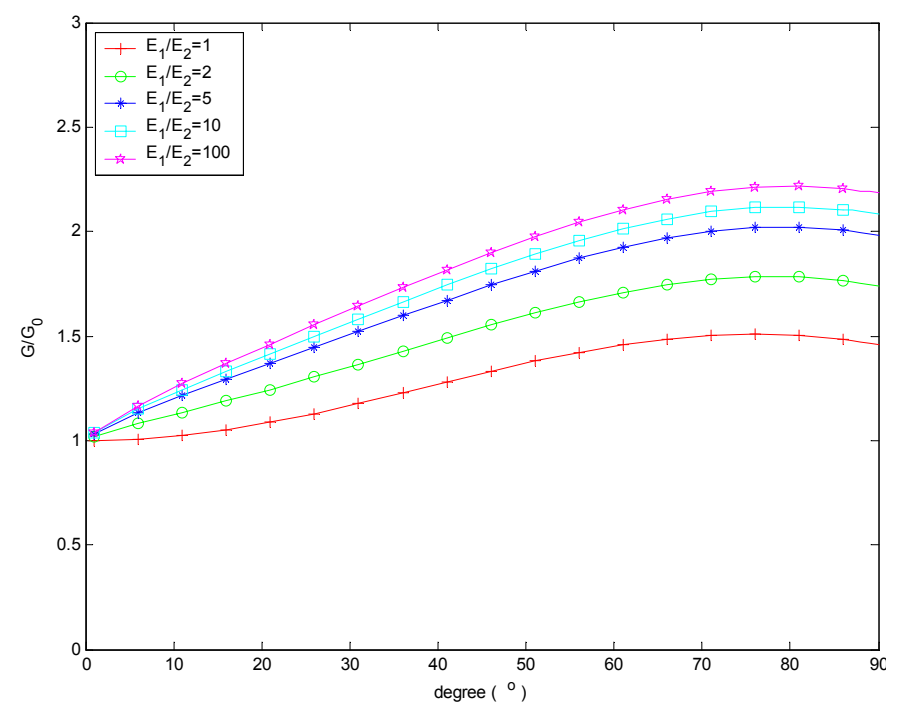

Figure 4. Energy release rate versus kinking angle.

Adopting the criterion of maximum energy release rate, we can find the kinking angle for each case. See the results in Table 2, which also lists, for comparison, the kinking angle evaluated from the criterion $K_{I I}=0$.

\section{Comparison with experiments and discussion}

For the homogeneous limit $(\alpha=\beta=0)$, here, $E_{1} / E_{2}=1$, we compute the kinking angle at $77.1^{\circ}$ using the criterion $K_{I I}=0$; see Table 2 . This result agrees well with the $77.3^{\circ}$ obtained by correlating of initial and local SIF by quadratures [Bilby and Cardew 1975] and the $77.8^{\circ}$ obtained by FEM analysis [Leblond and Frelat 2001; 2004], both of which use the criterion $K_{I I}=0$.

Pelegri and Chen [2000] performed experiments on cross-ply laminated composites (IM7/5260) using a Mixed Mode Bending (MMB) test facility. The kinking angles were found at different loading ratios. The experimental results show that the kinking angle is $80^{\circ}$ when the loading ratio of shear to tension is 4:1. From the fractographic images [Gilchrist and Svensson 1995; Partridge and Singh 1995; Gilchrist et al. 1996] of loading ratio experiments similar to those of Pelegri, the interfacial cracks show contact between the crack flanks at a loading ratio of 4:1. Here, the program is implemented to compute the kinking angle for those experiments. Considering the contact effect in our simulation, we predict the kinking angle of $80.4^{\circ}$ when the loading ratio of $4: 1$, which matches well the experimental result.

\begin{tabular}{lccccccc}
\hline$E_{1} / E_{2}$ & 1 & 2 & 5 & 10 & 20 & 50 & 100 \\
Kinking angle by maximum $G / G_{0}$ & $75.7^{\circ}$ & $77.9^{\circ}$ & $79.3^{\circ}$ & $79.8^{\circ}$ & $80.0^{\circ}$ & $80.2^{\circ}$ & $80.2^{\circ}$ \\
Kinking angle by $K_{2}=0$ & $77.1^{\circ}$ & $81.1^{\circ}$ & $84.3^{\circ}$ & $85.5^{\circ}$ & $86.1^{\circ}$ & $86.5^{\circ}$ & $86.7^{\circ}$ \\
\hline
\end{tabular}

Table 2. Kinking angles in the presence of contact, assuming $v_{1}=v_{2}$. 

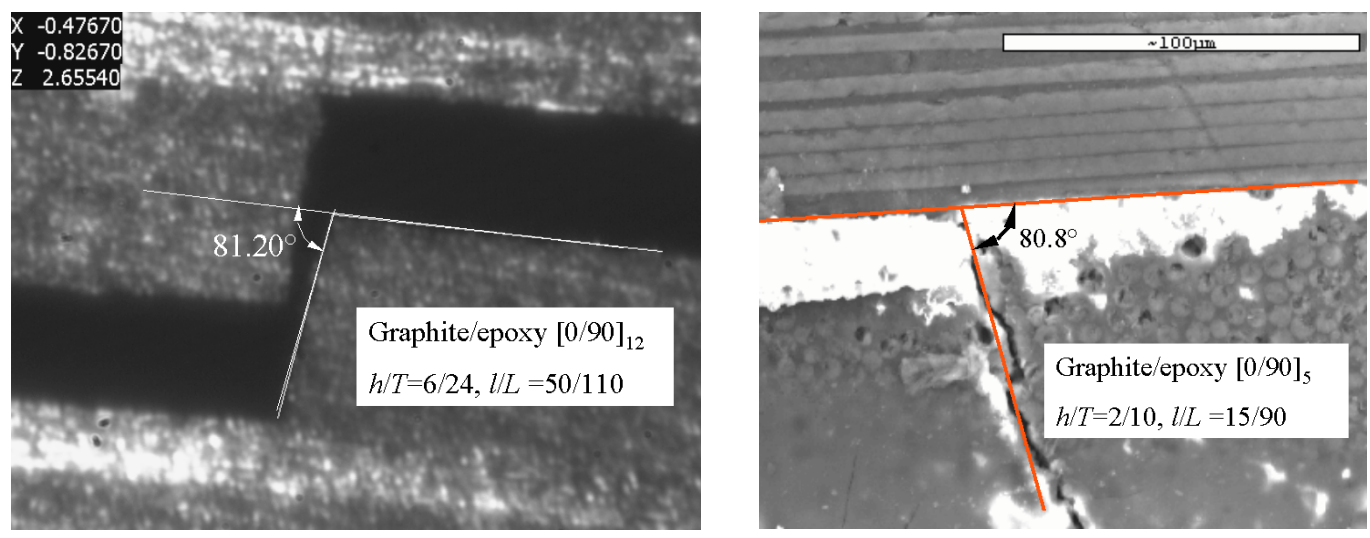

Figure 5. At left, the kinking angle for the cantilever bending experiment and, at right, for the microscopic three-point experiment.

We experiment with cantilever bending and microscopic three-point bending on cross-ply laminated composite (IM7/G8548) [Shan and Pelegri 2003]. Figure 5 shows images of the kinking crack in these two cases. In the cantilever bending experiment, the cantilever beam can withstand the shear resultant force and moment after its local buckling, so that the inner delamination tip is in local compressive and mode II dominant conditions, and the two surfaces are in contact near the tip. The dominance of mode II and the presence of contact are also verified by finite element analysis [Shan and Pelegri 2003], which Figure 6 shows as a large ratio of $K_{I I} / K_{I}$. This paper's program computes the kinking angle to be $80.4^{\circ}$ for the mode II dominant condition, which fits well with the experimental result of $81.2^{\circ}$; see Figure 5 at left. Figure 5, at right, illustrates a microscopic experiment by three-point bending; the results are also dominated by mode II and the presence of contact. The kinking angle of $80.8^{\circ}$ agrees well with our numerics.

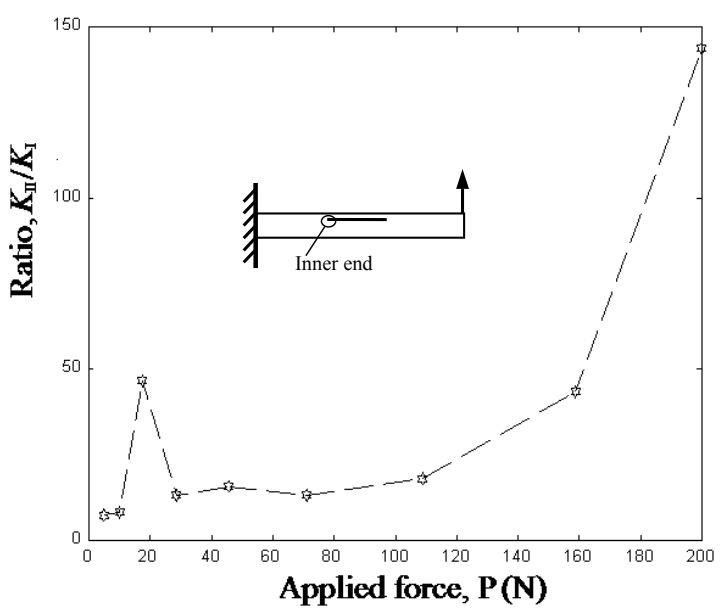

Figure 6. Ratio of stress intensity factors in mode II to mode I by FEM. 


\section{Conclusions}

We determined the kinking angle for an interfacial crack under the effects of contact by a numerical analysis using a complex SIF, a dislocation technique, and singular integration. The contact effects are important for evaluating the competition between the interlayer and intralayered cracks in laminate materials and composites.

When contact is present, our numerical analysis finds the kinking angle out of the interface into the more compliant material to be $75.7^{\circ}$ for homogeneous layered materials and around $80.2^{\circ}$ for a wide range of nonhomogeneous material combinations. According to the analytical, FEM, and experimental results, this angle is independent of the structural geometry, the loading type (be it static, fatigue, or dynamic), and loading history and has little relation to material elastic constants. Furthermore, the progressive crack tends to get trapped on the interface regardless of the loading and architectural configuration for the laminate composites. This it because the crack is eventually forced against interface and accordingly meet more resistance to its further propagation.

Our future work will focus on the crack growth law under effects of contact and friction and on how the crack progresses from pure mode I opening to mixed mode to pure mode II shearing. The size and pattern of heckles formed during as the crack propagates may serve to measure this phenomenon.

\section{Acknowledgements}

This work was sponsored by the National Science Foundation, Career Award CMS-9982023. The authors gratefully acknowledge this financial support and are thankful to the program manager, Dr. Jorn LarsenBasse, for his genuine interest and encouragement.

\section{Appendix A. Derivation of the functions $H_{1}$ and $H_{2}$ in the dislocation method}

In terms of the Muskhelishvili [1977] potentials, the stresses and displacements in two-dimensional infinite bimaterial system may be expressed as

$$
\begin{aligned}
\left(\sigma_{y y}-i \sigma_{x y}\right)_{j} & =\Phi_{j}(z)+\overline{\Phi_{j}(z)}+z \overline{\Phi_{j}^{\prime}(z)}+\overline{\Psi_{j}(z)}, \\
\left(\sigma_{y y}+\sigma_{x x}\right)_{j} & =2\left(\Phi_{j}(z)+\overline{\Phi_{j}(z)}\right), \\
2 \mu_{j}\left(\frac{\partial u}{\partial x}-i \frac{\partial v}{\partial x}\right)_{j} & =\kappa_{j} \Phi_{j}(z)-\left(\overline{\Phi_{j}(z)}+z \overline{\Phi_{j}^{\prime}(z)}+\overline{\Psi_{j}(z)}\right),
\end{aligned}
$$

where the subscript $j=1,2$ means "in the region $j$ " and where $\Phi_{j}$ and $\Psi_{j}$ are the potentials. Also, the complex $z$ is $x+i y$, the prime takes derivatives in $z$, the overbar denotes complex conjugation, $\kappa=3-4 v$ for plane strain, $\kappa=(3-v) /(1+v)$ for plane stress, and $v$ is Poisson's ratio.

Mukai et al. [1990] introduced two additional jump potentials $\Omega_{S}$ and $\Omega_{D}$ to solve the branching of interfacial crack of finite length within an infinite large body. Similarly to Mukai's method, Suo [1989] 
obtained the Muskhelishvili potentials for an infinite large body with a semiinfinite interfacial crack from

$$
\begin{aligned}
\Omega_{S} & = \begin{cases}\Phi_{1}(z)-\left[\bar{\Phi}_{2}(z)+z \bar{\Phi}_{2}(z)+\bar{\Psi}_{2}(z)\right], & z \in S_{1}, \\
\Phi_{2}(z)-\left[\bar{\Phi}_{1}(z)+z \bar{\Phi}^{\prime}{ }_{1}(z)+\bar{\Psi}_{1}(z)\right], & z \in S_{2},\end{cases} \\
\Omega_{D} & = \begin{cases}\frac{\kappa_{1}}{2 \mu_{1}} \Phi_{1}(z)+\frac{1}{2 \mu_{2}}\left[\bar{\Phi}_{2}(z)+z \bar{\Phi}_{2}^{\prime}(z)+\bar{\Psi}_{2}(z)\right], \quad z \in S_{1}, \\
\frac{\kappa_{2}}{2 \mu_{2}} \Phi_{2}(z)+\frac{1}{2 \mu_{1}}\left[\bar{\Phi}_{1}(z)+z \bar{\Phi}_{1}^{\prime}(z)+\bar{\Psi}_{1}(z)\right], \quad z \in S_{2},\end{cases} \\
\Phi_{1}(z) & =Q_{1}\left[\frac{1}{2 \mu_{2}} \Omega_{S 1}(z)+\Omega_{D 1}(z)\right], \\
\Psi_{1}(z) & =Q_{2}\left[\frac{-\kappa_{2}}{2 \mu_{2}} \bar{\Omega}_{S 2}+\bar{\Omega}_{D 2}\right]-\Phi_{1}(z)-z \Phi_{1}^{\prime}(z), \\
\Phi_{2}(z) & =Q_{2}\left[\frac{1}{2 \mu_{1}} \Omega_{S 2}(z)+\Omega_{D 2}(z)\right], \\
\Psi_{2}(z)= & Q_{1}\left[\frac{-\kappa_{1}}{2 \mu_{1}} \bar{\Omega}_{S 1}+\bar{\Omega}_{D 1}\right]-\Phi_{2}(z)-z \Phi_{2}^{\prime}(z),
\end{aligned}
$$

where

$$
Q_{1}=\frac{2 \mu_{1} \mu_{2}}{\mu_{1}+\kappa_{1} \mu_{2}}, \quad Q_{2}=\frac{2 \mu_{1} \mu_{2}}{\mu_{2}+\kappa_{2} \mu_{1}},
$$

and $\bar{f}(z) \equiv \overline{f(\bar{z})}$. If $f(z)$ is analytic for $z$ in region $S$, then $\bar{f}(z)$ is analytic for $\bar{z}$ in region $S$.

The interaction between an interface crack and a dislocation may be solved by superposing the solutions for (i) a dislocation in $S_{2}$ near an interface and (ii) an interface crack loaded with the negative of the stresses produced by (i). By simply replacing $\alpha$ with $-\alpha, \beta$ with $-\beta$ and by switching the subscripts 1 and 2 of $\Phi^{D}$ and $\Psi^{D}$ from Mukai's results [1990], we get the potentials

$$
\begin{aligned}
\Phi_{1, \text { singular }}^{D} & =\Psi_{1, \text { singular }}^{D}=0, \\
\Phi_{2, \text { singular }}^{D} & =\frac{B}{z-s_{0}}, \\
\Psi_{2, \text { singular }}^{D} & =\bar{B} \frac{1}{z-s_{0}}+B \frac{\bar{s}_{0}}{\left(z-s_{0}\right)^{2}}, \\
\Phi_{1, \text { continuation }}^{D} & =B\left(\frac{(1-\alpha)}{(1+\beta)\left(z-s_{0}\right)}\right), \\
\Psi_{1, \text { continuation }}^{D} & =A\left(\frac{(1-\alpha)\left(\bar{s}_{0}-s_{0}\right)}{(1-\beta)\left(z-s_{0}\right)^{2}}\right)+\bar{A}\left(\frac{(1-\alpha)}{(1-\beta)\left(z-s_{0}\right)}\right)-\Phi_{1, \text { continuation }}^{D}-z \Phi_{1, \text { continuation }}^{D^{\prime}}, \\
\Phi_{2, \text { continuation }}^{D} & =B\left(\frac{(\beta-\alpha)}{(1-\beta)\left(z-\bar{s}_{0}\right)}\right)+\bar{B}\left(\frac{(\beta-\alpha)\left(s_{0}-\bar{s}_{0}\right)}{(1-\beta)\left(z-\bar{s}_{0}\right)^{2}}\right), \\
\Psi_{2, \text { continuation }}^{D} & =\bar{A}\left(\frac{-(\alpha+\beta)}{(1+\beta)\left(z-\bar{s}_{0}\right)}\right)-\Phi_{2, \text { continuation }}^{D}-z \Phi_{2, \text { continuation }}^{D^{\prime}},
\end{aligned}
$$


where $B$ is the Burgers vector of Equation (2) and $\alpha$ and $\beta$ are Dundur's constants defined in Equation (6).

When the main crack is introduced, the stresses due to the dislocation near an interface will be removed from the crack faces by the two potentials $\Phi^{C}$ and $\Psi^{C}$. Many investigators [Suo 1989; Mukai et al. 1990; Rice et al. 1990] have presented the solutions for crack problems. For a semiinfinite crack on an interface, the interface boundary conditions at $y=0$ are

$$
\begin{aligned}
\left(\sigma_{y y}-i \sigma_{x y}\right)_{1}-\left(\sigma_{y y}-i \sigma_{x y}\right)_{2} & =0 & & \text { for }|x|<\infty, \\
\left(\frac{\partial u}{\partial x}-i \frac{\partial v}{\partial x}\right)_{1}-\left(\frac{\partial u}{\partial x}-i \frac{\partial v}{\partial x}\right)_{2} & =0 & & \text { for } x>0 \\
\left(\sigma_{y y}-i \sigma_{x y}\right) & =f(x) & & \text { for } x<0 .
\end{aligned}
$$

In terms of the jump potentials the first boundary condition is simply

$$
\Omega_{S 1}(x)-\Omega_{S 2}(x)=0 \text { for }|x|<\infty .
$$

Since $\Omega_{S}$ is analytic everywhere and bounded, by Liouville's theorem it must be constant. Moreover, for zero stress at infinity, $\Omega_{S}^{C}=0$.

The second boundary condition in terms of the jump potentials is

$$
\Omega_{D 1}(x)-\Omega_{D 2}(x)=0 \text { for } x>0 .
$$

The third boundary conditions in terms of the jump potentials is

$$
\begin{array}{ll}
Q_{1} \Omega_{D 1}\left(x^{+}\right)+Q_{2} \Omega_{D 2}\left(x^{-}\right)=f\left(x^{+}\right)=f(x) & \text { for } x<0, \\
Q_{2} \Omega_{D 2}\left(x^{-}\right)+Q_{1} \Omega_{D 1}\left(x^{+}\right)=f\left(x^{-}\right)=f(x) & \text { for } x<0 .
\end{array}
$$

or,

$$
\Omega_{D}^{+}+m \Omega_{D}^{-}(x)=\frac{1}{Q_{1}} f(x) \text { for } x<0, \text { where } m=\frac{Q_{2}}{Q_{1}}=\frac{1+\beta}{1-\beta} .
$$

These two last boundary conditions define a Hilbert problem with the solution

$$
\Omega_{D}(z)=\frac{\chi(z)}{2 \pi i} \int_{-\infty}^{0} \frac{1}{Q_{1}} \frac{f(x)}{\chi^{+}(x)(x-z)} d x+\chi(z) P(z)
$$

in which $\chi(z)=z^{\gamma-1}$ is the solution of the homogeneous Hilbert problem defined above,

$$
\gamma=\frac{1}{2}-\frac{i}{2 \pi} \log |m|=\frac{1}{2}+i \varepsilon, \quad \text { where } \quad \varepsilon=\frac{1}{2 \pi} \log \left|\frac{1}{m}\right|,
$$

and $P(z)$ is equal to zero [Suo 1989; Mukai et al. 1990]. After $\Omega_{D}$ is obtained, the jump potentials can be inverted back to standard potentials using Equations (A.6)-(A.9). To remove the stresses on the crack caused by the dislocation solution, the integration in Equation (A.17) is carried out with $f(x)$ opposite to the tractions due to a dislocation near the interface. These tractions are obtained by substituting the Equations (A.11), (A.12), (A.15) and (A.16) into Equation (A.1):

$$
\left(\sigma_{y y}-i \sigma_{x y}\right)_{c}=B\left(\frac{(1-\alpha)}{(1+\beta)\left(x-s_{0}\right)}+\frac{(1-\alpha)}{(1-\beta)\left(x-\bar{s}_{0}\right)}\right)+\bar{B}\left(\frac{(1-\alpha)\left(s_{0}-\bar{s}_{0}\right)}{(1-\beta)\left(x-\bar{s}_{0}\right)^{2}}\right) .
$$


So the jump potential is

$$
\Omega_{D}(z)=-\frac{\chi(z)}{2 \pi i Q_{1}} \int_{-\infty}^{0} \frac{\left(\sigma_{y y}-i \sigma_{x y}\right)_{c}}{\chi^{+}(x)(x-z)} d x
$$

and it can be obtained by following Suo's procedure [1989], giving

$$
\Omega_{D}^{C}(z)=-B \frac{(1-\alpha)(1-\beta)}{Q_{1}}\left(\frac{F\left(z, s_{0}\right)}{1+\beta}+\frac{F\left(z, \bar{s}_{0}\right)}{1-\beta}\right)-\bar{B} \frac{(1-\alpha)(1-\beta)}{Q_{1}}\left(\frac{\left(s_{0}-\bar{s}_{0}\right) G\left(z, \bar{s}_{0}\right)}{1-\beta}\right),
$$

where

$$
F(z, a)=\frac{1}{2(z-a)}\left(1-\frac{\chi(z)}{\chi(a)}\right) \quad \text { and } \quad G(z, a)=\frac{\partial F(z, a)}{\partial a} .
$$

Inverting these to standard potentials gives

$\Phi_{1}^{C}(z)=-B(1-\alpha)(1-\beta)\left[\frac{F\left(z, s_{0}\right)}{1+\beta}+\frac{F\left(z, \bar{s}_{0}\right)}{1-\beta}\right]-\bar{B}(1-\alpha)(1-\beta)\left[\frac{\left(s_{0}-\bar{s}_{0}\right) G\left(z, \bar{s}_{0}\right)}{1-\beta}\right]$
$\Phi_{2}^{C}(z)=-B(1-\alpha)(1+\beta)\left[\frac{F\left(z, s_{0}\right)}{1+\beta}+\frac{F\left(z, \bar{s}_{0}\right)}{1-\beta}\right]-\bar{B}(1-\alpha)(1+\beta)\left[\frac{\left(s_{0}-\bar{s}_{0}\right) G\left(z, \bar{s}_{0}\right)}{1-\beta}\right]$

$\Psi_{1}^{C}(z)=-B(1-\alpha)(1+\beta)\left[\frac{\left(\bar{s}_{0}-s_{0}\right) \bar{G}\left(z, \bar{s}_{0}\right)}{1-\beta}\right]-\bar{B}(1-\alpha)(1+\beta)\left[\frac{\bar{F}\left(z, s_{0}\right)}{1+\beta}+\frac{\bar{F}\left(z, \bar{s}_{0}\right)}{1-\beta}\right]-\Phi_{1}^{C}(z)-z \Phi_{1}^{C^{\prime}}(z)$

$\Psi_{2}^{C}(z)=-B(1-\alpha)(1-\beta)\left[\frac{\left(\bar{s}_{0}-s_{0}\right) \bar{G}\left(z, \bar{s}_{0}\right)}{1-\beta}\right]-\bar{B}(1-\alpha)(1-\beta)\left[\frac{\bar{F}\left(z, s_{0}\right)}{1+\beta}+\frac{\bar{F}\left(z, \bar{s}_{0}\right)}{1-\beta}\right]-\Phi_{2}^{C}(z)-z \Phi_{2}^{C^{\prime}}(z)$

The final potentials that solve the interaction between a discrete dislocation and an interface crack are

$$
\begin{aligned}
& \Phi=\Phi^{D}+\Phi^{C}=\Phi_{\text {singular }}^{D}+\Phi_{\text {continuation }}^{D}+\Phi_{C} \\
& \Psi=\Psi^{D}+\Psi^{C}=\Psi_{\text {singular }}^{D}+\Psi_{\text {continuation }}^{D}+\Psi^{C},
\end{aligned}
$$

where the dislocation $(D)$ potentials and the crack $(C)$ potentials are all defined above. Thus, the traction at $z$ on $\theta=-\omega$ can be written as

$$
\sigma_{\theta \theta}(t)+i \sigma_{r \theta}(t)=2 \bar{B} e^{-i \omega}(t-\eta)^{-1}+B H_{1}(t, \eta)+\bar{B} H_{2}(t, \eta)
$$

where

$$
\begin{aligned}
& H_{1}(t, \eta)=H_{10}(t, \eta)+H_{11}(t, \eta), \\
& H_{2}(t, \eta)=H_{20}(t, \eta)+H_{21}(t, \eta),
\end{aligned}
$$


and

$$
\begin{aligned}
& H_{10}=-\delta\left[\frac{1}{z-\bar{s}_{0}}+\frac{\left(\bar{s}_{0}-s_{0}\right)}{\left(\bar{z}-s_{0}\right)^{2}}+e^{-2 i \omega} \frac{\left(\bar{s}_{0}-s_{0}\right)}{\left(z-\bar{s}_{0}\right)^{2}}\right], \\
& H_{20}=-\delta\left[\frac{1}{\bar{z}-s_{0}}+\frac{\left(s_{0}-\bar{s}_{0}\right)}{\left(z-\bar{s}_{0}\right)^{2}}+e^{-2 i \omega} \frac{\left(s_{0}-\bar{s}_{0}\right)\left(z+\bar{s}_{0}-2 \bar{z}\right)}{\left(z-\bar{s}_{0}\right)^{3}}\right]-\frac{\lambda}{z-\bar{s}_{0}} e^{-2 i \omega}, \\
& H_{11}=-(1-\alpha)(1+\beta) L\left[\frac{F\left(z, s_{0}\right)}{1+\beta}+\frac{F\left(z, \bar{s}_{0}\right)}{1-\beta}, \frac{\left(s_{0}-\bar{s}_{0}\right) G\left(z, \bar{s}_{0}\right)}{1-\beta}\right], \\
& H_{21}=-(1-\alpha)(1+\beta) L\left[\frac{\left(s_{0}-\bar{s}_{0}\right) G\left(z, \bar{s}_{0}\right)}{1-\beta}, \frac{F\left(z, s_{0}\right)}{1+\beta}+\frac{F\left(z, \bar{s}_{0}\right)}{1-\beta}\right],
\end{aligned}
$$

where

$$
L(\phi(z), \varphi(z))=\phi(z)+\overline{\varphi(z)}+e^{-2 i \omega}\left[(\bar{z}-z) \phi^{\prime}(z)+\frac{1+\beta}{1-\beta} \bar{\varphi}(z)-\phi(z)\right] \quad \text { and } \begin{gathered}
\delta=\frac{\alpha-\beta}{1-\beta} \\
\lambda=\frac{\alpha+\beta}{1+\beta}
\end{gathered} .
$$

The functions $H_{10}$ and $H_{20}$ represent the effects of a dislocation below the interface where the material does not crack. The functions $H_{11}$ and $H_{21}$ are additional terms needed to satisfy the traction-free condition on the semiinfinite crack.

The traction expression is very similar to what had been reported by Hutchinson and Suo [1992]. Our expressions for $H_{10}$ and $H_{20}$ are the same as theirs, but our $H_{11}$ and $H_{21}$ are different, in that theirs are missing all terms related to $\left(s_{0}-\bar{s}_{0}\right) G\left(z, \bar{s}_{0}\right) /(1-\beta)$.

\section{Appendix B. Formula for asymptotic stresses of an interfacial crack}

When a crack branches into material 2, one can formulate $\sigma_{\theta \theta}^{0}(t)+i \sigma_{r \theta}^{0}(t)$ in terms of potential functions [Rice 1988; He and Hutchinson 1989] as

$$
\sigma_{\theta \theta}^{0}(t)+i \sigma_{r \theta}^{0}(t)=\phi_{0}^{\prime}(z)+\bar{\phi}_{0}^{\prime}(z)+e^{-2 i \omega}\left(\bar{z} \phi_{0}^{\prime \prime}(z)+\chi_{0}^{\prime}(z)\right),
$$

where

$$
\begin{aligned}
& \phi_{0}^{\prime}(z)=\frac{1}{2 \sqrt{2 \pi} \cosh (\pi \varepsilon)} e^{\varepsilon \pi} \bar{K} z^{-(1 / 2+i \varepsilon)}, \\
& \chi_{0}^{\prime}(z)=\frac{1}{2 \sqrt{2 \pi} \cosh (\pi \varepsilon)}\left(e^{-\varepsilon \pi} K z^{-1 / 2+i \varepsilon}-e^{\varepsilon \pi}\left(\frac{1}{2}-i \varepsilon\right) \bar{K} z^{-(1 / 2+i \varepsilon)}\right) .
\end{aligned}
$$

Sun and Qian [1996] demonstrated that this expression of the asymptotic stresses around an interfacial crack tip is appropriate for calculating the stress field in either the traditional linear fracture model or the contact model.

\section{References}

[Atkinson 1966] C. Atkinson, "The interaction between a dislocation and a crack”, Int. J. Fract. 2:4 (1966), $567-575$.

[Banks-Sills and Ashkenazi 2000] L. Banks-Sills and D. Ashkenazi, "A note on fracture criteria for interface fracture", Int. J. Fract. 103 (2000), 177-188. 
[Bilby and Cardew 1975] B. A. Bilby and G. E. Cardew, “The crack with a kinked tip", Int. J. Fract. 11 (1975), 708-712. [Comninou 1977] M. Comninou, "The interface crack”, J. Appl. Mech. (Trans. ASME) 44 (1977), 631-636.

[Comninou and Dundurs 1979a] M. Comninou and J. Dundurs, "An example for frictional slip progressing into a contact zone of a crack", Eng. Fract. Mech. 12 (1979), 191-197.

[Comninou and Dundurs 1979b] M. Comninou and J. Dundurs, "On the frictional contact in crack analysis”, Eng. Fract. Mech. 12 (1979), 117-123.

[Cotterell and Rice 1980] B. Cotterell and J. R. Rice, "Slightly curved or kinked cracks", Int. J. Fract. 16 (1980), 155-169.

[Erdogan and Gupta 1972] F. Erdogan and G. D. Gupta, "On the numerical solution of singular integral equation”, Quart. J. Appl. Math. 29 (1972), 525-534.

[Fan et al. 1998] H. Fan, Y. M. Sun, and Z. M. Xiao, "Contact zone in an interfacial zone Zener-Stroh crack”, Mech. Mater. 30 (1998), 151-159.

[Gautesen and Dundurs 1988] A. K. Gautesen and J. Dundurs, "The interface crack under combined loading”, J. Appl. Mech. 55 (1988), 580-586.

[Gilchrist and Svensson 1995] M. D. Gilchrist and N. Svensson, "A fractographic analysis of delamination within multidirectional carbon/epoxy laminates", Compos. Sci. Technol. 55:2 (1995), 195-207.

[Gilchrist et al. 1996] M. D. Gilchrist, A. J. Kinloch, and F. L. Matthews, "Mechanical performance of carbon-fibre and glassfiber-reinforced epoxy i-beam: ii. fractographic failure observation”, Compos. Sci. Technol. 56 (1996), 1031-1045.

[Hayashi and Nemat-Nasser 1981] K. Hayashi and S. Nemat-Nasser, "Energy-release rate and crack kinking under combined loading”, J. Appl. Mech. (Trans. ASME) 48 (1981), 520-524.

[He and Hutchinson 1989] Y. M. He and J. W. Hutchinson, "Kinking of a crack out of an interface", J. Appl. Mech. (Trans. ASME) 56 (1989), 270-278.

[Hills et al. 1996] D. A. Hills et al., Solution of crack problems: the distributed dislocation technique, edited by G. M. L. Gladwell, Solid mechanics and its application, Kluwer academic publishers, 1996.

[Hutchinson and Suo 1992] J. W. Hutchinson and Z. Suo, "Mixed mode cracking in layered materials", Adv. Appl Mech. 29 (1992), 63-187.

[Leblond and Frelat 2001] J. B. Leblond and J. Frelat, "Crack kinking from an interface crack with initial contact between the crack lips”, Eur. J. Mech. A: Solids 20 (2001), 937-951.

[Leblond and Frelat 2004] J. B. Leblond and J. Frelat, "Crack king from an initially closed, ordinary or interface crack, in the presence of friction", Eng. Fract. Mech. 71 (2004), 289-307.

[Li et al. 2004] J. Li, X. B. Zhang, and N. Recho, "J-Mp based criteria for bifurcation assessment of a crack in elastic-plastic materials under mixed mode I-II loading”, Eng. Fract. Mech. 71 (2004), 329-343.

[Lo 1978] K. K. Lo, “Analysis of branched Cracks”, J. Appl. Mech. (Trans. ASME) 45 (1978), 797-802.

[Mukai et al. 1990] D. J. Mukai, R. Ballarini, and G. R. Miller, “Analysis of branched interface cracks", J. Appl. Mech. (Trans. ASME) 57 (1990), 887-893.

[Mulville et al. 1978] D. Mulville, D. L. Hunston, and P. W. Mast, "Developing failure criteria for adhesive joints under complex loading”, J. Eng. Mater. Technol. (Trans. ASME) 100 (1978), 25-31.

[Muskhelishvili 1977] N. I. Muskhelishvili (editor), Some basic problems of the mathematical theory of elasticity, edited by N. I. Muskhelishvili, Noordhoff International Publishing, Lerden, Netherlands, 1977.

[Partridge and Singh 1995] I. K. Partridge and S. Singh, "Mixed-mode fracture in an interleaved carbon-fibre/epoxy composite", Compos. Sci. Technol. 55:4 (1995), 319-327.

[Pelegri and Chen 2000] A. A. Pelegri and I. Chen, "Mixed mode fatigue of fiber reinforced composites using a modified MMB fixture", pp. 1-8 in 41st AIAA/ASME/ASCE/AHS/ASC SDM (Structures), AIAA-2000-1404, Atlanta, GA, 2000.

[Rice 1988] J. R. Rice, "Elastic fracture concepts for interfacial cracks”, J. Appl. Mech. 55 (1988), 98-103.

[Rice et al. 1990] J. R. Rice, Z. Suo, and J. S. Wang, "Mechanics and thermodynamics of brittle interfacial failure in bimaterial systems”, pp. 269-294 in Metal-ceramic interface, edited by M. Ruhle et al., Pergamon Press, New York, 1990. 
[Sanford 1997a] R. J. Sanford (editor), Crack tip stress fields, edited by R. J. Sanford, Spie optical engineering press, Bellingham, Washington, 1997.

[Sanford 1997b] R. J. Sanford (editor), Foundations of linear elastic fracture mechanics, edited by R. J. Sanford, Spie optical engineering press, Bellingham, Washington, 1997.

[Shan and Pelegri 2003] B. X. Shan and A. A. Pelegri, "Assessment of the fracture behavior of an asymmetrically loaded cantilever composite structure”, J. Eng. Mater. Technol. (Trans. ASME) 125 (2003), 353-360.

[Sih 1977] G. C. Sih (editor), Mechanics of fracture, edited by G. C. Sih, Noordhoff International Publishing, Lerden, Netherlands, 1977.

[Sun and Jih 1987] C. Sun and C. Jih, "On strain energy release rates for interfacial cracks in bimaterial media", Eng. Fract. Mech. 28:1 (1987), 13-20.

[Sun and Qian 1996] C. T. Sun and W. Qian, "The use of finite extension strain energy release rates in fracture of interfacial cracks", Int. J. Solids Struct. 34 (1996), 2595-2609.

[Suo 1989] Z. Suo, "Singularities interacting with interfaces and cracks", Int. J. Solids Struct. 25:10 (1989), 1133-1142.

[Wang 1994] K. J. Wang, “Criteria for kinking out of interface crack”, Eng. Fract. Mech. 49 (1994), 587-598.

[Whitcomb 1981] J. D. Whitcomb, "Finite element analysis of instability related delamination growth", J. Compos. Mater. 15:5 (1981), 403-426.

[Xie et al. 2005] D. Xie, A. M. Waas, K. W. Shahwan, J. A. Schroeder, and R. G. Boeman, "Fracture. Criterion for kinking cracks in a tri-material adhesively bonded joint under mixed mode loading", Eng. Fract. Mech. 72:16 (2005), 2487-2504.

Received 26 Mar 2007. Accepted 30 Sep 2007.

BAOXIANG X. SHAN: bxshan@eden.rutgers.edu

Mechanical and Aerospace Engineering, School of Engineering, Rutgers, The State University of New Jersey, 98 Brett Road, Piscataway, NJ 08854-8058, United States

Assimina A. Pelegri: pelegri@jove.rutgers.edu

Mechanical and Aerospace Engineering, School of Engineering, Rutgers, The State University of New Jersey, 98 Brett Road, Piscataway, NJ 08854-8058, United States

YI PAN: yipan@eden.rutgers.edu

Mechanical and Aerospace Engineering, School of Engineering, Rutgers, The State University of New Jersey, 98 Brett Road, Piscataway, NJ 08854-8058, United States 


\title{
A VARIATIONAL DEDUCTION OF SECOND GRADIENT POROELASTICITY II: AN APPLICATION TO THE CONSOLIDATION PROBLEM
}

\author{
Angela Madeo, Francesco Dell'Isola, Nicoletta IANiro ANd Giulio SciarRa
}

\begin{abstract}
The second gradient model of poromechanics, introduced in Part I, is here linearized in the neighborhood of a prestressed reference configuration to be applied to the one-dimensional consolidation problem originally considered by Terzaghi and Biot. Second gradient models allow for the description of boundary layer effects both in the vicinity of the external surface and the impermeable wall.

The formulated differential problem involves linear pencils of ordinary differential operators on a finite interval, with boundary conditions depending on the spectral parameter. Taking into account the dependence of the differential problem on initial stresses a linear stability analysis is carried out. Finally, numerical solutions are compared with the corresponding classical Terzaghi solutions.
\end{abstract}

\section{Introduction}

This paper addresses a geotechnical application of the macroscopic second gradient poroelasticity theory presented in the first part; in particular we aim to treat the well known soil consolidation problem [Terzaghi 1943]. The consolidation of a soil layer of depth $L$ can be schematically described as follows:

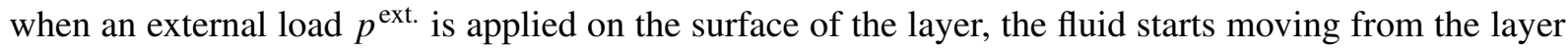
towards the surface, and it finally leaves the system. While the fluid keeps flowing, the external load is gradually distributed to the solid skeleton, which starts to deform.

Different theories which model consolidation have been developed in the literature [Biot 1941; Terzaghi 1943; Heinrich and Desoyer 1961], however the two due to Terzaghi and Biot are surely the most widespread ones. Actually, as it was noted also by de Boer [1996], the derivation of the Terzaghi differential equation [Terzaghi 1923] is obscure and essentially driven by the comparison between the phenomenon of soil consolidation and that of heat propagation, rather than from the statement of suitable mechanical principles. On the other hand Biot's theory seems to be well grounded from the mechanical point of view, even if directly restricted to the case of linear elasticity, in its earliest presentation [Biot 1941]. The two models collapse one into the other when considering one-dimensional problems; however, this is not the case when modeling, for instance, the behavior of a saturated porous slab [Mandel 1953] or a saturated porous sphere [Cryer 1963]. In both circumstances Biot's three-dimensional model provides time increasing values of the water pressure (and fluid mass density) at the center of the slab (or sphere) if the Lamé constant $\mu$ of the skeleton is different from zero; on the other hand the solution for $\mu=0$ coincides with the one derived from Terzaghi's three dimensional model. This localized pore-fluid segregation is known in the literature as the Mandel-Cryer effect.

Keywords: poromechanics, second gradient materials, consolidation. 
The occurrence of compaction localization phenomena has recently been discovered by Mollema and Antonellini [1996], who presented evidence of so called "compaction bands" in outcrops of the Jurassic Navajo sandstone in the Kaibab monocline, in Utah. These bands are characterized by volume loss due to microfracturing, but essentially no grain crushing or comminution. Later on, laboratory experiments have been developed [Olsson and Holcomb 2000; 2003] using triaxial compression tests to reproduce the formation of these bands. These tests proved that increasing axial stress $\sigma_{11}$ initially determines only homogeneous axial strain $\epsilon_{11}$, however, when a suitable stress threshold has been overwhelmed, tabular zones associated with nonhomogeneous strain can be detected close to the axial borders of the specimen. Nonuniform compaction also affects fluid flow in the porous material, being detrimental if permeability of the compacted material is much reduced with respect to the uncompacted zone.

The second gradient poromechanical model presented in Part I [Sciarra et al. 2008] is capable of describing fluid mass density boundary segregation even in the one dimensional model. Both in the vicinity of the external consolidating surface and the impermeable wall, suitable boundary layer effects can be predicted by the second gradient model. Formation of segregation bands enhances high gradients of density of the fluid entrapped in the pores of the solid skeleton. This can be explained by means of nonvanishing hyperstresses at the boundary (see [Sciarra et al. 2008, Equations (32) $\left.2-(32)_{3}\right]$ ); these last cause the pore pressure to differ from its reference initial value in a transient period, when dissipation does not yet dominate the evolution process.

Having in mind the classical Terzaghi's consolidation problem, whose space-time evolution is governed by the same equation as that of heat conduction, replacing temperature with pore-pressure, we claim that the second gradient model, presented in [Sciarra et al. 2008], is capable of regularizing the behavior of the Darcy flow inside the porous medium. Because of second gradient effects, the fluid mass density diffusion is smoothed. It is self evident that Terzaghi's theory does not model those phenomena occurring at the boundaries which oppose the fluid flow, for example, pore closure, solid-fluid capillarity, etc. The present model tries to macroscopically account for some of them and aims to establish the preliminary theoretical framework necessary for conceiving and designing any kind of experimental activity. In this paper it is shown that the overpressure occurring at the impermeable boundary actually depends on second gradient coefficients; therefore a more detailed analysis of these effects is recognized to be necessary.

Pore fluid segregation is probably the triggering effect for vertical drain or sand volcano formation, observed after liquefaction [Kolymbas 1998]; these last can indeed be interpreted as bifurcation modes of consolidation, which, in the case of the second gradient model should correspond to the boundary layer detected close to the impermeable wall becoming larger and larger. As it is not possible to identify this bifurcation mode in the case of linearized (small strain) theory, only the limit condition describing the stability/instability limit is detected. Further developments will be devoted to study of the onedimensional nonlinear problem.

From the mathematical point of view the present model, in which both second gradient conservative (relative to the behavior of the porous solid skeleton) and dissipative (relative to the flow of the saturating fluid) contributions are taken into account, implies that the newly formulated initial boundary value problem of consolidation fits in the framework of the theory of linear pencils of ordinary differential operators on a finite interval with boundary conditions depending on the spectral parameter. We refer 
to the general results presented, for example, in [Shkalikov 1986; Shkalikov and Tretter 1996; Marletta et al. 2003] for more details on this topic.

\section{Linearization of the one-dimensional differential problem}

We study here the aforementioned consolidation problem referring to the equations of motion for a second gradient porous medium as obtained in [Sciarra et al. 2008, Equations (30) and (31)], restricting our attention to the one-dimensional case. This will allow us to compare our results with the classical ones due to Terzaghi [1923]. Of course, Equations (30)-(31) can also be applied to treat three-dimensional problems, so extending classical Biot's equations.

Clearly, because of the one-dimensional hypothesis, all the gradient and divergence operations appearing in Equations (30) and (31) become simple derivatives with respect to the space variable $x$, and the deformation tensor $\varepsilon$ simply reduces to its only nonzero component $\varepsilon_{x x}$ along the $x$ axis. In the following we will indicate the component $\varepsilon_{x x}$ simply by $\varepsilon$.

From now on we assume the hypothesis of small deformations in the neighborhood of a suitable solid skeleton reference configuration. For the sake of simplicity we will therefore use the same notation as used in [Sciarra et al. 2008] for $\varepsilon$ and $m_{f}$ to indicate the corresponding incremental quantities with respect to the considered small deformation parameter.

In accordance with the aforementioned assumptions and the introduced nomenclature, the quadratic expression for the Hemholtz free energy density $\Psi$, in terms of the state parameters $\left(\varepsilon, m_{f}, \varepsilon^{I}, m_{f}^{I}\right)$ is adopted as,

$$
\begin{aligned}
\Psi=-p_{0}^{\text {ext. }} \varepsilon+\mu_{0}^{\text {ext. }} m_{f}+\frac{1}{2}(\lambda & \left.+2 \mu+b^{2} M\right) \varepsilon^{2}+\frac{1}{2} M\left(\frac{m_{f}}{m_{f}^{0}}\right)^{2} \\
& \quad b M \varepsilon \frac{m_{f}}{m_{f}^{0}}+\frac{1}{2}\left(K_{s s}+\mathbb{M} K_{s f}^{2}\right)\left(\varepsilon^{I}\right)^{2}+\mathbb{M} K_{s f} \frac{m_{f}^{I}}{m_{f}^{0}} \varepsilon^{I}+\frac{1}{2} \mathbb{M}\left(\frac{m_{f}^{I}}{m_{f}^{0}}\right)^{2},
\end{aligned}
$$

where $\varepsilon^{I}$ and $m_{f}^{I}$ indicate the first spatial derivatives of $\varepsilon$ and $m_{f}$, respectively.

The constant coefficients $p_{0}^{\text {ext. }}, \mu_{0}^{\text {ext. }}$, and $m_{f}^{0}$ account for the state of stress of the solid skeleton, the chemical potential of the fluid, and the initial apparent density of the fluid before any external perturbation is applied to the porous system. Moreover, $\lambda$ and $\mu$ are the classical Lamé coefficients, $b$ and $M$ the Biot parameters, and $\mathbb{M}, K_{s s}$, and $K_{s f}$ are the second gradient constitutive parameters.

The nonstandard energetic contributions associated with $\left(\varepsilon^{I}\right)^{2},\left(m_{f}^{I}\right)^{2}$, and $\varepsilon^{I} m_{f}^{I}$ are those responsible for the presence of hyperstresses in the balance equations of the overall material and the pure fluid. They allow for describing the compaction/dilatancy localization effects arising in the fluid-filled porous material when the fluid remains entrapped in the solid skeleton. In particular $K_{s s}$ and $\mathbb{M}$ provide nonvanishing hyperstress on the solid skeleton and the pure fluid if second gradient coupling is negligible. Following the interpretation of double forces given in [Sciarra et al. 2008, Section 2], these two constitutive parameters allow for describing internal actions working on the rate of dilatancy along the outward unit normal. The coupling coefficient $K_{s f}$ is labeled as the cocapillarity coefficient in analogy to standard second gradient theories for capillarity models [Seppecher 1987]. It describes second gradient solid-fluid interactions and can be assumed as vanishing in contrast with $K_{s s}$ and $\mathbb{M}$, which will be proved to be positive when positiveness of strain energy density is required (see Equation (2)). 
Values of the Lamé and Biot moduli can easily be recovered from the literature [Coussy 2004]; on the other hand no identification for the second gradient moduli is available up to now. It is not the purpose of this paper to set up a constitutive identification based on experiments or mathematical homogenization; conversely, our aim is that of exhibiting the capability of the model presented in [Sciarra et al. 2008] to catch compaction/dilatancy effects. Second gradient parameters therefore will be tuned so as to permit the one-dimensional model to show boundary layer effects for the solid strain and the fluid mass density in the vicinity of the external surfaces.

Requiring definite positiveness of the energy density $\Psi$ defined in Equation (1), the following conditions on the parameters must hold:

$$
\lambda+2 \mu>0, \quad M>0, \quad \text { and } \quad K_{s s}>0, \quad \mathbb{M}>0 .
$$

The first two conditions are well known in the framework of the classical Biot poromechanics; the second ones restrict the constitutive assumptions on the second gradient parameters.

2.1. Equations of motion. We will now deduce the linearized form of the equations of motion for the second gradient consolidation problem. In order to do so, it is worthwhile to recall that in the onedimensional, linearized problem the following chains of equalities hold:

$$
\mathbf{F}_{s} \simeq \mathbf{I}+\nabla_{s} \mathbf{u}=(1+\varepsilon) \mathbf{I},
$$

u being the infinitesimal solid displacement field,

$$
\mathbf{F}_{s}^{-1} \simeq(1-\varepsilon) \mathbf{I},
$$

and

$$
J_{s} \simeq \operatorname{det} \mathbf{F}_{s}=1+\operatorname{tr}\left(\nabla_{0} \mathbf{u}\right)=1+\varepsilon,
$$

where we recall that all the considered fields in the right hand side of (3)-(5) have to be regarded as incremental quantities with respect to the small deformation parameter. Taking into account (1) for the strain energy $\Psi$ and the one-dimensional form of (3) and (5), the linearized governing equations given in [Sciarra et al. 2008, Equations (30) and (31)], reduce to

$$
\left[\left(-p_{0}^{\text {ext. }}+\lambda+2 \mu+b^{2} M\right) \varepsilon-b M \frac{m_{f}}{m_{f}^{0}}-\left(K_{s s}+\mathbb{M} K_{s f}^{2}\right) \varepsilon^{I I}-\mathbb{M} K_{s f} \frac{m_{f}^{I I}}{m_{f}^{0}}\right]^{I}=0,
$$

and

$$
-m_{f}^{0}\left[M \frac{m_{f}^{I}}{\left(m_{f}^{0}\right)^{2}}-\frac{b M}{m_{f}^{0}} \varepsilon^{I}-\frac{M K_{s f}}{m_{f}^{0}} \varepsilon^{I I I}-\frac{M}{\left(m_{f}^{0}\right)^{2}} m_{f}^{I I I}\right]-D\left(v_{f}-v_{s}\right)+\left[\alpha\left(v_{f}-v_{s}\right)^{I}\right]^{I}=0,
$$

for the solid skeleton and the pure fluid, respectively. We have denoted by $D$ and $\alpha$ the only nonzero component of the Darcy and Darcy-like tensors $\mathbb{D}$ and $\mathbb{A}$, respectively (see [Sciarra et al. 2008, Equation (25)]), and by $v_{f}-v_{s}$ the vertical component of the relative velocity. In the absence of inertia forces the solid momentum conservation law, Equation (6), can be integrated in the form

$$
\left(\lambda+2 \mu+b^{2} M-p_{0}^{\text {ext. }}\right) \varepsilon-b M \frac{m_{f}}{m_{f}^{0}}-\left(K_{s s}+\mathbb{M} K_{s f}^{2}\right) \varepsilon^{I I}-\mathbb{M} K_{s f} \frac{m_{f}^{I I}}{m_{f}^{0}}=\text { const. }:=c_{0} .
$$


Moreover, considering that $v_{f}-v_{s}$ is related to the apparent fluid density $m_{f}$ by means of the linearized continuity equation $\dot{m}_{f}+m_{f}^{0}\left(v_{f}-v_{s}\right)^{I}=0$, (7) can be rewritten, performing a derivative with respect to the space variable $x$, as

$$
-m_{f}^{0}\left[M \frac{m_{f}^{I I}}{\left(m_{f}^{0}\right)^{2}}-\frac{b M}{m_{f}^{0}} \varepsilon^{I I}-\frac{\mathbb{M} K_{s f}}{m_{f}^{0}} \varepsilon^{I V}-\frac{\mathbb{M}}{\left(m_{f}^{0}\right)^{2}} m_{f}^{I V}\right]+\frac{D}{m_{f}^{0}} \dot{m}_{f}-\frac{\alpha}{m_{f}^{0}} \dot{m}_{f}^{I I}=0,
$$

where we have indicated by $\dot{m}_{f}$ the time derivative of $m_{f}$.

In order to write the linearized governing equations in a dimensionless form, the following quantities are introduced:

$$
\xi=\frac{x}{L}, \quad \tilde{m}_{f}=\frac{m_{f}}{m_{f}^{0}}, \quad \tilde{t}=\frac{t}{\tau}, \quad \text { with } \quad \tau=\frac{D L^{2}}{M},
$$

where $L$ is the depth of the solid layer.

According to these definitions, (8) and (9) can be rewritten in their dimensionless form as

$$
\frac{\left(\lambda+2 \mu+b^{2} M-p_{0}^{\text {ext. }}\right)}{\lambda+2 \mu} \varepsilon-\frac{b M}{\lambda+2 \mu} \tilde{m}_{f}-\frac{\left(K_{s s}+\mathbb{M} K_{s f}^{2}\right)}{(\lambda+2 \mu) L^{2}} \varepsilon^{I I}-\frac{\mathbb{M} K_{s f}}{(\lambda+2 \mu) L^{2}} \tilde{m}_{f}^{I I}=\frac{c_{0}}{\lambda+2 \mu},
$$

and

$$
\frac{\mathbb{M}}{M L^{2}} \tilde{m}_{f}^{I V}+\frac{\mathbb{M}}{M L^{2}} K_{s f} \varepsilon^{I V}-\tilde{m}_{f}^{I I}+b \varepsilon^{I I}-\frac{\alpha}{D L^{2}} \dot{\tilde{m}}_{f}^{I I}+\dot{\tilde{m}}_{f}=0,
$$

which represent the linearized equations of motion for the consolidation problem. For the sake of simplicity, we will no longer distinguish between $m_{f}$ and $\tilde{m}_{f}$, and, if not specified, $m_{f}$ will indicate the dimensionless quantity. Moreover, the dimensionless variables $\xi$ and $\tilde{t}$ will be also indicated by $x$ and $t$ if no confusion can arise.

2.2. Boundary conditions. The constant $c_{0}$ is deduced from the boundary condition (BC) in $x=0$ given in [Sciarra et al. 2008, Equation (32) $]$ which, in the linearized form, reads

$$
\frac{\left(\lambda+2 \mu+b^{2} M-p_{0}^{\text {ext. }}\right)}{\lambda+2 \mu} \varepsilon-\frac{b M}{\lambda+2 \mu} m_{f}-\frac{\left(K_{s s}+\mathbb{M} K_{s f}^{2}\right)}{(\lambda+2 \mu) L^{2}} \varepsilon^{I I}-\frac{\mathbb{M} K_{s f}}{(\lambda+2 \mu) L^{2}} m_{f}^{I I}=-\frac{\Delta p^{\text {ext. }}}{(\lambda+2 \mu)} ;
$$

here $\Delta p^{\text {ext. }}$ represents the incremental external pressure acting on the system, deriving from the lineariza-

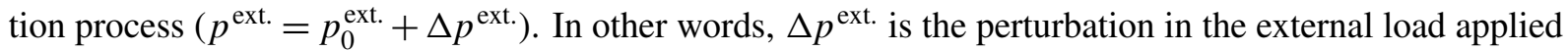
on the surface of the soil layer. Comparing this $\mathrm{BC}$ with (10) it is easy to recognize that $c_{0}=-\Delta p^{\text {ext. }}$.

Equations (10) and (11) represent a differential system of the sixth order in the space variable $x$ and of the first order in time, the integration of which requires therefore six boundary conditions and one initial condition. In classical poromechanics the Terzaghi consolidation problem does not take into account second gradient effects, and indeed it can be obtained from (10) and (11) when the second gradient parameters $\mathbb{M}, K_{s s}, K_{s f}$, and $\alpha$ are vanishing. Clearly, the problem reduces in this case to a second order system with respect to the space variable $x$.

The boundary conditions for the consolidation problem are derived from the general ones deduced in [Sciarra et al. 2008, Equation (32)]. In particular, since the given problem is one-dimensional, surface 
divergence and surface gradient operations do not contribute to the BCs; moreover no edge $\mathfrak{E}_{k}$ of the boundary exists. Extending the classical BCs stated in the Terzaghi consolidation problem we assume:

- Zero fluid traction in $x=0$. This BC states that the surface of the solid layer is kept drained, meaning that the fluid reaching the surface is continuously removed from the surface itself. This BC corresponds to the one given in [Sciarra et al. 2008, Equation (32) $)_{1}$ which, in its linearized, dimensionless form, reduces to

$$
m_{f}-b \varepsilon-\frac{\mathbb{M} K_{s f}}{M L^{2}} \varepsilon^{I I}-\frac{\mathbb{M}}{M L^{2}} m_{f}^{I I}+\frac{\alpha}{D L^{2}} \dot{m}_{f}=\frac{m_{f}^{0} \Delta \mu^{\text {ext. }}}{M}=0
$$

where $\Delta \mu^{\text {ext. }}$ represents the incremental chemical potential. In other words we have assumed the linearization $\mu^{\text {ext. }}=\mu_{0}^{\text {ext. }}+\Delta \mu^{\text {ext. }}$. Assuming that the fluid is continuously removed from the surface of the layer, this implies a restriction to the case $\Delta \mu^{\text {ext. }}=0$.

- Impermeable soil in $x=L$. With this BC we assume that the relative velocity is equal to zero in $x=L$, implying $v_{f}-v_{s}=0$. Using Equation (7), which holds everywhere in the interval $[0, L]$, the impermeability of the layer $x=L$ can be rewritten in its dimensionless form as

$$
-m_{f}^{I}+b \varepsilon^{I}+\frac{\mathbb{M} K_{s f}}{M L^{2}} \varepsilon^{I I I}+\frac{\mathbb{M}}{M L^{2}} m_{f}^{I I I}-\frac{\alpha}{D L^{2}} \dot{m}_{f}^{I}=0 .
$$

- Zero double force for the overall system in $x=0$ and $x=L$. These BCs are those ones given in [Sciarra et al. 2008, Equation (32) 2 , and can be rewritten in their linearized dimensionless form as

$$
m_{f}^{I}+\frac{\left(K_{s s}+\mathbb{M} K_{s f}^{2}\right)}{\mathbb{M} K_{s f}} \varepsilon^{I}=0
$$

We remind that the overall double forces are the contact forces introduced in the second gradient model, which work on the rate of pore opening/pore shrinkage. With the assumption of vanishing double forces on the boundary of the porous material, we claim that no external source of double force exists; internal double forces, on the contrary, allow for capturing the effects of pressure gradient concentration in the neighborhood of the external and impermeable surfaces [Holcomb and Olsson 2003].

- Zero fluid double force in $x=0$ and in $x=L$. These BCs are those ones given in [Sciarra et al. 2008, Equation $(32)_{3}$ ]. They can be rewritten in their linearized dimensionless form as

$$
m_{f}^{I}+K_{s f} \varepsilon^{I}=0
$$

The assumption on fluid double forces can be interpreted similarly to that considered for the overall double forces. In this case no external double forces are exerted on the fluid boundary, but internal double forces, associated with pressure gradient concentration, account for internal capillarity and wetting phenomena. 


\section{Initial boundary value problem}

The differential Equations (10) and (11) can be reduced to a unique differential equation introducing an auxiliary function $V(x, t)$ which satisfies the relationships

$$
\varepsilon=\frac{K_{s f} \mathbb{M}}{(\lambda+2 \mu) L^{2}} V^{I I}+\frac{b M}{(\lambda+2 \mu)} V,
$$

and

$$
m_{f}=\frac{\left(\lambda+2 \mu+b^{2} M-p_{0}^{\text {ext. }}\right)}{(\lambda+2 \mu)} V-\frac{K_{s s}+\mathbb{M} K_{s f}^{2}}{(\lambda+2 \mu) L^{2}} V^{I I}+\frac{\Delta p^{\text {ext. }}}{b M} .
$$

In such a way, Equation (10) is identically satisfied, while (11) can be rewritten, after some straightforward calculations, as

$$
C_{1} V^{V I}-C_{2}\left(p_{0}^{\text {ext. }}\right) V^{I V}-C_{3} \dot{V}^{I V}+C_{4}\left(p_{0}^{\text {ext. }}\right) V^{I I}+C_{5}\left(p_{0}^{\text {ext. }}\right) \dot{V}^{I I}-C_{6}\left(p_{0}^{\text {ext. }}\right) \dot{V}=0 ;
$$

on the other hand, the boundary conditions given in (12)-(14) read

$$
\begin{gathered}
\mathbb{C}_{1} V^{I V}-\mathbb{C}_{2}\left(p_{0}^{\text {ext. }}\right) V^{I I}-\mathbb{C}_{3} \dot{V}^{I I}+\mathbb{C}_{4}\left(p_{0}^{\text {ext. }}\right) V+\mathbb{C}_{5}\left(p_{0}^{\text {ext. }}\right) \dot{V}+\mathbb{C}_{6}=0 \quad \text { at } x=0, \\
V^{I}=0 \quad \text { at } x=0, L, \quad V^{I I I}=0 \quad \text { at } x=0, L, \quad V^{V}=0 \quad \text { at } x=L .
\end{gathered}
$$

Finally, the initial condition (corresponding to the instant in which the external load is applied) is deduced assuming that the apparent Lagrangian fluid density is vanishing for $t=0^{+}$. For instance, $m_{f}\left(x, t=0^{+}\right)=0$; this initial condition states, similarly to in classical consolidation, that, at the instant in which the external load is applied there is no instantaneous variation of the fluid density $m_{f}$ inside the soil layer. In terms of the auxiliary function $V$ the initial datum reads as

$$
V\left(x, 0^{+}\right):=V_{\text {in }}=-\frac{\Delta p^{\text {ext. }}}{b M} \frac{1}{C_{4}\left(\pi_{0}\right)+k_{6}},
$$

where (16) with $m_{f}=0$ has been solved using BCs given in (19).

All the coefficients appearing in the governing equation, (17), as well as in the initial and boundary conditions, (20) and (19), depend on the constitutive parameters, the solid initial stress $p_{0}^{\text {ext. }}$, and the increment of the external force $\Delta p^{\text {ext. }}$; their expressions are listed in Appendix A.

It must be remarked that the expression for the energy density $\Psi$ assumed in (1) would not allow the linearized differential problem to explicitly depend on the initial solid stress $p_{0}^{\text {ext. }}$ and on the initial chemical potential $\mu_{0}^{\text {ext. }}$. In fact, considering (1) we can write, in dimensionless form,

$$
\frac{\partial \Psi}{\partial \varepsilon}=\frac{1}{\lambda+2 \mu} p_{0}^{\text {ext. }}+\left(1+k_{6}\right) \varepsilon-b k_{5} m_{f}, \quad \frac{\partial \Psi}{\partial m_{f}}=\frac{m_{f}^{0}}{\lambda+2 \mu} \mu_{0}^{\text {ext. }}+k_{5} m_{f}-b k_{5} \varepsilon,
$$

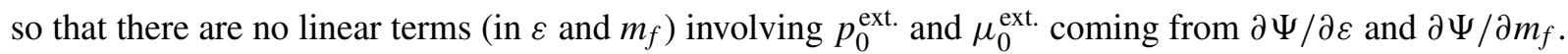
On the other hand the dependence of the differential system (17)-(19) on $p_{0}^{\text {ext. }}$ is due to so called geometrical nonlinearities; as matter of fact it is the presence of $\mathbf{F}_{s}$ in the balance of the total momentum (see [Sciarra et al. 2008]) which even in linearized problems implies a nontrivial dependence of the governing

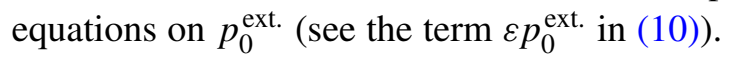


Considering the linearity of the differential problem and the nonhomogeneity appearing in the $\mathrm{BC}$, given in Equation (18), we will look for a solution $V(x, t)$ in the form

$$
V(x, t)=\bar{V}(x)+W(x, t),
$$

where $\bar{V}(x)$ is the solution of the stationary problem

$$
C_{1} \bar{V}^{V I}-C_{2} \bar{V}^{I V}+C_{4} \bar{V}^{I I}=0
$$

with nonhomogeneous $\mathrm{BCs}$

$$
\begin{gathered}
\mathbb{C}_{1} \bar{V}^{I V}-\mathbb{C}_{2} \bar{V}^{I I}+\mathbb{C}_{4} \bar{V}=-\mathbb{C}_{6} \quad \text { at } x=0, \\
\bar{V}^{I}=0 \quad \text { at } x=0, L, \quad \bar{V}^{I I I}=0 \quad \text { at } x=0, L, \quad \bar{V}^{V}=0 \quad \text { at } x=L,
\end{gathered}
$$

while the deviation $W(x, t)$ is the solution of the initial boundary value problem (IBVP)

$$
C_{1} W^{V I}-C_{2} W^{I V}-C_{3} \dot{W}^{I V}+C_{4} W^{I I}+C_{5} \dot{W}^{I I}-C_{6} \dot{W}=0,
$$

with homogeneous BCs

$$
\begin{gathered}
\mathbb{C}_{1} W^{I V}-\mathbb{C}_{2} W^{I I}-\mathbb{C}_{3} \dot{W}^{I I}+\mathbb{C}_{4} W+\mathbb{C}_{5} \dot{W}=0 \quad \text { at } x=0, \\
W^{I}=0 \quad \text { at } x=0, L, \quad W^{I I I}=0 \quad \text { at } x=0, L, \quad W^{V}=0 \quad \text { at } x=L,
\end{gathered}
$$

and nontrivial initial condition

$$
W\left(x, 0^{+}\right):=W_{i n}=V_{i n}-\bar{V}(x) .
$$

For the sake of simplicity we will no longer specify the dependence of the coefficients $C_{i}$ and $\mathbb{C}_{i}$ on the prestress parameter $p_{0}^{\text {ext. }}$.

It is easy to prove that the solution of the stationary problem given by (23)-(25) when $\mathbb{C}_{4} \neq 0$ is given by

$$
\bar{V}(x)=-\frac{\mathbb{C}_{6}}{\mathbb{C}_{4}}, \quad \mathbb{C}_{4} \neq 0,
$$

while if $\mathbb{C}_{4}=0$ the stationary solution $\bar{V}(x)$ exists if and only if $\mathbb{C}_{6}=0$; in this case a family of constant solutions of the stationary problem arises, so that we can write $\bar{V}(x)=K$, and $\mathbb{C}_{4}=\mathbb{C}_{6}=0$, where $\mathrm{K}$ is an undetermined constant. On the basis of the preliminary study of the stationary solution $\bar{V}(x)$ we can state that, according to the assumption (22), a solution of the given problem for the variable $V$ exists if and only if $\mathbb{C}_{4} \neq 0$ or $\mathbb{C}_{4}=\mathbb{C}_{6}=0$. We will now restrict our attention to the case $\mathbb{C}_{4} \neq 0$ and will analyze the case $\mathbb{C}_{4}=0$ later.

\section{Fourier series solution}

The initial boundary value problem given by (26)-(29) is solved using the method of separation of variables; in other words we assume that $W(x, t)=X(x) T(t)$. A straightforward calculation yields to the definition of the real eigenparameter $\lambda$ as $\lambda=\dot{T} / T$, which leads to $T(t)=T_{0} e^{\lambda t}$. Consequently, the function $X(x)$ must satisfy the eigenvalue problem

$$
C_{1} X^{V I}-C_{2} X^{I V}+C_{4} X^{I I}=\lambda\left(C_{3} X^{I V}-C_{5} X^{I I}+C_{6} X\right),
$$


endowed with the BCs

$$
\begin{gathered}
\mathbb{C}_{1} X^{I V}-\mathbb{C}_{2} X^{I I}+\mathbb{C}_{4} X=\lambda\left(\mathbb{C}_{3} X^{I I}-\mathbb{C}_{5} X\right), \quad \text { at } x=0, \\
X^{I}=0 \quad \text { at } x=0, L, \quad X^{I I I}=0 \quad \text { at } x=0, L, \quad X^{V}=0 \quad \text { at } x=L .
\end{gathered}
$$

This is a nonclassical spectral problem since the BCs also depend on the spectral parameter $\lambda$; in the literature this kind of spectral problem is referred to as a linear pencil $\mathbb{L}(X)=\lambda \mathbb{A}(X)$. Many authors investigate the spectral properties of the differential operators $\mathbb{L}$ and $\mathbb{A}$ in suitable function spaces in order to guarantee completeness and orthonormality for the eigenfunction system and discreteness of the spectrum [Shkalikov 1986; Shkalikov and Tretter 1996; Marletta et al. 2003]. Here we rely on these general results and numerically determine a subset of the eigenfunction space so as to approach the requirements of the Parseval equality [Kolmogorov and Fomin 1975].

According to the aforementioned properties of the eigensystem, the solution of the considered IBVP can be given in Fourier series form as

$$
W(x, t)=\sum_{k=0}^{+\infty} p_{k} X_{k}(x) e^{\lambda_{k} t}
$$

where $p_{k}$ denotes the $k$-th Fourier coefficient, and, in particular, $p_{0}$ the Fourier coefficient relative to the null eigenvalue $\lambda=0$ (if any). It is easy to prove that if $\mathbb{C}_{4} \neq 0$ the eigenfunction $X_{0}$ relative to the null eigenvalue is the trivial one $X_{0}=0$, so that in Equation (34) $k$ runs now from one to infinity.

The eigenfunctions $\left(X_{k}\right)_{k \in \mathbb{N}}$ are orthogonal with respect to the following bilinear form defined, in the Hilbert space $H^{3}([0, L]) \times H^{3}([0, L])$, as

$$
\left\langle X_{k}, X_{h}\right\rangle:=\alpha_{0} \int_{0}^{L} X_{k} X_{h} d x+\alpha_{1} \int_{0}^{L} X_{k}^{I} X_{h}^{I} d x+\alpha_{2} \int_{0}^{L} X_{k}^{I I} X_{h}^{I I} d x+\alpha_{3} \int_{0}^{L} X_{k}^{I I I} X_{h}^{I I I} d x,
$$

where the coefficients $\alpha_{i}$ are defined as

$$
\begin{array}{ll}
\alpha_{0}=\mathbb{C}_{4} C_{6}, & \alpha_{1}=\mathbb{C}_{4} C_{5}-C_{4} \mathbb{C}_{5}+\mathbb{C}_{2} C_{6}, \\
\alpha_{2}=\mathbb{C}_{4} C_{3}-\mathbb{C}_{3} C_{4}+\mathbb{C}_{2} C_{5}-C_{2} \mathbb{C}_{5}+\mathbb{C}_{1} C_{6}, & \alpha_{3}=\mathbb{C}_{2} C_{3}-C_{2} \mathbb{C}_{3}+\mathbb{C}_{1} C_{5}-C_{1} \mathbb{C}_{5} .
\end{array}
$$

It must be noted that expression (35) is indeed an inner product over the aforementioned function space if and only if all the coefficients $\alpha_{i}$ are positive definite.

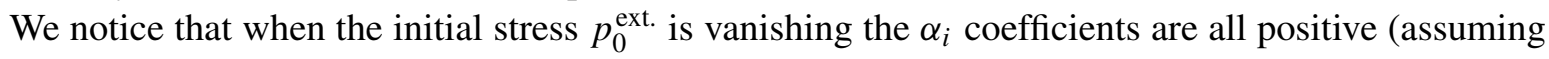
positiveness of the energy $\Psi$, see (2)), so that (35) always represents an inner product. On the other hand, it is easy to verify that in the presence of prestress the positiveness of the aforementioned coefficients $\alpha_{i}$ is guaranteed if and only if $p_{0}^{\text {ext. }}<\lambda+2 \mu\left(\Leftrightarrow \mathbb{C}_{4}>0\right)$.

The explicit form of the Fourier coefficients $p_{k}$ is determined by projecting the initial datum (29) on the $k$-th element of the Fourier series according to the inner product (35); in particular we can find

$$
\begin{aligned}
& \left\langle W_{i n}, X_{k}\right\rangle=\alpha_{0} W_{i n} \int_{0}^{L} X_{k} d x \\
& \left\langle W_{i n}, X_{k}\right\rangle=\left\langle\sum_{h=1}^{+\infty} p_{h} X_{h}, X_{k}\right\rangle=\sum_{h=1}^{+\infty} p_{h}\left\langle X_{h}, X_{k}\right\rangle=p_{k}\left\|X_{k}\right\|^{2},
\end{aligned}
$$


where we have noted by $\|\cdot\|=\langle\cdot, \cdot\rangle^{1 / 2}$ the norm induced by the inner product $\langle\cdot, \cdot\rangle$. Comparing Equation (36) with (37) it is easy to recognize that

$$
p_{k}=\frac{\alpha_{0} W_{i n} \int_{0}^{L} X_{k} d x}{\left\|X_{k}\right\|^{2}}
$$

so that, recalling (34), the final form of the solution is

$$
W(x, t)=\alpha_{0} W_{i n} \sum_{k=1}^{+\infty}\left[\frac{1}{\left\|X_{k}\right\|^{2}} \int_{0}^{L} X_{k}(\xi) d \xi\right] X_{k}(x) e^{\lambda_{k} t},
$$

and, according to (22) and (30), the solution for the variable $V(x, t)$ is finally given by

$$
V(x, t)=-\frac{\mathbb{C}_{6}}{\mathbb{C}_{4}}+\alpha_{0} W_{i n} \sum_{k=1}^{+\infty}\left[\frac{1}{\left\|X_{k}\right\|^{2}} \int_{0}^{L} X_{k}(\xi) d \xi\right] X_{k}(x) e^{\lambda_{k} t} .
$$

Finally, the fields $\varepsilon$ and $m_{f}$ can be evaluated using (38) with (15) and (16) respectively.

4.1. The limit case $\mathbb{C}_{\mathbf{4}}=\mathbf{0}$. We have already mentioned that when $\mathbb{C}_{4}=0\left(p_{0}^{\text {ext. }}=\lambda+2 \mu\right)$ the stationary solution $\bar{V}(x)$ exists if and only if $\mathbb{C}_{6}=0\left(\Leftrightarrow \Delta p^{\text {ext. }}=0\right)$, and it is an undetermined constant $K$. This means that, corresponding to a critical value of the prestress $p_{0}^{\text {ext. }}$, no solution can be found if perturbing the porous system with an external load $\Delta p^{\text {ext. }}$. The only possible solution is relative to the unloaded configuration of the porous system $\left(\Delta p^{\text {ext. }}=0\right)$. In this case the solution for $V(x, t)$ is found by solving the differential problem given by (17), (19), and (20) when $\mathbb{C}_{4}=\mathbb{C}_{6}=0$. Separating the variables, $V(x, t)=X(x) T(t)$, the solution can be found in Fourier series form as

$$
V(x, t)=\sum_{k=0}^{+\infty} p_{k} X_{k}(x) e^{\lambda_{k} t}
$$

It must be noticed that when $\mathbb{C}_{4}=0$ the inner product (35) reduces to

$$
\left\langle X_{k}, X_{h}\right\rangle_{\mathbb{C}_{4}=0}:=\alpha_{1} \int_{0}^{L} X_{k}^{I} X_{h}^{I} d x+\alpha_{2} \int_{0}^{L} X_{k}^{I I} X_{h}^{I I} d x+\alpha_{3} \int_{0}^{L} X_{k}^{I I I} X_{h}^{I I I} d x
$$

and it is still well defined over the quotient space of the $H^{3}([0, L])$ functions, differing at most by a constant. It follows that the Fourier coefficients $p_{k}$ are now determined on the basis of the reduced form $\langle\cdot, \cdot\rangle_{\mathbb{C}_{4}=0}$ of the inner product, according to the identities involving the initial condition $V_{i n}=$ constant,

$$
\begin{aligned}
& 0=\left\langle V_{i n}, X_{0}\right\rangle_{\mathbb{C}_{4}=0}=\left\langle p_{0} X_{0}+\sum_{k=1}^{+\infty} p_{k} X_{k}(x), X_{0}\right\rangle_{\mathbb{C}_{4}=0}=p_{0}\left\langle X_{0}, X_{0}\right\rangle_{\mathbb{C}_{4}=0}, \\
& 0=\left\langle V_{i n}, X_{k}\right\rangle_{\mathbb{C}_{4}=0}=\left\langle p_{0} X_{0}+\sum_{k=1}^{+\infty} p_{h} X_{h}(x), X_{k}\right\rangle_{\mathbb{C}_{4}=0}=p_{k}\left\|X_{k}\right\|_{\mathbb{C}_{4}=0}^{2}, \quad \text { for all } k \in \mathbb{N},
\end{aligned}
$$

where we have noted by $\|\cdot\|_{\mathbb{C}_{4}=0}=\langle\cdot, \cdot\rangle_{\mathbb{C}_{4}=0}^{1 / 2}$ the norm induced by the inner product $\langle\cdot, \cdot\rangle_{\mathbb{C}_{4}=0}$. Notice that $p_{0}$ and $X_{0}=\bar{V}=K$ are the Fourier coefficient and the constant eigenfunction corresponding to the 
null eigenvalue $\lambda_{0}=0$, respectively, while $\left(X_{k}\right)_{k \in \mathbb{N}}$ are the remaining eigenfunctions. Since $X_{0}=$ constant, Equation (40) reads $p_{0} 0=0 \Longrightarrow p_{0}$ undetermined, moreover, (41) gives $p_{k}\left\|X_{k}\right\|_{\mathbb{C}_{4}=0}^{2}=$ $0 \Longrightarrow p_{k}=0, \forall k \in \mathbb{N}$. According to (39), the solution for $V(x, t)$ is an undetermined constant, so $V(x, t)=p_{0} X_{0}:=p_{0} K=$ constant.

We want to remark that all the Fourier coefficients $p_{k}$ corresponding to nonvanishing eigenvalues turn to be zero only because the initial condition $V_{i n}$ has been assumed to be constant; if it was not the case, (41) would have stated the expression for the coefficients $p_{k}$, and the solution for $V(x, t)$ would have been known except for a constant $K$. The fact that a family of constant solutions for $V(x, t)$ arises can be seen as a sort of bifurcation phenomenon which is triggered when $p_{0}^{\text {ext. }}$ reaches the critical value $\lambda+2 \mu$.

Finally, we underline that the null eigenvalue $\lambda_{0}=0$ belongs to the spectrum of the differential problem only when $\mathbb{C}_{4}=0$; in the following section we will show that when $\mathbb{C}_{4}>0$ only negative eigenvalues exist, while if $\mathbb{C}_{4}<0$ some positive eigenvalues appear.

\section{Numerical results}

In this section we will show the numerical solution of the differential problem, given by (17)-(20), for a particular set of values of the constitutive parameters, which are listed in Table 1. The first gradient parameters are those relative to a water saturated clay, while the values of the second gradient dimensionless numbers are chosen in order to let boundary layer effects arise.

Fixing suitable values for the initial external pressure $\left(p_{0}^{\text {ext. }}=4.9 \mathrm{GPa}\right)$ and for the increment of this latter $\left(\Delta p^{\text {ext. }}=1 \mathrm{MPa}\right)$, so as to guarantee $\mathbb{C}_{4}>0$ and $\mathbb{C}_{6} \neq 0$, we look for a numerical solution $V(x, t)$ given by (38). In particular, we look for a numerical solution $X(x)$ of the differential problem, given by (31)-(33), in the form

$$
X(x)=\sum_{i=1}^{6} K_{i} e^{\beta_{i}(\lambda) x}
$$

where $K_{i}$ are the integration constants and $\beta_{i}(\lambda)$ are the solutions of the characteristic polynomial associated with the differential equation, (31). Consequently, BCs given by (32)-(33) yield

$$
\mathbf{A}(\lambda) \mathbf{v}=0,
$$

where $\mathbf{A}(\lambda)$ is a suitably defined $6 \times 6$ matrix and $\mathbf{v}:=\left(K_{1}, \ldots, K_{6}\right)$. We notice that the matrix $\mathbf{A}(\lambda)$ depends on the eigenparameter $\lambda$ both because it appears in the differential equation, (31), and in the boundary condition, (32). It follows that the resulting eigenvalue problem cannot be classified as a standard eigenvalue problem. The system of algebraic equations, (43), has a nontrivial solution if and only if $\operatorname{det}[\mathbf{A}(\lambda)]=0$, which leads to the calculation of the eigenvalues $\lambda_{k}$ (discrete spectrum). For

\begin{tabular}{|ccccccc|}
\hline$M(G P a)$ & $\lambda(G P a)$ & $\mu(G P a)$ & $k_{1}$ & $k_{2}$ & $k_{3}$ & $k_{4}$ \\
5 & 2.3 & 1.5 & $10^{-2}$ & $10^{-2}$ & $10^{-2}$ & $10^{-2}$ \\
\hline
\end{tabular}

Table 1. The values of first gradient elasticity parameters relative to a normally consolidated water saturated clay, together with trial values of second gradient dimensionless parameters. 


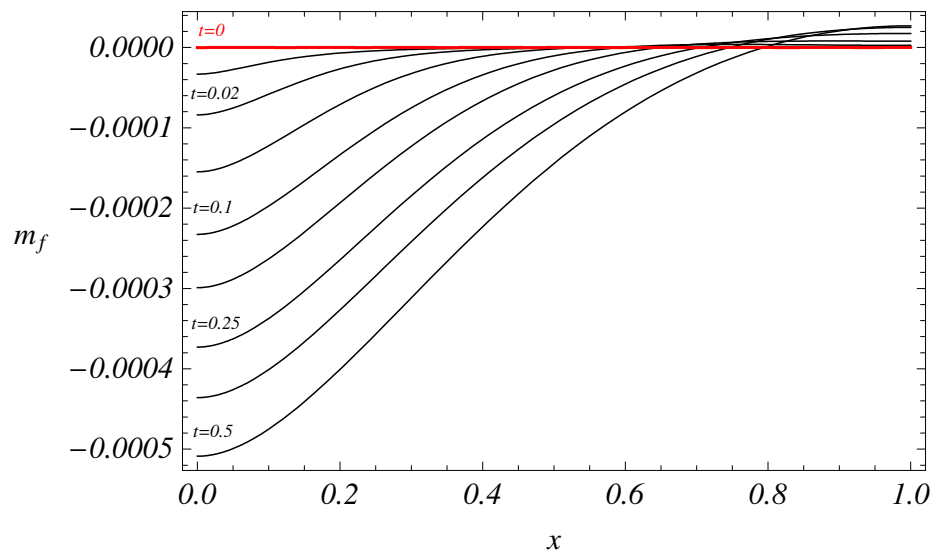

Figure 1. Behavior of the fluid mass density $m_{f}$ versus depth $x$ in the vicinity of the time $t=0^{+}$. A segregation of the fluid arises close to the impermeable wall at $x=1$.

each eigenvalue $\lambda_{k}$ an eigenfunction $X_{k}(x)$ is deduced in the form of Equation (42) so that a numerical solution for $V(x, t)$ can be found according to (38).

The numerical solution for $V(x, t)$ involves a finite number of eigenfunctions $N$, where the choice of $N$ is made so as to approach the condition stated by the Parseval equality [Kolmogorov and Fomin 1975]. Once the numerical solution for $V(x, t)$ has been found, we can deduce the corresponding solutions for the fields $\varepsilon$ and $m_{f}$ simply be referring to (15) and (16).

We now show the behavior of the fields $\varepsilon$ and $m_{f}$ corresponding to the aforementioned values of the constitutive parameters, and initial and incremental pressures. In Figure 1 the fluid apparent density $m_{f}$ versus $x$ is depicted for times in the very close neighborhood of $t=0^{+}$. It can be noticed that a critical depth $x_{c r} \simeq 0.8$ exists such that the density $m_{f}$ decreases for $0 \leq x<x_{c r}$, while it increases for $x_{c r}<x \leq 1$. This means that the fluid of the upper regions actually leaves the layer, while the fluid contained in the deeper regions remains entrapped in the pores whose deformation consequently increases the apparent density $m_{f}$. When increasing time (see Figure 2), the apparent density $m_{f}$ decreases along the whole depth of the layer, and finally approaches a constant value. This means that the fluid starts flowing out also from the deeper regions until the system reaches a new equilibrium and no fluid leaves the layer anymore. This effect is evidently related to viscosity, which dominates the evolution of the fluid density as time becomes larger and larger.

As far as the vertical deformation $\varepsilon$ is concerned, the same qualitative behavior as that of $m_{f}$ is detected (see Figure 3 and Figure 4). For times close to $t=0^{+}$(see Figure 3) the upper regions of the layer undergo to a vertical compression, which is connected to the fact that less fluid is present in the pores, while the deeper regions experience a sort of dilatancy which is connected to an over pressurization of the saturating fluid.

For increasing times (see Figure 4) a general further compression is detected along the whole depth of the layer (this is due to the fact that the fluid is uniformly flowing along the layer) until the layer does not deform anymore (equilibrium). 
We remark that the chosen values of the prestress $p_{0}^{\text {ext. }}$ are such that $\mathbb{C}_{4}>0$ so that the inner product Equation (35) is well defined. Consequently the solution for $V(x, t)$ (and thus for $\varepsilon$ and $m_{f}$ ) can be numerically evaluated.

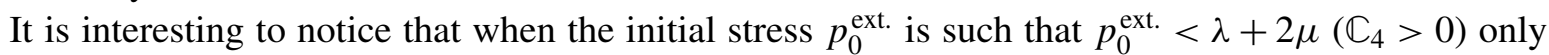
negative eigenvalues $\lambda_{k}<0$ have been found, while in the region where $\mathbb{C}_{4}<0$ a finite number of positive eigenvalues arise. In Figure 5 the behavior of the first eigenvalue $\lambda_{1}$ is shown when varying $p_{0}^{\text {ext. through }}$ the threshold $p_{0}^{\text {ext. }}=\lambda+2 \mu$. It is worth noticing that when $\lambda_{1}$ passes from negative to positive values, the solution $V(x, t)$ given in the form of Equation (38) blows up due to the presence of positive time exponentials; the solution thus experiences an unstable behavior related to the fact that $p_{0}^{\text {ext. }}$ reaches a critical value. This kind of instability is known as geometrical instability since the presence of $p_{0}^{\text {ext. in }}$ the differential problem is due to the geometry of the problem (see Equation (21)).

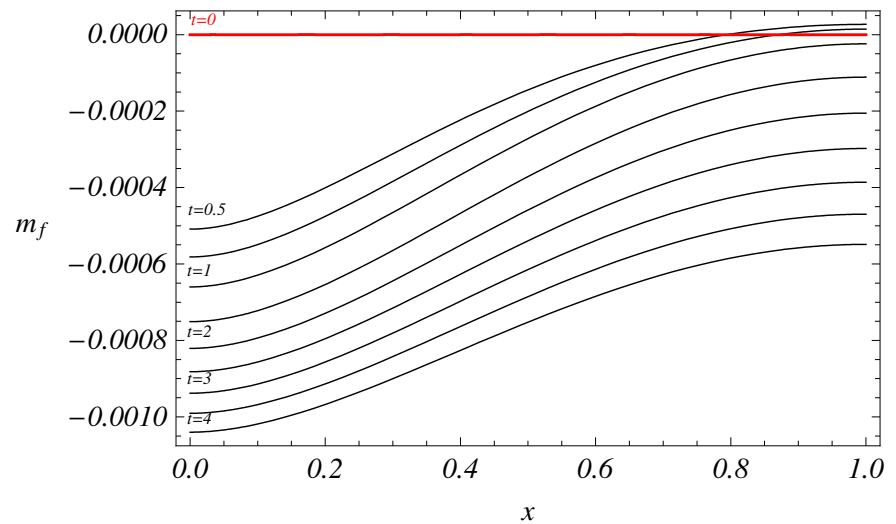

Figure 2. Profile of $m_{f}$ for further times. Notice that $m_{f}$ tends to assume a constant value for $t \rightarrow+\infty$, approaching equilibrium.

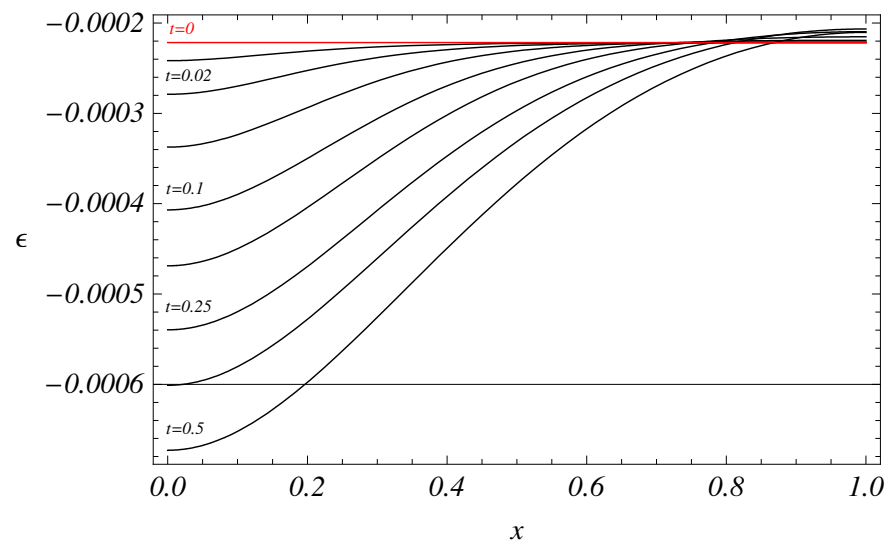

Figure 3. Profile of the vertical solid strain $\varepsilon$ versus $x$ for times close to $t=0^{+}$. A dilatancy of the solid skeleton is detected in the neighborhood of $x=1$. 
The linearity of the present model does not allow us to capture solutions associated with unstable conditions; this is evident when considering that the bilinear form given in (35) is no longer a well defined inner product.

In order to show the influence of the solid prestress on the behavior of $\varepsilon$ and $m_{f}$ we have found solutions for different values of $p_{0}^{\text {ext. }}$ and noticed changes in the solution when approaching the threshold $\mathbb{C}_{4}=0$. Figure 6 shows the behavior of $m_{f}$ when $p_{0}^{\text {ext. }}$ progressively approaches the critical value $p_{0}^{\text {ext. }}=\lambda+2 \mu$. When increasing the value of $p_{0}^{\text {ext. }}$ the fluid density decreases in the superficial regions of the layer, while increasing in the deeper ones. This means that the initial stress increases the capability of the fluid to flow out from the skeleton matrix close to the external surface, while pumping it in the deeper layers.

Let us now consider the second gradient constitutive parameters and the initial stresses to be vanishing. The resulting differential problem reduces to the classical Terzaghi consolidation problem. More particularly, Equation (10) reduces to

$$
\varepsilon=\frac{b M}{\lambda+2 \mu+b^{2} M} m_{f}-\frac{\Delta p^{\text {ext. }}}{\lambda+2 \mu+b^{2} M},
$$

which, substituted in (11), gives

$$
\dot{m}_{f}=a m_{f}^{I I}, \quad a=\frac{(\lambda+2 \mu)}{\lambda+2 \mu+b^{2} M} .
$$

The Terzaghi consolidation problem thus reduces to the differential equation, Equation (44), together with the initial datum $m_{f}\left(x, 0^{+}\right)=0$ and the BCs, (12) and (13), which simplify into

$$
m_{f}=\frac{\lambda+2 \mu+b^{2} M}{\lambda+2 \mu}\left(b \frac{\Delta p^{\text {ext. }}}{\lambda+2 \mu}\right):=c \quad \text { at } x=0 .
$$

and $m_{f}^{I}=0$ at $x=L$, respectively.

It is easy to notice that the $\mathrm{BC}$, (45), and the initial datum, $m_{f}\left(x, 0^{+}\right)=0$, are not consistent, so the Terzaghi solution for $m_{f}$ exhibits the well known behavior of the classical unidimensional heat equation.

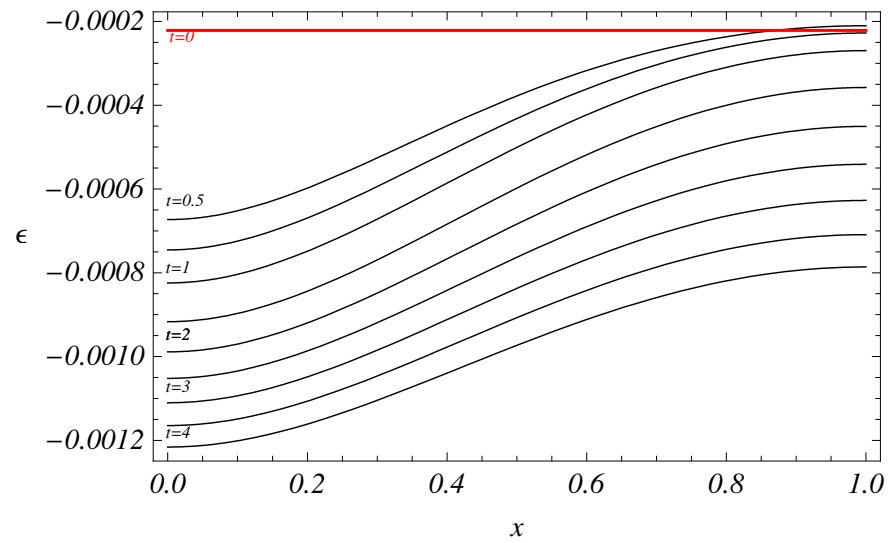

Figure 4. Behavior of $\varepsilon$ for further times. Notice that the system tends to reach a state of equilibrium for $t \rightarrow+\infty$. 


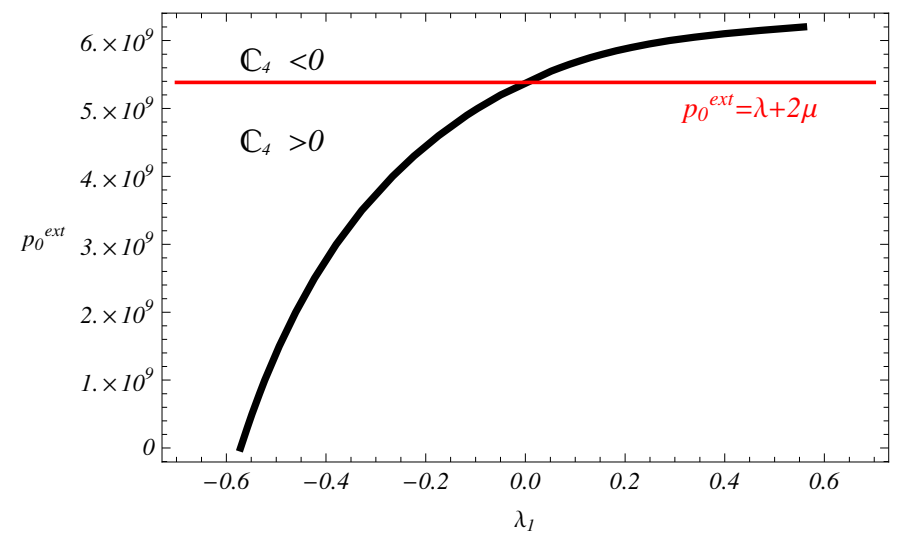

Figure 5. The value of the first eigenvalue $\lambda_{1}$ versus the prestress $p_{0}^{\text {ext. }}$.

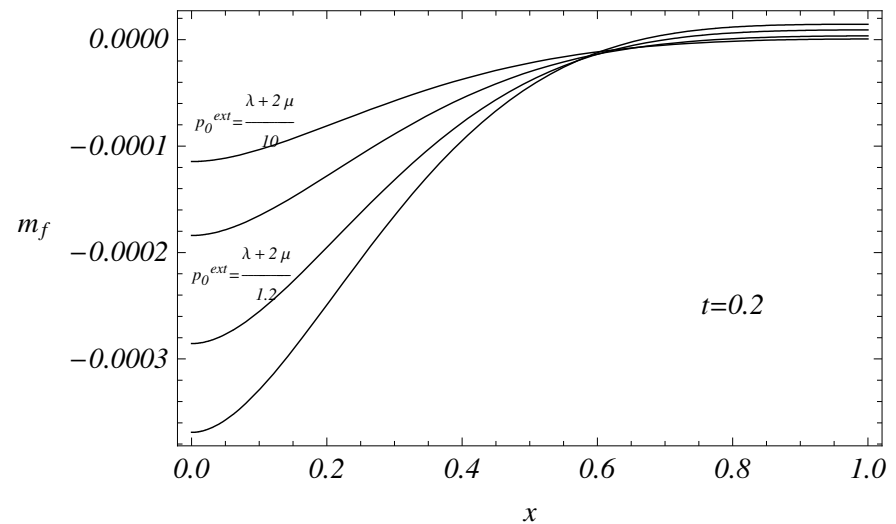

Figure 6. $m_{f}$ profile versus depth for $t=0.2$ and for different values of the prestress

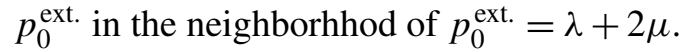

As it will be shown in the following, this discontinuity between the initial datum and the BC is cured by the second gradient model. The Terzaghi solution given in terms of the fluid mass density $m_{f}$ reads

$$
m_{f}(x, t)=c+\sum_{k=1}^{+\infty} \sqrt{2 a} \sin \left[\left(\frac{\pi}{2}+k \pi\right) x\right] e^{\lambda_{k} t}, \quad \lambda_{k}=a\left(\frac{\pi}{2}+k \pi\right)^{2} .
$$

In Figure 7 we show the comparison between the Terzaghi and the second gradient solutions for $m_{f}$ and $\varepsilon$, respectively (in absence of prestresses), corresponding to the initial condition. The Terzaghi solution (blue line) tends to a step function due to the discontinuity between the initial datum and the BC; on the other hand this discontinuity is not present in the second gradient solution (red line). Moreover, we underline that the second gradient Fourier series solution converges more quickly to its limit compared with the Terzaghi one.

Figure 8 shows the comparison between the Terzaghi and second gradient solutions for increasing time. It must be noted that, due to the continuity between the initial datum and the BC, the second gradient 
solution smoothly decreases with respect to the initial datum, while the Terzaghi solution is not able to describe the behavior of $m_{f}$ close to the external surface. The second gradient allows for describing compaction of the solid in the vicinity of the external surface, which contrasts instantaneous escape of the fluid out of the porous skeleton. This effect has been indeed recognized both in experiments and in situ measurements [Mollema and Antonellini 1996; Holcomb and Olsson 2003; 2000].

\section{Concluding remarks}

In this paper an application of the second gradient theory of poromechanics to the consolidation problem is discussed. In particular, we present some results within the hypothesis of small deformations around a prestressed reference configuration of the solid skeleton. Even in the framework of the linearized theory, the considered second gradient model gives rise to several interesting questions, concerning both the mathematical formulation of the problem and the mechanical interpretation of the results.

From the mathematical point of view the problem could be studied within the framework of linear pencils of ordinary differential operators on a finite interval, with boundary conditions depending on the spectral parameter. Several applications of this theory to physics and mechanics can be found in the literature [Tretter 2000; Marletta et al. 2003]; it is our purpose to investigate in the future how the very special problem we are dealing with can fit within the general theory.

From the mechanical point of view, the results presented also look quite interesting, in particular concerning the capability of the model to describe fluid segregation. It has to be remarked that second gradient models, in general, regularize the solutions of evolutionary or equilibrium equations (see, for example, Figure 8). In the case of their application to phase transition phenomena they allow for the coexistence of different phases at equilibrium, in the case of strain concentration phenomena for the description of shear and compaction bands, and in the case of wetting for the description of drop/film stability. In the first and third instances, the second gradient is necessary to describe capillarity, and in

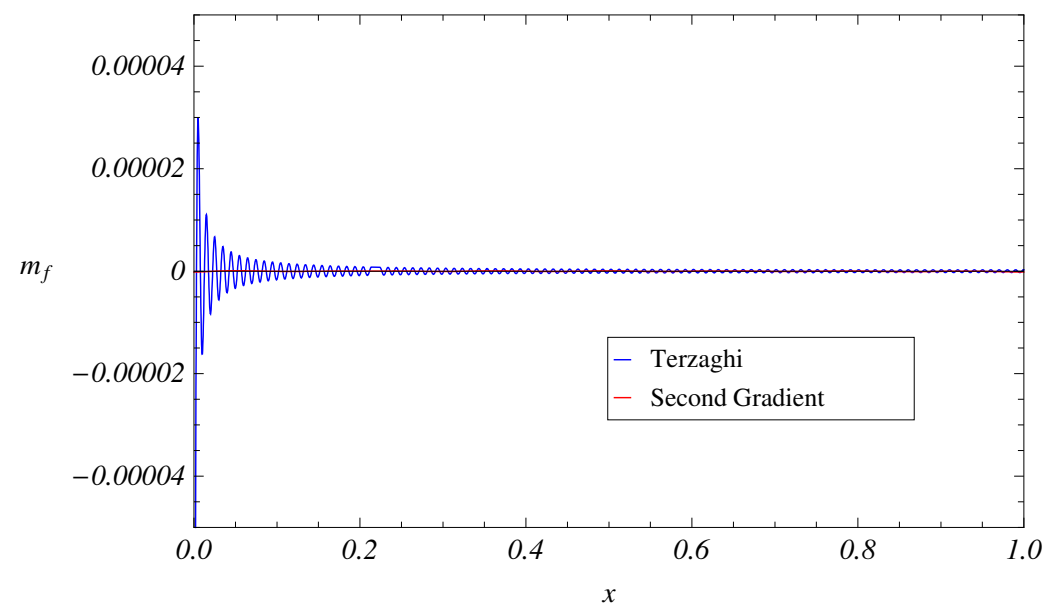

Figure 7. Comparison between the classical Terzaghi solution and the second gradient solution for $t=0^{+}$. 


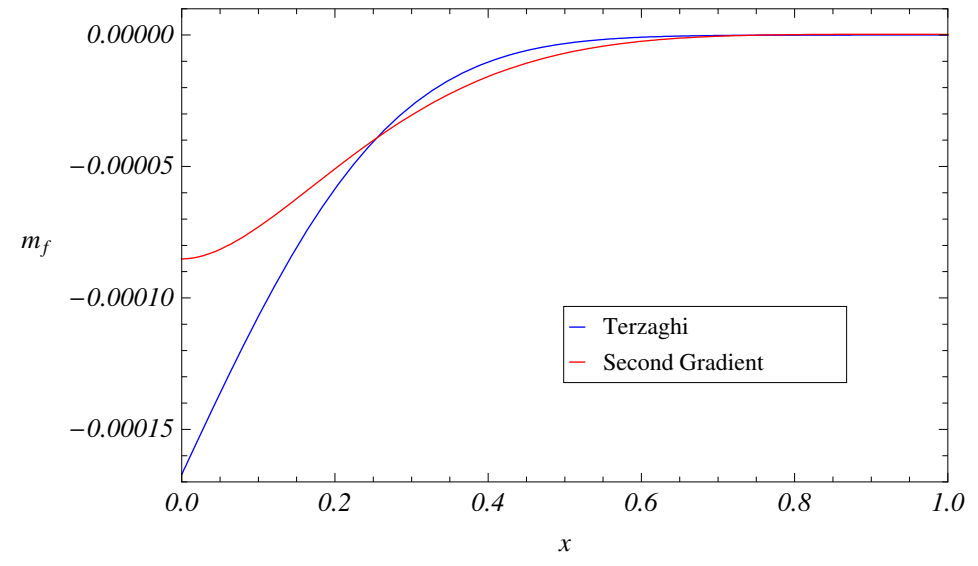

Figure 8. Comparison between the Terzaghi and the second gradient solutions for $t=0.1$.

the second plasticity. In the present instance we propose to use second gradient models to describe those phenomena occurring close to the drained boundary which contrasts fluid flow.

A linear stability analysis provides the limit value of the initial stress, which forces the first eigenvalues to become at least nonnegative. The goal in the future will be that of identifying which are the buckling modes, and in particular to corroborate the idea that bifurcated modes of consolidation can interpret liquefaction phenomena and occurrence of sand boils [Kolymbas 1998].

\section{Appendix A: Coefficients of the differential problem}

The constant coefficients $C_{i}$ and $\mathbb{C}_{i}$ appearing in the differential problem given by Equations (17) and (19) are defined as

$$
\begin{aligned}
C_{1} & =k_{1} k_{3}, & C_{2}\left(p_{0}^{\text {ext. }}\right) & =k_{1}+k_{3} k_{5}\left(k_{2}+b\right)^{2}+k_{3} C_{4}\left(p_{0}^{\text {ext. }}\right), \\
C_{3} & =k_{4}\left(k_{1}+k_{3} k_{5} k_{2}^{2}\right), & C_{4}\left(p_{0}^{\text {ext. }}\right) & =1-\frac{p_{0}^{\text {ext. }}}{\lambda+2 \mu}, \\
C_{5}\left(p_{0}^{\text {ext. }}\right) & =k_{4}\left[C_{4}\left(p_{0}^{\text {ext. }}\right)+k_{6}\right]+k_{1}+k_{3} k_{5} k_{2}^{2}, & & C_{6}\left(p_{0}^{\text {ext. }}\right)=C_{4}\left(p_{0}^{\text {ext. }}\right)+k_{6},
\end{aligned}
$$

with

$$
\begin{aligned}
& k_{1}=\frac{K_{s s}}{(\lambda+2 \mu) L^{2}}, \quad k_{2}=K_{s f}, \quad k_{3}=\frac{\mathbb{M}}{M L^{2}}, \\
& k_{4}=\frac{\alpha}{D L^{2}}, \quad k_{5}=\frac{M}{\lambda+2 \mu}, \quad k_{6}=b^{2} k_{5} .
\end{aligned}
$$

Moreover, the coefficients appearing in the BC, Equation (18), are defined as

$$
\begin{aligned}
& \mathbb{C}_{1}=C_{1}, \\
& \mathbb{C}_{2}\left(p_{0}^{\text {ext. }}\right)=C_{2}\left(p_{0}^{\text {ext. }}\right), \\
& \mathbb{C}_{3}=C_{3} \\
& \mathbb{C}_{4}\left(p_{0}^{\text {ext. }}\right)=C_{4}\left(p_{0}^{\text {ext. }}\right), \quad \mathbb{C}_{5}\left(\pi_{0}\right)=C_{5}-\left(k_{1}+k_{3} k_{5} k_{2}^{2}\right), \\
& \mathbb{C}_{6}\left(\pi_{f}^{0}\right)=\frac{\Delta p^{\text {ext. }}}{b M} \text {. }
\end{aligned}
$$


It must be noticed that the constants $k_{1}, \ldots, k_{4}$ are introduced by the second gradient model, while $k_{5}$ and $k_{6}$ are related to the first gradient parameters $M, \lambda$, and $\mu$, which represent the Biot bulk modulus and the Lamé coefficients of the considered material, respectively.

\section{References}

[Biot 1941] M. A. Biot, "General theory of three-dimensional consolidation", J. Appl. Phys. 12:2 (1941), 155-164.

[de Boer 1996] R. de Boer, "Highlights in the historical development of the porous media theory: toward a consistent macroscopic theory", Appl. Mech. Rev. (Trans. ASME) 49:4 (1996), 201-262.

[Coussy 2004] O. Coussy, Poromechanics, Wiley, Chichester, 2004.

[Cryer 1963] C. W. Cryer, "A comparison of the three-dimensional consolidation theories of Biot and Terzaghi", Q. J. Mech. Appl. Math. 16:4 (1963), 401-412.

[Heinrich and Desoyer 1961] G. Heinrich and K. Desoyer, "Theorie dreidimensionaler setznugsvorgänge in Tonschichten", Ing. Arch. 30:4 (1961), 225-253.

[Holcomb and Olsson 2003] D. J. Holcomb and W. A. Olsson, "Compaction localization and fluid flow", J. Geophys. Res. 108:B6 (2003), 2290.

[Kolmogorov and Fomin 1975] A. N. Kolmogorov and S. V. Fomin, Introductory real analysis, Dover, New York, 1975.

[Kolymbas 1998] D. Kolymbas, "Behaviour of liquified sand”, Phil. T. Roy. Soc. A 356:1747 (1998), 2609-2622.

[Mandel 1953] J. Mandel, "Consolidation des sols (étude mathématique)", Géothecnique 3 (1953), 287-299.

[Marletta et al. 2003] M. Marletta, A. Shkalikov, and C. Tretter, "Pencils of differential operators containing the eigenvalue parameter in the boundary conditions", Proc. R. Soc. Edin. A-Ma 133 (2003), 893-917.

[Mollema and Antonellini 1996] P. N. Mollema and M. A. Antonellini, "Compaction bands: a structural analog for anti-mode I cracks in aeolian sandstone", Tectonophysics 267:1-4 (1996), 209-228.

[Olsson and Holcomb 2000] W. Olsson and D. Holcomb, "Compaction localization in porous rock", Geophys. Res. Lett. 27:21 (2000), 3537-3540.

[Sciarra et al. 2008] G. Sciarra, F. dell'Isola, N. Ianiro, and A. Madeo, "A variational deduction of second gradient poroelasticity I: general theory", J. Mech. Mater. Struct. 3 (2008), 507-526.

[Seppecher 1987] P. Seppecher, Etude d'une modelisation des zones capillaires fluides: interfaces et lignes de contact, Ph.D. thesis, Thèse Université PARIS VI, 1987.

[Shkalikov 1986] A. A. Shkalikov, "Boundary problems for ordinary differential equations with parameter in the boundary conditions", J. Math. Sci. 33:6 (1986), 1311-1342.

[Shkalikov and Tretter 1996] A. A. Shkalikov and C. Tretter, "Spectral analysis for linear pencils $N-\lambda P$ of ordinary differential operators", Math. Nachr. 179:1 (1996), 275-305.

[Terzaghi 1923] K. v. Terzaghi, "Die Berechnung der Durchlässigkeitsziffer des tones aus dem verlauf der hydrodynamischen spanunngserscheinnungen", Technical report II a,132 N 3/4, 125,138, Akademie der Wissenschaften in Wien. Sitzungsberichte Mathnaturwiss Klasse Abt, 1923.

[Terzaghi 1943] K. v. Terzaghi, Theoretical soil mechanics, Wiley, New York, 1943.

[Tretter 2000] C. Tretter, “On buckling problems”, ZAMM 80:9 (2000), 633-639.

Received 8 Mar 2007. Revised 27 Jul 2007. Accepted 25 Nov 2007.

ANGELA MADEO: angela.madeo@uniroma1.it

Dipartimento di Metodi e Modelli Matematici per le Scienze Applicate, Università di Roma "La Sapienza”, Via Scarpa 16, 00161 Rome, Italy

FRANCESCO DELL'ISOLA: francesco.dellisola@uniroma1.it

Dipartimento di Ingegneria Strutturale e Geotecnica, Università di Roma "La Sapienza", Via Eudossiana 18, 00184 Rome, Italy

and

Laboratorio di Strutture e Materiali Intelligenti, Palazzo Caetani (Ala Nord), 04012 Cisterna di Latina, Italy 
NICOLETTA IANIRO: ianiro@dmmm.uniroma1.it

Dipartimento di Metodi e Modelli Matematici per le Scienze Applicate, Università di Roma "La Sapienza", Via Scarpa 16, 00161 Rome, Italy

GIULIO SCIARRA: giulio.sciarra@uniroma1.it

Dipartimento di Ingegneria Chimica Materiali Ambiente, Università di Roma “La Sapienza”, Via Eudossiana 18, 00184 Rome, Italy 


\title{
EFFECTS OF LAYER STACKING ORDER ON THE $V_{50}$ VELOCITY OF A TWO-LAYERED HYBRID ARMOR SYSTEM
}

\author{
Pankaj Kumar Porwal and Stuart Leigh Phoenix
}

\begin{abstract}
We develop a theoretical and computational model to investigate the ballistic response of a hybrid twolayered flexible armor system. In particular, we study the effects of stacking order of the two fibrous layers, which have distinctly different mechanical properties, on the $V_{50}$ limit velocity. A system consisting of Kevlar and Spectra fabrics is studied in detail. For this system, previous experimental results of Cunniff show nearly a factor of two difference in the $V_{50}$ velocities for the two possible stacking orders. The new model presented here extends our previous multilayer model by directly addressing interference effects between the two layers, treated here using length and tension compatibility along the radial direction away from the projectile. The primary task is to calculate strains in the individual layers in the presence of constraining interference that forces the nested layers to have a common impact cone shape different from what would be generated by the impact if the layers were allowed to deform freely. We show that this interference, together with relative areal densities of the layers, have a significant effect on the strain evolution in the layers, particularly near the edge of the projectile where failure initiates. As observed experimentally by Cunniff, our model predicts a large decrease in the $V_{50}$ velocity of the hybrid armor system when Spectra is the strike layer. However, to achieve this reduction it is necessary to use a lowered normalization velocity in multilayered Spectra systems than the theoretical value obtained from basic fiber properties. Besides matching the experimental results of Cunniff, the model reveals many subtle transitions in the onset and effects of interference between the layers. Somewhat surprising and contrary to conventional wisdom is the observation that layer interference can sometimes be beneficial depending on the relative mechanical properties and areal densities of the two layers.
\end{abstract}

\section{Introduction}

The ballistic performance of a body armor system is usually measured in terms of the $V_{50}$ velocity, the residual velocity when impacted above the $V_{50}$ velocity, and the maximum deflection of the projectile in the armor system perpendicular to the plane of armor panel. There are two main focus areas in the development of low weight and high performance body armor systems: (a) development of materials with superior mechanical properties, and (b) design optimization of integrated system to efficiently translate these superior properties into ballistic performance. Fibrous body armors are constructed by stacking the woven or nonwoven layers of high performance fibers like aramid (Kevlar®), high molecular weight polyethylene (Spectra $\left.{ }^{\circledR}\right)$, and until recently PBO (Zylon $\left.{ }^{\circledR}\right)$. Others are still in development such as DuPont PIPD (M5®) and carbon nanotube based yarns. Modeling efforts to optimize the performance

Keywords: multilayered hybrid armor systems, stacking order, thermal effects, layer interference, $V_{50}$ velocity, impact velocity versus residual velocity.

The authors acknowledge the support of the United States Air Force, contract no. USAF-5212-STI-SC-0013, monitored by Captain Todd J. Turner. 
of armor systems have not kept pace with developments in new materials. Trial and error experimental investigation is very expensive and often ineffective due to a fundamental lack of understanding of the physics of the impact event and the mechanisms of yarn and layer failure, especially subtle interactions.

In this paper we analyze the two possible stacking orders of a hybrid two-layered system where, in one stacking order, the layers interfere with each other in the cone wave region. This interference results when the underlying layer attempts to form an incompatible cone shape with the top layer, when both layers are driven by a projectile velocity history during deformation. In the model, the momentum exchange between the interfering layers over time occurs primarily around the ring formed by the coincident cone wavefronts in the layers. The strain evolution in the layers is determined using length compatibility in the conical and in-plane deformation zones extending from the projectile edge to the tension wavefronts in the layers and tension compatibility from dynamic effects analogous to those seen in a belt traveling over a pulley at modest wrap angles. The case of a noninterfering arrangement of layers was treated in a previous work by the authors [Porwal and Phoenix 2005] and those results are also used in the comparison of the two stacking orders.

\section{Literature survey}

Earlier efforts to model the performance of a multilayered fibrous soft body armor system focused on either extrapolating results from a single layer system or assuming sequential failure through widely spaced layers where only one layer at a time engages the projectile, that is, a decoupled system [Roylance et al. 1973; Hearle et al. 1981; 1984; Taylor Jr. and Vinson 1989; Parga-Landa and Hernandez-Olivers 1995; Chocron-Benloulo et al. 1997; Cunniff 1999a; 1999b; 1999c; Billon and Robinson 2001; Zohdi 2002; Zohdi and Powell 2006]. Recently the authors have developed analytical models in which layers respond to the impact of a projectile in a coupled and synergistic manner, as they do in reality, but the layers are arranged in such a way that they form nested cones but without interference in the cone wave region or elsewhere [Phoenix and Porwal 2003; Porwal and Phoenix 2005].

It has been shown experimentally as well as by computer simulation models [Roylance et al. 1995] that constraining the transverse deflection of fabric layers, especially near the cone wavefront, significantly alters the strain distribution and hence the ballistic performance of the system. In a hybrid multilayered armor, the stacking order of the layers becomes critically important because it dictates the extent of interference between the layers in the cone of transverse deflection. Perhaps the best known demonstration of this effect is due to Cunniff [1992] who stacked Kevlar and Spectra layers in two possible arrangements and showed that the $V_{50}$ velocity could be altered by about a factor of two.

\section{Theoretical background for multilayered system behavior}

The model developed here is based on the results from previous works by Phoenix and Porwal [2003] and Porwal and Phoenix [2005]. In those works, membranes with in-plane isotropic elastic properties are impacted normally by a flat-faced, right circular cylindrical (RCC) projectile with radius $r_{\mathrm{p}}$ and traveling at velocity $V_{\mathrm{p}}$ before the impact. Some of the results in those works will be the basis for the work here so we quote them without derivation. We note that all the results quoted below are not exact but are very accurate approximations, and should be understood as such. 
We let $\Gamma_{0 i}$ be the areal density ratio of the $i$ th layer relative to that of the projectile, that is,

$$
\Gamma_{0 i}=\frac{A_{\mathrm{d}, i}}{A_{\mathrm{d}, \mathrm{p}}}=\frac{m_{\mathrm{p} i}}{M_{\mathrm{p}}}
$$

where $A_{\mathrm{d}, i}$ and $A_{\mathrm{d}, \mathrm{p}}$ are the areal densities of the $i$ th layer and the projectile, $m_{\mathrm{p} i}=A_{\mathrm{d}, i} A_{\mathrm{p}}$ is the mass of the plug of the membrane material directly contacted by the projectile or right under it in the $i$ th layer, and $M_{\mathrm{p}}=A_{\mathrm{d}, \mathrm{p}} A_{\mathrm{p}}$ is the mass of the projectile. Here $A_{\mathrm{p}}$ is the projected area of the projectile onto the membrane plane, which is the same as the cross-sectional area of the RCC projectile impacting longitudinally. For a two-layered armor, the system areal density is

$$
\tilde{\Gamma}_{0}=\sum_{i=1}^{2} \Gamma_{0 i}
$$

Upon impact of the projectile, there is a virtually instantaneous momentum transfer to the circular patches of the layers right under the projectile. Thus, the velocity of the projectile just after impact is

$$
V_{0}=\frac{V_{\mathrm{p}}}{1+\tilde{\Gamma}_{0}}
$$

Note that both layers respond instantaneously to the impact because there are negligible gaps between the layers. For the $i$ th layer, the critical layer tensile strain just after impact occurs near the projectile edge, that is, at the projectile radius $r_{\mathrm{p}}$. This strain is given by

$$
\epsilon_{\mathrm{p} 0 i}=\left[\frac{V_{0}}{\sqrt{2} a_{0 i}}\right]^{4 / 3}
$$

where $a_{0 i}$ is the tension wave velocity in the $i$ th layer (approximately $\sqrt{E_{i} / 2 \rho_{i}}$ ), and $E_{i}$ and $\rho_{i}$ are the Young's modulus and density of the constituent yarns, respectively. The factor of 2 in the denominator comes from the added mass of crossing yarns that support no load in the direction of the wave propagation.

Two types of waves are formed just after the sudden local momentum transfer at time $t=0$. The first type consists of radially growing tensile waves, and these are followed by much slower transverse waves in the form of growing cones with the projectile at their apexes. The projectile is decelerated by the membrane forces generated as the waves propagate in the layers. The velocity profile of the projectile is given by

$$
V=\frac{V_{\mathrm{p}}}{1+\tilde{\Gamma}_{0}} \exp \left[-\frac{\sum_{i=1}^{2} \varphi_{i} \Gamma_{0 i} \psi_{i}^{2}+\sum_{i=1}^{2}\left(1-\varphi_{i}\right) \Gamma_{0 i} \psi_{i, \mathrm{fi}}^{2}-\tilde{\Gamma}_{0}}{1+\tilde{\Gamma}_{0}}\right],
$$

where $\psi_{i}=r_{\mathrm{c} i} / r_{\mathrm{p}}$ is the normalized cone wavefront position in the $i$ th layer (where $r_{\mathrm{c} i}$ is the radius of the base of the conical deflection in that layer), $\psi_{i, \mathrm{fi}}$ is the normalized position of the cone wavefront in the $i$ th layer when it fails, and

$$
\varphi_{i}= \begin{cases}0, & \text { for a failed layer } \\ 1, & \text { for an intact layer }\end{cases}
$$


As the cone waves propagate, the changing strains in the layers at the projectile edge can be obtained by solving

$$
\epsilon_{\mathrm{p}_{i}}=\left(\frac{V}{a_{0 i} \sqrt{2}}\right)^{4 / 3} \psi_{i}^{1 / 3}\left(\frac{\sqrt{\psi_{i} / \epsilon_{\mathrm{p}_{i}}}\left(\psi_{i}-1\right)}{\ln \left(1+\sqrt{\psi_{i} / \epsilon_{\mathrm{p}_{i}}}\left(\psi_{i}-1\right)\right)}\right)^{2 / 3} .
$$

The material behind the tension wavefront flows toward the impact region. At the cone wavefront, the magnitude of this inflow velocity at time $t$ is

$$
\dot{u}_{\mathrm{c} i}=\frac{a_{0 i} \epsilon_{\mathrm{c} i} r_{\mathrm{c} i}}{r_{\mathrm{p}}+a_{0 i} t}\left\{\ln \left(\frac{r_{\mathrm{c} i}}{r_{\mathrm{p}}+a_{0 i} t}\right)-1\right\},
$$

which happens to be different for the two layers. Here $\epsilon_{\mathrm{c} i}=\epsilon_{\mathrm{p} i} / \psi_{i}$ is the strain in the membrane at the cone wavefront. The cone wave generated by the transverse deformation of the membrane propagates into this inflowing material with velocity $c_{i}$ in the material coordinate system, which in terms of the instantaneous projectile velocity $V$ is given as

$$
c_{i}=r_{\mathrm{p}} \frac{d \psi_{i}}{d t}=\text { const } \times a_{0 i}\left(\frac{V}{a_{0 i} \sqrt{2}}\right)^{2 / 3}=\text { const } \times a_{0 i}^{1 / 3}\left(\frac{V}{\sqrt{2}}\right)^{2 / 3} .
$$

The constant 'const' is typically a number slightly larger than 1 depending on the impact velocity, but in the subsequent calculations it suffices to take const $=1$ (see [Phoenix and Porwal 2003]). The velocity of the cone wave in the ground coordinate system is $\tilde{c}_{i}=c_{i}+\dot{u}_{\mathrm{c} i}$, which is somewhat less than $c_{i}$ because $\dot{u}_{c i}$ is negative though much smaller. The tangential strain distribution in the membrane in terms of the strain in the membrane at the cone wavefront, $\epsilon_{\mathrm{c} i}$, can be written as

$$
\epsilon_{i} \approx \frac{\epsilon_{\mathrm{c} i} r_{\mathrm{c} i}}{r}, \quad r_{\mathrm{p}} \leq r \leq a_{0 i} t+r_{\mathrm{p}} .
$$

This can be integrated to estimate the change in length of the membrane material, $\Delta l$, in the radial direction due to the strain induced by the impact

$$
\Delta l=\epsilon_{\mathrm{c} i} r_{\mathrm{c} i} \ln \left(\frac{a_{0 i} t+r_{\mathrm{p}}}{r_{\mathrm{p}}}\right) .
$$

\section{Model for interfering two-layered system}

Let us consider a two-layered system deforming under a given projectile velocity history where both layers are in contact with each other under the projectile. Interference occurs when the transverse deflection of the top layer is hindered from forming its natural cone shape (when alone) by the underlying layer trying to form an incompatible cone shape. This is typically the case when the underlying layer has a lower Young's modulus and a higher material density and thus a lower tensile wave speed — though other factors are at play as well.

Figure 1 illustrates the situation in terms of the two nested layers. In particular, Figure 1(b) shows the case where the lower Kevlar layer exerts forces on the upper Spectra layer, causing the cone angle of Spectra to be larger than it would be if the layers were stacked in reverse order, as shown in Figure 1(a). Subscripts k and s denote the Kevlar and Spectra layers, respectively. An overbar is used for quantities corresponding to the interfering arrangement of the layers. In Figure 1, $a_{0}$ is the velocity of the tensile 


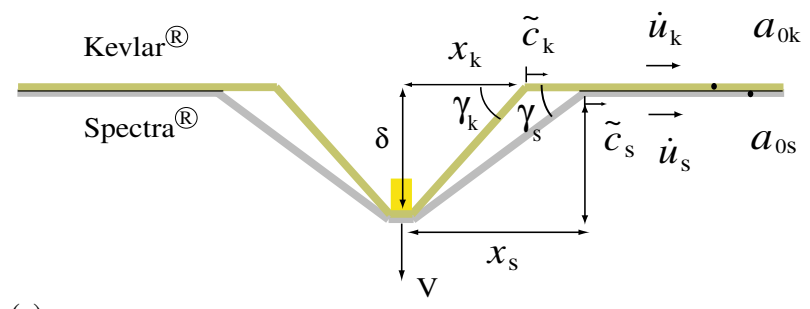

(a)

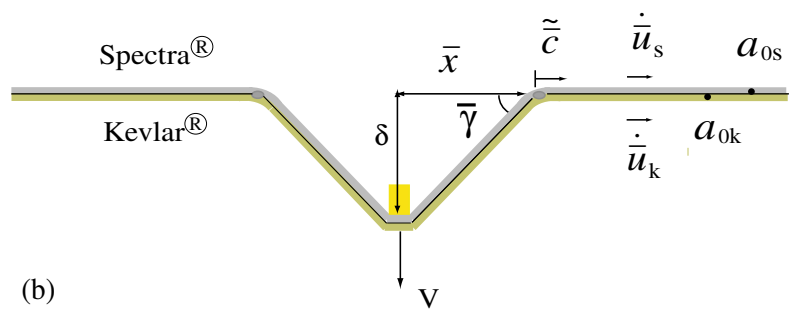

Figure 1. Effect of layer stacking order on the system response: (a) noninterfering arrangement (Kevlar-Spectra), versus (b) interfering arrangement (Spectra-Kevlar).

wave, $\tilde{c}$ is the velocity of the cone wavefront in the ground coordinate system, $\gamma$ is the cone angle of transverse deflection with respect to ground, $V$ is the instantaneous velocity of the projectile, and $\dot{u}$ is the material flow velocity, which turns out to be negative indicating that the material is flowing towards the impact region.

In the two-dimensional membrane model with constant projectile velocity, the portion of membrane that is right under the projectile moves with the projectile at the same velocity as the projectile, and with no velocity transverse to the projectile motion (that is, there is negligible slipping at the projectile edge). The material from the projectile edge to the cone wavefront forms a very mild curve [Phoenix and Porwal 2003] and can be approximated by a straight line to calculate its length. In the tensile wave region beyond the cone there is no significant interaction between the layers since there is no stitching or bonding. In the region of conical deformation, the material velocity is equal to that of the projectile. Thus, we can realistically assume that interaction forces between the layers only occur in the vicinity of the cone wavefront, that is, at the junction of the cone wave and tension wave (apart from the interaction between the plugs of layer material directly under the projectile).

For the interfering arrangement of the two layers, the basic assumption is that the cone wavefronts of both layers have the same velocity with respect to the ground coordinate system, and in fact the cone shapes are identical (Figure 1(b)). This, however, does not imply that the local strains along the cones and in the tension wave regions beyond the cones are the same in each layer; in fact, the tension wavefronts will be at different locations. In addition, each layer will be in tension out to its tensile wavefront, that is, there is no slack in either layer. 
Based on compatibility of length implied by the identical cone shapes of the deformed layers, two equations can be written, which are

$$
\begin{gathered}
\sqrt{\delta^{2}+\bar{x}^{2}}+\left(x_{\mathrm{s}}-\bar{x}\right)-\sqrt{\delta^{2}+x_{\mathrm{s}}^{2}}=\left(\bar{\epsilon}_{\mathrm{cs}} \bar{r}_{\mathrm{cs}}-\epsilon_{\mathrm{cs}} r_{\mathrm{cs}}\right) \ln \left(\frac{r_{\mathrm{p}}+a_{0 s} t}{r_{\mathrm{p}}}\right), \\
\sqrt{\delta^{2}+x_{\mathrm{k}}^{2}}+\left(\bar{x}-x_{\mathrm{k}}\right)-\sqrt{\delta^{2}+\bar{x}^{2}}=\left(\epsilon_{\mathrm{ck}} r_{\mathrm{ck}}-\bar{\epsilon}_{\mathrm{ck}} \bar{r}_{\mathrm{ck}}\right) \ln \left(\frac{r_{\mathrm{p}}+a_{0 \mathrm{k}} t}{r_{\mathrm{p}}}\right),
\end{gathered}
$$

where $\delta \int_{0}^{t} V(t) d t$ is the displacement of the projectile in the layers perpendicular to the plane of the armor panel. In the above equations $x_{\mathrm{s}}=\dot{c}_{\mathrm{s}} t, x_{\mathrm{k}}=\dot{c}_{\mathrm{k}} t$, and $\bar{x}=\tilde{\bar{c}} t$ are the bases of the approximately triangular shapes formed by the transverse deflection in the ground coordinate system, as shown in Figure 1. The radii of the bases of the cone wavefronts in the material coordinate system are

$$
\bar{r}_{\mathrm{cs}}=r_{\mathrm{p}}+\left(\tilde{\bar{c}}-\dot{\bar{u}}_{\mathrm{cs}}\right) t \quad \text { and } \quad \bar{r}_{\mathrm{ck}}=r_{\mathrm{p}}+\left(\tilde{\bar{c}}-\dot{\bar{u}}_{\mathrm{ck}}\right) t,
$$

for the Spectra and the Kevlar layers, respectively. These, however, are calculated incrementally because the velocities are not constant during the impact process. The left hand side of Equation (12), for the Spectra layer, is the extra length of material required to change its natural shape (as in Figure 1(a)) into the one that it is forced to form due to the interfering Kevlar back layer (as in Figure 1(b)). The right hand side of this equation represents the extra length of material generated by the increased strain in the layer, which is estimated using Equation (11). A similar interpretation can be applied to both sides of Equation (13). In Equation (14), the radially inward material flow velocities at the cone wavefronts of each layer are obtained by modifying Equation (8) as

$$
\begin{gathered}
\dot{\bar{u}}_{\mathrm{cs}}=\frac{a_{0 \mathrm{~s}} \bar{\epsilon}_{\mathrm{cs}} \bar{r}_{\mathrm{cs}}}{r_{\mathrm{p}}+a_{0 \mathrm{~s}} t}\left\{\ln \left(\frac{\bar{r}_{\mathrm{cs}}}{r_{\mathrm{p}}+a_{0 \mathrm{~s}} t}\right)-1\right\}, \\
\dot{\bar{u}}_{\mathrm{ck}}=\frac{a_{0 \mathrm{k}} \bar{\epsilon}_{\mathrm{ck}} \bar{r}_{\mathrm{ck}}}{r_{\mathrm{p}}+a_{0 \mathrm{k}} t}\left\{\ln \left(\frac{\bar{r}_{\mathrm{ck}}}{r_{\mathrm{p}}+a_{0 \mathrm{k}} t}\right)-1\right\} .
\end{gathered}
$$

A very helpful analogy for understanding the interaction forces between the two interfering cones is that of a pair of tensioned belts, one on top of the other and both running together over a pulley at modest wrap angle, as shown in Figure 2. We assume that the upper belt has a higher ratio of tension to linear density than the lower belt, which is in contact with the pulley. At a sufficiently high speed for the two

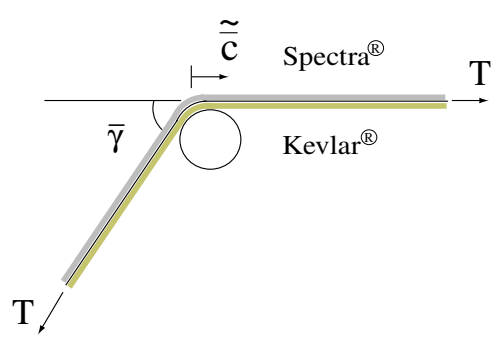

Figure 2. Belt-over-pulley analogy. 
belts, yet maintaining the same total tension, the centrifugal forces of the belts around the pulley will become large enough for the lower belt to loose contact with the pulley despite the contact forces between the upper belt and lower belt. To connect this analogy to the problem of two interfering cones, the frame of reference is changed such that the pulley is traveling at the speed of the cone wavefront and the belts are stationary with respect to ground (see Appendix A for more details on the belt-over-pulley analogy). It should be noted that the individual tensions in the belts at the lift off threshold are not arbitrary but must be consistent with the length compatibility condition described earlier.

The combined tension in the two layers is

$$
T=T_{\mathrm{s}}+T_{\mathrm{k}}=A_{\mathrm{s}} E_{\mathrm{s}} \bar{\epsilon}_{\mathrm{cs}}+A_{\mathrm{k}} E_{\mathrm{k}} \bar{\epsilon}_{\mathrm{ck}},
$$

where $A_{\mathrm{s}}=2 \pi\left(\bar{x}+r_{\mathrm{p}}\right) h_{\mathrm{s}}, A_{\mathrm{k}}=2 \pi\left(\bar{x}+r_{\mathrm{p}}\right) h_{\mathrm{k}}$, and $h_{\mathrm{s}}$ and $h_{\mathrm{k}}$ are the thicknesses of the Spectra and the Kevlar layers, respectively. From the belt-over-pulley analogy the tension in Equation (17) should also satisfy

$$
T=\bar{\rho} \bar{A} \tilde{\bar{c}}^{2},
$$

where $\bar{\rho}=\left(A_{\mathrm{s}} \rho_{\mathrm{s}}+A_{\mathrm{k}} \rho_{\mathrm{k}}\right) / \bar{A}$ and $\bar{A}=A_{\mathrm{s}}+A_{\mathrm{k}}$. Equations (17) and (18) can be combined to give

$$
\bar{\rho} \bar{A} \tilde{c}^{2}=A_{\mathrm{s}} E_{\mathrm{s}} \bar{\epsilon}_{\mathrm{cs}}+A_{\mathrm{k}} E_{\mathrm{k}} \bar{\epsilon}_{\mathrm{ck}}
$$

For a given impact velocity we can solve Equations (12), (13), (15), (16), and (19) for the unknowns $\tilde{\bar{c}}$, $\dot{\bar{u}}_{\mathrm{cs}}, \dot{\bar{u}}_{\mathrm{ck}}, \bar{\epsilon}_{\mathrm{cs}}$, and $\bar{\epsilon}_{\mathrm{ck}}$. If the impact velocity is high enough then the critical strain in one of the layers will reach its failure value. In this case we continue the analysis further depending on which layer fails first.

Case 1: Strike layer fails first. If the Spectra strike layer fails first, at an instantaneous projectile velocity $V_{\mathrm{f}, \mathrm{s}}$ and when the cone wavefront in the back layer is at $\psi_{\mathrm{k}, \mathrm{fs}}$, then tension in the Spectra layer is relaxed, it is left behind, and only the back layer can actively decelerate the projectile. To determine whether the second layer is subsequently penetrated we revert to single layer behavior for the back layer. We must calculate a hypothetical initial impact velocity for a single layer system consisting of only the Kevlar layer, that will give the projectile velocity $V_{\mathrm{f}, \mathrm{s}}$ with normalized position of the cone wave $\psi_{\mathrm{k}, \mathrm{fs}}$ :

$$
V_{\mathrm{p}, \mathrm{fs}}=V_{\mathrm{f}, \mathrm{k}}\left(1+\Gamma_{0}\right) \exp \left\{\frac{\Gamma_{0}}{1+\Gamma_{0}}\left(\psi_{\mathrm{k}, \mathrm{fs}}^{2}-1\right)\right\} .
$$

The Kevlar layer of the hybrid system is assumed to be penetrated if $V_{\mathrm{p}, \mathrm{fs}}$ is higher than its ballistic limit when impacted alone; otherwise the projectile is stopped.

Case 2: Back layer fails first. If the Kevlar back layer fails first, that is, a plug of material in front of the projectile is severed and a hole is formed, then this penetrated layer will still be pushed along by the Spectra layer. The Kevlar layer will tend to recover its tension some distance away from the projectile edge due to the hoop stresses in the cone wave region of the punctured layer. However, the tension originally supported by the Kevlar back layer in the vicinity of the projectile edge must now be carried by the Spectra layer, thus locally increasing its strain. This strain can be calculated as

$$
\bar{\epsilon}_{\mathrm{ps}, \mathrm{fk}}=\frac{A_{\mathrm{s}} E_{\mathrm{s}} \bar{\epsilon}_{\mathrm{ps}}+A_{\mathrm{k}} E_{\mathrm{k}} \bar{\epsilon}_{\mathrm{pk}}}{A_{\mathrm{s}} E_{\mathrm{s}}},
$$

and if it is higher than the failure strain of the Spectra layer then this layer is also penetrated and the armor is defeated. 
The smallest impact velocity at which both layers eventually fail, irrespective of which one fails first, is called the $V_{50}$ velocity of the system.

\section{Results and discussion}

The physical and mechanical properties used for calculations are representative of the Kevlar and Spectra fibers as given in Table 1. Note that for these calculations the areal densities $\Gamma_{0 i}$ are replaced by $\theta^{2} \Gamma_{0 i}$ to account for the uncertainty in the impact area. Here $\theta=1.3$ is used (this value was found applicable to a wide range of data and material types in [Phoenix and Porwal 2003]) to reflect an increase in the effective contact area relative to the radius of the RCC projectile nose. We also show some results from [Porwal and Phoenix 2005] for a noninterfering stacking of the layers. Readers are referred to this paper for more details.

Figure 3 plots the strain evolution in the two layers of the hybrid system for both interfering and noninterfering arrangements of the layers, at impact velocity $V_{\mathrm{p}}=140 \mathrm{~m} / \mathrm{s}$. The strains in each of the layers near the projectile edge first increase up to a maximum and then decrease as the cone wavefronts in both the layers propagate. This is similar to the case of a single layer or noninterfering arrangement of layers. Interference between the layers as well as their relative areal densities significantly alters the strains in both the Kevlar and Spectra layers. Interference decreases the strain in the Kevlar layer, in general, because of the widening cone base relative to its natural noninterfering shape. Conversely, the strain in the Spectra layer increases because of the smaller cone base radius formed in the interfering arrangement. In the case of an armor system consisting of predominantly Spectra, for example, $A_{\mathrm{d}, \mathrm{k}}=$ $0.01 \mathrm{~kg} / \mathrm{m}^{2}$ and $A_{\mathrm{d}, \mathrm{s}}=0.46 \mathrm{~kg} / \mathrm{m}^{2}$, the strain in the Kevlar at the projectile edge drops significantly without affecting the strain in the Spectra layer because Spectra can easily push the thin layer of Kevlar to widen its cone shape. On the other hand, for the system consisting of predominantly Kevlar , the Kevlar strain remains unchanged and the strain in Spectra increases significantly. However, when $A_{\mathrm{d}, \mathrm{k}}=$ $0.29 \mathrm{~kg} / \mathrm{m}^{2}$ and $A_{\mathrm{d}, \mathrm{s}}=0.18 \mathrm{~kg} / \mathrm{m}^{2}$ then strains in the Spectra and Kevlar are both altered significantly.

Figure 4 shows the effect of stacking order of the layers on the $V_{50}$ limit velocity as well as on residual velocity when impacted above the $V_{50}$ velocity for the specific combination of Kevlar areal density, $A_{\mathrm{d}, \mathrm{k}}=0.29 \mathrm{~kg} / \mathrm{m}^{2}$, and Spectra areal density, $A_{\mathrm{d}, \mathrm{s}}=0.18 \mathrm{~kg} / \mathrm{m}^{2}$. For the interfering arrangement of layers, the combined effects of strength loss due to thermal softening and an increase in the strain due to interference for the Spectra layer reduces the $V_{50}$ velocity from $216 \mathrm{~m} / \mathrm{s}$ (with $\epsilon_{\mathrm{s} \text {,fail }}=0.035$ ), for the noninterfering arrangement, to $156 \mathrm{~m} / \mathrm{s}$ (with $\epsilon_{\mathrm{s} \text {,fail }}=0.021$ due to thermal softening). Our theory confirms the experimental observation of Cunniff [1992], however, his results exhibited a greater

\begin{tabular}{cc||ccc}
\hline \multicolumn{2}{c||}{ Projectile } & Fibers \\
& Property & $\begin{array}{c}\text { Spectra } \\
\text { Radius }\end{array}$ & $2.76 \mathrm{~mm}$ & Kevlar \\
\hline Weight & 16 grain & Density, $\rho$ & $970 \mathrm{~kg} / \mathrm{m}^{3}$ & $1440 \mathrm{~kg} / \mathrm{m}^{3}$ \\
\hline
\end{tabular}

Table 1. Right circular cylindrical (RCC) projectile, with length to diameter ratio 1 , and fiber properties used for calculations. 

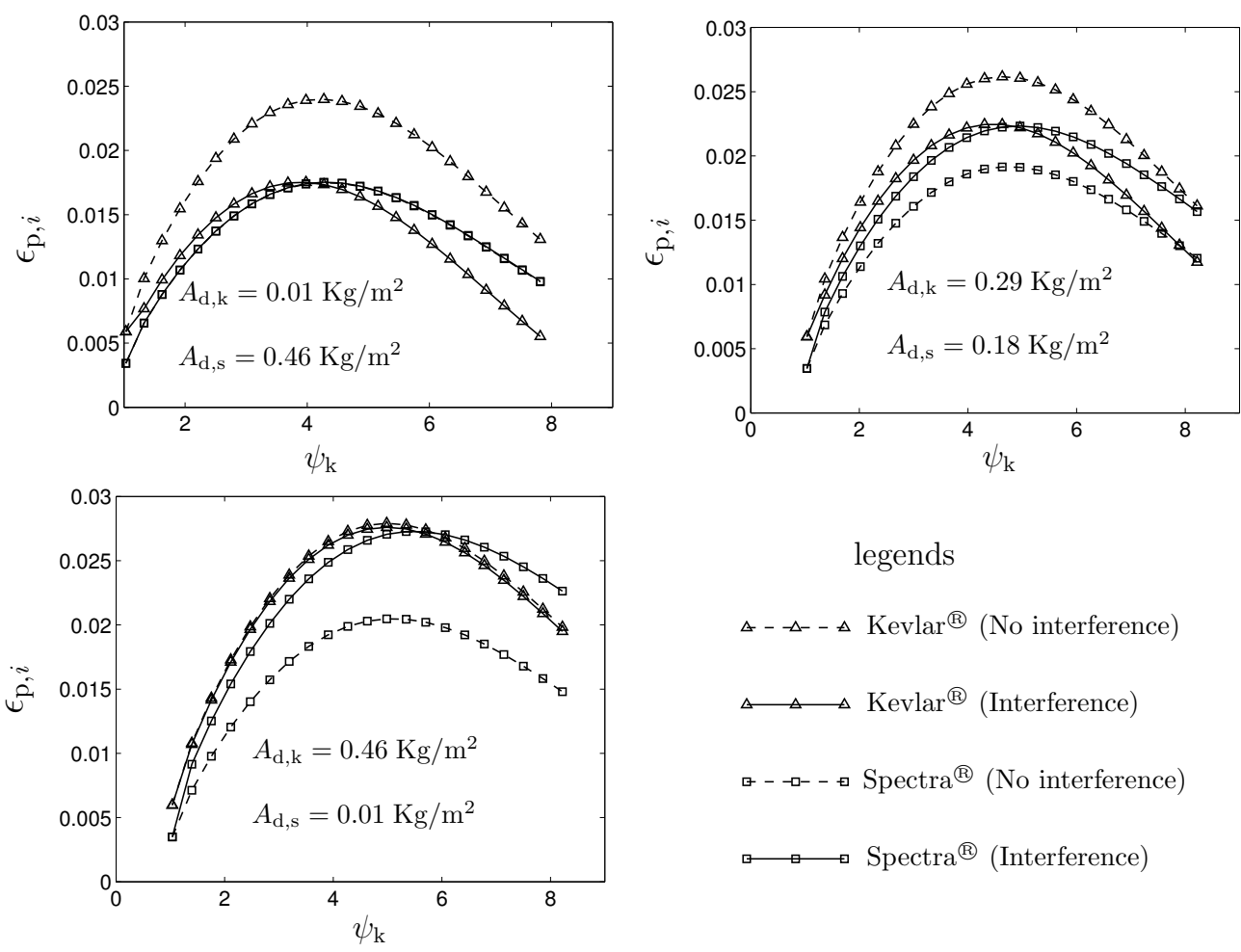

Figure 3. Strains in the layers around the projectile tip versus normalized cone wavefront for both interfering and noninterfering arrangements under impact velocity $V_{\mathrm{p}}=$ $140 \mathrm{~m} / \mathrm{s}$. The $\mathrm{x}$-axis coordinate, $\psi_{k}$, is the wavefront position of the Kevlar layer when in the noninterfering arrangement. In the first figure the strains for interfering and noninterfering arrangement coincide for Spectra layer.

difference in $V_{50}$ performance for the two possible arrangements, that is, $269 \mathrm{~m} / \mathrm{s}$ versus $114 \mathrm{~m} / \mathrm{s}$. Note that if softening does not occur then the difference in performance for the two possible arrangements is only slight. Thus, while we can create a scenario to match the results of [Cunniff 1992] it can only be done by invoking severe thermal softening of the thin Spectra layer.

We have also investigated the $V_{50}$ velocity for other areal density combinations of Spectra and Kevlar layers as shown in Figure 5, which plots the $V_{50}$ velocity versus the areal density of Kevlar layer. In the comparison, the total areal density of the system is kept constant, that is, $A_{\mathrm{d}}=A_{\mathrm{d}, \mathrm{k}}+A_{\mathrm{d}, \mathrm{s}}=0.47$ $\mathrm{kg} / \mathrm{m}^{2}$. The family of curves corresponds to different failure strains for the Spectra. Also shown are plots for the individual Kevlar and Spectra layers, when impacted alone, for the failure strains shown in the figure. Obviously, the effects of interference are modest unless one also invokes thermal softening effects in terms of reduced failure strain of the Spectra when it is the strike layer. When the failure strain is held fixed for both arrangements, the largest effect of interference compared to no interference is seen at $A_{\mathrm{d}, \mathrm{k}} \approx 0.16$ for $\epsilon_{\mathrm{s}, \text { fail }}=0.045$ and $\epsilon_{\mathrm{k}, \text { fail }}=0.036$. In this case the $V_{50}$ velocities for the interfering and noninterfering arrangement of the layers are 224 versus $289 \mathrm{~m} / \mathrm{s}$. Also, for a fixed $A_{d, k}\left(A_{d, k}=0.20\right.$, for 


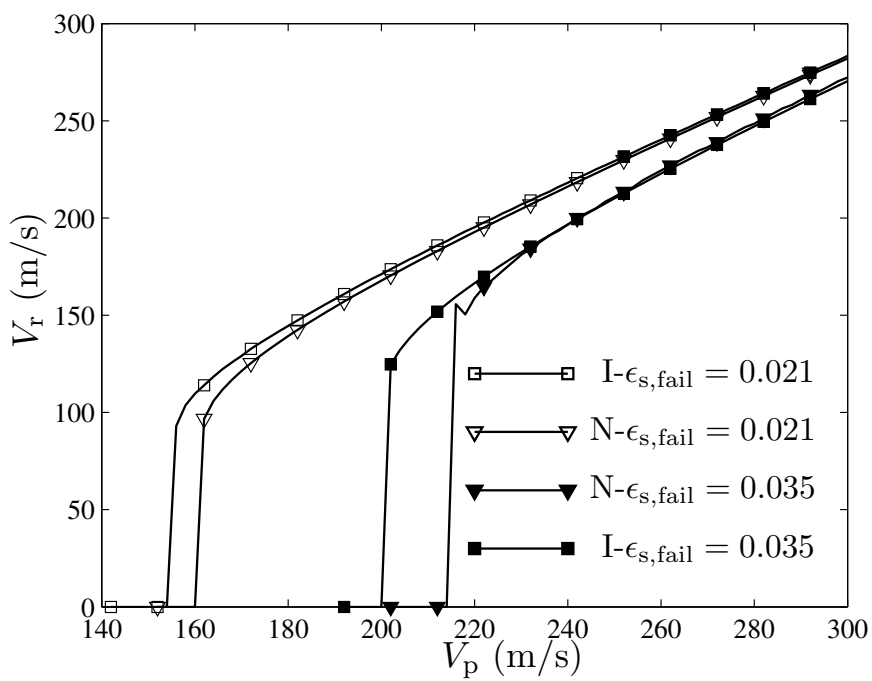

Figure 4. $V_{\mathrm{r}}$ versus $V_{\mathrm{p}}$ curves for a two-layered hybrid system consisting of Kevlar $\left(A_{\mathrm{d}, \mathrm{k}}=0.29 \mathrm{~kg} / \mathrm{m}^{2}\right)$ and Spectra $\left(A_{\mathrm{d}, \mathrm{k}}=0.18 \mathrm{~kg} / \mathrm{m}^{2}\right)$ layers. The failure strain of the Kevlar is $\epsilon_{\mathrm{k}, \text { fail }}=0.036$ for all cases. In legends I and $\mathrm{N}$ correspond to interfering and noninterfering arrangements of the layers, respectively.

example) and different failure strains, there are transitions between the situations in which interference degrades performance versus enhances performance, though only modestly. At these transitions, the sequential order in which the layers fail often reverses. The locations of these transitions depend on the relative failure strains of the layers. This is so because the ability a layer to withstand the load without penetration, for a given projectile impact velocity, depends not only on its own failure strain but also on contribution of other layers in decelerating the projectile before they are penetrated.

\section{Conclusions}

This paper is the third in the series of papers we have written to model the performance of multilayered fibrous soft body armor systems analytically. Here, we make an attempt to explain the experimental results of Cunniff [1992] using typical mechanical properties for the Kevlar and Spectra layers. The only fitting parameter used in the calculations is $\theta=1.3$ to reflect an expanded plug of fabric in the initial momentum exchange.

\section{Appendix A: Belt-over-pulley analogy}

Figure 6 shows a tensioned belt traveling over a pulley at a speed $c$. In the case of a membrane this would be a section of the membrane traveling over a ring in the plane of the membrane. Let us consider a small element $\mathrm{BO}^{\prime} \mathrm{B}^{\prime}$ of this belt that subtends an angle $d \phi$ such that $\sin d \phi \approx d \phi$ at the center of the pulley. This element experiences three different forces. The first is the tensile force from the belt, which is approximately the same at each end of the element. The resultant of these two tensile forces acts in 


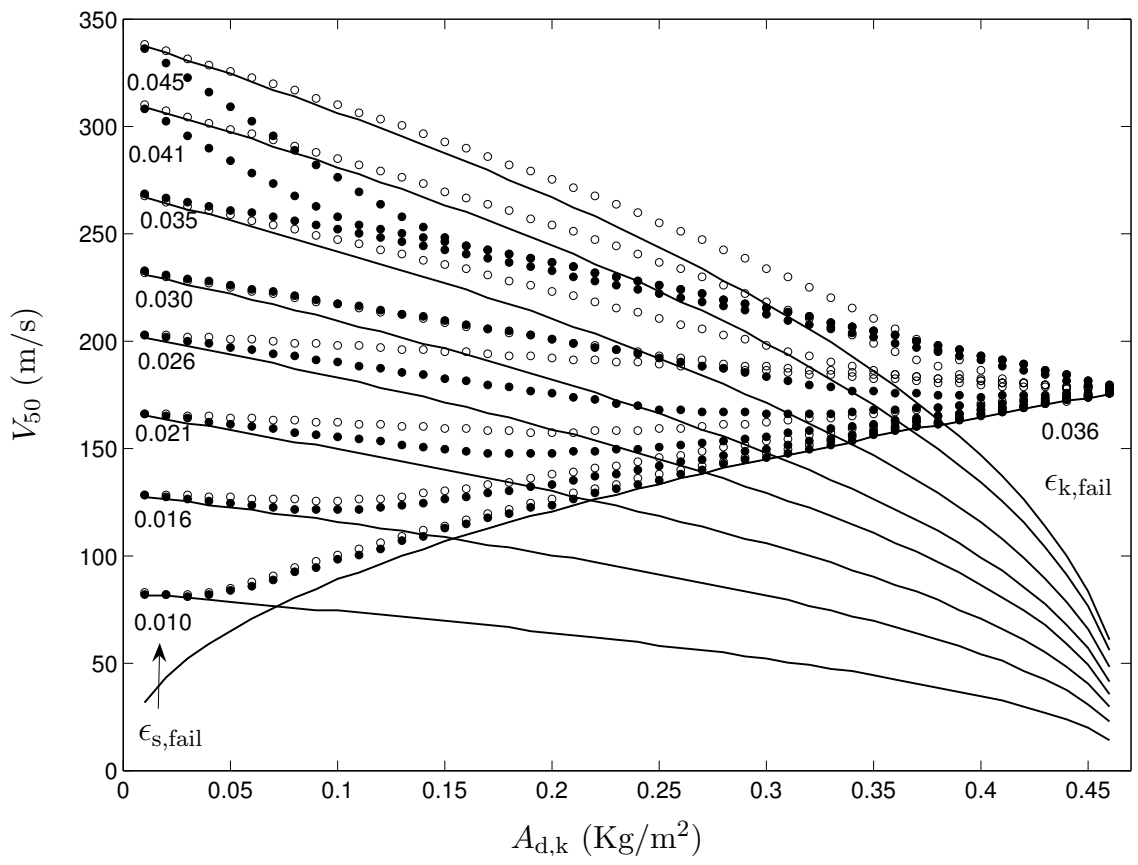

Figure 5. $V_{50}$ velocity of the hybrid (Kevlar and Spectra) armor system versus areal density of the Kevlar layer, $A_{\mathrm{d}, \mathrm{k}}$, for various failure strain combinations for the constituent layers. The total system areal density is $A_{\mathrm{d}}=A_{\mathrm{d}, \mathrm{k}}+A_{\mathrm{d}, \mathrm{s}}=0.47 \mathrm{~kg} / \mathrm{m}^{2}$. The solid and hollow circular markers correspond to interfering and noninterfering arrangements of the layers, respectively. The solid lines correspond to the $V_{50}$ velocity of the individual Kevlar and Spectra layers.
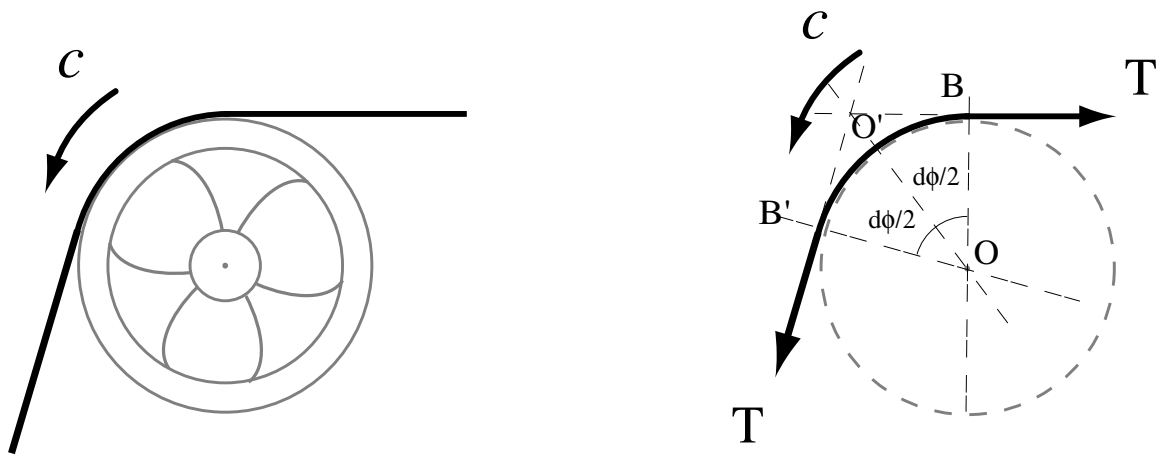

Figure 6. Illustration of the belt-over-pulley analogy. 
the $\mathrm{O}^{\prime} \mathrm{O}$ direction, which bisects the angle subtended by the element, and has a magnitude

$$
T_{\mathrm{O}^{\prime} \mathrm{O}}=2 T \sin d \phi / 2 \approx T d \phi=A E_{\mathrm{f}} \epsilon_{\mathrm{c}} d \phi,
$$

where $A$ is the cross-sectional area of the belt, $E_{\mathrm{f}}$ is the Young's modulus of the belt, and $\epsilon_{\mathrm{c}}$ is the longitudinal tensile strain in the belt at the point of contact with the pulley, that is, point $\mathrm{O}^{\prime}$. The second force is the centrifugal force that the element experiences because of its motion on a circular path at speed $c$. The centrifugal force acts in the $\mathrm{OO}^{\prime}$ direction, and its magnitude is given by $F_{\mathrm{c}}=\rho A c^{2} d \phi$, where $\rho$ is the density of the belt material. A contact force between the belt element and the pulley is the third force that acts on the element. For the purpose of our problem we can assume the contact between the belt and the pulley to be frictionless, as explained later. Under this assumption the contact force will act in a direction normal to the surface of the pulley at the contact point, that is, along $\mathrm{OO}^{\prime}$ direction. The magnitude of the normal contact force, $N$, can be obtained by considering the linear momentum balance for the element in the $\mathrm{OO}^{\prime}$ direction, which gives

$$
N=A E_{\mathrm{f}} \epsilon_{\mathrm{c}} d \phi-\rho A c^{2} d \phi .
$$

The component of the tensile force along $\mathrm{O}^{\prime} \mathrm{O}$ direction is independent of the speed $c$, whereas the centrifugal force is proportional to the square of this speed. Thus, the magnitude of the normal contact force decreases with $c$. There exists a speed at which the normal contact force becomes zero. At this speed the presence of the pulley is immaterial and the situation is equivalent to the cone wavefront in the ballistic impact problem. Furthermore, at this instance of zero normal contact force, the frictional forces will be zero for both the frictional and frictionless contact between the pulley and the belt.

Thus from Equation (A.2), with $N=0$, we have

$$
A E_{\mathrm{f}} \epsilon_{\mathrm{c}}=\rho A c^{2},
$$

or

$$
\epsilon_{\mathrm{c}}={\frac{c^{2} \rho}{E_{\mathrm{f}}}}=\left(\frac{c}{a_{0}}\right)^{2}=\alpha^{2},
$$

where $a_{0}=\sqrt{E_{\mathrm{f}} / \rho}$ is the tensile wave velocity in the belt and $\alpha=c / a_{0}$. Equation (A.4) gives the relationship between the strain in the belt at the cone wavefront, the cone wave velocity, and the tension wave velocity in the impact problem as obtained by Phoenix and Porwal [2003]. One thing to note is that in the impact problem, the velocity $c$ is not known exactly and hence the strain calculated using Equation (A.4) will be an approximation to the actual strain.

Of interest to the two-layered hybrid armor system is the case of two stacked belts passing over the pulley. In this case the top belt can press the bottom belt against the pulley even when the bottom belt has insufficient tension to maintain contact when by itself. This is the situation that arises in the case of interference. In this case, when the speed is high enough for the contact force between the bottom belt and pulley to vanish, Equation (A.3) can be modified to give

$$
A_{\mathrm{s}} E_{\mathrm{s}} \bar{\epsilon}_{\mathrm{cs}}+A_{\mathrm{k}} E_{\mathrm{k}} \bar{\epsilon}_{\mathrm{ck}}=\bar{\rho} \bar{A} \tilde{\bar{c}}^{2},
$$

where the left hand side is the component of tensile forces in the pair of stacked belt elements along the $\mathrm{O}^{\prime} \mathrm{O}$ direction, and the right hand side is the centrifugal force experienced by the same. The symbols have the usual meanings. 


\section{References}

[Billon and Robinson 2001] H. H. Billon and D. J. Robinson, "Model for the ballistic impact of fabric armour", International Journal of Impact Engineering 25:4 (2001), 411-422.

[Chocron-Benloulo et al. 1997] I. S. Chocron-Benloulo, J. Rodriguez, and V. Sanches-Gelvez, "A simple analytical model to simulate textile fabric ballistic impact behaviour", Textile Research Journal 67:7 (1997), 520-528.

[Cunniff 1992] P. M. Cunniff, "An analysis of the system effects in woven fabrics under ballistic impact", Textile Research Journal 62:9 (1992), 495-509.

[Cunniff 1999a] P. M. Cunniff, "Decoupled response of textile body armor", pp. 814-821 in Proceedings of 18th International Symposiam of Ballistics, 1999.

[Cunniff 1999b] P. M. Cunniff, "Dimensional parameters for optimization of textile-based body armor systems", pp. 13031310 in Proceedings of 18th International Symposiam of Ballistics, 1999.

[Cunniff 1999c] P. M. Cunniff, "Vs-Vr relationships in textile system impact", pp. 814-822 in Proceedings of 18th International Symposiam of Ballistics, 1999.

[Hearle et al. 1981] J. W. S. Hearle, C. M. Leech, A. Adeyefa, and C. R. Cork, "Ballistic impact resistance of multi-layer textile fabrics. Final Technical Report", Technical report AD-A128064, University of Manchester Institute of Science and Technology, 1981.

[Hearle et al. 1984] J. W. S. Hearle, C. M. Leech, and C. R. Cork, “Textile ballistic performance (Data Base). Final Technical Report”, Technical report AD-A143249, European Research Office, United States Army, 1984.

[Parga-Landa and Hernandez-Olivers 1995] B. Parga-Landa and F. Hernandez-Olivers, "An analytical model to predict impact behaviour of soft body armors", International Journal of Impact Engineering 16:3 (1995), 455-466.

[Phoenix and Porwal 2003] S. L. Phoenix and P. K. Porwal, "A new membrane model for the ballistic impact response and $V_{50}$ performance of multi-ply fibrous system”, International Journal of Solids and Structures 40 (2003), 6723-6765.

[Porwal and Phoenix 2005] P. K. Porwal and S. L. Phoenix, "Modeling of system effects in ballistic impact into multi-layered fibrous structures for soft body armors", International Journal of Fracture 135:1-4 (2005), 219-251.

[Roylance et al. 1973] D. Roylance, A. Wilde, and G. Tocci, "Ballistic impact of textile structures", Textile Research Journal 43:1 (1973), 34-41.

[Roylance et al. 1995] D. Roylance, P. Hammas, J. Ting, H. Chi, and B. Scott, "Numerical modeling of fabric impact. high strain rate effects on polymer, metal and ceramic matrix composite and other advanced materials", ASME 48 (1995), 155-160.

[Taylor Jr. and Vinson 1989] W. J. Taylor Jr. and J. R. Vinson, "Modeling ballistic impact into flexible materials", American Institute of Aeronautics and Astronautics Journal 28:12 (1989), 2098-2103.

[Zohdi 2002] T. I. Zohdi, "Modeling and simulation of progressive penetration of multilayered ballistic fabric shielding", Computational Mechanics 29 (2002), 61-67.

[Zohdi and Powell 2006] T. I. Zohdi and D. Powell, "Multiscale construction and large-scale simulation of structural fabric undergoing ballistic impact", Computer Methods in Applied Mechanics and Engineering 195:1-3 (2006), 94-109.

Received 28 Mar 2007. Revised 31 Jul 2007. Accepted 1 Aug 2007.

PANKAJ Kumar Porwal: pporwal@iitb.ac.in

227 Department of Civil Engineering, Indian Institute of Technology, Powai, Mumbai 400076, India

http://www.civil.iitb.ac.in/ pporwal

StUART Leigh Phoenix: slp6@cornell.edu

Department of Theoretical and Applied Mechanics, 321 Thurston Hall, Cornell University, Ithaca, NY 14853,

United States

http://www.tam.cornell.edu/Phoenix 1.html 


\title{
A FINITE ELEMENT FOR DYNAMIC ANALYSIS OF A CYLINDRICAL ISOTROPIC HELICAL SPRING
}

\author{
Mohamed Taktak, Fakhreddine Dammak, Said Abid and Mohamed Haddar
}

This paper presents a finite element for the dynamic analysis of the cylindrical isotropic helical spring. The hybrid-mixed formulation is used to compute the stiffness matrix. A simple approach is used to calculate the mass matrix. These matrices are used for solving the dynamic equation of the spring to calculate natural frequencies and the dynamic response of a simple or an assembled spring for different types of cross-section.

\section{Introduction}

The helical spring is one of fundamental mechanical elements used in various industrial applications such as balances, brakes, clutch, and valves. The investigation of its vibratory behavior in order to find its natural resonant frequencies permits a better conception of different dynamic conditions. The analysis of this type of element is complex due to the presence of bending, stretching, coupling, the effects of shear strain and the rotatory inertia, as well as the shape complexity of its structure. Neglecting one of these parameters to simplify the solution gives wrong results and erroneous frequencies. Since investigations in this area began in the 19th century with Michell [1890], researchers have predominantly investigated two aspects of springs.

The first field is the vibratory behavior of charged springs with purely axial compression or under compression and torsion [Haringx 1949; Pearson 1982; Becker and Cleghorn; 1992; 1993; 1994; Chassie et al. 1997; 2002]. Other investigations in this field are concentrated on the stability of this kind of structure after calculation of resonant frequencies. Mottershead [1982] and Pearson [1982] obtain governing equations by summing forces and moments on an element of the spring. Tabarrok and Xiong [1989; 1992] and Xiong and Tabarrok [1992] developed a finite element for the vibration and buckling of curved and twisted rods under loads, giving results which agree well with those given by Chassie et al. [1997].

The second field of research is the study of unloaded springs. In this study, many techniques are used to analyze the problem. The experimental method is used to determinate natural frequencies of the spring as in [Lin and Pisano 1987] and [Mottershead 1980], but these studies show the difficulty of finding these frequencies because they are close each to other, especially for higher modes. Other techniques are used to solve this problem, such as the analytical method. Wahl [1963] determined axial and torsional modes of cylindrical helical springs, but that approach is valid only for circular cross-sections with a small helix angle, and does not give realistic results, particularly for high frequencies. Pietra and Valle [1982] improve the last model by taking into account the effect of helix angle. Their model gives acceptable

Keywords: helical spring, hybrid mixed formulation, natural frequencies, dynamic response. 
results. Other work also uses the analytical method [Kagawa 1968; Philips and Castello 1972; Castello 1975; Pietra 1976; Guido et al. 1978]. Recent research is now based on the transfer matrix and stiffness matrix methods to solve the free vibration problem [Haktanir 1994; Yildirim; 1995; 1996; 1997; 1999a; 1999b; Lee and Thompson 2001]. These methods take into account the axial and shear strain and rotary inertia and give good results with less error than other approaches.

The research presented above is limited to academic applicactions; results can not be used directly by engineers in the phase of spring design. In fact, in practical problems the spring is not alone, but is in assembly with other types of mechanisms. To get reasonably good results for those problems, a large number of terms will have to be used and getting them may not always be easy; thus analytical methods are restored for the study of the simple spring behavior. To solve this problem, the finite element method is often used [Mottershead 1982; 1980; Sawanbori and Fukushima 1983; 1983; Pearson and Wittrick 1986]. This method is an approximate technique and obtains a solution for specific problems and is characterized by its versatility and capacity to solve practical problems found in engineering. It can easily model the behavior of a spring in a complex mechanism with minimum of calculation. Examples of springs in different boundary cases (such as fixed-fixed and fixed-free) and for various numbers of parameters (such as number of coils and helix angle) are studied. Results given by this model are close to those determined by analytical and experimental methods. After treating a static study of the spring [Taktak et al. 2005b] and a stress analysis [Dammak et al. 2005], in which we presented the method of determination of the stiffness matrix of the structure, we present in this paper a method for computing the mass matrix and solve the dynamic equation. These methods were presented in previous communications [Abid et al. 2005; Taktak et al. 2005a] in the case of a single spring, the first of which presented the dynamic behavior of a single helical spring and the second was a parametric study of geometrical and mechanical proprieties effects on the natural frequencies of the helical spring. The aim of this paper is to present a finite element which permits the reduction of the number of elements needed to study the structure that can be used with other finite elements in cases where the spring is assembled with others structures. To validate the developed element, natural frequencies and the dynamic response of single and assembled springs calculated by this element are presented in comparison with results given by a three-dimensional elastic beam finite element.

\section{Nomenclature}

$\begin{array}{ll}{\left[M_{T}\right]} & \text { Total mass matrix } \\ {\left[K_{T}\right]} & \text { Total stiffness matrix } \\ {\left[M_{G}\right]} & \text { Element global mass matrix } \\ {\left[K_{G}\right]} & \text { Element global stiffness matrix } \\ \{X\} & \text { Global displacements vector } \\ \{U\} & \text { Eigen vector } \\ \omega & \text { Eigen pulsation } \\ t & \text { Time } \\ {\left[C_{T}\right]} & \text { Damping matrix } \\ {\left[F_{T}\right]} & \text { External forces vector } \\ {[\Phi]} & \text { Modal matrix } \\ n & \text { Number of eigen modes }\end{array}$

$\delta e_{t}$
$\delta \gamma_{t n, t b}$
$\delta \chi_{t, n, b}$
$\langle\delta \varepsilon\rangle$
$N$
$T_{n}, T_{b}$
$M_{t}$
$M_{n}, M_{b}$
$\langle R\rangle$
$\sigma_{t, t b, t n}$
$E$
$G$

Virtual membrane strain

Virtual shear strain

Virtual strains

Generalized virtual strains vector

Normal force

Shearing forces

Torsional moment

Bending moments

Resulting forces vector

Stress tensor components

Young's module

Shearing's module 


$\begin{array}{llll}\{a(t)\} & \text { Generalized displacements vector } & A & \text { Section area } \\ {\left[M_{m}\right]} & \text { Generalized mass matrix } & J & \text { Inertia of torsion } \\ {\left[K_{m}\right]} & \text { Generalized stiffness matrix } & I_{y}, I_{z} & \text { Central quadratic moments } \\ {\left[C_{m}\right]} & \text { Generalized damping matrix } & k_{y}, k_{z} & \text { Shears correction factors } \\ \left\{F_{m}\right\} & \text { Generalized forces vector } & {[H]} & \text { Elastic behavior Matrix } \\ \omega_{i} & \text { Eigen pulsation } & \Pi & \text { Mixed functional of energy } \\ \xi_{i} & \text { Reduced damping coefficient } & \Pi_{e x t}^{e} & \text { External energy element functional } \\ X, Y, Z & \text { Global coordinate system } & \Pi_{\text {int }}^{e} & \text { internal energy element functional } \\ \vec{I}, \vec{J}, \vec{K} & \text { Global basis } & W_{\text {int }}^{e} & \text { Internal work } \\ s, y, z & \text { Local coordinate system } & e & \text { Numbers of elements } \\ \vec{t}, \vec{n}, \vec{b} & \text { Local basis } & \{f\} & \text { Distributed forces Vector } \\ s & \text { Curvilinear axis } & \langle u\rangle & \text { Displacements vector } \\ p & \text { Point belonging to the axis } " s & \{F\} & \text { Concentrated forces vector } \\ u, v, w & \text { Local displacements } & \left\langle u_{n}\right\rangle & \text { Element degrees of freedom vector } \\ \theta_{t}, \theta_{n}, \theta_{b} & \text { Local rotations } & {[P]} & \text { Approximation matrix } \\ U, V, W & \text { Global displacements } & \left\{\alpha_{n}\right\} & \text { Discontinuous parameters vector } \\ \Theta_{t}, \Theta_{n}, \Theta_{b} & \text { Global rotations } & {[A]} & \text { Binodal approximation matrix } \\ \vec{X} & \text { Position vector } & {[k]} & \text { Local stiffness matrix } \\ r & \text { Helix radius } & W_{\text {inertial }}^{e} & \text { Element inertial work } \\ P & \text { Helix Pitch } & \rho_{0} & \text { Mass per unit volume } \\ \theta & \text { Polar angle } & L & \text { Curvilinear length } \\ N_{s} & \text { Number of spires } & {[m]} & \text { Local mass matrix } \\ R & \text { Radius of curvature } & \xi & \text { Parametric variable } \\ T & \text { Radius of torsion } & \Delta \theta & \text { Difference of nodal angles } \\ q & \text { Point belonging to the beam } & {\left[K_{G}\right]} & \text { Global stiffness matrix } \\ \delta u, \delta v, \delta w & \text { Virtual displacements } & {\left[M_{G}\right]} & \text { Global mass matrix } \\ \delta \theta_{t, n, b} & \text { Virtual rotations } & {[T]} & \text { Transfer matrix } \\ \delta \varepsilon_{t} & \text { Axial virtual strain } & {\left[Q_{i}\right]} & \text { Rotation matrix } \\ \delta \gamma_{n}, b & \text { Virtual transverse shear strains } & & \\ & & & \\ & & & \end{array}$

\section{Dynamic analysis}

3.1. Modal analysis. The calculation of natural frequencies and modes is made by the resolution of the matrix system

$$
\left[M_{T}\right]\{\ddot{X}\}+\left[K_{T}\right]\{X\}=\{0\},
$$

where $\left[M_{T}\right]$ is the total mass matrix, $\left[K_{T}\right]$ is the total stiffness matrix. These matrices are obtained by an assembly of element matrices in the global coordinate system. $\left[M_{G}\right]$ and $\left[K_{G}\right]$ are defined in the following sections. $\{X\}$ is the global nodal displacements vector. For a harmonic solution having the expression

$$
\{X\}=\{U\} \exp (i \omega t)
$$

$\{U\}$ is the eigenvector and $\omega$ is the eigen pulsation $\left(\mathrm{rad} \mathrm{s}^{-1}\right)$. Equation (3-1) is reduced to the general eigenvalue problem

$$
\left(\left[K_{T}\right]-\omega^{2}\left[M_{T}\right]\right)\{U\}=\{0\} .
$$


This eigen problem can be solved with one of many methods which exist in the literature, such as subspace iteration.

3.2. Dynamic response: method of modal superposition. The equation of movement of the system is written as

$$
\left[M_{T}\right]\{\ddot{U}\}+\left[C_{T}\right]\{\dot{U}\}+\left[K_{T}\right]\{U\}=\left\{F_{T}\right\},
$$

where $\left[C_{T}\right]$ is the damping matrix and $\left\{F_{T}\right\}$ is the vector of external forces. The description of the movement of a system with several degrees of freedom can be made by its spatial coordinates or by its modal coordinates. The movement's equation of the structure without a second member admits a linear base of orthogonal real modes of the nondamped system. These eigen modes are characterized by the eigen pulsations $\omega$ and also by their eigenvectors $\left\{U_{i}\right\}$. We define the modal matrix [ $\left.\Phi\right]$ by

$$
[\Phi]=\left[\left\{U_{1}\right\},\left\{U_{2}\right\},\left\{U_{3}\right\}, \ldots,\left\{U_{n}\right\}\right],
$$

where $n$ is the number of considered eigen modes to describe the movement of the system. The projection of the movement's equation on the modal basis leads to a system of $n$ equations coupled only by the damping matrix. The equation of the movement according to the generalized parameters is written as

$$
\left[M_{m}\right]\{\ddot{a}(t)\}+\left[C_{m}\right]\{\dot{a}(t)\}+\left[K_{m}\right]\{a(t)\}=\left\{F_{m}\right\},
$$

where $a(t)$ is the generalized displacements vector defined as $\{U(t)\}=[\Phi]\{a(t)\},\left[M_{m}\right]$ is the generalized mass matrix $\left[M_{m}\right]=[\Phi]^{t}\left[M_{T}\right][\Phi]=\operatorname{diag}\left(m_{i}\right)$, and $\left[K_{m}\right]$ is the generalized stiffness matrix $\left[K_{m}\right]=$ $[\Phi]^{t}\left[K_{T}\right][\Phi]=\operatorname{diag}\left(m_{i} \omega_{i}^{2}\right) . \omega_{i}$ are the eigen pulsations of each mode. The actions of damping are small. The matrix $\left[C_{m}\right]$ is obtained by adopting a reduced modal damping coefficient on each eigen mode [Dahtt and Touzout 1984], as in $\left[C_{m}\right]=\operatorname{diag}\left(2 m_{i} \omega_{i} \xi_{i}\right)$, where $\xi_{i}$ is the reduced modal damping coefficient. $\left[F_{m}\right]$ is the vector of the generalized forces

$$
\left\{F_{m}\right\}=[\Phi]^{t}\left\{F_{T}\right\}=\left\{\begin{array}{c}
f_{1} \\
\vdots \\
f_{i} \\
\vdots \\
f_{n}
\end{array}\right\} .
$$

The matrices $\left[K_{m}\right],\left[M_{m}\right]$ and $\left[C_{m}\right]$ are diagonal, so we obtain a system of uncoupled $n$ oscillators with one degree of freedom for each. The equation of movement of each oscillator is written as

$$
m_{i} \ddot{a}_{i}+2 m_{i} \xi_{i} \omega_{i} \dot{a}_{i}+m_{i} \omega_{i}^{2} a_{i}=f_{i}, \quad i=1,2, \ldots, n .
$$

The advantage of this modal description is that it simplifies the resolution of the movement's equations, reducing them to a linear system of $n$ completely uncoupled equations.

\section{Finite element formulation}

4.1. Geometric presentation. A spring's beam is a three-dimensional curved beam defined in the global coordinate system $(O, X, Y, Z)$ of the basis $(\vec{I}, \vec{J}, \vec{K})$. This beam is generated by a succession of plane domains which are orthogonal to the middle fiber of the structure $s$. The dimensions of those domains 
are small in comparison to the beam's length. Two geometric hypotheses are taken. The first is that the orthogonal sections are identical along the curvilinear axis. The second is that studied beams have a full section. The position vector of any point $p$ belonging to the middle fiber of the spring's beam is defined in the global coordinate system by

$$
\vec{X}_{p}=\left\{\begin{array}{c}
r \cos \theta \\
r \sin \theta \\
\frac{P}{2 \pi} \theta
\end{array}\right\}_{(\vec{I}, \vec{J}, \vec{K})},
$$

where $r$ and $P$ are respectively the radius and pitch of the helix and $\theta$ is the polar angle. This angle is defined as

$$
\theta=2 \pi N_{s},
$$

where $N_{s}$ is the number of spires of the spring. The curvilinear coordinate $s$ is related to this angle by the relation

$$
d s=\rho d \theta \quad \rho=\sqrt{r^{2}+\left(\frac{P}{2 \pi}\right)^{2}} .
$$

Vectors of the local coordinate system, which are related to the point $p$, are defined as the tangential $\vec{t}$, the normal $\vec{n}$ and the binormal $\vec{b}$. The corresponding expression of each vector is

$$
\vec{t}=\frac{1}{\rho}\left\{\begin{array}{c}
-r \sin \theta \\
r \cos \theta \\
\frac{P}{2 \pi}
\end{array}\right\}_{(\vec{I}, \vec{J}, \vec{K})}, \quad \vec{n}=\left\{\begin{array}{c}
-\cos \theta \\
-\sin \theta \\
0
\end{array}\right\}_{(\vec{I}, \vec{J}, \vec{K})}, \quad \vec{b}=\frac{1}{\rho}\left\{\begin{array}{c}
\frac{P}{2 \pi} \sin \theta \\
-\frac{P}{2 \pi} \cos \theta \\
r
\end{array}\right\}_{(\vec{I}, \vec{J}, \vec{K})},
$$

where the radius of curvature $R$ of this helical beam defined as $R=\frac{\rho^{2}}{r}$ and the radius of torsion $T$ is $T=\rho^{2} \frac{2 \pi}{P}$. Figure 1 shows different parameters presented thus far.

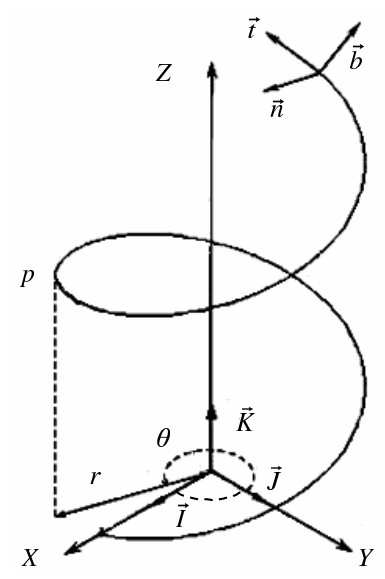

Figure 1. Geometric description of the helical spring and its two bases. 
4.2. Kinematic presentation. To determine the displacement's field, two hypotheses are used. First, Timoshenko's hypothesis that the shear effect is not neglected and the strain of the helical beam takes place such that cross section remains planar. Second, the St. Venant hypothesis that the torsion is uniform along the beam and all sections undergo the same warping. Then the axial stress resultants and distortions due to the moment of torsion are null. The position vector of a point $q$, different from $p$ in the section of the wire, is defined in local coordinate system as

$$
\vec{X}_{q}=\vec{X}_{p}+\vec{h}, \quad \vec{h}=\left\{\begin{array}{c}
0 \\
y \\
z
\end{array}\right\}_{(\vec{t}, \vec{n}, \vec{b})} .
$$

For the point $p$, the vector of the virtual displacements in the local coordinate system is expressed as

$$
\delta \vec{u}_{p}=\left\{\begin{array}{c}
\delta u \\
\delta v \\
\delta w
\end{array}\right\}_{(\vec{t}, \vec{n}, \vec{b})},
$$

where $\delta u, \delta v$, and $\delta w$ are the displacements parallel to the $\vec{t}, \vec{n}$ and $\vec{b}$ axes. The vector of the virtual displacements of the point $q$ is given by the expression

$$
\delta \vec{u}_{q}=\delta \vec{u}_{p}+\left\{\begin{array}{l}
\delta \theta_{t} \\
\delta \theta_{n} \\
\delta \theta_{b}
\end{array}\right\}_{(\vec{t}, \vec{n}, \vec{b})} \wedge \vec{h},
$$

where $\delta \theta_{t}, \delta \theta_{n}$, and $\delta \theta_{b}$ are respectively the rotations of the point $p$ around the $\vec{t}, \vec{n}$ and $\vec{b}$ axes. The expression of the vector of virtual displacements of the point $q$ in the local coordinate system $(\vec{t}, \vec{n}, \vec{b})$ is expressed as

$$
\delta \vec{u}_{q}=\left\{\begin{array}{c}
\delta u+z \delta \theta_{n}-y \delta \theta_{b} \\
\delta v-z \delta \theta_{t} \\
\delta w+y \delta \theta_{t}
\end{array}\right\}_{(\vec{t}, \vec{n}, \vec{b})} .
$$

The virtual displacement field in Equation (4-6) generates three components of virtual strain in any point of the beam, written as

$$
\begin{aligned}
& \delta \varepsilon_{t}=\delta \varepsilon_{t t}=\left(1-\frac{y}{R}\right)^{-1}\left(\delta e_{t}-y \delta \chi_{b}+z \delta \chi_{n}\right), \\
& \delta \gamma_{n}=2 \delta \varepsilon_{n t}=\left(1-\frac{y}{R}\right)^{-1}\left(\delta \gamma_{t n}-z \delta \chi_{t}\right), \\
& \delta \gamma_{b}=2 \delta \varepsilon_{b t}=\left(1-\frac{y}{R}\right)^{-1}\left(\delta \gamma_{t b}+y \delta \chi_{t}\right),
\end{aligned}
$$

where $\delta \varepsilon_{t}$ is the axial virtual strain and $\delta \gamma_{n}$ and $\delta \gamma_{b}$ are the virtual transverse shear strains.

$$
\begin{array}{lll}
\delta e_{t}=\frac{d(\delta u)}{d s}-\frac{\delta v}{R}, & \delta \gamma_{t n}=\frac{d(\delta v)}{d s}+\frac{\delta u}{R}-\frac{\delta w}{T}-\delta \theta_{b}, & \delta \gamma_{t b}=\frac{d(\delta w)}{d s}-\frac{\delta v}{T}+\delta \theta_{n}, \\
\delta \chi_{t}=\frac{d\left(\delta \theta_{t}\right)}{d s}-\frac{\delta \theta_{n}}{R}, & \delta \chi_{n}=\frac{d\left(\delta \theta_{n}\right)}{d s}+\frac{\delta \theta_{t}}{R}-\frac{\delta \theta_{b}}{T}, & \delta \chi_{b}=\frac{d\left(\theta_{b}\right)}{d s}-\frac{\delta \theta_{n}}{R} .
\end{array}
$$


$\delta e_{t}$ is the virtual membrane strain, $\delta \gamma_{t n}$ and $\delta \gamma_{t b}$ are the virtual shear strains, $\delta \chi_{t}$ is the virtual strain due to the effect of torsion, and $\delta \chi_{n}$ and $\delta \chi_{b}$ are respectively the virtual curvatures in the planes $(s, z)$ and $(y, s)$. These strains constitute the components of the generalized virtual strains vector

$$
\langle\delta \varepsilon\rangle=\left\langle\begin{array}{llllll}
\delta e_{t} & \delta \gamma_{t n} & \delta \gamma_{t b} & \delta \chi_{t} & \delta \chi_{n} & \delta \chi_{b}
\end{array}\right\rangle
$$

The system of equilibrium equations for the helical beam is written [Batoz and Dhatt 1993] in the local coordinate system as

$$
\left\{\begin{array} { l } 
{ \frac { d N } { d \theta } - a T _ { n } = 0 , } \\
{ \frac { d T _ { n } } { d \theta } + a N + b T _ { b } = 0 , } \\
{ \frac { d T _ { b } } { d \theta } - b T _ { n } = 0 , }
\end{array} \quad \left\{\begin{array}{l}
\frac{d M_{t}}{d \theta}-a M_{n}=0 \\
\frac{d M_{n}}{d \theta}+a M_{t}+b M_{b v}-\rho T_{b}=0, \\
\frac{d M_{b}}{d \theta}-b M_{n}+\rho T_{n}=0,
\end{array}\right.\right.
$$

where $a=\frac{r}{\rho}$ and $b=-\frac{P}{2 \pi \rho}$. $N$ is the normal force, $T_{n}$ and $T_{b}$ the normal and binormal shearing forces, $M_{t}$ the torsional moment, and $M_{n}$ and $M_{b}$ the bending moments around the normal and binormal axis. These resulting forces are defined as

$$
\begin{aligned}
& N=\int_{A} \sigma_{t} d A, \quad T_{n}=\int_{A} \sigma_{t n} d A, \quad T_{b}=\int_{A} \sigma_{t b} d A \\
& M_{n}=\int_{A} z \sigma_{t} d A, \quad M_{b}=\int_{A}-y \sigma_{t} d A, \quad M_{t}=\int_{A}\left(y \sigma_{t b}-z \sigma_{t n}\right) d A,
\end{aligned}
$$

with $d A=d y d z$. They constitute the components of the vector of generalized forces which correspond to the resulting forces

$$
\langle R\rangle=\left\langle\begin{array}{llllll}
N & T_{n} & T_{b} & M_{t} & M_{n} & M_{b}
\end{array}\right\rangle
$$

4.3. Constitutive relation. The spring has an isotropic material behavior, in which stresses are linearly related to the strains by the constitutive relations

$$
\sigma_{t}=E \varepsilon_{t}, \quad \sigma_{t n}=G \gamma_{n}, \quad \sigma_{t b}=G \gamma_{b},
$$

where $E$ is the Young's modulus and $G$ is the shear modulus of the material. The principal axes of inertia are assumed to be coincident with the local coordinate system. So, the resulting force vector $\{R\}$ is written $\{R\}=[H]\{\varepsilon\}$, with

$$
\langle\varepsilon\rangle=\left\langle\begin{array}{llllll}
e_{t} & \gamma_{t n} & \gamma_{t b} & \chi_{t} & \chi_{n} & \chi_{b}
\end{array}\right\rangle, \quad[H]=\operatorname{diag}\left(H_{m}, H_{c n}, H_{c b}, H_{t}, H_{f n}, H_{f b}\right),
$$

where $[H]$ is the matrix of the elastic comportment. Its components are given by

$$
\begin{aligned}
& H_{m} \simeq E A, \quad H_{t} \simeq G J, \quad H_{f n} \simeq E I_{y}, \\
& H_{f b} \simeq E I_{z}, \quad H_{c n} \simeq k_{y} G A, \quad H_{c b} \simeq k_{z} G A \text {, }
\end{aligned}
$$

where $A$ is the area of the section, $J$ the inertia of torsion, $I_{y}$ and $I_{z}$ the central quadratic moments regarding $(p, y)$ and $(p, z)$ axes, and $k_{y}$ and $k_{z}$ are the shear correction factors for $y$ and $z$ axis. 
4.4. The mixed formulation. The mixed functional of energy associated to the equilibrium equations is expressed by Batoz and Dhatt [1993] as

$$
\Pi=\sum_{e}\left(\Pi_{\mathrm{int}}^{e}-\Pi_{e x t}^{e}\right)
$$

where

$$
\left.\Pi_{\mathrm{int}}^{e}=\int_{0}^{L}\left(-\frac{1}{2}\langle R\rangle[H]^{-1}\{R\}+\langle\varepsilon\rangle\{R\}\right) d s\right), \quad \Pi_{\text {ext }}^{e}=\int_{0}^{L}\langle u\rangle\{f\} d s+(\langle u\rangle\{F\})_{S} .
$$

$\Pi_{\text {int }}^{e}$ is the element functional energy of internal forces, and $\Pi_{e x t}^{e}$ is the same for the external forces. $e$ is the number of elements, $\langle u\rangle$ is the vector of the displacements in the local coordinate system, $\{f\}$ the vector of distributed forces, $\{F\}$ is the vector of concentrated forces, and $S$ is the boundary of the wire.

4.5. Element stiffness matrix in the local coordinate system. The development of the element stiffness matrix in the local coordinate system was presented in [Taktak et al. 2005b]. In the case where an approximation of the generalized forces $\langle R\rangle$ verifies the equilibrium equations, the expression (4-15) becomes

$$
\Pi_{\mathrm{int}}^{e}=\int_{0}^{L}-\frac{1}{2}\langle R\rangle[H]^{-1}\{R\} d s+\left\langle u_{n}\right\rangle\left\{R_{n}\right\},
$$

where $\left\langle u_{n}\right\rangle$ and $\left\langle R_{n}\right\rangle$ represent respectively the vector of the degrees of freedom for the element and the vector of the nodal resulting forces

$$
\begin{aligned}
& \left\langle u_{n}\right\rangle=\left\langle\begin{array}{lllllllllllllll}
u_{1} & v_{1} & w_{1} & \theta_{t 1} & \theta_{n 1} & \theta_{b 1} & u_{2} & v_{2} & w_{2} & \theta_{t 2} & \theta_{n 2} & \theta_{b 2}
\end{array}\right\rangle \\
& \left\langle R_{n}\right\rangle=\left\langle\begin{array}{llllllllllll}
-N_{1} & -T_{n 1} & -T_{b 1} & -M_{t 1} & -M_{n 1} & -M_{b 1} & N_{2} & T_{n 2} & T_{b 2} & M_{t 2} & M_{n 2} & M_{b 2}
\end{array}\right) .
\end{aligned}
$$

The expression of internal virtual work is then written

$$
W_{\text {int }}^{e}=\int_{0}^{L}-<\delta R_{n}>[H]^{-1}\left\{R_{n}\right\} d s+<\delta u_{n}>\left\{R_{n}\right\}+<\delta R_{n}>\left\{u_{n}\right\} .
$$

The element stiffness matrix in the local coordinate system is determined using a mixed formulation where the equilibrium equations are enforced in the variational (4-18). The resolution of the equilibrium equations (4-10) permits choice for the resulting forces vector the approximation $\{R\}=[P]\left\{\alpha_{n}\right\}$, where $[P]$ is the approximation matrix of the resulting forces and $\left\{\alpha_{n}\right\}$ is the vector of the independent parameters, defined as

$$
\left\{\alpha_{n}\right\}^{T}=\left\langle\alpha_{1} \quad \alpha_{2} \quad \alpha_{3} \quad \alpha_{4} \quad \alpha_{5} \quad \alpha_{6}\right\rangle .
$$

The matrix $[P]$ is obtained by resolving the equilibrium equations while expressing the resulting forces in any point $p$ of the beam, according to the forces exerted on one of extremities expressed by $\left\{\alpha_{n}\right\}$. This matrix is given by

$$
[P]=\left[\begin{array}{cccccc}
P_{1,1} & P_{1,2} & P_{1,3} & 0 & 0 & 0 \\
-P_{1,2} & P_{2,2} & P_{2,3} & 0 & 0 & 0 \\
-P_{1,3} & P_{2,3} & P_{3,3} & 0 & 0 & 0 \\
P_{4,1} & P_{4,2} & P_{4,3} & -P_{1,1} & -P_{1,2} & -P_{1,3} \\
-P_{4,2} & P_{5,2} & P_{5,3} & P_{1,2} & -P_{2,2} & -P_{2,3} \\
-P_{4,3} & P_{5,3} & P_{6,3} & P_{1,3} & -P_{3,2} & -P_{3,3}
\end{array}\right],
$$




$$
\begin{aligned}
P_{1,1}= & a^{2} C+b, \quad P_{1,2}=a S, \\
P_{1,3}= & a b(1-C), \quad P_{2,2}=C, \\
P_{2,3}= & b S, \quad P_{3,3}=-b^{2} C-a^{2}, \\
& P_{4,1}=-2 \rho a^{2} b(1-C)+a^{2} b \rho \theta S, \\
& P_{4,2}=-a b(\rho \theta C-\rho S), \\
& P_{4,3}=-a b^{2} \rho \theta S-r\left(a^{2}+b^{2}\right)(1-C), \\
& P_{5,2}=b \rho \theta S, \\
& P_{5,3}=-b^{2} \rho \theta C-r a S, \\
& P_{6,3}=-b^{3} \rho \theta S-2 \rho b a^{2}(1-C),
\end{aligned}
$$

where $C=\cos \theta$ and $S=\sin \theta$. We define the matrix [A] joining the nodal resulting forces to the independent parameters $\alpha_{n}\left\langle R_{n}\right\rangle=\left\langle\alpha_{n}\right\rangle[A]$, expressed by $[A]=\left[-\left[P_{1}\right]^{T}\left[P_{2}\right]^{T}\right]$, where $\left[P_{1}\right]$ and $\left[P_{2}\right]$ are the approximation matrices of the resulting forces at nodes 1 and 2 of the element. We also define the matrix $[B]$ as

$$
[B]=\int_{0}^{L}[P]^{T}[H]^{-1}[P] d s .
$$

We express Equation (4-18) as

$$
W_{\text {int }}^{e}=-<\delta \alpha_{n}>[B]\left\{\alpha_{n}\right\}+<\delta u_{n}>[A]^{T}\left\{\alpha_{n}\right\}+<\delta \alpha_{n}>[A]\left\{u_{n}\right\}
$$

This leads to

$$
[A]\left\{u_{n}\right\}-[B]\left\{\alpha_{n}\right\}=0,
$$

which permits expression of the independent variables as

$$
\left\{\alpha_{n}\right\}=[B]^{-1}[A]\left\{u_{n}\right\}
$$

and the virtual internal work's expression becomes

$$
W_{\text {int }}^{e}=<\delta u_{n}>[k]\left\{u_{n}\right\},
$$

with the element stiffness matrix $[k]$ defined in the local coordinate system by

$$
[k]=[A]^{T}[B]^{-1}[A] .
$$

4.6. The element mass matrix in the local coordinate system. The term of element inertial work is written as

$$
W_{\text {inertial }}^{e}=\int_{V} \rho_{0}<\delta u_{q}>\left\{\ddot{u}_{q}\right\} d V
$$

where $\rho_{0}$ is the mass per unit volume of the spring's material, $\left\langle\delta u_{q}\right\rangle$ is the vector of virtual displacement in a point $q$ of the section, $\left\{\ddot{u}_{q}\right\}$ is the vector of acceleration at this point, and $V$ is the volume of the corresponding portion of the spring modelled by the element. 
By taking account of the field of displacement (4-7), the expression (4-28) becomes

$$
W_{\text {inertial }}^{e}=\int_{V}\left[\begin{array}{c}
\left(\delta u+z \delta \theta_{n}-y \delta \theta_{b}\right) \rho_{0}\left(\ddot{u}+z \ddot{\theta}_{n}-y \ddot{\theta}_{b}\right) \\
+\left(\delta v-z \delta \theta_{t}\right) \rho_{0}\left(\ddot{v}-z \ddot{\theta}_{t}\right) \\
+\left(\delta w+y \delta \theta_{t}\right) \rho_{0}\left(\ddot{w}+y \ddot{\theta}_{t}\right)
\end{array}\right] d V .
$$

We define the homogenized inertias as

$$
\left\{\begin{array} { l } 
{ \rho _ { m } = \int _ { A } \rho _ { 0 } ( 1 - \frac { y } { R } ) d A , } \\
{ \rho _ { 1 } = \int _ { A } \rho _ { 0 } z ( 1 - \frac { y } { R } ) d A , } \\
{ \rho _ { 2 } = \int _ { A } \rho _ { 0 } y ( 1 - \frac { y } { R } ) d A , }
\end{array} \quad \left\{\begin{array}{l}
\rho_{3}=\int_{A} \rho_{0} z^{2}\left(1-\frac{y}{R}\right) d A, \\
\rho_{4}=\int_{A} \rho_{0} y^{2}\left(1-\frac{y}{R}\right) d A, \\
\rho_{5}=\int_{A} \rho_{0} y z\left(1-\frac{y}{R}\right) d A,
\end{array}\right.\right.
$$

and the expression of $W_{\text {inertial }}^{e}$ becomes

$$
\begin{aligned}
W_{\text {inertial }}^{e}=\int_{s}\left(\delta u \left(\rho_{m} \ddot{u}\right.\right. & \left.+\rho_{1} \ddot{\theta}_{n}-\rho_{2} \ddot{\theta}_{b}\right)+\delta v\left(\rho_{m} \ddot{v}-\rho_{1} \ddot{\theta}_{t}\right)+\delta w\left(\rho_{m} \ddot{w}+\rho_{2} \ddot{\theta}_{t}\right) \\
& +\delta \theta_{t}\left(-\rho_{1} \ddot{v}+\rho_{3} \ddot{\theta}_{t}+\rho_{2} \ddot{w}+\rho_{4} \ddot{\theta}_{t}\right)+\delta \theta_{n}\left(\rho_{1} \ddot{u}+\rho_{3} \ddot{\theta}_{n}-\rho_{5} \ddot{\theta}_{b}\right) \\
& \left.+\delta \theta_{b}\left(-\rho_{2} \ddot{u}-\rho_{5} \ddot{\theta}_{n}+\rho_{4} \ddot{\theta}_{b}\right)\right) d s .
\end{aligned}
$$

The element mass matrix in the local coordinate system is obtained by discretization of the expression (4-31). We choose a linear interpolation for virtual displacements $\left(\delta u, \delta v, \delta w, \delta \theta_{t}, \delta \theta_{n}\right.$ and $\left.\delta \theta_{b}\right)$ and accelerations $\left(\ddot{u}, \ddot{v}, \ddot{w}, \ddot{\theta}_{t}, \ddot{\theta}_{n}\right.$ and $\left.\ddot{\theta}_{b}\right)$ and we follow the geometry of the spring during the integration. The expression (4-28) in the discretized form is written as

$$
\begin{aligned}
& W_{\text {inertial }}^{e}=<\delta u_{n}>[m]\left\{\ddot{u}_{n}\right\} \\
& <\delta u_{n}>=<\delta u_{1} \delta v_{1} \delta w_{1} \delta \theta_{t 1} \delta \theta_{n 1} \delta \theta_{b 1} \delta u_{2} \delta v_{2} \delta w_{2} \delta \theta_{t 2} \delta \theta_{n 2} \delta \theta_{b 2}>
\end{aligned}
$$

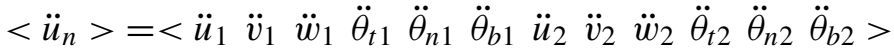

where $[m]$ is the element mass matrix in the local coordinate system.

The choice of the linear interpolation for the nodal variables is expressed as

$$
\left\{\begin{array} { l } 
{ \delta u = \delta u _ { 1 } N _ { 1 } + \delta u _ { 2 } N _ { 2 } , } \\
{ \delta v = \delta v _ { 1 } N _ { 1 } + \delta v _ { 2 } N _ { 2 } , } \\
{ \delta w = \delta w _ { 1 } N _ { 1 } + \delta w _ { 2 } N _ { 2 } , } \\
{ \delta \theta _ { t } = \delta \theta _ { t 1 } N _ { 1 } + \delta \theta _ { t 2 } N _ { 2 } , } \\
{ \delta \theta _ { n } = \delta \theta _ { n 1 } N _ { 1 } + \delta \theta _ { n 2 } N _ { 2 } , } \\
{ \delta \theta _ { b } = \delta \theta _ { b 1 } N _ { 1 } + \delta \theta _ { b 2 } N _ { 2 } , }
\end{array} \quad \left\{\begin{array}{l}
\ddot{u}=\ddot{u}_{1} N_{1}+\ddot{u}_{2} N_{2}, \\
\ddot{v}=\ddot{v}_{1} N_{1}+\ddot{v}_{2} N_{2}, \\
\ddot{w}=\ddot{w}_{1} N_{1}+\ddot{w}_{2} N_{2}, \\
\ddot{\theta}_{t}=\ddot{\theta}_{t 1} N_{1}+\ddot{\theta}_{t 2} N_{2}, \\
\ddot{\theta}_{n}=\ddot{\theta}_{n 1} N_{1}+\ddot{\theta}_{n 2} N_{2}, \\
\ddot{\theta}_{b}=\ddot{\theta}_{b 1} N_{1}+\ddot{\theta}_{b 2} N_{2},
\end{array}\right.\right.
$$

where $N_{1}=\frac{1-\xi}{2}$ and $N_{2}=\frac{1+\xi}{2}$ are the interpolation functions.

The transformation from the curvilinear variable $s$ to the parametric variable $\xi$ is done by analogy between the reference element and the real element. We suppose that $\xi$ follows a linear law according to $\theta$, as in

$$
\xi=\frac{2}{\Delta \theta} \theta-1
$$


where $\Delta \theta$ is the difference between the corresponding angles of each node, defined as

$$
\Delta \theta=\theta_{2}-\theta_{1} .
$$

The expression of element inertial work becomes

$$
W_{\text {inertial }}^{e}=\int_{0}^{L}<\delta u_{n}>[N]\left\{\ddot{u}_{n}\right\} d s=\frac{\rho \Delta \theta}{2} \int_{-1}^{1}<\delta u_{n}>[N]\left\{\ddot{u}_{n}\right\} d \xi,
$$

where $L$ is the curvilinear length of the corresponding portion of the spring modelled by the element.

$$
\begin{aligned}
& {[N]=\left[\begin{array}{ll}
{\left[N_{11}\right]} & {\left[N_{12}\right]} \\
{\left[N_{12}\right]} & {\left[N_{22}\right]}
\end{array}\right],} \\
& {\left[N_{11}\right]=\left[\begin{array}{cccccc}
N_{1}^{2} \rho_{m} & 0 & 0 & 0 & N_{1}^{2} \rho_{1} & -N_{1}^{2} \rho_{2} \\
& N_{1}^{2} \rho_{m} & 0 & -N_{1}^{2} \rho_{1} & 0 & 0 \\
& & N_{1}^{2} \rho_{m} & N_{1}^{2} \rho_{2} & 0 & 0 \\
& & & N_{1}^{2}\left(\rho_{3}+\rho_{4}\right) & 0 & 0 \\
& & & & N_{1}^{2} \rho_{3} & -N_{1}^{2} \rho_{5} \\
S y m & & & & & N_{1}^{2} \rho_{4}
\end{array}\right],} \\
& {\left[N_{22}\right]=\left[\begin{array}{cccccc}
N_{2}^{2} \rho_{m} & 0 & 0 & 0 & N_{2}^{2} \rho_{1} & -N_{2}^{2} \rho_{2} \\
& N_{2}^{2} \rho_{m} & 0 & -N_{2}^{2} \rho_{1} & 0 & 0 \\
& & N_{2}^{2} \rho_{m} & N_{2}^{2} \rho_{2} & 0 & 0 \\
& & & N_{2}^{2}\left(\rho_{3}+\rho_{4}\right) & 0 & 0 \\
& & & & N_{2}^{2} \rho_{3} & -N_{2}^{2} \rho_{5} \\
S y m & & & & & N_{2}^{2} \rho_{4}
\end{array}\right]}
\end{aligned}
$$

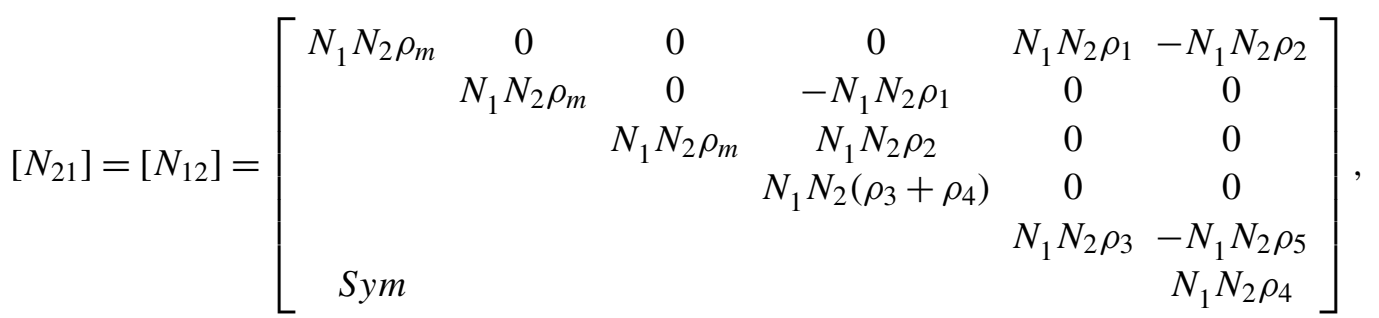

The analogy between expressions (4-32) and (4-36) defines the element mass matrix in the local coordinate system as

$$
[m]=\frac{\rho \Delta \theta}{2} \int_{-1}^{1}[N] d \xi .
$$

Then the element stiffness matrix $\left[K_{G}\right]$ and mass matrix $\left[M_{G}\right]$ in the global coordinate system are defined by

$$
\left[K_{G}\right]=[T]^{T}[k][T]
$$

go to line

$$
\left[M_{G}\right]=[T]^{T}[m][T],
$$




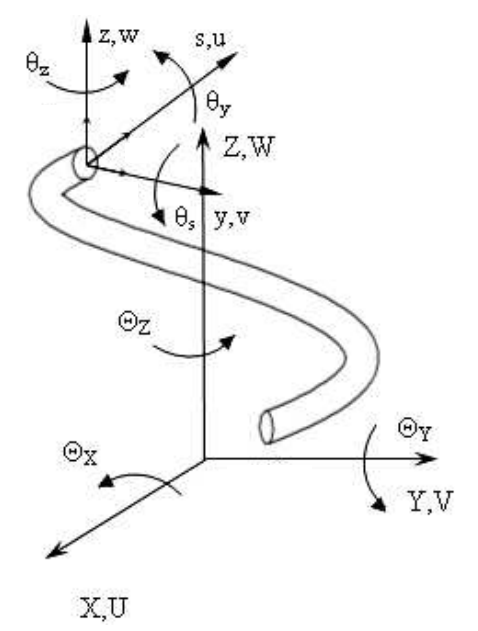

Figure 2. Local and global coordinate systems.

where $[T]$ is the transfer matrix from the local coordinate system to the global coordinate system, presented in Figure 2 and defined as

$$
[T]^{T}=[T]^{-1}=\left[\begin{array}{cccc}
{\left[Q_{1}\right]} & 0 & 0 & 0 \\
0 & {\left[Q_{1}\right]} & 0 & 0 \\
0 & 0 & {\left[Q_{2}\right]} & 0 \\
0 & 0 & 0 & {\left[Q_{2}\right]}
\end{array}\right] .
$$

$\left[Q_{1}\right]$ and $\left[Q_{2}\right]$ are the rotation matrixes defined by the leading cosines of local axes $x, y$ and $z$ in each of the elements

$$
\left[Q_{i}\right]=\left[\begin{array}{ccc}
-\frac{r}{\rho} \sin \left(\theta_{i}\right) & -\cos \left(\theta_{i}\right) & \frac{P}{2 \pi \rho} \sin \left(\theta_{i}\right) \\
\frac{r}{\rho} \cos \left(\theta_{i}\right) & -\sin \left(\theta_{i}\right) & -\frac{P}{2 \pi \rho} \cos \left(\theta_{i}\right) \\
\frac{P}{2 \pi \rho} & 0 & \frac{r}{\rho}
\end{array}\right] .
$$

\section{Numerical examples}

5.1. Natural frequencies of clamped-free spring. To verify the developed model, the natural frequencies are determined for three types of clamped-free springs $\left(S_{1}, S_{2}\right.$, and $\left.S_{3}\right)$ that differ in the nature of their cross-sections. The common proprieties of these springs are the follows: number of coils $N_{s}=10$ coils; mean diameter of the spring $D=113 \mathrm{~mm}$; pitch $P=26 \mathrm{~mm}$; Young's modulus $E=2.124810^{11} \mathrm{~N} / \mathrm{m}^{2}$; Poisson ratio $v=0.28$ and mass per unit volume of wire $\rho_{0}=8000 \mathrm{Kg} / \mathrm{m}^{3}$. The specific properties of each spring are:

spring $S_{1}$ : Circular cross-section with a wire diameter $d=15 \mathrm{~mm}$ and a shear correction ratio $k=0.886$ [Batoz and Dhatt 1993];

spring $S_{2}$ : Square cross-section with a thickness $h=15 \mathrm{~mm}$ and a shear correction ratio $k=0.833$ [Batoz and Dhatt 1993]; 


\begin{tabular}{|c|ccc|ccc|}
\hline \multirow{2}{*}{ Frequency } & \multicolumn{3}{|c|}{ Three-dimensional beam element } & \multicolumn{3}{|c|}{ Presented element } \\
& $\mathrm{e}=60$ & $\mathrm{e}=240$ & $\mathrm{e}=600$ & $\mathrm{e}=2$ & $\mathrm{e}=5$ & $\mathrm{e}=10$ \\
\hline 1 & 8.059 & 8.071 & 9.987 & 9.306 & 9.386 & 9.391 \\
2 & 9.461 & 9.297 & 10.001 & 9.465 & 9.598 & 9.611 \\
3 & 17.043 & 20.007 & 21.118 & 21.949 & 21.712 & 21.656 \\
\hline
\end{tabular}

\begin{tabular}{|c|ccc|ccc|}
\hline \multirow{2}{*}{ Frequency } & \multicolumn{3}{|c|}{ Three-dimensional beam element } & \multicolumn{3}{|c|}{ Presented element } \\
& $\mathrm{e}=60$ & $\mathrm{e}=240$ & $\mathrm{e}=600$ & $\mathrm{e}=2$ & $\mathrm{e}=5$ & $\mathrm{e}=10$ \\
\hline 1 & 8.837 & 10.181 & 10.950 & 10.243 & 10.328 & 10.333 \\
2 & 8.868 & 10.411 & 10.989 & 10.507 & 10.661 & 10.676 \\
3 & 18.123 & 20.881 & 22.457 & 23.544 & 23.313 & 23.258 \\
\hline
\end{tabular}

\begin{tabular}{|c|ccc|ccc|}
\hline Frequency & \multicolumn{3}{|c|}{ Three-dimensional beam element } & \multicolumn{3}{|c|}{ Presented element } \\
& $\mathrm{e}=60$ & $\mathrm{e}=240$ & $\mathrm{e}=600$ & $\mathrm{e}=2$ & $\mathrm{e}=5$ & $\mathrm{e}=10$ \\
\hline 1 & 11.199 & 12.903 & 13.877 & 12.748 & 12.885 & 12.897 \\
2 & 11.249 & 12.961 & 13.939 & 12.998 & 13.100 & 13.107 \\
3 & 22.407 & 25.8167 & 27.765 & 29.097 & 28.732 & 28.657 \\
\hline
\end{tabular}

Table 1. Natural frequencies (Hz) of the spring $S_{1}$ (top), $S_{1}$ (middle), and $S_{3}$ (bottom).

spring $S_{3}$ : Rectangular Cross-section with a thickness $e_{1}=15 \mathrm{~mm}$ and width $e_{2}=20 \mathrm{~mm}$ and a shear correction ratio $k=0.833$ [Batoz and Dhatt 1993].

Natural frequencies are determined using two types of finite elements: The first is a three-dimensional elastic beam finite element [Cosmos 1990]. The second is the finite element developed in this paper.

Table 1 presents the first three natural frequencies, given by the two elements, for each spring and for a different number of elements used. In fact, according to Abid et al. [2005], these frequencies are dangerous natural frequencies of the structure because they present high vibration amplitudes which can cause failure of the structure. Natural frequencies presented in Table 1 correspond to the first three simple modes of the spring which are

mode 1: bending mode around $Y$ axis;

mode 2: bending mode around $X$ axis;

mode 3: compression mode.

These modes are presented in Figure 3. The similarity between the two results is clear for each spring and mode. These results confirm the efficiency of the finite element developed.

5.2. Dynamic response of a helical spring. In this study, we are interested in determining the dynamic response of the free extremity of a clamped-free spring subjected at its free end to a harmonic compressive excitation. 

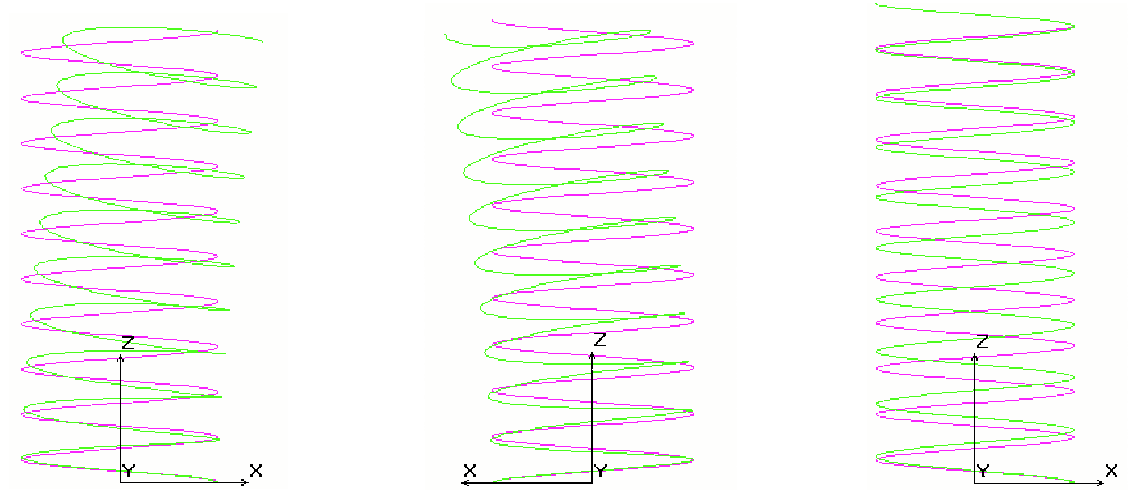

Figure 3. The three first spring's modes: Bending mode around $Y$ axis (left), bending mode around $X$ axis (middle) and compression mode (right). Pink represents the state case and green the vibrating case.

By varying the frequency of the excitation, the dynamic response of the free end of the spring is determined. The studied spring $S_{3}$ is subjected to a harmonic force with maximum amplitude $F_{\max }=100 \mathrm{~N}$. We suppose that the material of the spring has a modal damping coefficient $\xi=0.01$.

Results given by the present model are compared with those given by the three-dimensional elastic beam finite element [Cosmos 1990]. Figure 4 show this comparison respectively for the resultant displacement and rotation:

$$
R D=\sqrt{\left(U^{2}+V^{2}+W^{2}\right)}, \quad R R=\sqrt{\left(\Theta_{X}^{2}+\Theta_{Y}^{2}+\Theta_{Z}^{2}\right)}
$$

of the top end of the spring expressed in the global coordinate system. The chosen frequency band is between 0 and $100 \mathrm{~Hz}$.

Dynamic responses given by the two methods are close to each other. The developed finite element gives the right natural frequencies with a number of elements less than used in the first model (only 10 of the present elements for 600 of the three-dimensional elastic finite element). This means less calculation and programming needed for realistic results.

These figures indicate that the dangerous zone is especially located in the frequency band containing the three first natural frequencies. The difference between the vibration amplitude given by the two finite elements is due to the linear form of the developed finite element, but doesn't reduce the efficiency of the element. The helical spring is sensitive to its three first modes (two modes of bending and one of compression): If the spring is excited with these frequencies, resonance is produced and vibrations will have great amplitude that may damage the structure. At the other natural frequencies, the resonance

\begin{tabular}{|c|cccccccc|}
\hline Mode & 1 & 2 & 3 & 4 & 5 & 6 & 7 & 8 \\
Model 1 $(e=600)$ & 6.01424 & 6.61148 & 8.8772 & 9.41936 & 12.9729 & 14.0427 & 30.4497 & 31.1247 \\
Model 2 $(e=10)$ & 5.8720 & 6.1350 & 9.0642 & 9.6332 & 13.997 & 14.729 & 28.094 & 29.209 \\
\hline
\end{tabular}

Table 2. The first eight natural frequencies of the studied system $(\mathrm{Hz})$. 

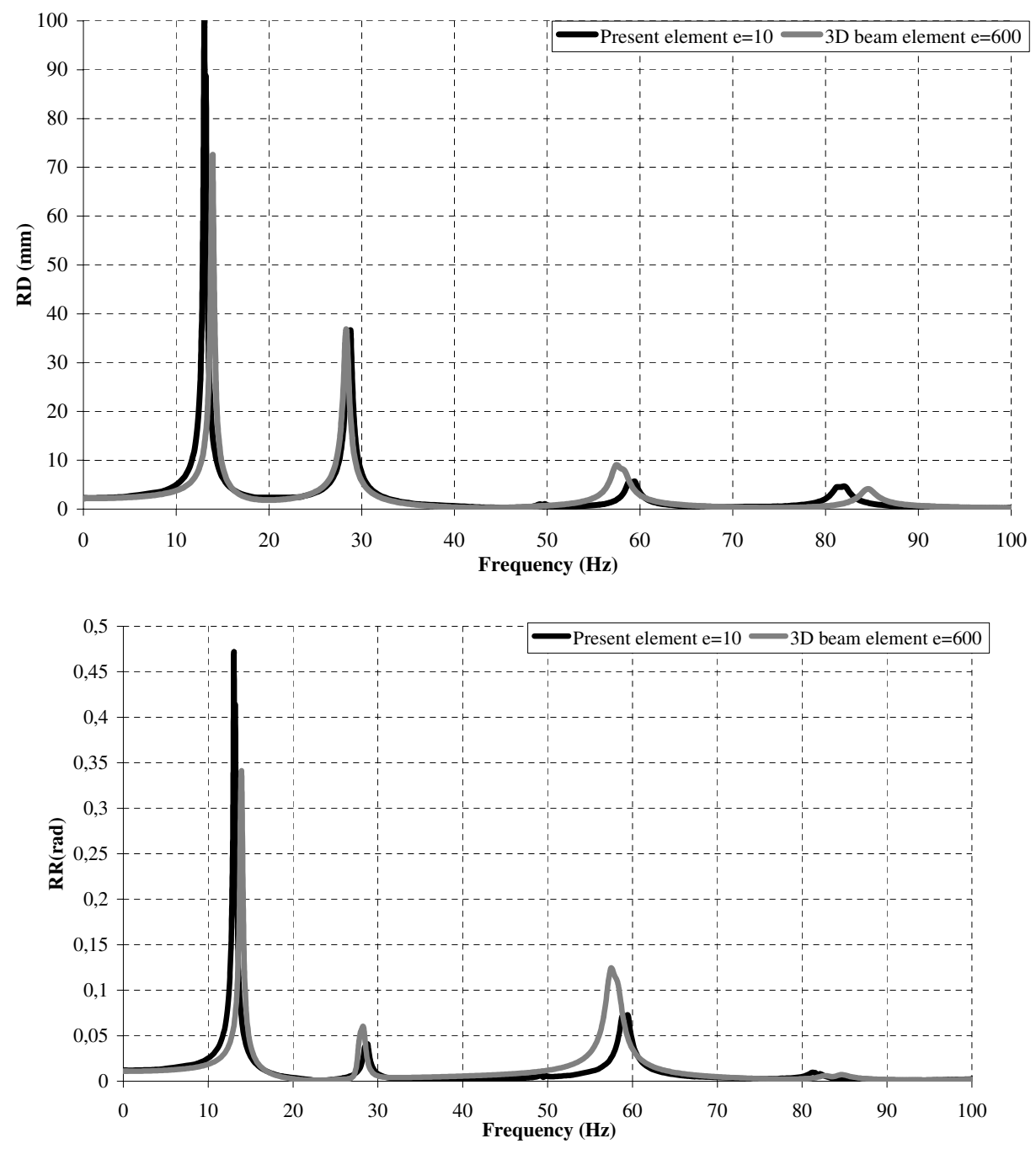

Figure 4. Resultant displacement of the free end of the studied spring (top) and resultant rotation of the free end of the studied spring (bottom).

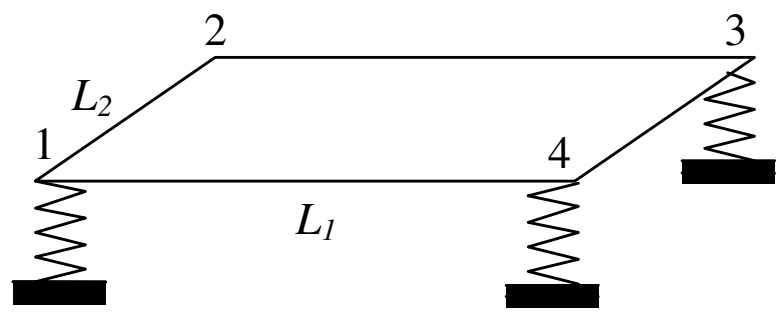

Figure 5. Studied system. 

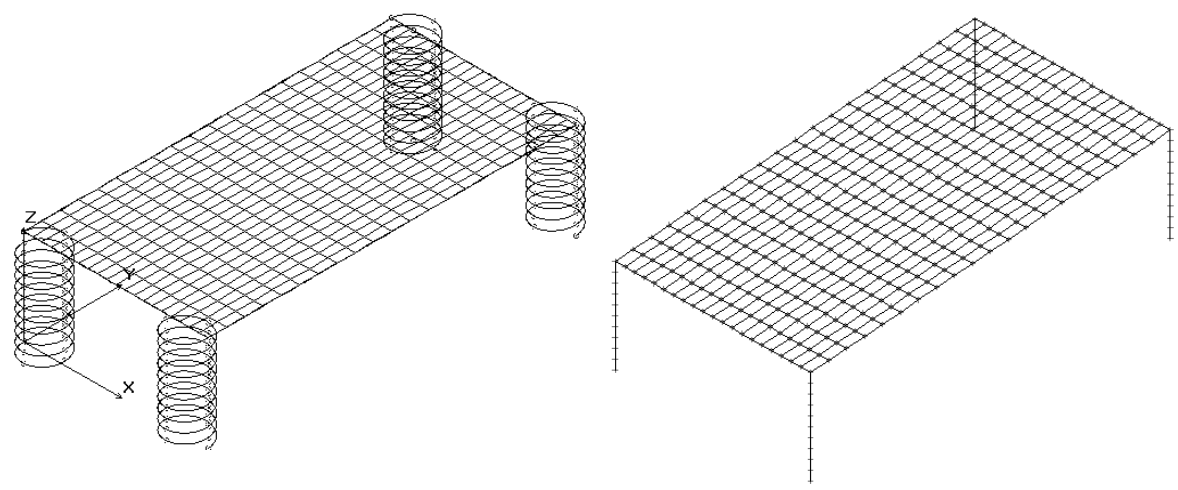

Figure 6. Meshing of the studied system. three-dimensional beam elements spring modeling (left); Present element modeling (right).

phenomena also manifests, but with smaller amplitudes. Thus, it is important to avoid the three first natural frequencies in practical applications of the spring and develop technological solutions to pass this frequency band without structural damage.

5.3. Vibrating plate. We next apply the developed and validated model to the practical example of a vibrating plate. This system consists of a thin plate supported at each extremity by a helical spring, as shown in Figure 5.

The thin plate has the characteristics:

$\begin{array}{llll}\text { length: } & L_{1}=1 \mathrm{~m}, & \text { width: } & L_{2}=0.5 \mathrm{~m}, \\ \text { thickness: } & c=7 \mathrm{~mm}, & \text { Young's modulus: } & E=2.110^{11} \mathrm{~N} / \mathrm{m}^{2}, \\ \text { Poisson ratio: } & v=0.28, & \text { mass per unit of volume } & \rho_{0}=8000 \mathrm{Kg} / \mathrm{m}^{3} .\end{array}$

With this plate we use four springs of type $S_{1}$.

The modeling of this plate is done by four-node quadrilateral thin shell elements with six degrees of freedom per node [Cosmos 1990]. The springs are modeled by the same two elements presented in Section 5.2 (proposed element + three-dimensional element). Figure 6 presents the meshing of the system in each method. The first eight natural frequencies of this system, given by the two modeling methods, are presented in Table 2.

Results given by the two methods are similar. This demonstrates the efficiency of the developed element in assembly with other types of structures. The element is not only capable of accurately the simple spring, but also the spring in practical applications.

\section{Conclusion}

In the present study, we develop a finite element for the dynamic analysis of a helical spring. The mixedhybrid formulation is established from geometric and cinematic hypothesis and takes into account the effect of shear strain to calculate the stiffness matrix. A simple approach is used for calculating the mass matrix. Comparison with an other types of finite elements shows the efficiency of the element to model simple and assembled springs. The study shows the sensitivity of the spring to its first three natural 
frequencies where the phenomena of resonance appears with large displacements and cause the damage to the structure.

\section{References}

[Abid et al. 2005] S. Abid, M. Taktak, F. Dammak, and M. Haddar, "Étude du comportement dynamique d'un ressort hélicoidal", pp. 65-67 in Proceeding of $7^{\text {th }}$ Congrès de Mécanique, Casablanca, Maroc, 2005.

[Batoz and Dhatt 1993] J.-L. Batoz and G. Dhatt, Modélisation des structures par éléments finis, vol. 2: Poutres et plaques, Hermès, Paris, 1993.

[Becker and Cleghorn 1992] L. E. Becker and W. L. Cleghorn, "On the buckling of helical compression springs", Int. J. Mech. Sci. 34:4 (1992), 275-282.

[Becker and Cleghorn 1993] L. E. Becker and W. L. Cleghorn, "The buckling behavior of hinged circular-bar compression springs", in Proceeding of the 14th Canadian Congress of Applied Mechanics, 1993.

[Becker and Cleghorn 1994] L. E. Becker and W. L. Cleghorn, "The buckling behavior of rectangular-bar helical compression springs", J. App. Mech. (Trans. ASME) 61:2 (1994), 491-493.

[Becker et al. 2002] L. E. Becker, G. G. Chassie, and W. L. Cleghorn, "On the natural frequencies of helical compression springs", Int. J. Mech. Sci. 44:4 (2002), 825-841.

[Castello 1975] G. A. Castello, "Radial expansion of impacted helical springs", J. App. Mech. (Trans. ASME) 42 (1975), 789.

[Chassie et al. 1997] G. G. Chassie, L. E. Becker, and W. L. Cleghorn, "On the buckling of helical springs under combined compression and torsion", Int. J. Mech. Sci. 39:6 (1997), 697-704.

[Cosmos 1990] User's manual, Structural Research and Analysis Corporation, Los Angeles, California, 1990.

[Dahtt and Touzout 1984] G. Dahtt and G. Touzout, Une présentation de la méthode des éléments finis, 2nd ed., Maloine, Paris, 1984.

[Dammak et al. 2005] F. Dammak, M. Taktak, S. Abid, A. Dhieb, and M. Haddar, "Finite element method for the stress analysis of isotropic cylindrical helical spring", Eur. J. Mech. A Solids 24:6 (2005), 1068-1078.

[Guido et al. 1978] A. R. Guido, L. D. Pietra, and S. D. Valle, "Transverse vibrations of cylindrical helical springs", Meccanica 13:2 (1978), 90-108.

[Haktanir 1994] V. Haktanir, "Analytical investigation of parameters affecting stiffness of helical springs of arbitrary shape under compression", pp. 473 in Proceeding of the 6th International Machine Design and Production Conference, Ankara, 1994.

[Haringx 1949] J. A. Haringx, "On highly compressible helical springs and rubber rods, and their application for vibration-free mountings, II", Philips Res. Rep. 4 (1949), 49-80.

[Kagawa 1968] Y. Kagawa, "On the dynamical properties of helical springs of finite length with small pitch", J. Sound Vib. 8:1 (1968), 1-15.

[Lee and Thompson 2001] J. Lee and D. J. Thompson, "Dynamic stiffness formulation, free vibration and wave motion of helical springs", J. Sound Vib. 239:2 (2001), 297-320.

[Lin and Pisano 1987] Y. Lin and A. P. Pisano, "General dynamic equations of helical springs with static solution and experimental verification", J. App. Mech. (Trans. ASME) 54 (1987), 910.

[Michell 1890] J. H. Michell, "The small deformation of curves and surfaces with applications to the vibrations of a helix and a circular ring", Mes. Math. 19 (1890), 68-82.

[Mottershead 1980] J. E. Mottershead, "Finite element for dynamical analysis of helical rods", Int. J. Mech. Sci. 22:5 (1980), 267-283.

[Mottershead 1982] J. E. Mottershead, "The large displacements and dynamic stability of springs using helical finite elements", Int. J. Mech. Sci. 24:9 (1982), 547-558.

[Pearson 1982] D. Pearson, "The transfer matrix method for the vibration of compressed helical springs", J. Mech. Eng. Sci. 24 (1982), 163.

[Pearson and Wittrick 1986] D. Pearson and W. H. Wittrick, "An exact solution for the vibration of helical springs using a Bernoulli-Euler model”, Int. J. Mech. Sci. 28:2 (1986), 83-96.

[Philips and Castello 1972] J. W. Philips and G. A. Castello, "Large deflection of impacted helical springs", J. Acoust. Soc. Am. 51:3B (1972), 967-973. 
[Pietra 1976] L. D. Pietra, "The dynamic coupling of torsional and flexural strains in cylindrical helical springs", Meccanica 11:2 (1976), 102-119.

[Pietra and Valle 1982] L. D. Pietra and S. D. Valle, "On the dynamic behavior of axially excited helical springs", Meccanica 17:1 (1982), 31-43.

[Sawanbori and Fukushima 1983] T. Sawanbori and Y. Fukushima, "Analysis of dynamic behavior of coil springs", Trans. Jpn. Soc. Mech. Eng. 49 (1983), 422.

[Sawanobori and Fukushima 1983] T. Sawanobori and Y. Fukushima, "A finite element approach to dynamic characteristics of helical springs (free vibration)", Bul. JMSE 26:221 (1983), 2002-2009.

[Tabarrok and Xiong 1989] B. Tabarrok and Y. Xiong, "On the buckling equations for spatial rods", Int. J. Mech. Sci. 31:3 (1989), 179-192.

[Tabarrok and Xiong 1992] B. Tabarrok and Y. Xiong, "A spatially curved and twisted rod element for buckling analysis", Int. J. Solids Struct. 29:23 (1992), 3011-3023.

[Taktak et al. 2005a] M. Taktak, S. Abid, F. Dammak, and M. Haddar, "Investigation of parameters affecting natural frequencies of isotropic helical spring", pp. 9-16 in Proceeding of the Second International Scientific and Pedagogical Days of Mechanics and Energitics, Gafsa, Tunisia, 2005.

[Taktak et al. 2005b] M. Taktak, F. Dammak, S. Abid, and M. Haddar, "A mixed-hybrid finite element for three-dimensional isotropic helical beam analysis", Int. J. Mech. Sci. 47:2 (2005), 209-229.

[Wahl 1963] A. M. Wahl, Mechanical springs, 2nd ed., McGraw-Hill, New York, 1963.

[Xiong and Tabarrok 1992] Y. Xiong and B. Tabarrok, "A finite element model for the vibration of spatial rods under various applied loads", Int. J. Mech. Sci. 34:1 (1992), 41-51.

[Yildirim 1995] V. Yildirim, "Investigation of free vibration of helical spring by the stiffness matrix method", Turkish J. Eng. Envi. Sci. 19 (1995), 343.

[Yildirim 1996] V. Yildirim, "Investigation of parameters affecting free vibration frequency of helical springs", Int. J. Numer. Meth. Eng. 39:1 (1996), 99-114.

[Yildirim 1997] V. Yildirim, "Free vibration analysis of non-cylindrical coil springs by combined use of the transfer matrix and the complementary functions methods", Commun. Numer. Meth. Eng. 13:6 (1997), 487-494.

[Yildirim 1999a] V. Yildirim, "An efficient numerical method for predicting the natural frequencies of cylindrical helical springs", Int. J. Mech. Sci. 41:8 (1999), 919-939.

[Yildirim 1999b] V. Yildirim, "A numerical study on the free vibration of symmetric cross-ply laminated cylindrical helical springs”, J. App. Mech. (Trans. ASME) 66:4 (1999), 1040-1043.

Received 17 Apr 2007. Accepted 30 Nov 2007.

MOHAMED TAKTAK: mohamed.taktak@utc.fr

Unit of Mechanics, Modelling and Manufacturing, Mechanical Engineering Department, National School of Engineers of Sfax, BP. W-3038 Sfax, Tunisia

FAKHREDDINE DAMMAK: Dammakf@yahoo.ca

Unit of Mechanics, Modelling and Manufacturing, Mechanical Engineering Department, National School of Engineers of Sfax, BP. W-3038 Sfax, Tunisia

SAID ABID: Said.abid@Issatgb.rnu.tn

Unit of Mechanics, Modelling and Manufacturing, Mechanical Engineering Department, National School of Engineers of Sfax, BP. W-3038 Sfax, Tunisia

MOHAMED HADDAR: mohamed.haddar@enis.rnu.tn

Unit of Mechanics, Modelling and Manufacturing, Mechanical Engineering Department, National School of Engineers of Sfax, BP. W-3038 Sfax, Tunisia 


\title{
PERTURBATION TECHNIQUE FOR WAVE PROPAGATION ANALYSIS IN A NOTCHED BEAM USING WAVELET SPECTRAL ELEMENT MODELING
}

\author{
Mira Mitra, S. Gopalakrishnan, Massimo Ruzzene, Nicole Apetre and S. Hanagud
}

\begin{abstract}
In this paper, spectral finite element is formulated for an Euler-Bernoulli beam with through-width notch type defect. In spectral finite element modeling, exact shape functions are derived and finite element procedure is followed in the transformed frequency domain. Here spectral finite element formulation is done using Daubechies scaling function bases for temporal approximation. In comparison to the conventional Fourier transform based spectral finite element method, the use of localized bases functions in the Daubechies scaling function based spectral finite element method allows accurate wave propagation analysis of finite length structures. The wave propagation response of the damaged beam is considered as a perturbation of the undamaged beam response within the restriction of small damage. First, numerical experiments are performed with narrow banded modulated pulse loading to obtain the location of damage from wave arrival time. Next, a broad banded impulse load is considered and effects of parameters like damage width, depth, and location on the responses are studied in time and frequency domains.
\end{abstract}

\section{Introduction}

Diagnostic waves are extensively used for structural health monitoring. Wave propagation problems deal with high frequency excitations and thus help to identify the presence of very small damages. These wave based techniques can thus be used to detect the minute defects which occur at the onset of the damage, and then propagate, causing failure of the structure.

Numerical solution of wave equations requires high accuracy in numerical differentiation and, for computational efficiency, it needs to have larger spatial grids and time steps. The conventional finite element (FE) technique widely used for modeling of structures is not suitable here, mainly because the element size should be comparable to the wavelength, which is very small at higher frequencies. This makes FE modeling computationally prohibitive for wave propagation problems. Alternative numerical techniques are generally adopted for such problems, and fast Fourier transform-based spectral finite element (FSFE) [Doyle 1999] is one such method. In short, the method follows FE technique in the transformed frequency domain. The governing partial differential equations are reduced to ordinary differential equations (ODEs) using fast Fourier transform (FFT) in time. These ODEs are solved exactly to derive the shape functions which are used to obtain the elemental dynamic stiffness matrix relating the transformed nodal forces and displacements.

However, the FSFE method possesses certain serious limitations as it uses bases with global support for temporal approximation, and thus requires the assumption of periodicity. As a consequence, the FSFE method does not allow time domain analysis of wave propagation in finite length structures. This problem is referred as "wrap around". To eliminate this drawback of FSFE, a Daubechies scaling function based

Keywords: wave propagation, Euler-Bernoulli beam, spectral element, Daubechies scaling functions, perturbation technique. 
spectral finite element, referred to as a wavelet based spectral finite element [Mitra and Gopalakrishnan 2005] (WSFE), is formulated using compactly supported Daubechies scaling as bases for approximation in time. The localized nature of the basis functions allows accurate simulation of wave propagation in finite length waveguides. In addition, similar to FSFE, the present method is computationally efficient and allows simultaneous frequency domain analysis [Mitra and Gopalakrishnan 2006].

In this paper, WSFE is formulated for a notched Euler-Bernoulli beam. The effect of the notch is introduced in the formulation using perturbation technique. The wave propagation response of the damaged beam is considered to be a perturbation of the response of the undamaged beam with the assumption of small damage. This perturbation technique has been developed to identify the presence of damage in beam [Luo and Hanagud 1998] and plates [Sharma et al. 2006] using curvature as the indicator; the effect of the notch on the curvature is studied using modal analysis. It should be mentioned here that as the analysis is restricted to damages with much smaller dimension, compared to that of the beam, the mode conversion resulting in axial and flexural coupling is not considered in the formulation. The present method is developed for pure flexural wave propagation and the effect of axial coupling due to the presence of the notch is neglected.

The paper is organized as follows. In Section 2, a brief overview of Daubechies compactly supported wavelets is provided. In the next two sections, the reduction of the wave equations and the perturbation techniques are elaborated. Section 5 concisely describes the spectral finite element formulation. Numerical experiments are presented in Section 6. The examples are provided for an aluminum cantilever beam with through-width notches of different configurations. The effects of the presence of the notch on the wave propagation responses due to both narrow and broad banded loading are studied in time and frequency domains. The influences of damage parameters like width, depth, and location on the responses are also analyzed.

\section{Daubechies compactly supported wavelets}

A concise review of the orthogonal bases of Daubechies wavelets [Daubechies 1992] is provided. Wavelets $\psi_{j, k}(t)$ form compactly supported orthonormal bases for $\boldsymbol{L}^{\mathbf{2}}(\boldsymbol{R})$. The wavelets and the associated scaling functions $\varphi_{j, k}(t)$ are obtained by translation and dilation of single functions $\psi(t)$ and $\varphi(t)$, respectively, as

$$
\begin{array}{rlrl}
\psi_{j, k}(t) & =2^{j / 2} \psi\left(2^{j} t-k\right), & j, k \in \boldsymbol{Z}, \\
\varphi_{j, k}(t) & =2^{j / 2} \varphi\left(2^{j} t-k\right), \quad j, k \in \boldsymbol{Z} .
\end{array}
$$

The scaling functions $\varphi(t)$ are derived from the dilation or scaling equation

$$
\varphi(t)=\sum_{k} a_{k} \varphi(2 t-k),
$$

and the wavelet function $\psi(t)$ is obtained as

$$
\psi(t)=\sum_{k}(-1)^{k} a_{1-k} \varphi(2 t-k) .
$$

$a_{k}$ are the filter coefficients, and they are fixed for specific wavelet or scaling function bases. For compactly supported wavelets only a finite number of $a_{k}$ are nonzero. The filter coefficients $a_{k}$ are derived by 
imposing certain constraints on the scaling functions, which are as follows: First, the area under scaling function is normalized to one. Second, the scaling function $\varphi(t)$ and its translates are orthonormal. Finally, the wavelet function $\psi(t)$ has $M$ vanishing moments. The number of vanishing moments $M$ denotes the order $N$ of the Daubechies wavelet, where $N=2 M$.

Let $P_{j}(f)(t)$ be the approximation of a function $f(t)$ in $\boldsymbol{L}^{\mathbf{2}}(\boldsymbol{R})$, using $\varphi_{j, k}(t)$ as bases, at a certain level (resolution) $j$, then

$$
P_{j}(f)(t)=\sum_{k} c_{j, k} \varphi_{j, k}(t), \quad k \in \boldsymbol{Z},
$$

where $c_{j, k}$ are the approximation coefficients.

\section{Reduction of wave equations to ODEs}

The governing differential equations for a Euler-Bernoulli beam are given as

$$
E I \frac{\partial^{4} w}{\partial x^{4}}+\rho A \frac{\partial^{2} w}{\partial t^{2}}=0
$$

where $w(x, t)$ is the transverse displacement. $E$ and $\rho$ are the Young's modulus and mass density, respectively, and $I$ and $A$ are area moment of inertia and area, respectively. The associated force boundary conditions are

$$
E I \frac{\partial^{2} w}{\partial x^{2}}=M \quad \text { and } \quad E I \frac{\partial^{3} w}{\partial x^{3}}=-V,
$$

where $M$ and $V$ are respectively the applied moment and the transverse force.

The first step of formulation of WSFE is the reduction of the governing differential equation given by Equation (4) to a set of coupled ODEs by Daubechies scaling function based transformation in time [Mitra and Gopalakrishnan 2005]. The procedure is described here in brief, for completeness. Let $w(x, t)$ be discretized at $n$ points in the time window $\left[0, t_{f}\right]$. Let $\tau=0,1, \ldots, n-1$ be the sampling points, then $t=\Delta t \tau$, where $\Delta t$ is the time interval between two sampling points. The function $w(x, t)$ can be approximated a by scaling function $\varphi(\tau)$ at an arbitrary scale as

$$
w(x, t)=w(x, \tau)=\sum_{k} w_{k}(x) \varphi(\tau-k), \quad k \in \boldsymbol{Z},
$$

where $w_{k}(x)$ (referred to as $w_{k}$ hereafter) is the approximation coefficient at a certain spatial dimension $x$. Thus (4) can be written as

$$
E I \sum_{k} \frac{d^{4} w_{k}}{d x^{4}} \varphi(\tau-k)+\frac{\rho A}{\triangle t^{2}} \sum_{k} w_{k} \varphi^{\prime \prime}(\tau-k)=0 .
$$

Taking the inner product of both sides of (6) with the translates of scaling functions $\varphi(\tau-j)$, where $j=0,1, \ldots, n-1$, and using their orthogonal properties, we get $n$ simultaneous ODEs as

$$
E I \frac{d^{4} w_{j}}{d x^{4}}+\frac{\rho A}{\Delta t^{2}} \sum_{k=j-N+2}^{j+N-2} \Omega_{j-k}^{2} w_{k}=0, \quad j=0,1, \ldots, n-1,
$$


where $N$ is the order of the Daubechies wavelet, and the connection coefficients for the $n$-th order derivative are defined as

$$
\Omega_{j-k}^{n}=\int \varphi^{n}(\tau-k) \varphi(\tau-j) d \tau .
$$

For compactly supported wavelets, the first and second order connection coefficients, $\Omega_{j-k}^{1}$ and $\Omega_{j-k}^{2}$, are nonzero only in the interval $k=j-N+2$ to $k=j+N-2$. The details for evaluation of the connection coefficients for different orders of the derivative are given by Beylkin [1992].

It can be observed from the ODEs given by Equation (7) that certain coefficients $w_{j}$ near the vicinity of the boundaries $(j=0$ and $j=n-1)$ lie outside the time window $\left[0, t_{f}\right]$ defined by $j=0,1, \ldots, n-1$. These coefficients must be treated properly for finite domain analysis; the wavelet based extrapolation scheme [Williams and Amaratunga 1997] is implemented for this purpose. The above method converts the ODEs given by (7) to a set of coupled ODEs given as

$$
E I\left\{\frac{d^{4} w_{j}}{d x^{4}}\right\}+\left[\Gamma^{1}\right]^{2} \rho A\left\{w_{j}\right\}=0,
$$

where $\Gamma^{1}$ is the first order connection coefficient matrix obtained after using the wavelet extrapolation technique. It should be mentioned here that though the connection coefficients matrix, $\Gamma^{2}$, for the second order derivative can be obtained independently, here it is written as $\left[\Gamma^{1}\right]^{2}$, as it helps to impose the initial conditions [Mitra and Gopalakrishnan 2005]. These coupled ODEs are decoupled using eigenvalue analysis as $\Gamma^{1}=\Phi \Pi \Phi^{-1}$, where $\Pi$ is the diagonal eigenvalue matrix and $\Phi$ is the eigenvectors matrix of $\Gamma^{1}$. Let the eigenvalues be $l \gamma_{j}(l=\sqrt{-1})$, then the decoupled ODEs corresponding to (8) are

$$
E I \frac{d^{4} \hat{w}_{j}}{d x^{4}}-\rho A \gamma_{j}^{2} \hat{w}_{j}=0, \quad j=0,1, \ldots, n-1,
$$

where $\hat{w}_{j}$ is defined as $\hat{w}_{j}=\Phi^{-1} w_{j}$.

Similarly, the transformed form of the force boundary conditions given by (5) are given as

$$
E I \frac{d^{2} \hat{w}_{j}}{d x^{2}}=\hat{M}_{j}, \quad E I \frac{d^{3} \hat{w}_{j}}{d x^{3}}=-\hat{V}_{j}, \quad j=0,1, \ldots, n-1,
$$

where $\hat{M}_{j}$ and $\hat{V}_{j}$ are the transformed $M(x, t)$ and $V(x, t)$, respectively. In the following sections, the subscript $j$ is dropped for simplified notation and all the following equations hold for $j=0,1, \ldots, n-1$.

\section{Perturbation analysis of damaged beams}

Here, a through-width notch is modeled as a localized decrease in the stiffness and inertia. For a notch of width $\Delta L$ and depth $h_{d}$, as shown in Figure 1, the area moment of inertia $I_{d}$ at the damaged location can be written as

$$
I_{d}=\frac{1}{12} b\left(h-h_{d}\right)^{3}=\frac{1}{12} b h^{3}\left(1-\frac{h_{d}}{h}\right)^{3},
$$

where $b$ and $h$ are the width and depth of the beam, respectively. Assuming small damage, meaning $\frac{h_{d}}{h} \ll 1$, the above Equation (11) can be written as

$$
I_{d} \approx \frac{1}{12} b h^{3}\left(1-3 \frac{h_{d}}{h}\right) \approx I_{0}\left(1-\epsilon_{p}\right), \quad \epsilon_{p}=3 \frac{h_{d}}{h},
$$




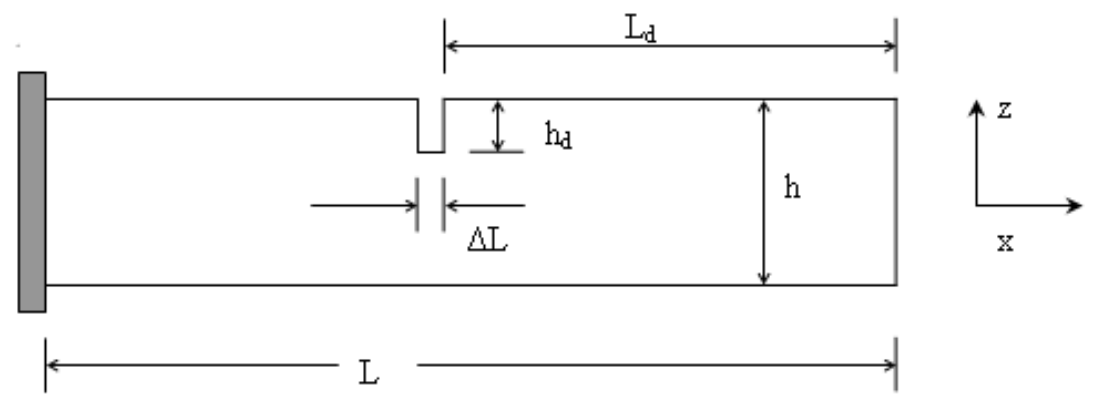

Figure 1. Schematic for the cantilever beam with notch.

where $I_{0}$ is the area moment of inertia of the undamaged beam and $\epsilon_{p}$ is used as the perturbation parameter later. Similarly, the mass per unit length at the damaged location, $m_{d}$, can be written as

$$
m_{d}=\rho b\left(h-h_{d}\right)=\rho b h\left(1-\frac{h_{d}}{h}\right)=m_{0}\left(1-\frac{1}{3} \epsilon_{p}\right),
$$

where $m_{0}$ is the mass per unit length of the undamaged beam. Thus, the overall flexural rigidity $E I(x)$ and mass per unit length $m(x)$ of the beam with a notch of width $\Delta L$, at $x=x_{d}$, can be written as a function of $x$ [Luo and Hanagud 1998]

$$
\begin{aligned}
E I(x) & =E I_{0}\left[1-\epsilon_{p} \Delta L \delta\left(x-x_{d}\right)\right], \\
m(x) & =m_{0}\left[1-\frac{1}{3} \epsilon_{p} \Delta L \delta\left(x-x_{d}\right)\right],
\end{aligned}
$$

where $\delta(x)$ is the Dirac delta function.

Next, the displacement $w(x, t)$ of the damaged beam is considered as a perturbation of that of the undamaged beam [Luo and Hanagud 1998], and is written as

$$
w=w^{0}-\epsilon_{p} w^{1},
$$

where $w^{0}$ is the displacement of the undamaged beam, while $w^{1}$ is the first approximation of the perturbation solution. The linear transformations used in the last section can be done for the variables in (13), and the transformed equation can be written as

$$
\hat{w}=\hat{w}^{0}-\epsilon_{p} \hat{w}^{1} .
$$

Substituting (12) and (14) into (9), the following equations are obtained:

$$
\begin{aligned}
1: E I_{0} \frac{d^{4} \hat{w}^{0}}{d x^{4}} & =\gamma^{2} m_{0} \hat{w}^{0}, \\
\epsilon_{p}: E I_{0} \frac{d^{4} \hat{w}^{1}}{d x^{4}} & =\gamma^{2} m_{0} \hat{w}^{1}+\frac{1}{3} \gamma^{2} m_{0} \Delta L \delta\left(x-x_{d}\right) \hat{w}_{d}^{0}-E I_{0} \frac{d^{2}}{d x^{2}}\left[\Delta L \delta\left(x-x_{d}\right) \frac{d^{2} \hat{w}_{d}^{0}}{d x^{2}}\right] .
\end{aligned}
$$

However, the terms associated with $\epsilon_{p}^{2}$ are neglected. $\hat{w}_{d}^{0}$ and $\frac{d^{2} \hat{w}_{d}^{0}}{d x^{2}}$ are the values of $\hat{w}^{0}$ and $\frac{d^{2} \hat{w}^{0}}{d x^{2}}$ at $x=x_{d}$, respectively. The solution of Equation (15) and the complimentary solution of Equation (16) are obtained through the spectral finite element method described in the next section. Here the particular 
solution of (16) is required for spectral element formulation. Due to the discontinuity present in (16) it is difficult to obtain the exact particular solution. Thus, the solution of the static part of (16) is taken as the approximate particular solution, which is of the form,

$$
\hat{w}^{1}=\Delta L\left\langle x-x_{d}\right\rangle \frac{d^{2} \hat{w}^{0}}{d x^{2}}-\frac{1}{18} \gamma^{2} k^{4} \Delta L\left\langle x-x_{d}\right\rangle^{3} \hat{w}^{0}+\frac{1}{6} C_{1} x^{3}+\frac{1}{2} C_{2} x^{2}+C_{3} x+C_{4},
$$

where $k^{4}=m_{0} / E I_{0}$ and \langle\rangle is the ramp function [Jones 1982; Lestari 2001]. $C_{1}, C_{2}, C_{3}$ and $C_{4}$ are the constants to be calculated from the four boundary conditions as

$$
\left\{\begin{array}{c}
\hat{w}_{1}^{1} \\
\frac{d \hat{w}_{1}^{1}}{d x} \\
\hat{w}_{2}^{1} \\
\frac{d \hat{w}_{2}^{1}}{d x}
\end{array}\right\}=\left[\begin{array}{cccc}
0 & 0 & 0 & 1 \\
0 & 0 & 1 & 0 \\
\frac{L^{3}}{6} & \frac{L^{2}}{2} & L & 1 \\
\frac{L^{2}}{2} & L & 1 & 0
\end{array}\right]\left\{\begin{array}{c}
C_{1} \\
C_{2} \\
C_{3} \\
C_{4}
\end{array}\right\}+\left\{\begin{array}{c}
0 \\
0 \\
\Delta L\left(L-x_{d}\right) \frac{d^{2} \hat{w}_{d}^{0}}{d x^{2}}-\frac{1}{18} \gamma^{2} k^{4} \Delta L\left(L-x_{d}\right)^{3} \hat{w}_{d}^{0} \\
\Delta L \frac{d^{2} \hat{w}_{d}^{0}}{d x^{2}}-\frac{1}{6} \gamma^{2} k^{4} \Delta L\left(L-x_{d}\right)^{2} \hat{w}_{d}^{0}
\end{array}\right\} .
$$

The above equation can be rewritten more simply as

$$
\left\{u_{e}^{1}\right\}=[G]\{C\}+\{R\},
$$

where

$\left\{u_{e}^{1}\right\}=\left\{\hat{w}_{1}^{1} \frac{d \hat{w}_{1}^{1}}{d x}\left(=\theta_{1}^{1}\right) \hat{w}_{2}^{1} \frac{d \hat{w}_{2}^{1}}{d x}\left(=\theta_{2}^{1}\right)\right\}, \quad \hat{w}_{1}^{1} \equiv \hat{w}^{1}(0) \frac{d \hat{w}_{1}^{1}}{d x} \equiv \frac{d \hat{w}^{1}(0)}{d x}, \quad \hat{w}_{2}^{1} \equiv \hat{w}^{1}(L) \frac{d \hat{w}_{2}^{1}}{d x} \equiv \frac{d \hat{w}^{1}(L)}{d x}$,

with $L$ being the length of the beam.

Substituting the particular solution given by Equation (17) in the transformed force boundary conditions, (10), we get

$$
\begin{aligned}
& \hat{M}^{1}=E I_{0}\left[\Delta L \delta\left(x-x_{d}\right) \frac{d^{2} \hat{w}_{d}^{0}}{d x^{2}}-\frac{1}{3} \gamma^{2} k^{4} \Delta L\left\langle x-x_{d}\right\rangle \hat{w}_{d}^{0}+C_{1} x+C_{2}\right], \\
& \hat{V}^{1}=-E I_{0}\left[\Delta L \frac{d}{d x} \delta\left(x-x_{d}\right) \frac{d^{2} \hat{w}_{d}^{0}}{d x^{2}}-\frac{1}{3} \gamma^{2} k^{4} \Delta L H\left(x-x_{d}\right) \hat{w}_{d}^{0}+C_{1}\right],
\end{aligned}
$$

where $H(x)$ is the step function. The above equations can be written in matrix form relating boundary forces and constants $\{C\}$ as

$$
\left\{\begin{array}{c}
\hat{V}_{1}^{1} \\
\hat{M}_{1}^{1} \\
\hat{V}_{2}^{1} \\
\hat{M}_{2}^{1}
\end{array}\right\}=E I_{0}\left[\begin{array}{cccc}
-1 & 0 & 0 & 0 \\
0 & 1 & 0 & 0 \\
1 & 0 & 0 & 0 \\
-L & -1 & 0 & 0
\end{array}\right]\left\{\begin{array}{c}
C_{1} \\
C_{2} \\
C_{3} \\
C_{4}
\end{array}\right\}+E I_{0}\left\{\begin{array}{c}
0 \\
0 \\
-\frac{1}{3} \gamma^{2} k^{4} \Delta L \hat{w}_{d}^{0} \\
\frac{1}{3} \gamma^{2} k^{4} \Delta L\left(L-x_{d}\right) \hat{w}_{d}^{0}
\end{array}\right\},
$$

or

$$
\left\{\hat{F}_{e}^{1}\right\}=[\bar{G}]\{C\}+\{\bar{R}\},
$$

where

$$
\left\{F_{e}^{1}\right\}=\left\{\hat{V}_{1}^{1} \hat{M}_{1}^{1} \hat{V}_{2}^{1} \hat{M}_{2}^{1}\right\}, \quad \hat{V}_{1}^{1} \equiv \hat{V}^{1}(0), \quad \hat{M}_{1}^{1} \equiv \hat{M}^{1}(0), \quad \hat{V}_{2}^{1} \equiv \hat{V}^{1}(L), \quad \hat{M}_{2}^{1} \equiv \hat{M}^{1}(L) .
$$


Using Equations (17) and (18), the boundary displacements $\left\{u_{e}^{1}\right\}$ can be written in terms of forces $\left\{\hat{F}_{e}^{1}\right\}$ as

$$
\left.\left\{\hat{F}_{e}^{1}\right\}=\left[\hat{K}^{1}\right]\left\{\hat{u}_{e}^{1}\right\}-\left[\hat{K}^{1}\right]\{R\}+\{\bar{R}\}, \quad \hat{K}^{1}\right]=[\bar{G}][G]^{-1} .
$$

However, it should be mentioned here that two spectral elements are required to model the notched beam, and one node is located at the damage position. This is needed to account for the discontinuity of the particular solution at $x=x_{d}$.

The solutions of Equation (15) and the complimentary solution of (16) using the spectral finite element method described in the next section is of similar form, and relates the boundary (nodal) forces and displacements through a matrix equation. Thus the total perturbed solution $\hat{w}^{1}$ is obtained by adding the particular solution given by (19) to the complimentary solution.

\section{Spectral finite element method}

As said earlier, (15) and the complimentary part of (16) are solved for $\hat{w}^{0}$ and $\hat{w}^{1}$, respectively, using a spectral finite element technique. Here the method is described in a general form considering $\hat{w}$ as the variable. The degrees of freedom (DOFs) associated with the element formulation are shown in Figure 2. The element has two DOFs per node, which are $\hat{w}$ and $d \hat{w} / d x(=\theta)$. From the previous sections, the set of decoupled ODEs (see (9)) are obtained, and are required to be solved exactly for $\hat{w}$. The actual solutions $w(x, t)$ are obtained using inverse wavelet transform. For finite length data, the wavelet transform and its inverse can be obtained using a transformation matrix [Williams and Amaratunga 1994]. Here, the spectral finite element technique is explained for the decoupled ODEs given by (9).

The exact interpolating functions for an element of length $L$, obtained by solving (9), are

$$
\{\hat{w}(x)\}^{T}=[\boldsymbol{R}][\boldsymbol{\Theta}]\{\boldsymbol{a}\},
$$

where $[\boldsymbol{\Theta}]$ is a diagonal matrix with the diagonal terms $\left[e^{-k_{1} x}, e^{-k_{1}\left(L_{X}-x\right)}, e^{-k_{2} x}\right.$, and $\left.e^{-k_{2}\left(L_{X}-x\right)}\right] ;[\boldsymbol{R}]$ is a $1 \times 4$ amplitude ratio matrix for each set of $k_{1}$ and $k_{2}$ as $[\boldsymbol{R}]=\left[\begin{array}{llll}R_{11} & \ldots & \ldots & R_{14}\end{array}\right] . k_{1}$ and $k_{2}$ are obtained by substituting (20) into (9) and posing it as a polynomial eigenvalue problem [Chakraborty and Gopalakrishnan 2005].

Here $\{\boldsymbol{a}\}=\{\mathrm{A}, \mathrm{B}, \mathrm{C}, \mathrm{D}\}$ are the unknown coefficients to be determined from transformed nodal displacements $\left\{\hat{\boldsymbol{u}}_{\boldsymbol{e}}\right\}$, where

$$
\begin{aligned}
\left\{\hat{\boldsymbol{u}}_{e}\right\} & =\left\{\hat{w}_{1} d \hat{w}_{1} / d x \hat{w}_{2} d \hat{w}_{2} / d x\right\}, & & \hat{w}_{1} \equiv \hat{w}(0), \\
d \hat{w}_{1} / d x & \equiv d \hat{w}(0) / d x, & & \hat{w}_{2} \equiv \hat{w}(L),
\end{aligned}
$$

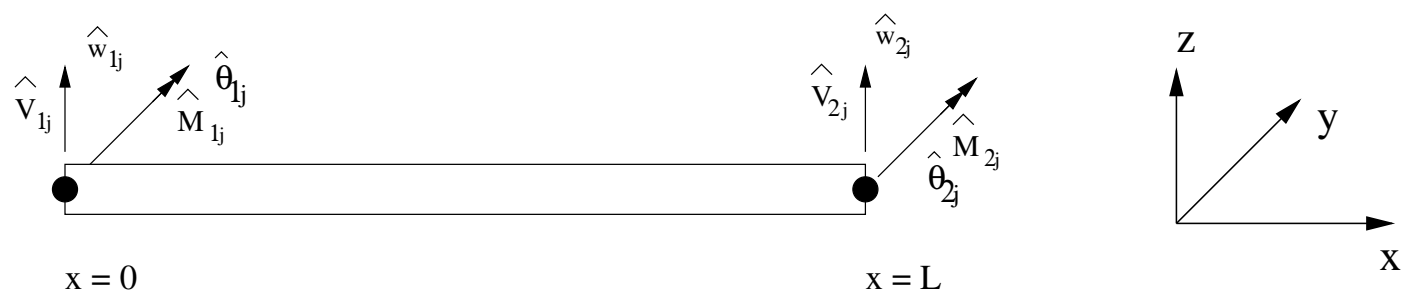

Figure 2. Spectral finite element with nodal displacements and forces. 
and $d \hat{w}_{2} / d x \equiv d \hat{w} / d x(L)$ (see Figure 6 for details on the degrees of freedom the element can support). Thus we can relate the nodal displacements and unknown coefficients as

$$
\left\{\hat{\boldsymbol{u}}_{\boldsymbol{e}}\right\}=[\boldsymbol{B}]\{\boldsymbol{a}\} .
$$

Substituting Equation (20) in the forced boundary conditions given by (10), the nodal forces can be related to the unknown coefficients as

$$
\left\{\hat{\boldsymbol{F}}_{\boldsymbol{e}}\right\}=[\boldsymbol{C}]\{\boldsymbol{a}\}
$$

where $\left\{\hat{\boldsymbol{F}}_{\boldsymbol{e}}\right\}=\left\{\hat{V}_{1} \hat{M}_{1} \hat{V}_{2} \hat{M}_{2}\right\}, \hat{V}_{1} \equiv-\hat{V}(0), \hat{M}_{1} \equiv-\hat{M}(0), \hat{F}_{2} \equiv \hat{F}(L), \hat{V}_{2} \equiv \hat{V}(L)$, and $\hat{M}_{2} \equiv \hat{M}(L)$ (see Figure 2). From Equations (21) and (22) we can obtain a relation between transformed nodal forces and displacements, similar to conventional FE,

$$
\left\{\hat{\boldsymbol{F}}_{\boldsymbol{e}}\right\}=[\boldsymbol{C}][\boldsymbol{B}]^{-1}\left\{\hat{\boldsymbol{u}}_{\boldsymbol{e}}\right\}=\left[\hat{\boldsymbol{K}}_{\boldsymbol{e}}\right]\left\{\hat{\boldsymbol{u}}_{\boldsymbol{e}}\right\},
$$

where $\left[\hat{\boldsymbol{K}}_{\boldsymbol{e}}\right]$ is the exact elemental dynamic stiffness matrix. After the constants $\{\boldsymbol{a}\}$ are known from the above equations, they can substituted back into Equation (20) to obtain the transformed displacements $\hat{w}, d \hat{w} / d x$ at any given $x$.

(23), corresponding to the perturbation solution, must be added to the particular solution, given by (19), to obtain total perturbation solution. Thus, we finally have two equations relating the transformed nodal forces to the transformed nodal displacements. For the perturbation solution, the boundary conditions for a cantilever beam are taken as

$$
\hat{w}^{1}(0)=\frac{d \hat{w}^{1}}{d x}(0)=0 \quad \text { and } \quad E I_{0} \frac{d^{2} \hat{w}^{1}}{d x^{2}}(L)=E I_{0} \frac{d^{3} \hat{w}^{1}}{d x^{3}}(L)=0 .
$$

The other boundary conditions can be similarly implemented.

\section{Numerical experiments}

In this section, flexural wave propagation in a damaged beam, due to a narrow banded modulated pulse and a broad banded impulse loading, are studied and compared with the responses of the undamaged beam. In the first case of modulated pulse loading, the wave propagates nondispersively and the locations of the damage are obtained from the arrival time of the wave reflected from the notch. The wave speeds corresponding to the loading frequencies are obtained from the dispersion relation. This in turn helps in validation of the present formulation. Next, the flexural wave propagation due to broad band impulse load is studied for different damage parameters like width, $\Delta L$, depth, $h_{d}$, and distance, $L_{d}$, from the free end or tip of the cantilever beam.

In all the cases the beam considered is an aluminum cantilever beam of length $L$, width $b=0.05 \mathrm{~m}$, and depth $h=0.01 \mathrm{~m}$. The material properties considered are Young's modulus, $E=70 \mathrm{GPa}$, Poisson's ratio, $v=0.3$, and mass density $\rho=2700 \mathrm{~kg} / \mathrm{m}^{3}$. The order of the Daubechies scaling function used in all the examples is $N=22$ unless otherwise mentioned.

First, the present method is validated by comparison with two-dimensional FE results. The comparison is made for the transverse wave velocity resulting from an impulse load of duration $500 \mu$ s and frequency content $4.4 \mathrm{kHz}$. The applied impulse load is very similar to that shown in Figure 3, however, there the impulse load has a duration of $50 \mu \mathrm{s}$ and frequency content of $44 \mathrm{kHz}$, used for the numerical experiments 


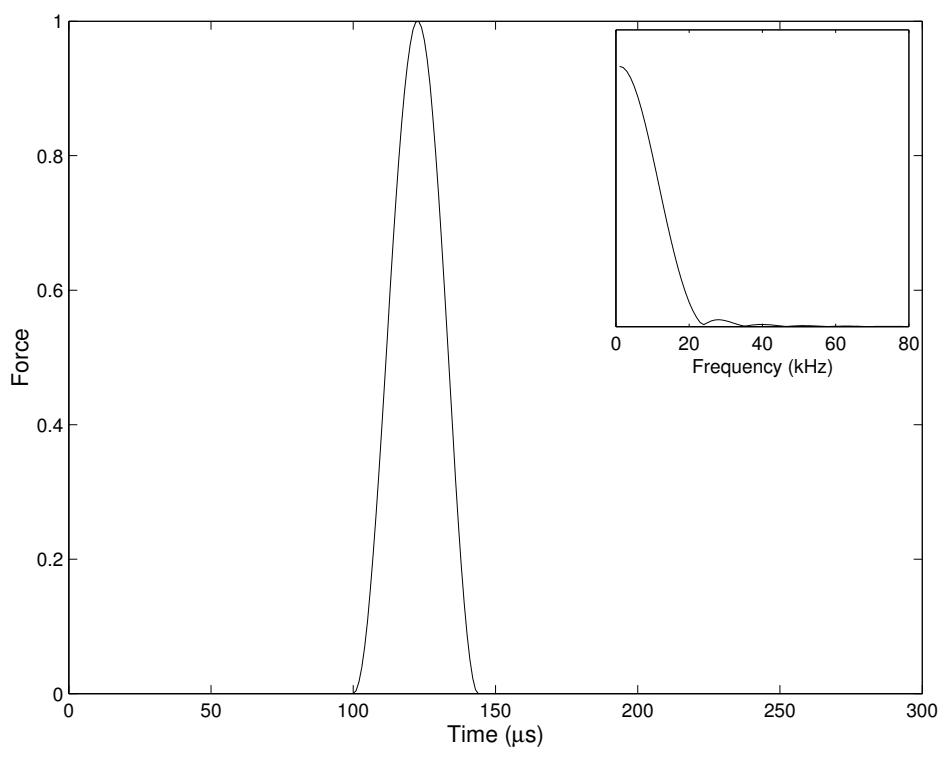

Figure 3. Impulse load in time and frequency (inset) domains.

presented in the later part of the paper. The responses simulated using the present method and twodimensional FE modeling is presented in Figure 4 which shows the transverse velocity measured at the tip of a fixed-free beam with the impulse load applied at the tip. The damage is located at a distance of $0.5 \mathrm{~m}$ from the free end and has a depth of $h_{d}=0.001 \mathrm{~m}$ ( $10 \%$ of the depth of the beam) and width $\triangle L=0.03 \mathrm{~m}$. The two-dimensional FE simulation is done using ANSYS 10.0, with 3994, 4-noded, plane stress quadrilateral elements and Newmark time integration with $\Delta t=2 \mu \mathrm{s}$. As mentioned before, the WSFE results are obtained using only two elements with the intermediate node at the damage location. It

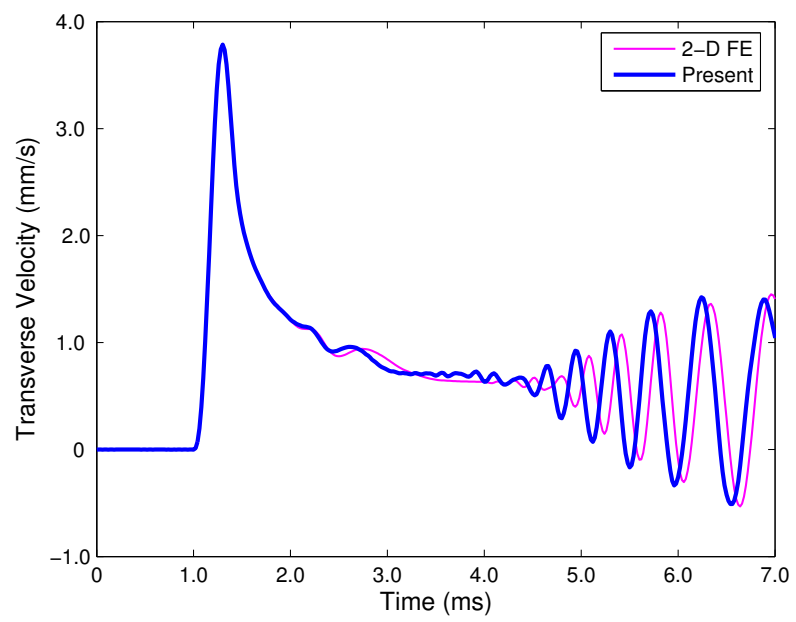

Figure 4. Transverse velocities at the tip of a cantilever beam with notch. 


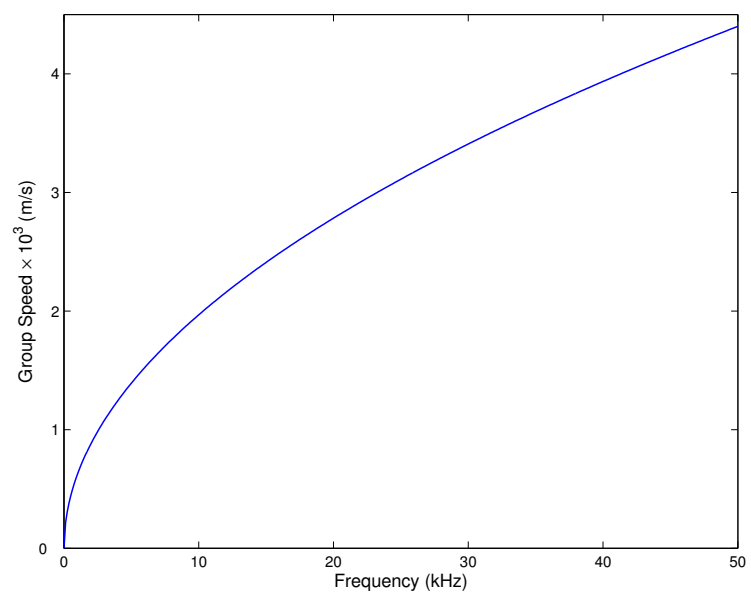

(a)

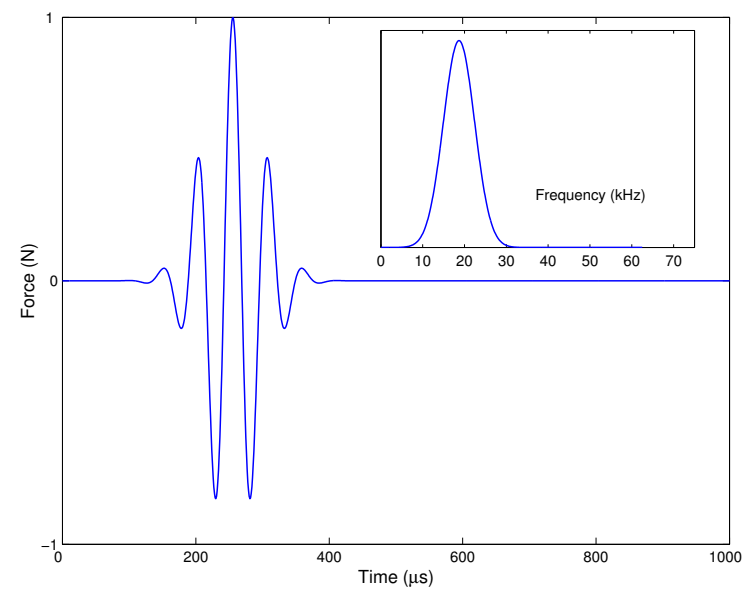

(b)

Figure 5. Dispersion relation for (a) Euler-Bernoulli beam (left) and (b) modulated sinusoidal pulse load in time and frequency (inset) domains.

can be seen that the responses match very well. The frequency content of the applied load is consciously kept lower than that used for the other examples. This is because the two-dimensional FE modeling of the beam always simulates the results of Timoshenko beam theory and thus predicts lower wave speed than the Euler-Bernoulli beam theory used for the WSFE modeling. The difference in the responses due to this difference in the wave speeds is not negligible even in the slender beam case. However, the difference will be less for lower frequency excitation, hence a lower range of frequency is considered for the comparison between the two-dimensional FE and present models. The small difference in the wave speeds predicted by the two methods, observed in Figure 4, can be attributed to the reason explained above.

In Figure 5a, the dispersion relation for the beam, the group speed with respect to the frequencies, is plotted. The narrow banded modulated sinusoidal pulse is shown in time and frequency domains in Figure 5b. It can be seen that this load has a very narrow frequency band and propagates nondispersively, retaining its shape. Such a modulated pulse is widely used in health monitoring studies. Here, additional waves result due to reflection from the damage. The damage location is obtained from the arrival time of such a reflected wave, and the wave speed derived from the dispersion relation is shown in Figure 5a. In the present case, the modulated pulse has a central frequency of nearly $20 \mathrm{kHz}$, and the group speed of the flexural wave in the beam corresponding to this frequency is approximately $C_{g}=2716 \mathrm{~m} / \mathrm{s}$, as calculated from Figure 5a. In Figure 6 the transverse velocities measured at the tip of the cantilever beam with length $L=2.0 \mathrm{~m}$, due to the modulated pulse applied at the tip in the transverse direction, are plotted. Figure 6 shows these velocities for beams with though-width notches at distances $L_{d}=0.1,0.25$, and $0.4 \mathrm{~m}$ from the free end, respectively. In all cases the notch width $\Delta L=0.02$ and the depth $h_{d}=$ $10 \%$ for the beam depth $h$. The arrival time of the waves reflected from the notch, as obtained from Figure 6, are, respectively, $T=75,185$, and $300 \mu$ s. The locations of the damages, calculated inversely from these arrival times, and the group speed $C_{g}$ are nearly equal to those assumed for the simulations, $L_{d}=0.1,0.25$, and $0.4 \mathrm{~m}$. This also validates the present formulation. 

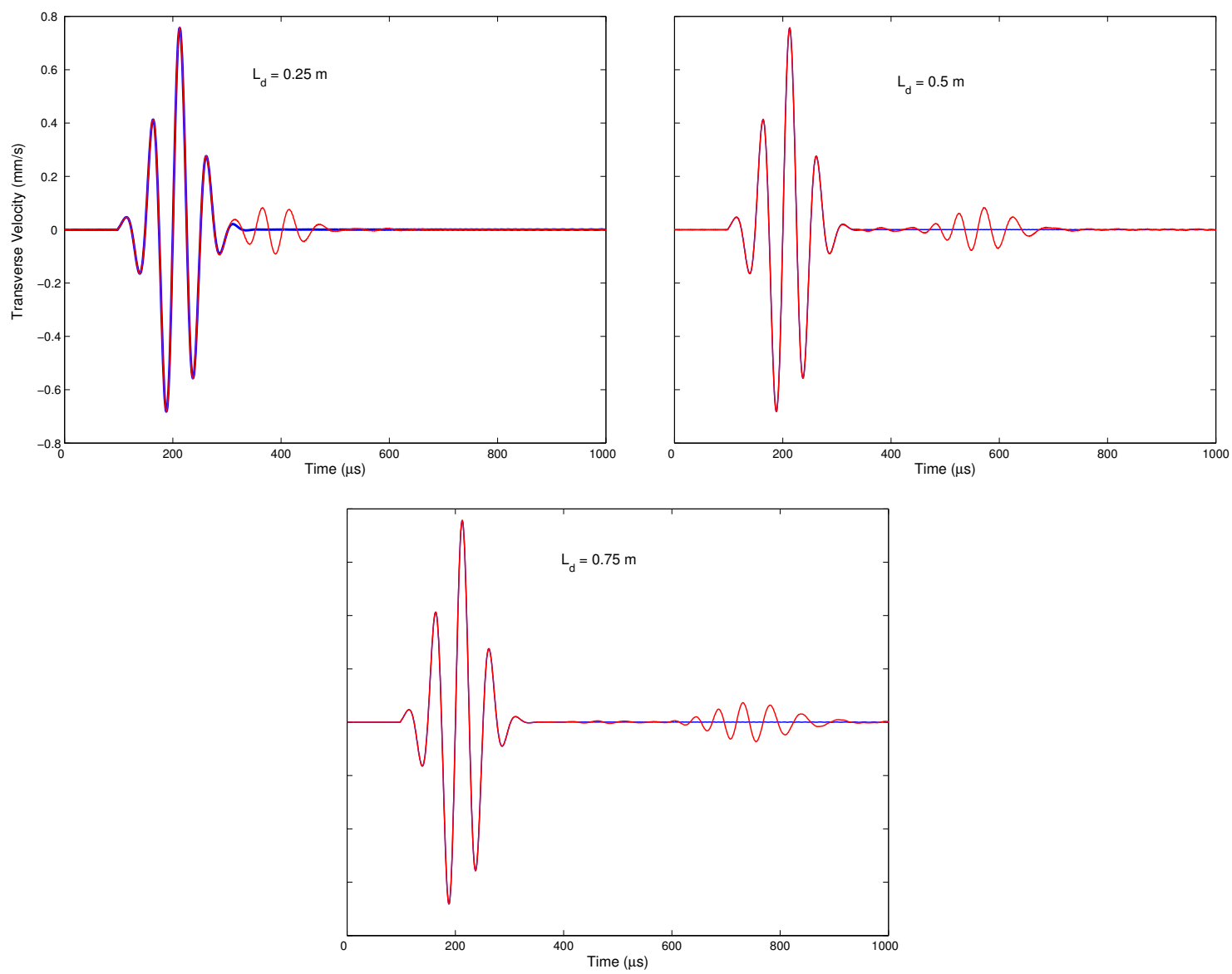

Figure 6. Transverse velocities at the tip of a cantilever beam of length $L=2.0 \mathrm{~m}$ with notch of $\Delta L=0.02 \mathrm{~m}$, depth $h_{d}=10 \%$, at $L_{d}=0.25 \mathrm{~m}$ (upper left), $L_{d}=0.5 \mathrm{~m}$ (upper right), and $L_{d}=0.75 \mathrm{~m}$ (lower), compared with the response of an undamaged beam.

Next, examples are presented to study the effect of notch on the flexural wave propagation due to broad band impulse loading. The unit impulse load is shown in Figure 3 in time and frequency domains. The load has a time duration of $50 \mu \mathrm{s}$ and a frequency content of $44 \mathrm{kHz}$. The load is again applied at the tip of a cantilever beam of length $L=1.0 \mathrm{~m}$ and the transverse velocities are measured at the tip. The effects of damage parameters, including damage width $\Delta L$, depth $h_{d}$, and location $L_{d}$ from the tip, on such responses are studied in the time and frequency domains. In Figure 7 the transverse tip velocities in three damaged beam configurations with varying damage width $\Delta L$ are plotted in the time and frequency domains, respectively. In all cases the notch is at a distance $L_{d}=0.25 \mathrm{~m}$ from the tip and the depth is $h_{d}=0.1 h$. The $\Delta L$ are varied as $0.01,0.02$, and $0.03 \mathrm{~m}$. These responses are also compared with that of the undamaged beam. It can be seen from Figure 7a that the presence of the notch results in an early reflection of the flexural wave, which appears before the arrival of the wave reflected from the fixed end of the cantilever beam. In addition, the increase in $\Delta L$ results in an increase of the amplitude of the wave reflected from the notch. Similar responses are studied in frequency domain in Figure $7 \mathrm{~b}$. It can be 
observed from the frequency domain response of the damaged and undamaged beams that the presence of notch results in a very small change only at the higher natural frequencies, while the amplitude changes considerably even at the lower frequencies. This is to be expected, since we are considering very small notch dimensions, length $\Delta L$ and depth $h_{d}$, consistent with the assumptions made in the perturbation analysis. The frequency responses are plotted in Figure $7 \mathrm{~b}$ only within a small frequency range around 16.5 to $19.0 \mathrm{kHz}$. However, a small shift in the frequencies can also be observed at other frequencies. The figure shows that the increase in $\Delta L$ increases the amplitude and the shift in the frequency is nearly the same for all the damaged responses.

Figure 8 shows the responses of the damaged beam with different depths $h_{d}$ of damage, with the other parameters, $\Delta L$ and $L_{d}$, remaining the same. In Figure 8a, the transverse velocities measured at the tip of the undamaged and damaged cantilever beams with a notch of $\Delta L=0.01 \mathrm{~m}$ at $L_{d}=0.25 \mathrm{~m}$ from the tip are presented. The depth of the notch is varied as $h_{d}=0.1 h, 0.2 h$, and $0.3 h$. Similar to the previous example, here the amplitude of the wave resulting from reflection from the notch increases with the increase in the depth $h_{d}$. In Figure 8b, the responses are plotted in the frequency domain. Even here, similarly to the previous example, a prominent change in the natural frequencies occurs at the higher values of 17.0 and $18.4 \mathrm{kHz}$. Interestingly, it can be seen that the pattern of the frequency response function within the plotted interval is very similar to that of the previous example. This is mainly because the damage model takes into account only the overall reduction in stiffness due to the notch, instead of the separate effect of $h_{d}$ and $\Delta L$.

Finally, numerical experiments are performed to study the responses of undamaged and damaged beams with the notch at different locations, due to the broad band impulse load (see Figure 3) applied at the tip in transverse direction. The transverse velocities measured at the tip are presented in Figure 9a. Here the notches have a width $\Delta L=0.02 \mathrm{~m}$ and depth $h_{d}=0.1 h$, but are located at distances $L_{d}=$ $0.1,0.25$, and $0.4 \mathrm{~m}$ from the tip. It can be observed that for $L_{d}=0.1 \mathrm{~m}$ the reflection from the notch

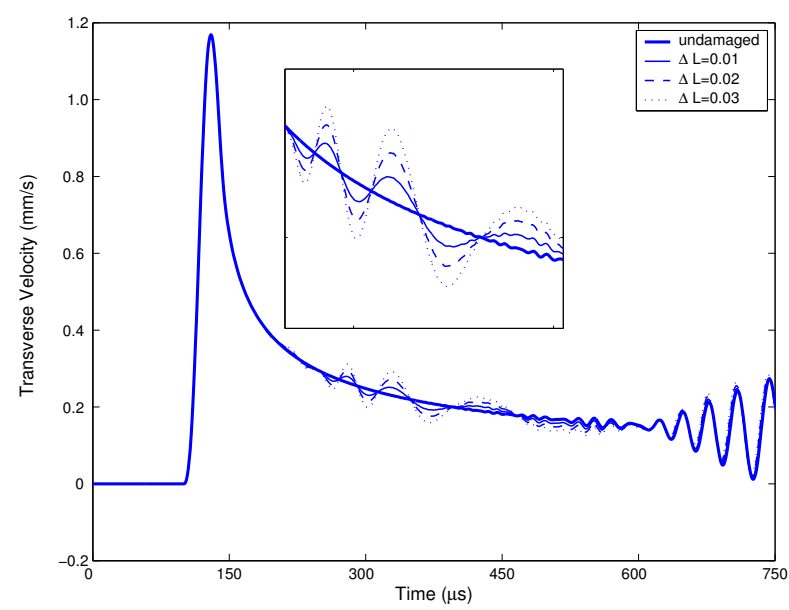

(a)

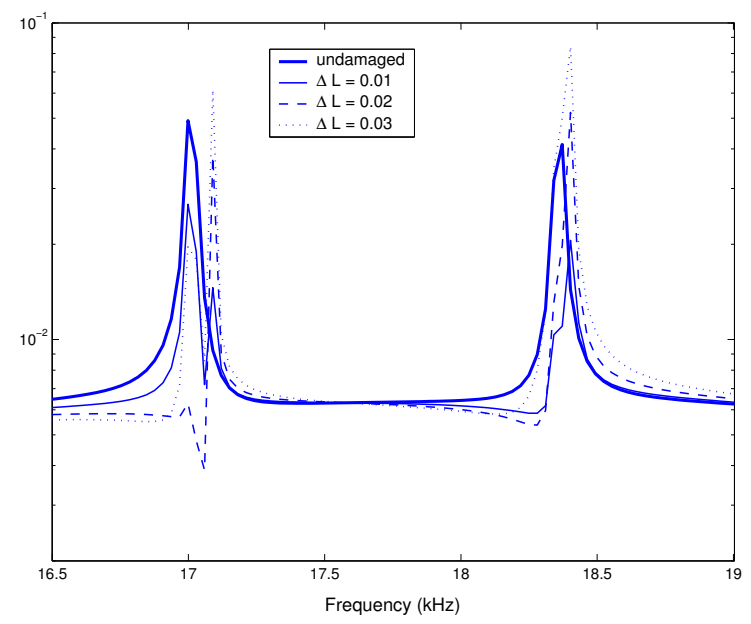

(b)

Figure 7. Transverse velocities at the tip of a cantilever beam of length $L=1.0 \mathrm{~m}$ with $L_{d}=0.25 \mathrm{~m}, \Delta L=0.01,0.02$, and $0.03 \mathrm{~m}$, and depth $h_{d}=10 \%$ compared with response of the undamaged beam in (a) time and (b) frequency domains. 


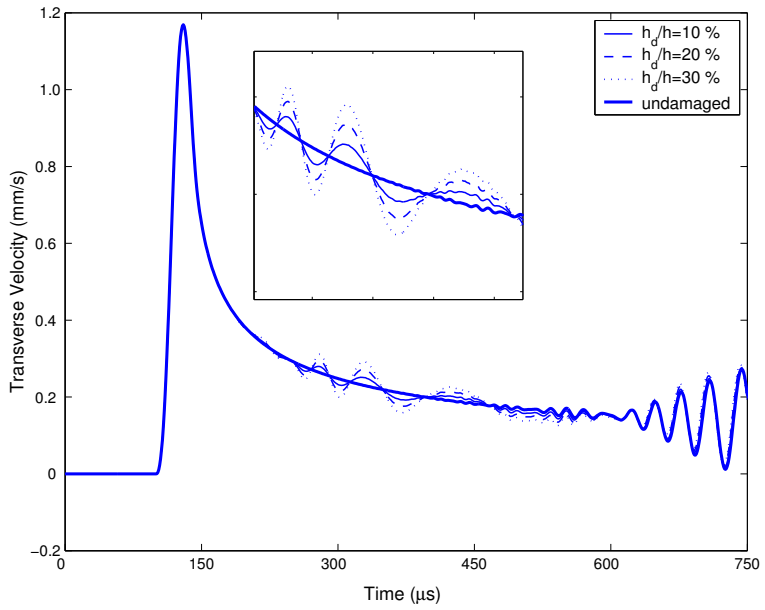

(a)

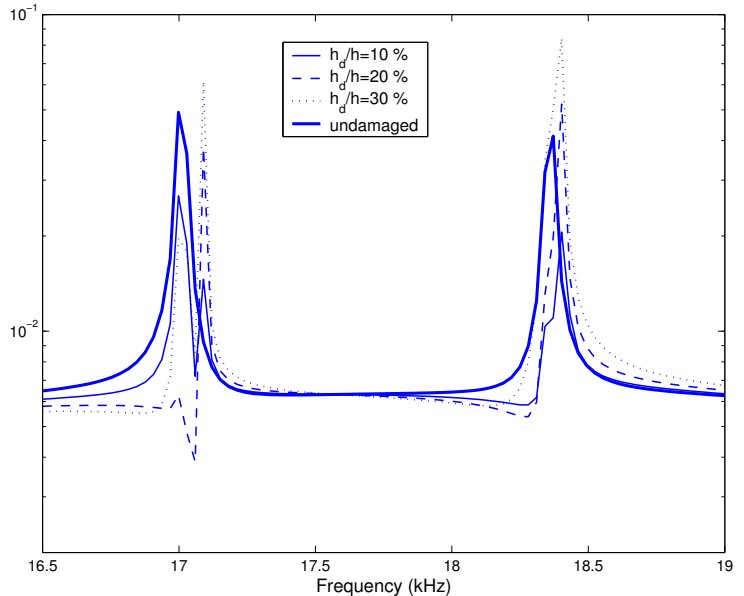

(b)

Figure 8. Transverse velocities at the tip of a cantilever beam of length $L=1.0 \mathrm{~m}$ with $L_{d}=0.25 \mathrm{~m}, \Delta L=0.01 \mathrm{~m}$, and depth $h_{d}=10,20$, and $30 \%$, compared with the response of an undamaged beam in (a) time and (b) frequency domains.

arrives much earlier than expected. However, in the case of $L_{d}=0.4 \mathrm{~m}$, the difference in the arrival time between the reflections from the damage and the fixed end is much less, and they are not quite distinguishable. In addition, for the same damage size, the amplitude of the reflection from the damage at $L_{d}=0.4 \mathrm{~m}$ is least. This is because the flexural waves are dispersive in nature, and thus the amplitude decreases as it travels more. The corresponding frequency domain responses are shown in Figure 9b, for frequency ranges around $21.5 \mathrm{kHz}$ and $25 \mathrm{kHz}$ respectively. In this case, unlike the two previous

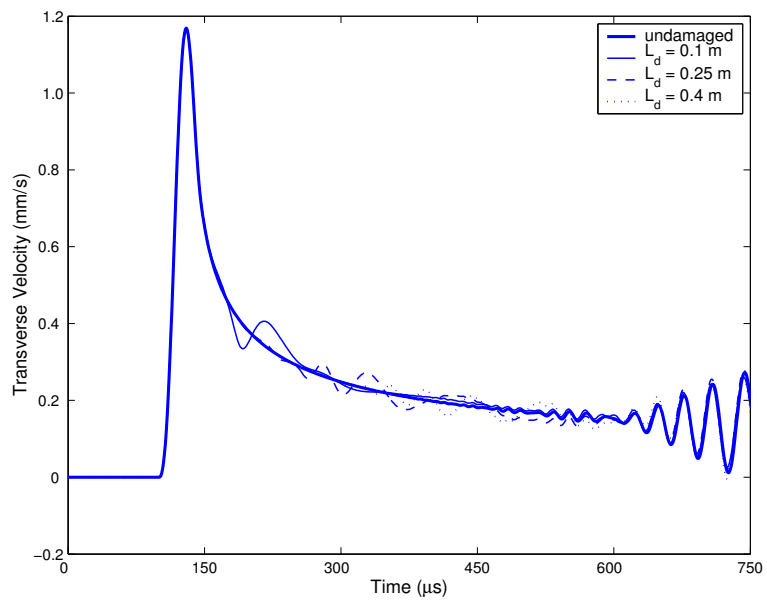

(a)

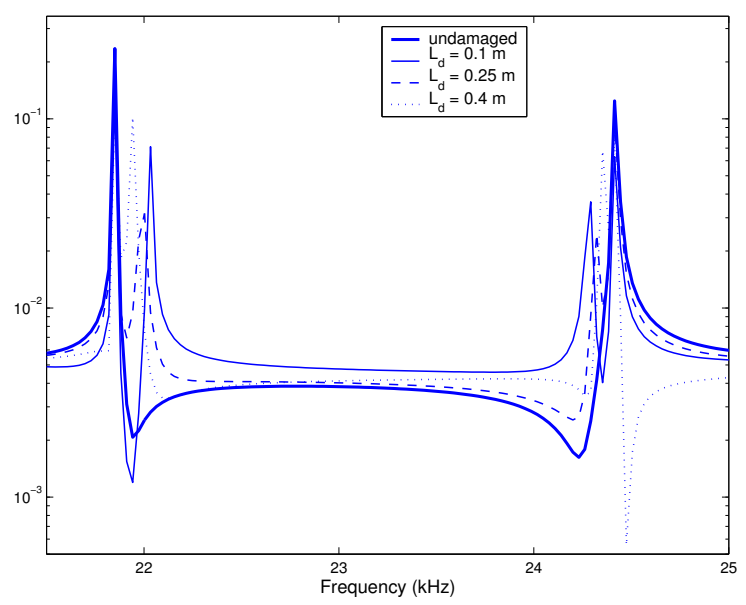

(b)

Figure 9. Transverse velocities at the tip of a cantilever beam of length $L=1.0 \mathrm{~m}$ with $L_{d}=0.1,0.25$, and $0.4 \mathrm{~m}, \Delta L=0.01 \mathrm{~m}$, and depth $h_{d}=10 \%$, compared with the response of an undamaged beam in (a) time and (b) frequency domains. 
examples, considerable shift in frequencies occurs at two other frequencies, approximately 22.1 and $23.4 \mathrm{kHz}$.

\section{Conclusions}

In this paper, wave propagation characteristics in a damaged beam modeled as a Euler-Bernoulli beam with through-width notches are studied and compared with the response of the corresponding undamaged beam. The wave propagation of the notched beam is considered as a perturbation of the undamaged beam response with the assumption of small damage. The modeling is done using the wavelet based spectral finite element technique, including the perturbation solution. The wavelet based method allows efficient analysis of finite length structure due to the use of localized Daubechies scaling functions as bases, unlike the Fourier transform based spectral finite element method. Here the flexural wave propagation in the damaged beam is studied for both narrow and broad banded excitations in both time and frequency domains. For narrow banded modulated pulse loading the location of the damages, calculated inversely from the simulated response, using the arrival time and wave speeds obtained from the dispersion relations, matches very well with that considered for the simulation. Next, the effects of different damage parameters, including damage width, depth, and locations, on the response, due to broad band impulse loading, are analyzed in the time and frequency domains.

In the present work, coupling of axial and flexural modes due to the presence of damage is not considered. The model is developed for pure flexural wave propagation, neglecting the effect of axial mode conversion that may arise due to the damage. This is justified for damages with small dimensions, where this effect is negligible. However, further work lies in including such coupling in the formulation and studying its effects. It should also be mentioned that Timoshenko theory is more suited for the study of wave characteristics. However, the extension of the present method to Timoshenko theory will involve solution of a set of coupled partial differential equations, unlike Euler-Bernoulli beam theory. The formulation for a Timoshenko beam can be considered for future work.

\section{References}

[Beylkin 1992] G. Beylkin, "On the representation of operators in bases of compactly supported wavelets", SIAM Journal of Numerical Analysis 6:6 (1992), 1716-1740.

[Chakraborty and Gopalakrishnan 2005] A. Chakraborty and S. Gopalakrishnan, "A spectrally formulated plate element for wave propagation analysis in anisotropic material", Computer Methods in Applied Mechanics and Engineering 194 (2005), 4425-4446.

[Daubechies 1992] I. Daubechies, Ten lectures on wavelets, CBMS-NSF Series in Applied Mathematics (SIAM, Philadelphia), 1992.

[Doyle 1999] J. F. Doyle, Wave Propagation in Structures, Springer, New York, 1999.

[Jones 1982] D. S. Jones, The Theory of Generalized Functions, Cambridge University Press, Cambridge, UK, 1982.

[Lestari 2001] W. Lestari, Damage of composite structures : detection technique, dynamic response and residual strength, Ph.D. thesis, Georgia Institute of Technology, 2001.

[Luo and Hanagud 1998] H. Luo and S. Hanagud, "An integral equation for changes in the structural characteristics of damaged structures", International Journal of Solids and Structures 34(35-36) (1998), 4557-4579.

[Mitra and Gopalakrishnan 2005] M. Mitra and S. Gopalakrishnan, "Spectrally Formulated Wavelet Finite Element for Wave Propagation and Impact Force Identification in Connected 1-D Waveguides", International Journal of Solids and Structures 42 (2005), 4695-4721. 
[Mitra and Gopalakrishnan 2006] M. Mitra and S. Gopalakrishnan, "Extraction of wave charateristics from wavelet based spectral finite element formulation", Mechanical Systems and Signal Processing 20 (2006), 2046-2079.

[Sharma et al. 2006] V. K. Sharma, M. Ruzzene, and S. Hanagud, "Perturbation methods for the analysis of the dynamic behavior of damaged plates", International Journal of Solids and Structures 43 (2006), 4648-4672.

[Williams and Amaratunga 1994] J. R. Williams and K. Amaratunga, "Introduction to wavelets in engineering", International Journal for Numerical Methods in Engineering 37 (1994), 2365-2388.

[Williams and Amaratunga 1997] J. R. Williams and K. Amaratunga, "A Discrete wavelet transform without edge effects using wavelet extrapolation”, Journal of Fourier Analysis and Applications 3:4 (1997), 435-449.

Received 2 May 2007. Revised 18 Aug 2007. Accepted 14 Dec 2007.

MIRA MITRA: mira@aero.iitb.ac.in

Department of Aerospace Engineering, Indian Institute of Technology, Bombay 400 076, India

http://www.aero.iitb.ac.in/ mira

S. GOPALAKRISHNAN: krishnan@aero.iisc.ernet.in

Department of Aerospace Engineering, Indian Institute of Science, Bangalore 560 012, India

http://www.aero.iisc.ernet.in/ krishnan

MASSIMO RUZZENE: Massimo.ruzzene@ae.gatech.edu

School of Aerospace Engineering, Georgia Institute of Technology, 270 Ferst Drive, Atlanta, GA, United States

NiCOLE APETRE: nicole.apetre@gatech.edu

School of Aerospace Engineering, Georgia Institute of Technology, 270 Ferst Drive, Atlanta, GA, United States

S. HANAGUD: hanagud@ae.gatech.edu

School of Aerospace Engineering, Georgia Institute of Technology, 270 Ferst Drive, Atlanta, GA, United States 


\title{
INTEGRATION OF MEASURES AND ADMISSIBLE STRESS FIELDS FOR MASONRY BODIES
}

\author{
Massimiliano LuCChesi, Miroslav ŠIlhaVÝ AND NiCOla Zani
}

\begin{abstract}
We study the compatibility of loads for bodies made of a no-tension (masonry) material. Loads are defined as weakly compatible if they can be equilibrated by an admissible stress field represented by a tensor valued measure, and strongly compatible if they can be equilibrated by a square integrable function. In the present study, we examine situations in which weak compatibility implies strong compatibility. For families of loads that depend on a parameter and the families of measures that equilibrate these loads, we find that, under some conditions, averaging with respect to the parameter leads to a measure with a square integrable density that equilibrates the loads. We illustrate the procedure on twodimensional rectangular panels free from gravity, clamped at the bottom, and subjected to various loads on the free part of the boundary.
\end{abstract}

\section{Introduction}

We study the equilibrium problem of a body made of a no-tension (or masonry-like) material [Di Pasquale 1984; Anzellotti 1985; Giaquinta and Giusti 1985; Del Piero 1989; Lucchesi et al. 1994] under given loads $(\boldsymbol{s}, \boldsymbol{b})$ where $\boldsymbol{s}$ is the force applied to the free part of the boundary and $\boldsymbol{b}$ is the body force. The existence of equilibrium states, or at least the weaker property that the total energy functional of the masonry body be bounded from below, is closely related to the existence of a stress field $\boldsymbol{T}$ that is equilibrated with the applied loads and compatible with the incapability of the material to withstand traction (see Proposition 3.1, below). The problem of finding such an admissible equilibrating stress field $\boldsymbol{T}$ is a central problem of limit analysis [Temam 1983, Chapter 1, Section 5; Del Piero 1998; Lucchesi et al. 2008] because these stresses can be used to determine lower bounds for the collapse load and sometimes the collapse load itself. The loads admitting such a stress field are called compatible.

It has been shown in [Lucchesi et al. 2004; 2005a; 2005b; 2006; 2007] that the solution in concrete cases simplifies considerably if instead of admissible equilibrating stress fields represented by ordinary functions $\boldsymbol{T}$ one admits also stress fields $\boldsymbol{T}$ represented by tensor valued measures. This amounts to allowing for singularities of the stress field on one or more surfaces or curves of concentrated stress. In this paper, loads that admit an admissible equilibrating stress represented by a measure are called weakly compatible to distinguish them from loads that admit admissible equilibrating stresses represented by a square integrable function, which we call strongly compatible. These notions are not equivalent, as the examples show.

Keywords: masonry bodies, compatibility of loads, stresses represented by measures.

This research has been supported by Project SP2 Metodi e modelli predittivi del comportamento strutturale di manufatti in presenza e in assenza di danno. The support is gratefully acknowledged. 
Nevertheless, the existence of solutions to the equilibrium problem, and in particular the existence of a lower bound for the total energy functional, is strictly related to the existence of the admissible equilibrating stress field represented by a square integrable function by Proposition 3.1.

In the present paper, we describe a procedure that in certain cases allows us to use the information that loads are weakly compatible to show that they are actually strongly compatible. Crucial to the procedure is the fact that, in applications, both the loads $\left(\boldsymbol{s}^{\lambda}, \boldsymbol{b}^{\lambda}\right)$ and the admissible equilibrating stress measure $\mathrm{T}^{\lambda}$ depend on a real parameter $\lambda$. The identification of $\lambda$ depends on the nature of the problem. The idea is to take the average of the stress measure over any set $(\mu-\epsilon, \mu+\epsilon)$, where $\epsilon>0$ is sufficiently small as dictated by the nature of the solution $\mathbf{T}^{\lambda}$ and $\mu$ is any point in the set of parameters. Averaging gives the measure

$$
\mathbf{T}=\frac{1}{2 \epsilon} \int_{\mu-\epsilon}^{\mu+\epsilon} \mathbf{T}^{\lambda} d \lambda
$$

and it may happen that this measure, in contrast to $\mathrm{T}^{\mu}$, is absolutely continuous (with respect to the Lebesgue measure) with the density $\boldsymbol{T}$, which is square integrable. If the loads $\left(\boldsymbol{s}^{\lambda}, \boldsymbol{b}^{\lambda}\right)$ depend linearly on the parameter $\lambda$, as is often the case, then it is automatic that $\boldsymbol{T}$ equilibrates the loads $\left(\boldsymbol{s}^{\mu}, \boldsymbol{b}^{\mu}\right)$. It is intuitively plausible that the averaging procedure smears out the singularities in $\mathbf{T}^{\lambda}$ if the set of singularities changes its position with changing $\lambda$. Mathematically, the procedure is based on the coarea formula of the geometric measure theory, which also gives the conditions under which it is really the case.

This paper illustrates the general procedure on rectangular two-dimensional panels. We assume that the panel is free from body forces, clamped at its bottom, and subjected to loads prescribed on the boundary. We consider three types of the boundary loads:

(i) vertical top loads and horizontal loads on one side of the panel,

(ii) uniform vertical top loads and oblique side loads on one side of the panel,

(iii) and uniform vertical top loads and vanishing side loads on a panel with a symmetric opening.

In all cases we use the admissible equilibrating stresses represented by measures constructed in [Lucchesi et al. 2006], and combine them with averaging to produce equilibrating stress fields represented by square integrable functions (in fact, they are bounded in these three cases).

In Section 2 we consider families of vector valued measures, called parametric measures, that are mappings from the set of real parameters to the space of vector valued measures. We define an integral with respect to the parameter of such a mapping, which is the abstract counterpart of the averaging procedure mentioned above. The result of integration is again a measure. Section 3 introduces bodies and the loads applied to them. We define weakly and strongly equilibrating stress fields, and in Propositions 3.2 and 3.3 we describe the averaging procedure. The rest of the paper is devoted to the treatment of the loads (i)-(iii) listed above: Sections 4-5 deal with (i), Section 6 with (ii) and Section 7 with (iii). In general, the average of the parametric measure is difficult to calculate explicitly, and for applications it wholly suffices to know that averaging leads to the existence of a square integrable admissible stress field equilibrating the loads. Such is the case of the loads (i)-(iii). However, in a special subcase of case (i), treated in Section 5, we explicitly determine the result of the averaging.

Throughout, we use the conventions for vectors and second order tensors given in [Gurtin 1981]. Thus Lin denotes the set of all second order tensors on $\mathbb{R}^{n}$, that is, linear transformations from $\mathbb{R}^{n}$ into itself; 
Sym is the subspace of symmetric tensors; $\mathrm{Sym}^{+}$the set of all positive semidefinite elements of Sym; $\mathrm{Sym}^{-}$is the set of all negative semidefinite elements of Sym. The scalar product of $\boldsymbol{A}, \boldsymbol{B} \in \mathrm{Lin}$ is defined by $\boldsymbol{A} \cdot \boldsymbol{B}=\operatorname{tr}\left(\boldsymbol{A} \boldsymbol{B}^{\top}\right)$ and $|\cdot|$ denotes the associated Euclidean norm on Lin.

\section{Families of measures}

If $\Omega \subset \mathbb{R}^{n}$ is a Borel set and $V$ a finite-dimensional inner product space, we denote by $M(\Omega, V)$ the set of all $V$ valued measures (of finite total variation) supported by $\Omega$ [Ambrosio et al. 2000, Chapter 1]. If $\mathbf{m} \in \mathcal{M}\left(\mathbb{R}^{n}, V\right)$, we denote by $|\mathbf{m}|$ the total variation measure of $\mathbf{m}$, and by $\mathrm{M}(\mathbf{m})$ the mass of $\mathbf{m}$, defined by $\mathbf{M}(\mathbf{m})=|\mathbf{m}|\left(\mathbb{R}^{n}\right)$. We call the elements of $\mathcal{M}\left(\Omega\right.$, Sym) tensor measures. We denote by $\mathscr{L}^{n}$ the Lebesgue measure in $\mathbb{R}^{n}$ [Ambrosio et al. 2000, Definition 1.52], and if $k$ is an integer, $0 \leq k \leq n$, we denote by $\mathscr{g}^{k}$ the $k$-dimensional Hausdorff measure in $\mathbb{R}^{n}$ [Ambrosio et al. 2000, Section 2.8]. If $\phi$ is a nonnegative measure or a $V$ valued measure, we denote by $\phi L A$ the restriction of $\phi$ to a Borel set $A \subset \mathbb{R}^{n}$; if $\phi$ is a nonnegative measure, we denote by $f \phi$ the product of the measure $\phi$ by a $\phi$ integrable $V$ valued function $f$ on $\mathbb{R}^{n}$; we refer to [Lucchesi et al. 2006, Section 2] for details. If $\Omega$ is an open subset of $\mathbb{R}^{n}$, we denote by $C_{0}(\Omega, V)$ the space of all continuous $V$ valued functions on $\mathbb{R}^{n}$ with compact support that is contained in $\Omega$, and denote by $|\cdot|_{C_{0}}$ the maximum norm on $C_{0}\left(\mathbb{R}^{n}, V\right)$.

An integrable parametric measure is a family $\left\{\mathbf{m}^{\lambda}: \lambda \in \Lambda\right\}$ of $V$ valued measures on $\mathbb{R}^{n}$ where $\Lambda \subset \mathbb{R}$ is a $\mathscr{L}^{1}$ measurable set of parameters such that

(i) for every $f \in C_{0}\left(\mathbb{R}^{n}, V\right)$ the function $\lambda \mapsto \int_{\mathbb{R}^{n}} f \cdot d \mathbf{m}^{\lambda}$ is $\mathscr{L}^{1}$ measurable on $\Lambda$;

(ii) we have

$$
c:=\int_{\Lambda} \mathrm{M}\left(\mathbf{m}^{\lambda}\right) d \lambda<\infty
$$

We note that the function $\lambda \mapsto \mathrm{M}\left(\mathbf{m}^{\lambda}\right)$ is $\mathscr{L}^{1}$ measurable on $\Lambda$ as a consequence of condition (i): if $K \subset C_{0}\left(\mathbb{R}^{n}, V\right)$ is a countable dense set then

$$
\mathbf{M}\left(\mathbf{m}^{\lambda}\right)=\sup \left\{\int_{\mathbb{R}^{n}} f \cdot d \mathbf{m}^{\lambda}: f \in K,|f|_{C_{0}} \leq 1\right\},
$$

and thus the function $\lambda \mapsto \mathrm{M}\left(\mathbf{m}^{\lambda}\right)$ is a supremum of a countable family of $\mathscr{L}^{1}$ measurable functions. Hence, $\mathscr{L}^{1}$ measurable.

We note that parametric measures similar to those defined above occur in the contexts of disintegration (slicing) of measures [Ambrosio et al. 2000, Section 2.5] and Young's measures [Müller 1999, Chapter 5].

Proposition 2.1. If $\left\{\mathbf{m}^{\lambda}: \lambda \in \Lambda\right\}$ is an integrable parametric measure, then there exists a unique $V$ valued measure $\mathbf{m}$ on $\mathbb{R}^{n}$ such that

$$
\int_{\mathbb{R}^{n}} f \cdot d \mathbf{m}=\int_{\Lambda} \int_{\mathbb{R}^{n}} f \cdot d \mathbf{m}^{\lambda} d \lambda
$$

for each $f \in C_{0}\left(\mathbb{R}^{n}, V\right)$.

We write

$$
\mathbf{m}=\int_{\Lambda} \mathbf{m}^{\lambda} d \lambda
$$

and call $\mathbf{m}$ the integral of the family $\left\{\mathbf{m}^{\lambda}: \lambda \in \Lambda\right\}$ with respect to $\lambda$. 
Proof. We note that for each $f \in C_{0}\left(\mathbb{R}^{n}, V\right)$, the right hand side of Equation (1) is a well defined real number. Indeed,

$$
\begin{aligned}
\left|\int_{\Lambda} \int_{\mathbb{R}^{n}} f \cdot d \mathbf{m}^{\lambda} d \lambda\right| & \leq \int_{\Lambda} \int_{\mathbb{R}^{n}}|f| d\left|\mathbf{m}^{\lambda}\right| d \lambda \\
& \leq|f|_{C_{0}} \int_{\Lambda} \mathbf{M}\left(\mathbf{m}^{\lambda}\right) d \lambda \\
& \leq c|f|_{C_{0}} .
\end{aligned}
$$

Thus, by the Riesz representation theorem [Ambrosio et al. 2000, Theorem 1.54], there exists a measure m such that Equation (1) holds.

The following two propositions give two important examples of integrable parametric measures. In both cases the corresponding integral, Equation (2), is absolutely continuous with respect to the Lebesgue measure.

Proposition 2.2. Let $\left\{h^{\lambda}: \lambda \in \Lambda\right\}$ be a family of $V$ valued functions on $\Omega \subset \mathbb{R}^{n}$ defined for all $\lambda$ from a $\mathscr{L}^{1}$ measurable set $\Lambda \subset \mathbb{R}$ such that the mapping $(\boldsymbol{x}, \lambda) \mapsto h^{\lambda}(\boldsymbol{x})$ is $\mathscr{L}^{n+1}$ integrable on $\Omega \times \Lambda$, that is,

$$
\int_{\Lambda} \int_{\Omega}\left|h^{\lambda}(\boldsymbol{x})\right| d \boldsymbol{x} d \lambda<\infty .
$$

If we define a $V$ valued measure $\mathbf{m}^{\lambda}$ by

$$
\mathrm{m}^{\lambda}=h^{\lambda} \mathscr{L}^{n} \mathrm{~L} \Omega
$$

then $\left\{\mathbf{m}^{\lambda}: \lambda \in \Lambda\right\}$ is an integrable parametric measure, and we have

$$
\int_{\Lambda} \mathbf{m}^{\lambda} d \lambda=k \mathscr{L}^{n}\llcorner\Omega,
$$

where $k(\boldsymbol{x})=\int_{\Lambda} h^{\lambda}(\boldsymbol{x}) d \lambda$, for $\mathscr{L}^{n}$ a.e. $\boldsymbol{x} \in \Omega$.

Proof. This follows directly from Fubini's theorem.

Proposition 2.3. Let $\Omega_{0} \subset \mathbb{R}^{n}$ be open, let $\varphi: \Omega_{0} \rightarrow \mathbb{R}$ be locally Lipschitz continuous, and let $g: \Omega_{0} \rightarrow V$ be $\mathscr{L}^{n}$ measurable on $\Omega_{0}$, with

$$
\int_{\Omega_{0}}|g||\nabla \varphi| d \mathscr{L}^{n}<\infty .
$$

Then for $\mathscr{L}^{1}$ a.e. $\lambda \in \mathbb{R}$, the function $g$ is $\mathscr{H}^{n-1} L^{-1}(\lambda)$ integrable. Denoting by $\Lambda$ the set of all such $\lambda$, we define the measure $\mathbf{m}^{\lambda}$ by

$$
\mathbf{m}^{\lambda}:=g \mathscr{H}^{n-1}\left\llcorner\varphi^{-1}(\lambda),\right.
$$

for each $\lambda \in \Lambda$. Then $\left\{\mathrm{m}^{\lambda}: \lambda \in \Lambda\right\}$ is an integrable parametric measure, and we have

$$
\int_{\Lambda} \mathbf{m}^{\lambda} d \lambda=g|\nabla \varphi| \mathscr{L}^{n}\left\llcorner\Omega_{0} .\right.
$$


Proof. Let $\mathbf{m}$ be given by Equation (2). If $f \in C_{0}\left(\mathbb{R}^{n}, V\right)$, then by the coarea formula [Ambrosio et al. 2000, Section 2.12] we have

$$
\begin{aligned}
\int_{\Omega_{0}} f \cdot g|\nabla \varphi| d \mathscr{L}^{n} & =\int_{\mathbb{R}} \int_{\varphi^{-1}(\lambda)} f \cdot g d \mathscr{H}^{n-1} d \lambda \\
& =\int_{\Lambda} \int_{\mathbb{R}^{n}} f \cdot d \mathbf{m}^{\lambda} d \lambda \\
& =\int_{\mathbb{R}^{n}} f \cdot d \mathbf{m} .
\end{aligned}
$$

\section{Equilibrated loads}

We consider a continuous body represented by a Lipschitz domain [Adams and Fournier 2003] $\Omega \subset \mathbb{R}^{n}$ and assume that $\mathscr{D}, \mathscr{Y}$ are two disjoint Borel subsets of $\partial \Omega$ such that $\mathscr{D} \cup \mathscr{S}=\partial \Omega$, where $\mathscr{D}, \mathscr{Y}$ will be identified below as the set of prescribed boundary displacement and prescribed boundary force.

We set

$$
V_{0}=\left\{\boldsymbol{v} \in C^{1}\left(\mathrm{cl} \Omega, \mathbb{R}^{n}\right): \boldsymbol{v}=\mathbf{0} \text { on } \mathscr{D}\right\}
$$

and

$$
V=\left\{\boldsymbol{v} \in W^{1,2}\left(\Omega, \mathbb{R}^{n}\right): \boldsymbol{v}=\mathbf{0} \text { a.e. on } \mathscr{D}\right\},
$$

where $C^{1}\left(\operatorname{cl} \Omega, \mathbb{R}^{n}\right)$ is the set of all continuously differentiable mappings $v: \Omega \rightarrow \mathbb{R}^{n}$ such that $v$ and its derivative $\nabla \boldsymbol{v}$ have a continuous extension to the closure $\operatorname{cl} \Omega$ of $\Omega$, and $W^{1,2}\left(\Omega, \mathbb{R}^{n}\right)$ is the Sobolev space of all $\mathbb{R}^{n}$ valued maps such that $\boldsymbol{v}$ and the distributional derivative $\nabla \boldsymbol{v}$ of $\boldsymbol{v}$ are square integrable on $\Omega$ [Adams and Fournier 2003]. We have $V_{0} \subset V$. For any $v \in V$ we define the infinitesimal strain tensor $\hat{\boldsymbol{E}}(\boldsymbol{v})$ of $\boldsymbol{v}$ by

$$
\hat{\boldsymbol{E}}(\boldsymbol{v})=\frac{1}{2}\left(\nabla \boldsymbol{v}+\nabla \boldsymbol{v}^{\top}\right) .
$$

The loads of the body are a pair $\mathfrak{L}=(\mathbf{s}, \mathbf{b})$ where $\mathbf{s} \in \mathcal{M}\left(\varphi, \mathbb{R}^{n}\right), \mathbf{b} \in \mathcal{M}\left(\Omega, \mathbb{R}^{n}\right)$. Here $\mathbf{s}$ represents the force applied to the boundary $\mathscr{S}$ and $\mathbf{b}$ the force applied to the bulk $\Omega$ of the body. Since both $\mathbf{s}$ and $\mathbf{b}$ are measures, the definition admits concentrated forces on $\mathscr{S}$ and in $\Omega$ [Podio-Guidugli 2004; Lucchesi et al. 2006]. See Equation (42) for an example. Below we also consider the special case when these two measures are absolutely continuous with respect to the measures $\mathscr{H}^{n-1}$ and $\mathscr{L}^{n}$.

We interpret the measures $T \in \mathcal{M}(\Omega$, Sym $)$ as stresses. Again, concentration effects are possible. We say that $\mathbf{T} \in \mathcal{M}(\Omega$, Sym $)$ is admissible if $\mathbf{T}$ takes the values in the set $\mathrm{Sym}^{-}$of the negative semidefinite symmetric tensors, that is, if $\mathbf{T}(A) \boldsymbol{a} \cdot \boldsymbol{a} \leq 0$ for any Borel set $A \subset \Omega$ and for any $\boldsymbol{a} \in \mathbb{R}^{n}$. We say that $\mathbf{T}$ weakly equilibrates the loads $(\mathbf{s}, \mathbf{b})$ if

$$
\int_{\Omega} \hat{\boldsymbol{E}}(\boldsymbol{v}) \cdot d \mathbf{T}=\int_{\Omega} \boldsymbol{v} \cdot d \mathbf{b}+\int_{\mathscr{\varphi}} \boldsymbol{v} \cdot d \mathbf{s}
$$

for any $\boldsymbol{v} \in V_{0}$. We say that the loads $\mathfrak{L}=(\mathbf{s}, \mathbf{b})$ are weakly compatible if there exists an admissible $\mathbf{T} \in \mathcal{M}(\Omega$, Sym) which weakly equilibrates them.

One can consider, in particular, the loads $\mathfrak{L}=(\mathbf{s}, \mathbf{b})$ of the form

$$
\mathbf{s}=\boldsymbol{s}^{\mathscr{H}^{n-1}}\left\llcorner\mathscr{S}, \quad \mathbf{b}=\boldsymbol{b} \mathscr{L}^{n}\llcorner\Omega,\right.
$$


where

$$
\boldsymbol{s} \in L^{2}\left(\mathscr{S}, \mathbb{R}^{n}\right), \quad \boldsymbol{b} \in L^{2}\left(\Omega, \mathbb{R}^{n}\right),
$$

with the first $L^{2}$ space taken relative to the measure $\mathscr{H}^{n-1}$ on $\mathscr{Y}$ and the second relative to $\mathscr{L}^{n}$ on $\Omega$. In this case, we often identify the pair $\mathfrak{L}=(\mathbf{s}, \mathbf{b})$ with the pair $\mathfrak{L}=(\boldsymbol{s}, \boldsymbol{b})$.

One can consider, in particular, the measure $\mathbf{T}$ of the form $\boldsymbol{T}=\boldsymbol{T} \mathscr{L}^{n} L \Omega$, where $\boldsymbol{T} \in L^{2}(\Omega$, Sym). We say that $\boldsymbol{T}$ is admissible if $\boldsymbol{T}(\boldsymbol{x}) \in \mathrm{Sym}^{-}$for $\mathscr{L}^{n}$ a.e. $\boldsymbol{x} \in \Omega$. This is equivalent to saying that the measure $\mathbf{T}=\boldsymbol{T} \mathscr{L}^{n} L \Omega$ is admissible in the sense defined above. We say that $\boldsymbol{T}$ strongly equilibrates the loads $\mathfrak{L}=(\boldsymbol{s}, \boldsymbol{b})$ if

$$
\int_{\Omega} \hat{\boldsymbol{E}}(\boldsymbol{v}) \cdot \boldsymbol{T} d \mathscr{L}^{n}=\int_{\Omega} \boldsymbol{v} \cdot \boldsymbol{b} d \mathscr{L}^{n}+\int_{\mathscr{S}} \boldsymbol{v} \cdot \boldsymbol{s} d \mathscr{H}^{n-1},
$$

for each $\boldsymbol{v} \in V$. Note that this notion applies only to the special loads represented by $\boldsymbol{s}, \boldsymbol{b}$ as in Equation (6). We say that the loads $\mathfrak{L}=(\boldsymbol{s}, \boldsymbol{b})$ satisfying Equation (7) are strongly compatible if there exists an admissible stress field $\boldsymbol{T} \in L^{2}(\Omega$, Sym) strongly equilibrating them. In [Šilhavý 2008, Example 9.4], an example is given of loads $(\boldsymbol{s}, \boldsymbol{b})$ satisfying Equation (7) (even with $\boldsymbol{s}$ bounded and $\boldsymbol{b} \equiv \mathbf{0}$ ) such that $(\boldsymbol{s}, \boldsymbol{b})$ are weakly compatible but not strongly compatible.

The importance of the strong compatibility arises from the following statement.

Proposition 3.1 ([Padovani et al. 2007]). Let $\mathfrak{L}=(\boldsymbol{s}, \boldsymbol{b})$ be the loads satisfying Equation (7). Define the total energy functional $I: V \rightarrow \mathbb{R}$ by

$$
I(\boldsymbol{v})=\int_{\Omega} \hat{w}(\hat{\boldsymbol{E}}(\boldsymbol{v})) d \mathscr{L}^{n}-\int_{\Omega} \boldsymbol{v} \cdot \boldsymbol{b} d \mathscr{L}^{n}-\int_{\mathscr{S}} \boldsymbol{v} \cdot \boldsymbol{s} d \mathscr{H}^{n-1}
$$

$\boldsymbol{v} \in V$, where $\hat{w}: \operatorname{Sym} \rightarrow[0, \infty)$ is the stored energy of a no-tension material [Del Piero 1989]. Then the loads are strongly compatible if and only if

$$
I_{0}:=\inf \{I(\boldsymbol{v}): \boldsymbol{v} \in V\}>-\infty .
$$

The condition $I_{0}>-\infty$, in turn, has a dynamical significance [Padovani et al. 2007]: If $I_{0}>-\infty$ then any dynamical process of a masonry body with dissipation stabilizes in the sense that the kinetic energy tends to 0 , and if the set of equilibrium states is nonempty, the process asymptotically approaches the set of all equilibrium states. If, on the contrary, $I_{0}=-\infty$, then any dynamical process exhibits a (dynamical) collapse in the sense that the total energy approaches $-\infty$, and the $W^{1,1}$ norm of the state at large times converges to $\infty$ (at least if $\boldsymbol{s}$ and $\boldsymbol{b}$ are bounded).

One often encounters the situation in which the loads depend on a parameter $\lambda$ from a subset $\Lambda$ of $\mathbb{R}$; that is, one deals with the family of loads $\mathfrak{L}^{\lambda}=\left(\mathbf{s}^{\lambda}, \mathbf{b}^{\lambda}\right), \lambda \in \Lambda$, where

$$
\left\{\mathbf{s}^{\lambda}, \lambda \in \Lambda\right\}, \quad\left\{\mathbf{b}^{\lambda}, \lambda \in \Lambda\right\}
$$

are integrable parametric measures with values in $\mathbb{R}^{n}$, with $\Lambda$ an $\mathscr{L}^{1}$ measurable subset of $\mathbb{R}$. A more specific situation arises when the loads $\mathfrak{L}^{\lambda}$ are of the form

$$
\mathbf{s}^{\lambda}=\boldsymbol{s}(\cdot, \lambda) \mathscr{H}^{n-1}\left\llcorner\mathscr{Y}, \quad \mathbf{b}^{\lambda}=\boldsymbol{b}(\cdot, \lambda) \mathscr{L}^{n}\llcorner\Omega,\right.
$$

$\lambda \in \Lambda$, where

$$
\boldsymbol{s} \in L^{2}\left(\mathscr{Y} \times \Lambda, \mathbb{R}^{n}\right), \quad \boldsymbol{b} \in L^{2}\left(\Omega \times \Lambda, \mathbb{R}^{n}\right),
$$


with the first $L^{2}$ space taken with respect to the measure $\mathscr{H}^{n-1} \otimes \mathscr{L}^{1}$ on $\mathscr{S} \times \Lambda$ and the second relative to the $n+1$ dimensional Lebesgue measure on $\Omega \times \Lambda$. Each load $\mathfrak{L}^{\lambda}$ is often weakly equilibrated by a stress field $\mathbf{T}^{\lambda} \in \mathcal{M}(\Omega$, Sym $)$ in such a way that

$$
\left\{\mathbf{T}^{\lambda}, \lambda \in \Lambda\right\}
$$

is an integrable parametric measure. In this situation, we have:

Proposition 3.2. Let $\Lambda \subset \mathbb{R}$ be $\mathscr{L}^{1}$ measurable, and consider the parametric measures as in Equation (8) and Equation (11). Suppose that for $\mathscr{L}^{1}$ a.e. $\lambda \in \Lambda$ the stress field $\mathbf{T}^{\lambda}$ weakly equilibrates the loads $\mathfrak{L}^{\lambda}=\left(\mathbf{s}^{\lambda}, \mathbf{b}^{\lambda}\right)$. Then

(i) the stress field $\overline{\mathbf{T}}:=\int_{\Lambda} \mathbf{T}^{\lambda} d \lambda$ weakly equilibrates the loads $\overline{\mathfrak{L}}:=(\overline{\mathbf{s}}, \overline{\mathbf{b}})$, where

$$
\overline{\mathbf{s}}=\int_{\Lambda} \mathbf{s}^{\lambda} d \lambda, \quad \overline{\mathbf{b}}=\int_{\Lambda} \mathbf{b}^{\lambda} d \lambda ;
$$

(ii) if $\mathscr{L}^{1}(\Lambda)<\infty$ and if the loads are of the form Equation (9)-Equation (10), then the loads $\overline{\mathfrak{L}}$ defined in (i) are given by $\overline{\mathfrak{L}}=(\overline{\boldsymbol{s}}, \overline{\boldsymbol{b}})$, where

$$
\overline{\boldsymbol{s}} \in L^{2}\left(\mathscr{Y}, \mathbb{R}^{n}\right), \quad \overline{\boldsymbol{b}} \in L^{2}\left(\Omega, \mathbb{R}^{n}\right),
$$

are given by

$$
\overline{\boldsymbol{s}}(\boldsymbol{r})=\int_{\Lambda} \boldsymbol{s}(\boldsymbol{r}, \lambda) d \lambda, \quad \boldsymbol{r} \in \mathscr{Y}, \quad \overline{\boldsymbol{b}}(\boldsymbol{r})=\int_{\Lambda} \boldsymbol{b}(\boldsymbol{r}, \lambda) d \lambda, \quad \boldsymbol{r} \in \Omega .
$$

If, additionally,

$$
\int_{\Lambda} \mathbf{T}^{\lambda} d \lambda=\boldsymbol{T} \mathscr{L}^{n}\llcorner\Omega
$$

where $\boldsymbol{T} \in L^{2}(\Omega$, Sym $)$ and $V_{0}$ is dense in $V$, then $\boldsymbol{T}$ strongly equilibrates the loads $\overline{\mathfrak{L}}=(\overline{\boldsymbol{s}}, \overline{\boldsymbol{b}})$.

We note that $V_{0}$ is dense in $V$ if $\Omega$ has Lipschitz boundary, and if $\mathscr{D}$ is closed in $\partial \Omega$ with Lipschitz boundary.

Proof. (i): We have

$$
\int_{\Omega} \hat{\boldsymbol{E}}(\boldsymbol{v}) \cdot d \mathbf{T}^{\lambda}=\int_{\Omega} \boldsymbol{v} \cdot d \mathbf{b}^{\lambda}+\int_{\mathscr{S}} \boldsymbol{v} \cdot d \mathbf{s}^{\lambda}
$$

for any $v \in V_{0}$ and $\mathscr{L}^{1}$ a.e. $\lambda \in \Lambda$. Integrating over $\Lambda$ and invoking the definitions of integrals of measures, we obtain

$$
\int_{\Omega} \hat{\boldsymbol{E}}(\boldsymbol{v}) \cdot d \overline{\mathbf{T}}=\int_{\Omega} \boldsymbol{v} \cdot d \overline{\mathbf{b}}+\int_{\mathscr{\varphi}} \boldsymbol{v} \cdot d \overline{\mathbf{s}}
$$

and thus $\overline{\mathbf{T}}$ weakly equilibrates the loads $\overline{\mathfrak{L}}$.

(ii): The formulas in Equation (13) are obtained by invoking the definitions of integrals of parametric measures, and exchanging the orders of integration with respect to $\boldsymbol{r}$ and $\lambda$. The inclusions Equation (12) follow from the assumption Equation (10) by using Hölder's inequality. If we have Equation (14), then by (i),

$$
\int_{\Omega} \hat{\boldsymbol{E}}(\boldsymbol{v}) \cdot \boldsymbol{T} d \mathscr{L}^{n}=\int_{\Omega} \boldsymbol{v} \cdot \overline{\boldsymbol{b}} d \mathscr{L}^{n}+\int_{\mathscr{S}} \boldsymbol{v} \cdot \overline{\boldsymbol{s}} d \mathscr{L}^{n-1}
$$


for each $\boldsymbol{v} \in V_{0}$; this extends by density to all $\boldsymbol{v} \in V$.

Consider, finally, the situation in which the loads $\mathfrak{L}^{\lambda}$ are of the form Equation (9), and the functions $\boldsymbol{s}(\cdot, \lambda), \boldsymbol{b}(\cdot, \lambda)$ depend on $\lambda$ linearly [Del Piero 1998]. Thus $\mathfrak{L}^{\lambda}:=\left(\boldsymbol{s}^{\lambda}, \boldsymbol{b}^{\lambda}\right)$ where

$$
\boldsymbol{s}^{\lambda}=\boldsymbol{s}_{0}+\lambda \boldsymbol{s}_{1}, \quad \boldsymbol{b}^{\lambda}=\boldsymbol{b}_{0}+\lambda \boldsymbol{b}_{1}, \quad \lambda \in \mathbb{R},
$$

where

$$
\boldsymbol{s}_{0}, \boldsymbol{s}_{1} \in L^{2}\left(\mathcal{Y}, \mathbb{R}^{n}\right), \quad \boldsymbol{b}_{0}, \boldsymbol{b}_{1} \in L^{2}\left(\Omega, \mathbb{R}^{n}\right) .
$$

We call $\boldsymbol{s}_{0}, \boldsymbol{b}_{0}$ the permanent part of the loads, $\boldsymbol{s}_{1}, \boldsymbol{b}_{1}$ the variable part of the loads, and $\lambda$ the loading multiplier.

If $\Lambda \subset \mathbb{R}$ is an $\mathscr{L}^{1}$ measurable set with $0<\mathscr{L}^{1}(\Lambda)<\infty$, we abbreviate

$$
f_{\Lambda} \boldsymbol{O} d \lambda:=\frac{1}{\mathscr{L}^{1}(\Lambda)} \int_{\Lambda} \boldsymbol{O} d \lambda
$$

for any $\lambda$ integrable function $\boldsymbol{O}$ on $\Lambda$.

Proposition 3.3. Assume that the loads $\mathfrak{L}^{\lambda}:=\left(\boldsymbol{s}^{\lambda}, \boldsymbol{b}^{\lambda}\right)$ are given by Equation (15). Let $\Lambda \subset \mathbb{R}$ be a $\mathscr{L}^{1}$ measurable set such that $0<\mathscr{L}^{1}(\Lambda)<\infty$, put

$$
\mu:=f_{\Lambda} \lambda d \lambda
$$

and let $\left\{\mathrm{T}^{\lambda}: \lambda \in \Lambda\right\}$ be an integrable parametric measure such that for $\mathscr{L}^{1}$ a.e. $\lambda \in \Lambda$ the measure $\mathbf{T}^{\lambda}$ weakly equilibrates the loads $\mathfrak{L}^{\lambda}$. Then

(i) the measure

$$
\mathbf{T}:=\int_{\Lambda} \mathbf{T}^{\lambda} d \lambda
$$

weakly equilibrates the loads $\mathfrak{L}^{\mu}$;

(ii) if $V_{0}$ is dense in $V$ and $\boldsymbol{T}=\boldsymbol{T} \mathscr{L}^{n} L \Omega$ where $\boldsymbol{T} \in L^{2}(\Omega$, Sym) then $\boldsymbol{T}$ strongly equilibrates the loads $\mathfrak{L}^{\mu}$.

Proof. (i): This follows from Proposition 3.2 (i) if one notes that

$$
f_{\Lambda} s^{\lambda} d \lambda=s^{\mu}, \quad f_{\Lambda} b^{\lambda} d \lambda=b^{\mu} .
$$

(ii): This follows from Proposition 3.2 (ii).

\section{A panel under vertical top loads and horizontal side loads}

We consider the panel

$$
\Omega=(0, b) \times(0, h) \subset \mathbb{R}^{2}
$$

and introduce a coordinate system $x, y$ in $\mathbb{R}^{2}$ with the origin in the upper right corner of $\Omega$ and with the orientation of axes as shown in Figure 1. We denote a general point of $\Omega$ by $\boldsymbol{r}=(x, y)$ and let $\boldsymbol{i}, \boldsymbol{j}$ be the coordinate vectors along the axes $x, y$, respectively. We set

$$
\mathscr{D}=(0, b) \times\{h\}, \quad \mathscr{S}=\partial \Omega \backslash \mathscr{D},
$$




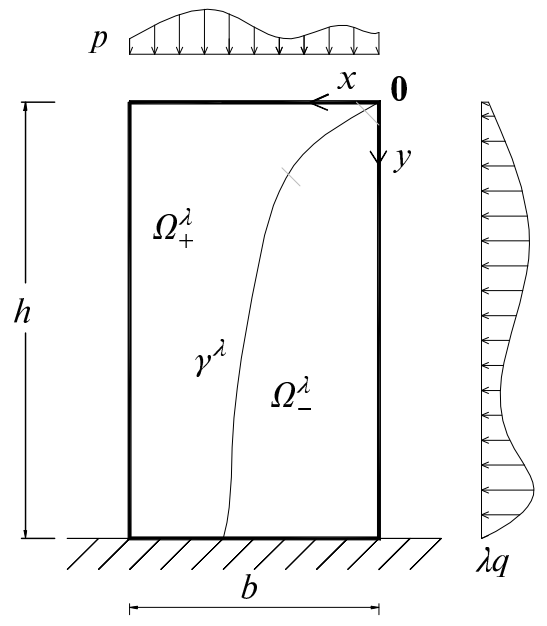

Figure 1. The panel under vertical top loads and horizontal side loads.

and consider the loads $\mathfrak{L}^{\lambda}=\left(\boldsymbol{s}^{\lambda}, \boldsymbol{b}^{\lambda}\right)$, where $\boldsymbol{b}^{\lambda}=\mathbf{0}$ in $\Omega$, and, for $\boldsymbol{r}=(x, y) \in \mathscr{Y}$,

$$
\boldsymbol{s}^{\lambda}(\boldsymbol{r})= \begin{cases}p(x) \boldsymbol{j}, & \text { on }(0, b) \times\{0\}, \\ \lambda q(y) \boldsymbol{i}, & \text { on }\{0\} \times(0, h), \\ \mathbf{0}, & \text { elsewhere, }\end{cases}
$$

where $p, q$ are nonnegative continuous functions on $[0, b]$ and $[0, h]$, respectively. We assume that

$$
p_{0}:=p(0)>0, \quad q_{0}:=q(0)>0 .
$$

We denote by $P, Q$ the primitives of $p, q$, respectively, satisfying $P(0)=Q(0)=0$, and by $\mathscr{P}, 2$ the second primitives of $p, q$, respectively, satisfying $\mathscr{P}(0)=\mathscr{P}^{\prime}(0)=2(0)=\mathscr{L}^{\prime}(0)=0$. We set

$$
\lambda_{c}=\mathscr{P}(b) / 2(h) .
$$

Since $p, q$ are nonnegative and $p_{0}>0, q_{0}>0$, the functions $P, Q$ are strictly positive and nondecreasing on the intervals $(0, b)$ and $(0, h)$, respectively. Consequently, $\mathscr{P}, \mathcal{Q}$ are strictly positive and strictly increasing on the intervals $(0, b)$ and $(0, h)$, respectively. If $0<\lambda \leq \lambda_{c}$, then the range $[0, \lambda 2(h)]$ of $\lambda 2$ is contained in the range $[0, \mathscr{P}(b)]$ of $\mathscr{P}$. It follows that the set

$$
\gamma^{\lambda}=\{\boldsymbol{r}=(x, y) \in \operatorname{cl} \Omega: \lambda 2(y)=\mathscr{P}(x)\},
$$

is a graph of an increasing function $\omega^{\lambda}:\left[0, t^{\lambda}\right] \rightarrow[0, h]$, where $t^{\lambda}$ is determined from the equation $\lambda \mathscr{Q}(h)=\mathscr{P}\left(t^{\lambda}\right)$. One easily finds that $\omega^{\lambda}$ is continuously differentiable, and from $\omega^{\lambda}(0)=0, \omega^{\lambda}\left(t^{\lambda}\right)=h$ one deduces that $\gamma^{\lambda}$ is a smooth curve with one endpoint the origin $\mathbf{0} \in \mathbb{R}^{2}$ and the other endpoint $\left(t^{\lambda}, h\right)$. Moreover, except for the endpoints, the curve $\gamma^{\lambda}$ is contained in $\Omega$. If $\boldsymbol{r}=(x, y) \in \gamma^{\lambda}$, we denote by $\boldsymbol{t}^{\lambda}(\boldsymbol{r})$ the unit tangent vector to $\gamma^{\lambda}$ at $\boldsymbol{r}$, given by

$$
\boldsymbol{t}^{\lambda}(\boldsymbol{r})=\frac{\lambda Q(y) \boldsymbol{i}+P(x) \boldsymbol{j}}{\sqrt{P^{2}(x)+\lambda^{2} Q^{2}(y)}} .
$$


We note that if $\varphi: \Omega \rightarrow \mathbb{R}$ is defined by

$$
\varphi(\boldsymbol{r})=\mathscr{P}(x) / 2(y),
$$

$\boldsymbol{r}=(x, y) \in \Omega$, then for any $\lambda \in\left(0, \lambda_{c}\right)$ the curve $\gamma^{\lambda}$ is the level set of $\varphi$ corresponding to the value $\lambda$, that is,

$$
\gamma^{\lambda}=\varphi^{-1}(\lambda):=\{\boldsymbol{r} \in \Omega: \varphi(\boldsymbol{r})=\lambda\} .
$$

We note, for future use, that $\varphi$ is continuously differentiable, and

$$
|\nabla \varphi(\boldsymbol{r})|=\tau(\boldsymbol{r}),
$$

where

$$
\tau(\boldsymbol{r})=\frac{\sqrt{\mathscr{P}^{\prime 2}(x) \mathscr{2}^{2}(y)+\mathscr{P}^{2}(x) \mathscr{2}^{\prime 2}(y)}}{2^{2}(y)},
$$

$\boldsymbol{r}=(x, y) \in \Omega$. The system of curves $\gamma^{\lambda}, \lambda \in\left(0, \lambda_{c}\right)$ forms a nonintersecting family that fully covers the region

$$
\Omega_{0}=\left\{\boldsymbol{r}=(x, y) \in \Omega: \mathscr{P}(x) / \mathscr{Q}(y) \in\left(0, \lambda_{c}\right)\right\} \equiv \varphi^{-1}\left(0, \lambda_{c}\right) .
$$

For a $\lambda \in\left(0, \lambda_{c}\right]$ the curve $\gamma^{\lambda}$ divides $\Omega$ into two open sets $\Omega_{ \pm}^{\lambda}$ defined by

$$
\begin{gathered}
\Omega_{+}^{\lambda}=\left\{\boldsymbol{r}=(x, y) \in \Omega: \text { either } t^{\lambda} \leq x<b \text { or } 0<x<t^{\lambda} \text { and } y<\omega^{\lambda}(x)\right\}, \\
\Omega_{-}^{\lambda}=\left\{\boldsymbol{r}=(x, y) \in \Omega: 0<x<t^{\lambda} \text { and } y>\omega^{\lambda}(x)\right\}, .
\end{gathered}
$$

Proposition 4.1. Let $0<\lambda \leq \lambda_{c}$ and let $\boldsymbol{T}_{r}^{\lambda}: \Omega \rightarrow \operatorname{Sym}$ and $\boldsymbol{T}_{s}^{\lambda}: \gamma^{\lambda} \rightarrow$ Sym be defined by

$$
\boldsymbol{T}_{r}^{\lambda}(\boldsymbol{r})= \begin{cases}-p(x) \boldsymbol{j} \otimes \boldsymbol{j}, & \text { if } \boldsymbol{r} \in \Omega_{+}^{\lambda}, \\ -\lambda q(y) \boldsymbol{i} \otimes \boldsymbol{i}, & \text { if } \boldsymbol{r} \in \Omega_{-}^{\lambda},\end{cases}
$$

for $\boldsymbol{r}=(x, y) \in \Omega$ and by

$$
\boldsymbol{T}_{s}^{\lambda}(\boldsymbol{r})=\sigma^{\lambda}(\boldsymbol{r}) \boldsymbol{t}^{\lambda}(\boldsymbol{r}) \otimes \boldsymbol{t}^{\lambda}(\boldsymbol{r}),
$$

for $\boldsymbol{r} \in \gamma^{\lambda}$, where $\sigma^{\lambda}: \gamma^{\lambda} \rightarrow \mathbb{R}$ is the unique continuously differentiable function satisfying

$$
\begin{gathered}
\frac{d \sigma^{\lambda}(\boldsymbol{r})}{d s}=\rho^{\lambda}(\boldsymbol{r}), \quad \boldsymbol{r} \in \gamma^{\lambda}, \\
\sigma^{\lambda}(\mathbf{0})=0,
\end{gathered}
$$

where $\rho^{\lambda}: \gamma^{\lambda} \rightarrow \mathbb{R}$ is defined by

$$
\rho^{\lambda}(\boldsymbol{r})=-\frac{\lambda P(x) Q(y)(p(x)+\lambda q(y))}{P^{2}(x)+\lambda^{2} Q^{2}(y)},
$$

$\boldsymbol{r}=(x, y) \in \gamma^{\lambda}$, and where $d / d s$ denotes the derivative with respect to the arc length parameter son $\gamma^{\lambda}$, measured from the origin $\mathbf{0}$. Then $\boldsymbol{T}_{r}^{\lambda}$ and $\boldsymbol{T}_{s}^{\lambda}$ are bounded functions on $\Omega$ and $\gamma^{\lambda}$, respectively, and the measure

$$
\mathbf{T}^{\lambda}=\boldsymbol{T}_{r}^{\lambda} \mathscr{L}^{2}\left\llcorner\Omega+\boldsymbol{T}_{s}^{\lambda} \mathscr{H C}^{1}\left\llcorner\gamma^{\lambda},\right.\right.
$$

is an admissible stress field weakly equilibrating the loads $\mathfrak{L}^{\lambda}$. 
Proof. We note that the continuity of $p, q$ on the closed intervals $[0, b]$ and $[0, h]$ implies that $\boldsymbol{T}_{r}^{\lambda}$ is a bounded function, hence the first term in the right hand side of Equation (25) is a well defined measure. We note that $\rho^{\lambda}$ is a continuous function on $\gamma^{\lambda}$. Using the fact that for $x \rightarrow 0, y \rightarrow 0$ we have

$$
\mathscr{P}(x) \sim \frac{1}{2} p_{0} x^{2}, \quad 2(y) \sim \frac{1}{2} q_{0} y^{2},
$$

to within the errors $\mathrm{o}\left(x^{2}\right), \mathrm{o}\left(y^{2}\right)$, respectively, and that

$$
\lim _{\substack{r=(x, y) \rightarrow \mathbf{0} \\ r \in \gamma^{\lambda}}} y / x=\sqrt{p_{0} / \lambda q_{0}}
$$

one finds that

$$
\lim _{\substack{\boldsymbol{r} \rightarrow \mathbf{0} \\ \boldsymbol{r} \in \gamma^{\lambda}}} \rho^{\lambda}(\boldsymbol{r})=-\sqrt{\lambda p_{0} q_{0}}
$$

Furthermore, trivially,

$$
\lim _{\substack{\boldsymbol{r} \rightarrow\left(t^{\lambda}, h\right) \\ \boldsymbol{r} \in \gamma^{\lambda}}} \rho^{\lambda}(\boldsymbol{r})=-\frac{\lambda P\left(t^{\lambda}\right) Q(h)\left(p\left(t^{\lambda}\right)+\lambda q(h)\right)}{P^{2}\left(t^{\lambda}\right)+\lambda^{2} Q^{2}(h)} .
$$

Hence $\sigma^{\lambda}$ is well defined, bounded, and continuous on $\gamma^{\lambda}$. This shows that $\boldsymbol{T}_{s}^{\lambda}$ is a bounded function on $\gamma^{\lambda}$, and the second term in the right hand side of Equation (25) is a well defined measure. We further note that $\mathbf{T}_{r}^{\lambda}$ is admissible since its density $\boldsymbol{T}_{r}^{\lambda}$ is a negative semidefinite tensor for $\mathscr{L}^{2}$ a.e. $\boldsymbol{r} \in \Omega$. The measure $\mathbf{T}_{s}^{\lambda}$ is admissible as well: clearly, $\rho^{\lambda}$ is nonpositive everywhere on $\gamma^{\lambda}$, and hence the integration of Equation (22)-Equation (23) shows that $\sigma^{\lambda}$ is a nonincreasing nonpositive function. Thus Equation (21) shows that the density $\boldsymbol{T}_{s}^{\lambda}$ is a negative semidefinite tensor. Consequently, $\boldsymbol{T}^{\lambda}$ is also admissible. Finally, one has to show that $\mathrm{T}^{\lambda}$ weakly equilibrates the loads $\mathfrak{L}^{\lambda}$. Referring for the details to [Lucchesi et al. 2006, Section 6], we note that this amounts to showing that the normal trace of $\mathbf{T}^{\lambda}$ equals $\boldsymbol{s}^{\lambda}$ on $\mathscr{Y}$, and that the weak divergence of $\mathbf{T}^{\lambda}$ in $\Omega$ vanishes. The last is equivalent to proving that the classical divergence of $\boldsymbol{T}_{r}$ vanishes on $\Omega \backslash \gamma^{\lambda}$ (which is immediate), and that along $\gamma^{\lambda}$ the jump condition

$$
\left[\boldsymbol{T}_{r}\right] \boldsymbol{n}-\operatorname{div} \boldsymbol{T}_{s}=\mathbf{0},
$$

holds where $\left[\boldsymbol{T}_{r}\right] \boldsymbol{n}$ is the jump of the normal component of $\boldsymbol{T}_{r}$ across $\gamma^{\lambda}$ and div $\boldsymbol{T}_{s}$ is the linear divergence of $\boldsymbol{T}_{s}$ along $\gamma^{\lambda}$. Equation (29) leads to the above described shape of $\gamma^{\lambda}$ and to the differential equation, Equation (22)-Equation (23). We omit the details.

Proposition 4.1 is now used to establish the following:

Proposition 4.2. If $0<\mu<\lambda_{c}$, then the loads $\mathfrak{L}^{\mu}$ are strongly compatible. In fact if $\Lambda \subset\left(0, \lambda_{c}\right)$ is any $\mathscr{L}^{1}$ measurable set with $\mathscr{L}^{1}(\Lambda)>0$ such that

$$
\mu=f_{\Lambda} \lambda d \lambda
$$

then $\left\{\mathbf{T}^{\lambda}: \lambda \in \Lambda\right\}$ is an integrable parametric measure, and the measure $\mathbf{T}=f_{\Lambda} \mathbf{T}^{\lambda} d \lambda$ is of the form

$$
\mathbf{T}=\boldsymbol{T} \mathscr{L}^{2}\llcorner\Omega,
$$


where $\boldsymbol{T}$ is a bounded admissible stress field on $\Omega$ that strongly equilibrates the loads $\mathfrak{L}^{\mu}$. We have $\boldsymbol{T}=\boldsymbol{T}_{r}+\boldsymbol{T}_{s}$, where for $\boldsymbol{r} \in \Omega$,

$$
\begin{gathered}
\boldsymbol{T}_{r}(\boldsymbol{r})=f_{\Lambda} \boldsymbol{T}_{r}^{\lambda}(\boldsymbol{r}) d \lambda, \\
\boldsymbol{T}_{s}(\boldsymbol{r})= \begin{cases}\frac{\sigma^{\lambda}(\boldsymbol{r}) \tau(\boldsymbol{r})}{\mathscr{L}^{1}(\Lambda)} \boldsymbol{t}^{\lambda}(\boldsymbol{r}) \otimes t^{\lambda}(\boldsymbol{r}), & \text { if } \varphi(\boldsymbol{r}) \in \Lambda, \text { where } \lambda:=\varphi(\boldsymbol{r}), \\
\mathbf{0}, & \text { otherwise, }\end{cases}
\end{gathered}
$$

where $\varphi$ and $\tau$ are defined by Equation (17) and Equation (19).

For $\mu=\lambda_{c}$ we have the weak compatibility of the loads $\mathfrak{L}^{\mu}$ by Proposition 4.1 , but the above proposition says nothing about the strong compatibility for this limiting value.

Proof. We write

$$
\mathbf{T}^{\lambda}=\mathbf{T}_{r}^{\lambda}+\mathbf{T}_{s}^{\lambda},
$$

where

$$
\mathbf{T}_{r}^{\lambda}=\boldsymbol{T}_{r}^{\lambda} \mathscr{L}^{2}\left\llcorner\Omega, \quad \mathbf{T}_{s}^{\lambda}=\boldsymbol{T}_{s}^{\lambda} \mathscr{H}^{1}\left\llcorner\gamma^{\lambda} .\right.\right.
$$

We note that $\mathbf{T}_{r}^{\lambda}$ is of the form considered in Proposition 2.2, where $h^{\lambda}$ is to be identified with $\boldsymbol{T}_{r}^{\lambda}$. One sees that the integrability condition of Equation (3) is satisfied, and hence for any $\mathscr{L}^{1}$ measurable set $\Lambda \subset\left[0, \lambda_{c}\right]$, the measure

$$
\mathbf{T}_{r}:=f_{\Lambda} \mathbf{T}_{r}^{\lambda} d \lambda
$$

is a measure absolutely continuous with respect to $\mathscr{L}^{2} L \Omega$. Moreover, since the density $h^{\lambda}$ is a bounded function on $\Omega \times \Lambda$, we see that the density of $\mathbf{T}_{r}$ with respect to $\mathscr{L}^{2}$ is a bounded function. Thus

$$
\mathbf{T}_{r}=\boldsymbol{T}_{r} \mathscr{L}^{2}\llcorner\Omega
$$

where $\boldsymbol{T}_{r}$ is a bounded function on $\Omega$ given by Equation (30).

The measure $\mathbf{T}_{s}^{\lambda}$ is of the form

$$
\mathbf{T}_{s}^{\lambda}=\boldsymbol{G} \mathscr{H}^{1}\left\llcorner\varphi^{-1}(\lambda),\right.
$$

where $\boldsymbol{G}: \Omega_{0} \rightarrow$ Sym is defined by

$$
\boldsymbol{G}(\boldsymbol{r})=\boldsymbol{T}_{s}^{\lambda}(\boldsymbol{r})
$$

for any $\boldsymbol{r} \in \Omega_{0}$, and where in the last formula $\lambda$ is an abbreviation for $\varphi(\boldsymbol{r})$.

We now wish to verify that the function $g:=\boldsymbol{G}$ satisfies the integrability condition of Equation (4). We shall actually prove that the product $|\nabla \varphi \| \boldsymbol{G}|$ is bounded on $\Omega_{0}$. For this it suffices to prove that for each $\lambda \in\left(0, \lambda_{c}\right)$, the limit

$$
L(\lambda):=\lim _{\substack{\boldsymbol{r} \rightarrow \mathbf{0} \\ \boldsymbol{r} \in \gamma^{\lambda}}}|\nabla \varphi(\boldsymbol{r})||\boldsymbol{G}(\boldsymbol{r})|
$$

exists, and the function $L$ is bounded on $\left(0, \lambda_{c}\right)$.

Recalling Equation (26) and Equation (27), we infer from Equation (18) and Equation (19) that

$$
\lim _{\substack{\boldsymbol{r}=(x, y) \rightarrow \mathbf{0} \\ \boldsymbol{r} \in \gamma^{\lambda}}} x|\nabla \varphi(\boldsymbol{r})|=2 \lambda \frac{\sqrt{p_{0}+\lambda q_{0}}}{\sqrt{p_{0}}} .
$$


Furthermore, combining Equation (22), Equation (23), Equation (28) and $d s / d x=\sqrt{1+\mathscr{P}^{\prime 2}(x) / \lambda^{2} \mathscr{Q}^{\prime 2}(y)}$ one finds that

$$
\lim _{\substack{\boldsymbol{r}=(x, y) \rightarrow \mathbf{0} \\ \boldsymbol{r} \in \gamma^{\lambda}}}|\boldsymbol{G}(\boldsymbol{r})| / x=\sqrt{p_{0}^{2}+\lambda p_{0} q_{0}},
$$

and hence

$$
\lim _{\substack{\boldsymbol{r} \rightarrow \mathbf{0} \\ \boldsymbol{r} \in \gamma^{\lambda}}}|\nabla \varphi(\boldsymbol{r})||\boldsymbol{G}(\boldsymbol{r})|=2 \lambda\left(p_{0}+\lambda q_{0}\right) .
$$

This shows that the function $L$ is bounded on $\left(0, \lambda_{c}\right)$, and consequently that $|\nabla \varphi \| \boldsymbol{G}|$ is bounded on $\Omega_{0}$.

In particular, the integrability condition of Equation (4) and Proposition 2.3 say that for any $\mathscr{L}^{1}$ measurable set $\Lambda \subset \mathbb{R}$ the measure

$$
\mathrm{T}_{s}:=\int_{\Lambda} \mathrm{T}_{s}^{\lambda} d \lambda
$$

is $\mathscr{L}^{2}$ absolutely continuous over $\Omega$, with the density given by Equation (5). In the present case, this gives $\mathbf{T}_{s}=\boldsymbol{T}_{s} \mathscr{L}^{2}\left\llcorner\Omega\right.$, where $\boldsymbol{T}_{s}$ is given by Equation (31). Noting that $V_{0}$ is dense in $V$, we see that a combination of Propositions 4.1 and 3.3 completes the proof.

\section{Example: Explicit determination of the averaged stress field}

The goal of this section is to determine explicitly the density $\boldsymbol{T}=\boldsymbol{T}_{r}+\boldsymbol{T}_{s}$ of the measure $\boldsymbol{T}$ from Proposition 4.2 in a special case. The formula is in Equation (39), below.

We consider the situation of Section 4 and take in particular

$$
p=\text { const on }[0, b], q \equiv 1 \text { on }[0, h]
$$

Hence

$$
\boldsymbol{s}^{\lambda}(\boldsymbol{r})= \begin{cases}p \boldsymbol{j}, & \text { on }(0, b) \times\{0\} \\ \lambda \boldsymbol{i}, & \text { on }\{0\} \times(0, h) \\ \mathbf{0}, & \text { elsewhere on } \mathscr{S}\end{cases}
$$

see Figure 2. The results of Section 4 apply directly.

We find

$$
\mathscr{P}(x)=\frac{1}{2} p x^{2}, \quad 2(y)=\frac{1}{2} y^{2}, \quad 0 \leq x \leq b, 0 \leq y \leq h,
$$

and Equation (16) gives $\lambda_{c}=p b^{2} / h^{2}$. Furthermore, if $0 \leq \lambda \leq \lambda_{c}$, then $\gamma^{\lambda}$ is the line segment

$$
\gamma^{\lambda}=\{(x, y) \in \Omega: y=\sqrt{p / \lambda} x\}
$$

The regions $\Omega_{ \pm}^{\lambda}$ are given by

$$
\Omega_{ \pm}^{\lambda}=\{\boldsymbol{r}=(x, y) \in \Omega: \pm(\sqrt{p / \lambda} x-y)>0\} .
$$

The region $\Omega_{0}$ covered by the segments $\gamma^{\lambda}, \lambda \in\left(0, \lambda_{c}\right)$ is delimited by the main diagonal of $\Omega$; in fact

$$
\Omega_{0}=\{\boldsymbol{r}=(x, y) \in \Omega: y / x>h / b\} .
$$




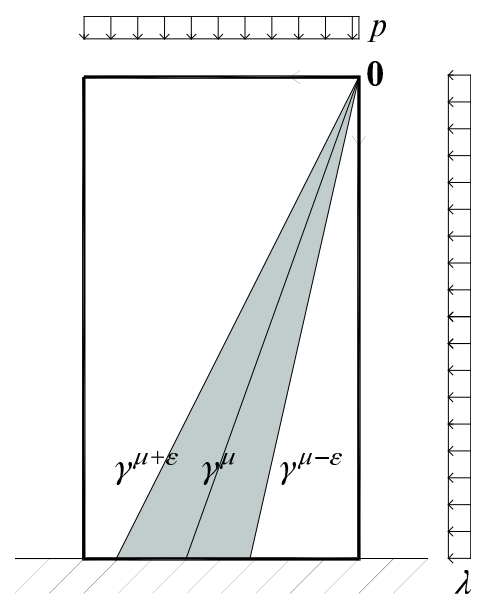

Figure 2. The panel under special load conditions.

We consider the measure $\mathbf{T}^{\lambda}$ given by Equation (25). In the present special case we find from Equation (20) that for $\boldsymbol{r}=(x, y) \in \Omega$,

$$
\boldsymbol{T}_{r}^{\lambda}(\boldsymbol{r})= \begin{cases}-p \boldsymbol{j} \otimes \boldsymbol{j}, & \text { if } \boldsymbol{r} \in \Omega_{+}^{\lambda}, \\ -\lambda \boldsymbol{i} \otimes \boldsymbol{i}, & \text { if } \boldsymbol{r} \in \Omega_{-}^{\lambda} .\end{cases}
$$

Furthermore, Equation (24) and Equation (34) give $\rho^{\lambda}(\boldsymbol{r})=-p x / y, \boldsymbol{r}=(x, y) \in \gamma^{\lambda}$, and hence

$$
\sigma^{\lambda}(\boldsymbol{r})=-p x|\boldsymbol{r}| / y
$$

by Equation (22) and Equation (23). Consequently,

$$
\boldsymbol{T}_{s}^{\lambda}(\boldsymbol{r})=-\sqrt{p \lambda} \boldsymbol{r} \otimes \boldsymbol{r} /|\boldsymbol{r}|
$$

for $\boldsymbol{r} \in \gamma^{\lambda}$, where we note that $\boldsymbol{t}^{\lambda}(\boldsymbol{r})=\boldsymbol{r} /|\boldsymbol{r}|$ is the tangent vector to $\gamma^{\lambda}$.

We now wish to determine the density $\boldsymbol{T}=\boldsymbol{T}_{r}+\boldsymbol{T}_{s}$ of the measure $\mathbf{T}$. Recall that the functions $\boldsymbol{T}_{r}, \boldsymbol{T}_{s}$ are given by Equations (30)and (31). Let $0<\mu<\lambda_{c}$, and let $\epsilon>0$ be such that

$$
\Lambda:=(\mu-\epsilon, \mu+\epsilon) \subset\left(0, \lambda_{c}\right),
$$

and let

$$
A=\left\{\boldsymbol{r}=(x, y): p x^{2} / y^{2} \in \Lambda\right\} .
$$

We refer to Figure 2, where $A$ is the shaded region delimited by segments $\gamma^{\mu-\epsilon}, \gamma^{\mu+\epsilon}$, and where $\gamma^{\mu}$ is the middle segment.

Let us show that from Equation (30) one obtains

$$
\boldsymbol{T}_{r}(\boldsymbol{r})= \begin{cases}-p \boldsymbol{j} \otimes \boldsymbol{j}, & \text { if } \boldsymbol{r} \in \Omega_{+}^{\lambda} \backslash A, \\ -\mu \boldsymbol{i} \otimes \boldsymbol{i}, & \text { if } \boldsymbol{r} \in \Omega_{-}^{\lambda} \backslash A, \\ (2 \epsilon)^{-1}(\alpha(\boldsymbol{r}) \boldsymbol{i} \otimes \boldsymbol{i}+\beta(\boldsymbol{r}) \boldsymbol{j} \otimes \boldsymbol{j}), & \text { if } \boldsymbol{r} \in A,\end{cases}
$$


$\boldsymbol{r} \in \Omega$, where for $\boldsymbol{r}=(x, y) \in A$ we set

$$
\alpha(\boldsymbol{r})=\frac{1}{2}\left(p^{2} x^{4} / y^{4}-(\mu+\epsilon)^{2}\right), \quad \beta(\boldsymbol{r})=p\left(\mu-\epsilon-p x^{2} / y^{2}\right) .
$$

Let us derive the third regime of Equation (36); the derivation of the first two regimes is similar and simpler. Thus let $\boldsymbol{r}=(x, y) \in A$, and set $a=p x^{2} / y^{2}$. We have

$$
\boldsymbol{T}_{r}(\boldsymbol{r})=(2 \epsilon)^{-1} \int_{\mu-\epsilon}^{\mu+\epsilon} \boldsymbol{T}_{r}^{\lambda}(\boldsymbol{r}) d \lambda=(2 \epsilon)^{-1}\left(\int_{\mu-\epsilon}^{a} \boldsymbol{T}_{r}^{\lambda}(\boldsymbol{r}) d \lambda+\int_{a}^{\mu+\epsilon} \boldsymbol{T}_{r}^{\lambda}(\boldsymbol{r}) d \lambda\right) .
$$

If $\mu-\epsilon<\lambda<a$, then $\boldsymbol{T}_{r}^{\lambda}(\boldsymbol{r})=-p \boldsymbol{j} \otimes \boldsymbol{j}$; if $a<\lambda<\mu+\epsilon$ then $\boldsymbol{T}_{r}^{\lambda}(\boldsymbol{r})=-\lambda \boldsymbol{i} \otimes \boldsymbol{i}$. Inserting these values into the integrals in Equation (37), and recalling $a=p x^{2} / y^{2}$, we obtain the value giving the third regime.

To determine $\boldsymbol{T}_{s}$, we note that from Equation (19) we obtain

$$
\tau(\boldsymbol{r})=2 p x|\boldsymbol{r}| / y^{3}
$$

$\boldsymbol{r}=(x, y) \in \Omega_{0}$. Consequently, we deduce from Equation (35) and Equation (31) that for $\boldsymbol{r}=(x, y) \in \Omega$,

$$
\boldsymbol{T}_{s}(\boldsymbol{r})= \begin{cases}-2 p^{2} x^{2} \boldsymbol{r} \otimes \boldsymbol{r} / 2 \epsilon y^{4}, & \text { if } \boldsymbol{r} \in A \\ \mathbf{0}, & \text { otherwise. }\end{cases}
$$

From Equation (36) and Equation (38) we obtain finally

$$
\boldsymbol{T}(\boldsymbol{r})= \begin{cases}-p \boldsymbol{j} \otimes \boldsymbol{j}, & \text { if } \boldsymbol{r} \in \Omega_{+}^{\lambda} \backslash A, \\ -\mu \boldsymbol{i} \otimes \boldsymbol{i}, & \text { if } \boldsymbol{r} \in \Omega_{-}^{\lambda} \backslash A, \\ \boldsymbol{S}(\boldsymbol{r}), & \text { if } \boldsymbol{r} \in A,\end{cases}
$$

$r \in \Omega$, where

$$
\boldsymbol{S}(\boldsymbol{r})=(2 \epsilon)^{-1}\left(\left(p^{2} x^{4} / y^{4}-(\mu+\epsilon)^{2}\right) \boldsymbol{i} \otimes \boldsymbol{i} / 2+p\left(\mu-\epsilon-p x^{2} / y^{2}\right) \boldsymbol{j} \otimes \boldsymbol{j}-2 p^{2} x^{2} \boldsymbol{r} \otimes \boldsymbol{r} / y^{4}\right) .
$$

Thus, by Proposition 3.3, the function $\boldsymbol{T}$ satisfies

$$
\boldsymbol{T} \boldsymbol{n}=\boldsymbol{s}^{\mu} \quad \text { on } \mathscr{S}, \quad \operatorname{div} \boldsymbol{T}=\mathbf{0} \quad \text { in } \Omega,
$$

which can be also verified directly.

\section{A panel with vertical top loads and oblique side loads}

We again consider the panel

$$
\Omega=(0, b) \times(0, h),
$$

and assume that the top of the panel is subjected to a uniform pressure $p_{0}$ while the right side of the panel is subjected to oblique loads to be described below. We set

$$
\mathscr{D}=(0, b) \times\{h\}, \quad \mathscr{S}=\partial \Omega \backslash \mathscr{D},
$$




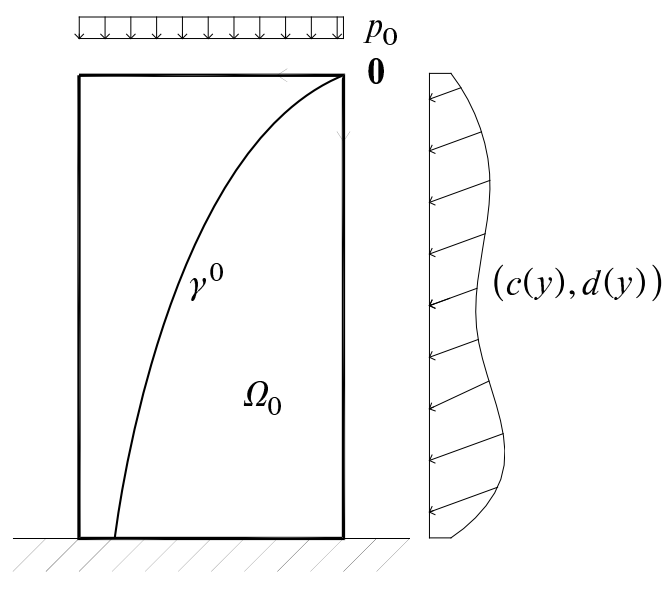

Figure 3. Oblique side loads.

$\boldsymbol{b}=\mathbf{0}$ in $\Omega$, and

$$
\boldsymbol{s}(\boldsymbol{r})= \begin{cases}p_{0} \boldsymbol{j}, & \text { if } \boldsymbol{r} \in(0, b) \times\{0\}, \\ c(y) \boldsymbol{i}+d(y) \boldsymbol{j}, & \text { if } \boldsymbol{r}=(x, y) \in\{0\} \times[0, h], \\ \mathbf{0}, & \text { if } \boldsymbol{r} \in\{b\} \times(0, h),\end{cases}
$$

$\boldsymbol{r} \in \mathscr{S}$, where $p_{0}>0$ and

$$
c:[0, h] \rightarrow(0, \infty), \quad d:[0, h] \rightarrow(0, \infty)
$$

are continuously differentiable functions, see Figure 3. We make a permanent assumption that the functions

$$
y \mapsto 1 / c(y), \quad y \mapsto d(y) / c(y) \text { are nondecreasing on }[0, h]
$$

If $0 \leq \lambda \leq h$, let $\omega^{\lambda}: \mathbb{R} \rightarrow \mathbb{R}$ be given by

$$
\omega^{\lambda}(x)=\alpha(\lambda) x^{2}+\beta(\lambda) x+\lambda
$$

$x \in \mathbb{R}$, where

$$
\alpha(\lambda)=p_{0} / 2 h c(\lambda), \quad \beta(\lambda)=d(\lambda) / c(\lambda),
$$

and let $\gamma^{\lambda}$ be given by

$$
\gamma^{\lambda}=\left\{\boldsymbol{r}=\left(x, \omega^{\lambda}(x)\right) \in \Omega: 0<x<b\right\} .
$$

In the following proposition we consider an auxiliary problem in which $\lambda \in[0, h]$ is fixed and the body is subjected to the loads $\left(\mathbf{s}^{\lambda}, \mathbf{0}\right)$ with $\mathbf{s}^{\lambda}$ given by the measure

$$
\mathbf{s}^{\lambda}=\boldsymbol{s}_{0} \mathscr{H}^{1}\left\llcorner\mathscr{S}+(c(\lambda) \boldsymbol{i}+d(\lambda) \boldsymbol{j}) \delta_{(0, \lambda)},\right.
$$


where $\delta_{(0, \lambda)}$ is the Dirac measure at the point $(0, \lambda)$ and where

$$
\boldsymbol{s}_{0}(\boldsymbol{r})= \begin{cases}p_{0} \boldsymbol{j} / h, & \text { if } \boldsymbol{r} \in(0, b) \times\{0\} \\ \mathbf{0}, & \text { if } \boldsymbol{r} \in \mathscr{S} \backslash(0, b) \times\{0\}\end{cases}
$$

$\boldsymbol{r} \in \mathscr{S}$

Proposition 6.1. Let $0 \leq \lambda \leq h$, and let $\mathrm{T}^{\lambda}$ be the measure defined by

$$
\mathbf{T}^{\lambda}=\boldsymbol{T}_{r}^{\lambda} \mathscr{L}^{2}\left\llcorner\Omega+\boldsymbol{T}_{s}^{\lambda} \mathscr{H}^{1}\left\llcorner\gamma^{\lambda}\right.\right.
$$

where $\boldsymbol{T}_{r}^{\lambda}, \boldsymbol{T}_{s}^{\lambda}$ are bounded functions on $\Omega$ and $\gamma^{\lambda}$, respectively, given by

$$
\boldsymbol{T}_{r}^{\lambda}(\boldsymbol{r})= \begin{cases}-p_{0} \boldsymbol{j} \otimes \boldsymbol{j} / h, & \text { if } y<\omega^{\lambda}(x), \\ \mathbf{0}, & \text { if } y>\omega^{\lambda}(x),\end{cases}
$$

$\boldsymbol{r}=(x, y) \in \Omega$, and

$$
\boldsymbol{T}_{s}^{\lambda}(\boldsymbol{r})=\sigma^{\lambda}(\boldsymbol{r}) \boldsymbol{t}^{\lambda}(\boldsymbol{r}) \otimes \boldsymbol{t}^{\lambda}(\boldsymbol{r})
$$

$\boldsymbol{r}=(x, y) \in \gamma^{\lambda}$, where $\boldsymbol{t}^{\lambda}(\boldsymbol{r})$ is the unit tangent vector to $\gamma^{\lambda}$ at $\boldsymbol{r}$ and

$$
\sigma^{\lambda}(\boldsymbol{r})=-\sqrt{c^{2}(\lambda)+\left(p_{0} x / h+d(\lambda)\right)^{2}}
$$

If $\omega^{\lambda}(b) \geq h$ then $\mathrm{T}^{\lambda}$ is an admissible stress field weakly equilibrating the loads $\left(\mathbf{s}^{\lambda}, \mathbf{0}\right)$.

Note that one endpoint of $\gamma^{\lambda}$ is always $(0, \lambda)$; the other endpoint can be either on the side $\{b\} \times(0, h)$ or on the base $[0, b] \times\{h\}$. The condition $\omega^{\lambda}(b) \geq h$ then says that the latter possibility occurs.

Proof. This follows from the considerations in [Lucchesi et al. 2006, Example 2].

Proposition 6.2. If $\omega^{0}(b) \geq h$ then the loads $(\boldsymbol{s}, \mathbf{0})$ are strongly compatible. In fact, there exists a bounded admissible tensor field $\boldsymbol{T}$ on $\Omega$ strongly equilibrating them.

The condition $\omega^{0}(b) \geq h$ says that the initial curve $\gamma^{0}$ ends on the base $[0, b] \times\{h\}$ of $\Omega$.

Proof. One easily finds that

$$
\left\{\mathrm{T}^{\lambda}: 0 \leq \lambda \leq h\right\}, \quad\left\{\mathrm{s}^{\lambda}: 0 \leq \lambda \leq h\right\},
$$

are integrable parametric measures. From conditions Equation (41), one finds that $\omega^{\lambda}(b) \geq \omega^{0}(b)$. Thus the hypothesis $\omega^{0}(b) \geq h$ implies that $\omega^{\lambda}(b) \geq h$ for all $\lambda \in[0, h]$. Consequently, $\mathrm{T}^{\lambda}$ weakly equilibrates the loads $\left(\mathbf{s}^{\lambda}, \mathbf{0}\right)$ whenever $0 \leq \lambda \leq h$ by Proposition 6.1. By Proposition 3.2 (i), the stress field $\mathbf{T}=$ $\int_{0}^{h} \mathrm{~T}^{\lambda} d \lambda$ weakly equilibrates the loads $(\overline{\mathbf{s}}, \mathbf{0})$, where $\overline{\mathbf{s}}=\int_{0}^{h} \mathbf{s}^{\lambda} d \lambda$. If $0 \leq \lambda \leq h$ and $\boldsymbol{v} \in C_{0}\left(\mathbb{R}^{2}, \mathbb{R}^{2}\right)$, then comparing Equation (42) with Equation (40) we obtain

$$
\int_{\mathscr{S}} \boldsymbol{v} \cdot d \mathbf{s}^{\lambda}=\int_{\mathscr{S}} \boldsymbol{v} \cdot \boldsymbol{s}_{0} d \mathscr{H}^{1}+\boldsymbol{v}(0, \lambda) \cdot \boldsymbol{s}(0, \lambda)
$$

Hence,

$$
\int_{0}^{h} \int_{\mathscr{S}} \boldsymbol{v} \cdot d \mathbf{s}^{\lambda} d \lambda=h \int_{\mathscr{S}} \boldsymbol{v} \cdot \boldsymbol{s}_{0} d \mathscr{H}^{1}+\int_{0}^{h} \boldsymbol{v}(0, \lambda) \cdot \boldsymbol{s}(0, \lambda) d \lambda=\int_{\mathscr{S}} \boldsymbol{v} \cdot \boldsymbol{s} d \mathscr{H}^{1}
$$


which shows that

$$
\overline{\mathbf{s}} \equiv \int_{0}^{h} \mathbf{s}^{\lambda} d \lambda=s \mathscr{H}^{1}\llcorner\mathscr{S} .
$$

Thus, we conclude that $\mathrm{T}$ weakly equilibrates the loads $(\boldsymbol{s}, \mathbf{0})$.

Let us now show that $\mathbf{T}=\boldsymbol{T} \mathscr{L}^{2} \mathrm{~L} \Omega$, where $\boldsymbol{T}$ is a bounded function on $\Omega$. Decompose $\mathrm{T}^{\lambda}$ into $\mathbf{T}_{r}^{\lambda}, \mathbf{T}_{s}^{\lambda}$ as in Equation (32) and Equation (33). Then $\mathbf{T}=\mathbf{T}_{r}+\mathbf{T}_{s}$, where

$$
\mathbf{T}_{r}=\int_{0}^{h} \mathbf{T}_{r}^{\lambda} d \lambda, \quad \mathbf{T}_{s}=\int_{0}^{h} \mathbf{T}_{s}^{\lambda} d \lambda .
$$

Since $\boldsymbol{T}_{r}^{\lambda}$ is bounded independently of $\lambda$, it is found that $\mathrm{T}_{r}=\boldsymbol{T}_{r} \mathscr{L}^{2}\left\llcorner\Omega\right.$ where $\boldsymbol{T}_{r}$ is a bounded function in the same way as in the proof of Proposition 4.2.

Next, we prove that

$$
\mathbf{T}_{s}=\boldsymbol{T}_{s} \mathscr{L}^{2}\llcorner\Omega
$$

where $\boldsymbol{T}_{S}$ is a bounded function. Let

$$
\Omega_{0}=\bigcup\left\{\gamma^{\lambda}: 0<\lambda<h\right\} \equiv\left\{\boldsymbol{r}=(x, y) \in \Omega: y>\omega^{0}(x)\right\} .
$$

The assumption Equation (41) and the form of $\omega^{\lambda}$ imply that for each $\boldsymbol{r}=(x, y) \in \Omega_{0}$ there exists exactly one $\lambda$ such that

$$
y=\omega^{\lambda}(x) .
$$

We define $\varphi: \Omega_{0} \rightarrow \mathbb{R}$ by setting $\varphi(\boldsymbol{r})=\lambda$, that is, by

$$
y=\alpha(\varphi(\boldsymbol{r})) x^{2}+\beta(\varphi(\boldsymbol{r})) x+\varphi(\boldsymbol{r}),
$$

$\boldsymbol{r}=(x, y) \in \Omega_{0}$. The implicit function theorem and the differentiability of $\alpha, \beta$ imply that $\varphi$ is continuously differentiable and the derivatives of $\varphi$ at $\boldsymbol{r}=(x, y)$ are given by

$$
\frac{\partial \varphi}{\partial x}=-\frac{2 \alpha x+\beta}{\alpha^{\prime} x^{2}+\beta^{\prime} x+1}, \quad \frac{\partial \varphi}{\partial y}=\frac{1}{\alpha^{\prime} x^{2}+\beta^{\prime} x+1},
$$

where $\alpha, \beta, \alpha^{\prime}, \beta^{\prime}$ are evaluated at $\varphi(\boldsymbol{r})$. We have $\alpha^{\prime} \geq 0, \beta^{\prime} \geq 0$ by Equation (41) and hence the denominators in Equation (45) are $\geq 1$. Since the numerators are bounded as $\alpha, \beta$ are continuous on $[0, h]$, we see that the partial derivatives Equation (45) are bounded on $\Omega_{0}$. Hence $|\nabla \varphi|$ is also bounded. We have

$$
\mathbf{T}_{s}^{\lambda}=\boldsymbol{G} \mathscr{H}^{1}\left\llcorner\varphi^{-1}(\lambda),\right.
$$

where $\boldsymbol{G}: \Omega_{0} \rightarrow$ Sym is given by

$$
\boldsymbol{G}(\boldsymbol{r})=\boldsymbol{T}_{s}^{\lambda}(\boldsymbol{r})
$$

$\boldsymbol{r} \in \Omega_{0}$, and where $\lambda$ stands for $\varphi(\boldsymbol{r})$. From the expression Equation (43), we find that $\boldsymbol{G}$ is bounded on $\Omega_{0}$. Proposition 2.3 then says that we have Equation (44), where

$$
\boldsymbol{T}_{s}(\boldsymbol{r})= \begin{cases}|\nabla \varphi(\boldsymbol{r})| \boldsymbol{G}(\boldsymbol{r}), & \text { if } \boldsymbol{r} \in \Omega_{0}, \\ \mathbf{0}, & \text { if } \boldsymbol{r} \in \Omega \backslash \Omega_{0},\end{cases}
$$

$\boldsymbol{r} \in \Omega$. Thus $\boldsymbol{T}_{s}$ is bounded. Noting that $V_{0}$ is dense in $V$, we see that a combination of Propositions 6.1 and 3.2 (ii) completes the proof. 


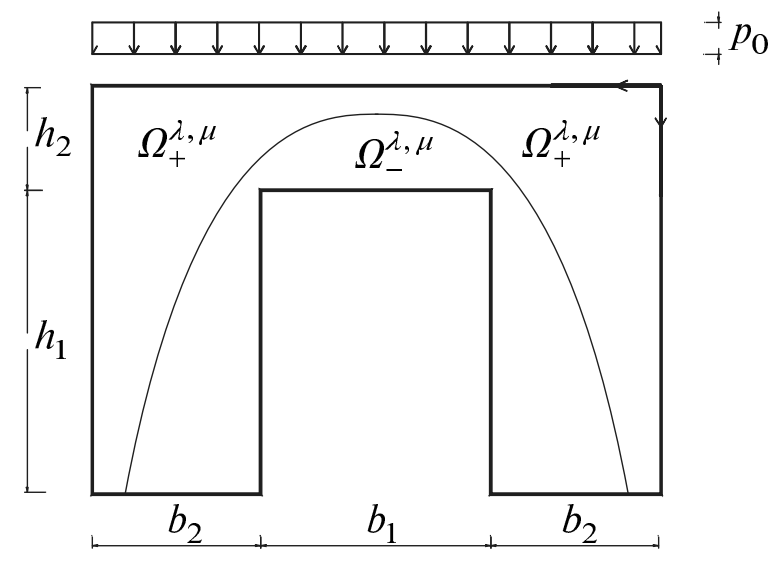

Figure 4. Panel with a symmetric opening.

\section{A panel with a symmetric opening}

Let us consider a rectangular panel $\Omega$ with base $b=b_{1}+2 b_{2}$, height $h=h_{1}+h_{2}$, and a symmetric opening of dimensions $b_{1}$ and $h_{1}$ (Figure 4), that is clamped at its base and subjected to a vertical load $p_{0}$, uniformly distributed on its top. We set

$$
\mathscr{D}=\left(0, b_{2}\right) \times\{h\} \cup\left(b_{1}+b_{2}, b\right) \times\{h\}, \quad \mathscr{S}=\partial \Omega \backslash \mathscr{D},
$$

$\boldsymbol{b}=\mathbf{0}$ in $\Omega$,

$$
\boldsymbol{s}= \begin{cases}p_{0} \boldsymbol{j}, & \text { on }(0, b) \times\{0\}, \\ \mathbf{0}, & \text { on } \mathscr{Y} \backslash(0, b) \times\{0\},\end{cases}
$$

$p_{0}>0$.

Let $\lambda>0, \mu>0$, and consider the parabola

$$
\gamma^{\lambda, \mu}=\left\{\left(x, \omega^{\lambda, \mu}(x)\right) \in \mathbb{R}^{2}: b / 2-\mu<x<b / 2+\mu\right\},
$$

where $\omega^{\lambda, \mu}:(b / 2-\mu, b / 2+\mu) \rightarrow \mathbb{R}$ is defined by

$$
\omega^{\lambda, \mu}(x)=\lambda+(h-\lambda)(x-b / 2)^{2} / \mu^{2},
$$

$b / 2-\mu<x<b / 2+\mu$. Let

$$
\mathscr{A}=\left\{(\lambda, \mu) \in(0, \infty) \times(0, \infty): \gamma^{\lambda, \mu} \subset \Omega\right\}
$$

be the set of all pairs $(\lambda, \mu)$ for which the parabola $\gamma^{\lambda, \mu}$ is wholly contained in the panel $\Omega$. One has [Lucchesi et al. 2006, Section 6]:

$$
\mathscr{A} \text { is nonempty } \Leftrightarrow \quad \zeta \leq 4 \xi(\xi+1),
$$

$\mathscr{A}$ has a nonempty interior $\Leftrightarrow \quad \zeta<4 \xi(\xi+1)$, 
where $\xi:=b_{2} / b_{1}, \zeta:=h_{1} / h_{2}$. If $(\lambda, \mu) \in \mathscr{A}$, we define the sets $\Omega_{ \pm}^{\lambda, \mu}$ by

$$
\begin{gathered}
\Omega_{-}^{\lambda, \mu}=\left\{\boldsymbol{r}=(x, y) \in \Omega:|x-b / 2|<\mu, y>\omega^{\lambda, \mu}(x)\right\}, \\
\Omega_{+}^{\lambda, \mu}=\Omega \backslash\left(\Omega_{-}^{\lambda, \mu} \cup \gamma^{\lambda, \mu}\right) .
\end{gathered}
$$

Proposition 7.1. Let $(\lambda, \mu) \in \mathscr{A}$ and define the measure $\mathrm{T}^{\lambda, \mu}$ by

$$
\mathbf{T}^{\lambda, \mu}=\boldsymbol{T}_{r}^{\lambda, \mu} \mathscr{L}^{2}\left\llcorner\Omega+\boldsymbol{T}_{s}^{\lambda, \mu} \mathscr{H}^{1}\left\llcorner\gamma^{\lambda, \mu},\right.\right.
$$

where $\boldsymbol{T}_{r}^{\lambda, \mu}$ and $\boldsymbol{T}_{s}^{\lambda, \mu}$ are bounded functions on $\Omega$ and $\gamma^{\lambda, \mu}$, respectively, given by

$$
\begin{gathered}
\boldsymbol{T}_{r}^{\lambda, \mu}(\boldsymbol{r})= \begin{cases}-p_{0} \boldsymbol{j} \otimes \boldsymbol{j}, & \text { if } \boldsymbol{r} \in \Omega_{+}^{\lambda, \mu}, \\
\mathbf{0}, & \text { otherwise, }\end{cases} \\
\boldsymbol{r} \in \Omega, \quad \text { and } \quad \boldsymbol{T}_{s}^{\lambda, \mu}(\boldsymbol{r})=\sigma^{\lambda, \mu}(\boldsymbol{r}) \boldsymbol{T}^{\lambda, \mu}(\boldsymbol{r}) \otimes \boldsymbol{T}^{\lambda, \mu}(\boldsymbol{r}),
\end{gathered}
$$

where

$$
\sigma^{\lambda, \mu}(\boldsymbol{r})=-\frac{p_{0} \sqrt{\mu^{4}+4(h-\lambda)^{2}(x-b / 2)^{2}}}{2(h-\lambda)},
$$

$\boldsymbol{r}=(x, y) \in \gamma^{\lambda, \mu}$. Then $\mathbf{T}^{\lambda, \mu}$ is an admissible stress field weakly equilibrating the loads (s, $\left.\mathbf{0}\right)$.

We emphasize that for all $(\lambda, \mu) \in \mathscr{A}$ the stress field $\mathrm{T}^{\lambda, \mu}$ equilibrates the same loads.

Proof. This follows from the considerations in [Lucchesi et al. 2006, Examples 3 and 4].

Proposition 7.2. If $\mathscr{A}$ has a nonempty interior, then the loads $(\boldsymbol{s}, \mathbf{0})$ are strongly compatible. In fact, there exists a bounded admissible stress field $\boldsymbol{T}$ on $\Omega$ strongly equilibrating them.

Proof. Let $\left(\lambda_{0}, \mu_{0}\right)$ be an interior point of $\mathscr{A}$, hence $(\lambda, \mu) \in \mathscr{A}$ for all $(\lambda, \mu)$ sufficiently close to $\left(\lambda_{0}, \mu_{0}\right)$. Therefore, setting

$$
\alpha:=\left(h-\lambda_{0}\right) / \mu_{0}^{2}, \quad \hat{\lambda}(\mu)=h-\alpha \mu^{2},
$$

we have $(\hat{\lambda}(\mu), \mu) \in \mathscr{A}$ for all $\mu \in \Lambda:=\left(\mu_{0}-\epsilon, \mu_{0}+\epsilon\right)$, where $\epsilon>0$ is sufficiently small. If $\mathrm{T}^{\lambda, \mu}$ denotes the measure Equation (46), then by Proposition 3.2 (i), the measure

$$
\mathbf{T}:=\int_{\Lambda} \mathbf{T}^{\hat{\lambda}(\mu), \mu} d \mu
$$

weakly equilibrates the loads $(\boldsymbol{s}, \mathbf{0})$. We write $\mathbf{T}=\mathbf{T}_{r}+\mathbf{T}_{s}$, where

$$
\begin{gathered}
\mathbf{T}_{r}=f_{\Lambda} \mathbf{T}_{r}^{\mu} d \mu, \quad \mathbf{T}_{s}=\int_{\Lambda} \mathbf{T}_{s}^{\mu} d \mu, \\
\mathbf{T}_{r}^{\mu}=\boldsymbol{T}_{r}^{\hat{\lambda}(\mu), \mu} \mathscr{L}^{2} \mathrm{~L} \Omega, \quad \mathbf{T}_{s}^{\mu}=\boldsymbol{T}_{s}^{\hat{\lambda}(\mu), \mu \mathscr{H}^{1}} \mathrm{~L} \gamma^{\hat{\lambda}(\mu), \mu} .
\end{gathered}
$$

By Proposition 2.2, $\mathrm{T}_{r}=\boldsymbol{T}_{r} \mathscr{L}^{2} \mathrm{~L} \Omega$, where

$$
\boldsymbol{T}_{r}(\boldsymbol{r})=\int_{\Lambda} \boldsymbol{T}_{s}^{\hat{\lambda}(\mu), \mu}(\boldsymbol{r}) d \mu
$$

$\boldsymbol{r} \in \Omega$. Since $\boldsymbol{T}_{s}^{\hat{\lambda}(\mu), \mu}$ is bounded independently of $\mu$ if $\mu_{0}-\epsilon<\mu<\mu_{0}+\epsilon$, we see that $\boldsymbol{T}_{r}$ is bounded on $\Omega$. 
Furthermore, one finds that $\gamma^{\hat{\lambda}(\mu), \mu}=\varphi^{-1}(\mu)$, where $\varphi: \Omega \rightarrow \mathbb{R}$ is defined by

$$
\varphi(\boldsymbol{r})=\sqrt{(x-b / 2)^{2}+(h-y) / \alpha},
$$

$\boldsymbol{r}=(x, y) \in \Omega$. Let

$$
\Omega_{0}=\bigcup\left\{\gamma^{\hat{\lambda}(\mu), \mu}: \mu_{0}-\epsilon<\mu<\mu_{0}+\epsilon\right\} \equiv \varphi^{-1}\left(\left(\mu_{0}-\epsilon, \mu_{0}+\epsilon\right)\right) .
$$

The measure $\mathbf{T}_{s}^{\mu}$ can be written as

$$
\mathbf{T}_{s}^{\mu}=\boldsymbol{G} \mathscr{H}^{1}\left\llcorner\varphi^{-1}(\mu)\right.
$$

where $\boldsymbol{G}: \Omega_{0} \rightarrow$ Sym is given by

$$
\boldsymbol{G}(\boldsymbol{r})=\boldsymbol{T}_{s}^{\hat{\lambda}(\mu), \mu}(\boldsymbol{r}),
$$

$\boldsymbol{r} \in \Omega_{0}$, where $\mu$ stands for $\varphi(\boldsymbol{r})$. One easily finds that $\varphi$ is continuously differentiable on $\Omega$ with bounded derivatives on $\Omega_{0}$; in particular, $|\nabla \varphi|$ is bounded on $\Omega_{0}$. Furthermore, one has

$$
\left|\sigma^{\hat{\lambda}(\mu), \mu}(\boldsymbol{r})\right| \leq \frac{p_{0}}{2 \alpha} \sqrt{1+\alpha^{2} b^{2}}, \quad \boldsymbol{r} \in \gamma^{\hat{\lambda}(\mu), \mu},
$$

which implies that $\boldsymbol{G}$ is bounded on $\Omega_{0}$. Proposition 2.3 then says that $\mathbf{T}_{s}=\boldsymbol{T}_{s} \mathscr{L}^{2}\llcorner\Omega$, where

$$
\boldsymbol{T}_{s}(\boldsymbol{r})= \begin{cases}(2 \epsilon)^{-1}|\varphi(\boldsymbol{r})| \boldsymbol{G}(\boldsymbol{r}), & \text { if } \boldsymbol{r} \in \Omega_{0}, \\ \mathbf{0}, & \text { otherwise, }\end{cases}
$$

$\boldsymbol{r} \in \Omega$, which is a bounded function by the above.

We thus conclude that

$$
\mathbf{T}=\boldsymbol{T} \mathscr{L}^{2}\llcorner\Omega,
$$

where $\boldsymbol{T}=\boldsymbol{T}_{r}+\boldsymbol{T}_{s}$ is a bounded function on $\Omega$. A reference to the density of $V_{0}$ in $V$ and to Propositions 7.1 and 3.2 (ii) then completes the proof.

\section{References}

[Adams and Fournier 2003] A. Adams and J. J. F. Fournier, Sobolev spaces, 2nd ed., Academic Press, New York, 2003.

[Ambrosio et al. 2000] L. Ambrosio, N. Fusco, and D. Pallara, Functions of bounded variation and free discontinuity problems, Oxford Mathematical Monographs, The Clarendon Press Oxford University Press, New York, 2000. MR 2003a:49002

[Anzellotti 1985] G. Anzellotti, "A class of convex noncoercive functionals and masonry-like materials", Ann. Inst. H. Poincaré Anal. Non Linéaire 2:4 (1985), 261-307. MR 87f:49056

[Del Piero 1989] G. Del Piero, "Constitutive equation and compatibility of the external loads for linear elastic masonry-like materials”, Meccanica 24:3 (1989), 150-162. MR 91i:73004

[Del Piero 1998] G. Del Piero, "Limit analysis and no-tension materials", Int. J. Plasticity 14 (1998), 259-271.

[Di Pasquale 1984] S. Di Pasquale, "Statica dei solidi murari: teorie ed esperienze", Pubblicazione 27, 1984.

[Giaquinta and Giusti 1985] M. Giaquinta and E. Giusti, "Researches on the equilibrium of masonry structures", Arch. Rational Mech. Anal. 88:4 (1985), 359-392. MR 86h:73012

[Gurtin 1981] M. E. Gurtin, An introduction to continuum mechanics, vol. 158, Mathematics in Science and Engineering, Academic Press Inc. [Harcourt Brace Jovanovich Publishers], New York, 1981. MR 84c:73001

[Lucchesi et al. 1994] M. Lucchesi, C. Padovani, and A. Pagni, "A numerical method for solving equilibrium problems of masonry-like solids", Meccanica 24 (1994), 175-193. 
[Lucchesi et al. 2004] M. Lucchesi, M. Šilhavý, and N. Zani, "Stress state for heavy masonry panels", in Proceedings of the Colloquium Lagrangianum (Venice, Italy), Springer, 2004. To appear.

[Lucchesi et al. 2005a] M. Lucchesi, M. Šilhavý, and N. Zani, "Singular equilibrated stress fields for no-tension panels", pp. 255-265 in Lecture notes in applied and computational mechanics, vol. 23, Springer, 2005.

[Lucchesi et al. 2005b] M. Lucchesi, M. Šilhavý, and N. Zani, "Stress fields for axisymmetric no-tension bodies", in Proceedings of XVII ${ }^{\text {th }}$ AIMETA Congress (Florence, Italy), 2005.

[Lucchesi et al. 2006] M. Lucchesi, M. Šilhavý, and N. Zani, "A new class of equilibrated stress fields for no-tension bodies", Journal of Mechanics of Materials and Structures 1 (2006).

[Lucchesi et al. 2007] M. Lucchesi, M. Šilhavý, and N. Zani, "A note on equilibrated stress fields for no-tension bodies under gravity", Quarterly of Applied Mathematics (2007), 605-624.

[Lucchesi et al. 2008] M. Lucchesi, C. Padovani, and M. Šilhavý, "On the energetic justification of the limit analysis for masonry bodies", (2008). In preparation.

[Müller 1999] S. Müller, "Variational models for microstructure and phase transitions", pp. 85-210 in Calculus of variations and geometric evolution problems (Cetraro, 1996), vol. 1713, Lecture Notes in Math., Springer, Berlin, 1999. MR 2001b:49019

[Padovani et al. 2007] C. Padovani, G. Pasquinelli, and M. Šilhavý, "Processes in masonry bodies and the dynamical significance of collapse", Math. Mech. Solids (2007). In press.

[Podio-Guidugli 2004] P. Podio-Guidugli, "Examples of concentrated contact interactions in simple bodies", J. Elasticity 75:2 (2004), 167-186. MR 2006e:74003

[Temam 1983] R. Temam, Problèmes mathématiques en plasticité, vol. 12, Méthodes Mathématiques de l'Informatique [Mathematical Methods of Information Science], Gauthier-Villars, Montrouge, 1983. MR 85k:73031

[Šilhavý 2008] M. Šilhavý, "Normal currents: structure, duality pairings and divcurl lemmas", Milan Journal of Mathematics (2008). In press.

Received 31 May 2007. Accepted 28 Nov 2007.

MASSIMILIANO LUCCHESI: massimiliano.lucchesi@unifi.it

Dipartimento di Costruzioni, Università di Firenze, Piazza Brunelleschi 6, 50121 Firenze, Italy

MIROSLAV ŠILHAVÝ: silhavy@math.cas.cz

Mathematical Institute of the AV ČR, Zitná 25, 115 67, Prague 1, Czech Republic

NICOLA ZANI: nicola.zani@unifi.it

Dipartimento di Costruzioni, Università di Firenze, Piazza Brunelleschi 6, 50121 Firenze, Italy 


\title{
CONCENTRATED FORCE ACTING ON A POWER LAW CREEP HALF-PLANE
}

\author{
JEFFREy JORDAN, IWONA JASIUK AND ALEKSANDER ZUBELEWICZ
}

\begin{abstract}
The problem of concentrated force acting on a half-plane made of a power-law creep material is solved analytically. In our approach, the constitutive equation that describes the process of dilatational deformation is omitted. The incomplete material description is used for constructing a solution by bringing the dilatational deformation to zero and, in this manner, making the material incompressible. We find solutions for two cases; one solution is for a linear viscous material, while the second is for a power-law material where the power exponent is equal to three. Solutions of the two problems are found to be very different. While the linear viscous solution is found to be the same as the linear elastic solution, the nonlinear solution is found to be significantly different. This result may give rise to a new experimental technique for characterization of materials with a nonlinear creep behavior.
\end{abstract}

\section{Introduction}

The problem of concentrated force acting on a linear elastic and isotropic half-space was solved by Boussinesq [1885] in three dimensions and by Flamant in 1892 (see [Love 1944; Malvern 1969; Timoshenko and Goodier 1970] for details) in two dimensions. Some recent solutions addressed a concentrated force acting on a linear elastic half-space [Jager 1997; Levy 2002; Unger 2002; Marzocchi and Musesti 2004], a transversely isotropic elastic half-space [Liao and Wang 1999], an inhomogeneous transversely isotropic elastic half-space [Wang et al. 2003], an elastic nonlocal half-plane [Artan 1996], a gradient elasticity half-space [Zhou and Jin 2003; Li et al. 2004; Lazar and Maugin 2006], an elastic linear hardening half-plane [Gao 1999], and a piezoelectric half-plane [Sosa and Castro 1994].

In this paper we focus on the problem of concentrated force acting on a half-plane made of a powerlaw creep material in the context of plane strain (see Figure 1). In our approach, following [Zubelewicz 2005], we omit the constitutive equation that describes dilatancy. In this manner we are able to examine various kinematically admissible solutions, from which we narrow our search to the solution for an incompressible material.

Recall that in the Flamant solution the only nonzero stress term is

$$
\sigma_{r r}=-\frac{2 P}{\pi} \frac{\cos \theta}{r} .
$$

For the two dimensional case, the equivalent Tresca stress (the maximum shear stress) is

$$
\sigma_{e q}=\left|\frac{\sigma_{r r}}{2}\right|=\frac{P}{\pi} \frac{\cos \theta}{r} \text {. }
$$

Keywords: concentrated force, power-law material, creep, viscous material, incompressible material.

The authors acknowledge funding from the National Science Foundation, grant no. CMS-0085137 (grant monitor Dr. Ken Chong). 


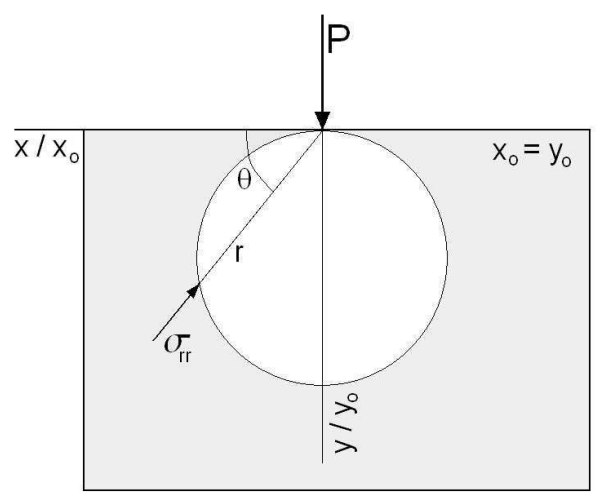

Figure 1. Force acting on a half-plane.

In this paper, the radial stress $\sigma_{r r}$ for a linear elastic case will be compared to the radial stress for a power-law material, where the power is assumed to be one and three.

\section{Problem statement}

We study the problem involving a concentrated force acting on a half-plane made of a power-law creep incompressible material in the context of plane strain (see Figure 1). Thus, the applied load $P$ has units of load per unit thickness. Following the technique presented in [Zubelewicz 2005], we convert all functions from a real into a complex domain, where the complex variables are defined, as usual, by $z=x+i y$ and $z^{*}=x-i y$.

The complex shear strains can be defined as

$$
\eta=\left(\varepsilon_{x x}-\varepsilon_{y y}\right)+2 i \varepsilon_{x y}, \quad \eta^{*}=\left(\varepsilon_{x x}-\varepsilon_{y y}\right)-2 i \varepsilon_{x y} .
$$

The complex displacements, $v=\left(u_{x}+i u_{y}\right)$ and $v^{*}=\left(u_{x}-i u_{y}\right)$, and the strains are coupled and satisfy the relations

$$
\eta=2 \frac{\partial v}{\partial z^{*}}, \quad \eta^{*}=2 \frac{\partial v^{*}}{\partial z} .
$$

Using the above equations it is possible to express the displacements $u_{x}$ and $u_{y}$ in terms of complex shear strains as

$u_{x}=\frac{1}{4}\left[\int \eta d z^{*}+\int \eta^{*} d z\right]+\frac{1}{2}\left[\psi_{v}(z)+\psi_{v}^{*}\left(z^{*}\right)\right], \quad u_{y}=\frac{1}{4 i}\left[\int \eta d z^{*}-\int \eta^{*} d z\right]+\frac{1}{2 i}\left[\psi_{v}(z)-\psi_{v}^{*}\left(z^{*}\right)\right]$,

where the additional displacement functions $\psi_{v}$ and $\psi_{v}^{*}$ must satisfy kinematic boundary conditions. In order to find $u_{x}$ and $u_{y}$, a path-dependent integration is chosen such that one of the complex variables is kept constant while integrating with respect to the other [Vekua 1962]. Then, the rate of volumetric change (dilatational deformation) can be defined as

$$
\dot{I}_{\varepsilon}=\frac{1}{2}\left[\int \frac{\partial \dot{\eta}}{\partial z} d z^{*}+\int \frac{\partial \dot{\eta}^{*}}{\partial z^{*}} d z\right]+\dot{\psi}_{v}^{\prime}(z)+\dot{\psi}_{v}^{*^{\prime}}\left(z^{*}\right), \quad \psi_{v}^{\prime}=\frac{\partial \psi_{v}}{\partial z} .
$$


Equation (1) is written in a rate form, where the dot indicates that it is a time derivative. It has been shown in [Zubelewicz 2005] that kinematical compatibility is always satisfied when the dilatant deformation is expressed through Equation (1).

Next, the complex shear stresses are defined as $\tau=\Delta \sigma+2 i \sigma_{x y}$ and $\tau^{*}=\Delta \sigma-2 i \sigma_{x y}$, where $\Delta \sigma=\sigma_{x x}-\sigma_{y y}$. The Tresca stress is equal to $\sigma_{e q}=\frac{1}{2} \sqrt{\tau \tau^{*}}$.

The power-law creep equation which couples the equivalent strain rate and equivalent stress is

$$
\dot{e}_{e q}=\Lambda\left(\frac{\sigma_{e q}}{\sigma_{o}}\right)^{p}
$$

Assuming associated flow rules, we can express the constitutive Equation (2) in terms of $\dot{\eta}$ and $\tau$ such that

$$
\dot{\eta}=\frac{\Lambda}{\left(2 \sigma_{o}\right)^{p}} \tau^{\frac{p+1}{2}}\left(\tau^{*}\right)^{\frac{p-1}{2}}, \quad \dot{\eta}^{*}=\frac{\Lambda}{\left(2 \sigma_{o}\right)^{p}} \tau^{\frac{p-1}{2}}\left(\tau^{*}\right)^{\frac{p+1}{2}} .
$$

The proposed formulation allows for a separation of the material response due to shear and dilatancy. As we notice, neither the hydrostatic stress $\left(I_{\sigma}=\sigma_{x x}+\sigma_{y y}\right)$ nor the rate of volumetric change (1) is involved in the constitutive (3).

For completeness, the equilibrium equations are

$$
\frac{\partial \tau}{\partial z}+\frac{\partial I_{\sigma}}{\partial z^{*}}=0, \quad \frac{\partial \tau^{*}}{\partial z^{*}}+\frac{\partial I_{\sigma}}{\partial z}=0
$$

We solve the equilibrium equations (4) with the use of two stress functions, $\psi_{\sigma}(z)$ and $\Omega\left(z, z^{*}\right)$, where $\Omega$ is a real function of complex arguments. We satisfy these equations by representing the complex stresses as

$$
\tau=\psi_{\sigma}^{*}\left(z^{*}\right)+\int \frac{\partial \Omega}{\partial z^{*}} d z, \quad \tau^{*}=\psi_{\sigma}(z)+\int \frac{\partial \Omega}{\partial z} d z^{*}, \quad I_{\sigma}=-\Omega .
$$

Thus there are six variables to be determined: three complex stresses $\left(\tau, \tau^{*}, I_{\sigma}\right)$ and three complex strain rates $\left(\dot{\eta}, \dot{\eta}^{*}, \dot{I}_{\varepsilon}\right)$. On the other hand, there are five equations available: the kinematical compatibility equation (1), two constitutive equations (3), and two equilibrium equations (4). The sixth governing equation is the requirement of material incompressibility. We enforce the condition assuming that the dissipation energy due to volumetric change is equal to zero, while the dilatational deformation is nonnegative in all points of the material. First, we calculate the total rate of dissipation

$$
\dot{W}^{t}=\int_{\Delta V} \sigma_{e q} \dot{e}_{e q} d V+\frac{1}{2} \int_{\Delta V} I_{\sigma} \dot{I}_{\varepsilon} d V
$$

Then, we bring the second term in (6) to zero. This assures that there is no volumetric change and, therefore, the material is incompressible. Our chosen stress functions are

$$
\psi_{\sigma}(z)=\sum_{m} C_{m} z^{\lambda_{m}}, \quad \Omega=\sum_{n, m}\left[D_{n m} z^{\alpha_{m}^{n}}\left(z^{*}\right)^{\beta_{m}^{n}}+D_{n m}^{*} z^{\beta_{m}^{n}}\left(z^{*}\right)^{\alpha_{m}^{n}}\right]
$$

where $\lambda_{m}$ satisfies the condition $\lambda_{m}=\alpha_{m}+\beta_{m}$ for any $n, m=1,2, \ldots \infty$. For an asymptotic analysis, $n$ is equal to 1 , and can be omitted in further equations. 
Now, substituting Equation (7) into (5) leads to

$$
\begin{aligned}
\tau & =C^{*}\left(z^{*}\right)^{\lambda}+\sum_{m}\left(\frac{\beta_{m} D_{m}}{\alpha_{m}+1} z^{\alpha_{m}+1}\left(z^{*}\right)^{\beta_{m}-1}+\frac{\alpha_{m} D_{m}^{*}}{\beta_{m}+1} z^{\beta_{m}+1}\left(z^{*}\right)^{\alpha_{m}-1}\right), \\
\tau^{*} & =C z^{\lambda}+\sum_{m}\left(\frac{\beta_{m} D_{m}^{*}}{\alpha_{m}+1}\left(z^{*}\right)^{\alpha_{m}+1} z^{\beta_{m}-1}+\frac{\alpha_{m} D_{m}}{\beta_{m}+1}\left(z^{*}\right)^{\beta_{m}+1} z^{\alpha_{m}-1}\right), \\
I_{\sigma} & =-\Omega=-\sum_{m}\left(D_{m} z^{\alpha_{m}}\left(z^{*}\right)^{\beta_{m}}+D_{m}^{*} z^{\beta_{m}}\left(z^{*}\right)^{\alpha_{m}}\right),
\end{aligned}
$$

where $C$ is a real constant, $C^{*}=C, D_{m}=D_{m}^{0} e^{i \phi_{m}}$, and $D_{m}^{*}=D_{m}^{0} e^{-i \phi_{m}}$, where $\phi_{m}$ need to be defined together with $D_{m}^{0}$. Thus, in summary, we will find these constants through the process of minimization of the plastic dissipation rate due to volumetric change (the second term in (6)) which results in solutions for incompressible materials.

\section{General solution}

In the case of concentrated force acting on a half plane (1), there are zero tractions along the free surfaces, thus $\sigma_{y y} \pm i \sigma_{x y}=0$, at $\theta=0, \pi$. In the complex domain, the conditions are $\sigma_{y y}+i \sigma_{x y}=\frac{1}{2}\left(I_{\sigma}-\tau^{*}\right)=0$ and $\sigma_{y y}-i \sigma_{x y}=\frac{1}{2}\left(I_{\sigma}-\tau\right)=0$, where the second equation is redundant. Using the definitions of the complex stresses given in (8), we find

$$
\begin{aligned}
-C z^{\lambda}-\sum_{m}\left[D_{m}^{*}\left(\frac{\beta_{m}}{\alpha_{m}+1} z^{\beta_{m}-1}\left(z^{*}\right)^{\alpha_{m}+1}+z^{\beta_{m}}\left(z^{*}\right)^{\alpha_{m}}\right)\right. & \\
& \left.+D_{m}\left(\frac{\alpha_{m}}{\beta_{m}+1} z^{\alpha_{m}-1}\left(z^{*}\right)^{\beta_{m}+1}+z^{\alpha_{m}}\left(z^{*}\right)^{\beta_{m}}\right)\right]=0 .
\end{aligned}
$$

In a polar coordinate system $z=R(\cos \theta+i \sin \theta)$, and $z^{*}=R(\cos \theta-i \sin \theta)$. For $\theta=0$, (9) reduces to

$$
C+\sum_{m}(\lambda+1)\left(\frac{D_{m}}{\beta_{m}+1}+\frac{D_{m}^{*}}{\alpha_{m}+1}\right)=0
$$

When $\theta=\pi$, the equation becomes

$$
\begin{aligned}
C(\cos \lambda \pi+i \sin \lambda \pi)+\sum_{m}\left[\frac{D_{m}}{\beta_{m}+1}(\lambda+1)\left\{\cos \left[\left(\alpha_{m}-\beta_{m}\right) \theta\right]+i \sin \left[\left(\alpha_{m}-\beta_{m}\right) \theta\right]\right\}\right. \\
\left.+\frac{D_{m}^{*}}{\alpha_{m}+1}(\lambda+1)\left\{\cos \left[\left(\alpha_{m}-\beta_{m}\right) \theta\right]+i \sin \left[\left(\alpha_{m}-\beta_{m}\right) \theta\right]\right\}\right]=0 .
\end{aligned}
$$

Equation (10) is used to solve for $C$, and then (11) becomes

$$
\begin{array}{r}
\sum_{m}(\lambda+1)\left[-\left(\frac{D_{m}^{*}}{\alpha_{m}+1}+\frac{D_{m}}{\beta_{m}+1}\right)(\cos \lambda \pi+i \sin \lambda \pi)+\frac{D_{m}}{\beta_{m}+1}\left\{\cos \left[\left(\alpha_{m}-\beta_{m}\right) \theta\right]+i \sin \left[\left(\alpha_{m}-\beta_{m}\right) \theta\right]\right\}\right. \\
\left.+\frac{D_{m}^{*}}{\alpha_{m}+1}\left\{\cos \left[\left(\alpha_{m}-\beta_{m}\right) \theta\right]+i \sin \left[\left(\alpha_{m}-\beta_{m}\right) \theta\right]\right\}\right]=0 .
\end{array}
$$

It follows from (12) that $\lambda=-1$. Then, from (10), $C$ is found to be equal to zero. 
Applying these conditions to the complex stresses we find

$$
\begin{aligned}
\tau & =-\sum_{m} D_{m}^{0}\left[e^{i \phi_{m}} z^{\alpha_{m}+1}\left(z^{*}\right)^{\beta_{m}-1}+e^{-i \phi_{m}} z^{\beta_{m}+1}\left(z^{*}\right)^{\alpha_{m}-1}\right], \\
\tau^{*} & =-\sum_{m} D_{m}^{0}\left[e^{i \phi_{m}}\left(z^{*}\right)^{\alpha_{m}+1} z^{\beta_{m}-1}+e^{-i \phi_{m}}\left(z^{*}\right)^{\beta_{m}+1} z^{\alpha_{m}-1}\right], \\
I_{\sigma} & =-\sum_{m} D_{m}^{0}\left[e^{i \phi_{m}} z^{\alpha_{m}}\left(z^{*}\right)^{\beta_{m}}+e^{-i \phi_{m}} z^{\beta_{m}}\left(z^{*}\right)^{\alpha_{m}}\right] .
\end{aligned}
$$

At this point it is convenient to convert the complex stresses into the real domain. The stress components are found as

$$
\begin{aligned}
& \sigma_{x x}=\frac{1}{2} R^{-1} \sum_{m}-2 D_{m}^{0}\left[e^{i \phi_{m}} \cos \left[\left(\alpha_{m}-\beta_{m}+2\right) \theta\right]-e^{i \phi_{m}} \sin \left[\left(\alpha_{m}-\beta_{m}-2\right) \theta\right]\right], \\
& \sigma_{y y}=-R^{-1} \sum_{m} D_{m}^{0}\left[(-\cos 2 \theta+1)\left(e^{i \phi_{m}} \cos \left[\left(\alpha_{m}-\beta_{m}\right) \theta\right]-e^{i \phi_{m}} \sin \left[\left(\alpha_{m}-\beta_{m}\right) \theta\right]\right],\right. \\
& \sigma_{x y}=-R^{-1} \sum_{m} D_{m}^{0}\left[\sin 2 \theta\left(e^{i \phi_{m}} \cos \left[\left(\alpha_{m}-\beta_{m}\right) \theta\right]-e^{i \phi_{m}} \sin \left[\left(\alpha_{m}-\beta_{m}\right) \theta\right]\right],\right.
\end{aligned}
$$

and in the polar coordinate system, there is only one nonzero stress component, namely

$$
\sigma_{r r}=-2 R^{-1} \sum_{m} D_{m}^{0}\left[e^{i \phi_{m}} \cos \left[\left(\alpha_{m}-\beta_{m}\right) \theta\right]-e^{-i \phi_{m}} \sin \left[\left(\alpha_{m}-\beta_{m}\right) \theta\right]\right] .
$$

Recalling that $\alpha_{n}+\beta_{n}=\lambda_{n}=-1$ and knowing that $\sigma_{r r}$ is symmetric with respect to $y$ (at $\theta=\pi / 2$ ), this allows us to determine the parameters $\alpha_{n}$ and $\beta_{n}$ such that

$$
\left(\alpha_{n}-\beta_{n}\right)+\frac{2 \phi_{n}}{\pi}=2 n, \quad \alpha_{n}+\beta_{n}=-1 .
$$

In the final form we find

$$
\alpha_{n}=n-\frac{\phi_{n}}{\pi}-\frac{1}{2}, \quad \beta_{n}=-n+\frac{\phi_{n}}{\pi}-\frac{1}{2},
$$

where $n=1,2,3, \ldots, \infty$.

3.1. Linear case. When $p=1$, the rate of volumetric change

$$
\dot{I}_{\varepsilon}=\frac{\Lambda}{4 \sigma_{o}}\left(\int \frac{\partial \tau}{\partial z} d z^{*}+\int \frac{\partial \tau^{*}}{\partial z^{*}} d z\right)+\dot{\psi}_{v}^{\prime}(z)+\dot{\psi}_{v}^{*^{\prime}}\left(z^{*}\right)
$$

is simplified and takes the form

$$
\dot{I}_{\varepsilon}=\frac{\Lambda}{\sigma_{o}} R^{-1} \sum_{m} D_{m}^{0}\left(\cos \left[\phi_{m}+\delta_{m} \theta\right]\right)+A_{1} \sin \lambda \theta,
$$

where the constant $A_{1}$ comes from the functions $\psi_{v}^{\prime}(z) \psi$ and $\psi_{v}^{*^{\prime}}\left(z^{*}\right)$. The solution is obtained by selecting the parameters $\left(A_{1}, D_{m}^{0}\right.$, and $\left.\phi_{m}\right)$ such that the condition for material incompressibility is satisfied. These constants are listed for reference in Table 1. 
3.2. Non-linear case $(\boldsymbol{p}=3)$. In the nonlinear case, the expression for the rate of volumetric change is

$$
\begin{aligned}
\dot{I}_{\varepsilon}=-\frac{\Lambda}{8 \sigma_{o}^{3}} R^{3 \lambda} \sum_{n, m, k} D_{m}^{0} D_{m}^{0} D_{m}^{0}\left[\left(\frac{\alpha_{n}+\alpha_{m}+\alpha_{k}+1}{\beta_{n}+\beta_{m}+\beta_{k}}+\frac{\beta_{n}+\beta_{m}+\beta_{k}+1}{\alpha_{n}+\alpha_{m}+\alpha_{k}}\right) \times \cos \left[\phi_{n}+\phi_{m}+\phi_{k}+\left(\delta_{n}+\delta_{m}+\delta_{k}\right) \theta\right]\right. \\
+2\left(\frac{\beta_{n}+\alpha_{m}+\alpha_{k}+1}{\alpha_{n}+\beta_{m}+\beta_{k}}\right) \times \cos \left[\phi_{n}-\phi_{m}-\phi_{k}+\left(\delta_{n}-\delta_{m}-\delta_{k}\right) \theta\right] \\
+2\left(\frac{\beta_{n}+\alpha_{m}+\alpha_{k}+1}{\alpha_{n}+\beta_{m}+\beta_{k}}\right) \times \cos \left[\phi_{n}+\phi_{m}-\phi_{k}+\left(\delta_{n}+\delta_{m}-\delta_{k}\right) \theta\right] \\
\left.+\left(\frac{\alpha_{n}+\beta_{m}+\alpha_{k}+1}{\beta_{n}+\alpha_{m}+\beta_{k}}+\frac{\beta_{n}+\alpha_{m}+\beta_{k}+1}{\alpha_{n}+\beta_{m}+\alpha_{k}}\right) \times \cos \left[\phi_{n}+\phi_{m}-\phi_{k}+\left(\delta_{n}+\delta_{m}-\delta_{k}\right) \theta\right]\right] \\
+A_{1} \sin [3 \lambda \theta],
\end{aligned}
$$

where $\delta_{m}=\alpha_{m}-\beta_{m}$. Again, as in the linear case, the solution is obtained by determining $A_{1}, D_{m}^{0}$, and $\phi_{m}$ for a material that exhibits zero dissipation energy due to dilatation. These constants are again given in Table 1.

\section{Discussion}

Since incompressibility is our requirement, we evaluate the solutions at each point by comparing the rate of the volumetric change (an error of the solution) to the shear strain rates. We generated solutions assuming a three term series $\left(D_{m}^{0}, \phi_{m}\right)$ for $m=1,2$, and 3 .

The calculated constants for cases $p=1$ and $p=3$ are given in Table 1 . A contour plot of the maximum shear strain rate and the rate of volumetric change is presented in Figure 2 (left). The large curve is the strain rate, while the small curve near the origin is the rate of volumetric change. As can be seen (for example, in Figure 2, left), the rate of volumetric change is negligible compared with the shear strain rate, with an error less than $3.5 \%$. In the case of $p=3$, the contours are very different (compare Figure 2, left and right). As before, we are satisfied with an error less than 3.5\%.

In our next step we examine stress $\sigma_{r r}$. The contour plot for the case of $p=1$ is essentially the same as that for Flamant's solution (see Figure 3). The slight difference in the shapes is due to numerical error. Taking higher order terms in $D_{m}^{0}$ and $\phi_{m}$ would reduce the discrepancies even further. Thus the solution for a linear viscous case, having linear stress-strain rate relations, is the same, within numerical accuracy, as for the linear elastic case, having linear stress-strain constitutive relations. This is expected since the governing equations for these two cases are analogous, and thus can serve as a check of our method. Contours of the stress $\sigma_{r r}$ for a power-law material, where the power is equal to one and

\begin{tabular}{|c|ccrcrcc|}
\hline$p$ & $A_{1}$ & \multicolumn{1}{c}{$D_{1}^{0}$} & \multicolumn{1}{c}{$D_{2}^{0}$} & \multicolumn{1}{c}{$D_{3}^{0}$} & \multicolumn{1}{c}{$\phi_{1}$} & $\phi_{2}$ & $D_{3}$ \\
\hline 1 & -0.80 & -8.16 & -5.16 & 3.83 & -0.19 & 3.49 & 5.70 \\
3 & -6.70 & -149.59 & -158.42 & -9.42 & 0.78 & 3.89 & 6.65 \\
\hline
\end{tabular}

Table 1. Calculated constants used to solve cases $p=1$ and $p=3$. 

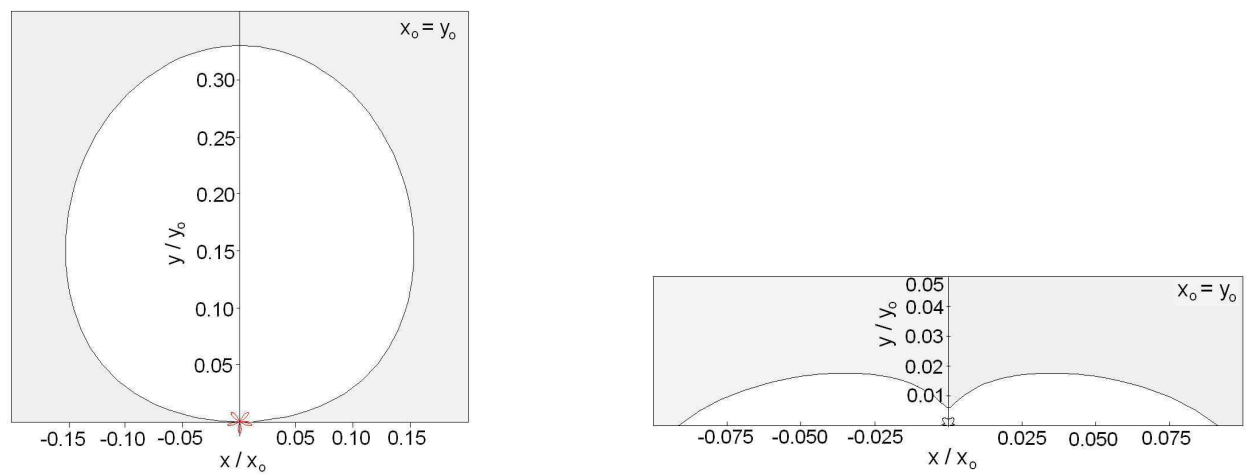

Figure 2. Contours of maximum strain rate (large curve) versus change of volume (small curve near origin) for $p=1$ (left) and $p=3$ (right).

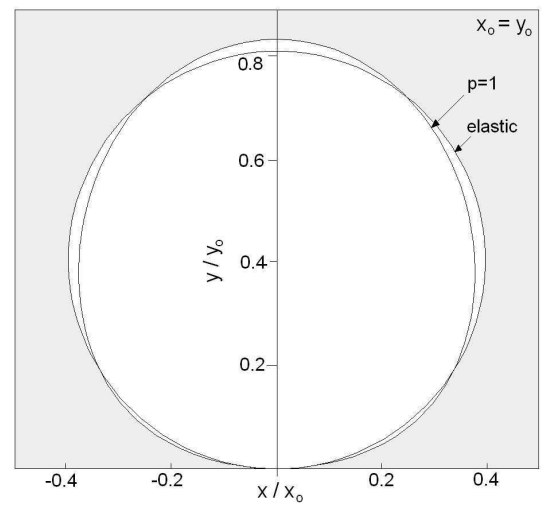

Figure 3. $\sigma_{r r}$ for $p=1$ versus elastic case.

three, are shown in Figure 4. Again, the contours for $p=1$ and $p=3$ are very different. Thus, the contours of the equivalent shear strain rate and stress for the two materials are surprisingly different (see Figure 2 and Figure 4). In summary, the new technique provides a new way to look at the problem of a concentrated force on a half-plane. Although the methods of finding the linear elastic and linear viscous solutions are completely different, the results are the same. That proves the validity of the proposed technique.

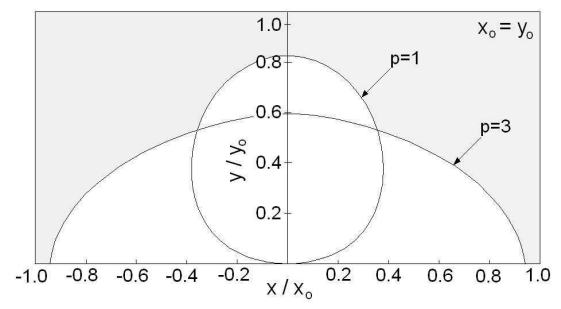

Figure 4. $\sigma_{r r}$ for $p=3$ versus $p=1$. 
The power-law material with power three $(p=3)$ represents materials which have viscous-like behavior, and thus are close to their melting point. One can construct a solution for higher powers such as five, which would represent a ductile material at room temperature. The solution would be obtained in the same manner but would involve more terms in the series and thus a more extensive algebra. The solution obtained in this paper for a power of three could be suitable for nonNewtonian fluids, solder pastes used in electronic packaging industry, and in geological applications involving sand or clay saturated in oil, or other oil-like fluids with suspensions, for example.

The problem solved in this paper could also be studied experimentally. The set-up would involve a material under a plane strain geometry constrained at the ends, with one side having a transparent frictionless plate. The loading would be a line load perpendicular to the plane of observation. Such a set-up would allow us to measure experimentally the rate of surface subsidence (surface velocity). In power-law materials, given by Equation (2), where the power $p$ is greater than one, the profile of the out of plane surface velocity is proportional to $r^{-(p-1)}$. This relation between surface velocity and $p$ could be used to obtain $p$ for a given viscous material from surface velocity measurements. Thus, we conjecture that such an experimental set-up, if done successfully (meaning plane strain conditions and frictionless boundary conditions would be achieved) could serve as a new experimental method, a two dimensional analog to indentation techniques, to characterize a constitutive law of viscous (fluid-like) materials which are difficult to test otherwise. We plan to test this conjecture in the future.

\section{Summary}

We examined a new solving scheme for ductile materials that obeyed a power-law creep behavior. We constructed solutions for a viscous linear $(p=1)$ and power-law nonlinear $(p=3)$ material. We compared the linear elastic (Flamant) and linear viscous solutions and have shown that both solutions produce the same distribution of stresses, and that the strain rate corresponds one to one to strain in an elastic material. We used this comparison to validate our approach. We are intrigued with the solution for the nonlinear power-law material where the stress exponent $p$ is equal to three. The two materials $(p=1$ and $p=3$ ) have very different stress contours. The theoretical result suggests the feasibility of a two dimensional indentation test which could be used for characterization of constitutive laws of viscous materials.

\section{Acknowledgments}

The authors thank Los Alamos National Laboratory Science Campaigns for active support and encouragement.

\section{References}

[Artan 1996] R. Artan, "Nonlocal elastic half plane loaded by a concentrated force", Int. J. Eng. Sci. 34:8 (1996), 943-950. [Boussinesq 1885] J. Boussinesq, Application des potentiels a l'étude de l'équilibre et du mouvement des solides elastiques, Gauthier-Villars, Paris, 1885.

[Gao 1999] X.-L. Gao, "An exact elastoplastic solution for the plane wedge problem of an elastic linear-hardening material", Math. Mech. Solids 4:3 (1999), 289-306.

[Jager 1997] J. Jager, "Half-planes without coupling under contact loading”, Arch. Appl. Mech. 67:4 (1997), 247-259.

[Lazar and Maugin 2006] M. Lazar and G. A. Maugin, "A note on line forces in gradient elasticity", Mech. Res. Commun. 33:5 (2006), 674-680. 
[Levy 2002] A. J. Levy, "A note on the application of the Flamant solution of classical elasticity to circular domains", J. Appl. Mech. (Trans. ASME) 69:6 (2002), 856-859.

[Li et al. 2004] S. Li, I. Miskioglu, and B. S. Altan, "Solution to line loading of a semi-infinite solid in gradient elasticity", Int. J. Solids Struct. 41:13 (2004), 3395-4310.

[Liao and Wang 1999] J. J. Liao and C. D. Wang, "Elastic solutions for a transversely isotropic half-space subjected to a point load", Int. J. Numer. Anal. Met. 22:6 (1999), 425-447.

[Love 1944] A. E. H. Love, A treatise on the mathematical theory of elasticity, 4th ed., Dover Publications, New York, 1944.

[Malvern 1969] L. E. Malvern, Introduction to the mechanics of a continuous medium, Prentice-Hall, Englewood Cliffs, NJ, 1969.

[Marzocchi and Musesti 2004] A. Marzocchi and A. Musesti, "Balance laws and weak boundary conditions in continuum mechanics", J. Elasticity 74:3 (2004), 239-248.

[Sosa and Castro 1994] H. A. Sosa and M. A. Castro, "On concentrated loads at the boundary of a piezoelectric half-plane", $J$. Mech. Phys. Solids 42:7 (1994), 1105-1122.

[Timoshenko and Goodier 1970] S. Timoshenko and J. N. Goodier, Theory of elasticity, McGraw Hill, New York, 1970.

[Unger 2002] D. J. Unger, "Similarity solution of the Flamant problem by means of a one-parameter group transformation", $J$. Elasticity 66:1 (2002), 93-97.

[Vekua 1962] I. N. Vekua, Generalized analytic functions, vol. 25, Int. series of monographs in pure and applied mathematics, Pergamon Press, New York, 1962.

[Wang et al. 2003] C. D. Wang, C. S. Tzeng, E. Pan, and J. J. Liao, "Displacements and stresses due to a vertical point load in an inhomogeneous transversely isotropic half-space", Int. J. Rock. Mech. Min. 40:5 (2003), 667-685.

[Zhou and Jin 2003] D. Zhou and B. Jin, "Boussinesq-flamant problem in gradient elasticity with surface energy", Mech. Res. Commun. 30:5 (2003), 463-468.

[Zubelewicz 2005] A. Zubelewicz, "New method to study dilatant deformation mechanisms in ductile materials", Int. J. Solids Struct. 42:9-10 (2005), 2755-2770.

Received 19 Jun 2007. Revised 4 Dec 2007. Accepted 5 Dec 2007.

JEFFREY JORDAN: j11mjordan@yahoo.com

The G. W. W. School of Mechanical Engineering, Georgia Institute of Technology, 801 Ferst Drive, Atlanta, GA 30332-0405, United States

IWONA JASIUK: ijasiuk@uiuc.edu

Department of Mechanical Science and Engineering, University of Illinois at Urbana-Champaign, Urbana, IL 61801-2906, United States

http://www.mechse.uiuc.edu/faculty/ijasiuk

ALEKSANDER ZubELEWICZ: alek@lanl.gov

Los Alamos National Laboratory, Los Alamos, NM 87545, United States 


\title{
OPTIMIZATION OF PENETRATION INTO GEOLOGICAL AND CONCRETE SHIELDS BY IMPACTOR WITH JET THRUSTER
}

\author{
GABi Ben-Dor, ANATOLy DUBinsky AND TOV ELPERIN
}

\begin{abstract}
High-speed penetration into soil, rock, concrete, and ice by impactors equipped with a jet thruster is optimized using analytical and numerical methods. It is shown that using a jet thruster with optimum burning programs bears considerable promise for increasing the depth of penetration. In this study, we used modified Young's penetration equations with a smooth approximation of the dependence between the depth of penetration and impact velocity for the description of impactor-shield interaction.
\end{abstract}

\section{Introduction}

Optimization of jet propulsion in media with drag was considered mainly with applications to planes and missiles. Surveys of the obtained results and references can be found in the studies by Leitmann [1962], Kosmodemiansky [1966], and Tertychny-Dauri [2004]. Using jet thrusters for increasing the depth of penetration into solid media was analyzed in only a few publications. Sagomonyan [1988] formulated two problems. In the first problem, a jet thruster was assumed to operate during a fixed time interval, and, in order to maximize the depth of penetration (DOP) of penetrator into soil, it was necessary to determine the moment at which the jet thruster must be switched on. In the second problem, a jet thruster could operate along a fixed length of the trajectory, and, in order to provide the maximum DOP, it was necessary to determine the depth at which the jet thruster must be switched on. The second problem was solved for a penetrator with a conical nose, assuming that the mass of the penetrator remains constant. Gould [1997] suggested engineering designs whereby a rocket motor is attached to the penetrator and operates during penetration. Ben-Dor et al. [2007] considered maximization of the DOP as an optimization problem for a penetrator with a variable mass. They noticed a similarity between this problem and maximization of the distance of a horizontal flight in the atmosphere. Various formulations of the latter problem were considered in the past [Hibbs 1952; Cicala and Miele 1956; Miele 1957; Miele 1962; Krotov 1995]. However, it transpired that this similarity had limited applications because of different drag laws in the atmosphere and soil. Only general properties of the solutions obtained for an arbitrary dependence of drag force upon the instantaneous mass and velocity, $D=D(m, v)$, can be used for solving penetration optimization problems. Consequently, optimization of the penetrator with a jet thruster must be analyzed separately. In the study by Ben-Dor et al. [2007], the authors employed the simplest penetration model in which the drag force is a linear function of a squared velocity. Combining analytical and numerical methods, they determined the optimum burning programs and compared the obtained results with more simple burning programs for controlling the motion of a penetrator.

Keywords: rock, concrete, soil, penetration, optimization, jet thruster. 
In the present study, we further develop the approach suggested in [Ben-Dor et al. 2007] for a penetration model with a drag force depending upon the instantaneous velocity and the instantaneous mass of the impactor, whereby, as often happens in practice, the dependence of the drag force in different intervals of its arguments may be determined by different formulas. As a base model, we employed the well known set of models suggested by Young [1997] which is widely used for calculating the DOP in soil, rock, concrete, ice, and frozen soil. The shortcoming of these models is a nonsmooth variation of the DOP with impact velocity which is especially inconvenient in solving optimization problems. Consequently, we suggested a new approximation which is practically as simple and accurate as the original one but has continuous first and second derivatives. The latter renders this approximation more convenient, not only for the goals of the present study, but also for general applications. For a drag force determined by this modified Young's model (MYM), we analytically found the optimal burning programs for the case without an upper bound on the mass flux of the thruster. We suggested a numerical procedure based on dynamic programming for the optimization of the burning program for the general case of $D=D(m, v)$ and a tailored version of this procedure for (MYM). Calculations presented here demonstrate that the appropriate choice of parameters for the jet thruster allows achieving a considerable increase of the DOP.

\section{Formulation of the problem}

Consider a high speed normal penetration of a rigid sharp striker (a body of revolution) with a jet thruster into a semiinfinite shield along the axis $h$. The coordinate $h$, the instantaneous depth of penetration, is defined as the distance between the nose of the impactor and the front surface of the shield.

Since the effect of gravity during high speed penetration in a dense medium can be neglected, motion of the impactor is governed by the following equation of motion of a projectile with a variable mass:

$$
m \frac{d v}{d t}+c \frac{d m}{d t}=-D(m, v), \quad D(m, v)>0,
$$

where $v$ is the velocity of the impactor, $c$ is the relative exit velocity of gases at the nozzle of a jet thruster, $m$ is the instantaneous mass of the impactor that varies in the range between the initial value $m_{\text {imp }}$ to the final value $m_{\text {res }}$, that is,

$$
m_{\text {res }} \leq m \leq m_{\text {imp }}
$$

and $D$ is the drag force depending not only on $m$ and $v$, but also on parameters determining mechanical properties of the shield and the shape of the projectile.

It is assumed that the thruster is capable of delivering all mass fluxes in the range between zero and the maximum value $\mu_{\max }$ :

$$
-\mu_{\max } \leq \frac{d m}{d t} \leq 0
$$

The constraint imposed on the acceleration is as follows:

$$
|d v / d t| \leq \chi_{\max } g
$$

where $g$ is the acceleration of gravity, and $\chi_{\max }$ is the given upper bound for the overload of the impactor. 
Since $d / d t=(d / d h) \cdot(d h / d t)=v d / d h=v(d m / d h) \cdot(d / d m)$, Equation (1) can be rewritten as follows:

$$
v \frac{d m}{d h}\left(m v^{\prime}+c\right)=-D(m, v), \quad v^{\prime}=\frac{d v}{d m} .
$$

The DOP, $H$, for a given impact velocity, $v_{\text {imp }}$, is defined as the depth at which the velocity of the impactor vanishes. Equation (5) implies the following expression for $H$ :

$$
H=\int_{m_{\mathrm{res}}}^{m_{\mathrm{mmp}}} \frac{\left(m v^{\prime}+c\right) v}{D(m, v)} d m
$$

We consider $v$ to be a function of $m$. This is convenient because penetration is associated with a decrease of $m$, and $v$ is a single-valued function of $m$ except for the case in which $m=$ const (inertial, or passive motion of the penetrator). The following conditions are valid for the initial and terminal points of the impactor's path:

$$
v\left(m_{\mathrm{imp}}\right)=v_{\mathrm{imp}}, \quad v\left(m_{\mathrm{res}}\right)=0 .
$$

Using Equation (5) we obtain that

$$
\frac{d m}{d t}=v \frac{d m}{d h}=-\frac{D(m, v)}{m v^{\prime}+c},
$$

and Equation (3) can be rewritten as

$$
0 \leq \frac{D(m, v)}{m v^{\prime}+c} \leq \mu_{\max } .
$$

Using the relationship $d t=-\left(c+m v^{\prime}\right) d m / D(m, v)$ obtained from Equation (8) we can rewrite Equation (3) in the form:

$$
\left|\frac{D(m, v) v^{\prime}}{m v^{\prime}+c}\right| \leq \chi_{\max } g
$$

We also assume that

$$
v \geq 0, \quad v \leq v_{\max },
$$

where $v_{\max }$ is the maximum velocity of the impactor.

The problem is to determine the function $v(m)$ that provides the maximum DOP, $H$, in Equation (6), taking into account the constraints given by Equations (7), (9), (10) and (11). Function $D(m, v)$ and the parameters $\mu_{\max }, \chi_{\max }, v_{\max }, m_{\mathrm{imp}}, m_{\mathrm{res}}$, and $v_{\text {imp }}$ are assumed to be given.

Inequalities in Equation (9) imply two situations: the possibility of the vertical subarcs, $m=\mathrm{const}$ when $v^{\prime}=\infty$, and the restrictions $m v^{\prime}+c \geq D(m, v) / \mu_{\max }$ and $m v^{\prime}+c \geq 0$ when $v^{\prime}<\infty$. Clearly, the latter inequality can be omitted and we can write this constraint as follows:

$$
m v^{\prime}+c \geq D(m, v) / \mu_{\max }, \quad \text { if } v^{\prime}<\infty .
$$

For $m=$ const $\left(v^{\prime}=\infty\right)$, the constraint in Equation (10) can be simplified:

$$
D(m, v) / m \leq \chi_{\max } g, \quad \text { if } m=\text { const } .
$$




\section{Generalized Young's penetration model}

Numerous simplified models were proposed for high speed penetration into different media. An overview of these models may be found, for instance, in the recent monograph [Ben-Dor et al. 2006] and the review [Ben-Dor et al. 2005]. In this study are employed the well known Young's penetration equations [Young 1997] which determine the DOP into soil, rock, and concrete (SRC) shields as well as into ice and frozen soil (IFS) in the following unified form:

$$
\frac{P\left(v_{\mathrm{imp}}\right)}{k}= \begin{cases}\tilde{P}_{a}\left(v_{\mathrm{imp}}\right) & \text { if } v_{\mathrm{imp}}<\tilde{v}_{*} \\ \tilde{P}_{b}\left(v_{\mathrm{imp}}\right) & \text { if } v_{\mathrm{imp}} \geq \tilde{v}_{*},\end{cases}
$$

where

$$
\begin{aligned}
& \tilde{P}_{a}\left(v_{\mathrm{imp}}\right)=\tilde{\alpha}_{1} \ln \left(1+\tilde{\alpha}_{2} v_{\mathrm{imp}}^{2}\right), \quad \tilde{P}_{b}\left(v_{\mathrm{imp}}\right)=k_{1}\left(v_{\mathrm{imp}}-v_{0}\right), \\
& k=k_{0} \theta(m), \quad k_{0}=\tilde{S} \tilde{N} / \tilde{A}^{\kappa_{1}}, \\
& \theta(m)= \begin{cases}\sigma m^{\kappa_{1}+\kappa_{2}} & \text { if SRC } \\
m^{\kappa_{1}} \ln \left(50+0.29 m^{2}\right) & \text { if IFS, }\end{cases}
\end{aligned}
$$

$P$ is the DOP, $\tilde{S}$ is a coefficient depending on the mechanical properties of the shield, $\tilde{N}$ depends on the shape of the impactor, $\tilde{A}$ is the cross sectional area of the impactor, $v_{0}=30.5, \tilde{\alpha}_{2}=0.000215$, $\tilde{v}_{*}=2 v_{0}=61$, other coefficients are presented in Table 1. It is assumed that the mass of the projectile is constant. The parameters of the model are chosen such that the variables are measured in SI units [Young 1997]. Unfortunately, this widely used model is inconvenient in theoretical analysis because it employs the dimensional coefficients and because it is described by a discontinuous function.

Indeed, Young's dependence in the range between $P / k=\hat{P}$ and $v_{\text {imp }}$ for SRC is described by a function that is discontinuous at $v_{\text {imp }}=\tilde{v}_{*}, \tilde{P}_{a}\left(\tilde{v}_{*}\right)=0.000470, P_{b}\left(\tilde{v}_{*}\right)=0.000549$. Inaccuracy in calculation is a plausible reason for this discontinuity, and can be corrected by choosing $\tilde{\alpha}_{1}$ such that $\tilde{P}_{a}\left(\tilde{v}_{*}\right)=\tilde{P}_{b}\left(\tilde{v}_{*}\right)$. Then we obtain that

$$
\tilde{\alpha}_{1} / k_{1}=v_{0} / \ln \left(1+\tilde{\alpha}_{2} v_{*}^{2}\right)=51.89 .
$$

The corrected values $\tilde{\alpha}_{1}$ that enforce the continuity of $\hat{P}\left(v_{\text {imp }}\right)$ are presented in Table 1 . However, in our study we need a more smooth approximation of the function $\hat{P}\left(v_{\text {imp }}\right)$, namely, it must have the

\begin{tabular}{|c|ccccccc|}
\hline Shield's material & $m$ & $\kappa_{1}$ & $\kappa_{2}$ & $\sigma$ & $\tilde{\alpha}_{1}$ & $k_{1}$ & $\tilde{\alpha}_{1}$, corrected \\
\hline \multirow{2}{*}{ Soil } & $2 \leq m<27$ & 0.7 & 0.4 & 0.27 & 0.0008 & 0.000018 & 0.0009340 \\
& $m \geq 27$ & 0.7 & 0 & 1 & 0.0008 & 0.000018 & 0.0009340 \\
\hline \multirow{2}{*}{ Rock, Concrete } & $5 \leq m<182$ & 0.7 & 0.15 & 0.46 & 0.0008 & 0.000018 & 0.0009340 \\
& $m \geq 182$ & 0.7 & 0 & 1 & 0.0008 & 0.000018 & 0.0009340 \\
\hline Ice, Frozen Soil & & 0.6 & & & 0.00024 & 0.0000046 & 0.0002387 \\
\hline
\end{tabular}

Table 1. Parameters of the models. 
continuous second derivative for all $v_{\text {imp }}>0$. Toward this end, we modify the model for the relatively small $v_{\text {imp }}$ keeping the Young's approximation for large $v_{\text {imp. }}$. We look for this approximation of the function $P\left(v_{\text {imp }}\right)$ in the following form:

$$
\frac{P\left(v_{\mathrm{imp}}\right)}{k}= \begin{cases}P_{a}\left(v_{\mathrm{imp}}\right) & \text { if } v_{\mathrm{imp}}<v_{*} \\ \tilde{P}_{b}\left(v_{\mathrm{imp}}\right) & \text { if } v_{\mathrm{imp}} \geq v_{*}\end{cases}
$$

where

$$
\begin{aligned}
P_{a}\left(v_{\mathrm{imp}}\right) & =\left(\alpha_{2} v_{\mathrm{imp}}^{2}+\alpha_{1} v_{\mathrm{imp}}+\alpha_{0}\right) v_{\mathrm{imp}}^{2}, \quad v_{\mathrm{imp}} \leq v_{*} \\
v_{*} & =s v_{0}, \quad s \geq 2 .
\end{aligned}
$$

The constraint in Equation (21) follows from the fact that the function $\tilde{P}_{b}\left(v_{\text {imp }}\right)$ is defined only for $v_{\text {imp }} \geq \tilde{v}_{*}=2 v_{0}$.

The problem is to find such $\alpha_{0}, \alpha_{1}, \alpha_{2}$, and $v_{*}$ (or $s$ ) that

$$
P_{a}\left(v_{*}\right)=\tilde{P}_{b}\left(v_{*}\right), \quad P_{a}^{\prime}\left(v_{*}\right)=\tilde{P}_{b}^{\prime}\left(v_{*}\right), \quad P_{a}^{\prime \prime}\left(v_{*}\right)=\tilde{P}_{b}^{\prime \prime}\left(v_{*}\right), \quad v_{*}=s v_{0},
$$

where

$$
\begin{array}{ll}
P_{a}^{\prime}\left(v_{\mathrm{imp}}\right)=4 \alpha_{2} v_{\mathrm{imp}}^{3}+3 \alpha_{1} v_{\mathrm{imp}}^{2}+2 \alpha_{0} v_{\mathrm{imp}}, & \tilde{P}_{b}^{\prime}\left(v_{\mathrm{imp}}\right)=k_{1}, \\
P_{a}^{\prime \prime}\left(v_{\mathrm{imp}}\right)=12 \alpha_{2} v_{\mathrm{imp}}^{2}+6 \alpha_{1} v_{\mathrm{imp}}+2 \alpha_{0}, & \tilde{P}_{b}^{\prime \prime}\left(v_{\mathrm{imp}}\right)=0 .
\end{array}
$$

In addition, by physical reasoning, $P_{a}$ must be an increasing function.

Equation (22) yields a system of linear equations with respect to $\alpha_{0}, \alpha_{1}$, and $\alpha_{2}$ which has the following solution:

$$
\alpha_{0}=\frac{3 k_{1}(s-2)}{s^{2} v_{0}}, \quad \alpha_{1}=\frac{k_{1}(8-3 s)}{s^{3} v_{0}^{2}}, \quad \alpha_{2}=\frac{k_{1}(s-3)}{s^{4} v_{0}^{3}} .
$$

Let us prove that for any $s \geq 2$, the function $P_{a}\left(v_{\text {imp }}\right)$ increases when $0<v_{\text {imp }} \leq s v_{0}$. This occurs if $P_{a}^{\prime}\left(v_{\text {imp }}\right)>0$ in the same range of $v_{\text {imp }}$, or if $\omega(V)>0$ for $0<V \leq 1$, where

$$
\omega(V)=\frac{1}{k_{1} v_{0} V} P_{a}^{\prime}\left(s v_{0} V\right)=4(s-3) V^{2}+3(8-3 s) V+6(s-2), \quad V=\frac{v_{\text {imp }}}{s v_{0}} .
$$

Let $2 \leq s<3$. Since $\omega(0) \geq 0$ and $\omega(1)=s>0$, the convex function $\omega(V)>0$ for $0<V \leq 1$.

The discriminant of the quadratic equation $\omega(V)=0$ is $\Delta(s)=3 s(16-5 s)$. Since $\Delta<0$ and $s-3>0$ when $s>16 / 5$, then $\omega(V)>0$ for $s>16 / 5=3.2$.

Let $3 \leq s<3.2$. Then there are two different roots of the equation $\omega(V)=0, V_{1}$ and $V_{2}$. The Viet formula implies that both the roots are positive. The smaller root, $V_{1}$, is

$$
V_{1}(s)=[3(3 s-8)-\sqrt{\Delta(s)}] /[8(s-3)] .
$$

It is easy to prove that $V_{1}>1$ if $3 \leq s<3.2$. Since $\omega(0)>0$ and all the roots are larger than $1, \omega(V)>0$ for $0<V \leq 1$.

In order to complete the analysis, let us consider $\omega(V)$ for two remaining values $s$. If $s=3$ then $\omega(V)=3(2-V)>0$, and if $s=3.2$ then $\omega(V)=0.8(V-3)^{2}>0$, for $0<V \leq 1$.

Thus we proved that $P_{a}\left(v_{\text {imp }}\right)$ is an increasing function for $0<v_{\text {imp }} \leq s v_{0}$ when $s \geq 2$. 
Smooth approximation of the function $P=P\left(v_{\text {imp }}\right)$ in Equation (19) allows one to determine the dependence of the drag force, $D$, on the instantaneous velocity, $v$, that yields the following correlation for motion of the impactor with a constant mass $m$ :

$$
D=\frac{m}{k} \times \begin{cases}D_{a} & \text { if } v<v_{*}, \\ D_{b} & \text { if } v \geq v_{*},\end{cases}
$$

where

$$
\begin{aligned}
& D_{a}(v)=v / P_{a}^{\prime}(v)=\left(4 \alpha_{2} v^{2}+3 \alpha_{1} v+2 \alpha_{0}\right)^{-1}, \\
& D_{b}(v)=v / \tilde{P}_{b}^{\prime}(v)=v / k_{1} .
\end{aligned}
$$

The validity of this statement can be verified directly by determining the DOP from the equation of motion of the impactor, $m v d v / d h=-k D$. Equation (22) implies that the function $D$ has a continuous derivative with respect to $v$. Note that a similar approach was used in [Eisler et al. 1998] for the case in which the dependence of the DOP on $v_{\text {imp }}$ is determined by one analytical expression.

By physical reasoning, $D_{a}(v)$ must be a positive increasing function for $0<v_{\text {imp }} \leq s v_{0}$. Since $v / P_{a}^{\prime}(v)=1 / D_{a}(v)=\left(s / k_{1}\right) \omega(V)$, where $V=v /\left(s v_{0}\right)$, it is sufficient to show that $\omega(0)>0, \omega(1)>0$ and $\omega^{\prime}(V)=8(s-3) V+3(8-3 s)<0$ for $0<V \leq 1$. The first and the second inequalities are valid for $s>2$ because $\omega(0)=6(s-2)>0$ and $\omega(1)=s>0$. Since $\omega^{\prime}(V)$ is a linear function, the third inequality is equivalent to the conditions $\omega^{\prime}(0)=3(8-3 s) \leq 0, \omega^{\prime}(1)=-s \leq 0$ and $\omega^{\prime}(0)+\omega^{\prime}(1) \neq 0$. Consequently, $s \geq 8 / 3$ are the permissible values for $s$.

In order to obtain the most convenient approximation we set $s=8 / 3$ when $\alpha_{1}=0$. Then

$$
D=\frac{m}{k k_{1}} \times \begin{cases}1 /\left(\gamma_{0}-\gamma_{2} v^{2}\right) & \text { if } v<v_{*}, \\ v & \text { if } v \geq v_{*},\end{cases}
$$

where

$$
\begin{aligned}
& \gamma_{0}=\frac{2 \alpha_{0}}{k_{1}}=\frac{6(s-2)}{s^{2} v_{0}}=0.01844, \\
& \gamma_{2}=-\frac{4 \alpha_{2}}{k_{1}}=\frac{4(3-s)}{s^{4} v_{0}^{3}}=0.929318 \cdot 10^{-6}, \\
& v_{*}=s v_{0}=81.33 .
\end{aligned}
$$

The modified Young's approximation and the proposed approximation are compared in Figure 1. Inspection of Figure 1 shows that the difference between them is negligibly small.

We assume that Equation (28) for the drag force remains valid for motion of the penetrator with a variable mass.

We assume also that the mass of the impactor does not exceed $m \geq 27 \mathrm{~kg}$ or $m \geq 182 \mathrm{~kg}$ in the case of penetration into soil or rock and concrete, correspondingly. This restriction can be relaxed using a smoother approximation of the function $\theta(m)$.

\section{Analytical investigation of the limiting case}

In order to estimate the upper bound for increasing the DOP by using the jet thruster, let us consider the problem with the minimum number of constraints. In this version of the statement of the problem, 


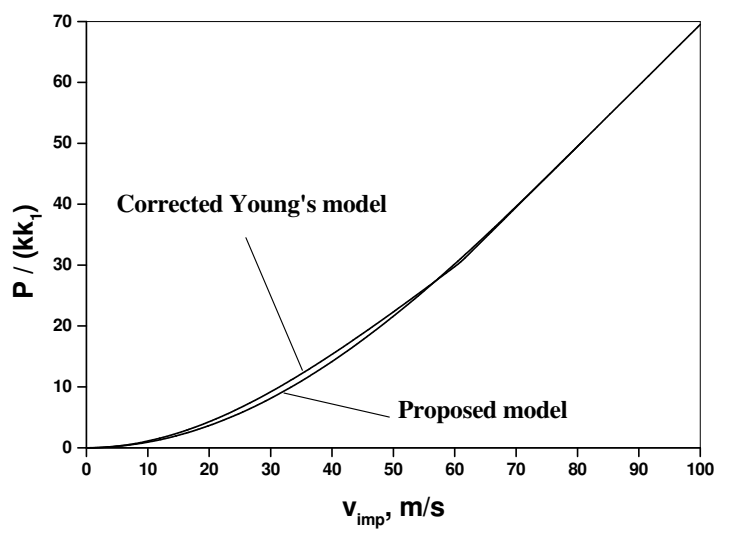

Figure 1. Comparison of the corrected Young's model and the proposed smooth model.

Equation (6) remains valid, and the constraints given by Equation (7), the first equation in Equation (11), and Equation (12) with $\mu_{\max }=\infty$, that is,

$$
m v^{\prime}+c \geq 0
$$

are taken into account.

We consider first penetration into nonfrozen soil, rock, or concrete. Then the formula for $\theta(m)$ in Equation (17) reads:

$$
\theta(m)=\sigma m^{\kappa}, \quad \kappa=\kappa_{1}+\kappa_{2},
$$

where the values $\kappa_{1}, \kappa_{2}$, and $\sigma$ are presented in Table 1 . We assume that $m$ varies in the range where $\sigma$ is constant.

Using Equations (16) and (31), Equation (28) can be written as follows:

$$
D=\frac{m^{1-\kappa}}{\sigma k_{0} k_{1}} \times \begin{cases}1 /\left(\gamma_{0}-\gamma_{2} v^{2}\right) & \text { if } v<v_{*}, \\ v & \text { if } v \geq v_{*} .\end{cases}
$$

Using the results of [Cicala and Miele 1956] (see also [Miele 1962]) the optimum curve must consist of the following subarcs:

subarc 1: $\Omega(m, v)=0$, where

$$
\Omega(m, v)=(v-c) D(m, v)+v(c d D / d v-m d D / d m),
$$

subarc 2: the subarc $m=$ const, and

subarc 3: the subarc $\dot{m}=-\mu_{\max }$ where $\dot{m}=d m / d t$.

Subarc 1 represents the solution of the Euler-Lagrange equation; subarc 3 is described by Equation (30) after replacing $\geq$ by $=$.

The above subarcs can be joined in the following sequences: 
(a) if $\Omega>0$ in some point then the only sequence $m=$ const $\Rightarrow \dot{m}=-\mu_{\max }$ is possible in this point;

(b) if $\Omega<0$ then the inverse sequence $\dot{m}=-\mu_{\max } \Rightarrow m=$ const is permissible;

(c) if $\Omega=0$ then the joints $\Omega=0 \Leftrightarrow \bar{m}=$ const and $\Omega=0 \Leftrightarrow \dot{m}=-\mu_{\max }$ are possible.

The arrows indicate the direction of the motion of the penetrator along the subarcs on the $m, v$ plane. Substituting $D$ from Equation (32) into Equation (33), we obtain:

$$
\Omega=\frac{\kappa m^{1-\kappa}}{\sigma k_{0} k_{1}} \times \begin{cases}-\gamma_{2}\left(\gamma_{0}-\gamma_{2} v^{2}\right)^{-2} \Omega_{a}(v) & \text { if } v<v_{*}, \\ \Omega_{b}(v) & \text { if } v \geq v_{*},\end{cases}
$$

where

$$
\Omega_{a}(v)=v^{3}-3 \hat{c} v^{2}-\hat{\gamma}_{0} v+\hat{\gamma}_{0} \hat{c},
$$

with $\Omega_{b}(v)=v^{2}, \hat{c}=c / \kappa$, and $\hat{\gamma}_{0}=\gamma_{0} / \gamma_{2}$. Since

$$
\begin{aligned}
\Omega_{a}(-\infty) & <0, & \Omega_{a}(0) & >0, \\
\Omega_{a}\left(v_{*}\right) & =-\Omega_{b}\left(v_{*}\right)\left(\gamma_{0}-\gamma_{2} v^{2}\right)^{2} / \gamma_{2}<0, & \Omega_{a}(+\infty) & >0,
\end{aligned}
$$

the cubic equation $\Omega_{a}(v)=0$ has 3 real roots, but only one root, $v_{\times}$, is located between 0 and $v_{*}$ :

$$
v_{\times}=\hat{c}+2\left(\frac{\hat{\gamma}_{0}+3 \hat{c}^{2}}{3}\right)^{1 / 2} \cos \left(\frac{\zeta}{3}+\frac{4 \pi}{3}\right), \quad \zeta=\cos ^{-1}\left(\frac{3 \hat{c}^{2}}{\hat{\gamma}_{0}+3 \hat{c}^{2}}\right)^{3 / 2} .
$$

The dependence $v_{\times}$versus $\hat{c}=c / \kappa$ is shown in Figure 2 .

Taking into account this result, and that $\Omega_{b}(v)>0$ for $v \geq v_{*}$, we conclude that $\Omega(v)<0$ when $0 \leq v<v_{\times}, \Omega\left(v_{\times}\right)=0$, and $\Omega(v)>0$ when $v>v_{\times}$. Therefore, the conditions $\Omega(v)<0, \Omega(v)=0$, and $\Omega(v)>0$ correspond to the conditions $\bar{v}<\bar{v}_{\times}, \bar{v}=\bar{v}_{\times}$, and $\bar{v}>\bar{v}_{\times}$, respectively.

The equation of the arc 1 reads:

$$
v=v_{\times}
$$

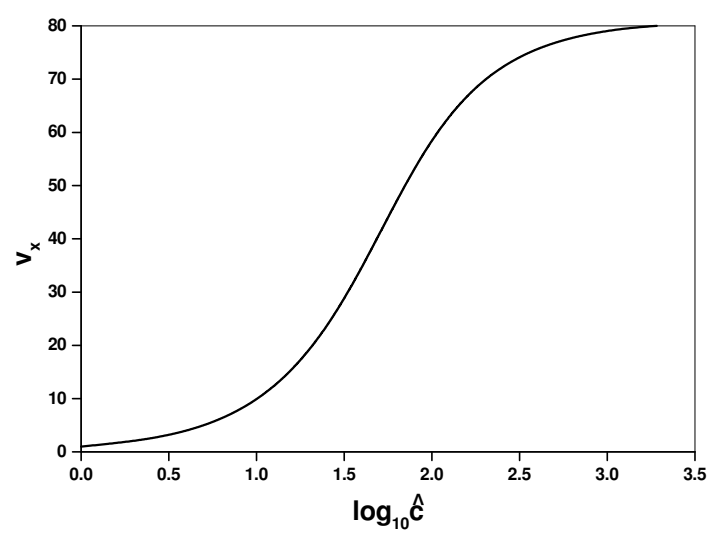

Figure 2. Solution of the equation $\Omega(v)=0$ as a function of the parameter $\hat{c}=c \kappa$. 
Consider a point moving along the curve $v=v_{\times}$, from the location with coordinates $\left(m_{b}, v_{\times}\right)$to the

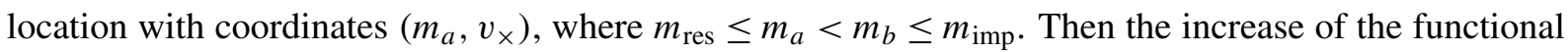
given by Equation (6) with $D$ determined by Equation (32) along this path reads:

$$
\Delta H /\left(k_{0} k_{1}\right)=\Gamma_{m}\left(m_{a}, m_{b}\right)=c v_{\times}\left(\gamma_{0}-\gamma_{2} v_{\times}^{2}\right) \int_{m_{a}}^{m_{b}} \frac{d m}{m^{1-\kappa}}=c \hat{v}_{\times}\left(\gamma_{0}-\gamma_{2} v_{\times}^{2}\right)\left(m_{b}^{\kappa}-m_{a}^{\kappa}\right) .
$$

Along the line $v=v_{\times}<v_{*}$, Equation (1) reads:

$$
c \frac{d m}{d t}=-\frac{m^{1-\kappa}}{k_{0} k_{1}\left(\gamma_{0}-\gamma_{2} v_{\times}^{2}\right)} .
$$

The solution to Equation (40) with the initial conditions $m\left(t_{b}\right)=m_{b}$ is as follows:

$$
m=\left[m_{b}^{\kappa}-\tau\left(t-t_{b}\right)\right]^{1 / \kappa}, \quad \tau=\frac{\kappa}{c k_{0} k_{1}\left(\gamma_{0}-\gamma_{2} v_{\times}^{2}\right)} .
$$

Increase of the mass of the penetrator from $m_{b}$ to $m_{a}$ occurs up to the time $t=t_{a}$ where $t_{a}=t_{b}+$ $\left(m_{b}^{\kappa}-m_{a}^{\kappa}\right) / \tau$.

Subarc 2 corresponds to the motion with a constant mass. Assume that the velocity of the impactor decreases from $v=v_{b}$ to $v=v_{a}$ while its mass remains constant, $m=m_{0}$. Then the increase of the functional given by Equation (6) is as follows:

$$
\begin{aligned}
\Delta H /\left(k_{0} k_{1}\right) & =\Gamma_{v}^{(1)}\left(m_{0}, v_{a}, v_{b}\right) \\
& =m_{0}^{\kappa} \int_{v_{a}}^{v_{b}} v\left(\gamma_{0}-\gamma_{2} v^{2}\right) d v \\
& =0.25 m_{0}^{\kappa}\left(v_{b}^{2}-v_{a}^{2}\right)\left[2 \gamma_{0}-\gamma_{2}\left(v_{b}^{2}+v_{a}^{2}\right)\right] \quad \text { if } v_{b} \leq v_{\times},
\end{aligned}
$$

and

$$
\Delta H /\left(k_{0} k_{1}\right)=\Gamma_{v}^{(2)}\left(m_{0}, v_{a}, v_{b}\right)=m_{0}^{\kappa} \int_{v_{a}}^{v_{b}} d v=m_{0}^{\kappa}\left(v_{b}-v_{a}\right) \quad \text { if } v_{b} \geq v_{\times} .
$$

Along subarc $3, \dot{m}=-\mu_{\max }=-\infty$. The equation of this subarc in the coordinates $(m, v)$ is determined by Equation (30) after replacing $\geq$ by $=$ :

$$
m v+c=0
$$

The solution of this ODE with separable variables passing through the point $\left(m_{\mathrm{imp}}, v_{0}\right)$ can be written as follows:

$$
v=v_{0}-c \ln \left(m / m_{\text {imp }}\right) .
$$

This trajectory corresponds to the step change of the mass of the penetrator (the pulse burning). This change of the mass and of the velocity of the penetrator occurs without change of its location.

Analysis of the conditions presented at the beginning of this section shows that three versions of burning program (BP) are possible, depending on the values of the parameters $\hat{c}$ and $v_{\text {imp }}$. 

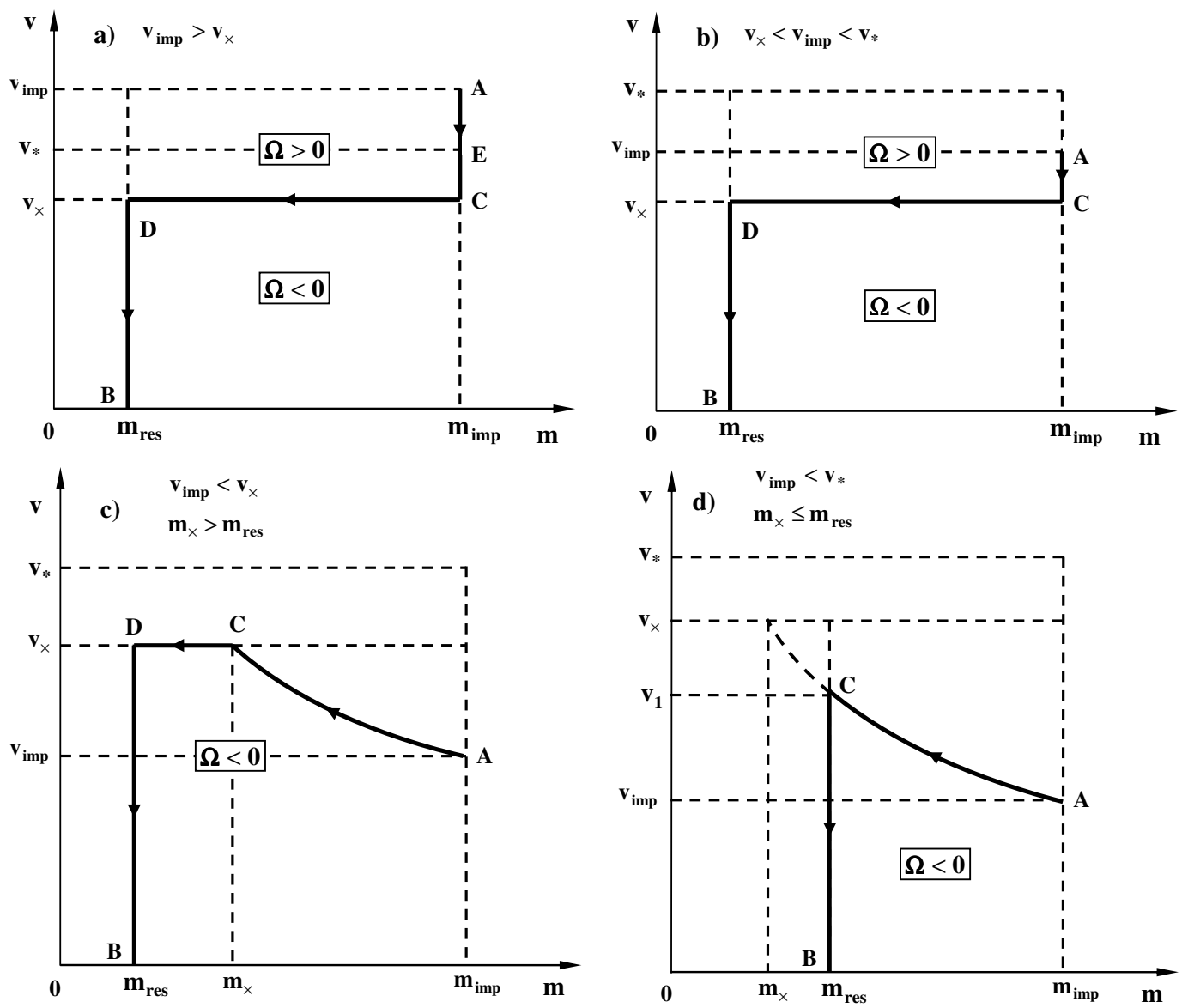

Figure 3. Stages of optimal burning programs for penetration into nonfrozen soil, rock, and concrete shields, $\bar{\beta}_{\min }=0$.

Consider the case when $v_{\text {imp }}>v_{*}$. Taking into account that $v_{*}>v_{\times}$, the optimal BP includes the following subarcs (Figure 3a) in the coordinates $(m, v)$ :

$$
\begin{cases}A E: m=m_{\mathrm{imp}}, & v: v_{\mathrm{imp}} \rightarrow v_{*}, \\ E C: m=m_{\mathrm{imp}}, & v: v_{*} \rightarrow v_{\times}, \\ C D: v=v_{\times}, & m: m_{\mathrm{imp}} \rightarrow m_{\mathrm{res}}, \\ D B: m=m_{\mathrm{res}}, & v: v_{\times} \rightarrow 0 .\end{cases}
$$

The corresponding expression for the DOP reads:

$$
H /\left(k_{0} k_{1} m_{\mathrm{imp}}^{\kappa}\right)=\Gamma_{v}^{(2)}\left(1, v_{*}, v_{\mathrm{imp}}\right)+\Gamma_{v}^{(1)}\left(1, v_{\times}, v_{*}\right)+\Gamma_{m}\left(\bar{m}_{\mathrm{res}}, 1\right)+\Gamma_{v}^{(1)}\left(\bar{m}_{\mathrm{res}}, 0, v_{\times}\right),
$$

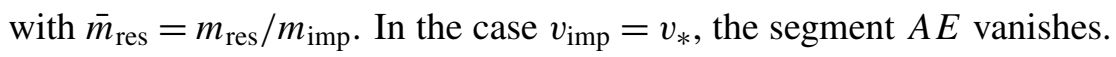


If $v_{\times}<v_{\text {imp }}<v_{*}$, then the optimal BP includes the subarcs (Figure $3 \mathrm{~b}$ ):

$$
\begin{cases}A C: m=m_{\mathrm{imp}}, & v: v_{\mathrm{imp}} \rightarrow v_{\times} \\ C D: v=v_{\times}, & m: m_{\mathrm{imp}} \rightarrow m_{\mathrm{res}}, \\ D B: m=m_{\mathrm{res}}, & v: v_{\times} \rightarrow 0\end{cases}
$$

and the DOP is as follows:

$$
H /\left(k_{0} k_{1} m_{\mathrm{imp}}^{\kappa}\right)=\Gamma_{v}^{(1)}\left(1, v_{\times}, v_{\mathrm{imp}}\right)+\Gamma_{m}\left(\bar{m}_{\mathrm{res}}, 1\right)+\Gamma_{v}^{(1)}\left(\bar{m}_{\mathrm{res}}, 0, v_{\times}\right) .
$$

In the case $v_{\text {imp }}=v_{\times}$, the segment $A C$ vanishes.

Motion of a penetrator which is described by Equations (46) and (48) includes (in a general case) the following stages: passive penetration when a jet thruster is turned off until the velocity slows down to the magnitude $v_{\times}$(segment $A C$ of the trajectory), motion with a constant velocity $v_{\times}$with an operating jet thruster until complete exhaustion of fuel supply (segment $C D$ of the trajectory), and passive penetration until a penetrator slows down to zero velocity (segment $D B$ of the trajectory).

Consider now the case when $v_{\text {imp }}<v_{\times}$and the curve determined by Equation (45) with $v_{0}=v_{\text {imp }}$ intersects with the line $v=v_{\times}$in the point $\left(m_{\times}, v_{\times}\right)$, where $m_{\times}>m_{\text {res }}$ (Figure $3 \mathrm{c}$ ). Then the optimal BP includes three subarcs:

$$
\begin{cases}A C: v=v_{0}-c \ln \left(m / m_{\mathrm{res}}\right), & m: m_{\mathrm{imp}} \rightarrow m_{\times}, \\ C D: v=v_{\times}, & m: m_{\times} \rightarrow m_{\mathrm{res}}, \\ D B: m=m_{\mathrm{res}}, & v: v_{\times} \rightarrow 0,\end{cases}
$$

where $m_{\times}=m_{\text {imp }} \exp \left(\left(1-v_{\times}\right) / c\right)$.

Equation (50) describes the following stages of the controlled motion of a penetrator: operation of a jet thruster in the impulse regime whereby the penetrator's velocity instantaneously changes from the initial value, $v_{\text {imp }}$, to $v_{\times}$by spending a mass of fuel equal to $m_{\text {imp }}-m_{\times}$without the motion of a penetrator (subarc $A C$ ); operation of a jet thruster for providing a constant velocity to the penetrator, $v_{\times}$, until exhaustion of the fuel supply (segment $C D$ ); and passive penetration until a penetrator slows down to zero velocity (segment $D B$ of the trajectory).

The corresponding expression for the DOP is as follows:

$$
H /\left(k_{0} k_{1} m_{\mathrm{imp}}^{\kappa}\right)=\Gamma_{m}\left(\bar{m}_{\mathrm{res}}, \bar{m}_{\times}\right)+\Gamma_{v}^{(1)}\left(\bar{m}_{\mathrm{res}}, 0, v_{\times}\right),
$$

with $\bar{m}_{\times}=m_{\times} / m_{\mathrm{imp}}$.

If $v_{\text {imp }}<v_{\times}$and $m_{\times} \leq m_{\text {res }}$ then the optimal BP includes two subarcs (Figure $3 \mathrm{~d}$ ):

$$
\begin{cases}A C: v=v_{0}-c \ln \left(m / m_{\mathrm{res}}\right), & m: m_{\mathrm{imp}} \rightarrow m_{\mathrm{res}}, \\ C B: m=m_{\mathrm{res}}, & v: v_{1} \rightarrow 0,\end{cases}
$$

where $v_{1}=v_{\text {imp }}-c \ln \bar{m}_{\text {res }}$.

Here, a jet thruster operates in the impulse regime until exhausting the whole fuel supply. In this case the velocity of the penetrator instantaneously changes from the initial velocity to $v_{1}$ (segment $A C$ of the trajectory). Afterwards, this penetrator continues its motion until it slows down to zero velocity (segment $C B$ of the trajectory). 

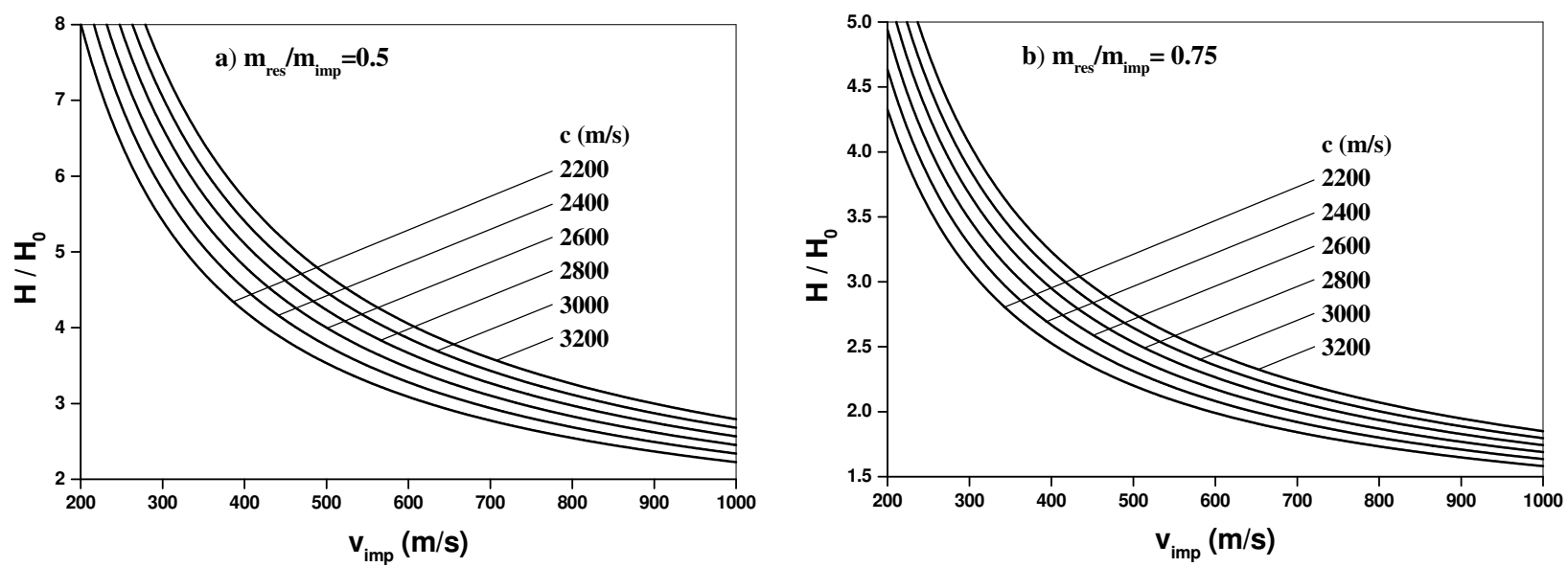

Figure 4. Normalized optimal DOP into nonfrozen soil, rock, and concrete shields versus impact velocity; $H$ is DOP for the optimal burning program, $H_{0}$ is DOP for a passive trajectory, $\bar{\beta}_{\min }=0, \kappa=0.7$.

The DOP is determined using only the subarc $C B$ :

$$
H /\left(k_{0} k_{1} m_{\mathrm{imp}}^{\kappa}\right)=\Gamma_{v}^{(1)}\left(\bar{m}_{\mathrm{res}}, 0, v_{1}\right) .
$$

The depth of passive penetration of the impactor with the mass $m_{\mathrm{imp}}$ is as follows:

$$
\frac{H_{0}}{k_{0} k_{1} m_{\text {imp }}^{K}}= \begin{cases}\Gamma_{v}^{(1)}\left(1,0, v_{\text {imp }}\right) & \text { if } v_{\text {imp }} \leq v_{*}, \\ \Gamma_{v}^{(2)}\left(1, v_{*}, v_{\text {imp }}\right)+\Gamma_{v}^{(1)}\left(1,0, v_{*}\right), & \text { if } v_{\text {imp }}>v_{*} .\end{cases}
$$

Some of the above relationships can be simplified by taking into account that $v_{\times} \approx v_{*}$ for $c>2000 \mathrm{~m} / \mathrm{s}$ (Figure 2).

We use the ratio

$$
\eta=H / H_{0},
$$

which depends on $v_{\text {imp }}, c, \bar{m}_{\text {res }}$, and $\kappa$, for comparing the DOP with the optimal BP and the depth of passive penetration (Figure 4). Although $\eta$ can attain very large values for relatively small impact velocities, the absolute values of the DOP, $H$, are reasonable. The results for the relatively high impact velocities are the most interesting because of the feasibility for increasing the absolute DOP by using the jet thruster.

The optimum BP for the impact velocities, $v_{\text {imp }}>v_{*} \approx 81 \mathrm{~m} / \mathrm{s}$, is simple: passive penetration up to velocity $v_{\times}$, motion with constant velocity $v_{\times}$while the propellant is available (the consumption of the propellant is described by Equation (41)) with $m_{a}=m_{\mathrm{res}}$ and $\left.m_{b}=m_{\mathrm{imp}}, m=\left[m_{\mathrm{res}}^{\kappa}-\tau\left(t-t_{\mathrm{res}}\right)\right]^{1 / \kappa}\right)$, and passive penetration until rest. This BP is valid if the constraints on mass fluxes and penetrator's acceleration are not taken into account. Let us determine the conditions under which these constraints do not affect this optimum BP.

The constraints given by Equation (3) are relevant only on the path of the trajectory where $v=v_{\times}$. Equation (1) implies that $d m / d t=-D\left(m, v_{\times}\right) / c=-(\tau / \kappa) m^{1-\kappa}$. Substituting this relationship into 


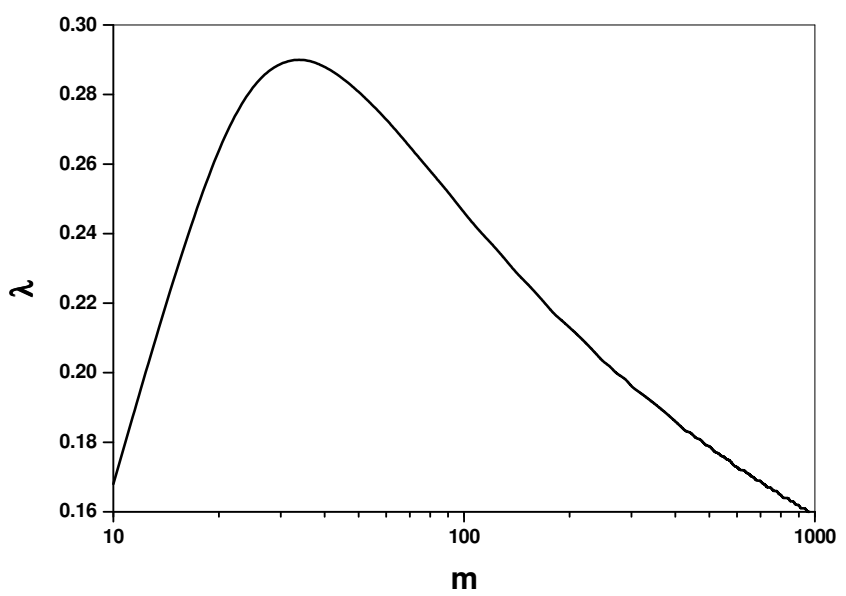

Figure 5. Dependence $\lambda=\lambda(m)$ in the model of penetration into ice and frozen soil.

Equation (3) we obtain the inequality $m \leq\left(\kappa \mu_{\max } / \tau\right)^{1 /(1-\kappa)}$ that must be satisfied for $m_{\text {res }} \leq m \leq m_{\text {imp }}$. Since the mass decreases on this interval, the above inequality can be substituted by the following:

$$
m_{\text {imp }} \leq\left(\kappa \mu_{\max } / \tau\right)^{1 /(1-\kappa)} .
$$

The constraints given by Equation (3) are relevant only on the segment of the trajectory with a constant mass, $m=\tilde{m}$. Equation (1) implies that $d v / d t=-D(\tilde{m}, v) / \tilde{m}$. Substituting this relationship into Equation (3) we obtain:

$$
\begin{array}{cl}
D\left(m_{\mathrm{imp}}, v\right) \leq m_{\mathrm{imp}} g \chi_{\max } & \text { if } v_{\times} \leq v \leq v_{\mathrm{imp}}, \\
D\left(m_{\mathrm{res}}, v\right) \leq m_{\mathrm{res}} g \chi_{\max } & \text { if } 0 \leq v \leq v_{\times} .
\end{array}
$$

Since $D$ is an increasing function of $v$, these constraints are valid if

$$
D\left(m_{\text {imp }}, v_{\text {imp }}\right) \leq m_{\text {imp }} g \chi_{\max } \quad \text { and } \quad D\left(m_{\text {res }}, v_{\times}\right) \leq m_{\text {res }} g \chi_{\max } .
$$

The two latter inequalities imply that

$$
m_{\mathrm{imp}} \geq \frac{v_{\text {imp }}}{k_{0} k_{1} g \chi_{\max }}, \quad m_{\text {res }} \geq \frac{1}{k_{0} k_{1}\left(\gamma_{0}-\gamma_{2} v_{\times}^{2}\right) g \chi_{\max }} .
$$

Let us consider the case of penetration into ice and frozen soil shields. Then

$$
\theta(m)=m^{0.6} \ln \left(50+0.29 m^{2}\right)
$$

and

$$
D=\frac{m^{0.4}}{k_{0} k_{1} \ln \left(50+0.29 m^{2}\right)} \times \begin{cases}1 /\left(\gamma_{0}-\gamma_{2} v^{2}\right) & \text { if } v<v_{*} \\ v & \text { if } v \geq v_{*}\end{cases}
$$


Substituting $D$ from this equation into Equation (33) we obtain:

$$
\Omega=\frac{m^{0.4}[0.6+\lambda(m)]}{k_{0} k_{1} \ln \left(50+0.29 m^{2}\right)} \times \begin{cases}-\gamma_{2}\left(\gamma_{0}-\gamma_{2} v^{2}\right)^{-2} \Omega_{a}(v) & \text { if } v<v_{*} \\ \Omega_{b}(v) & \text { if } v \geq v_{*}\end{cases}
$$

where

$$
\begin{array}{rlrl}
\Omega_{a}(m, v)=v^{3}-3 \hat{c} v^{2} & -\hat{\gamma}_{0} v+\hat{\gamma_{0}} \hat{c}, \\
\Omega_{b}(v) & =v^{2}, \quad \hat{\gamma}_{0} & =\gamma_{0} / \gamma_{2}, \\
\hat{c}=\frac{c}{0.6+\lambda(m)}, & \lambda(m) & =\frac{0.58 m^{2}}{\left(50+0.29 m^{2}\right) \ln \left(50+0.29 m^{2}\right)} .
\end{array}
$$

The equation $\Omega(m, v)=0$ determines the subarc $v=v_{\times}(m)$ where $v_{\times}(m)$ is determined by Equation (37) with $\hat{c}=\hat{c}(m)$ from Equation (60). In contrast to the case of SRC shields, the subarc is curvilinear in this case.

Let us investigate now the behavior of the function $\lambda(m)$. Changing the variable $50+0.29 m^{2}=x$ we reduce the problem to analyzing the function

$$
\Theta(x)=\lambda\left(\sqrt{\frac{x-50}{0.29}}\right)=\frac{2(x-50)}{x \ln x} .
$$

Let us calculate the derivative:

$$
\begin{aligned}
\Theta^{\prime}(x) & =\frac{2}{x \ln x} \Theta_{0}(x), \\
\Theta_{0}(x) & =50(\ln x+1)-x, \\
\Theta_{0}^{\prime}(x) & =\frac{50}{x}-1 .
\end{aligned}
$$

For $x>50, \Theta_{0}^{\prime}(x)<0$. Hence, $\Theta_{0}(x)$ decreases in this semiinfinite interval. Since $\Theta_{0}(50)>0$ and $\Theta_{0}(350)<0$ we conclude that the equation $\Theta_{0}(x)=0$ has a single root which can be easily determined, $x=x_{0} \approx 341.7$. This point is the maximum of the function $\Theta(x)$ that increases from $\Theta(50)=0$ to $\Theta\left(x_{0}\right) \approx 0.293$ and then decreases to 0 . Therefore, $0<\lambda(m) \leq 0.293$, and the maximum is attained for $m=m_{0} \approx 31.7 \mathrm{~kg}$ (Figure 5). Equation (60) shows that $1.12 c<\hat{c} \leq 1.67 c$ and, for $c>2000 \mathrm{~m} / \mathrm{s}$, the value $v_{\times}$is very close to $v_{*}$ and practically does not vary (Figure 2). Consequently, the subarc which is determined by equation $\Omega(m, v)=0$ is only slightly different from a straight line. Therefore, the trajectory which is close to the optimum for all considered shield materials is the following $\left(v_{\text {imp }}>v_{*} \approx\right.$ $81 \mathrm{~m} / \mathrm{s}$ ): passive penetration until to velocity $v_{*}$ is attained, motion with a constant velocity $v_{*}$ while the propellant is available, and passive penetration until rest. The analysis shows that the effect of the material of the shield on the coefficient $\eta$ is insignificant.

\section{Application of dynamic programming for numerical investigation of the problem}

Equations (6), (7), (10), (11), (12), and (13) describe the variational problem considered here in the general case, and imply the following expressions which can be written using, for convenience, the 
dimensionless variables that are defined below:

$$
\begin{aligned}
& \bar{H}=\int_{\bar{m}_{\mathrm{res}}}^{1} \frac{\left(\bar{m} \bar{v}^{\prime}+\bar{c}\right) \bar{v}}{\bar{D}(\bar{m}, \bar{v})} d \bar{m}, \\
& \bar{v}(1)=1, \quad \bar{v}\left(\bar{m}_{\text {res }}\right)=0, \quad 0 \leq \bar{v}(\bar{m}) \leq v_{\max }, \quad \bar{m}_{\text {res }} \leq \bar{m} \leq 1, \\
& \bar{m} \bar{v}^{\prime}+\bar{c} \geq \bar{\beta}_{\min } \bar{D}(\bar{m}, \bar{v}) \quad \text { if } \bar{v}^{\prime}<\infty, \\
& \left|\frac{\bar{D}(\bar{m}, \bar{v}) \bar{v}^{\prime}}{\bar{m} \bar{v}^{\prime}+\bar{c}}\right| \leq \bar{\chi}_{\max } \quad \text { if } \bar{v}^{\prime}<\infty, \\
& D(\bar{m}, \bar{v}) / \bar{m} \leq \bar{\chi}_{\max } \quad \text { if } \bar{m}=\mathrm{const}
\end{aligned}
$$

where

$$
\begin{array}{cccc}
\bar{v}=\frac{v}{v_{\mathrm{imp}}}, & \bar{c}=\frac{c}{v_{\mathrm{imp}}}, \quad \bar{v}_{\max }=\frac{v_{\mathrm{max}}}{v_{\mathrm{imp}}}, & \bar{m}_{\mathrm{res}}=\frac{m_{\mathrm{res}}}{m_{\mathrm{imp}}}, \\
\bar{m}=\frac{m}{m_{\mathrm{imp}}}, & \bar{\beta}_{\min }=\frac{v_{\mathrm{imp}} m_{\mathrm{imp}}}{L \mu_{\mathrm{max}}}, & \bar{\chi}_{\max }=\frac{L g \chi_{\max }}{v_{\mathrm{imp}}^{2}}, & \bar{h}=\frac{h}{L}, \\
\bar{H} & =\frac{H}{L}, \quad \bar{v}^{\prime}=\frac{d \bar{v}}{d \bar{m}}, \\
\bar{D}(\bar{m}, \bar{v}) & =\frac{L}{v_{\mathrm{imp}}^{2} m_{\mathrm{imp}}} D\left(m_{\mathrm{imp}} \bar{m}, v_{\mathrm{imp}} \bar{v}\right),
\end{array}
$$

and $L$ is some characteristic length.

The problem is reduced to the optimization of the functional $\bar{H}$ in Equation (63), whereas the solution must satisfy the constraints given by Equations (64)-(66).

Dynamic programming for the general model of a drag force. Dynamic programming is an appropriate method for determining the optimal BP. Note that optimization of the flight of an aircraft with a jet engine was mentioned in one of the first books on dynamic programming [Bellman et al. 1958] as an example of a possible application of dynamic programming. This method allows determining a global extremum while taking into account constraints. In the following, we describe an application of dynamic programming to the above formulated optimization problem that has some characteristic properties.

In order to solve the variational problem for the functional given by Equation (63), the function $\bar{v}(\bar{m})$ can be approximated as a piecewise linear function determined by the values (Figure 6)

$$
\begin{array}{lll}
\bar{v}^{(0)}=0, & \bar{v}^{(1)}, \ldots, \bar{v}^{(j)}, \ldots, \bar{v}^{\left(N_{m}-1\right)}, & \bar{v}^{\left(N_{m}\right)} \\
\bar{w}^{(0)}, & \bar{w}^{(1)}, \ldots, \bar{w}^{(j)}, \ldots, \bar{w}^{\left(N_{m}-1\right)}, & \bar{w}^{\left(N_{m}\right)}=1
\end{array}
$$

in $N_{m}+1$ equally spaced mesh points of interpolation

$$
\bar{m}^{(0)}=\bar{m}_{\mathrm{res}}, \bar{m}^{(1)}, \ldots, \bar{m}^{(j)}, \ldots, \bar{m}^{\left(N_{m}-1\right)}, \quad \bar{m}^{\left(N_{m}\right)}=1,
$$

where $\bar{m}^{(j)}=\bar{m}_{\text {res }}+j \Delta \bar{m}, \Delta \bar{m}=\left(1-\bar{m}_{\text {res }}\right) / N_{m}, j=0,1,2, \ldots, N_{m}$. The unknown values of the function, $\bar{v}^{(j)}$ and $\bar{w}^{(j)}$ are chosen among the finite set of values $0, \Delta \bar{v}, 2 \Delta \bar{v}, \ldots, N_{v} \Delta \bar{v}$, where $\Delta \bar{v}=$ 


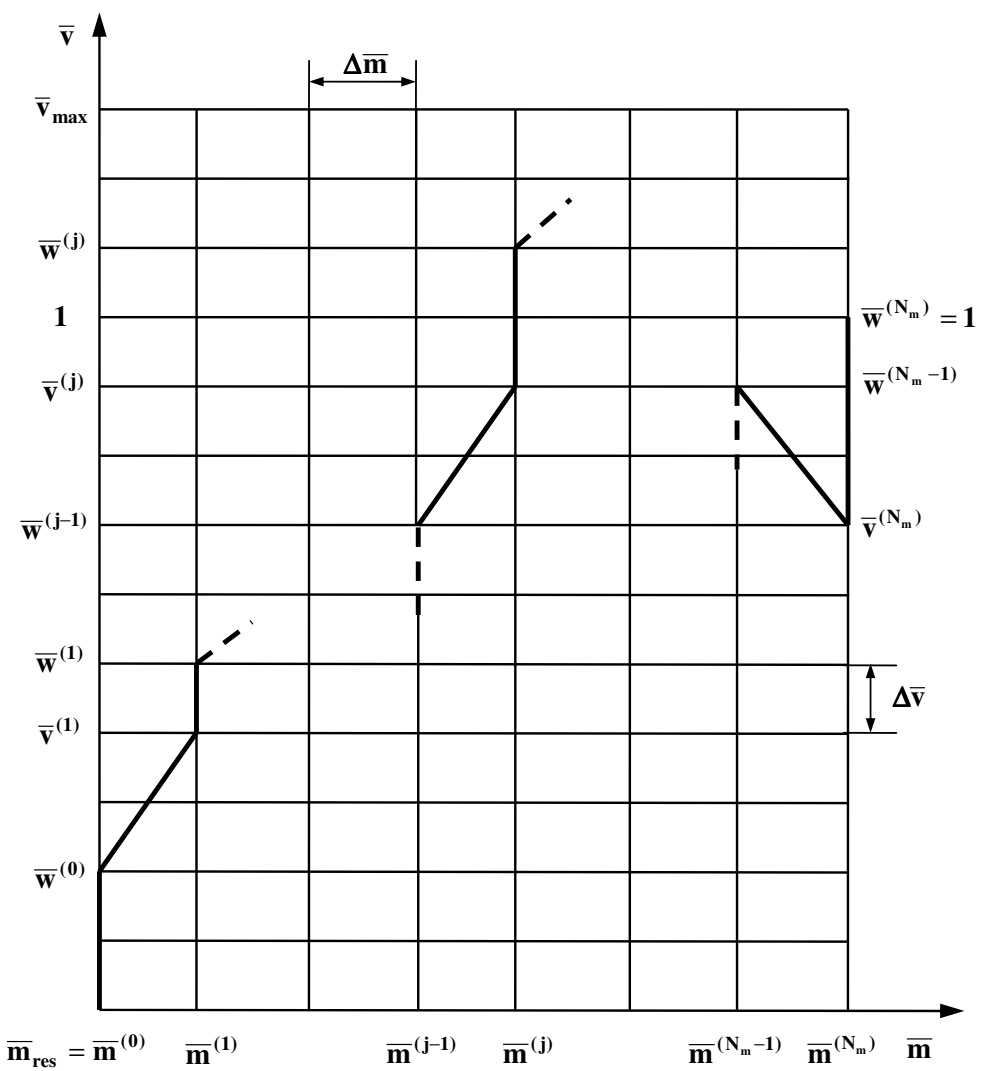

Figure 6. Discretization of the problem.

$\bar{v}_{\max } / N_{v}$ and $v_{\max }$ is a given upper bound for the velocity of the penetrator. Penetration of the impactor is associated with the trajectory

$$
\begin{aligned}
\left(\bar{m}^{\left(N_{m}\right)}, \bar{w}^{\left(N_{m}\right)}\right) \rightarrow\left(\bar{m}^{\left(N_{m}\right)}, \bar{v}^{\left(N_{m}\right)}\right) & \rightarrow\left(\bar{m}^{\left(N_{m}-1\right)}, \bar{w}^{\left(N_{m}-1\right)}\right) \rightarrow\left(\bar{m}^{\left(N_{m}-1\right)}, \bar{v}^{\left(N_{m}-1\right)}\right) \rightarrow \cdots \\
& \rightarrow\left(\bar{m}^{(1)}, \bar{w}^{(1)}\right) \rightarrow\left(\bar{m}^{(1)}, \bar{v}^{(1)}\right) \rightarrow\left(\bar{m}^{(0)}, \bar{w}^{(0)}\right) \rightarrow\left(\bar{m}^{(0)}, \bar{v}^{(0)}\right) .
\end{aligned}
$$

Since the velocity of the impactor cannot increase without burning fuel, $\bar{v}^{(j)} \leq \bar{w}^{(j)}$ for all $j$.

The equation of the straight line between the points $\left(\bar{m}^{(j-1)}, \bar{w}^{(j-1)}\right)$ and $\left(\bar{m}^{(j)}, \bar{v}^{(j)}\right)$ can be written as follows:

$$
\begin{gathered}
\bar{v}=e^{(j)} \bar{m}+f^{(j)}, \quad e^{(j)}=\frac{\bar{v}^{(j)}-\bar{w}^{(j-1)}}{\bar{m}^{(j)}-\bar{m}^{(j-1)}}, \\
f^{(j)}=\frac{\bar{m}^{(j)} \bar{w}^{(j-1)}-\bar{m}^{(j-1)} \bar{v}^{(j)}}{\bar{m}^{(j)}-\bar{m}^{(j-1)}},
\end{gathered}
$$


$j=1,2, \ldots, N_{m}$. Then the integral in Equation (63) along the piecewise linear contour can be written as follows:

$$
\bar{H}=Q_{v}^{(0)}+\sum_{j=1}^{N_{m}}\left(Q_{m}^{(j)}+Q_{v}^{(j)}\right)
$$

where $Q_{m}^{(j)}$ and $Q_{v}^{(j)}$ are the components of the integral over vertical and nonvertical segments of the trajectory, correspondingly (Figure 6),

$$
\begin{gathered}
Q_{v}^{(j)}=\int_{\bar{v}^{(j)}}^{\bar{w}^{(j)}} G_{v}^{(j)}(\bar{v}) d \bar{v}, \\
G_{v}^{(j)}(\bar{v})=\frac{\bar{m}^{(j)} \bar{v}}{\bar{D}\left(\bar{m}^{(j)}, \bar{v}\right)}, \quad j=0,1,2, \ldots, N_{m}, \\
Q_{m}^{(j)}=\int_{\bar{m}^{(j-1)}}^{\bar{m}^{(j)}} G_{m}^{(j)}(\bar{m}) d \bar{m}, \\
G_{m}^{(j)}(\bar{m})=\frac{\left(e^{(j)} \bar{m}+\bar{c}\right)\left(e^{(j)} \bar{m}+f^{(j)}\right)}{\bar{D}\left(\bar{m}, e^{(j)} \bar{m}+f^{(j)}\right)}, \quad j=1,2, \ldots, N_{m} .
\end{gathered}
$$

Integrals in Equation (71) can be often calculated in explicit form. Otherwise, one can use, for instance, the trapezoid rule formula with points of interpolation, $\bar{v}_{\xi}^{(j)}$, in nodes of the $(\bar{m}, \bar{v})$ mesh. Then

$$
Q_{v}^{(j)} / \Delta \bar{v}=0.5\left[G_{v}^{(j)}\left(\bar{v}_{0}^{(j)}\right)+G_{v}^{(j)}\left(\bar{v}_{n_{v}^{(j)}}^{(j)}\right)\right]+\sum_{\substack{\xi=1 \\ n_{v}^{(j)}>1}}^{n_{v}^{(j)}-1} G_{v}^{(j)}\left(\bar{v}_{\xi}^{(j)}\right),
$$

where $n_{v}^{(j)}$ is the integral part $\left(\bar{w}^{(j)}-\bar{v}^{(j)}\right) / \Delta \bar{v}$ and

$$
\bar{v}_{\xi}^{(j)}=\bar{v}^{(j)}+\xi \Delta \bar{v}, \quad \xi=0,1, \ldots, n_{v}^{(j)} .
$$

In a similar manner, integrals in Equation (72) can be represented as

$$
Q_{m}^{(j)} / \Delta \bar{m}^{(j)}=0.5\left[G_{m}^{(j)}\left(\bar{m}_{0}^{(j)}\right)+G_{m}^{(j)}\left(\bar{v}_{n_{m}^{(j)}}^{(j)}\right)\right]+\sum_{\substack{\xi=1 \\ n_{m}^{(j)}>1}}^{n_{m}^{(j)}-1} G_{m}^{(j)}\left(\bar{v}_{\xi}^{(j)}\right)
$$

where $n_{m}^{(j)}+1$ is the selected number of points of interpolations at the interval $\left[\bar{m}^{(j-1)}, \bar{m}^{(j)}\right]$,

$$
\bar{m}_{\xi}^{(j)}=m^{(j)}+\xi \Delta \bar{m}^{(j)}, \quad \xi=0,1, \ldots, n_{m}^{(j)}, \quad \Delta \bar{m}^{(j)}=\Delta m / n_{m}^{(j)} .
$$

Inequalities in Equations (65) and (66) can be written in the points of interpolations in the following form:

$$
A_{\xi}^{(j)} \geq \bar{\beta}_{\min }, \quad\left|e^{(j)}\right| \leq \bar{\chi}_{\max }\left|A_{\xi}^{(j)}\right|,
$$


where

$$
A_{\xi}^{(j)}=\frac{e^{(j)} \bar{m}_{\xi}^{(j)}+\bar{c}}{\bar{D}\left(\bar{m}_{\xi}^{(j)}, e^{(j)} \bar{m}_{\xi}^{(j)}+f^{(j)}\right)}, \quad j=1,2, \ldots, N_{m}, \quad \xi=0,1, \ldots, n_{m}^{(j)} .
$$

Inequality (67) implies the following constraints:

$$
\bar{D}\left(\bar{m}^{(j)}, \bar{v}_{\xi}^{(j)}\right) / \bar{m}^{(j)} \leq \bar{\chi}_{\max }, \quad j=1,2, \ldots, N_{m}, \xi=0,1, \ldots, n_{v}^{(j)}
$$

We do not allow regimes whereby fuel is exhausted while the velocity of the penetrator remains zero, that is, the following condition must be satisfied:

$$
\bar{v}^{(j)}+\bar{w}^{(j-1)}>0, \quad j=1,2, \ldots, N_{m} .
$$

Unlike the standard approach (see, for example, [Pedregal 2003]), we allow vertical segments in the optimal curve, use a more accurate approximation of the criterion on the subintervals, and take into account the constraints in the intermediate points of the subintervals. The second factor enhances the reliability of the analysis.

The case of the generalized Young's penetration model. In the case of the generalized Young model we obtain:

$$
\bar{D}(\bar{m}, \bar{v})=\frac{L \psi(\bar{v})}{k_{0} k_{1} v_{\mathrm{imp}}^{2} \varphi(\bar{m})},
$$

where

$$
\begin{aligned}
\psi(\bar{v}) & = \begin{cases}1 /\left(\gamma_{0}-\gamma_{2} v_{\mathrm{imp}}^{2} \bar{v}^{2}\right) & \text { if } \bar{v}<\bar{v}_{*}, \\
v_{\mathrm{imp}} \bar{v} & \text { if } \bar{v} \geq \bar{v}_{*},\end{cases} \\
\varphi(\bar{m}) & = \begin{cases}\sigma m_{\mathrm{imp}}^{\gamma_{1}+\gamma_{2}} \bar{m}^{\gamma_{1}+\gamma_{2}-1} & \text { if SRC, } \\
m_{\mathrm{imp}}^{\gamma_{1}} \bar{m}^{\gamma_{1}-1} \ln \left(50+0.29 m_{\mathrm{imp}}^{2} \bar{m}^{2}\right) & \text { if IFS, }\end{cases}
\end{aligned}
$$

$\bar{v}_{*}=v_{*} / v_{\text {imp }}$, and it is assumed that the substitution $m=m_{\mathrm{imp}} \bar{m}$ is made in Table 1 .

For the vertical segments of the trajectory, the analysis can be performed in the exact analytical form. Clearly, the integral in Equation (71) can be calculated:

$$
Q_{v}^{(j)}=\frac{k_{0} k_{1} v_{\mathrm{imp}}^{2} \bar{m}^{(j)} \varphi\left(\bar{m}^{(j)}\right)}{L} \int_{\bar{v}^{(j)}}^{\bar{w}^{(j)}} \frac{\bar{v} d \bar{v}}{\psi(\bar{v})}, \quad j=0,1,2, \ldots, N_{m}
$$

where

$$
\int_{\bar{v}^{(j)}}^{\bar{w}^{(j)}} \frac{\bar{v} d \bar{v}}{\psi(\bar{v})}= \begin{cases}\Phi\left(\bar{v}^{(j)}, \bar{w}^{(j)}\right) & \text { if } \bar{w}^{(j)}<\bar{v}_{*}, \\ \Phi\left(\bar{v}^{(j)}, \bar{v}_{*}\right)+\Psi\left(\bar{v}_{*}, \bar{w}^{(j)}\right) & \text { if } \bar{v}^{(j)} \leq \bar{v}_{*} \leq \bar{w}^{(j)} \\ \Psi\left(\bar{v}^{(j)}, \bar{w}^{(j)}\right) & \text { if } \bar{v}_{*}<\bar{v}^{(j)},\end{cases}
$$




$$
\begin{aligned}
\Phi\left(\bar{V}_{a}, \bar{V}_{b},\right) & =\int_{\bar{V}_{a}}^{\bar{V}_{b}}\left(\gamma_{0}-\gamma_{2} v_{\mathrm{imp}}^{2} \bar{v}^{2}\right) \bar{v} d \bar{v} \\
& =0.25\left(\bar{V}_{b}^{2}-\bar{V}_{a}^{2}\right)\left[2 \gamma_{0}-\gamma_{2} v_{\mathrm{imp}}^{2}\left(\bar{V}_{b}^{2}+\bar{V}_{a}^{2}\right)\right] \\
\Psi\left(\bar{V}_{a}, \bar{V}_{b}\right) & =\frac{1}{v_{\mathrm{imp}}} \int_{\bar{V}_{a}}^{\bar{V}_{b}} d \bar{v}=\left(\bar{V}_{b}-\bar{V}_{a}\right) / v_{\mathrm{imp}}
\end{aligned}
$$

Instead of Equation (79), the constraints in Equation (67) can be written as follows:

$$
\bar{w}^{(j)} \leq \psi^{-1}\left(\frac{k_{0} k_{1} v_{\mathrm{imp}}^{2} \bar{m}^{(j)} \varphi\left(\bar{m}^{(j)}\right) \bar{\chi}_{\max }}{L}\right), \quad j=0,1,2, \ldots, N_{m}
$$

where

$$
\psi^{-1}(z)= \begin{cases}\sqrt{\frac{\gamma_{0} z-1}{\gamma_{2} v_{\mathrm{imp}}^{2} z}} & \text { if } z \leq v_{\mathrm{imp}} \bar{v}_{*}, \\ z / v_{\mathrm{imp}} & \text { if } z>v_{\mathrm{imp}} \bar{v}_{*},\end{cases}
$$

\section{Results of numerical optimization}

Figure 7 shows typical results of calculations for which we selected the following data: penetration into nonfrozen soil, rock, and concrete shields, $m_{\text {imp }}=400 \mathrm{~kg}, m_{\text {res }}=200 \mathrm{~kg}, v_{\text {imp }}=700 \mathrm{~m} / \mathrm{s}>v_{*}, \chi_{\max }=\infty$.

Figure $7 \mathrm{a}, \mathrm{b}$ shows optimal BPs. For small values of $\bar{\beta}_{\min }$ when the constraint on the fuel flow rate is irrelevant, the regime of motion is as follows: inertial motion until velocity $\bar{v}_{\times}$is attained; motion with a constant velocity $\bar{v}_{\times}$until exhausting fuel supply, and, finally, inertial motion until rest. From some value, the effect of $\bar{\beta}_{\min }$ on the optimal BP becomes pronounced: the regime with a maximum fuel flow rate appears between the initial passive regime and the regime with a constant velocity $\bar{v}_{\times}$. Further increase of $\bar{\beta}_{\text {min }}$ results in the following changes: transition from a passive penetration to the motion with the maximum fuel flow rate at larger velocities, the regime of motion with a constant velocity $\bar{v}_{\times}$ gradually vanishes, and the regime with the maximum fuel flow rate switches to the regime of inertial penetration with mass $\bar{m}_{\text {res }}$. From some magnitude of $\bar{\beta}_{\min }$, a solution does not exist. The latter means that the penetrator slows down to zero velocity before exhausting the fuel supply for any BP.

In Figure 7c, we showed the dependencies of the maximum normalized DOP, $\eta=H / H_{0}$, as a function of parameter $\bar{\beta}_{\min }$ for different values of the dimensionless relative exit velocity of gases at the nozzle of the thruster, $c$; clearly, $H_{0}$ is the same when the variants with different $\bar{\beta}_{\text {min }}$ and $c$ are compared. The maximum effect of using a jet thruster is observed for small $\bar{\beta}_{\text {min }}$. An increase of $\bar{\beta}_{\text {min }}$ implies stronger constraints, namely, reducing the upper bound for the admissible fuel consumption rate of the thruster. The curves $\eta=\eta\left(\bar{\beta}_{\min }\right)$ are located higher, with an increase of $c$, that is, a negative effect of $\bar{\beta}_{\text {min }}$ can be compensated by increasing $c$. The fact that some curves of the dependencies $\eta=\eta\left(\bar{\beta}_{\min }\right)$ are terminated implies that the capabilities of the thruster are inadequate, and penetration terminates before exhausting fuel supply. 

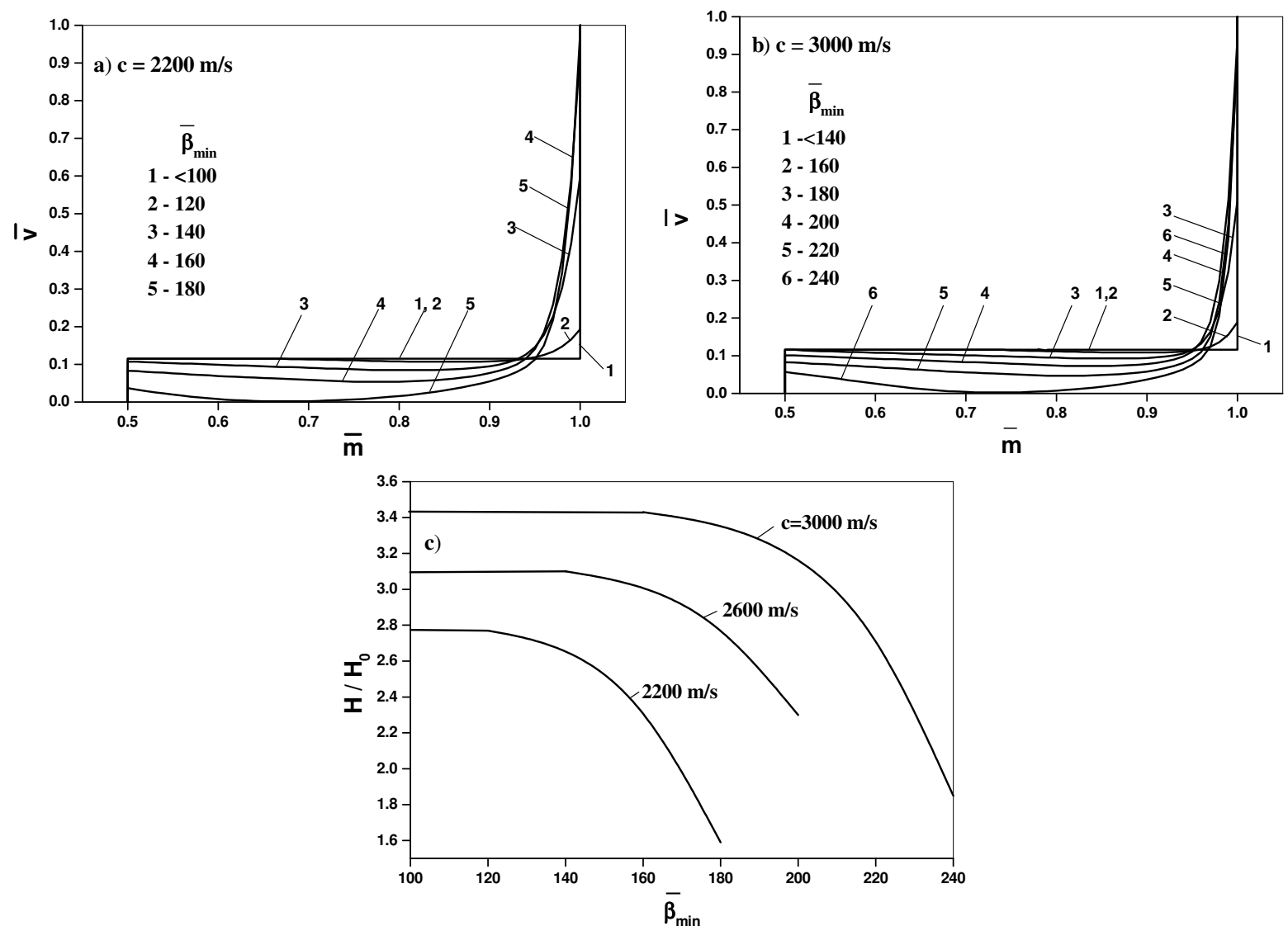

Figure 7. Typical optimal solution for relatively high impact velocities (penetration into nonfrozen soil, rock, and concrete shields, $m_{\text {imp }}=400 \mathrm{~kg}, m_{\text {res }}=200 \mathrm{~kg}, v_{\text {imp }}=700 \mathrm{~m} / \mathrm{s}$ ); (a-b) optimal burning programs; (c) normalized optimal DOP versus $\bar{\beta}_{\min }$. ( $H$ is DOP for the optimal burning program, $H_{0}$ is DOP for a passive trajectory.)

\section{Concluding remarks}

We suggested a mechanical model of a penetrator equipped with a jet thruster and demonstrated that appropriate choice of the parameters of a jet thruster allows increasing the depth of penetration considerably into different media. We showed that for relatively small impact velocities (about $100 \mathrm{~m} / \mathrm{s}$ ), penetration at the maximum depth is attained with velocities lower than the impact velocity. Nelson [2002] emphasized that there is a limitation on increasing impact velocity in order to attain a higher penetration depth because of the constraints on the impact velocity required for the survival of a penetrator on impact. Using a jet thruster is one of the possible solutions to overcome this constraint.

\section{References}

[Bellman et al. 1958] R. E. Bellman, I. Glicksberg, and O. A. Gross, Some aspects of the mathematical theory of control processes, Rand Corp., Santa Monica, Calif., 1958. 
[Ben-Dor et al. 2005] G. Ben-Dor, A. Dubinsky, and T. Elperin, "Ballistic impact: recent advances in analytical modeling of plate penetration dynamics. A review”, Appl. Mech. Rev. (Trans. ASME) 58:6 (2005), 355-371.

[Ben-Dor et al. 2006] G. Ben-Dor, A. Dubinsky, and T. Elperin, Applied high-speed plate penetration dynamics, Springer, Dordrecht, 2006.

[Ben-Dor et al. 2007] G. Ben-Dor, A. Dubinsky, and T. Elperin, "Optimization of high-speed penetration by impactor with jet thruster", Mech. Based Des. Struct. Mach. 35:3 (2007), 205-228.

[Cicala and Miele 1956] P. Cicala and A. Miele, "Generalized theory of the optimum thrust programming for the level flight of a rocket-powered aircraft”, ARSJ Am. Rocket Soc. J. 26:6 (1956), 443-455.

[Eisler et al. 1998] R. D. Eisler, A. K. Chatterjee, G. H. Burghart, and P. Loan, "Simulates the tissue damage from small arms projectiles and fragments penetrating the musculoskeletal system", Final report, Mission Research Corp, Fountain Valley, CA, 1998.

[Gould 1997] R. L. Gould, “Penetrating vehicle with rocket motor”, U.S.A. Patent 5596166, 1997.

[Hibbs 1952] A. R. Hibbs, “Optimum burning program for horizontal flight”, ARSJ Am. Rocket Soc. J. 22:4 (1952), 204-212.

[Kosmodemiansky 1966] A. A. Kosmodemiansky, Course on theoretical mechanics. Part 2, Prosveschenije, Moscow, 1966. in Russian.

[Krotov 1995] V. F. Krotov, Global methods in optimal control theory, Marcel Dekker, New York, 1995.

[Leitmann 1962] G. Leitmann, Optimization techniques with applications to aerospace systems, Academic Press, New York, London, 1962.

[Miele 1957] A. Miele, "An extension of the theory of the optimum burning program for the level flight of a rocket-powered aircraft", J. Aerospace Eng. 24:12 (1957), 874-884.

[Miele 1962] A. Miele, "The calculus of variations in applied aerodynamics and flight mechanics", pp. 258-301 in Optimization techniques with applications to aerospace systems, edited by G. Leitmann, Academic Press, New York, London, 1962.

[Nelson 2002] R. W. Nelson, "Low-yield earth-penetrating nuclear weapons", Sci. Global Secur. 10:1 (2002), 1-20.

[Pedregal 2003] P. Pedregal, Introduction to optimization, Springer, New York, 2003.

[Sagomonyan 1988] A. Y. Sagomonyan, Dynamics of barriers perforation, Moscow Univ. Publ., Moscow, 1988. in Russian.

[Tertychny-Dauri 2004] V. Y. Tertychny-Dauri, Hyperreactive mechanics, Fizmatlit, Moscow, 2004. in Russian.

[Young 1997] C. W. Young, "Penetration equations”, Report No. SAND972426, Sandia Nat. Lab., Albuquerque, NM, 1997.

Received 13 Aug 2007. Accepted 21 Jan 2008.

GABI BEN-DOR: bendorg@bgu.ac.il

Pearlstone Center for Aeronautical Engineering Studies, Department of Mechanical Engineering, Ben-Gurion University of the Negev, P.O. Box 653, Beer-Sheva, 84105, Israel

http://eng2.bgu.ac.il/engineering/profile.aspx?id=juesiMMt

AnATOLY Dubinsky: dubin@bgu.ac.il

Pearlstone Center for Aeronautical Engineering, Department of Mechanical Engineering, Ben-Gurion University of the Negev, P.O. Box 653, Beer-Sheva, 84105, Israel

http://www.bgu.ac.il/ dubin

TOV ELPERIN: elperin@bgu.ac.il

Pearlstone Center for Aeronautical Engineering, Department of Mechanical Engineering, Ben-Gurion University of the Negev, P.O. Box 653, Beer-Sheva, 84105, Israel

http://www.bgu.ac.il/me/staff/tov/index.html 


\title{
A NEW TAILORING OPTIMIZATION APPROACH FOR IMPROVING STRUCTURAL RESPONSE AND ENERGY ABSORPTION CAPABILITY OF LAMINATED AND SANDWICH COMPOSITES
}

\author{
UGO ICARDI AND LAURA FERRERO
}

This paper is dedicated to the memory of Liviu Librescu.

\begin{abstract}
In this paper a technique for tuning the energy absorption properties of laminated and sandwich composites through a new tailoring concept is presented. The purpose is to minimize the energy absorbed through unwanted modes (ones involving interlaminar strengths) and maximize that absorbed through desired modes (ones involving membrane strengths) by finding a suited in-plane variable distribution of stiffness properties. Herein mode is intended as a strain energy contribution, such as bending energy, in-plane and out-of-plane shear energy, etc., and no vibration mode. This distribution is obtained making extremal certain strain energy contributions of interest (for example, membrane, bending, in-plane, and out-of-plane shear energies) under in-plane variation of the plate stiffness properties. The effect of this technique is to act as an energy absorption tuning, since it minimizes or maximizes the amount of energy absorbed by specific modes. Although the present technique could be applied to laminates or to the face sheets of sandwich composites, in this paper a preliminary application is presented to single plies with variable stiffness coefficients over their plane. Once incorporated into a laminate or a sandwich composite, these layers are shown to have beneficial effects on the strength at the onset of delamination in sample cases where laminated and sandwich composites are subjected to low velocity, low energy impacts.
\end{abstract}

\section{Introduction}

As is well known, fiber reinforced and sandwich composites with laminated faces offer advantages over conventional metallic structures in terms of specific strength and stiffness, impact resistance, containment of explosions, protection against fragments' projection, survivability, noise, and vibration suppression. Beside many other, not cited favorable properties, they also offer the remarkable advantage of being tailored to fulfill design requirements.

Unfortunately, these materials absorb a large amount of the incoming energy through local failures. The effect of this damage accumulation usually appears at the global level as an embedded delamination. A significant accumulation is detrimental, since it could degrade strength and stiffness, cause a deleterious load redistribution, and reduce the service life. Obviously, an accurate assessment of the local damage mechanisms and postfailure behavior is mandatory, in order to fully exploit the potential advantages of these materials. The reader is referred to the review papers [Rowlands 1985; Tennyson and Wharam 1985;

Keywords: optimization of laminated and sandwich composites, impact induced damage, delamination. 
Nahas 1986; Bolotin 1996; Echaabi and Trochu 1996; Paris 2001; Icardi et al. 2007] for a comprehensive discussion of the local failure mechanisms and residual properties of these materials, these being outside the scope of this paper.

Various techniques have been recently published with the aim of preventing the damage accumulation, reducing its effects, and obtaining an improved structural performance. Functionally graded materials [Fuchiyama and Noda 1995] avoid unwise stress concentrations at interfacial material discontinuities that conventional fiber reinforced and sandwich composites exhibit, by virtue of the gradual variation of their physical properties. Technological skills such as stitching, lap and $\mathrm{T}$ joints, or short rods have been suggested in order to improve the transverse shear strength of multilayered materials and limit the detrimental effects of local damage and delamination propagation. These skills oppose cracks and sliding displacements by inducing bridging tractions, as shown by Cox [1999], although at the expense of the stress concentrations induced by the local tractions that oppose the propagation of the delamination. An improvement in the impact and delamination resistance and in dissipation can be achieved by stacking layers with different absorption and dissipation properties, namely, plies with customary properties and viscoelastic layers [Suzuky et al. 2003]. Since the viscoelastic layers must be as thick and numerous as the structural layers, this method unfortunately makes the structures too flexible and is not effective for sandwich composites, because the number of damping layers being incorporated is lower than in laminated composites. Moreover, the stresses in the adhesive film which bonds the viscoelastic layers to the host structure could limit the strength and the service life.

Several studies dealing with methods which seek to simultaneously improve stiffness, energy absorption, and dissipation have been recently published. Jung [2001] seeks to comply stiffness and energy dissipation by combining different materials with different absorption and stiffness properties. Lakes [2002] shows that the structural hierarchy makes it possible to obtain of both the desired stiffness and damping properties. McCoucheon [2004] shows that the energy dissipation can be increased without remarkable stiffness loss by inserting fluid filled microtubes into a matrix material, where the fluid flow is induced by the composite deformation. Actual tailoring, meaning the optimization of the reinforcement orientation and constituent materials, is preferred to these techniques because they introduce technological complications. Recently, variable stiffness composites in which the orientation of the fibers minimizes the stresses were studied, for example, by Pedersen [2003] and Setoodeh et al. [2005]. Other recent studies by Zinoviev and Ermakov [1994] and Georgi [1979] investigated the effects of the fibers" orientation (constant over the plate) for finding configurations able to dissipate a large amount of the incoming energy, while keeping the wanted strength and stiffness properties.

In the present paper a new approach based on a variable spatial distribution of stiffness properties is presented. Its aim is to limit the detrimental effects of damage and improve the structural performance of fiber reinforced and sandwich composites by optimizing the energy absorption properties. With this approach, contrasting objectives for currently available optimization techniques such as improvement of stiffness and, contemporaneously, of delamination strength, can be conjugated. The idea proposed is finding a variable spatial distribution that makes stationary the strain energy contributions as desired (for example, bending, in-plane, and out-of-plane modes), in order to allow a maximization of the energy stored in wanted modes (for example, in-plane modes and membrane strengths) and a minimization of that stored in unwanted modes (for example, out-of-plane shears, etc.). The appropriate in-plane distribution of plate stiffness coefficients is obtained making extremal the strain energy contributions of 
interest under in-plane variation of the plate stiffness properties, and enforcing conditions which range from the imposition of the thermodynamic constraints, to the choice of a convex or a concave shape (in order to minimize or maximize the energy contributions of interest), to the imposition of a mean value for these coefficients. To account for these optimized stiffness variations either the fiber orientation, the constituent materials, the volumetric rate of fibers, or the thickness of plies could be varied.

In this paper, a preliminary application to single plies with optimized variable stiffness coefficients is presented, but the actual technique could be applied also to laminates or to the face sheets of sandwich composites as well, numerically solving the Euler-Lagrange equations. Two optimized ply stiffness distributions with complementary properties are proposed; they are based on an approximate parabolic solution of these equations. Their mean stiffness properties are chosen to be the same as that of the corresponding plies made of the same constituent materials having constant stiffness. The first type allows an increased bending stiffness, at the expense of a moderate increase of transverse shear stresses; the second type does just the opposite. In this way, the delamination damage could be increased and used to absorb the incoming energy. The replacement of a couple of conventional, constant stiffness layers in laminated and sandwich composites with these layers will appear able to consistently reduce the through the thickness interlaminar stress, either keeping the bending stiffness substantially unchanged or improving it, for all the sample cases considered in the numerical applications. In the case of low velocity, low energy impacts they will be shown to always produce beneficial effects on the strength at the onset of delamination for both laminated and sandwich composites with laminated faces.

The case of low velocity, low energy impacts was chosen because it is dominated by delamination and matrix cracking and is always responsible for a relevant strength degradation, even when the barely visible impact damage is not evident. There is a general agreement that for this kind of impact the energy is mainly absorbed as strain energy and through local failures, thus strain rate dependent formulations and microstructure level considerations are reputed as unnecessary; in addition, the deformation of the projectile is considered negligible [Davies and Olsson 2004]. Since the contact duration is higher then the stress waves' lateral transit time, transverse shear waves reflect off the edges several times while the contact load is still being applied, so the plate structural modeling, the plate size, and the boundary conditions affect the response. Stress based criteria are used to predict the onset of delamination, since it is a common opinion that they are accurate enough for this task. Three different delamination criteria, which appeared accurate in literature, are considered in order to have mutual assessments. The effects of the accumulated damage are accounted for, reducing the elastic properties of the layers that failed, within the framework of the ply-discount theory. As customary for low velocity impacts, the time history of the contact force is computed using modified versions of Hertz's contact law; the dynamic equations are integrated through Newmark's technique.

Since the numerical results show that the interlaminar stresses can be consistently reduced through a variable in-plane distribution of stiffness properties, keeping a high stiffness, future applications are expected where the local effects are accounted for in a much more detailed way. In subsequent sections the features of the models used will be briefly summarized, the energy storage optimization process discussed in detail, and the numerical applications presented. 


\section{Structural modeling}

No attempt is made to review the ample literature about the modeling of laminated and sandwich composites. We refer to the available survey papers and monographs for a comprehensive discussion of this topic [Reddy 1982; 1990; 2003; Bert 1984; Librescu and Reddy 1986; Noor and Burton 1989; 1990; 1992; Noor et al. 1996; Reddy and Robbins 1994]. We just remind the reader that to accurately model the energy contributions involved, the layerwise kinematics and their inherent stress fields have to be accounted for as accurately as possible and, to limit the computational costs and storage, as efficiently as possible. To this purpose, in this paper a three-dimensional zigzag model [Icardi 1998; 2001], and the corresponding $\mathrm{C}^{\circ}$ eight node plate element [Icardi 2005; Icardi and Zardo 2005], both based on the five DOF of customary plate models, are employed as structural models. The three-dimensional zigzag model has the advantage of requiring a low computational effort, like conventional single layer plate models, with higher accuracy. This makes its use affordable within the optimization process for finding the stiffness distribution. The finite element is based on a conventional first order shear deformation plate element, to which a procedure for updating the strain energy to that of the three-dimensional zigzag model and a postprocessing technique based on a high order spline representation of the nodal DOF (which is used in place of the low order representation by shape function for obtaining integrated stresses) are applied, which makes the computation of interlaminar stresses accurate, but saves computational and memory storage costs. The mixed brick element with three displacements and three interlaminar stresses as nodal DOF developed by Icardi and Atzori [2004] is used to compute the local effects of the optimized solutions with the highest accuracy.

The capability of the present computational model to predict the local failures and the impact induced delamination damage has been successfully assessed in a number of previously published papers [Icardi and Zardo 2005; Icardi and Ferrero 2005; 2006a; 2006b; 2006c; 2007a; 2007b; Ferrero and Icardi 2006; 2007; Icardi 2007]. It appears from the comparison with ultrasonic inpsection detections of impact induced delamination damage and force time history detections that this simple modeling of the impact with a low computational effort is capable of accurate predictions of the delamination damage.

A variety of models, the so called layerwise models, have been developed. They have different features, degree of accuracy, and computational effort. In a broad outline, they subdivide into discrete layer and zigzag models, as aforementioned, if they impose the interfacial continuity requirements as constraint conditions, or if they postulate an appropriate piecewise variation of displacements, respectively. Both kinds of models are used in this paper, to exploit their complementary performance in terms of accuracy and computational effort. Their characteristic features are briefly summarized.

Perhaps the most general zigzag representation of displacements is the one developed by Icardi [2001] and subsequently improved in [Icardi 2007]. Unfortunately, this model is difficult to treat within the optimization process, since a big mathematical effort is required. For this reason, a third order zigzag model with a constant transverse displacement, which only accounts for the discrete layer effects of transverse shears, is particularized and used within the optimization process. In order to improve the accuracy of this model, its strain energy is updated to that of the previous model in the applications where the response of laminates incorporating optimized layers is studied using an approach based on the method of weighted residuals, instead of the finite element model described in the next section. This updating is carried out as outlined by Icardi [2005], using the postprocessing technique presented therein 
to obtain a realistic prediction of interlaminar stresses. The mixed, eight node solid element used in this study was developed by Icardi and Atzori [2004]. It has the three interlaminar stresses and the three elastic displacements as nodal DOF, in order to fulfill the interfacial stress and displacement continuity requirements. Characteristic feature, $C^{\circ}$, trilinear, and standard serendipity shape functions are used for interpolating the DOF across the element volume. In this way, the intraelement equilibria are met in an approximate integral form, according to Zienkiewicz and Taylor [1994]. As customary, the required level of variation of the layerwise quantities is obtained by increasing the number of subdivisions across the thickness. This makes the structural model computationally intensive, but accurate, as shown by Icardi and Atzori [2004]. The readers will find a compendium on hybrid and mixed (we reserve the term mixed for the case where the master fields are internal fields and the term hybrid for when surface fields are involved) finite elements for composites in the recent book by Hoa and Feng [1998], and the omitted details on development of the present element and an assessment of its accuracy in [Icardi and Atzori 2004].

Since displacements and stresses are interpolated with the same functions, the intraelement equilibrium conditions are met in an approximate integral form. This approach, seldom used, though efficient, makes easier the development of mixed elements and does not compromise the accuracy [Loubignac et al. 1978; Nakazawa 1984]. The generation of the stiffness matrix and the vector of nodal forces follows the standard path.

The representation of the chosen nodal DOF makes the computational effort required by the present element similar to that of displacement based counterpart solid elements, while accuracy and convergence are dramatically improved [Icardi and Atzori 2004]. Furthermore, it enables the element to be implemented into commercial finite element codes.

\section{Optimization of the energy storage}

As previously mentioned, the present paper aims to improve the structural performance of laminated and sandwich composites, optimizing the energy absorption properties of the constituent layers. The concept involved is finding optimized spatially variable stiffness properties which make stationary the bending, in-plane, and out-of-plane contributions to the strain energy, as desired. In this way, the energy absorbed by modes having detrimental effects (meaning, involving weak properties) is transferred to acceptable modes by minimizing the energy contributions involved in the unwanted modes (for example, transverse shears) and maximizing those of the acceptable ones (for example, membrane loading). As a result of this energy "tuning", stiffness and delamination strength, which are contrasting objectives for currently available constant stiffness optimization techniques, can both be improved.

The optimization of the energy storage starts writing the membrane, bending, in-plane, and out-ofplane strain energy contributions appearing in the zigzag model, for a generic laminate (herein sandwich composites are viewed as multilayered materials). Since standard variational calculus techniques are used, no details of these techniques are here discussed. It is just reminded that setting to zero the first variation of a functional coming from a weak form produces a set of governing equations, the so called Euler-Lagrange equations, that recover the strong forms of the weakened field equations and natural boundary conditions. In the present case, the stiffness properties will represent the master field, while the other variables not subjected to variation will represent the slave and data fields. Therefore, the 


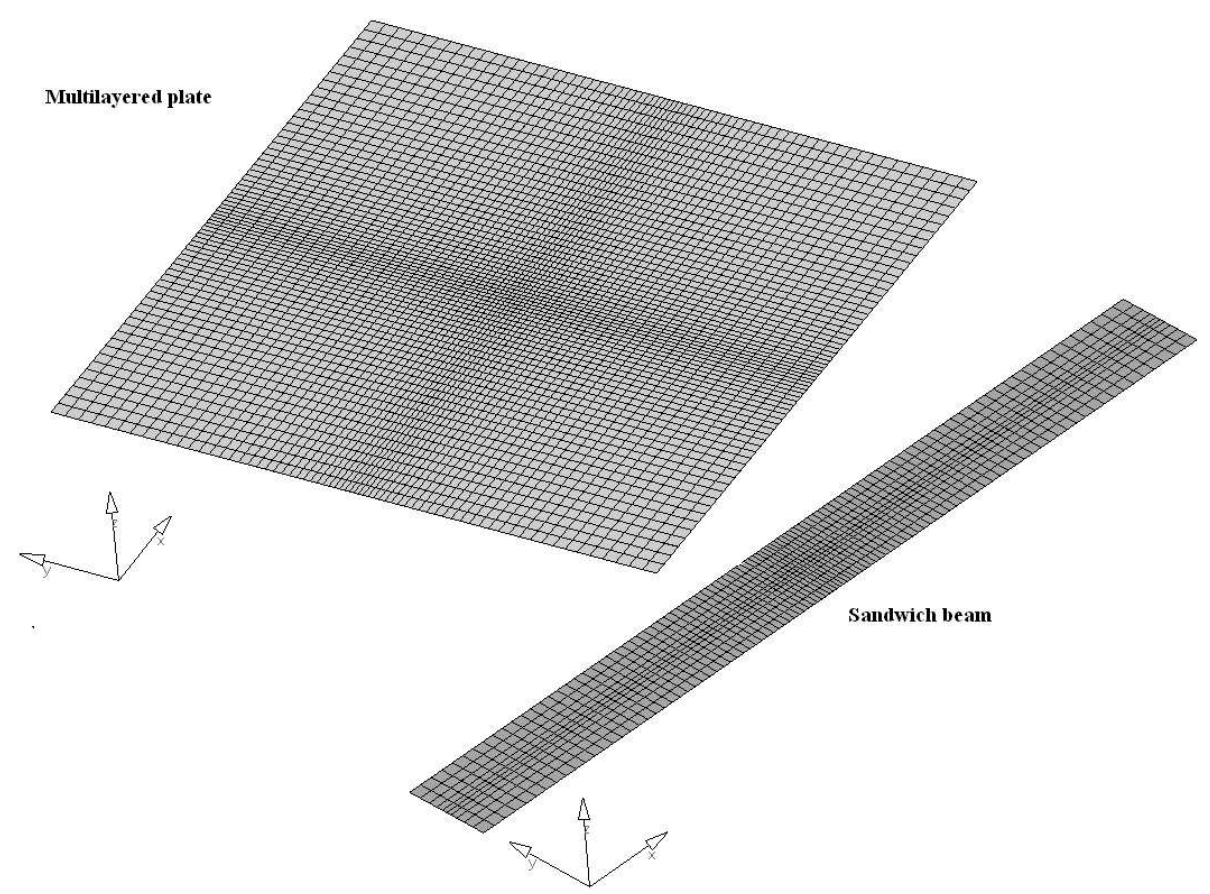

Figure 1. Discretizations used in the optimization process with the zig zag finite element. The multilayered plate is described in Section 4.2 and the sandwich beam analyzed in Section 4.4.

Euler-Lagrange equations will represent the relations enabling the stiffness properties to make the strain energy contributions extremal. The solution of these equations finds the stiffness distributions making minimal or maximal the desired energy contributions, meaning the in-plane variable distribution of the reinforcement fibers' orientation, the fiber volume rate, and the constituent materials.

3.1. Stationary conditions for the strain energy contributions. The basic step for the optimization of the energy absorption is to write the first variation of the strain energy for the zigzag model and to set it to zero, since it represents the equilibrium condition that the optimized solutions must fulfill. This variation is expressed in terms of stress resultants. The readers are referred to the paper by Icardi [1998] for the explicit expressions of the numerous and quite complex stress resultants in terms of the stiffness quantities of the zigzag model and of the continuity functions they involve.

According to the present paper's aim, assume the elastic stiffness coefficients $Q_{i j}$ of each lamina to be functions of position in the lamina $(x, y)$ plane. Since the three principal material directions can have a different orientation point to point, the thermodynamic constraints of Appendix A will need to be checked and, if necessary, enforced in a pointwise sense, as outlined subsequently. Assume the orthotropic relations hold locally at any point.

We list below the stationary conditions for each of the energy contributions of the zigzag model, obtained under variation of the stiffness properties of layers in the $x$ and $y$ directions. The conditions are expressed in terms of stiffness quantities and functional DOF derivatives, using the notation defined 
in Appendix B. The equations can be applied irrespectively of the lay-up (that is, symmetric and nonsymmetric can both be considered), since they are coupled by the large number of coupling stiffness coefficients appearing in Appendix B.

For notational convenience, we set $\eta=h^{-} 1$.

(i) Stationary condition for the bending energy contribution:

$-W R 1 \delta u^{(0)}-W R 2 \delta v^{(0)}-W R 3 \delta w^{(0)}+\left(W R 4-\frac{4}{3} W R 5+W R 6+W R 7\right) \delta \gamma_{x}^{(0)}$

$$
+\left(W R 8-\frac{4}{3} W R 9+W R 10+W R 11\right) \delta \gamma_{y}{ }^{(0)}=0 .
$$

(ii) Stationary condition for the shear energy contribution in the $(x, z)$ plane:

$$
\begin{aligned}
& \left\{X R_{R 1}+X R_{R 1}^{a}+X R_{R 1}^{d}+X R_{R 44}+X R_{R 44}^{a}+X R_{S 44}^{d}-\frac{4}{3} \eta^{2}\left(X R_{P 1}-X R_{P 6}\right)\right. \\
& \left.\quad-\frac{1}{2} \eta\left(X R_{26 X 2}+X R_{31 X 2}+X R_{36 X 2}+X R_{41 X 2}\right)-\frac{2}{3} \eta^{2}\left(X R_{26 X 3}+X R_{31 X 3}+X R_{36 X 3}+X R_{41 X 3}\right)\right\} \delta u^{(0)} \\
& +\left\{X R_{R 2}+X R_{R 2}^{a}+X R_{R 2}^{d}+X R_{R 55}+X R_{R 55}^{a}+X R_{S 55}^{d}-\frac{4}{3} \eta^{2}\left(X R_{P 2}-X R_{P 7}\right)\right. \\
& \left.\quad-\frac{1}{2} \eta\left(X R_{27 X 2}+X R_{32 X 2}+X R_{37 X 2}+X R_{41 X 2}\right)-\frac{2}{3} \eta^{2}\left(X R_{27 X 3}+X R_{32 X 3}+X R_{37 X 3}+X R_{42 X 3}\right)\right\} \delta v^{(0)} \\
& +\left\{X R_{R 3}+X R_{R 3}^{a}+X R_{R 3}^{d}+X R_{R 66}+X R_{R 66}^{a}+X R_{S 66}+X R_{S 66}^{d}-\frac{4}{3} \eta^{2}\left(X R_{P 3}+X R_{P 8}\right)\right. \\
& \left.-\frac{1}{2} \eta\left(X R_{28 X 2}+X R_{33 X 2}+X R_{38 X 2}+X R_{43 X 2}\right)-\frac{2}{3} \eta^{2}\left(X R_{28 X 3}+X R_{33 X 3}+X R_{38 X 3}+X R_{43 X 3}\right)\right\} \delta w^{(0)} \\
& +\left\{X R_{R 4}+X R_{R 4}^{a}+X R_{R 4}^{d}+X R_{R 88}+X R_{R 88}^{a}+X R_{S 88}^{d}-X R_{T 88}-X R_{T 88}^{a}-X R_{T 88}^{d}\right. \\
& \quad-\frac{4}{3} \eta^{2}\left(X R_{P 4}+X R_{P 9}\right)+4 \eta^{2} X R_{R R 1}-\frac{1}{2} \eta\left(X R_{29 X 2}+X R_{34 X 2}+X R_{39 X 2}+X R_{44 X 2}\right) \\
& \left.-\frac{2}{3} \eta^{2}\left(X R_{29 X 3}+X R_{34 X 3}+X R_{39 X 3}+X R_{44 X 3}\right)+\eta\left(X R_{46 X 1}+X R_{48 X 1}\right)+2 \eta^{2}\left(X R_{46 X 2}+X R_{48 X 2}\right)\right\} \delta \gamma_{x}{ }^{(0)} \\
& +\left\{X R_{R 5}+X R_{R 5}^{a}+X R_{R 5}^{d}+X R_{R 99}+X R_{R 99}^{a}+X R_{S 99}^{d}-X R_{T 99}-X R_{T 99}^{a}-X R_{T 99}^{d}\right. \\
& \quad-\frac{4}{3} \eta^{2}\left(X R_{P 5}+X R_{P 10}\right)+4 \eta^{2} X R_{R R 2}-\frac{1}{2} \eta\left(X R_{30 X 2}+X R_{35 X 2}+X R_{40 X 2}+X R_{45 X 2}\right) \\
& \left.-\frac{2}{3} \eta^{2}\left(X R_{30 X 3}+X R_{35 X 3}+X R_{40 X 3}+X R_{45 X 3}\right)+\eta\left(X R_{47 X 1}+X R_{49 X 1}\right)+2 \eta^{2}\left(X R_{47 X 2}+X R_{49 X 2}\right)\right\} \delta \gamma_{y}{ }^{(0)} .
\end{aligned}
$$

(iii) Stationary condition for the shear energy contribution in the $(y, z)$ plane:

$$
\begin{gathered}
\left\{Y R_{R 1}+Y R_{R 1}^{b}+Y R_{R 1}^{b}+Y R_{R 44}+Y R_{R 44}^{b}+Y R_{S 44}^{b}-\frac{4}{3} \eta^{2}\left(Y R_{P 1}-Y R_{P 6}\right)\right. \\
\left.-\frac{1}{2} \eta\left(Y R_{26 Y 2}+Y R_{31 Y 2}+Y R_{36 Y 2}+Y R_{41 Y 2}\right)-\frac{2}{3} \eta^{2}\left(Y R_{26 Y 3}+Y R_{31 Y 3}+Y R_{36 Y 3}+Y R_{41 Y 3}\right)\right\} \delta u^{(0)} \\
+\left\{Y R_{R 2}+Y R_{R 2}^{b}+Y R_{R 2}^{c}+Y R_{R 55}+Y R_{R 55}^{b}+Y R_{S 55}^{c}-\frac{4}{3} \eta^{2}\left(Y R_{P 2}-Y R_{P 7}\right)\right. \\
\left.-\frac{1}{2} \eta\left(Y R_{27 X 2}+Y R_{32 X 2}+Y R_{37 X 2}+Y R_{41 X 2}\right)-\frac{2}{3} \eta^{2}\left(Y R_{27 X 3}+Y R_{32 X 3}+Y R_{37 X 3}+Y R_{42 X 3}\right)\right\} \delta v^{(0)} \\
+\left\{Y R_{R 3}+Y R_{R 3}^{b}+Y R_{R 3}^{c}+Y R_{R 66}+Y R_{R 66}^{b}+Y R_{S 66}+Y R_{S 66}^{c}-\frac{4}{3} \eta^{2}\left(Y R_{P 3}+Y R_{P 8}\right)\right. \\
\left.\quad-\frac{1}{2} \eta\left(Y R_{28 Y 2}+Y R_{33 Y 2}+Y R_{38 Y 2}+Y R_{43 Y 2}\right)-\frac{2}{3} \eta^{2}\left(Y R_{28 Y 3}+Y R_{33 Y 3}+Y R_{38 Y 3}+Y R_{43 Y 3}\right)\right\} \delta w^{(0)} \\
+\left\{Y R_{R 4}+Y R_{R 4}^{b}+Y R_{R 4}^{c}+Y R_{R 88}+Y R_{R 88}^{b}+Y R_{S 88}^{c}-Y R_{T 88}-Y R_{T 88}^{b}-Y R_{T 88}^{c}-\frac{4}{3} \eta^{2}\left(Y R_{P 4}+Y R_{P 9}\right)\right. \\
+4 \eta^{2} Y R_{R R 1}-\frac{1}{2} \eta\left(Y R_{29 Y 2}+Y R_{34 Y 2}+Y R_{39 Y 2}+Y R_{44 Y 2}\right)-\frac{2}{3} \eta^{2}\left(Y R_{29 Y 3}+Y R_{34 Y 3}+Y R_{39 Y 3}+Y R_{44 Y 3}\right) \\
\left.+\eta\left(Y R_{46 Y 1}+Y R_{48 Y 1}\right)+2 \eta^{2}\left(Y R_{46 Y 2}+Y R_{48 Y 2}\right)\right\} \delta \gamma_{x}^{(0)} \\
+\left\{Y R_{R 5}+Y R_{R 5}^{b}+Y R_{R 5}^{c}+Y R_{R 99}+Y R_{R 99}^{b}+Y R_{S 99}^{c}-Y R_{T 99}-Y R_{T 99}^{b}-Y R_{T 99}^{c}-\frac{4}{3} \eta^{2}\left(Y R_{P 5}+Y R_{P 10}\right)\right. \\
+4 \eta^{2} Y R_{R R 2}-\frac{1}{2} \eta\left(Y R_{30 Y 2}+Y R_{35 Y 2}+Y R_{40 Y 2} Y R_{45 Y 2}\right)-\frac{2}{3} \eta^{2}\left(Y R_{30 Y 3}+Y R_{35 Y 3}+Y R_{40 Y 3}+Y R_{45 Y 3}\right) \\
\left.+\eta\left(Y R_{47 Y 1}+Y R_{49 Y 1}\right)+2 \eta^{2}\left(Y R_{47 Y 2}+Y R_{49 Y 2}\right)\right\} \delta \gamma_{y}{ }^{(0)} .
\end{gathered}
$$


The quantities $X R_{i j X 3}, Y R_{i j Y 3}$ (for $i=2$ and $j=6,7,8,9 ; i=3$ and $j=0$ to $9 ; i=4$ and $j=0$ to 9), which are not defined in Appendix B, are obtained in a straightforward way changing Equation (2) with Equation (3) into the definitions therein reported for $X R_{i j X 2}$ and $Y R_{i j Y 2}$.

Homogenizing the virtual variations, so as to convert the derivatives of virtual displacements, the Euler-Lagrange equations making extremal each of the desired strain energy contributions are obtained in the form of partial differential equations in terms of plate stiffness quantities of the laminate.

These extremal equations have to be elaborated in order to collect all the contributions that multiply a specific virtual displacement, since it is arbitrary inside the domain. As a result a rather intricate system of coupled, partial differential equations in terms of the laminate stiffness quantities is obtained.

For practical reasons, since the displacements, the number of layers in the laminate, and the constituent materials are arbitrary, as a preliminary application the optimization procedure is next applied to a single constituent lamina of a laminated or sandwich composite. The problem turns into an extremal problem at the ply level where we try to find optimized plies which, once incorporated in place of preexisting layers, can improve the structural performance of laminated and sandwich composites, whatever the lay-up, boundary conditions, and loading might be.

In the next part of this section, the stiffness distributions which make extremal the bending and shear energy contributions of a single ply will be searched.

3.2. Optimized single ply. An approximate solution, of technical interest, to the resulting set of partial differential equations (1)-(3) for a single constituent ply is the following second order polynomial approximation for the transformed reduced stiffness coefficients,

$$
\begin{aligned}
& Q_{11}=A_{1}+A_{2} x+A_{3} x^{2} \\
& Q_{22}=B_{1}+B_{2} y+B_{3} y^{2} \\
& Q_{12}=C_{1}+C_{2} x+C_{3} y+C_{4} x^{2}+C_{5} y^{2}+C_{6} x y, \\
& Q_{66}=D_{1}+D_{2} x+D_{3} y+D_{4} x^{2}+D_{5} y^{2}+D_{6} x y, \\
& Q_{16}=E_{1}+E_{2} x+E_{3} x^{2}+E_{4} x y \\
& Q_{26}=F_{1}+F_{2} y+F_{3} y^{2}+F_{4} x y, \\
& Q_{44}=G \\
& Q_{55}=L \\
& Q_{45}=M,
\end{aligned}
$$

where $A_{1}, A_{2}, \ldots, B_{1}, \ldots, F_{1}, G, L$, and $M$ are coefficients to be determined by enforcing desired conditions. Although many other conditions could be enforced, those arising from the imposition of means stiffness properties are considered in this paper. In consequence of their enforcement, the mean stiffness of optimized layers is made equal to that of the customary layers they replace. In this way, the comparison between the lay-up with optimized layers and the conventional ones can be carried out keeping the average properties unchanged. In addition, the thermodynamic constraints listed in Appendix A have to be enforced, in order to make the optimized ply a real material [Jones 1999]. Finally, convexity or concavity have to be chosen, in order to minimize or maximize the energy contributions whose variation is set to zero. Note that Equations (4), (5), and (8)-(12) provide an exact solution and identically fulfill 
the thermodynamic constraints, while (6) and (7) are approximate and require these constraints to be enforced. The formulas (6) and (7) exactly fulfill the extremal conditions in the equations where only second order derivatives of the stiffness coefficients appear. However almost all the terms involving third and higher order derivatives vanish for an insulated lamina. As a consequence, the present solution is a suboptimal approximation; it is motivated by the need to find a solution of technical interest, compatible with the current technologies.

In Section 4, the performance of laminates incorporating few layers that minimize the bending strain energy and maximize the transverse shear energy, or vice versa, will be assessed. Since the features of the solution could depend on the model used, the set of governing equations being different from one structural model to another, the performance of the present approximation will be tested using the mixed solid element of Section 2 and, in a few cases, the zigzag plate model and the related energy updating, within the framework of Galerkin's approach. The basic features concerning the modeling of the contact force used in the impact studies of Section 4 will be summarized.

3.3. Modeling of the impulsive loads. The impact problems can be classified according to the impactor velocity (or energy) as a low velocity, an intermediate, a ballistic, or a hyper velocity impact [Davies and Olsson 2004]. A different modeling has to be used in each of these cases.

Although the velocities and the energies indicated as threshold in literature can vary consistently, the researchers agree that for low velocity impacts, the object of this paper, since they are always responsible for a relevant strength degradation even when the so called barely visible impact damage is not evident, the incoming energy is mainly absorbed as strain energy and through local failures. This kind of impact is dominated by delamination and matrix cracking, rather than by penetration induced fiber breakage like in the other cases [Joshi and Sun 1987].

Since the contact duration is higher then the stress waves' lateral transit time, transverse shear waves reflect off the edges several times while the contact load is still being applied. Due to this, the accuracy of the structural modeling, the plate size, and the boundary conditions strongly affect the response. On the contrary, strain rate effects are marginal due to the low speed, so the material properties can be assumed unchanged from the static ones in the low velocity impacts. Although more sophisticated approaches could be used where the impactor and the structure are discretized by three-dimensional finite elements, the contact force can also be successfully simulated simply using modified versions of the Hertzian contact law, provided that the involved parameters are appropriately set either by numerical and analytical techniques, or by experiments. This is what appears in a large number of studies published in the literature [Tan and Sun 1985; Wu and Shyu 1993; Matemilola and Stronge 1995; Yigit and Christoforou 1995; Carvalho and Guedes Soares 1996; Lee et al. 1997; Liou 1997; Choi 2006]. The Hertzian contact law correlates the contact force $\mathscr{F}$ with the indentation depth $\alpha$, by a parameter called the contact stiffness $K_{c}$. The contact region is required to be small, while the vibration of the striking mass and the frictional forces must be negligible. The problem can be divided into three phases: loading, unloading, and reloading. In the present paper, in the loading phase we use the relation $\mathscr{F}(t)=\mathscr{K}_{c} \alpha(t)^{3 / 2}$. The exponent $3 / 2$ was chosen because it appears in very good correlation with a great amount of experimental tests published in the literature for carbon, Kevlar, glass reinforced, and sandwich composites (see the aforementioned relevant samples), while $K_{c}$ needs to be estimated for each case (the target and the projectile materials, etc.). Various techniques for evaluating $K_{c}$ have been proposed, which are either based on analytical 


\begin{tabular}{|l|ccccccc|}
\hline & $\mathrm{E}_{11}$ & $\mathrm{E}_{22}\left(\mathrm{E}_{33}\right)$ & $\mathrm{G}_{12}$ & $\mathrm{G}_{13}$ & $\mathrm{G}_{23}$ & $v_{12}\left(v_{23}\right)$ & Density \\
\hline Faces & 142 & 9.8 & 7.1 & 7.1 & 3.3 & $0.3(0.5)$ & 1617.3 \\
Cores & & $(0.138)$ & & 0.041 & 0.024 & $0.5(0.02)$ & 48 \\
\hline
\end{tabular}

Table 1. Material properties of the sandwich panel, with units of GPa, except for $v$, unitless, and density, in $\mathrm{kg} / \mathrm{m}^{3}$.

and numerical approaches or on direct derivation from laboratory tests. With the advent of recent threedimensional nonlinear finite elements able to accurately simulate the contact problem, the available commercial finite element codes can be conveniently used to determine the relation between force and indentation depth. In the present paper, the finite element code Mecalog Radioss was used for this purpose; the constituent layers were discretized by a three-dimensional meshing, where the fibers and matrix were described by solid elements. As the loading phase ends, a significant permanent indentation takes place even when the composites are relatively thin. In this phase the relative motion between the target structure and projectile changes sign, thus a new law has to be used; in the present paper, we apply the formula, first suggested by Crook [1952], $\mathscr{F}_{(}(t)=\mathscr{F}_{m}\left(\left(\alpha-\alpha_{0}\right) /\left(\alpha_{m}-\alpha_{0}\right)\right)^{q} ._{\mathscr{F}_{m}}$ is the load at which the unload phase starts, $\alpha_{m}$ is the relative indentation depth, both easily determined from the loading phase curve, while $\alpha_{0}$ is the permanent indentation depth, which in the present paper has been estimated by Mecalog Radioss. The exponent $q$ is set to 2.5, because this value fits the experiments presented in the literature for a variety of laminated and sandwich composites. This unloading curve has to be used every time the contact force decreases; moreover, in the event of bounces, a reloading law is also required.

The indentation used in the reloading phase formula is the difference between the permanent and the current one. Because the properties are degraded after the first loading phase, the transverse material properties, and consequently the contact rigidity, change; this is indicated by $\mathscr{K}_{c}$. We use the reloading law $\mathscr{F}(t)=\mathscr{K}_{c^{\prime}}\left(\alpha-\alpha_{0}\right)^{p}$, with the exponent $p$ set to 1.5 , according to experiments. The contact force computed as outlined above is transformed into an equivalent force distributed at the nodes in the finite element discretization.

This simplified approach for modeling the contact force was chosen because it provides results always in a good agreement with the experiments in the case of low velocity impacts, as shown by many researchers [Tan and Sun 1985; Wu and Shyu 1993; Matemilola and Stronge 1995; Yigit and Christoforou 1995; Carvalho and Guedes Soares 1996; Lee et al. 1997; Liou 1997; Choi 2006; Icardi and Zardo 2005; Icardi and Ferrero 2005; 2006a; 2006b; 2006c; 2007a; 2007b; Ferrero and Icardi 2006; 2007; Icardi 2007], and is easy to implement.

The Newmark implicit time integration scheme is used for solving the contact problem, because the explicit time integration schemes need extremely small time steps to be stable. The reader is referred to Icardi and Zardo [2005] for details about this method.

In the former applications [Icardi and Ferrero 2005; 2006a; 2006; 2006b; 2006c; 2007a; 2007b; Ferrero and Icardi 2007; Icardi 2007] this impact model always provided a contact force time history in good agreement with the experiments, and, where available, with the damage detected via ultrasonic inspection. As a further assessment, in this paper the impact problem on a sandwich plate is considered. 
The sample case here examined was formerly investigated by Choi [2006], who assessed the accuracy of the spring element method, with or without considering von Karman nonlinear strains, since locally deformations can be rather large. The material properties for this sample case are reported in Table 1. The sandwich panel has sides of $102 \times 102$, the constituent layers have a thickness of $0.175 \mathrm{~mm}$, the face sheets have a $\left[0^{\circ} / 90^{\circ}\right]$ lay-up, the core is $25.4 \mathrm{~mm}$ thick, and all the edges are fixed. It is impacted by a $1.61 \mathrm{~kg}$ steel sphere with a velocity of $1.2 \mathrm{~m} / \mathrm{s}$. The contact force time history obtained by the present model is reported in Figure 2, where it is compared with that of Choi [2006]. The results from the present model have been obtained either using linear or nonlinear strain displacement relations of the von Karman type, and two structural models, the present zigzag model and the FSDPT model. It appears the accuracy of the zigzag model improved and the effects of nonlinearity on it were negligible. In contrast, the accuracy of the simplified structural model by Choi [2006] is consistently improved using nonlinear strains. Since nonlinearity was shown to have a mild effect on the zigzag model,the next results will refer to linear strains. Many results are available in the literature which confirm that a linear approach is also quite accurate for sandwich composites, because they have a relevant crushing at the impact point only if the face sheets are very thin. Checks made using the model of Goldberg [2001] have shown the strain rate effects to be negligible, due to the low velocity and energy involved in all the sample cases considered; thus these effects will not be accounted for in the next section.

\section{Numerical applications}

The technique for tuning the energy absorption mechanism previously illustrated is now applied to several sample cases of practical interest. The aim is to assess whether it enables a simultaneous improvement

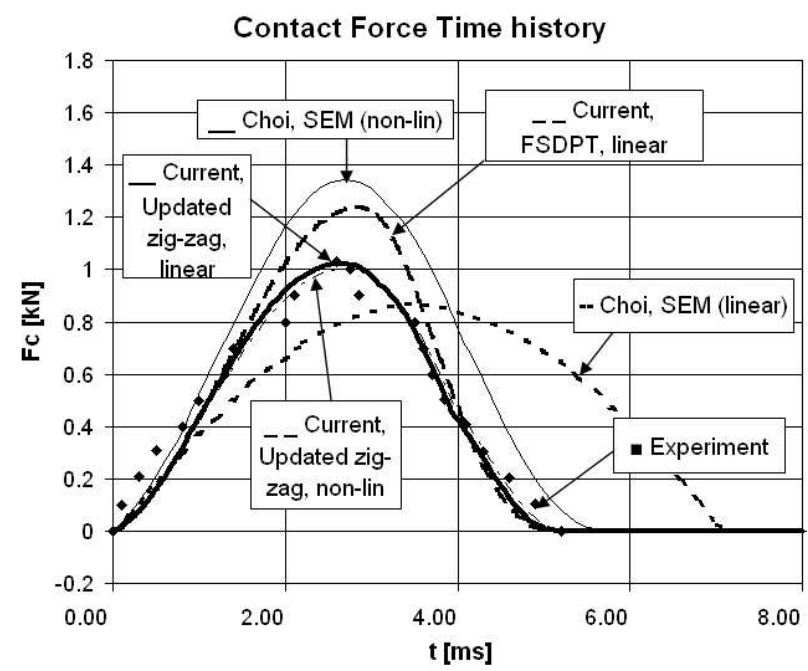

Figure 2. Contact force time history for a sandwich plate. Comparison between experimental test and some numerical results, obtained with five different techniques: the spring element method, linear and nonlinear, by Choi [2006], the current procedure with low refined structural model (FSDPT) and refined model (zigzag), in linear and nonlinear formulation. 
of strength, stiffness and delamination resistance pursuing a magnitude reduction of interlaminar shear stresses, which are partly converted into membrane stresses. So, an advantage of the current technique is to operate on quantities (such as the interlaminar stresses) which affect the delamination strength without reducing beneficial properties such as the stiffness, as occurs with the currently available techniques for improving the delamination strength through use of viscoelastic layers, since they can make too deformable the laminates where they are incorporated. In this technique, the bending, shear, and membrane stiffness coefficients are locally changed, keeping the global stiffness unchanged. In other words, since the current stress based criteria consider the interlaminar stresses of primary importance for delamination, the current energy tuning technique works on these stresses. Whether it can reduce them and whether this fact has beneficial effects over the failure index, it can be intended as a technique for improving delamination. Future studies have to be carried out using much more sophisticated delamination models in order to understand the role played by damping and to better describe the damage process. Since the present technique seems to have relevant advantages, the use of much more accurate, but also computationally more intensive, models seems justified. It is however expected that the current technique will not considerably change the damping, because the variation of the fiber orientation involved occurs in a range where experimental results in the literature show mild variations of damping, and because these variations interest a couple, or very few, layers in a laminate. The loadings of interest here are impact loadings because they cause a variety of local failures and large delaminated zones across the thickness.

The present technique is illustrated through the following specific samples:

- Before applying them to the cases of interest, preliminary tests are presented in the Section 4.1 to assess the present technique and to illustrate its capability. A comparison is presented between a simply supported panel in cylindrical bending, made with a classical metallic material, and its carbon fiber, epoxy resin optimized counterpart. The aim is to limit deflections, although the mean bending stiffness is the same in the two cases.

- In Section 4.2 multilayered, simply supported plates undergoing impact loads are compared. Displacements and stresses of a lay-up with customary constant stiffness constituent layers are compared with those of counterpart plates in which two layers with optimized features and the same mean stiffness of the layers they substitute are incorporated at various positions across the thickness.

- In Section 4.3 a parametric study is reported in order to show the beneficial effects of the optimized lay-ups for some advantageous solutions to the aforementioned problems.

- In Section 4.4 the optimized layers are incorporated in the faces of a multicore sandwich beam in several positions, in order to further test the effectiveness of the present technique. The sandwich beam has two external faces and an internal one, splitting the core in two parts. It is subjected to an impulsive load and its performances are compared to those obtainable with classical layers, in terms of transverse stress field and local failure index.

4.1. Single layer panel. Consider a simply supported, metallic plate in cylindrical bending under a uniformly distributed transverse loading, which will enable us to draw observations of general validity. Let the goal be to minimize the deflection by an optimized composite layer with the same mean properties of the metallic beam. Assume these to correspond to an Al7075 alloy (see Table 2). The equilibrium 


\begin{tabular}{|l|ccccc|}
\hline Type of material & Application & $E_{L}(\mathrm{GPa})$ & $E_{T}(\mathrm{GPa})$ & $G_{L T}(\mathrm{GPa})$ & $v_{L T}$ \\
\hline AL7075 alloy & Isotropic plate & 71.00 & 26.69 & 0.33 & \\
Carbon-HM & Fibers constituent (1) & 380.00 & NA & NA & 0.69 \\
Carbon-A & Fibers constituent (2) & 210.00 & NA & NA & 0.69 \\
Kevlar149 & Fibers constituent (3) & 130.00 & NA & NA & 0.30 \\
Epoxy resin & Matrix constituent (1) & 5.00 & 5.00 & 1.85 & 0.35 \\
Polyethylene & Matrix constituent (2) & 0.05 & 0.05 & 0.02 & 0.30 \\
Carbon-epoxy (1) & Composite ply & 138.00 & 138.00 & 5.99 & 0.28 \\
Carbon-epoxy (2) & Composite ply & 205.00 & 50.00 & 82.00 & 0.25 \\
\hline
\end{tabular}

Table 2. Materials used in the numerical applications of Section 4.

equations are solved using Gelerkin's method and the updating process. The solution that minimizes bending is

$$
D_{11}=6.35 \times 10^{2}+2.0 \times 10^{2} \frac{x}{L}-2.0 \times 10^{2}\left(\frac{x}{L}\right)^{2} .
$$

The higher order stiffness contribution $F_{11}$ is treated in the same way. The terms $D_{22}, D_{12}, D_{16}, D_{26}, D_{66}$, $F_{12}$, etc., are not involved in this problem since a cylindrical deformation is considered. An application to a plate under bidirectional sinusoidal loading which considers these contributions will be presented later. Consider various length to thickness ratios to have an indication of the gain obtainable, defined as the ratio gain $=\left(w_{\max }-w_{\max }^{\mathrm{opt}}\right) /\left(w_{\max }\right), w_{\max }$ being the maximum, or central, deflection of the metallic plate and $w_{\max }^{\mathrm{opt}}$ that of the optimized composite plate. Choose a ratio between the minimum (at the bound) and the maximum (at the center) bending stiffnesses close to zero. The result obtained is that the gain increases till it reaches an asymptote, observed at about $L / h=100$, corresponding to a gain of $18 \%$, which shows that the optimization is always effective in the range of thickness variation of technical interest. The optimized stiffness distribution of Equation (13) could be obtained by varying the thickness of the lamina, or, more interestingly by the practical point of view, by varying the fibers and matrix constituent materials, their relative volume fraction, or varying point by point the orientation of fibers. If the fiber volume fraction is varied, while all the other parameters remain unchanged, the optimal stiffness distribution is obtained as

$$
V_{\text {fibers }}=\left[\frac{h^{3}}{2.20 \times 10^{1}+4.8 \times 10^{4} x / L-4.8 \times 10^{4}(x / L)^{2}} \frac{1}{1-v^{2}}-\frac{1}{E_{m}}\right]\left(\frac{1}{E_{f}}-\frac{1}{E_{m}}\right),
$$

according to the mixture rule, where $V_{\text {fibers }}$ is the volumetric rate of the fibers, while $E_{m}$ and $E_{f}$ are the elastic moduli of the matrix and of the fibers. According to previous considerations, an optimized distribution of stiffness is conveniently obtained using low cost fibers, but a high quality matrix.

Figure 3 shows an assessment of a suboptimal, step distribution of different materials. This simple option appears of technical interest since it decreases deflection by $40 \%$. Furthermore, the results of an analysis by the mixed solid element, not reported here for brevity, show that the local effects at the transition points are mild, although $E_{11}$ of the fibers steeply changes from 40 to $517 \mathrm{GPa}$. This result shows that the use of high quality materials is effective at the center of plates, while at the bounds low quality materials can be used. 


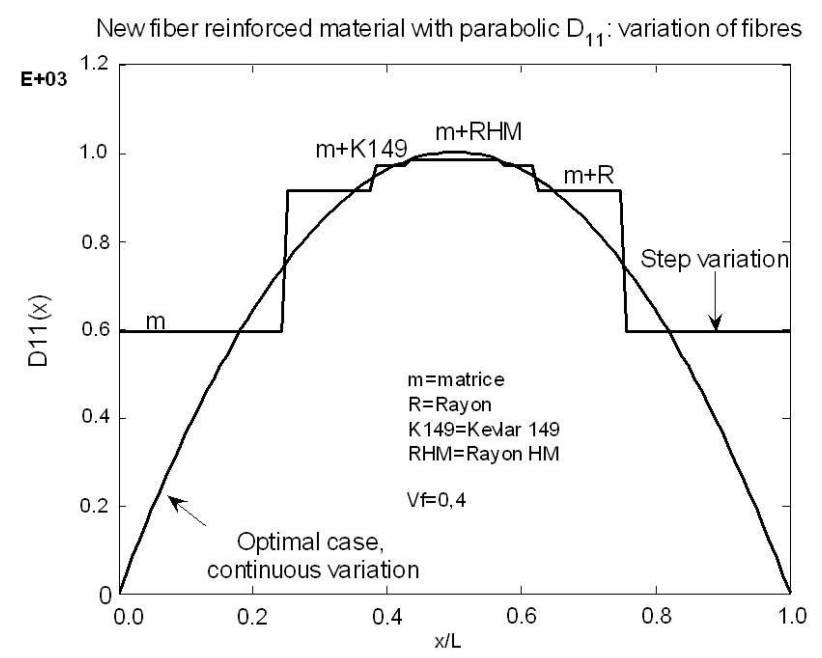

Figure 3. Optimized and step variation of bending stiffness, as obtained by varying the reinforcement fibers.

Here no chemical compatibility considerations are discussed; they are left to a future study. Finally, the optimal orientation $\theta$ of the reinforcement fibers, keeping unchanged the other material parameters, is represented in Figure 4. It features a variation from $0^{\circ}$ to $24^{\circ}$, thus compatible with braiding and filament winding techniques. It can be smoothed at the bound with no remarkable performance loss (less than $3 \%$ ), as numerically assessed.

4.2. Impacted multilayered plate. Consider a panel of length $10 \mathrm{~cm}$, width $5 \mathrm{~cm}$, and thickness $1 \mathrm{~cm}$, with a $\left[45^{\circ} /-45^{\circ} / 0_{2}^{\circ} / 45^{\circ} /-45^{\circ}\right]_{s}$ symmetric and balanced lay-up, which is simply supported at the bounds and subjected to a center point impact. For this panel, the contact force time history measured

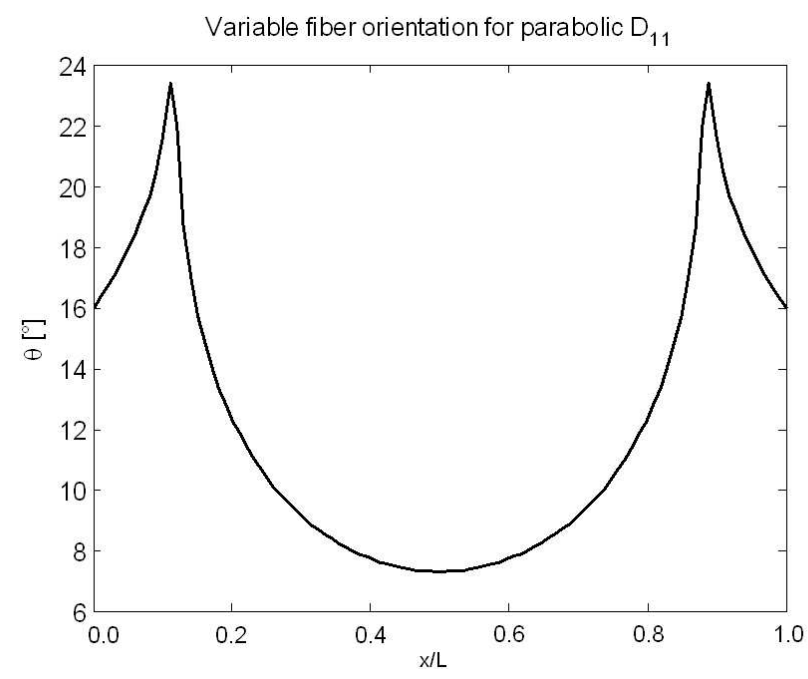

Figure 4. Variable reinforcement fiber orientation for minimizing bending. 


\begin{tabular}{|c|ccccccc|}
\hline Panel & $E_{11}$ & $E_{22}$ & $G_{12}$ & $G_{13}$ & $G_{23}$ & $v_{12}$ & Density \\
& 130 & 8 & 5 & 5 & 2.5 & 0.3 & 1557 \\
\hline \multirow{2}{*}{ Impactor } & & Nose diameter & Mass & Impact energy & $E$ & $v$ & \\
& & 25.4 & 5.451 & 40 & 210 & 0.3 & \\
\hline
\end{tabular}

Table 3. Material properties of the laminated panel and impactor characteristics, with $E$ and $G$ in $\mathrm{GPa}, v$ dimensionless, density in $\mathrm{kg} / \mathrm{m}^{3}$, nose diameter in $\mathrm{mm}$, mass in $\mathrm{kg}$, and impact energy in $\mathrm{J}$.

during an experiment is reported in the papers by Icardi [2005] and Icardi and Zardo [2005], to which the readers are referred for the details here omitted. Being rather thick, this panel has interlaminar stresses nearly of the same magnitude of the membrane stresses, so it is suited for applying the present tailoring optimization. The material properties, the impact energy, and the impactor characteristics are reported in Table 3, while the material strengths are reported in Table 4. The FE discretization is depicted in Figure 1. This sample has to be ascribed to the class of low velocity impacts, for which the strain rate effects

\begin{tabular}{|l|ccccccccc|}
\hline & $X_{t}$ & $Y_{t}$ & \multicolumn{1}{c}{$Z_{t}$} & \multicolumn{1}{c}{$X_{c}$} & $Y_{c}$ & $Z_{c}$ & \multicolumn{1}{c}{$S_{12}$} & \multicolumn{1}{c}{$S_{13}-S_{23}$} & $S_{i}$ \\
\hline A & 0.167 & 0.06 & 0.101 & 0.108 & 0.17 & 0.23 & 0.07 & $0.0700-0.069$ & 0.069 \\
B & 0.210 & 0.074 & 0.074 & 0.11 & 0.18 & 0.24 & 0.086 & $0.0860-0.064$ & 0.064 \\
C & 0.009 & 0.009 & 0.0014 & & & 0.022 & & $0.0086-0.0064$ & 0.064 \\
\hline
\end{tabular}

Table 4. In plane and out-of-plane material strengths, in GPa, for the laminated and sandwich panels. Row $\mathrm{A}$ is the strengths of the laminated panel, row B those of the sandwich panel faces, and row $\mathrm{C}$ those of the sandwich panel cores (foam).

\begin{tabular}{|ccccccccc|}
\hline Lam 0 & Lam A & Lam B & Lam C & Lam D & Lam E & Lam F & Lam G & Lam H \\
\hline 45 & $\mathrm{a}$ & $\mathrm{a}$ & $\mathrm{c}$ & $\mathrm{c}$ & 45 & 45 & 45 & $\mathrm{a}$ \\
-45 & $\mathrm{c}$ & $\mathrm{a}$ & $\mathrm{a}$ & $\mathrm{c}$ & -45 & -45 & -45 & -45 \\
0 & 0 & 0 & 0 & 0 & $\mathrm{a}$ & $\mathrm{a}$ & 0 & $\mathrm{~b}$ \\
0 & 0 & 0 & 0 & 0 & $\mathrm{c}$ & $\mathrm{a}$ & 0 & 0 \\
45 & 45 & 45 & 45 & 45 & 45 & 45 & $\mathrm{a}$ & 45 \\
-45 & -45 & -45 & -45 & -45 & -45 & -45 & $\mathrm{a}$ & $\mathrm{a}$ \\
-45 & -45 & -45 & -45 & -45 & -45 & -45 & $\mathrm{a}$ & $\mathrm{a}$ \\
45 & 45 & 45 & 45 & 45 & 45 & 45 & $\mathrm{a}$ & 45 \\
0 & 0 & 0 & 0 & 0 & $\mathrm{c}$ & $\mathrm{a}$ & 0 & 0 \\
0 & 0 & 0 & 0 & 0 & $\mathrm{a}$ & $\mathrm{a}$ & 0 & $\mathrm{~b}$ \\
-45 & $\mathrm{c}$ & $\mathrm{a}$ & $\mathrm{a}$ & $\mathrm{c}$ & -45 & -45 & -45 & -45 \\
45 & $\mathrm{a}$ & $\mathrm{a}$ & $\mathrm{c}$ & $\mathrm{c}$ & 45 & 45 & 45 & $\mathrm{a}$ \\
\hline
\end{tabular}

Table 5. Stack-ups considered in the problem of Figure 8. 

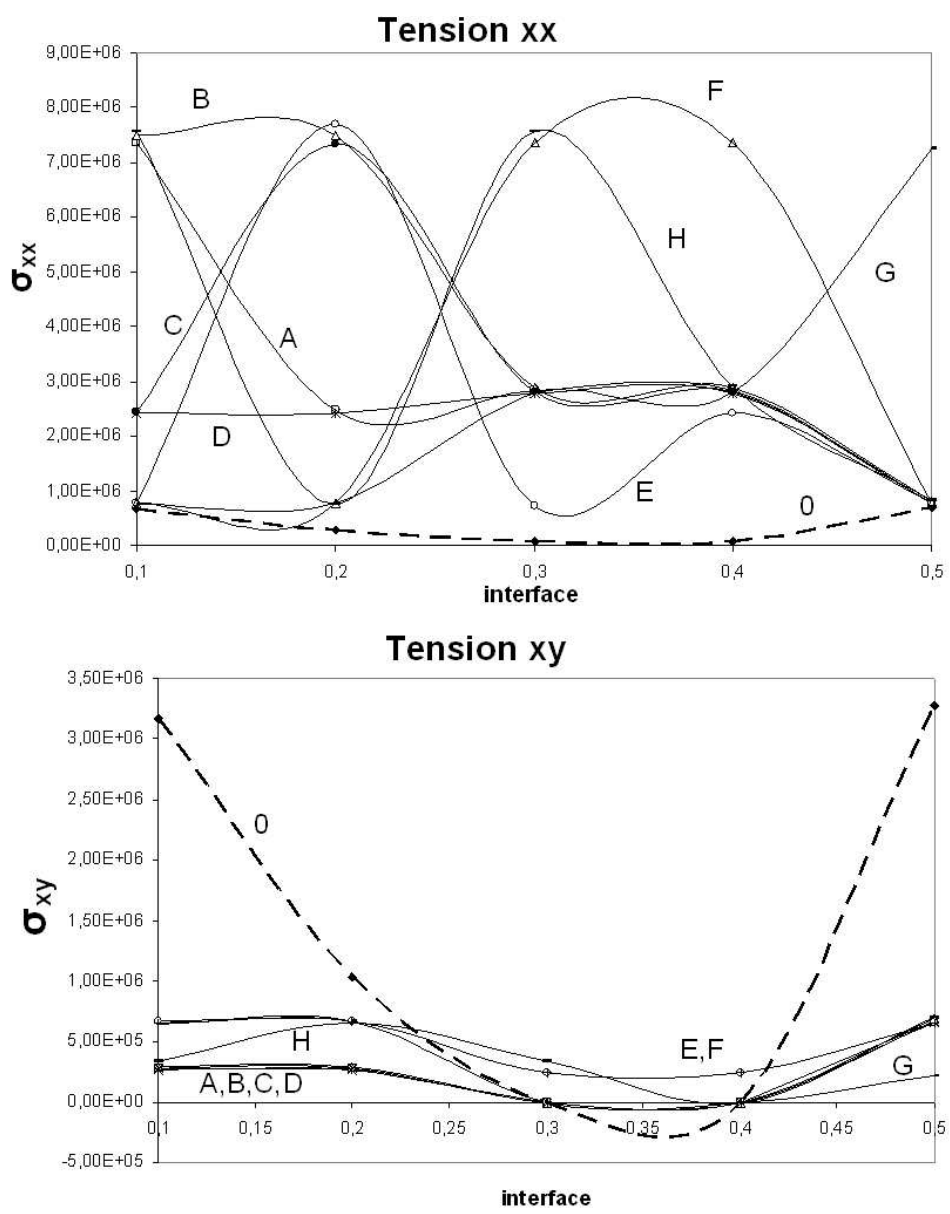

Figure 5. Panels subjected to an impact force: lay-ups considered, membrane stress, and in-plane shear stress. The dashed line shows unoptimized lay-up.

are negligible, as universally agreed by the most representative authors in the field. To assess whether the use of optimized layers can beneficially modify unwise stress fields, we will compare its structural performance with those of plates obtained substituting a couple of layers with optimized layers, in various positions across the thickness. The contact force is recomputed for these stack-ups using the approach outlined above. Consider three kinds of optimized layers:

(i) A layer that minimizes bending and maximizes membrane energy,

(ii) A layer that minimizes bending and maximizes transverse shear,

(iii) A layer that maximizes bending and minimizes transverse shear.

In any case, the mean stiffness properties are maintained equal to those of the preexisting layers that are replaced with these optimized layers.

Type (a) is obtainable with the optimization technique shown in Section 3.1, acting on bending and membrane energy first variations so that an energy transfer takes place from the bending mode (the 
unwanted one) to the membrane mode (the preferred one). Types (b) and (c) have been formulated with the same technique, but the energy contributions involved are now the bending and the transverse shear modes. In the first case the energy is transferred form bending to shear, while in the second case the transfer takes place in the opposite direction. The choice of these layers has specific reasons, which we will explain subsequently, since each optimized layer has some beneficial effects on the panel performances, depending on the type and the position in the stack-up.

Now consider the effects on the impact behavior of substituting the preexisting layers in the $\left[45^{\circ} /-45^{\circ}\right.$ $\left./ 0_{2}^{\circ} / 45^{\circ} /-45^{\circ}\right]_{s}$ laminate with optimized layers (types (a) and (c)). Incorporate symmetric layers of the previously described types across the thickness of the laminate, according to the scheme of Table 5. In this first example, the transfer from shear to membrane energy should be underlined, so the graphs shown in Figure 5 concern membrane stresses (they are just plotted in the $x$ direction) and in-plane shear. The dashed line represents the unoptimized panel (for example, Lam 0), while a label describes which laminate has been considered (for example, from Lam A to Lam G); the stresses have been calculated at each internal interface, at the impact point. Every optimized laminate shows an higher membrane stress field, but a lower in-plane shear field, especially at the most critical interface, the outer internal one. Similar observations can be drawn for the $y$ direction.

Now consider a plate with the same dimensions of the previous one and the lay-up $\left[0^{\circ} / 45^{\circ} / 90^{\circ} /-45^{\circ}\right.$ $\left./ 0^{\circ}\right]_{s}$. Consider in this case layers (a), (b), and (c) one type at a time, and incorporate them according to the scheme presented in Table 6 . Figure 6 shows four plots pertaining to the center deflection, the transverse normal stress across the thickness, and the transverse shear stresses $\sigma_{x z}$ and $\sigma_{y z}$ in the spanwise direction, respectively, when the two outer plies are substituted with minimum bending layers of type (a). In this case, the effect of incorporating optimized layers is to strongly reduce deflections and interlaminar shears. In addition, it increases the membrane stresses (which help to suppress the damage spreading, like in pressurized structures) and the in-plane shear, but these results are here omitted for length, since their variations are not remarkable.

\begin{tabular}{|c|c|c|c|c|c|c|c|c|c|c|c|c|c|c|c|}
\hline Basic & Var1a & $1 b$ & $1 \mathrm{c}$ & Var2a & $2 b$ & $2 c$ & Var3a & $3 b$ & $3 c$ & Var4a & $4 b$ & $4 c$ & Var5a & $5 b$ & $5 c$ \\
\hline $0^{\circ}$ & Type a & $\mathrm{b}$ & $\mathrm{c}$ & & & & & & & & & & & & \\
\hline $45^{\circ}$ & & & & Type a & $\mathrm{b}$ & $\mathrm{c}$ & & & & & & & & & \\
\hline $90^{\circ}$ & & & & & & & Type a & $\mathrm{b}$ & $\mathrm{c}$ & & & & & & \\
\hline$-45^{\circ}$ & & & & & & & & & & Type a & $\mathrm{b}$ & $\mathrm{c}$ & & & \\
\hline $0^{\circ}$ & & & & & & & & & & & & & Type a & $\mathrm{b}$ & $\mathrm{c}$ \\
\hline $0^{\circ}$ & & & & & & & & & & & & & Type a & $\mathrm{b}$ & $\mathrm{c}$ \\
\hline$-45^{\circ}$ & & & & & & & & & & Type a & $\mathrm{b}$ & $\mathrm{c}$ & & & \\
\hline $90^{\circ}$ & & & & & & & Type a & $\mathrm{b}$ & $\mathrm{c}$ & & & & & & \\
\hline $45^{\circ}$ & & & & Type a & $\mathrm{b}$ & $\mathrm{c}$ & & & & & & & & & \\
\hline $0^{\circ}$ & Type a & $\mathrm{b}$ & c & & & & & & & & & & & & \\
\hline
\end{tabular}

Table 6. Stack-ups considered for the impact problem. Blank cases represent unchanged data with respect to the basic case with constant stiffness properties. Optimized layers are: type (a) with minimum bending and maximum membrane; type (b) with minimum bending and maximum shear; and type (c) with maximum bending and minimum shear. 

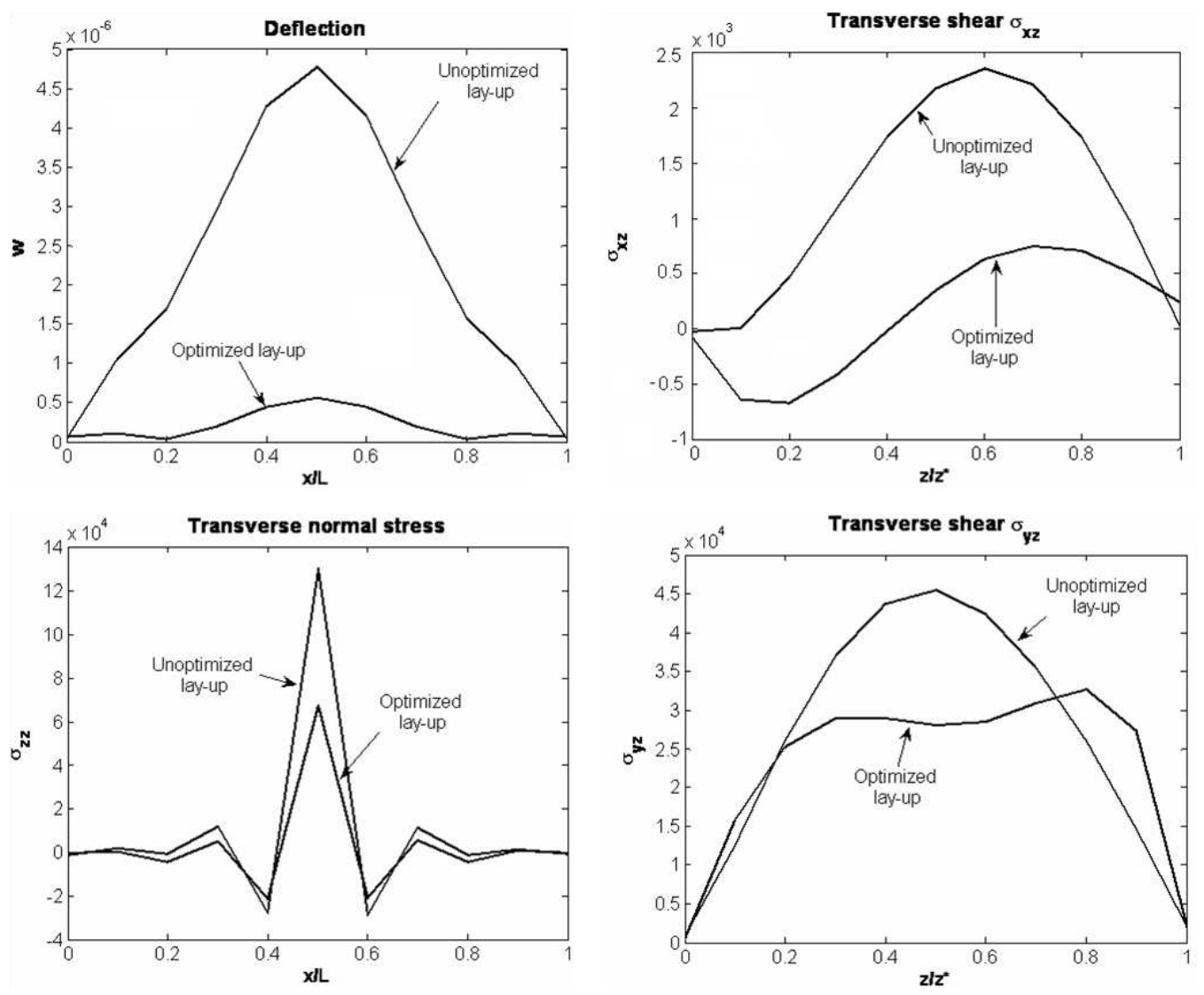

Figure 6. Deflection, transverse normal stress, and shear in the spanwise direction, and transverse shear across the thickness when the two outer plies are substituted with two minimum bending plies (type (a)). Comparison with the unoptimized lay-up.

The impact induced damage for this panel is computed at each interface nearby the impact point using three different stress based delamination criteria: the Hou, Petrinic, Ruiz, and Hallet criteria [Hou et al. 2000; 2001]; the Choi-Chang criteria [Choi and Chang 1992]; and the Chai-Gädke criteria [Chai and Gädke 1999]. These criteria have been chosen because they have found a number of successful applications in the literature to solution of this problem, and because they are easy to implement into finite element codes. To be concise, the failure index formulae are not reported. They can be found in [Icardi et al. 2007], to which the readers are referred also for a discussion of available delamination criteria. It is reminded that the stress based criteria are held by the leading scientists in this field to be accurate enough for predicting the delamination onset, but not the delamination growth. In the latter case, accurate results can be obtained only using fracture mechanics or progressive delamination models. The Choi-Chang criterion, which makes use of in situ properties, appears to be the most accurate criterion in all the published applications. Thus it will be used in all the numerical applications of this paper. In this section, the criterion of [Hou et al. 2000; 2001] will be also used, while in Section 4.4, the criterion of Chai-Gädke, which also makes use of in situ properties, is used. These additional criteria are considered 
in order to see whether the proposed technique is effective, irrespective of the criteria used, and whether there can be a relevant variation of the strength at the onset of delamination using different criteria.

The strength at the onset of delamination of the basic panel with constant stiffness layers is compared to fifteen variations of it, which are obtained by replacing the preexisting layers with optimized layers, according to the scheme reported in Table 6 (for example, var1a, var $1 \mathrm{~b}, \ldots$, var5a, . , var5c, and layers (a), (b), and (c)). Table 7 reports the results by the Choi-Chang criterion, while Figure 7 presents those by the Hou, Petrinic, Ruiz, and Hallet criteria. It appears by these results that the effects on the impact induced delamination are beneficial across the thickness of embodying the optimized layers. It appears that the majority of the solutions embodying optimized panels bring significant improvement in the impact resistance with respect to the classical lay-up. It appears that improvements are obtained especially at the most critical interfaces.

4.3. Parametric study for the laminated plate. Finally, a parametric study about the effects of variation of thickness and stack-up is presented. This analysis is aimed at defining what thickness range allows the best exploitation of the optimized layers and which position in the stacking sequence grants more evident effects. Let first focus on the thickness. Figures 8 and 9 report the results pertaining a variation of the overall thickness from 0.5 to $2 \mathrm{~cm}$, with the width fixed at a value of $20 \mathrm{~cm}$. In Figure 8 the deflection in the unoptimized plate and in the optimized plate varla (refer to Table 6) are compared; the effects of the optimization is more evident for thin structures, but it is always effective for the deflection control. Figure 9 shows the transverse normal stress in the unoptimized plate and in the plate varlb; it appears that the beneficial effects of optimized plies are evident in the central region, while near the edges the tensional field is approximately unchanged for thick samples; on the contrary, the thin plates show lower

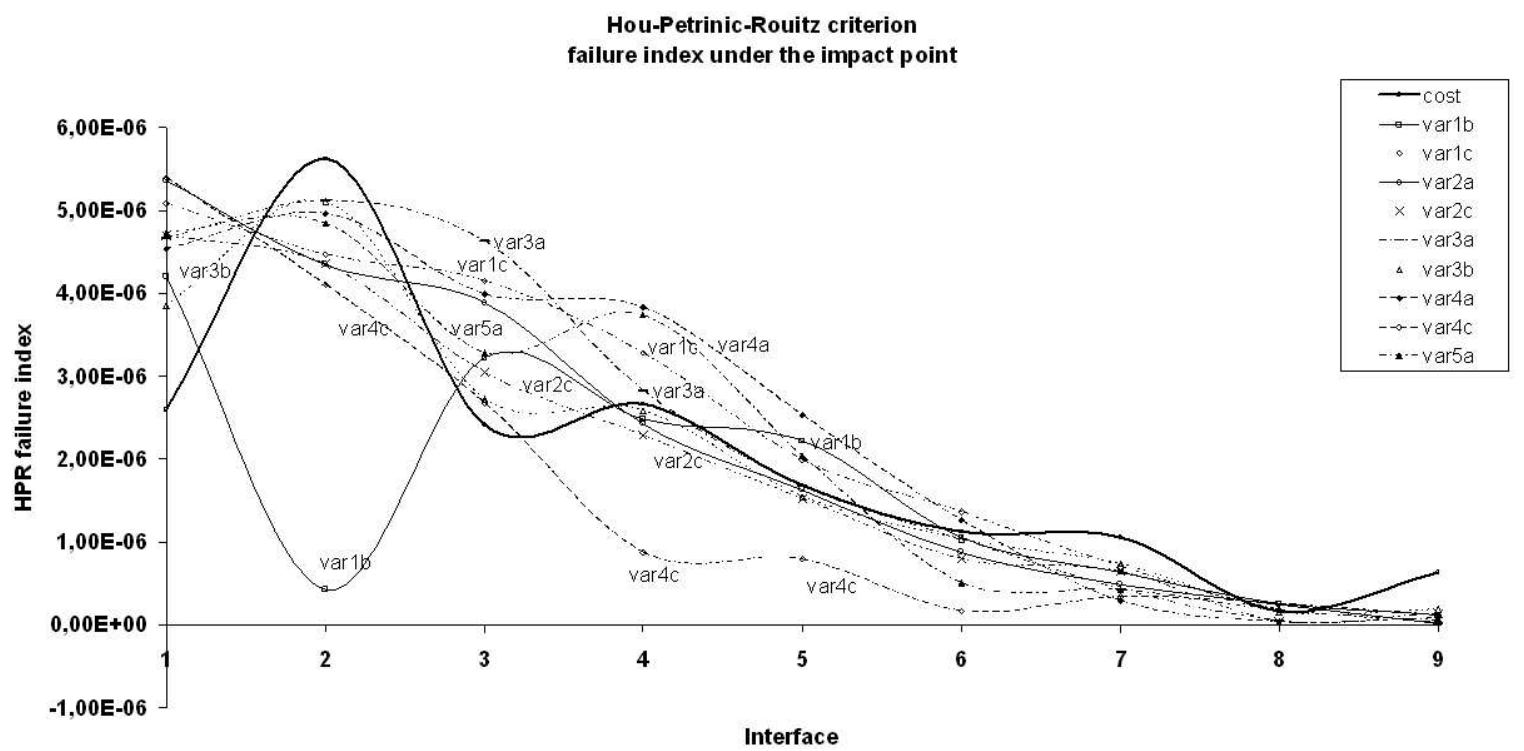

Figure 7. Failure index (dimensionless) according to the Hou-Petrinic-Ruiz criterion, at each interface, for the unoptimized lay-up (thick line) and for its variants, as defined in Table 7 (only the improved cases are reported). 


\begin{tabular}{|c|ccccccccc|}
\hline Case & 1 & 2 & 3 & 4 & 5 & 6 & 7 & 8 & 9 \\
\hline Basic & $1.10 \mathrm{E}-08$ & $5.56 \mathrm{E}-08$ & $1.22 \mathrm{E}-07$ & $2.18 \mathrm{E}-07$ & $2.62 \mathrm{E}-07$ & $2.44 \mathrm{E}-07$ & $2.15 \mathrm{E}-07$ & $1.41 \mathrm{E}-07$ & $4.92 \mathrm{E}-08$ \\
var1a & $3.93 \mathrm{E}-08$ & $1.15 \mathrm{E}-07$ & $1.07 \mathrm{E}-07$ & $1.23 \mathrm{E}-07$ & $1.25 \mathrm{E}-07$ & $1.09 \mathrm{E}-07$ & $1.05 \mathrm{E}-07$ & $8.87 \mathrm{E}-08$ & $5.49 \mathrm{E}-08$ \\
var1b & $1.80 \mathrm{E}-08$ & $7.42 \mathrm{E}-08$ & $1.25 \mathrm{E}-07$ & $1.98 \mathrm{E}-07$ & $2.32 \mathrm{E}-07$ & $2.14 \mathrm{E}-07$ & $1.92 \mathrm{E}-07$ & $1.37 \mathrm{E}-07$ & $6.06 \mathrm{E}-08$ \\
var1c & $4.95 \mathrm{E}-09$ & $7.17 \mathrm{E}-09$ & $1.29 \mathrm{E}-07$ & $3.48 \mathrm{E}-07$ & $4.38 \mathrm{E}-07$ & $4.12 \mathrm{E}-07$ & $3.63 \mathrm{E}-07$ & $1.62 \mathrm{E}-07$ & $4.65 \mathrm{E}-98$ \\
var2a & $1.07 \mathrm{E}-09$ & $5.41 \mathrm{E}-08$ & $1.71 \mathrm{E}-07$ & $1.90 \mathrm{E}-07$ & $1.94 \mathrm{E}-07$ & $1.73 \mathrm{E}-07$ & $1.63 \mathrm{E}-07$ & $1.40 \mathrm{E}-07$ & $3.91 \mathrm{E}-08$ \\
var2b & $7.45 \mathrm{E}-09$ & $5.52 \mathrm{E}-08$ & $1.34 \mathrm{E}-07$ & $2.16 \mathrm{E}-07$ & $2.44 \mathrm{E}-07$ & $2.23 \mathrm{E}-07$ & $1.95 \mathrm{E}-07$ & $1.24 \mathrm{E}-07$ & $4.13 \mathrm{E}-08$ \\
var2c & $4.84 \mathrm{E}-08$ & $1.18 \mathrm{E}-07$ & $1.36 \mathrm{E}-07$ & $2.14 \mathrm{E}-07$ & $2.22 \mathrm{E}-07$ & $1.95 \mathrm{E}-07$ & $1.41 \mathrm{E}-07$ & $5.55 \mathrm{E}-08$ & $1.55 \mathrm{E}-08$ \\
var3a & $6.80 \mathrm{E}-09$ & $1.26 \mathrm{E}-08$ & $9.37 \mathrm{E}-08$ & $2.80 \mathrm{E}-07$ & $2.92 \mathrm{E}-07$ & $2.69 \mathrm{E}-07$ & $2.57 \mathrm{E}-07$ & $1.02 \mathrm{E}-07$ & $9.27 \mathrm{E}-09$ \\
var3b & $1.38 \mathrm{E}-08$ & $6.57 \mathrm{E}-08$ & $1.22 \mathrm{E}-07$ & $1.99 \mathrm{E}-07$ & $2.36 \mathrm{E}-07$ & $2.16 \mathrm{E}-07$ & $1.85 \mathrm{E}-07$ & $1.24 \mathrm{E}-07$ & $4.53 \mathrm{E}-08$ \\
var3c & $9.36 \mathrm{E}-08$ & $3.58 \mathrm{E}-07$ & $3.85 \mathrm{E}-07$ & $2.73 \mathrm{E}-07$ & $1.67 \mathrm{E}-07$ & $8.55 \mathrm{E}-08$ & $3.64 \mathrm{E}-08$ & $1.86 \mathrm{E}-08$ & $7.54 \mathrm{E}-09$ \\
var4a & $4.68 \mathrm{E}-09$ & $1.46 \mathrm{E}-08$ & $2.07 \mathrm{E}-08$ & $2.38 \mathrm{E}-07$ & $6.20 \mathrm{E}-07$ & $5.96 \mathrm{E}-07$ & $2.48 \mathrm{E}-07$ & $4.21 \mathrm{E}-08$ & $1.69 \mathrm{E}-08$ \\
var4b & $1.03 \mathrm{E}-08$ & $5.47 \mathrm{E}-08$ & $1.31 \mathrm{E}-07$ & $2.22 \mathrm{E}-07$ & $2.52 \mathrm{E}-07$ & $2.47 \mathrm{E}-07$ & $2.09 \mathrm{E}-07$ & $1.31 \mathrm{E}-07$ & $4.66 \mathrm{E}-08$ \\
var4c & $1.12 \mathrm{E}-07$ & $5.33 \mathrm{E}-07$ & $6.27 \mathrm{E}-07$ & $3.87 \mathrm{E}-07$ & $2.02 \mathrm{E}-07$ & $4.47 \mathrm{E}-08$ & $1.34 \mathrm{E}-08$ & $1.90 \mathrm{E}-09$ & $4.65 \mathrm{E}-09$ \\
var5a & $7.10 \mathrm{E}-09$ & $3.09 \mathrm{E}-08$ & $5.93 \mathrm{E}-08$ & $1.15 \mathrm{E}-07$ & $4.97 \mathrm{E}-07$ & $4.77 \mathrm{E}-07$ & $1.20 \mathrm{E}-07$ & $8.61 \mathrm{E}-08$ & $3.32 \mathrm{E}-08$ \\
var5b & $1.01 \mathrm{E}-08$ & $5.38 \mathrm{E}-08$ & $1.19 \mathrm{E}-07$ & $2.17 \mathrm{E}-07$ & $2.67 \mathrm{E}-07$ & $2.46 \mathrm{E}-07$ & $2.13 \mathrm{E}-07$ & $1.43 \mathrm{E}-07$ & $5.02 \mathrm{E}-08$ \\
var5c & $1.37 \mathrm{E}-07$ & $7.59 \mathrm{E}-07$ & $9.53 \mathrm{E}-07$ & $4.81 \mathrm{E}-07$ & $1.13 \mathrm{E}-07$ & $1.20 \mathrm{E}-07$ & $2.76 \mathrm{E}-08$ & $3.80 \mathrm{E}-08$ & $5.18 \mathrm{E}-08$ \\
\hline
\end{tabular}

Table 7. Effects of optimized layer incorporation on delamination, according to the Choi-Chang criterion. Failure index (dimensionless) computed at each interface (the cases in the table) at the impact point for the basic panel with $\left[0^{\circ} / 45^{\circ} / 90^{\circ} /-45^{\circ} / 0^{\circ}\right]_{s}$ lay-up and fifteen variants embodying optimized layers.
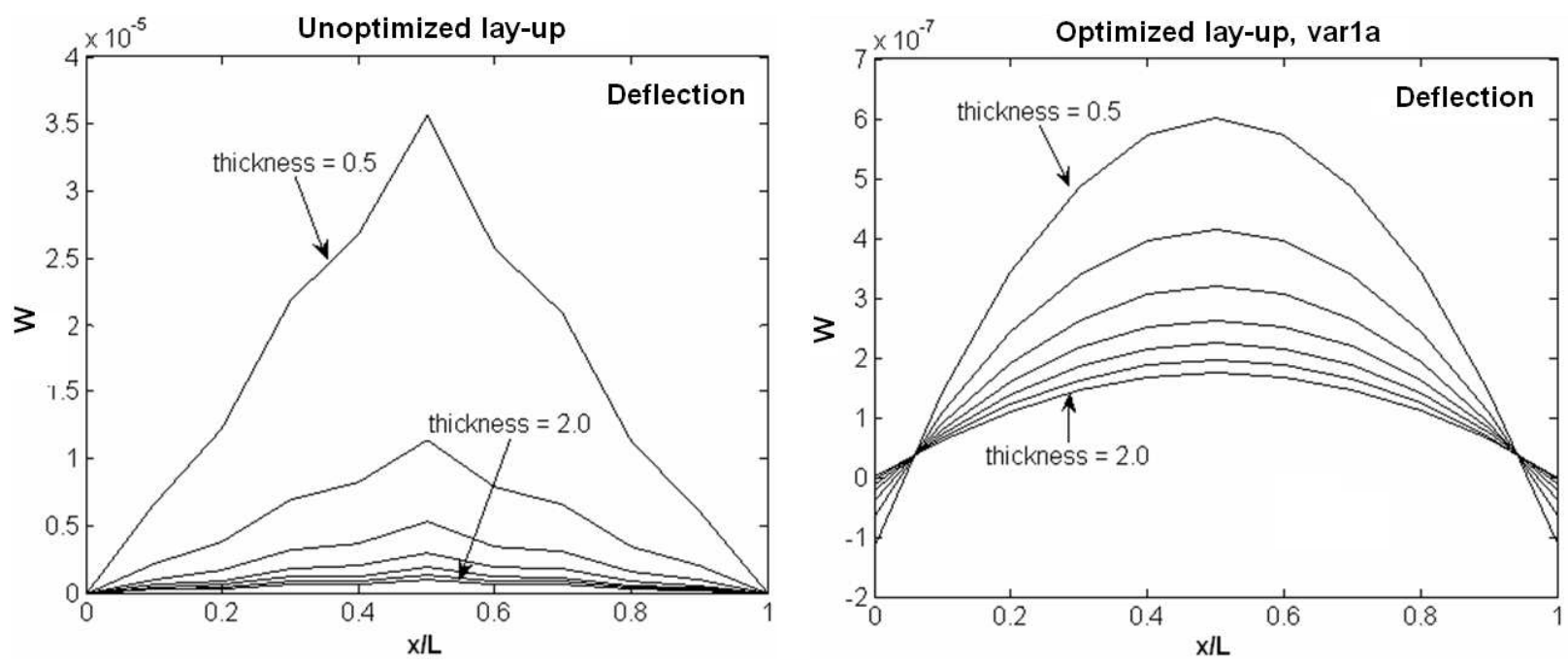

Figure 8. Deflection in the unoptimized plate and in the optimized plate (var1a), with increasing the total thickness. 

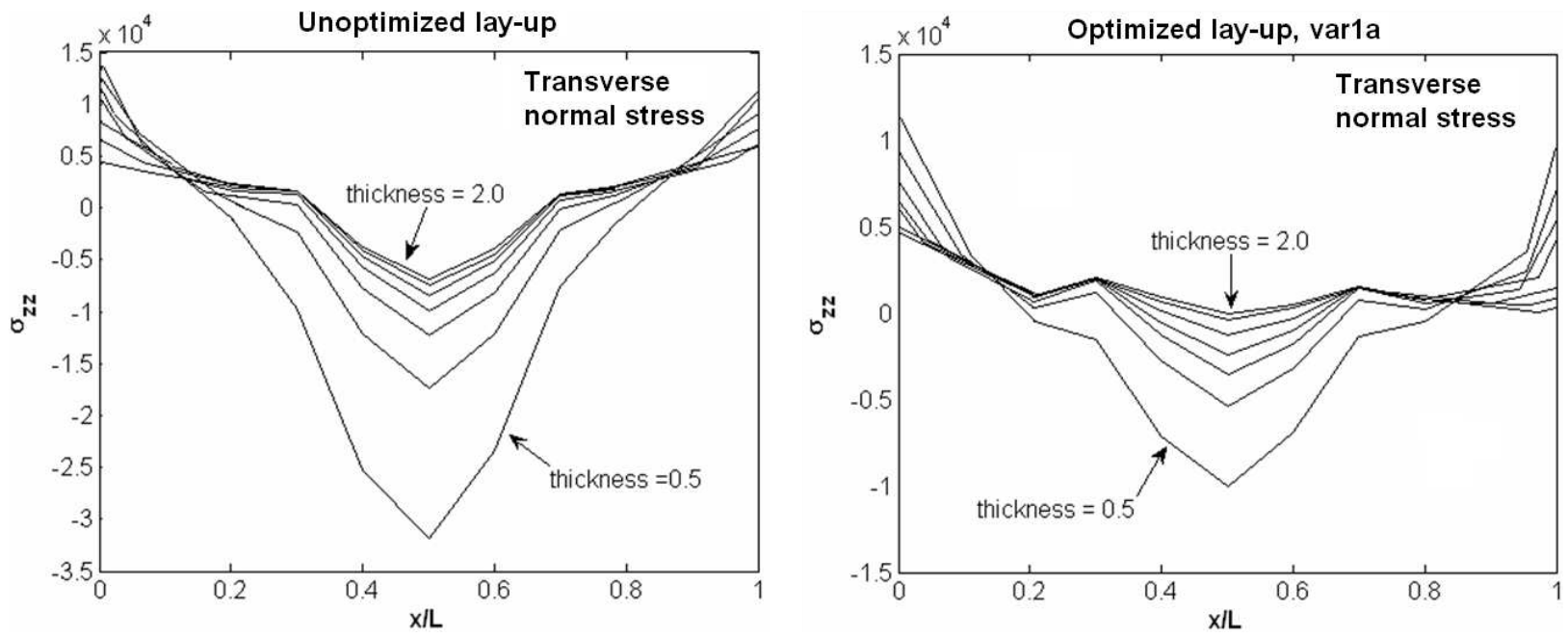

Figure 9. Transverse normal stress in the unoptimized plate and in the optimized plate (varlb) with increasing the total thickness.

tensional field also in the edges. Since the central region is the most damaged part, it can be guessed that the optimized (type (b)) layers are able to cut down the transverse normal stress.

Some considerations are now made about the influence of the stack-up. An extensive study has been performed in order to estimate the coupling effects, and it is possible to state that optimized structures show a good behavior even in nonsymmetric stack-ups. Moreover symmetric stack-ups are able to fulfill alternatively one of the two discussed aims, either the deflection control or the impact resistance improvement, with high gain compared to the classical plate. For this purpose, in Figure 10 four cases

Transverse shear Sxz

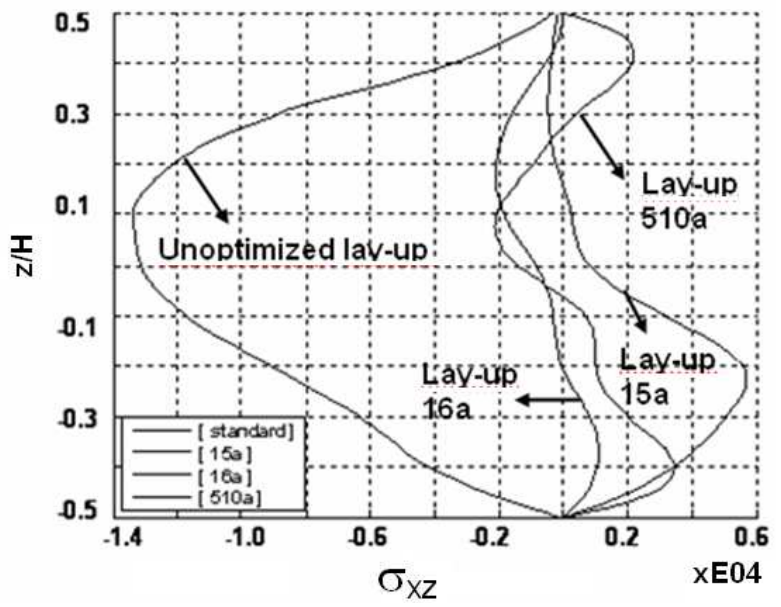

XE05

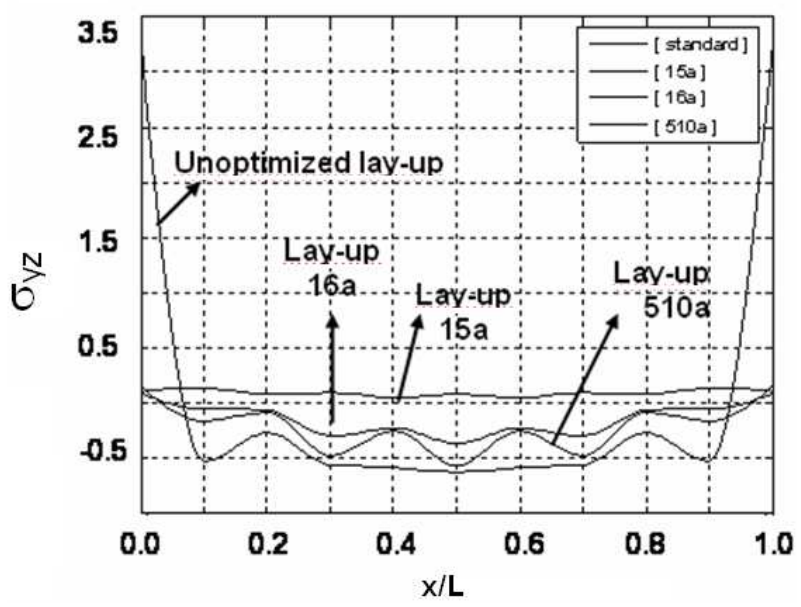

Figure 10. Transverse shear stress across the thickness and in the spanwise direction for the unoptimized plate and for three nonsymmetric optimized lay-ups. 
are explored. The unoptimized plate, previously studied, is now subjected to a patch load in the four central nodes; starting from this basic stack-up, minimum shear plies have been introduced in positions 1 and 5, 1 and 6, and 5 and 10, in nonsymmetric lay-ups. In the transverse shears plotted against both directions, the classical plate develops the worst stress field. Meanwhile asymmetric stack-ups allow us to fulfill both the requirements, deflection reduction and impact resistance improvement, with quite good improvement compared to the unoptimized plates performances, but lower than what is obtainable with symmetric ones. In particular, across the thickness the optimized panels develop a lower shear field in the central region, where the unoptimized one reaches its maximum. Similarly, if the transverse shear is observed in the spanwise direction, at the most critical interface, the beneficial effects of the current technique is particularly clear near the edges, where the classical plate has a stress concentration. However, the most important result underlined in the present section is the good behavior of the optimized layers also when they are introduced in nonsymmetric positions, thanks to their capability to decouple bending and shear deformations.

4.4. Impacted sandwich beam. The optimization process was above applied to laminates in order to assess the advantages in terms of impact strength, deflection, and interlaminar stress distributions. Now the optimization process is be applied to sandwich composites with laminated faces, which exhibit a critical impact behavior due to their high thickness and to the deep heterogeneity of their constituent materials. The aim of this section is to investigate the potential improvement of sandwich structures' critical behavior due to the introduction of the optimized plies. The sample here considered is a multicore sandwich structure made of two external faces, an internal one, and two cores (mechanical properties given in Table 1), which is clamped at the edges and impacted at the center with a steel sphere with a mass of $1.61 \mathrm{~kg}$ at speed $1.2 \mathrm{~m} / \mathrm{s}$. A $20 \times 1 \mathrm{~cm}$ sandwich beam made up of 4 ply external faces, a 2 ply internal face, and two cores is considered. Every ply is $0.025 \mathrm{~cm}$ thick, while the cores are $0.5 \mathrm{~cm}$ thick, thus the overall thickness is $1.3 \mathrm{~cm}$. Stack-up and geometry are described in Figure 11. The FE discretization is reported in Figure 1. Since this sample is still referring to a low velocity impact, again the strain rate effects are negligible. The optimized plies incorporated are minimum shear layers with properties which vary in the spanwise direction according to the law of Figure 12, where also the ratio between optimized and mean stiffnesses is plotted. As a first step, the transverse deflection is assessed, since it could have potential beneficial effects on the noise and vibration behavior. As shown in Figure 13, the transverse deflection can be radically cut off when optimized minimum shear layers replace classical plies, with a maximum gain of $35 \%$ when all the $0^{\circ}$ plies are substituted. In addition, the interlaminar stresses should be monitored, in order to define whether the optimization is effective for enhancing the impact strength of sandwich plates. Looking at Figure 14, the transverse shear stress under the impact point can be compared among the three analyzed beams; it can be reckoned a strong reduction in the transverse shear, especially at the most critical interfaces, while the transverse normal stress is slightly increased, as shown in Figure 15. The transverse shear field appears to be lowered by the optimized ply introduction, since these plies appear able to minimize the energy stored in the shear mode and of maximize the bending and membrane mode. This action can be seen as an energy transfer from the unwanted out-of-plane modes to the membrane mode, as already occurred for the sample case of Section 4.2. Finally, the impact induced damage is estimated by computing stress based failure indexes close to the impact point. For this sample case the criteria of Choi-Chang, already used in Section 4.2, and those 


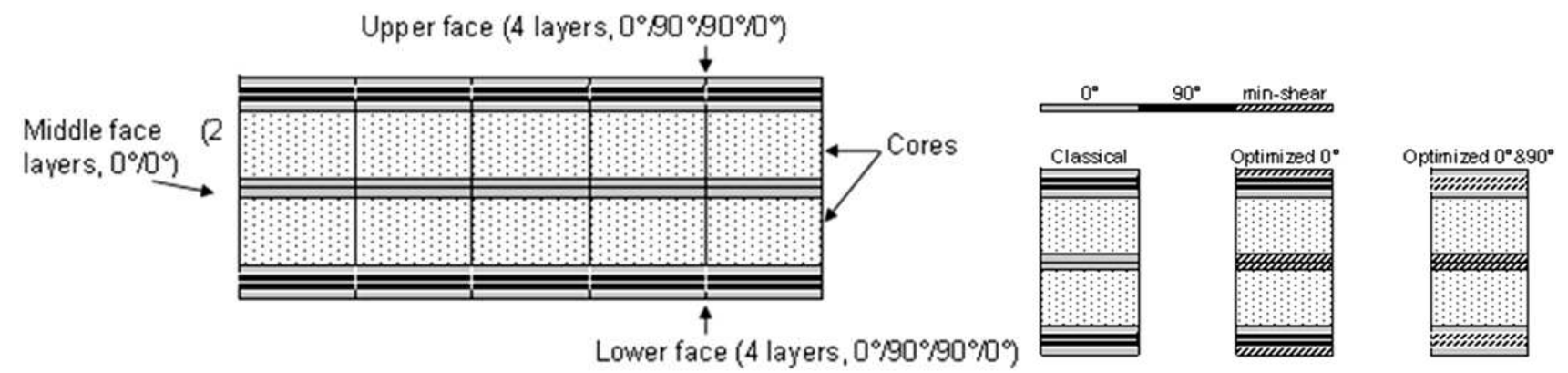

Figure 11. Geometry and stack-up of the dual-core sandwich beam; classical case with constant stiffness plies, optimized cases with minimum shear plies introduced in place of classical ones.

$$
Q_{\text {opt }} / Q_{\text {mean }}
$$

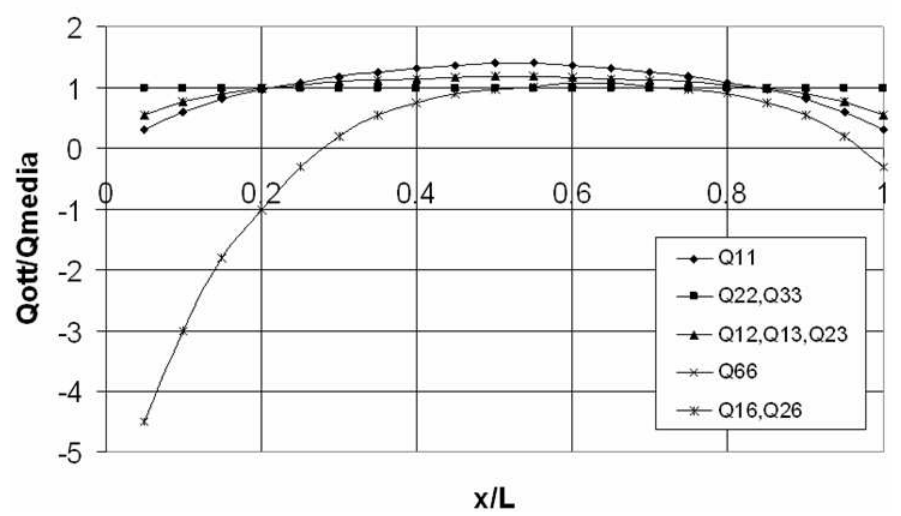

Figure 12. Stiffness law of variation in the spanwise direction for the minimum shear optimized layers.

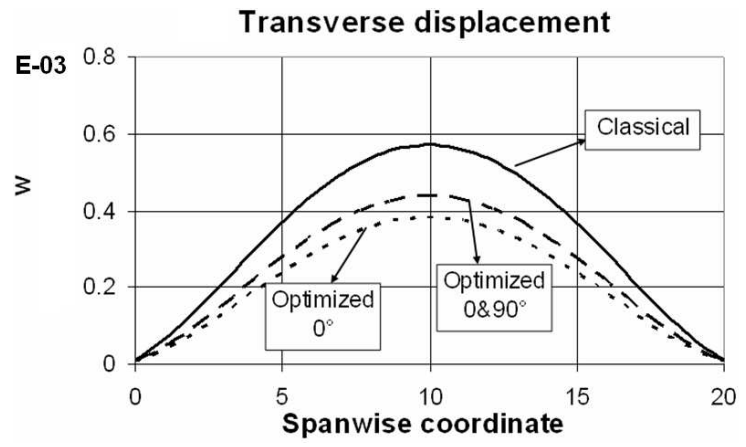

Figure 13. Transverse deflection of the dual core sandwich beam, in the classical configuration and in the optimized ones, obtained with minimum shear layers. 


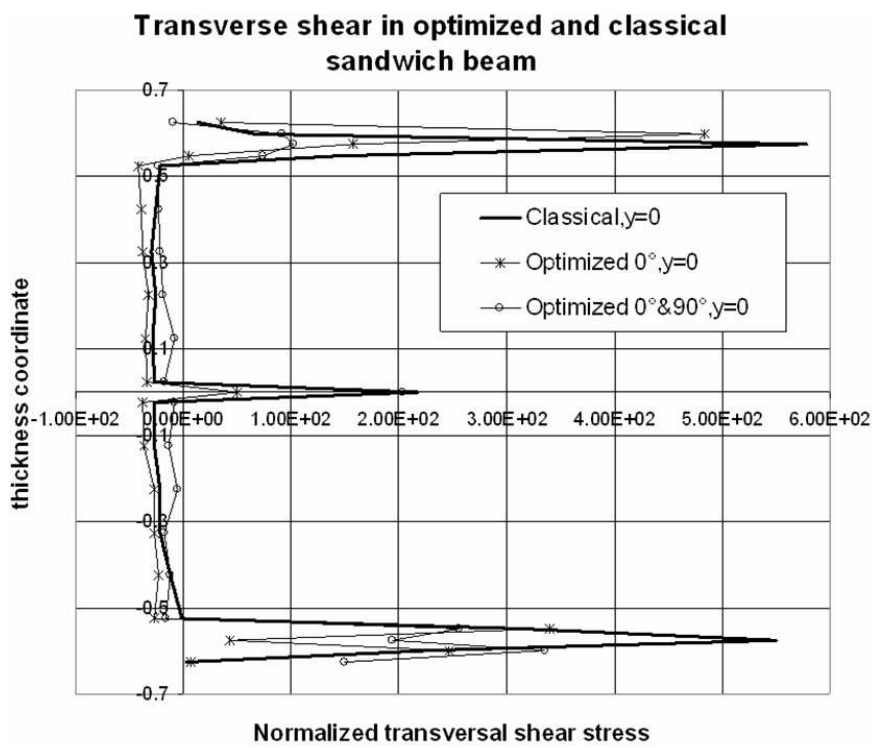

Figure 14. Transverse shear stress across the interfaces under the impact point; results for dual core sandwich beam, in the classical configuration and in the optimized ones, obtained with minimum shear layers.

of Chai-Gädke are considered, which are both based on in situ properties (see [Icardi et al. 2007] for a discussion and the explicit expressions of these criteria). In Figure 16, the damage computed by the above mentioned criteria is reported across the various interfaces under the impact point for the three analyzed structures. Comparing these cases, the effectiveness of the optimized layers can be assessed. It appears that a failure index reduction of 50\% in the internal face and of $70 \%$ in the external ones can be obtained incorporating the optimized layers. Since the most critical aspect of sandwich structures is the damage arising at the core face interfaces, we can guess by these results that the current optimization technique has a beneficial effect in this region through a strain energy manipulation that reduces the transverse shears.

\section{Concluding remarks}

A theoretical study dealing with a technique for tuning the energy absorbed by laminated and sandwich composites with laminated faces in the bending, in-plane, and out-of-plane shear modes has been presented. The basic idea is to minimize the energy absorbed through unwanted modes (those involving interlaminar strengths) and maximize that absorbed through desired modes (those involving membrane strengths), by finding a suitable in-plane variable distribution of stiffness properties. This optimal distribution is found making the energy contributions of interest extremal under the properties' spatial variation. Therefore this new tailoring concept requires either the orientation of the reinforcement fibers, the constituent materials, or the fiber volume fraction to be varied. In this way, the incoming energy can be transferred among the modes which are made extremal, obtaining an increase or a decrease, as desired, of the single contributions with respect to the case of customary constant stiffness constituent plies, 


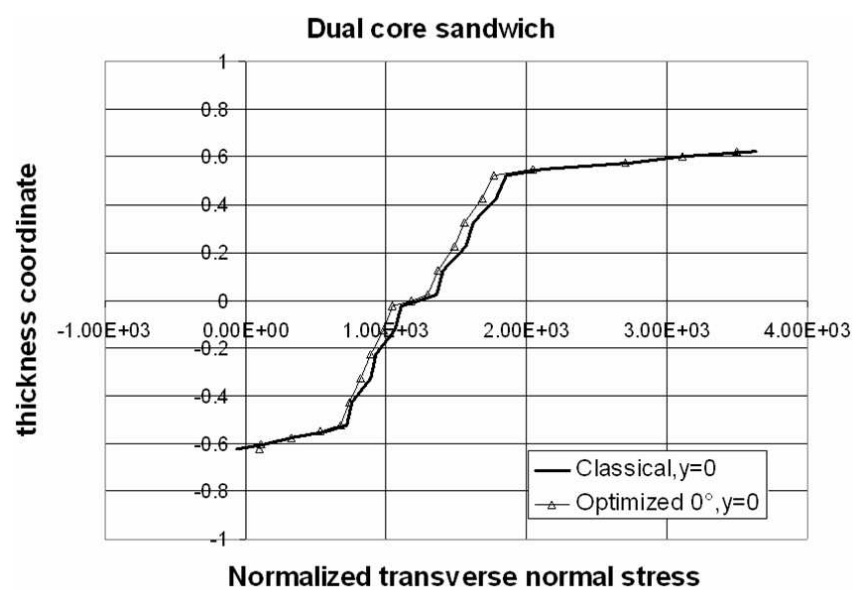

Figure 15. Transverse normal stress across the interfaces under the impact point; results for dual core sandwich beam, in the classical configuration and in the optimized ones, obtained with minimum shear layers.

assuming the mean stiffness properties of variable stiffness layers coincide with those of these layers. As a result, one can obtain an energy transfer from bending to in-plane and out-of-plane shear energy modes,

Choi-Chang and Chai-Gadke criterion, failure index criteria under the impact point

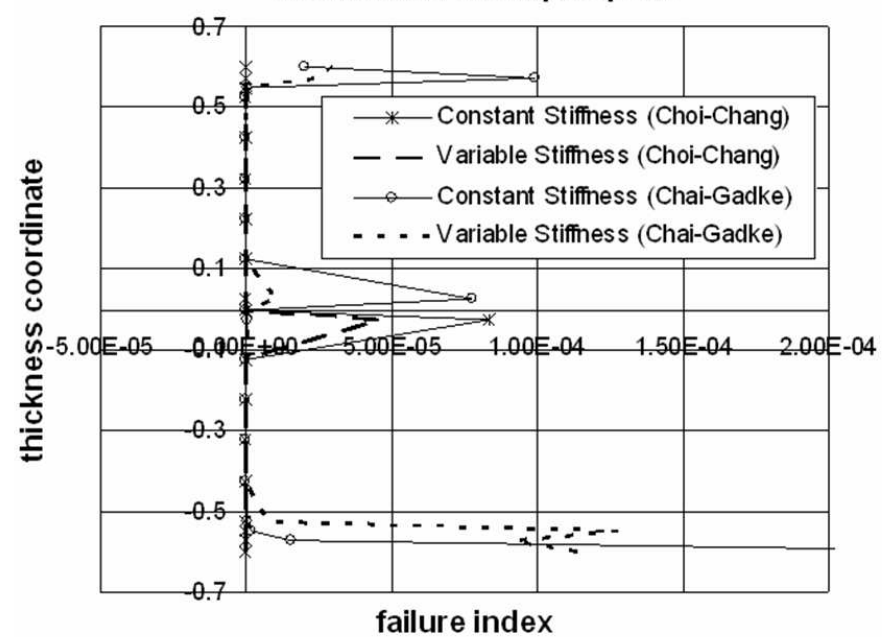

Figure 16. Failure index computed across the interfaces under the impact point; two stress based criteria have been used, Choi-Chang and Chai-Gädke, both involving in situ properties. Comparison between the optimized beam (with locally variable stiffness) and the classical one, with constant stiffness. 
or vice versa, with respect to layers with customary properties. The optimized stiffness distribution is obtained enforcing conditions which range from the imposition of thermodynamic constraints to the choice of a convex or a concave shape (in order to minimize or maximize the energy contributions of interest), to the imposition of their mean value. The present technique has its main advantage in the possibility of conjugating reduced interlaminar stresses with a high stiffness, that is precluded to the currently available methods based on interposition of compliant layers. Two structural models with complementary features are used: a zigzag model, which is inexpensive but accurate, is used in the optimization process, while a mixed, eight node solid element is used to accurately assess the local effects of the optimized solutions. A preliminary application to single plies with variable stiffness coefficients is presented. Two types are considered: the first is a layer that strongly reduces bending without increasing the shear stresses, and the second is a layer which strongly reduces shear stresses without increasing deflections. Both can be obtained by current manufacturing technologies, a smooth variation of the reinforcement fibers orientation being required over their plane. The numerical applications presented concern laminated and sandwich composites subjected to low velocity impacts. These layers appear able to consistently reduce the through the thickness interlaminar stress concentrations and to keep the bending stiffness substantially unchanged, when appropriately positioned across the thickness. These preliminary results encourage future developments of the present technique.

\section{Appendix A: Thermodynamic constraints}

The stiffness coefficients are subjected to the thermodynamic constraints conditions hereafter reported (see, Jones (1999) for details). To fulfill conservation of energy, the stiffness $\left[Q_{i j}\right]$ and compliance $\left[Q_{i j}^{-1}\right]$ matrices must be positive definite. Thereby $E_{L}, E_{T}, G_{L T}$ and $G_{T T}$ must be positive. Likewise, the following condition by Lempriere:

$$
\left[1-v_{32}^{2}\left(\frac{E_{2}}{E_{3}}\right)\right]\left[1-v_{13}^{2}\left(\frac{E_{3}}{E_{1}}\right)\right]-\left[v_{21}\left(\frac{E_{1}}{E_{2}}\right)^{1 / 2}+v_{13} v_{32}\left(\frac{E_{2}}{E_{1}}\right)^{1 / 2}\right]^{2}>0
$$

must be fulfilled. The compliance coefficients $S_{i j}$ also must fulfill following relations by Lekhnitski:

$$
S_{66}=\frac{1}{G_{12}} ; \quad S_{16}=\frac{\eta_{12,1}}{E_{1}}=\frac{\eta_{1,12}}{G_{12}} ; \quad S_{26}=\frac{\eta_{12,2}}{E_{2}}=\frac{\eta_{2,12}}{G_{12}}
$$

where

$$
\eta_{i, i j}=\frac{\epsilon_{i i}}{\epsilon_{i j}} ; \quad \eta_{i j, i}=\frac{\epsilon_{i j}}{\epsilon_{i i}}
$$

while the transverse shears must fulfill following relations in terms of Chentsov's coefficients $\eta_{i j, k l}=$ $\epsilon_{i j} / \epsilon_{k l}$ :

$$
\frac{\eta_{i j, k l}}{G_{k l}}=\frac{\eta_{k l, i j}}{G_{i j}}
$$




\section{Appendix B: Stiffness derivatives}

Recall that $\eta$ is defined as $h^{-1}$.

$$
\begin{aligned}
W R 1 & =B_{11}, 111+B_{12}, 122+3 B_{16}, 112+B_{26}, 222+2 B_{66}, 122 \\
W R 2 & =B_{12}, 112+B_{16}, 111+3 B_{26}, 112+B_{22}, 222+2 B_{66}, 112 \\
W R 3 & =D_{11}, 1111+2 D_{12}, 1122+4 D_{16}, 1112+4 D_{26}, 1222+4 D_{66}, 1122+D_{22,222} \\
W R 4 & =D_{11}, 111+D_{12,122}+3 D_{16}, 112+D_{26}, 222+2 D_{66}, 122 \\
W R 5 & =\left(F_{11} \eta^{2}\right), 111+\left(F_{12} \eta^{2}\right), 122+3\left(F_{16} \eta^{2}\right), 112+\left(F_{26} \eta^{2}\right), 222+2\left(F_{66} \eta^{2}\right), 221 \\
W R 6 & =D_{11}^{a}, 111+D_{12}^{a}, 122+3 D_{16}^{a}, 112+D_{26}^{a}, 222+2 D_{66}^{a}, 122 \\
W R 7 & =D_{12}^{a}, 112+D_{22}^{a}, 222+3 D_{26}^{a}, 122+D_{16}^{a}, 111+2 D_{66}^{a}, 112 \\
W R 8 & =D_{16}, 111+3 D_{26}, 122+2 D_{66}, 112+D_{12}, 112+D_{22}, 222 \\
W R 9 & =\left(F_{16} \eta^{2}\right), 111+3\left(F_{26} \eta^{2}\right),{ }_{122}+2\left(F_{66} \eta^{2}\right), 112+\left(F_{12} \eta^{2}\right), 112+\left(F_{22} \eta^{2}\right), 222 \\
W R 10 & =D_{16}^{b}, 111+3 D_{26}^{b}, 122+2 D_{66}^{b}, 112+D_{12}^{b}, 112+D_{22}^{b}, 222 \\
W R 11 & =D_{11}^{c}, 111+D_{12}^{c}, 122+3 D_{16}^{c}, 112+D_{26}^{c}, 222+2 D_{66}^{c}, 122
\end{aligned}
$$

Define the following quantities for the shear energy contribution in the plane $(x, z)$ :

$$
\begin{aligned}
& X R_{R 1}=B_{11,11}+B_{16,12} ; \quad X R_{R 2}=B_{16,11}+B_{12,12} \\
& X R_{R 3}=D_{11,111}+2 D_{16}, 112+D_{12,122} \\
& X R_{R 4}=R_{1 X},{ }_{11}+R_{2 X}, 12 ; \quad X R_{R 5}=R_{3 X}, 11+R_{4 X}, 12 \\
& R_{1 X}=D_{11}+D_{11}^{a}+D_{16}^{d}-\frac{4}{3} \eta^{2} F_{11} ; \quad R_{2 X}=D_{16}+D_{16}^{a}+D_{12}^{d}-\frac{4}{3} \eta^{2} F_{16} \\
& R_{3 X}=D_{16}+D_{16}^{b}+D_{11}^{c}-\frac{4}{3} \eta^{2} F_{16} ; \quad R_{4 X}=D_{12}+D_{12}^{b}+D_{16}^{c}-\frac{4}{3} \eta^{2} F_{12} \\
& X R_{R 1}^{a}=B_{11}^{a}, 11+B_{16}^{a}, 12 ; \quad X R_{R 2}^{a}=B_{16}^{a}, 11+B_{12}^{a}, 12 \\
& X R_{R 3}^{a}=D_{11}^{a}, 111+2 D_{16}^{a}, 112+D_{12}^{a}, 122 \\
& X R_{R 4}^{a}=R_{1 X}^{a}, 11+R_{2 X}^{a}, 12 ; \quad X R_{R 5}^{a}=R_{3 X}^{a}, 11+R_{4 X}^{a}, 12 \\
& R_{1 X}^{a}=D_{11}^{a}+D_{11}^{a a}+D_{16}^{a d}-\frac{4}{3} \eta^{2} F_{11}^{a} ; \quad R_{2 X}^{a}=D_{16}^{a}+D_{16}^{a a}+D_{12}^{a d}-\frac{4}{3} \eta^{2} F_{16}^{a} \\
& R_{3 X}^{a}=D_{16}^{a}+D_{16}^{a b}+D_{11}^{a c}-\frac{4}{3} \eta^{2} F_{16}^{a} ; \quad R_{4 X}^{a}=D_{12}^{a}+D_{12}^{a b}+D_{16}^{a c}-\frac{4}{3} \eta^{2} F_{12}^{a} \\
& X R_{R 1}^{d}=B_{16}^{d}, 11+B_{66}^{d}, 12 ; \quad X R_{R 2}^{d}=B_{66}^{d}, 11+B_{26}^{d}, 12 \\
& X R_{R 3}^{d}=D_{16}^{d}, 111+2 D_{66}^{d}, 112+D_{12}^{d}, 122 \\
& X R_{R 4}^{d}=R_{1 X}^{d}, 11+R_{2 X}^{d}, 12 ; \quad X R_{R 5}^{d}=R_{3 X}^{d}, 11+R_{4 X}^{d}, 12 \\
& R_{1 X}^{d}=D_{16}^{d}+D_{16}^{a d}+D_{66}^{d d}-\frac{4}{3} \eta^{2} F_{11}^{d} ; \quad R_{2 X}^{d}=D_{66}^{d}+D_{66}^{d d}+D_{26}^{d d}-\frac{4}{3} \eta^{2} F_{66}^{d} \\
& R_{3 X}^{d}=D_{66}^{d}+D_{66}^{b d}+D_{16}^{a d}-\frac{4}{3} \eta^{2} F_{16}^{d} ; \quad R_{4 X}^{d}=D_{26}^{d}+D_{26}^{b d}+D_{66}^{c d}-\frac{4}{3} \eta^{2} F_{26}^{d}
\end{aligned}
$$




$$
\begin{aligned}
& X R_{R 44}=B_{16,12}+B_{66,22} ; \quad X R_{R 55}=B_{66,12}+B_{26,22} \\
& X R_{R 66}=D_{16,112}+2 D_{66,122}+D_{26,222} \\
& X R_{R 88}=R_{9 X}, 12+R_{10 X}, 22 ; \quad X R_{R 99}=R_{11 X},{ }_{12}+R_{12 X}, 22 \\
& R_{9 X}=D_{16}+D_{16}^{a}+D_{66}^{d}-\frac{4}{3} \eta^{2} F_{16} ; \quad R_{10 X}=D_{66}+D_{26}^{a}+D_{26}^{d}-\frac{4}{3} \eta^{2} F_{66} \\
& R_{11 X}=D_{66}+D_{66}^{b}+D_{16}^{c}-\frac{4}{3} \eta^{2} F_{66} ; \quad R_{12 X}=D_{26}+D_{26}^{b}+D_{66}^{c}-\frac{4}{3} \eta^{2} F_{26} \\
& X R_{R 44}^{a}=B_{16}^{a}, 12+B_{66}^{a}, 22 ; \quad X R_{R 55}^{a}=B_{66}^{a}, 12+B_{26,22}^{a} \\
& X R_{R 66}^{a}=D_{16}^{a}, 112+2 D_{66}^{a}, 122+D_{26}^{a}, 222 \\
& X R_{R 88}^{a}=R_{9 X}^{a}, 12+R_{10 X}^{a}, 22 ; \quad X R_{R 99}^{a}=R_{11 X}^{a}, 12+R_{12 X}^{a}, 22 \\
& R_{9 X}^{a}=D_{16}^{a}+D_{16}^{a a}+D_{66}^{a d}-\frac{4}{3} \eta^{2} F_{16}^{a} ; \quad R_{10 X}^{a}=D_{66}^{a}+D_{26}^{a a}+D_{26}^{a d}-\frac{4}{3} \eta^{2} F_{66}^{a} \\
& R_{11 X}^{a}=D_{66}^{a}+D_{66}^{a b}+D_{16}^{a c}-\frac{4}{3} \eta^{2} F_{66}^{a} ; \quad R_{12 X}^{a}=D_{26}^{a}+D_{26}^{a b}+D_{66}^{a c}-\frac{4}{3} \eta^{2} F_{26}^{a} \\
& X R_{S 44}^{d}=B_{12}^{d}, 12+B_{26}^{d}, 22 ; \quad X R_{S 55}^{d}=B_{26}^{d}, 12+B_{22}^{d}, 22 \\
& X R_{S 66}^{d}=D_{11}^{d}, 112+2 D_{66}^{d}, 122+D_{22}^{d}, 222 \\
& X R_{S 88}^{d}=R_{13 X}^{d}, 12+R_{14 X}^{a}, 22 ; \quad X R_{S 99}^{d}=R_{15 X}^{d}, 12+R_{16 X}^{d}, 22 \\
& R_{13 X}^{d}=D_{12}^{d}+D_{12}^{a d}+D_{26}^{d d}-\frac{4}{3} \eta^{2} F_{16}^{a} ; \quad R_{14 X}^{d}=D_{26}^{d}+D_{26}^{a d}+D_{22}^{d d}-\frac{4}{3} \eta^{2} F_{66}^{a} \\
& R_{15 X}^{d}=D_{26}^{d}+D_{26}^{b d}+D_{12}^{c d}-\frac{4}{3} \eta^{2} F_{66}^{a} ; \quad R_{16 X}^{d}=D_{26}^{d}+D_{22}^{b d}+D_{66}^{c d}-\frac{4}{3} \eta^{2} F_{22}^{d} \\
& X R_{T 88}=A_{44}-4 \eta^{2} D_{44}+A_{44}^{a}+A_{45}^{d} ; \quad X R_{T 99}=A_{45}-4 \eta^{2} D_{45}+A_{44}^{c}+A_{45}^{b} \\
& X R_{T 88}^{a}=A_{44}^{a}-4 \eta^{2} D_{44}^{a}+A_{44}^{a a}+A_{45}^{a d} ; \quad X R_{T 99}^{a}=A_{45}^{a}-4 \eta^{2} D_{45}^{a}+A_{44}^{a c}+A_{45}^{a b} \\
& X R_{T 88}^{d}=A_{45}^{d}-4 \eta^{2} D_{45}^{d}+A_{45}^{a d}+A_{55}^{d d} ; \quad X R_{T 99}^{d}=A_{55}^{d}-4 \eta^{2} D_{55}^{d}+A_{45}^{c d}+A_{55}^{b d} \\
& X R_{P 1}=E_{11,11}+E_{16,12} ; \quad X R_{P 2}=E_{16,11}+E_{12,12} \\
& X R_{P 3}=F_{11,111}+2 F_{16,112}+F_{12,122} ; \quad X R_{P 4}=R_{18 X, 11}+R_{19 X}, 12 \\
& X R_{P 5}=R_{20 X}, 11+R_{21 X}, 12 \\
& R_{18 X}=F_{11}+F_{11}^{a}+F_{16}^{d}-\frac{4}{3} \eta^{2} H_{11} ; \quad R_{19 X}=F_{16}+F_{16}^{a}+F_{12}^{d}-\frac{4}{3} \eta^{2} H_{16} \\
& R_{20 X}=F_{16}+F_{16}^{b}+F_{11}^{c}-\frac{4}{3} \eta^{2} H_{16} ; \quad R_{21 X}=F_{12}+F_{12}^{b}+F_{16}^{c}-\frac{4}{3} \eta^{2} H_{12} \\
& X R_{P 6}=E_{16,12}+E_{66,22} ; \quad X R_{P 7}=E_{66,12}+E_{26,22} \\
& X R_{P 8}=F_{16,112}+2 F_{66,122}+F_{26,222} ; \quad X R_{P 9}=R_{22 X}, 12+R_{23 X}, 22 \\
& X R_{P 10}=R_{24 X}, 12+R_{25 X}, 22 \\
& R_{22 X}=F_{16}+F_{16}^{a}+F_{66}^{d}-\frac{4}{3} \eta^{2} H_{16} ; \quad R_{23 X}=F_{66}+F_{66}^{a}+F_{26}^{d}-\frac{4}{3} \eta^{2} H_{66} \\
& R_{24 X}=F_{66}+F_{66}^{b}+F_{16}^{c}-\frac{4}{3} \eta^{2} H_{66} ; \quad R_{25 X}=F_{26}+F_{26}^{b}+F_{66}^{c}-\frac{4}{3} \eta^{2} H_{26} \\
& X R_{R R 1 X}=D_{44}+D_{44}^{a}+D_{45}^{d}-4 \eta^{2} F_{44} ; \quad X R_{R R 2 X}=D_{45}+D_{44}^{c}+D_{45}^{b}-4 \eta^{2} F_{45}
\end{aligned}
$$




$$
\begin{aligned}
& X R_{26 X 2}=\mathscr{D}_{11}^{a} 2,,_{11}+\mathscr{D}_{16}^{a} 2,,_{12} ; \quad X R_{27 X 2}=\mathscr{D}_{16}^{a} 2,_{11}+\mathscr{D}_{12}^{a} 2,{ }_{12} \\
& X R_{28 X 2}=\mathscr{E}_{11}^{a} 2,{ }_{111}+2 \mathscr{E}_{16}^{a}{ }_{16},_{112} \mathscr{E}_{12}^{a} 2,{ }_{122} \\
& X R_{29 X 2}=R 2_{R 1 X, 11}+R 2_{R 2 X, 12} ; \quad X R_{30 X 2}=R 2_{R 3 X, 11}+R 2_{R 4 X, 12} \\
& R 2_{R 1 X}=\mathscr{D}_{11}^{a a} 2+\mathscr{D}_{16}^{a d} 2+\mathscr{E}_{11}^{a} 2+\mathscr{E}_{11}^{a a} 2+\mathscr{E}_{16}^{a d} 2+\mathscr{F}_{11}^{a} 2+\mathscr{F}_{11}^{a a} 2+\mathscr{F}_{16}^{a d} 2 \\
& R 2_{R 2 X}=\mathscr{D}_{12}^{a d} 2+\mathscr{D}_{16}^{a a} 2+\mathscr{E}_{12}^{a d} 2+\mathscr{E}_{16}^{a} 2+\mathscr{E}_{16}^{a a} 2+\mathscr{F}_{12}^{a d} 2+\mathscr{F}_{16}^{a} 2+\mathscr{F}_{16}^{a a} 2 \\
& R 2_{R 3 X}=\mathscr{D}_{11}^{a c} 2+\mathscr{D}_{16}^{a b} 2+\mathscr{E}_{12} a c 2+\mathscr{E}_{16} a+\mathscr{E}_{16} a b 2+\mathscr{F}_{16}^{a} 2+\mathscr{F}_{11}^{a c} 2+\mathscr{F}_{16}^{a b} 2 \\
& R 2_{R 4 X}=\mathscr{D}_{12}^{a b} 2+\mathscr{D}_{16}^{a c} 2+\mathscr{E}_{12}^{a b} 2+\mathscr{E}_{16} a c 2+\mathscr{F}_{12}^{a} 2+\mathscr{F}_{16}^{a c} 2 \\
& X R_{31 X 2}=\mathscr{D}_{12}^{d} 2,12+\mathscr{D}_{26}^{d} 2,22 ; \quad X R_{32 X 2}=\mathscr{D}_{26}^{d} 2,12+\mathscr{D}_{22}^{d} 2,22 \\
& X R_{33 X 2}=\mathscr{E}_{12}^{d} 2,{ }_{112}+2 \mathscr{E}_{26} d{ }^{d} 2,122+\mathscr{E}_{22}^{d} 2,222 \\
& X R_{34 X 2}=R 2_{R 5 X, 12}+R 2_{R 6 X, 22} ; \quad X R_{35 X 2}=R 2_{R 7 X, 12}+R 2_{R 8 X, 22} \\
& R 2_{R 5 X}=\mathscr{D}_{12}^{a d} 2+\mathscr{D}_{26}^{d d} 2+\mathscr{E}_{12}^{d} 2+\mathscr{E}_{12}^{a d} 2+\mathscr{E}_{26}^{d d} 2+\mathscr{F}_{21}^{d} 2+\mathscr{F}_{21}^{a d} 2+\mathscr{F}_{26}^{d d} 2 \\
& R 2_{R 6 X}=\mathscr{D}_{22}^{d d} 2+\mathscr{D}_{26}^{a d} 2+\mathscr{E}_{22}^{d d} 2+\mathscr{E}_{26}^{d} 2+\mathscr{E}_{26}^{a d} 2+\mathscr{F}_{22}^{d d} 2+\mathscr{F}_{26}^{d} 2+\mathscr{F}_{26}^{a d} 2 \\
& R 2_{R 7 X}=\mathscr{D}_{21}^{c d} 2+\mathscr{D}_{26}^{b d} 2+\mathscr{E}_{12}^{c d} 2+\mathscr{E}_{26}^{d} 2+\mathscr{E}_{26}^{b d} 2+\mathscr{F}_{26}^{d} 2+\mathscr{F}_{12}^{c d} 2+\mathscr{F}_{26}^{b d} 2 \\
& R 2_{R 8 X}=\mathscr{D}_{22}^{b d} 2+\mathscr{D}_{26}^{c d} 2+\mathscr{E}_{22}^{b d} 2+\mathscr{E}_{26}^{c d} 2+\mathscr{F}_{22}^{d} 2+\mathscr{F}_{26}^{c d} 2 \\
& X R_{36 X 2}=\mathscr{D}_{16}^{a} 2,{ }_{12}+\mathscr{D}_{66}^{a} 2,22 ; \quad X R_{37 X 2}=\mathscr{D}_{66}^{a} 2,{ }_{12}+\mathscr{D}_{26}^{a} 2,22 \\
& X R_{38 X 2}=\mathscr{E}_{16}^{a} 2,{ }_{112}+2 \mathscr{E}_{66} a{ }_{66} 2,{ }_{122}+\mathscr{E}_{26} a{ }_{222} \\
& X R_{39 X 2}=R 2_{R 10 X},{ }_{12}+R 2_{R 11 X}, 22 ; \quad X R_{40 X 2}=R 2_{R 12 X},{ }_{12}+R 2_{R 13 X}, 22 \\
& R 2_{R 10 X}=\mathscr{D}_{16}^{a a} 2+\mathscr{D}_{66}^{a d} 2+\mathscr{E}_{26}^{a} 2+\mathscr{E}_{16}^{a a} 2+\mathscr{E}_{66}^{a d} 2+\mathscr{F}_{16}^{a} 2+\mathscr{F}_{16}^{a a} 2+\mathscr{F}_{66}^{a d} 2 \\
& R 2_{R 11 X}=\mathscr{D}_{26}^{a d} 2+\mathscr{D}_{66}^{a a} 2+\mathscr{E}_{26}^{a d} 2+\mathscr{E}_{66}^{a} 2+\mathscr{E}_{66}^{a a} 2+\mathscr{F}_{26}^{a d} 2+\mathscr{F}_{66}^{a} 2+\mathscr{F}_{66}^{a a} 2 \\
& R 2_{R 12 X}=\mathscr{D}_{16}^{a c} 2+\mathscr{D}_{66}^{a b} 2+\mathscr{E}_{16}^{a c} 2+\mathscr{E}_{66}^{a} 2+\mathscr{E}_{66}^{a b} 2+\mathscr{F}_{66}^{a} 2+\mathscr{F}_{16}^{a c} 2+\mathscr{F}_{66}^{a b} 2 \\
& R 2_{R 13 X}=\mathscr{D}_{26}^{a b} 2+\mathscr{D}_{66}^{a c} 2+\mathscr{E}_{26}^{a b} 2+\mathscr{E}_{66}^{a c} 2+\mathscr{F}_{26}^{a} 2+\mathscr{F}_{66}^{a c} 2 \\
& X R_{41 X 2}=\mathscr{D}_{16}^{d} 2,,_{11}+\mathscr{D}_{66}^{d} 2,{ }_{12} ; \quad X R_{42 X 2}=\mathscr{D}_{66}^{d} 2,{ }_{11}+\mathscr{D}_{26}^{d} 2,12 \\
& X R_{43 X 2}=\mathscr{E}_{16}^{d} 2,{ }_{111}+2 \mathscr{E}_{66} d{ }^{d} 2,112+\mathscr{E}_{26}^{d} 2,222 \\
& X R_{44 X 2}=R 2_{R 14 X}, 11+R 2_{R 15 X}, 12 ; \quad X R_{45 X}=R 2_{R 16,11 X}+R 2_{R 17 X, 12} \\
& R 2_{R 14 X}=\mathscr{D}_{16}^{a d} 2+\mathscr{D}_{66}^{d d} 2+\mathscr{E}_{16}^{d} 2+\mathscr{E}_{16} a d+\mathscr{E}_{66} d d 2+\mathscr{F}_{16}^{d} 2+\mathscr{F}_{16}^{a d} 2+\mathscr{F}_{66} d d \\
& R 2_{R 15 X}=\mathscr{D}_{26}^{d d} 2+\mathscr{D}_{66}^{a d} 2+\mathscr{E}_{26}^{d d} 2+\mathscr{E}_{66}^{d} 2+\mathscr{E}_{66}^{a d} 2+\mathscr{F}_{26}^{d d} 2+\mathscr{F}_{66}^{d} 2+\mathscr{F}_{66}^{a d} 2 \\
& R 2_{R 16 X}=\mathscr{D}_{16}^{c d} 2+\mathscr{D}_{66}^{b d} 2+\mathscr{E}_{16}^{c d} 2+\mathscr{E}_{66}^{d} 2+\mathscr{E}_{66}^{b d} 2+\mathscr{F}_{66}^{d} 2+\mathscr{F}_{16}^{c d} 2+\mathscr{F}_{66}^{b d} 2 \\
& R 2_{R 17 X}=\mathscr{D}_{26}^{b d} 2+\mathscr{D}_{66}^{c d} 2+\mathscr{E}_{26}^{b d} 2+\mathscr{E}_{66}^{c d} 2+\mathscr{F}_{26}^{d} 2+\mathscr{F}_{66}^{c d} 2 \\
& X R_{46 X 2}=\mathscr{P}_{44}^{a} 2+\mathscr{S}_{44}^{a a} 2+\mathscr{B}_{45}^{a d} 2+\mathscr{D}_{44}^{a a} 2+\mathscr{D}_{45}^{a d} 2+\mathscr{E}_{44}^{a} 2+\mathscr{F}_{44}^{a a} 2+\mathscr{F}_{45}^{a d} 2 \\
& X R_{47 X 2}=\mathscr{B}_{45}^{a} 2+\mathscr{B}_{44}^{a c} 2+\mathscr{B}_{45}^{a b} 2+\mathscr{D}_{44}^{a c} 2+\mathscr{D}_{45}^{a b} 2+\mathscr{E}_{45}^{a} 2+\mathscr{F}_{44}^{a c} 2+\mathscr{F}_{45}^{a b} 2 \\
& X R_{48 X 2}=\mathscr{B}_{45}^{d} 2+\mathscr{S}_{45}^{a d} 2+\mathscr{B}_{55}^{d d} 2+\mathscr{D}_{45}^{a d} 2+\mathscr{D}_{55}^{d d} 2+\mathscr{E}_{45}^{d} 2+\mathscr{F}_{45}^{a d} 2+\mathscr{F}_{55}^{d d} 2 \\
& X R_{49 X 2}=\mathscr{B}_{55}^{d} 2+\mathscr{B}_{45}^{c d} 2+\mathscr{B}_{55}^{b d} 2+\mathscr{D}_{45}^{c d} 2+\mathscr{D}_{55}^{b d} 2+\mathscr{E}_{55}^{d} 2+\mathscr{F}_{45}^{c d} 2+\mathscr{F}_{55}^{b d} 2
\end{aligned}
$$


The quantities for the shear energy contribution in the plane $(y, z)$ are not reported, since they are similar to those for the plane $(x, z)$, replacing $x$ with $y$.

\section{References}

[Bert 1984] C. W. Bert, "A critical review of new plate theories applied to laminated composites", Compos. Struct. 2 (1984), 329-347.

[Bolotin 1996] V. V. Bolotin, "Delaminations in composite structures: its origin, buckling, growth and stability", Compos. B: Eng. 27 (1996), 129-145.

[Carvalho and Guedes Soares 1996] A. Carvalho and C. Guedes Soares, "Dynamic response of rectangular plates of composite materials subjected to impact loads", Compos. Struct. 34 (1996), 55-63.

[Chai and Gädke 1999] Y. Chai and M. Gädke, "Impact damage simulation and compression after impact of composite stiffened panels”, Dlr report 1b 20, DLR — German Aerospace Center, Institute of Structural Mechanics,, 1999. 131-199.

[Choi 2006] I. H. Choi, "Contact force history analysis of composite sandwich plates subjected to low-velocity impact", Compos. Struct. 75 (2006), 582-586.

[Choi and Chang 1992] H. Y. Choi and F. K. Chang, "A model for predicting damage in graphite/epoxy laminated composites resulting from low-velocity point impact", J. Compos. Mater. 26 (1992), 2134-2169.

[Cox 1999] B. N. Cox, "Constitutive model for a fiber tow bridging a delamination crack", Mech. Adv. Mater. Struct. 6:2 (1999), $117-151$.

[Crook 1952] A. W. Crook, "A study of some impacts between metal bodies by a piezoelectric method", pp. 377-380 in Proceeding of the Royal Society Series A, vol. 212, London, 1952.

[Davies and Olsson 2004] G. A. Davies and R. Olsson, "Impact on composite structures", The Aeronautical Journal 11 (2004), 541-564.

[Echaabi and Trochu 1996] J. F. Echaabi and F. Trochu, "Review of failure criteria of fibrous composite materials", Polym. Compos. 17 (1996), 786-798.

[Ferrero and Icardi 2006] L. Ferrero and U. Icardi, "Improving energy absorption and dissipation of composites through optimized tailoring", in Proceedings of the International Mechanical Engineering Congress and Exposition, IMECE, IMECE200613329, ASME, Chicago, IL, 2006.

[Ferrero and Icardi 2007] L. Ferrero and U. Icardi, "Optimization of multi-core sandwich composites undergoing impact loads", in Proceeding of the International Mechanical Engineering Congress and Exposition, IMECE, IMECE2007-42851, ASME, Seattle, WA, 2007.

[Fuchiyama and Noda 1995] T. Fuchiyama and N. Noda, "Analysis of thernal stress in a plate of functionally gradient material", JSAE Rev. 6 (1995), 263-268.

[Georgi 1979] H. Georgi, "Dynamic damping investigations on composites", pp. 9.1-9.20 in Proceeding of the 48th Meeting AGARD, Williamsbourg, 1979.

[Goldberg 2001] K. R. Goldberg, "Implementation of fiber substructuring into strain rate dependent micromechanics analysis of polymer matrix composites", Technical report NASA/TM-2001-210822, NASA, 2001.

[Hoa and Feng 1998] S. V. Hoa and W. Feng, Hybrid finite element method for stress analysis of laminated composites, Kluwer Academic Publications, Boston, 1998.

[Hou et al. 2000] J. P. Hou, N. Petrinic, C. Ruiz, and S. R. Hallet, "Prediction of impact damage in composite plates", Compos. Sci. Technol. 60 (2000), 273-281.

[Hou et al. 2001] J. P. Hou, N. Petrinic, and C. Ruiz, "A delamination criterion for laminated composites under low velocity impact”, Compos. Sci. Technol. 61 (2001), 2069-2074.

[Icardi 1998] U. Icardi, "Eight-noded zig-zag element for deflection and stress analysis of plates with general lay-up", Compos. B: Eng. 29 (1998), 435-41.

[Icardi 2001] U. Icardi, "Higher-order zig-zag model for analysis of thick composite beams with inclusion of transverse normal stress and sublaminates approximations", Compos. B: Eng. 32 (2001), 343-354.

[Icardi 2005] U. Icardi, " $C^{o}$ plate element for global/local analysis of multilayered composites, based on a 3D zig-zag model and strain energy updating”, Int. J. Mech. Sci. 47 (2005), 1561-1594. 
[Icardi 2007] U. Icardi, " $C^{o}$ plate element based on strain energy updating and spline interpolation, for analysis of impact damage in laminated composites", Int. J. Impact Eng. 34:11 (2007), 1835-1868.

[Icardi and Atzori 2004] U. Icardi and A. Atzori, "Simple, efficient mixed solid element for accurate analysis of local effects in laminated and sandwich composites", Adv. Eng. Softw. 32:12 (2004), 843-859.

[Icardi and Ferrero 2005] U. Icardi and L. Ferrero, "A study of energy absorption in fiber-reinforced composites: transfer form bending to shear", in Proceedings of the 3rd International Conference on Structural Stability and Dynamics, ICSSD05, Orlando, FL, 2005.

[Icardi and Ferrero 2006a] U. Icardi and L. Ferrero, "Impact and blast pulse: improving energy absorption of fibre-reinforced composites through optimized tailoring", in Proceedings of the Engineering Systems and Design Analysis Conference, ESDA, ESDA2006-95772, ASME, Torino, Italy, 2006.

[Icardi and Ferrero 2006b] U. Icardi and L. Ferrero, "Optimization of energy absorption and dissipation of composites", in International E-conference Of Computer Science 2006, edited by T. Simos, Lectures Series on Computer and Computational Sciences, Brill, 2006.

[Icardi and Ferrero 2006c] U. Icardi and L. Ferrero, "Optimized tailoring reducing interlaminar stresses accumulation in fiber-reinforced composites", in Proceeding of the 2nd International Congress on Computational Mechanics and Simulation, ICCMS-06, Guwahati, India, 2006.

[Icardi and Ferrero 2007a] U. Icardi and L. Ferrero, "Modeling assessment and optimization of impacted multi-core sandwich composites", in Proceeding of the Society for Advancement of Material and Process Engineering Conference, SAMPE, Baltimore, MD, 2007.

[Icardi and Ferrero 2007b] U. Icardi and L. Ferrero, "Modeling techniques assessment and optimization of laminated and sandwich composites undergoing impact loads", in Proceeding of the 12th Aerospace and Sciences and Aviation Technology, ASAT, 144, Cairo, Egypt, 2007.

[Icardi and Zardo 2005] U. Icardi and G. Zardo, " $C^{o}$ plate element for delmination damage analysis, based on a zig-zag model and strain energy updating", Int. J. Impact Eng. 31 (2005), 579-606.

[Icardi et al. 2007] U. Icardi, S. Locatto, and A. Longo, "Assessment of recent theories for predicting failure of composite laminates", Appl. Mech. Rev. 60:2 (2007), 76-86.

[Jones 1999] R. M. Jones, Mechanics of composite maerials, Taylor and Francis, Philadelfia, 1999.

[Joshi and Sun 1987] S. P. Joshi and C. T. Sun, "Impact-indiced fracture initiation and detailed dynamic stress field in the vicinity of impact", pp. 177-185 in Proceeding of the 2nd American Society of Composites Technical Conference, Newmark, DE, 1987.

[Jung 2001] W. Y. Jung, “A combined honeycomb and solid viscoelastic material for structural damping applications”, pp. 41-43 in Thrust area 2: seismic retrofit of acute care facilities, Department of Civil, Structural \& Environmental Engineering, University at Buffalo, 2001.

[Lakes 2002] R. S. Lakes, "High damping composite materials: effect of structural hierarchy”, J. Compos. Mater. 36:3 (2002), 287-297.

[Lee et al. 1997] Y. S. Lee, K. H. Kang, and O. Park, "Response of Hybrid laminated Composite Plates under loa-velocity Impact", Comput. Struct. 65:6 (1997), 965-974.

[Librescu and Reddy 1986] L. Librescu and J. N. Reddy, "A critical review and generalization of transverse shear deformable anisotropic plate theories", pp. 32-43 in Euromech Colloqioum 219, Refined dynamical theories of beams, plates and shells and their applications, Kassel, edited by I. Elishakoff and H. Irretier, Springer Verlag, Heidelberg, September 23-26 1986.

[Liou 1997] W. J. Liou, "Contact laws of carbon/epoxy laminated composite plate”, J. Reinf. Plast. Compos. 16:2 (1997), $155-166$.

[Loubignac et al. 1978] C. Loubignac, C. Cantin, and C. Touzot, "Continuous stress fields in finite element analysis", AIAA J. 15 (1978), 1645-1647.

[Matemilola and Stronge 1995] S. A. Matemilola and W. J. Stronge, "Impact induced dynamic deformations and stresses in CFRP composite laminates", Compos. Eng. 5:2 (1995), 211-222.

[McCoucheon 2004] D. M. McCoucheon, "Machine augmented composite materials for damping purposes", Degree of master of science thesis, Texas A\&M University, 2004. 
[Nahas 1986] M. N. Nahas, "Survey of failure and post-failure theories of laminated fibre reinforced composites", J. Compos. Tech. Res. 8 (1986), 138-153.

[Nakazawa 1984] S. Nakazawa, Mixed finite elements and iterative solution procedures, Iterative Methods in Non-Linear Problems, Pineridge, 1984.

[Noor and Burton 1989] A. K. Noor and W. S. Burton, "Assessment of shear deformation theories for multilayered composite plates", Appl. Mech. Rev. 42 (1989), 1-13.

[Noor and Burton 1990] A. K. Noor and W. S. Burton, "Assessment of computational models for multilayered composite shells", Appl. Mech. Rev. 43 (1990), 67-97.

[Noor and Burton 1992] A. K. Noor and W. S. Burton, "Computational models for high-temperature multilayered composite plates and shells", Appl. Mech. Rev. 45 (1992), 419-444.

[Noor et al. 1996] A. K. Noor, W. S. Burton, and C. W. Bert, "Computational models for sandwich panels and shells”, Appl. Mech. Rev. 49 (1996), 155-199.

[Paris 2001] F. Paris, "A study of failure criteria of fibrous composite materials", Technical report NASA/CR-2001-210661, NASA, 2001.

[Pedersen 2003] P. Pedersen, “A note on design of fiber-nets for maximum stiffness”, J. Elasticity 73:1-3 (2003), 127-145.

[Reddy 1982] J. N. Reddy, "Survey of recent research in the analysis of composite plates", Compos. Technol. Rev. 4 (1982), 101-104.

[Reddy 1990] J. N. Reddy, “A review of refined theories of laminated composite plates”, Shock Vibr. Dig. 22 (1990), 3-17.

[Reddy 2003] J. N. Reddy, Mechanics of laminated composite plates and shells: Theory and analysis, 2nd Edition ed., CRC Press, Boca Raton, FL, 2003.

[Reddy and Robbins 1994] J. N. Reddy and D. H. J. Robbins, "Theories and computational models for composite laminates", Appl. Mech. Rev. 47 (1994), 147-169.

[Rowlands 1985] R. E. Rowlands, "Strength (failure) theories and their experimental correlations", pp. 71-125 in Handbook of Composites, vol. 3, Elsevier Science Publ., 1985.

[Setoodeh et al. 2005] S. Setoodeh, M. M. Abdalla, Z. Gurdal, and B. Tatting, "Design of variable-stiffness composite laminates for maximum in-plane stiffness using lamination parameters", pp. 3473-3481 in Proceeding of the 46th AIAA/ASME/ASCE/ AHS/ASC Struc., Struct. Dynam and Appl. Conf., 13th AIAA/ ASME/ AHS Adap. Struc. Conf., 7th AIAA Non-Determ Appr. Forum, 2005.

[Suzuky et al. 2003] K. Suzuky, K. Kageyama, I. Kimpara, and S. Hotta, "Vibration and damping prediction of laminates with constrained viscoelastic layers", Mech. Adv. Mater. Struct. 10:2 (2003), 43-73.

[Tan and Sun 1985] T. M. Tan and C. T. Sun, "Use of statical indentation laws in the impact analysis of laminated composite plates", J. Appl. Mech. (Trans. ASME) 52 (1985), 6-12.

[Tennyson and Wharam 1985] R. C. Tennyson and G. E. Wharam, "Evaluation of failure criterion for graphite-epoxy", Technical report NASA CR-172547, NASA, 1985.

[Wu and Shyu 1993] E. Wu and K. Shyu, "Response of composite laminates of contact loads and relationship to low-velocity impact", J. Compos. Mater. 27:15 (1993), 1443-1464.

[Yigit and Christoforou 1995] A. S. Yigit and A. P. Christoforou, "Impact dynamics of composite beams", Compos. Struct. 32 (1995), 187-195.

[Zienkiewicz and Taylor 1994] O. C. Zienkiewicz and R. L. Taylor, The finite element method, vol. 1, 4th Ed. ed., McGraw-Hill, London, UK, 1994.

[Zinoviev and Ermakov 1994] P. A. Zinoviev and Y. N. Ermakov, Energy dissipation in composite materials, Technomic Pub. Co., Lancaster, UK, 1994.

Received 21 Aug 2007. Revised 24 Jan 2008. Accepted 29 Jan 2008.

UGo ICARDI: ugo.icardi@polito.it

DIASP, Politecnico di Torino, Corso Duca degli Abruzzi 24, 10129 Torino, Italy

LAURA FERRERO: laura.ferrero@polito.it

DIASP, Politecnico di Torino, Corso Duca degli Abruzzi 24, 10129 Torino, Italy 


\title{
INTERACTION BETWEEN A SCREW DISLOCATION AND A PIEZOELECTRIC CIRCULAR INCLUSION WITH VISCOUS INTERFACE
}

\author{
Xu Wang, ERnian Pan and A. K Roy
}

\begin{abstract}
Exact closed-form solutions in terms of elementary functions are derived for the problem of a screw dislocation embedded in an unbounded piezoelectric matrix interacting with a piezoelectric circular inclusion with a linear viscous interface. By means of the complex variable method, the original boundary value problem is reduced to an inhomogeneous first-order partial differential equation whose solution can be expressed in terms of elementary functions. The time dependent electroelastic fields such as stresses, strains, electric fields, and electric displacements are then obtained. In particular the image force acting on the piezoelectric screw dislocation, due to its interaction with the circular viscous interface, is presented. Some special cases of practical importance are discussed to verify and to illustrate the obtained solution. Finally we present a specific example of a screw dislocation located in a piezoelectric PZT-5 matrix interacting with a piezoelectric $\mathrm{BaTiO}_{3}$ fiber to graphically demonstrate the influence of the viscosity of the interface on the mobility of the screw dislocation.
\end{abstract}

\section{Introduction}

Due to their intrinsic electromechanical coupling behaviors, piezoelectric ceramics have been widely used in applications such as sensors, filters, ultrasonic generators, and actuators. More recently, the use of piezoelectric materials has gone beyond the traditional application domain of small electric devices due to the emergence of piezoelectric composites. Nowadays, piezoelectric materials have been employed as integrated active structural elements. These adaptive structures are capable of monitoring and adapting to their environments, providing a smart response to external conditions. Investigations on piezoelectric materials in the presence of defects such as dislocations, cracks, and inclusions are many [Pak 1990a; 1990b; 1992; Meguid and Deng 1998; Liu et al. 1999; Ru and Mao 1999; Lee et al. 2000; Chen et al. 2002; Wang et al. 2003; Wang and Pan 2007; Xiao et al. 2007] due to the fact that these defects can adversely influence the performance of the piezoelectric devices. Recently He and Lim [2003] analyzed the electromechanical response of a piezoelectric fibrous composite with a viscous interface described by the linear law of rheology [Ray and Ashby 1971; Suo 1997]. Their results demonstrated that the interfacial sliding could alter the local mechanical and electrical fields in the piezoelectric composite, and could further lead to significant change in overall electromechanical response of the composites with time.

The present paper investigates the interaction between a screw dislocation and a piezoelectric circular inclusion with a viscous interface described by the linear law of rheology [Ray and Ashby 1971; Suo 1997; He and Lim 2003]. The viscosity of the interface investigated in this research originates from the

Keywords: piezoelectricity, circular inclusion, screw dislocation, viscous interface, image force.

This work is supported in part by Air Force Research Laboratory (06-S531-060-C1). 
microscopically diffusion controlled sliding mechanism [Ray and Ashby 1971; Suo 1997], or from an artificially introduced thin viscous layer for damping purpose. This study is confined to the quasistatic assumption, ignoring the inertial force in the piezoelectric inclusion and matrix. By means of the complex variable method, the original boundary value problem is reduced to an inhomogeneous first-order partial differential equation for an analytic function defined within the circular inclusion. A closed-form solution in terms of elementary functions to the partial differential equation is obtained after a transformation is introduced. It is stressed that the usage of the complex variable combined with the real time variable is very novel in the literature.

\section{Basic formulations}

Consider a circular piezoelectric inclusion (or fiber) of radius $R$ embedded in an unbounded piezoelectric matrix, as shown in Figure 1. Both the inclusion and matrix are of $6 \mathrm{~mm}$ material with symmetry about the fiber axis. The inclusion/matrix interface is a viscous one characterized with a law of linear rheology [Ray and Ashby 1971; Suo 1997; He and Lim 2003] (or equivalently modeled by linear dashpot [Fan and Wang 2003; Wang and Schiavone 2007]). At time $t=0$, a piezoelectric screw dislocation is introduced into the piezoelectric matrix and fixed at point $\left(x_{0}, y_{0}\right)$. The screw dislocation is assumed to be straight and infinitely long in the $x_{3}$ direction (the fiber axis), experiencing a displacement jump $b$ and an electric potential jump $\Delta \phi$ across the slip plane. The dislocation also has a line force $p$ and line charge $q$ along its core. In this configuration the governing equations and constitutive equations can be simplified considerably as follows.

- Governing field equations:

$$
\sigma_{z x, x}+\sigma_{z y, y}=0, \quad D_{x, x}+D_{y, y}=0,
$$

- Electric field/electric potential relations:

$$
E_{x}=-\phi_{, x}, \quad E_{y}=-\phi_{, y},
$$

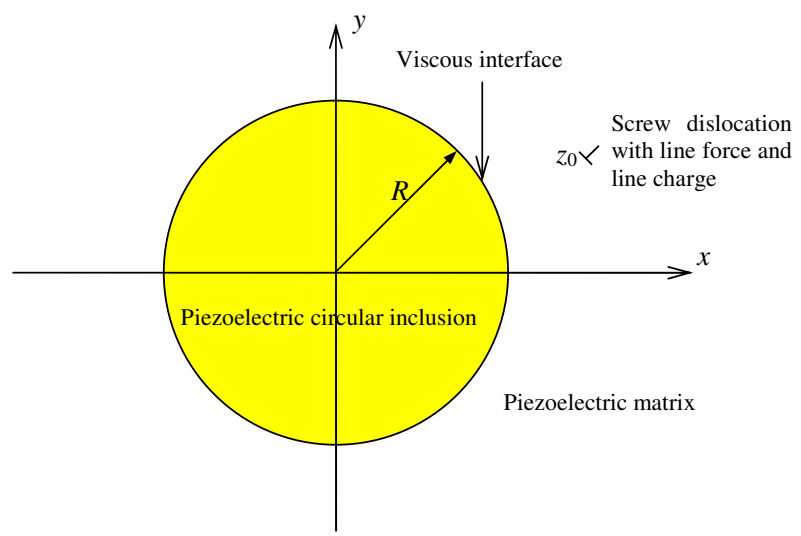

Figure 1. A screw dislocation with a line force and a line charge near a piezoelectric circular inclusion with a viscous interface. 
- Linear piezoelectric constitutive equations:

$$
\left[\begin{array}{c}
\sigma_{z y} \\
D_{y}
\end{array}\right]=\left[\begin{array}{cc}
c_{44} & -e_{15} \\
e_{15} & \epsilon_{11}
\end{array}\right]\left[\begin{array}{c}
w_{, y} \\
E_{y}
\end{array}\right], \quad\left[\begin{array}{c}
\sigma_{z x} \\
D_{x}
\end{array}\right]=\left[\begin{array}{cc}
c_{44} & -e_{15} \\
e_{15} & \epsilon_{11}
\end{array}\right]\left[\begin{array}{c}
w_{, x} \\
E_{x}
\end{array}\right]
$$

where a comma followed by $x$ or $y$ denotes partial derivatives with respect to $x$ or $y$, respectively. $\sigma_{z x}$ and $\sigma_{z y}$ are the shear stress components, $D_{x}$ and $D_{y}$ the electric displacement components, $E_{x}$ and $E_{y}$ the electric fields, $w$ the out of plane displacement, $\phi$ the electric potential, and $c_{44}, e_{15}$, and $\epsilon_{11}$ are, respectively, the elastic modulus, piezoelectric constant, and dielectric permittivity. In the following analysis the piezoelectrically stiffened elastic constant $\tilde{c}_{44}=c_{44}+e_{15}^{2} / \epsilon_{11}$ will be also used. In Equation (1) we have neglected the inertial effect of the piezoelectric material due to the fact that the viscous response comes from the interface only.

The displacement and electric potential can be expressed in terms of an analytic function vector $\boldsymbol{f}(z, t)=\left[f_{1}(z, t), f_{2}(z, t)\right]^{T}, z=x+i y$, as

$$
\left[\begin{array}{l}
w \\
\phi
\end{array}\right]=\operatorname{im}\{f(z, t)\}
$$

where im stands for the imaginary part. Since the viscous interface exhibits the time effect, the analytic function vector $f(z, t)$ depends not only on the complex variable $z$ but also on the time $t$. In terms of the analytic function vector $f(z, t)$, the strains, electric fields, stresses, and electric displacements in the Cartesian coordinate system can be expressed as

$$
\left[\begin{array}{c}
\gamma_{z y}+i \gamma_{z x} \\
-E_{y}-i E_{x}
\end{array}\right]=\frac{\partial \boldsymbol{f}(z, t)}{\partial z}, \quad\left[\begin{array}{c}
\sigma_{z y}+i \sigma_{z x} \\
D_{y}+i D_{x}
\end{array}\right]=\boldsymbol{C} \frac{\partial \boldsymbol{f}(z, t)}{\partial z}
$$

where the strains $\gamma_{z x}$ and $\gamma_{z y}$ are related to the out of plane displacement $w$ through $\gamma_{z x}=w_{, x}$, and $\gamma_{z y}=$ $w_{, y}$, and $\boldsymbol{C}$ is the extended stiffness matrix given by

$$
\boldsymbol{C}=\left[\begin{array}{cc}
c_{44} & e_{15} \\
e_{15} & -\epsilon_{11}
\end{array}\right]
$$

The strains, electric fields, stresses, and electric displacements in the polar coordinate system can also be expressed in terms of the analytic function vector $\boldsymbol{f}(z, t)$ as

$$
\left[\begin{array}{c}
\gamma_{z \theta}+i \gamma_{z r} \\
-E_{\theta}-i E_{r}
\end{array}\right]=\frac{z}{|z|} \frac{\partial \boldsymbol{f}(z, t)}{\partial z}, \quad\left[\begin{array}{c}
\sigma_{z \theta}+i \sigma_{z r} \\
D_{\theta}+i D_{r}
\end{array}\right]=\boldsymbol{C} \frac{z}{|z|} \frac{\partial \boldsymbol{f}(z, t)}{\partial z}
$$

In this paper, the superscripts (1) and (2) (or the subscripts 1 and 2) will be used to denote, respectively, the physical quantities in the inclusion and matrix. The analytic function vector defined in the inclusion is denoted by $\boldsymbol{g}(z, t)=\left[g_{1}(z, t), g_{2}(z, t)\right]^{T}$, whilst that in the unbounded matrix is denoted by $\boldsymbol{h}(z, t)=$ $\left[h_{1}(z, t), h_{2}(z, t)\right]^{T}$. 


\section{Complex potentials and field components}

The boundary conditions on the viscous interface $r=R$ can be expressed as [He and Lim 2003]

$$
\begin{aligned}
& \sigma_{z r}^{(1)}=\sigma_{z r}^{(2)}, D_{r}^{(1)}=D_{r}^{(2)}, \quad r=R \quad \text { and } t>0, \\
& \phi^{(1)}=\phi^{(2)}, \quad r(1)=\eta\left(\dot{w}^{(2)}-\dot{w}^{(1)}\right), \\
& \sigma_{z r}^{(1)}
\end{aligned}
$$

where a dot over the quantity denotes differentiation with respect to time $t$, and $\eta$ is the interface slip coefficient, which can be measured through properly designed experiment.

Equation $(6)_{1}$ for the continuity condition of traction and normal electric displacement across the interface can be equivalently expressed as

$$
\boldsymbol{C}_{1} \boldsymbol{g}^{+}(z, t)+\boldsymbol{C}_{1} \overline{\boldsymbol{g}}^{-}\left(R^{2} / z, t\right)=\boldsymbol{C}_{2} \boldsymbol{h}^{-}(z, t)+\boldsymbol{C}_{2} \overline{\boldsymbol{h}}^{+}\left(R^{2} / z,\right), \quad|z|=R,
$$

It follows from the above expression that

$$
\begin{aligned}
\boldsymbol{h}(z, t) & =\boldsymbol{C}_{2}^{-1} \boldsymbol{C}_{1} \overline{\boldsymbol{g}}\left(R^{2} / z, t\right)+\boldsymbol{h}_{0}(z)-\overline{\boldsymbol{h}}_{0}\left(R^{2} / z\right), \\
\overline{\boldsymbol{h}}\left(R^{2} / z, t\right) & =\boldsymbol{C}_{2}^{-1} \boldsymbol{C}_{1} \boldsymbol{g}(z, t)-\boldsymbol{h}_{0}(z)+\overline{\boldsymbol{h}}_{0}\left(R^{2} / z\right),
\end{aligned}
$$

where

$$
\begin{gathered}
\boldsymbol{h}_{0}(z)=\left[h_{10}(z), h_{20}(z)\right]^{T}=\frac{\hat{\boldsymbol{b}}-i \boldsymbol{C}_{2}^{-1} \hat{\boldsymbol{f}}}{2 \pi} \ln \left(z-z_{0}\right), \\
z_{0}=x_{0}+i y_{0}, \quad \hat{\boldsymbol{b}}=\left[\begin{array}{ll}
b & \Delta \phi
\end{array}\right]^{T}, \quad \hat{\boldsymbol{f}}=\left[\begin{array}{ll}
p & -q
\end{array}\right]^{T},
\end{gathered}
$$

which is time independent, is the complex potential for a piezoelectric screw dislocation in a homogeneous piezoelectric material [Pak 1990b].

Equation $(6)_{2}$ for the continuity condition of the electric potential across the interface can be equivalently expressed as

$$
g_{2}^{+}(z, t)-\bar{g}_{2}^{-}\left(R^{2} / z, t\right)=h_{2}^{-}(z, t)-\bar{h}_{2}^{+}\left(R^{2} / z, t\right), \quad|z|=R .
$$

It follows from the above expression that

$$
\begin{aligned}
h_{2}(z, t) & =-\bar{g}_{2}\left(R^{2} / z, t\right)+h_{20}(z)+\bar{h}_{20}\left(R^{2} / z\right), \quad|z|=R . \\
\bar{h}_{2}\left(R^{2} / z, t\right) & =-g_{2}(z, t)+h_{20}(z)+\bar{h}_{20}\left(R^{2} / z\right),
\end{aligned}
$$


In view of (7) and (8), the three analytic functions $h_{1}(z, t), h_{2}(z, t)$, and $\bar{g}_{2}\left(R^{2} / z, t\right)$ defined in the matrix can be expressed in terms of a single analytic function $\bar{g}_{1}\left(R^{2} / z, t\right)$ defined in the matrix as

$$
\begin{gathered}
h_{1}(z, t)=\frac{c_{44}^{(1)}\left(\epsilon_{11}^{(1)}+\epsilon_{11}^{(2)}\right)+e_{15}^{(1)}\left(e_{15}^{(1)}+e_{15}^{(2)}\right)}{c_{44}^{(2)}\left(\epsilon_{11}^{(1)}+\epsilon_{11}^{(2)}\right)+e_{15}^{(2)}\left(e_{15}^{(1)}+e_{15}^{(2)}\right)} \bar{g}_{1}\left(R^{2} / z, t\right) \\
+h_{10}(z)-\bar{h}_{10}\left(R^{2} / z\right)+\frac{2\left(\epsilon_{11}^{(2)} e_{15}^{(1)}-\epsilon_{11}^{(1)} e_{15}^{(2)}\right)}{c_{44}^{(2)}\left(\epsilon_{11}^{(1)}+\epsilon_{11}^{(2)}\right)+e_{15}^{(2)}\left(e_{15}^{(1)}+e_{15}^{(2)}\right)} \bar{h}_{20}\left(R^{2} / z\right), \\
\begin{array}{c}
h_{2}(z, t)=\frac{c_{44}^{(1)} e_{15}^{(2)}-c_{44}^{(2)} e_{15}^{(1)}}{c_{44}^{(2)}\left(\epsilon_{11}^{(1)}+\epsilon_{11}^{(2)}\right)+e_{15}^{(2)}\left(e_{15}^{(1)}+e_{15}^{(2)}\right)} \bar{g}_{1}\left(R^{2} / z, t\right) \\
\bar{g}_{2}\left(R^{2} / z, t\right)=\frac{h_{20}^{(2)}(z)+\frac{c_{44}^{(2)}\left(\epsilon_{11}^{(1)}-\epsilon_{11}^{(2)}\right)+e_{15}^{(2)}\left(e_{15}^{(1)}-e_{15}^{(2)}\right)}{c_{44}^{(2)}\left(\epsilon_{11}^{(1)}+\epsilon_{11}^{(2)}\right)+e_{15}^{(2)}\left(e_{15}^{(1)}+e_{15}^{(2)}\right)} \bar{h}_{20}\left(R^{2} / z\right),}{c_{44}^{(2)}\left(\epsilon_{11}^{(1)}+\epsilon_{11}^{(2)}\right)+e_{15}^{(2)}\left(e_{15}^{(1)}+e_{15}^{(2)}\right)} \bar{g}_{1}\left(R^{2} / z, t\right) \\
+\frac{2 \tilde{c}_{44}^{(2)} \epsilon_{11}^{(2)}}{c_{44}^{(2)}\left(\epsilon_{11}^{(1)}+\epsilon_{11}^{(2)}\right)+e_{15}^{(2)}\left(e_{15}^{(1)}+e_{15}^{(2)}\right)} \bar{h}_{20}\left(R^{2} / z\right) .
\end{array}
\end{gathered}
$$

Similarly the three analytic functions $\bar{h}_{1}\left(R^{2} / z, t\right), \bar{h}_{2}\left(R^{2} / z, t\right)$, and $g_{2}(z, t)$ defined in the inclusion can be expressed in terms of a single analytic function $g_{1}(z, t)$ defined in the inclusion as

$$
\begin{aligned}
& \bar{h}_{1}\left(R^{2} / z, t\right)=\frac{c_{44}^{(1)}\left(\epsilon_{11}^{(1)}+\epsilon_{11}^{(2)}\right)+e_{15}^{(1)}\left(e_{15}^{(1)}+e_{15}^{(2)}\right)}{c_{44}^{(2)}\left(\epsilon_{11}^{(1)}+\epsilon_{11}^{(2)}\right)+e_{15}^{(2)}\left(e_{15}^{(1)}+e_{15}^{(2)}\right)} g_{1}(z, t) \\
& +\bar{h}_{10}\left(R^{2} / z\right)-h_{10}(z)+\frac{2\left(\epsilon_{11}^{(2)} e_{15}^{(1)}-\epsilon_{11}^{(1)} e_{15}^{(2)}\right)}{c_{44}^{(2)}\left(\epsilon_{11}^{(1)}+\epsilon_{11}^{(2)}\right)+e_{15}^{(2)}\left(e_{15}^{(1)}+e_{15}^{(2)}\right)} h_{20}(z), \\
& \bar{h}_{2}\left(R^{2} / z, t\right)=\frac{c_{44}^{(1)} e_{15}^{(2)}-c_{44}^{(2)} e_{15}^{(1)}}{c_{44}^{(2)}\left(\epsilon_{11}^{(1)}+\epsilon_{11}^{(2)}\right)+e_{15}^{(2)}\left(e_{15}^{(1)}+e_{15}^{(2)}\right)} g_{1}(z, t) \\
& +\bar{h}_{20}\left(R^{2} / z\right)+\frac{c_{44}^{(2)}\left(\epsilon_{11}^{(1)}-\epsilon_{11}^{(2)}\right)+e_{15}^{(2)}\left(e_{15}^{(1)}-e_{15}^{(2)}\right)}{c_{44}^{(2)}\left(\epsilon_{11}^{(1)}+\epsilon_{11}^{(2)}\right)+e_{15}^{(2)}\left(e_{15}^{(1)}+e_{15}^{(2)}\right)} h_{20}(z), \\
& g_{2}(z, t)=\frac{c_{44}^{(2)} e_{15}^{(1)}-c_{44}^{(1)} e_{15}^{(2)}}{c_{44}^{(2)}\left(\epsilon_{11}^{(1)}+\epsilon_{11}^{(2)}\right)+e_{15}^{(2)}\left(e_{15}^{(1)}+e_{15}^{(2)}\right)} g_{1}(z, t)+\frac{2 \tilde{c}_{44}^{(2)} \epsilon_{11}^{(2)}}{c_{44}^{(2)}\left(\epsilon_{11}^{(1)}+\epsilon_{11}^{(2)}\right)+e_{15}^{(2)}\left(e_{15}^{(1)}+e_{15}^{(2)}\right)} h_{20}(z) \text {. }
\end{aligned}
$$

Equation $(6)_{3}$ for the law of linear rheology can be equivalently expressed as

$$
\begin{aligned}
& c_{44}^{(1)} z g_{1}^{\prime+}(z, t)+e_{15}^{(1)} z g_{2}^{g^{+}}(z, t)-c_{44}^{(1)} R^{2} z^{-1}{\overline{g^{\prime}}}_{1}^{-}\left(R^{2} / z, t\right) \\
& \quad-e_{15}^{(1)} R^{2} z^{-1}{\overline{g^{\prime}}}_{2}^{-}\left(R^{2} / z, t\right)=R \eta\left[\frac{\partial h_{1}^{-}(z, t)}{\partial t}-\frac{\partial \bar{h}_{1}^{+}\left(R^{2} / z, t\right)}{\partial t}-\frac{\partial g_{1}^{+}(z, t)}{\partial t}+\frac{\partial \bar{g}_{1}^{-}\left(R^{2} / z, t\right)}{\partial t}\right],
\end{aligned}
$$

where $|z|=R$, and where the superscript comma means the derivative with respect to the complex variable. 
Inserting (9) and (10) into (11), and with $|z|=R$, we finally obtain

$$
\begin{gathered}
\frac{\tilde{c}_{44}^{(1)} c_{44}^{(2)} \epsilon_{11}^{(1)}+c_{44}^{(1)} \tilde{c}_{44}^{(2)} \epsilon_{11}^{(2)}}{c_{44}^{(2)}\left(\epsilon_{11}^{(1)}+\epsilon_{11}^{(2)}\right)+e_{15}^{(2)}\left(e_{15}^{(1)}+e_{15}^{(2)}\right)} z g_{1}^{\prime+}(z, t)+R \eta \frac{\left(c_{44}^{(1)}+c_{44}^{(2)}\right)\left(\epsilon_{11}^{(1)}+\epsilon_{11}^{(2)}\right)+\left(e_{15}^{(1)}+e_{15}^{(2)}\right)^{2}}{c_{44}^{(2)}\left(\epsilon_{11}^{(1)}+\epsilon_{11}^{(2)}\right)+e_{15}^{(2)}\left(e_{15}^{(1)}+e_{15}^{(2)}\right)} \frac{2 \tilde{c}^{+}(z, t)}{\partial t} \\
+\frac{\tilde{c}_{44}^{(2)} \epsilon_{11}^{(2)} e_{15}^{(1)}}{c_{44}^{(2)}\left(\epsilon_{11}^{(1)}+\epsilon_{11}^{(2)}\right)+e_{15}^{(2)}\left(e_{15}^{(1)}+e_{15}^{(2)}\right)} z h_{20}^{\prime}(z) \\
=\frac{\tilde{c}_{44}^{(1)} c_{44}^{(2)} \epsilon_{11}^{(1)}+c_{44}^{(1)} \tilde{c}_{44}^{(2)} \epsilon_{11}^{(2)}}{c_{44}^{(2)}\left(\epsilon_{11}^{(1)}+\epsilon_{11}^{(2)}\right)+e_{15}^{(2)}\left(e_{15}^{(1)}+e_{15}^{(2)}\right)} R^{2} z^{-1} \bar{g}^{\prime}{ }_{1}^{-}\left(R^{2} / z, t\right) \\
+R \eta \frac{\left(c_{44}^{(1)}+c_{44}^{(2)}\right)\left(\epsilon_{11}^{(1)}+\epsilon_{11}^{(2)}\right)+\left(e_{15}^{(1)}+e_{15}^{(2)}\right)^{2}}{c_{44}^{(2)}\left(\epsilon_{11}^{(1)}+\epsilon_{11}^{(2)}\right)+e_{15}^{(2)}\left(e_{15}^{(1)}+e_{15}^{(2)}\right)} \frac{\left.2 R^{2} / z, t\right)}{\partial t} \\
+\frac{2 \tilde{c}_{44}^{(2)} \epsilon_{11}^{(2)} e_{15}^{(1)}}{c_{44}^{(2)}\left(\epsilon_{11}^{(1)}+\epsilon_{11}^{(2)}\right)+e_{15}^{(2)}\left(e_{15}^{(1)}+e_{15}^{(2)}\right)} R^{2} z^{-1} \bar{h}_{20}^{\prime}\left(R^{2} / z\right) .
\end{gathered}
$$

Apparently the left hand side of Equation (12) is analytic within the circle $|z|=R$, while the right hand side of (12) is analytic outside the circle including the point at infinity. By employing Liouville's theorem, the left and right hand sides should be identically zero. Consequently we obtain the inhomogeneous firstorder partial differential equation for $g_{1}(z, t)$

$$
z \frac{\partial g_{1}(z, t)}{\partial z}+t_{0} \frac{\partial g_{1}(z, t)}{\partial t}=-\frac{\beta z}{z-z_{0}}, \quad|z| \leq R
$$

where $t_{0}$ is the characteristic time and $\beta$ a constant, defined, respectively, by

$$
t_{0}=R \eta \frac{\left(c_{44}^{(1)}+c_{44}^{(2)}\right)\left(\epsilon_{11}^{(1)}+\epsilon_{11}^{(2)}\right)+\left(e_{15}^{(1)}+e_{15}^{(2)}\right)^{2}}{\tilde{c}_{44}^{(1)} c_{44}^{(2)} \epsilon_{11}^{(1)}+c_{44}^{(1)} \tilde{c}_{44}^{(2)} \epsilon_{11}^{(2)}}>0, \quad \beta=\frac{e_{15}^{(1)}\left[\tilde{c}_{44}^{(2)} \epsilon_{11}^{(2)} \Delta \phi-i\left(e_{15}^{(2)} p+c_{44}^{(2)} q\right)\right]}{\pi\left(\tilde{c}_{44}^{(1)} c_{44}^{(2)} \epsilon_{11}^{(1)}+c_{44}^{(1)} \tilde{c}_{44}^{(2)} \epsilon_{11}^{(2)}\right)} .
$$

It is of interest to notice that the resulting first-order partial differential equation (13) for a circular viscous interface is different from that for a straight viscous interface [Wang et al. 2007; Wang and Pan 2008]. Once we introduce the transformation $\zeta=\ln z$, (13) changes into

$$
\frac{\partial g_{1}(\zeta, t)}{\partial \zeta}+t_{0} \frac{\partial g_{1}(\zeta, t)}{\partial t}=-\frac{\beta e^{\zeta}}{e^{\zeta}-z_{0}}
$$

whose structure is in a sense similar to that of the resulting differential equation for a straight viscous interface [Wang et al. 2007; Wang and Pan 2008].

Equation (14) demonstrates that it has a homogeneous solution in the form $g_{1}\left(\zeta-t / t_{0}\right)$. In view of the form of the homogeneous solution to (14), the solution to the original partial differential equation (13) can be conveniently given by

$$
g_{1}(z, t)=\alpha \ln \left(z-e^{t / t_{0}} z_{0}\right)-\beta \ln \left(z-z_{0}\right), \quad|z| \leq R
$$

where $\alpha$ is an unknown constant to be determined, and the term $-\alpha t / t_{0}$ in $g_{1}(z, t)$ representing the rigid body displacement and equipotential has been ignored. It is mentioned that the first term in $g_{1}(z, t)$,

$$
\alpha \ln \left(z-e^{t / t_{0}} z_{0}\right)-a t / t_{0}=\alpha \ln \left(e^{-t / t_{0}} z-z_{0}\right),
$$


is a homogeneous solution to (13), while the second term in $g_{1}(z, t),-\beta \ln \left(z-z_{0}\right)$, is a particular solution to (13). At the initial moment $t=0$, when the piezoelectric screw dislocation is just introduced into the matrix, the displacement across the interface has no time to experience a jump due to the dashpot [Fan and Wang 2003; Wang and Schiavone 2007]. Therefore at $t=0$ the interface is a perfect one. As a result we have

$$
g_{1}(z, 0)=\frac{\boldsymbol{J}_{1}\left(\boldsymbol{C}_{1}+\boldsymbol{C}_{2}\right)^{-1}\left(\boldsymbol{C}_{2} \hat{\boldsymbol{b}}-i \hat{\boldsymbol{f}}\right)}{\pi} \ln \left(z-z_{0}\right), \quad \boldsymbol{J}_{0}=\left[\begin{array}{ll}
1 & 0
\end{array}\right], \quad \boldsymbol{J}_{2}=\left[\begin{array}{ll}
0 & 1
\end{array}\right]
$$

In view of Equations (15) and (16), the constant $\alpha$ can be uniquely determined to be

$$
\alpha=\beta+\frac{\boldsymbol{J}_{1}\left(\boldsymbol{C}_{1}+\boldsymbol{C}_{2}\right)^{-1}\left(\boldsymbol{C}_{2} \hat{\boldsymbol{b}}-i \hat{\boldsymbol{f}}\right)}{\pi} .
$$

Once $g_{1}(z, t)$ has been determined, after some tedious but straightforward mathematical operations we finally arrive at $g_{2}(z, t)$ defined in the inclusion and $h_{1}(z, t)$ and $h_{2}(z, t)$ defined in the matrix as

$$
\begin{gathered}
g_{2}(z, t)=\frac{\alpha\left(c_{44}^{(2)} e_{15}^{(1)}-c_{44}^{(1)} e_{15}^{(2)}\right)}{c_{44}^{(2)}\left(\epsilon_{11}^{(1)}+\epsilon_{11}^{(2)}\right)+e_{15}^{(2)}\left(e_{15}^{(1)}+e_{15}^{(2)}\right)} \ln \left(z-e^{t / t_{0}} z_{0}\right)+\frac{\beta c_{44}^{(1)}}{e_{15}^{(1)}} \ln \left(z-z_{0}\right), \\
h_{1}(z, t)=\frac{\bar{\alpha}\left[c_{44}^{(1)}\left(\epsilon_{11}^{(1)}+\epsilon_{11}^{(2)}\right)+e_{15}^{(1)}\left(e_{15}^{(1)}+e_{15}^{(2)}\right)\right]}{c_{44}^{(2)}\left(\epsilon_{11}^{(1)}+\epsilon_{11}^{(2)}\right)+e_{15}^{(2)}\left(e_{15}^{(1)}+e_{15}^{(2)}\right)} \ln \left(\frac{z-e^{-t / t_{0}} R^{2} / \bar{z}_{0}}{z}\right) \\
-\left[\frac{\tilde{c}_{44}^{(1)} \epsilon_{11}^{(1)} e_{15}^{(2)} \boldsymbol{J}_{2}}{\tilde{c}_{44}^{(1)} c_{44}^{(2)} \epsilon_{11}^{(1)}+c_{44}^{(1)} \tilde{c}_{44}^{(2)} \epsilon_{11}^{(2)}}+\frac{\boldsymbol{J}_{1}}{2}\right] \frac{\hat{\boldsymbol{b}}+i \boldsymbol{C}_{2}^{-1} \hat{\boldsymbol{f}}}{\pi} \ln \left(\frac{z-R^{2} / \bar{z}_{0}}{z^{2}}\right) \\
+\frac{\boldsymbol{J}_{1}\left(\hat{\boldsymbol{b}}-i \boldsymbol{C}_{2}^{-1} \hat{\boldsymbol{f}}\right)}{2 \pi} \ln \left(z-z_{0}\right),
\end{gathered}
$$

$$
\begin{aligned}
h_{2}(z, t)=\frac{\bar{\alpha}\left(c_{44}^{(1)} e_{15}^{(2)}-c_{44}^{(2)} e_{15}^{(1)}\right)}{c_{44}^{(2)}\left(\epsilon_{11}^{(1)}+\epsilon_{11}^{(2)}\right)+e_{15}^{(2)}\left(e_{15}^{(1)}+e_{15}^{(2)}\right)} \ln \left(\frac{z-e^{-t / t_{0}} R^{2} / \bar{z}_{0}}{z}\right) & \\
+\frac{\tilde{c}_{44}^{(1)} c_{44}^{(2)} \epsilon_{11}^{(1)}-c_{44}^{(1)} \tilde{c}_{44}^{(2)} \epsilon_{11}^{(2)}}{\tilde{c}_{44}^{(1)} c_{44}^{(2)} \epsilon_{11}^{(1)}+c_{44}^{(1)} \tilde{c}_{44}^{(2)} \epsilon_{11}^{(2)}} \frac{\boldsymbol{J}_{2}\left(\hat{\boldsymbol{b}}+i \boldsymbol{C}_{2}^{-1} \hat{\boldsymbol{f}}\right)}{2 \pi} \ln \left(\frac{z-R^{2} / \bar{z}_{0}}{z}\right) & +\frac{\boldsymbol{J}_{2}\left(\hat{\boldsymbol{b}}-i \boldsymbol{C}_{2}^{-1} \hat{\boldsymbol{f}}\right)}{2 \pi} \ln \left(z-z_{0}\right) .
\end{aligned}
$$

Notice that the last term in the expressions of $h_{1}(z, t)$ and $h_{2}(z, t)$ is the singular part due to the screw dislocation. If both the inclusion and the matrix are purely elastic materials, that is, $e_{15}^{(1)}=e_{15}^{(2)}=0$, then 
$g_{1}(z, t), g_{2}(z, t)$ defined in the inclusion and $h_{1}(z, t), h_{2}(z, t)$ defined in the matrix are reduced to

$$
\begin{aligned}
& g_{1}(z, t)=\frac{c_{44}^{(2)} b-i p}{\pi\left(c_{44}^{(1)}+c_{44}^{(2)}\right)} \ln \left(z-e^{t / t_{0}} z_{0}\right), \quad g_{2}(z, t)=\frac{\epsilon_{11}^{(2)} \Delta \phi-i q}{\pi\left(\epsilon_{11}^{(1)}+\epsilon_{11}^{(2)}\right)} \ln \left(z-z_{0}\right) \\
& h_{1}(z, t)=\frac{c_{44}^{(1)}\left(c_{44}^{(2)} b+i p\right)}{\pi c_{44}^{(2)}\left(c_{44}^{(1)}+c_{44}^{(2)}\right)} \ln \left(\frac{z-e^{-t / t_{0}} R^{2} / \bar{z}_{0}}{z}\right)-\frac{c_{44}^{(2)} b+i p}{2 \pi c_{44}^{(2)}} \ln \left(\frac{z-R^{2} / \bar{z}_{0}}{z}\right)+\frac{c_{44}^{(2)} b-i p}{2 \pi c_{44}^{(2)}} \ln \left(z-z_{0}\right), \\
& h_{2}(z, t)=\frac{\left(\epsilon_{11}^{(1)}-\epsilon_{11}^{(2)}\right)\left(\epsilon_{11}^{(2)} \Delta \phi+i q\right)}{2 \pi \epsilon_{11}^{(2)}\left(\epsilon_{11}^{(1)}+\epsilon_{11}^{(2)}\right)} \ln \left(\frac{z-R^{2} / \bar{z}_{0}}{z}\right)+\frac{\epsilon_{11}^{(2)} \Delta \phi-i q}{2 \pi \epsilon_{11}^{(2)}} \ln \left(z-z_{0}\right)
\end{aligned}
$$

where

$$
t_{0}=\frac{R \eta\left(c_{44}^{(1)}+c_{44}^{(2)}\right)}{c_{44}^{(1)} c_{44}^{(2)}} .
$$

In this special case $g_{2}(z, t)$ and $h_{2}(z, t)$ are in fact independent of the time $t$ due to the fact that there is no piezoelectric effect.

Substituting Equations (15) and (18) into (4) or (5), we can arrive at the explicit expressions of strains, stresses, electric fields, and electric displacements induced by the piezoelectric screw dislocation, which are listed in the online supplement. For example the strains, electric fields, stresses, and electric displacements within the piezoelectric circular inclusion are given by

$$
\begin{aligned}
\gamma_{z y}^{(1)}+i \gamma_{z x}^{(1)} & =\frac{\alpha}{z-e^{t / t_{0}} z_{0}}-\frac{\beta}{z-z_{0}}, \\
E_{y}^{(1)}+i E_{x}^{(1)} & =\frac{\alpha\left(c_{44}^{(1)} e_{15}^{(2)}-c_{44}^{(2)} e_{15}^{(1)}\right)}{\left[c_{44}^{(2)}\left(\epsilon_{11}^{(1)}+\epsilon_{11}^{(2)}\right)+e_{15}^{(2)}\left(e_{15}^{(1)}+e_{15}^{(2)}\right)\right]\left(z-e^{t / t_{0}} z_{0}\right)}-\frac{\beta c_{44}^{(1)}}{e_{15}^{(1)}\left(z-z_{0}\right)}, \\
\sigma_{z y}^{(1)}+i \sigma_{z x}^{(1)} & =\frac{\alpha\left(\tilde{c}_{44}^{(1)} c_{44}^{(2)} \epsilon_{11}^{(1)}+c_{44}^{(1)} \tilde{c}_{44}^{(2)} \epsilon_{11}^{(2)}\right)}{\left[c_{44}^{(2)}\left(\epsilon_{11}^{(1)}+\epsilon_{11}^{(2)}\right)+e_{15}^{(2)}\left(e_{15}^{(1)}+e_{15}^{(2)}\right)\right]\left(z-e^{t / t_{0}} z_{0}\right)}, \\
D_{y}^{(1)}+i D_{x}^{(1)} & =\frac{\alpha\left(\tilde{c}_{44}^{(2)} \epsilon_{11}^{(2)} e_{15}^{(1)}+\tilde{c}_{44}^{(1)} \epsilon_{11}^{(1)} e_{15}^{(2)}\right)}{\left[c_{44}^{(2)}\left(\epsilon_{11}^{(1)}+\epsilon_{11}^{(2)}\right)+e_{15}^{(2)}\left(e_{15}^{(1)}+e_{15}^{(2)}\right)\right]\left(z-e^{t / t_{0}} z_{0}\right)}-\frac{\beta \tilde{c}_{44}^{(1)} \epsilon_{11}^{(1)}}{e_{15}^{(1)}\left(z-z_{0}\right)} .
\end{aligned}
$$

It is clearly observed from the above expression that in general the strains, electric fields, stresses, and electric displacements inside the piezoelectric circular inclusion are time dependent due to appearance of the term $e^{t / t_{0}}$. In addition it is found from $(20)_{2}$ that the electric fields within the piezoelectric circular inclusion will be time independent when the condition $c_{44}^{(1)} e_{15}^{(2)}=c_{44}^{(2)} e_{15}^{(1)}$ is satisfied. As time elapses, the strains, electric fields, stresses, and electric displacements within the piezoelectric circular inclusion will finally arrive at the steady state

$$
\begin{aligned}
\gamma_{z y}^{(1)}+i \gamma_{z x}^{(1)} & =-\frac{\beta}{z-z_{0}}, & E_{y}^{(1)}+i E_{x}^{(1)} & =-\frac{c_{44}^{(1)}}{e_{15}^{(1)}} \frac{\beta}{z-z_{0}}, \\
\sigma_{z y}^{(1)}+i \sigma_{z x}^{(1)} & =0, & D_{y}^{(1)}+i D_{x}^{(1)} & =-\frac{\beta \tilde{c}_{44}^{(1)} \epsilon_{11}^{(1)}}{e_{15}^{(1)}\left(z-z_{0}\right)}, \quad|z| \leq R, t \rightarrow \infty .
\end{aligned}
$$


It is observed from the above expression that the internal stresses will eventually vanish due to the dashpot. The relationship $E_{y}^{(1)}+i E_{x}^{(1)}=\left(\gamma_{z y}^{(1)}+i \gamma_{z x}^{(1)}\right) c_{44}^{(1)} / e_{15}^{(1)}$ observed in the above expression is just in agreement with the vanishing internal stress condition when $t \rightarrow \infty$.

\section{Image force on the screw dislocation}

Furthermore, by employing the Peach-Koehler formulation [Pak 1990b; Lee et al. 2000] and the previously derived field components in the piezoelectric matrix (see Equation A2 of the the online supplement), it is also convenient to arrive at the image force acting on the screw dislocation due to its interaction with the circular viscous interface. For example if we assume that the piezoelectric screw dislocation with $b \neq 0, p=q=\Delta \phi=0$ lies on the positive real $x$ axis (that is, $x_{0}>R, y_{0}=0$ ), then a rather concise closed-form expression of the time dependent image force on the screw dislocation can be finally derived as

$$
F_{x}(t)=\frac{b^{2} R^{2}}{2 \pi x_{0}}\left[\frac{2\left(\tilde{c}_{44}^{(1)} c_{44}^{(2)} \epsilon_{11}^{(1)}+c_{44}^{(1)} \tilde{c}_{44}^{(2)} \epsilon_{11}^{(2)}\right)}{\left[\left(c_{44}^{(1)}+c_{44}^{(2)}\right)\left(\epsilon_{11}^{(1)}+\epsilon_{11}^{(2)}\right)+\left(e_{15}^{(1)}+e_{15}^{(2)}\right)^{2}\right]\left(e^{t / t_{0}} x_{0}^{2}-R^{2}\right)}-\frac{c_{44}^{(2)}}{x_{0}^{2}-R^{2}}\right],
$$

where $F_{x}$ is the $x$ component of the image force (the $y$ component of the image force is zero).

It is of interest to discuss several special cases to verify and to illustrate the obtained solution.

Case 1. If the inclusion and the matrix have the same material properties and poling direction, meaning $c_{44}^{(1)}=c_{44}^{(2)}=c_{44}, e_{15}^{(1)}=e_{15}^{(2)}=e_{15}$, and $\epsilon_{11}^{(1)}=\epsilon_{11}^{(2)}=\epsilon_{11}$, then it follows from Equation (21) that the image force on the screw dislocation is

$$
F_{x}(t)=\frac{c_{44} b^{2} R^{2} x_{0}\left(1-e^{t / t_{0}}\right)}{2 \pi\left(x_{0}^{2}-R^{2}\right)\left(e^{t / t_{0}} x_{0}^{2}-R^{2}\right)} \leq 0,
$$

with $t_{0}=2 R \eta / c_{44}$.

The above expression indicates that: there is no image force on the screw dislocation at the initial moment $t=0$; the screw dislocation will always be attracted to the piezoelectric inclusion when $t>0$; and the image force is independent of the piezoelectric and dielectric properties $e_{15}$ and $\epsilon_{11}$.

Case 2. If the inclusion and matrix have the same material property but are poled in opposite directions, implying $c_{44}^{(1)}=c_{44}^{(2)}=c_{44}, e_{15}^{(1)}=-e_{15}^{(2)}=e_{15}$, and $\epsilon_{11}^{(1)}=\epsilon_{11}^{(2)}=\epsilon_{11}$, then it follows from (21) that the image force on the screw dislocation is

$$
F_{x}(t)=\frac{b^{2} R^{2}}{2 \pi x_{0}}\left[\frac{\tilde{c}_{44}}{e^{t / t_{0}} x_{0}^{2}-R^{2}}-\frac{c_{44}}{x_{0}^{2}-R^{2}}\right],
$$

with $t_{0}=2 R \eta / \tilde{c}_{44}$.

It is observed from the above expression that when

$$
0 \leq t<t_{1}, \quad t_{1}=t_{0} \ln \left(\frac{\tilde{c}_{44} x_{0}^{2}-\left(\tilde{c}_{44}-c_{44}\right) R^{2}}{c_{44} x_{0}^{2}}\right),
$$

the screw dislocation will be repelled from the inclusion, meaning $F_{x}(t)>0,\left(0 \leq t<t_{1}\right)$. At the moment $t=t_{1}$, there is no image force on the dislocation, that is, $F_{x}\left(t_{1}\right)=0$. When $t>t_{1}$, the screw dislocation will always be attracted to the inclusion, meaning $F_{x}(t)<0,\left(t>t_{1}\right)$. 
Case 3. If both the inclusion and matrix are purely elastic, that is, $e_{15}^{(1)}=e_{15}^{(2)}=0$, then it follows from (21) that the image force on the screw dislocation is

$$
F_{x}(t)=\frac{c_{44}^{(2)} b^{2} R^{2}}{2 \pi x_{0}}\left(\frac{2 c_{44}^{(1)}}{c_{44}^{(1)}+c_{44}^{(2)}} \frac{1}{e^{t / t_{0}} x_{0}^{2}-R^{2}}-\frac{1}{x_{0}^{2}-R^{2}}\right),
$$

with $t_{0}$ given by Equation (19). We have carefully checked that our closed-form expression of the image force, (22), is consistent with the numerical results from Fan and Wang [2003, Figure 7, Equation (4.12)].

At the initial time $t=0,(22)$ for the image force is

$$
F_{x}(0)=\frac{c_{44}^{(2)} b^{2}}{2 \pi} \frac{c_{44}^{(1)}-c_{44}^{(2)}}{c_{44}^{(1)}+c_{44}^{(2)}} \frac{R^{2}}{x_{0}\left(x_{0}^{2}-R^{2}\right)},
$$

which is just the result for a screw dislocation interacting with a circular inclusion with a perfect interface [Dundurs 1967; Fan and Wang 2003]. At the time $t=\infty$, (22) for the image force is

$$
F_{x}(\infty)=-\frac{c_{44}^{(2)} R^{2} b^{2}}{2 \pi x_{0}\left(x_{0}^{2}-R^{2}\right)}<0,
$$

which is the result for a dislocation interacting with a traction free circular hole. It is observed from (22)-(24) that if the inclusion is stiffer than the matrix (that is, $c_{44}^{(1)}>c_{44}^{(2)}$ ), there always exists a time $t=t_{2}\left(t_{2}>0\right)$, at which $F_{x}\left(t_{2}\right)=0$ due to the fact that $F_{x}(0)>0$ and $F_{x}(\infty)<0$. In addition $t_{2}$ can be determined from (22) as

$$
t_{2}=t_{0} \ln \left(\frac{2 c_{44}^{(1)} x_{0}^{2}-R^{2}\left(c_{44}^{(1)}-c_{44}^{(2)}\right)}{x_{0}^{2}\left(c_{44}^{(1)}+c_{44}^{(2)}\right)}\right), \quad c_{44}^{(1)}>c_{44}^{(2)} .
$$

Furthermore, when $0 \leq t<t_{2}$ the screw dislocation will be repelled from the inclusion, while when $t>t_{2}$ the screw dislocation will be attracted to the inclusion.

Case 4. Lastly we consider a straight interface. The straight interface can be considered as a limit of the circular interface if we let $\delta=x_{0}-R$ and assume that $R \rightarrow \infty$. Under this condition and after some derivations, (21) is finally reduced to

$$
F_{x}(t)=\frac{b^{2}}{4 \pi \delta}\left[\frac{2\left(\tilde{c}_{44}^{(1)} c_{44}^{(2)} \epsilon_{11}^{(1)}+c_{44}^{(1)} \tilde{c}_{44}^{(2)} \epsilon_{11}^{(2)}\right)}{\left(c_{44}^{(1)}+c_{44}^{(2)}\right)\left(\epsilon_{11}^{(1)}+\epsilon_{11}^{(2)}\right)+\left(e_{15}^{(1)}+e_{15}^{(2)}\right)^{2}}\left(1+\frac{t}{2 \tilde{t}_{0}}\right)^{-1}-c_{44}^{(2)}\right],
$$

where

$$
\tilde{t}_{0}=\delta \eta \frac{\left(c_{44}^{(1)}+c_{44}^{(2)}\right)\left(\epsilon_{11}^{(1)}+\epsilon_{11}^{(2)}\right)+\left(e_{15}^{(1)}+e_{15}^{(2)}\right)^{2}}{\tilde{c}_{44}^{(1)} c_{44}^{(2)} \epsilon_{11}^{(1)}+c_{44}^{(1)} \tilde{c}_{44}^{(2)} \epsilon_{11}^{(2)}} .
$$

(25) is just the result derived in [Wang and Pan 2008, Equation (40)] for a straight interface.

Finally we consider a piezoelectric composite with the piezoelectric $\mathrm{BaTiO}_{3}$ being the fiber and the piezoelectric PZT-5 being the matrix. The material properties of $\mathrm{BaTiO}_{3}$ and PZT-5 are listed in Table 1.

Figure 2 illustrates the normalized image force $\tilde{F}=\left(R F_{x}\right) /\left(b^{2} c_{44}^{(2)}\right)$ on the screw dislocation at the four different times $t / t_{0}=0,0.05,0.5$, and $\infty$. It is observed that at the initial time $t=0$ the screw dislocation is always repelled from the inclusion $\left(F_{x}>0\right)$, while at the times $t=0.5 t_{0}$ and $t=\infty$ the 


\begin{tabular}{|ccccc|}
\hline Compound & $c_{44}\left(10^{10} \mathrm{~N} / \mathrm{m}^{2}\right)$ & $e_{15}\left(\mathrm{C} / \mathrm{m}^{2}\right)$ & $\epsilon_{11}\left(10^{-9} \mathrm{~F} / \mathrm{m}\right)$ & $\tilde{c}_{44}\left(10^{10} \mathrm{~N} / \mathrm{m}^{2}\right)$ \\
\hline $\mathrm{PZT}-5$ & 2.11 & 12.3 & 8.1103 & 3.9754 \\
$\mathrm{BaTiO}_{3}$ & 4.4 & 11.4 & 9.8722 & 5.7164 \\
\hline
\end{tabular}

Table 1. The material properties of PZT-5 and $\mathrm{BaTiO}_{3}$ [Wang et al. 2003].

screw dislocation is always attracted to the inclusion $\left(F_{x}<0\right)$. At the time $t=0.05 t_{0}$ we observe that there exists a transient equilibrium position $\left(F_{x}=0\right)$ at the point $x_{0}=1.066 R$ very close to the circular interface. In addition, the equilibrium position is unstable due to the fact that $F_{x}<0$ for $x_{0}<1.066 R$ and $F_{x}>0$ for $x_{0}>1.066 R$. In fact, the relationship between time and the transient unstable equilibrium position $\left(F_{x}=0\right)$ can be easily determined from Equation (21) as

$$
\frac{x_{0}}{R}=\sqrt{\frac{c-1}{c-e^{t / t_{0}}}}, \quad c>1,0^{+} \leq t \leq t_{0} \ln (c),
$$

where

$$
c=\frac{2\left(\tilde{c}_{44}^{(1)} c_{44}^{(2)} \epsilon_{11}^{(1)}+c_{44}^{(1)} \tilde{c}_{44}^{(2)} \epsilon_{11}^{(2)}\right)}{c_{44}^{(2)}\left[\left(c_{44}^{(1)}+c_{44}^{(2)}\right)\left(\epsilon_{11}^{(1)}+\epsilon_{11}^{(2)}\right)+\left(e_{15}^{(1)}+e_{15}^{(2)}\right)^{2}\right]} .
$$

In this example $c=1.4277$. Then it is found that when $0^{+} \leq t \leq 0.3561 t_{0}$ there always exists a transient unstable equilibrium position for the screw dislocation. We demonstrate in Figure 3 the transient unstable equilibrium position as a function of time. One can observe from Figure 3 that as the time evolves from $t=0^{+}$to $t=0.3561 t_{0}$ the transient unstable equilibrium position moves along the positive $x$ direction from $x_{0}=R$ to infinity.

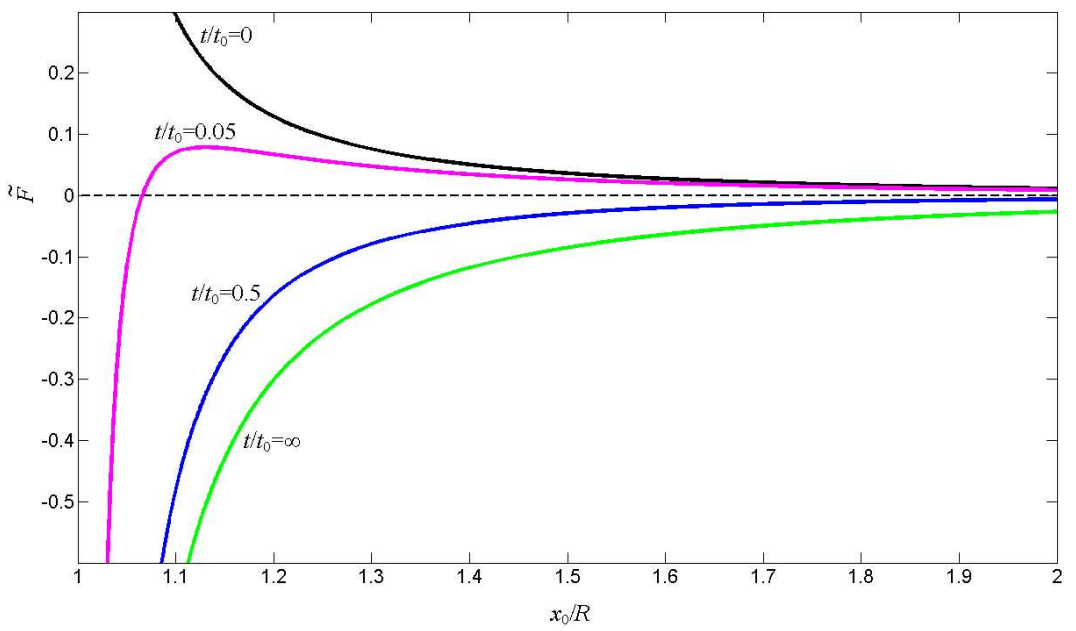

Figure 2. The normalized image force $\tilde{F}=\left(R F_{x}\right) /\left(b^{2} c_{44}^{(2)}\right)$ on the screw dislocation at the four times $t / t_{0}=0,0.05,0.5$, and $\infty$. The piezoelectric composite is composed of the piezoelectric $\mathrm{BaTiO}_{3}$ fiber and the piezoelectric PZT-5 matrix. 


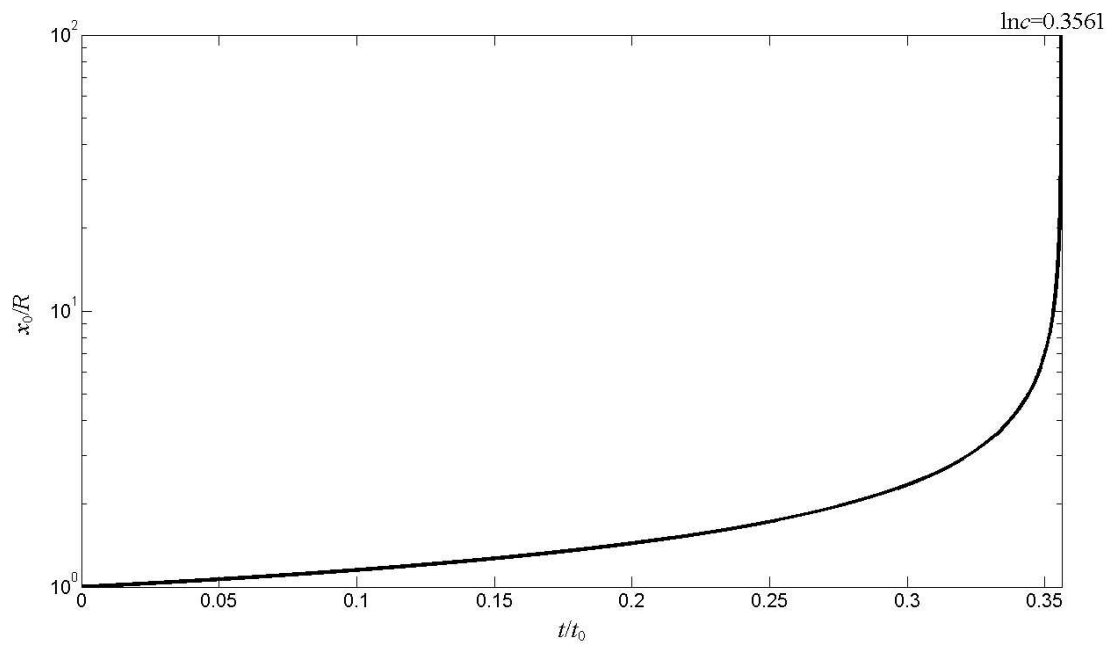

Figure 3. The transient unstable equilibrium position for the screw dislocation as a function of time. The piezoelectric composite is composed of the piezoelectric $\mathrm{BaTiO}_{3}$ fiber and the piezoelectric PZT-5 matrix.

\section{Conclusions}

A theoretical analysis was performed for a screw dislocation with a line force and a line charge interacting with a piezoelectric circular inclusion with a viscous interface described by a linear dashpot. The exact closed-form solutions were obtained by the complex variable and analytical continuation technique. In this investigation the screw dislocation was assumed to be within the matrix, whilst the solution to the situation in which the dislocation is located within the circular inclusion can be identically derived. Starting from the derived closed-form solution, we can further investigate the interaction of a matrix crack with the circular viscous interface. Finally we mention that if the viscoelastic effect modeled by both the linear spring and dashpot is introduced into the circular interface, a closed-form solution cannot be obtained for the interaction problem due to the additional introduction of the linear spring. In this case, however, infinite series form solutions to the interaction problem can be derived (see solutions in [Fan and Wang 2003] for the Kelvin and Maxwell type viscoelastic interfaces). In fact, it is in principle impossible to obtain closed-form solutions for the dislocation/inclusion interaction problem even when only the linear spring model is introduced into the circular interface [Ru and Schiavone 1997].

\section{Acknowledgements}

The authors are greatly indebted to the reviewers for their very helpful comments and suggestions.

\section{References}

[Chen et al. 2002] B. J. Chen, Z. M. Xiao, and K. M. Liew, "A screw dislocation in a piezoelectric bi-material wedge”, Int. J. Eng. Sci. 40:15 (2002), 1665-1685.

[Dundurs 1967] J. Dundurs, "On the interaction of a screw dislocation with inhomogeneities", Recent Adv. Eng. Sci. 2 (1967), 223-233. 
[Fan and Wang 2003] H. Fan and G. F. Wang, "Interaction between a screw dislocation and viscoelastic interfaces", Int. J. Solids Struct. 40:4 (2003), 763-776.

[He and Lim 2003] L. H. He and C. W. Lim, "Electromechanical responses of piezoelectric fiber composites with sliding interface under anti-plane deformations", Compos. Part B Eng. 34:4 (2003), 373-381.

[Lee et al. 2000] K. Y. Lee, W. G. Lee, and Y. E. Pak, "Interaction between a semi-infinite crack and a screw dislocation in a piezoelectric material”, J. Appl. Mech. (Trans. ASME) 67:1 (2000), 165-170.

[Liu et al. 1999] J. Liu, S. Du, and B. Wang, "A screw dislocation interacting with a piezoelectric bimaterial interface", Mech. Res. Commun. 26:4 (1999), 415-420.

[Meguid and Deng 1998] S. A. Meguid and W. Deng, "Electro-elastic interaction between a screw dislocation and an elliptical inhomogeneity in piezoelectric materials", Int. J. Solids Struct. 35:13 (1998), 1467-1482.

[Pak 1990a] Y. E. Pak, “Crack extension force in a piezoelectric material”, J. Appl. Mech. (Trans. ASME) 57 (1990), 647-653. [Pak 1990b] Y. E. Pak, "Force on a piezoelectric screw dislocation", J. Appl. Mech. (Trans. ASME) 57 (1990), 863-869.

[Pak 1992] Y. E. Pak, "Circular inclusion problem in antiplane piezoelectricity", Int. J. Solids Struct. 29:19 (1992), $2403-2419$. [Ray and Ashby 1971] R. Ray and M. F. Ashby, "On grain boundary sliding and diffusional creep”, Metall. Mater. Trans. B 2:4 (1971), 1113-1127.

[Ru and Mao 1999] C. Q. Ru and X. Mao, "Conducting cracks in a piezoelectric ceramic of limited electrical polarization", $J$. Mech. Phys. Solids 47:10 (1999), 2125-2146.

[Ru and Schiavone 1997] C. Q. Ru and P. Schiavone, "A circular inclusion with circumferentially inhomogeneous interface in antiplane shear", P. Roy. Soc. Lond. A Mat. 453:1967 (1997), 2551-2572.

[Suo 1997] Z. Suo, "Motion of microscopic surface in materials", Adv. Appl. Mech. 33 (1997), 193-294.

[Wang and Pan 2007] X. Wang and E. Pan, "A moving screw dislocation interacting with an imperfect piezoelectric bimaterial interface”, Phys. Status Solidi B 244:6 (2007), 1940-1956.

[Wang and Pan 2008] X. Wang and E. Pan, "Interaction between a screw dislocation and a viscoelastic piezoelectric bimaterial interface", Int. J. Solids Struct. 45:1 (2008), 245-257.

[Wang and Schiavone 2007] G. F. Wang and P. Schiavone, "Influence of thin film on a screw dislocation in a substrate with viscoelastic interface", Math. Mech. Solids 12:1 (2007), 119-128.

[Wang et al. 2003] X. Wang, Z. Zhong, and F. L. Wu, "A moving conducting crack at the interface of two dissimilar piezoelectric materials", Int. J. Solids Struct. 40:10 (2003), 2381-2399.

[Wang et al. 2007] X. Wang, E. Pan, and W. J. Feng, “Time-dependent Green's functions for an anisotropic bimaterial with viscous interface”, Eur. J. Mech. A Solids 26:5 (2007), 901-908.

[Xiao et al. 2007] Z. M. Xiao, H. X. Zhang, and B. J. Chen, "A piezoelectric screw dislocation interacts with interfacial collinear rigid lines in piezoelectric bimaterials", Int. J. Solids Struct. 44:1 (2007), 255-271.

Received 27 Sep 2007. Accepted 3 Feb 2008.

XU WANG: xuwang@uakron.edu

Deparment of Civil Engineering University of Akron, Akron, $\mathrm{OH} 44325-3905$, United States

and

Department of Applied Mathematics, University of Akron, Akron, OH 44325-3905, United States

ERNIAN PAN: pan2@uakron.edu

Department of Civil Engineering University of Akron, Akron, OH 44325-3905, United States

and

Department of Applied Mathematics, University of Akron, Akron, OH 44325-3905, United States

A. K RoY: Ajit.roy@wpafb.af.mil

Air Force Research Laboratory, AFRL/MLBCM, Bldg 654, 2941 Hobson Way, Wright-Patterson AFB, OH 45433-7750,

United States 


\title{
OPTIMAL BUCKLING DESIGN OF ANISOTROPIC RINGS/LONG CYLINDERS UNDER EXTERNAL PRESSURE
}

\begin{abstract}
KARAM Y. MAALAWI
Structural buckling failure due to high external hydrostatic pressure is a major consideration in designing rings and long cylindrical shell-type structures. This paper presents a direct approach for enhancing buckling stability limits of thin-walled rings/long cylinders that are fabricated from multiangle fibrous laminated composite lay-ups. The mathematical formulation employs the classical lamination theory for calculating the critical buckling pressure, where an analytical solution that accounts for the effective axial and flexural stiffness separately as well as the inclusion of the coupling stiffness terms is presented. The associated design optimization problem of maximizing the critical buckling pressure has been formulated in a standard nonlinear mathematical programming problem with the design variables encompassing the fiber orientation angles and the ply thicknesses as well. The physical and mechanical properties of the composite material are taken as preassigned parameters. The proposed model deals with dimensionless quantities in order to be valid for thin shells having different thickness-to-radius ratios. Useful design charts are given for several types of anisotropic rings/long cylinders showing the functional dependence of the buckling pressure on the selected design variables. Excellent results have been obtained for cases of filament wound rings/long cylinders fabricated from three different types of materials: $E$-glass/vinylester, graphite/epoxy and $S$-glass/epoxy. It was shown that significant improvement in the overall stability level can be attained as compared with a baseline shell design. In fact, the developed methodology has been proved to be a useful design tool for selecting an optimal stacking sequence of a thin-walled anisotropic ring/long cylinder having arbitrary thickness-to-radius ratio.
\end{abstract}

\section{Introduction}

Many mechanical and structural elements made of fiber reinforced composites are increasingly utilized in aerospace, marine and civil engineering applications [Vinson 1992; Daniel and Ishai 2006]. The most important benefits from using such advanced materials in the various structural types are the attainment of high stiffness-to-weight ratio and long fatigue life. One common application is the design of composite cylindrical shells under the action of external hydrostatic pressure, which might cause collapse by buckling instability [Simitses 1996; Sridharan and Kasagi 1997]. Examples are the underground and underwater pipelines, rocket motor casing, boiler tubes subjected to external steam pressure, and reinforced submarine structures. The composite cylindrical vessels for underwater applications [Davies and Chauchot 1999] are intended to operate at high external hydrostatic pressure (sometimes up to $60 \mathrm{MPa}$ ). For deep-submersible long-unstiffened vessels, the hulls are generally realized using multilayered, cross-ply, composite cylinders obtained following the filament winding process [Graham 1995]. On the other hand, previous numerical and experimental studies have shown that failure due to

Keywords: buckling instability, structural optimization, fibrous composite, laminated ring/cylindrical shell, external hydrostatic pressure. 
structural buckling is also a major risk factor for thin laminated cylindrical shells. Anastasiadis and Simitses [1993] studied the buckling of long laminated cylindrical shells under external radial pressure using higher order deformation theory. Their formulation, however, was restricted to symmetric lay-ups with respect to the mid-surface, to eliminate the coupling terms, as well as constant-directional pressure. More conservative results for a true fluid pressure were given by Rasheed and Yousif [2001; 2005] who applied standard energy formulation to derive the kinematics and equilibrium equations and the classical lamination theory to express the needed constitutive equations. They developed a powerful generalized closed form analytical formula for calculating stability limits of thin anisotropic rings/long cylinders subject to hydrostatic pressure. Another refined treatment of the inplane buckling of rings was given by Hodges [1999] and Hodges and Harursampath [2002]. Formulation was based on a nonlinear theory for stretching and bending of anisotropic beams having constant initial curvature in their plane of symmetry with the only restriction of small strain in the prebuckling state.

Considering next structural optimization, several papers appeared on the topic of buckling and stability optimization. Maalawi [2002] presented a piecewise structural model for buckling optimization of elastic columns under mass equality constraint. He showed that the most effective design variables that have a bearing on buckling optimization are the cross sectional area, radius of gyration and length of each segment composing the column. Another work by Maalawi and El Chazly [2002] dealt with both stability and dynamic optimization of multielement beam type structures. They formulated the associated optimization problems in a standard mathematical programming solved by the interior penalty function technique. More recently, Librescu and Maalawi [2007] considered optimization of aeroelastic stability of composite wings. They applied the concept of material grading with the implementation of both continuous and piecewise structural models. For fibrous laminated composite structures, the optimization of ply angles and thicknesses could allow the properties of the laminate to be tailored to a specific application. ZitzEvancih [1985] applied NASA buckling equations for the optimization of orthotropic cylinders against buckling. Balanced symmetric plies, consisting of $0^{\circ}, \pm 45^{\circ}$ and $90^{\circ}$ fiber orientations, were used to construct the laminates. The relative volume ratio of the laminates to each other and the stacking sequence were used as the optimization design variables. Chattopadhyay and Ferreira [1993] performed a study to investigate the maximum buckling load of a cylinder subject to ply stress constraints using material and geometric design variables. A closed form shell equation was utilized for the buckling load calculation. Laminates were constrained to be symmetric, and the number of plies was included in the design variables. Results for graphite/epoxy, glass/epoxy and Kevlar/epoxy models were found using the computer code CONMIN. Considering optimization of underwater cylindrical vessels, Tanguy et al. [2002] dealt with the optimal design of deep submarine vehicles. They developed a genetic algorithm procedure coupled with an analytical model to determine the laminate stacking sequences that maximize the critical external buckling pressure. They also showed that the measured buckling pressures for glass/epoxy and carbon/epoxy cylinders appear to be in good agreement with numerical results and demonstrated the gains due to the optimized laminations.

The aim of the present study is to achieve enhanced stability limits of anisotropic ring/long cylindrical shell structures subjected to hydrostatic external pressure. Based on the analytical buckling model developed by Rasheed and Yousif [2005], a useful optimization tool has been built for designing efficient configurations with improved buckling stability. This allows the search for the stacking sequences that maximize the buckling pressure and at the same time takes into account the manufacturing requirements. The 
corresponding increases in the buckling pressures calculated with respect to a baseline design have been evaluated for several configurations, including cases of orthotropic, filament wound rings/long cylinders fabricated from three different types of composite materials, namely $E$-glass/vinyl-ester, graphite/epoxy and $S$-glass/epoxy. It is assumed that the volume fractions of the constituent materials of the composite structure remain constant during optimization, so that the total structural mass is held at its reference value corresponding to the baseline design. The final results demonstrated the usefulness of the given methodology in attaining substantial improvement in the overall stability level of thin-walled anisotropic rings/long cylinders having arbitrary thickness-radius ratio, which is a major contribution of this paper.

\section{Structural analysis}

In this section, the basic structural analysis of multiangle laminated composite lay-ups that are widely used in filament wound rings/long cylinders are considered. In order to restrict the time of calculation to acceptable values for the developed optimization tool, the analytical formulation shall be based on the derivation in two fruitful papers by Rasheed and Yousif [2001; 2005], which are based on the assumption of small hoop strain and rotation of circumferential elements. Such an approach provides good sensitivity to lamination parameters, and allows the search for the needed optimal stacking sequences, which maximize the buckling pressure in a reasonable computational time.

Following the standard procedures of the classical lamination theory [Soden et al. 1998; Reddy 2004], the matrix equation, which relates the resultant, distributed forces $\left(N_{x x}, N_{s s}, N_{x s}\right)$ and moments $\left(M_{x x}, M_{s s}, M_{x s}\right)$ to the strains $\left(\varepsilon_{x x}^{0}, \varepsilon_{s s}^{0}, \gamma_{x a}^{0}\right)$ and curvatures $\left(\kappa_{x x}, \kappa_{s s}, \kappa_{x s}\right)$ at the middle surface of the shell structure, can be written as

$$
\left(\begin{array}{l}
N_{x x} \\
N_{s s} \\
N_{x s} \\
M_{x x} \\
M_{s s} \\
M_{x s}
\end{array}\right)=\left(\begin{array}{llllll}
A_{11} & A_{12} & A_{16} & B_{11} & B_{12} & B_{16} \\
A_{12} & A_{22} & A_{26} & B_{12} & B_{22} & B_{26} \\
A_{16} & A_{26} & A_{66} & B_{16} & B_{26} & B_{66} \\
B_{11} & B_{12} & B_{16} & D_{11} & D_{12} & D_{16} \\
B_{12} & B_{22} & B_{26} & D_{12} & D_{22} & D_{26} \\
B_{16} & B_{26} & B_{66} & D_{16} & D_{26} & D_{66}
\end{array}\right)\left(\begin{array}{c}
\varepsilon_{x x}^{0} \\
\varepsilon_{s s}^{0} \\
\gamma_{x s}^{0} \\
\kappa_{x x} \\
\kappa_{s s} \\
\kappa_{x s}
\end{array}\right),
$$

where $(x, s, z)$ are the axial, tangential and radial coordinates, respectively, and the matrix elements $\left(A_{i j}, B_{i j}, D_{i j}\right)$ are called the extensional, coupling and bending stiffness coefficients, respectively. They are all defined in Appendix A, along with the necessary kinematical relations and constitutive equations utilized in deriving (1). Actually, the kinematical relations follow the same expressions derived for thin isotropic rings [Brush and Almroth 1975; Simitses 1976]. Both cases of laminated composite rings and long cylindrical shells are considered. It was shown by Rasheed and Yousif [2005] that the only significant strain components in both cases are the hoop strain $\varepsilon_{s s}^{0}$ and the circumferential curvature $\kappa_{s s}$ of the mid-surface; see Appendix A. The reduced form of (1) for the two cases was shown to be

$$
\left(\begin{array}{c}
N_{s s} \\
M_{s s}
\end{array}\right)=\left(\begin{array}{cc}
A_{\text {ani }} & B_{\text {ani }} \\
B_{\text {ani }} & D_{\text {ani }}
\end{array}\right)\left(\begin{array}{c}
\varepsilon_{s s}^{0} \\
\kappa_{s s}
\end{array}\right) \text {. }
$$

In the case of thin rings the axial and shear forces $\left(N_{x x}, N_{x s}\right)$ must vanish along the free edges. The bending and twisting moments $\left(M_{x x}, M_{x s}\right)$ may also be neglected. Therefore, the first, third, fourth and 
sixth rows of (1) are solved for the strains and curvatures in terms of $\left(\varepsilon_{s s}^{0}, \kappa_{s s}\right)$ to give the following matrix relation

or

$$
S_{1}\left(\begin{array}{c}
\varepsilon_{x x}^{0} \\
\gamma_{x s}^{0} \\
\kappa_{x x} \\
\kappa_{x s}
\end{array}\right)=-S_{2}\left(\begin{array}{c}
\varepsilon_{s s}^{0} \\
\kappa_{s s}
\end{array}\right)
$$

$$
\left(\begin{array}{c}
\varepsilon_{x x}^{0} \\
\gamma_{x s}^{0} \\
\kappa_{x x} \\
\kappa_{x s}
\end{array}\right)=-S_{1}^{-1} S_{2}\left(\begin{array}{c}
\varepsilon_{s s}^{0} \\
\kappa_{s s}
\end{array}\right)
$$

where

$$
S_{1}=\left(\begin{array}{llll}
A_{11} & A_{16} & B_{11} & B_{16} \\
A_{16} & A_{66} & B_{16} & B_{66} \\
B_{11} & B_{16} & D_{11} & D_{16} \\
B_{16} & B_{66} & D_{16} & D_{66}
\end{array}\right), \quad \text { and } \quad S_{2}=\left(\begin{array}{ll}
A_{12} & B_{12} \\
A_{26} & B_{26} \\
B_{12} & D_{12} \\
B_{26} & D_{26}
\end{array}\right) \text {. }
$$

Substituting (3) back into (1), we can show that

$$
\left(\begin{array}{ll}
A_{\text {ani }} & B_{\text {ani }} \\
B_{\text {ani }} & D_{\text {ani }}
\end{array}\right)_{\text {ring }}=\left(\begin{array}{ll}
A_{22} & B_{22} \\
B_{22} & D_{22}
\end{array}\right)-S_{2}^{T} S_{1}^{-1} S_{2} .
$$

For the case of a long cylinder, the out-of-plane displacements are restrained, that is,

$$
\varepsilon_{x x}^{0}=\gamma_{x s}^{0}=\kappa_{x s}=0 .
$$

Therefore, the only strains to be taken into considerations are the in-plane hoop strain $\varepsilon_{s s}^{0}$ and the circumferential curvature $\kappa_{s s}$. Accordingly, the reduced matrix of (2) takes the following form

$$
\left(\begin{array}{ll}
A_{\text {ani }} & B_{\text {ani }} \\
B_{\text {ani }} & D_{\text {ani }}
\end{array}\right)_{\text {cylinder }}=\left(\begin{array}{ll}
A_{22} & B_{22} \\
B_{22} & D_{22}
\end{array}\right) .
$$

\section{Analytical buckling model}

The governing differential equations of anisotropic rings/long cylinders subjected to external pressure are similar to those of the isotropic case [Brush and Almroth 1975; Simitses 1976].

They are cast in the following:

$$
\begin{aligned}
M_{s s}^{\prime}+R\left(N_{s s}^{\prime}-\beta N_{s s}\right) & =\beta p R^{2}, \\
M_{s s}^{\prime \prime}-R\left(N_{s s}+\left(\beta N_{s s}\right)^{\prime}+p\left(w_{0}+v_{0}^{\prime}\right)\right) & =p R^{2},
\end{aligned}
$$

where the prime denotes differentiation with respect to angular position $\varphi$, and

$$
\beta=\frac{1}{R}\left(v_{0}-w_{0}^{\prime}\right)
$$

Definitions of other parameters are given in Appendix A. Rasheed and Yousif [2001; 2005] presented two solutions for (6): one for the prebuckled state and the other termed as the bifurcation solution obtained 

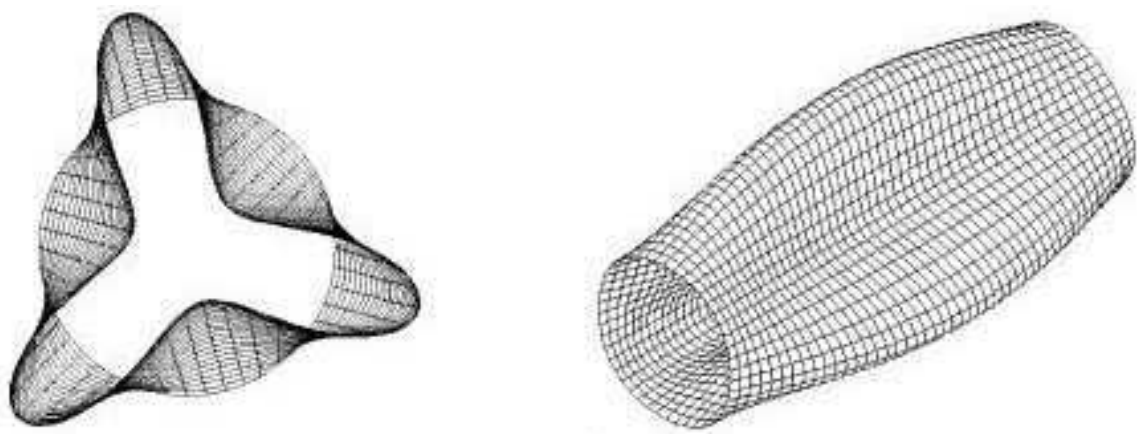

Figure 1. Characteristic buckling mode of a laminated long cylinder [Simitses 1996].

by perturbing the displacements about the prebuckling solution. They finally arrived at a closed form solution for the critical buckling pressure given by

$$
p_{c r}=3 \frac{D_{\mathrm{ani}}}{R^{3}}\left(\frac{1-\frac{\psi^{2}}{\alpha}}{1+\alpha+2 \psi}\right), \quad \psi=\frac{1}{R} \frac{B_{\mathrm{ani}}}{A_{\mathrm{ani}}}, \quad \alpha=\frac{1}{R^{2}} \frac{D_{\mathrm{ani}}}{A_{\mathrm{ani}}},
$$

where the stiffness coefficients $A_{\text {ani }}, B_{\text {ani }}$ and $D_{\text {ani }}$ can be calculated from (4) for the case of circular rings and from (5) for long cylinders. It is to be noticed here that the formula given in (7) for calculating $p_{c r}$ is only valid for thin rings/cylinders with thickness-to-radius ratio $h / R \leq 0.1$ [Rasheed and Yousif 2001]. A typical buckling mode of laminated long cylinder is shown in Figure 1.

The reduced forms of (7) for some limiting cases where $\psi=0$ and $\alpha \ll 1$ are given in Table 1 .

\section{Optimization problem statement}

The associated optimization problem shall seek maximization of the external hydrostatic pressure $p_{c r}$ at which buckling instability might occur. Optimization variables include the total number of plies $n$,

\begin{tabular}{|l|l|}
\hline \multicolumn{1}{|c|}{ Limiting Cases } & $\begin{array}{l}\text { Critical buckling pressure } p_{c r}=3 D / R^{3} \\
\text { Bending stiffness, } D\end{array}$ \\
\hline $\begin{array}{l}\text { Thin isotropic rings [Brush and Almroth 1975]. } \\
\text { Thin isotropic long cylinders [Simitses 1976]. }\end{array}$ & $E h^{3} / 12$ \\
Thin orthotropic rings [Anastasiadis and Simitses 1993] & $E_{22} / 12\left(1-v^{3}\right)$ \\
Thin orthotropic long cylindrical shells with fibers parallel & \\
to the shell axis $x$ [Anastasiadis and Simitses 1993] & $E_{22} h^{3} / 12\left(1-v_{12} v_{21}\right)$ \\
\hline
\end{tabular}

Table 1. Buckling pressure formulas for limiting cases $\psi=0$ and $\alpha \ll 1$. $E, v=$ isotropic modulus of elasticity and Poisson's ratio, $E_{22}=$ hoop modulus, $v_{12}=$ Poisson's ratio for axial load, $v_{21}=v_{12} E_{22} / E_{11}$ (in cases with fibers perpendicular to the shell axis, $E_{22}$ should be replaced by $E_{11}$ ) 
thickness $h_{k}$ and fiber orientation angle $\theta_{k}$ of the individual $k$-th ply. Side constraints are always imposed on the design variables for geometrical, manufacturing or logical reasons to avoid having unrealistic odd shaped optimum designs.

4A. Definition of the baseline design. It is convenient first to normalize all variables and parameters with respect to a baseline design, which has been selected to be a unidirectional orthotropic laminated ring/long cylinder with the fibers parallel to the shell axis $x$. Optimized shell designs shall have the same material properties, mean radius $R$ and total shell thickness $h$ of the baseline design. Therefore, the preassigned parameters, which are not subject to change in the optimization process, ought to be the type of material of construction, mean radius and total thickness of the shell.

Using the formulas given in Table 1 for cases of orthotropic shells, we define expressions for calculating the critical buckling pressure $P_{\text {cro }}$ of the baseline design in Table 2, which depend upon the type of composite material utilized and the shell thickness-to-radius ratio $h / R$ as well.

4B. Proposed optimization model. The search for the optimized lamination can be performed by coupling the analytical buckling shell model to a standard nonlinear mathematical programming procedure. The design variable vector $\underline{X}_{d}$, which is subject to change in the optimization process, is defined as $\underline{X}_{d}=\left(\hat{h}_{k}, \theta_{k}\right)_{k=1,2, \ldots, n}$, where the dimensionless thickness of the $k$-th lamina is defined by $\hat{h}_{k}=h_{k} / h$. Therefore, the buckling optimization problem considered herein may be cast in the following standard mathematical programming form:

$$
\begin{array}{ll}
\text { Maximize } & \hat{p}_{c r} \\
\text { subject to } & h_{L} \leq \hat{h}_{k} \leq h_{U}, \\
& \theta_{L} \leq \theta_{k} \leq \theta_{U} \quad k=1,2, \ldots, n \\
& \sum_{k=1}^{n} \hat{h}_{k}=1,
\end{array}
$$

where $\hat{p}_{c r}=p_{c r} / p_{\text {cro }}$ is the dimensionless critical buckling pressure and $h_{L}, h_{U}$ are the lower and upper bounds imposed on the individual dimensionless ply thicknesses. According to the filament-winding manufacturing process, each ply is characterized by its filament-winding angle $\theta_{k}$ with respect to the

\begin{tabular}{|c|cccc|cc|}
\hline & \multicolumn{4}{|c|}{$\begin{array}{c}\text { Orthotropic mechanical } \\
\text { properties* }(\mathrm{GPa})\end{array}$} & \multicolumn{2}{c|}{$p_{\text {cro }} x(h / R)^{3}(\mathrm{GPa})$} \\
\hline Material Type & $E_{11}$ & $E_{22}$ & $G_{12}$ & $v_{12}$ & Rings & Cylinders \\
\hline$E$-glass/vinyl-ester & 41.06 & 6.73 & 2.5 & 0.299 & 1.683 & 1.708 \\
graphite/epoxy & 130.0 & 7.0 & 6.0 & 0.28 & 1.75 & 1.757 \\
$S$-glass/epoxy & 57.0 & 14.0 & 5.7 & 0.277 & 3.50 & 3.567 \\
\hline
\end{tabular}

Table 2. Material properties and critical buckling pressure of the baseline design ( $\left.p_{\text {cro }}\right)$. *Taken from [Rasheed and Yousif 2001]. 
cylinder axis $x$. The stacking sequence is denoted by $\left[\theta_{1} / \theta_{2} / \cdots / \theta_{n}\right]$, where the angles are given in degrees, starting from the outer surface of the shell. In addition, in a real-world manufacturing process, the filament-winding angles $\theta_{k}$ must be chosen from a limited range of allowable lower $\left(\theta_{L}\right)$ and upper $\left(\theta_{U}\right)$ values according to technology references. It is important to mention here that the volume fractions of the constituent materials of the composite structure are assumed not to significantly change during optimization, so that the total structural mass remains constant at its reference value of the baseline design. The effect of changing the volume fractions is now under study by the author, where the concept of material grading will be considered [Librescu and Maalawi 2007].

This optimization problem may be thought as a search in a $2 n$-dimensional space for a point corresponding to the maximum value of the objective function such that it lies within the region bounded by subspaces representing the constraint functions [Vanderplaats 1994; Venkataraman 2002]. The usefulness and efficiency of penalty methods (see Appendix B) for solving this kind of optimization problems have been explored intensively in the literature [Maalawi and El Chazly 2002]. The constraints are taken into account indirectly by transforming the constrained problem into a series of unconstrained problems. Several software packages are available now for solving mathematical programming problems. The MATLAB optimization toolbox [Venkataraman 2002] offers routines that implement the interior penalty function method, which has a wide applicability in many engineering applications, via a built-in function named "fminsearch".

\section{Results and discussions}

The given approach discussed in previous sections shall be applied here to several cases of study of thin-walled anisotropic rings/long cylinders subjected to external hydrostatic pressure. The materials of construction are chosen to be $E$-glass/vinyl-ester, graphite/epoxy and $S$-glass/epoxy. The functional behavior of the candidate objective function, as represented by maximization of the dimensionless buckling pressure $\hat{p}_{c r}$, is thoroughly investigated in order to see how it is changed with the optimization variables in the selected design space. The final optimum designs recommended by the model will directly depend on the mathematical form and behavior of the objective function.

5A. Two-layer anisotropic long cylinder. The first case study to be considered herein is a long thinwalled cylindrical shell fabricated from $E$-glass/vinyl-ester composites with the lay-up composed of only two plies $(n=2)$ having equal thicknesses $\left(\hat{h}_{1}=\hat{h}_{2}=0.5\right)$ and different fiber orientation angles. Figure 2 shows the developed level curves of the dimensionless buckling pressure $\hat{p}_{c r}$ (also named isomerits or isobars) in the $\theta_{1}-\theta_{2}$ design space. It is seen that the objective function is well behaved in the selected design space with a symmetrical-shaped contours about the two lines $\theta_{1}=0$ and $\theta_{2}=0$ corresponding to the baseline design in which $\hat{p}_{c r}=1.0$, representing a point of global minima.

With the special case of $\pm 63^{\circ}$ angle-ply $E$-glass/vinyl-ester cylinder, the present model gives $\hat{p}_{c r}=$ 4.23, that is, $p_{c r}=4.23 \times 1.708 \times(h / R)^{3} \mathrm{GPa}$, depending on the shell thickness-to-radius ratio (refer to Table 2). The actual dimensional values of the critical buckling pressure for the different thickness ratios have been calculated from just one point in the design space of Figure 2: a significant contribution and gain from the given optimization formulation. The corresponding values are given in Table 3 for the cases of baseline design, helically wound $\left[ \pm 63^{\circ}\right]$ and $\left[ \pm 90^{\circ}\right]$ hoop layers. It is seen that the results compare very well with those given by Rasheed and Yousif [2001]. The unconstrained maximum value 


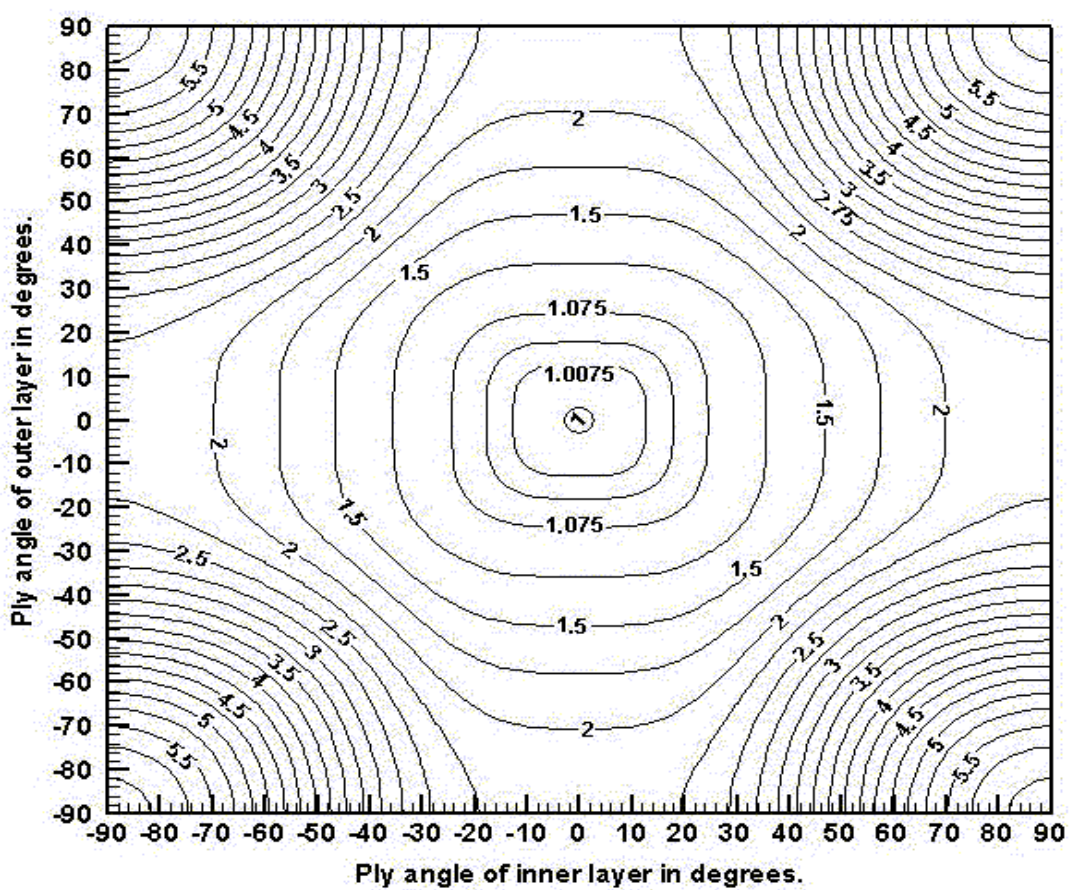

Figure 2. Dimensionless buckling pressure $\hat{p}_{c r}$ in the $\left[\theta_{1} / \theta_{2}\right]$ design space. Two-ply cylinder made of $E$-glass/vinyl-ester $\left(\hat{h}_{1}=\hat{h}_{2}=0.5\right)$.

of the critical buckling pressure occurs at any one of the corners of Figure 2 corresponding to the four design points $\left[\theta_{1} / \theta_{2}\right]=\left[ \pm 90^{\circ} / \pm 90^{\circ}\right]$ where $\hat{p}_{c r}=6.1$.

To examine the effect of using another type of constructional material, we show in Figure 3 the developed isomerits for a two-ply long cylinder fabricated from graphite/epoxy composites. As seen the shape of the level curves is similar to that of Figure 2, but with higher stability levels, reaching a maximum value of $\hat{p}_{c r}=18.57$ for a hoop wound construction.

Table 4 presents the solutions for the $\left[ \pm 45^{\circ}\right]$ angle-ply and the $\left[90^{\circ}\right]$ cross-ply constructions for different thickness-to-radius ratios. Results are compared with those in [Rasheed and Yousif 2001], which

\begin{tabular}{|cccc|}
\hline & $\begin{array}{c}\text { Baseline }\left[0^{\circ}\right] \\
\hat{p}_{c r}=1.00\end{array}$ & $\begin{array}{r}\text { Helically wound }\left[ \pm 63^{\circ}\right] \\
4.23\end{array}$ & Hoop plies $\left[ \pm 90^{\circ}\right]$ \\
$h / R$ & & & \\
\hline $1 / 15$ & 506.07 & 2140.69 & 3087.05 \\
$1 / 20$ & 213.50 & 903.11 & 1302.35 \\
$1 / 25$ & 109.31 & 462.39 & 666.80 \\
$1 / 50$ & 13.66 & 57.80 & 83.35 \\
\hline
\end{tabular}

Table 3. Critical buckling pressure for $E$-glass/vinyl-easter cylinders with different layups $\left(p_{c r}=\hat{p}_{c r} \times 1.708(h / R)^{3} \mathrm{GPa}\right)$. 


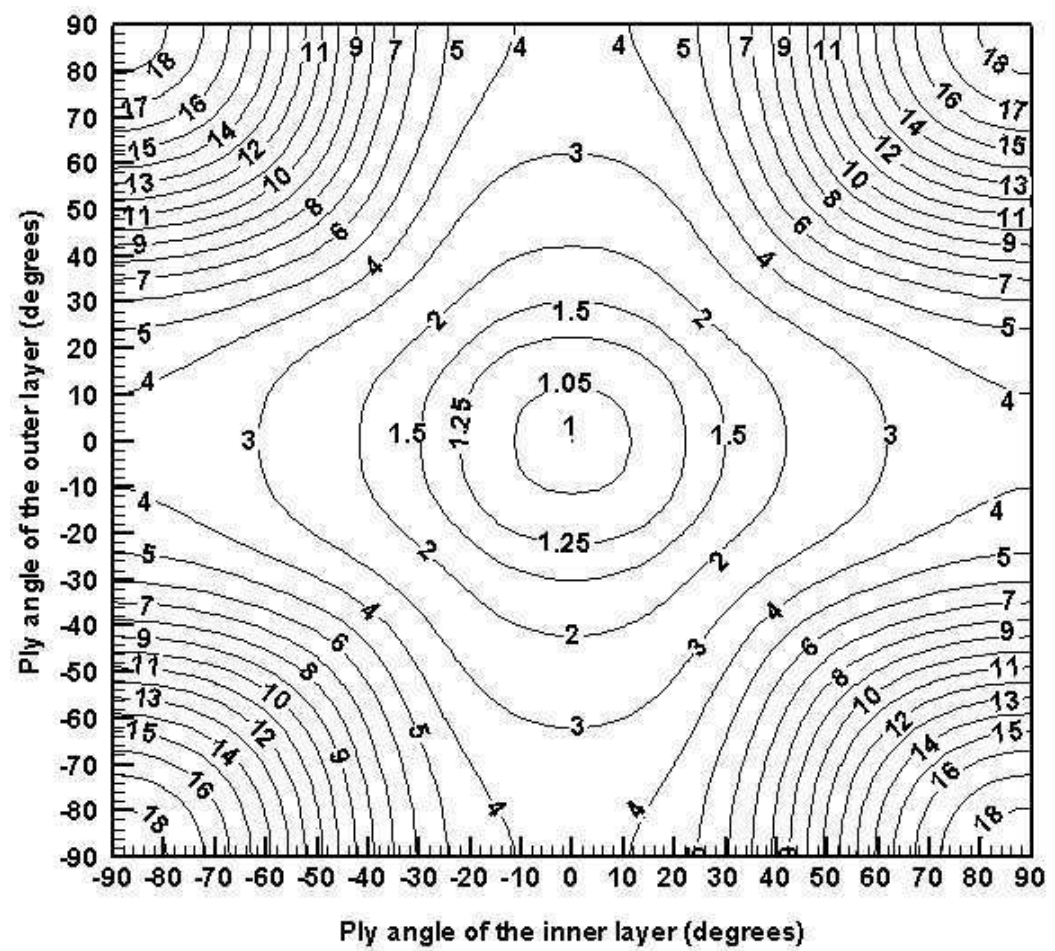

Figure 3. $\hat{p}_{c r}$-isomerits for a graphite/epoxy, two-layer cylinder in $\left[\theta_{1} / \theta_{2}\right]$ design space $\left(\hat{h}_{1}=\hat{h}_{2}=0.5\right)$.

were based on the assumption that adjacent $[ \pm \theta]$ layers are merged together with the stiffness coefficients taken as average values from the $(+\theta)$ and $(-\theta)$ plies. These solutions are also valid for lay-ups $\left[0_{3}^{\circ}\right]_{s}$, $\left[90_{3}^{\circ}\right]_{s},\left[45_{2}^{\circ} /-45_{2}^{\circ}\right]_{s}$ and $\left[45^{\circ} /-45^{\circ} / 45^{\circ} /-45^{\circ}\right]_{s}$, which were numbered $1,8,20$ and 21 in the papers by Anastasiadis and Simitses [1993] and Rasheed and Yousif [2001].

The case of a helically wound lay-up construction $[+\theta /-\theta]$ with unequal play thicknesses $\hat{h}_{1}$ and $\hat{h}_{2}$, such that their sum is held fixed at a value of unity, has also been investigated. Computer solutions have shown that no significant change in the resulting values of the critical buckling pressure can be remarked in spite of the wide change in the ply thicknesses. This is a natural expected result since

\begin{tabular}{|c|c|c|c|}
\hline$h / R$ & $\begin{array}{c}\text { Baseline }\left[0^{\circ}\right] \\
\hat{p}_{c r}=1.00\end{array}$ & $\begin{array}{l}\text { Helically wound }\left[ \pm 45^{\circ}\right] \\
5.9\end{array}$ & Hoop plies $\left[ \pm 90^{\circ}\right]$ \\
\hline $1 / 15$ & 520.59 & 3071.50 & 9667.40 \\
\hline $1 / 50$ & 14.06 & 82.93 & 261.02 \\
\hline $1 / 120$ & 1.02 & 5.99 & 18.88 \\
\hline
\end{tabular}

Table 4. Critical buckling pressure for graphite/epoxy cylinders with different lay-ups $\left(p_{c r}=\hat{p}_{c r} \times 1.757(h / R)^{3} \mathrm{GPa}\right)$. 


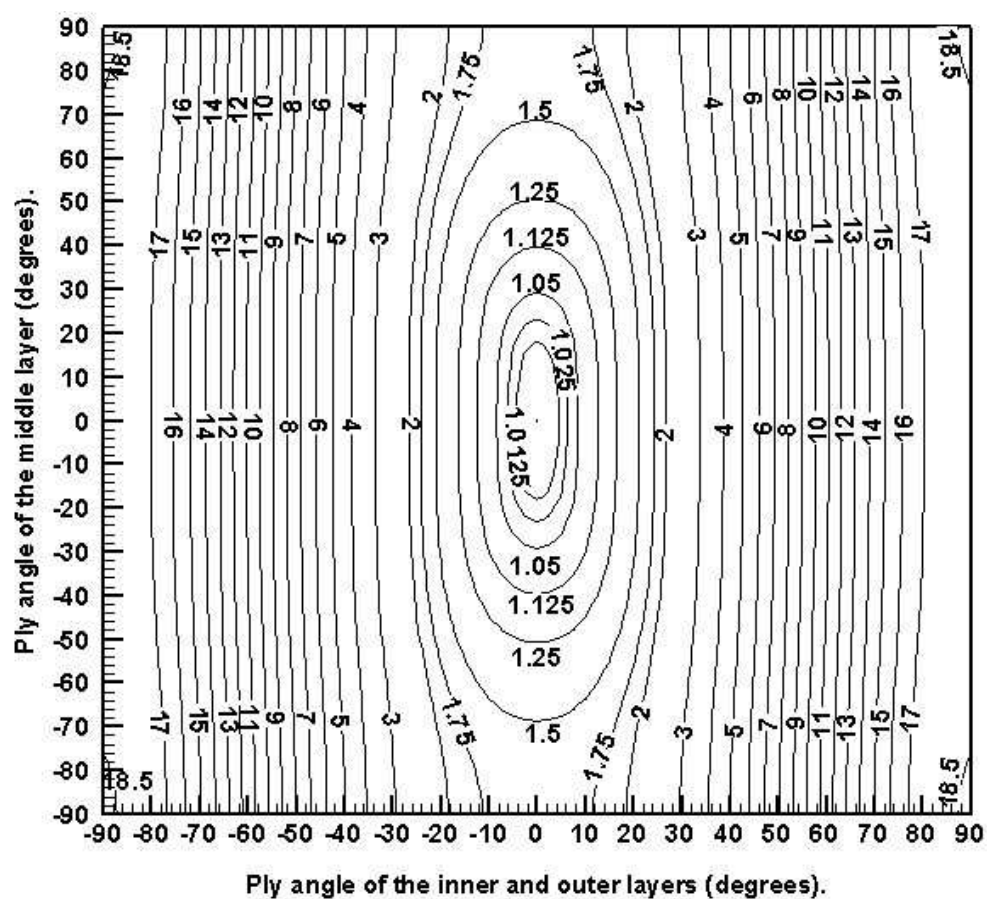

Figure 4. $\hat{p}_{c r}$ - isomerits for three-ply graphite/epoxy cylinder $\left[\theta_{1} / \theta_{2} / \theta_{1}\right]$.

the stiffness coefficients $A_{\text {ani }}, B_{\text {ani }}$ and $D_{\text {ani }}$ remain unchanged for such lay-up construction. Also, it should be mentioned here that the optimal buckling pressure for a cylinder having stacking sequence $\left[+\theta_{1} /-\theta_{1} /+\theta_{2} /-\theta_{2}\right]$ is identical to that obtained before for stacking sequence $\left[\theta_{1} / \theta_{2}\right]$, as given in Figures 2 and 3.

5B. Three-layer anisotropic long cylinder. Figure 4 shows the developed isomerits for a cylinder constructed from three equally-thicked layers with stacking sequence denoted by $\left[\theta_{1} / \theta_{2} / \theta_{1}\right]$. The same behavior can be observed as before, but with slight flattening in the $\theta_{2}$-direction. The contours are fully symmetrical about the mid-point corresponding to the minimal baseline value of unity. Two distinct zones can be seen: the closed middle one containing the global minima, and the open one covering the two ranges $\theta_{1}<-30^{\circ}$ and $\theta_{1}>30^{\circ}$ in which the critical buckling pressure is not much affected by variation in the ply angle $\theta_{2}$.

Other computational results for cross-ply lamination are given in Table 5, where substantial increase in the critical bucking pressure by changing the ply angles can be observed. Similar solutions were obtained for the stacking sequences $\left[0_{2}^{\circ} / 90^{\circ}\right]_{s}$ and $\left[90_{2}^{\circ} / 0^{\circ}\right]_{s}$, which corresponds to lay-up numbers 2 and 7 considered by Anastasiadis and Simitses [1993].

5C. Four-layer sandwiched anisotropic cylinder. The same graphite/epoxy cylinder is reconsidered here with changing the stacking sequence to become $\pm 20^{\circ}$ equal-thickness layers sandwiched in between outer and inner $90^{\circ}$ hoop layers with unequal thicknesses $\hat{h}_{2}=\hat{h}_{3}, \hat{h}_{1} \neq \hat{h}_{4}$, such that the thickness equality 


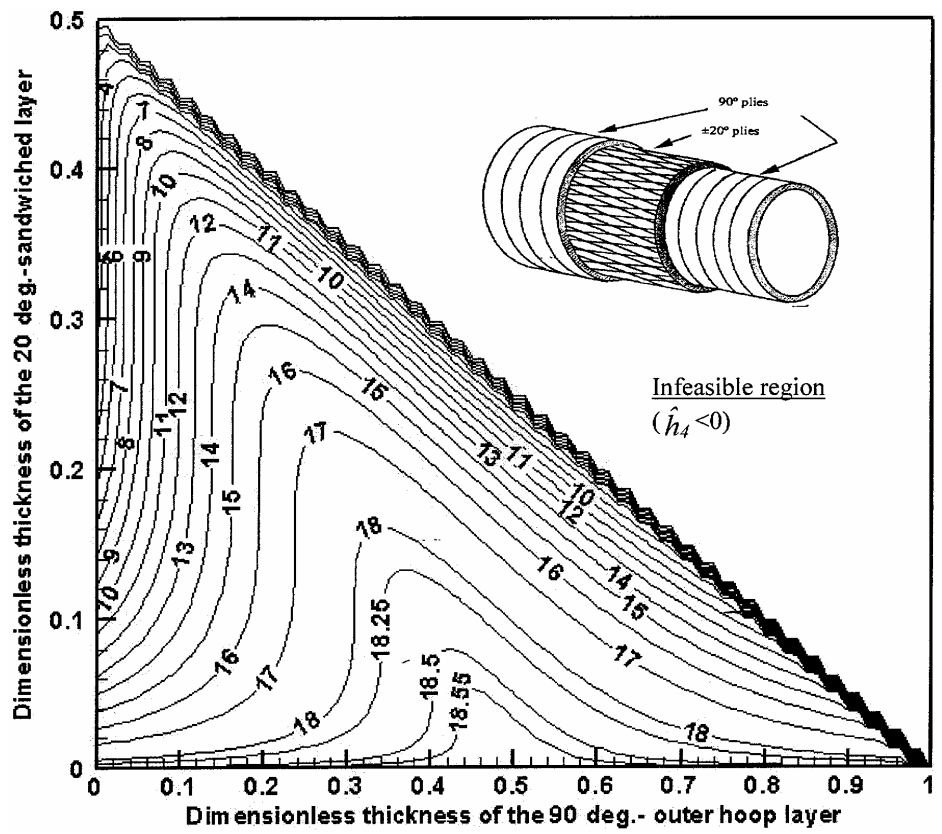

Figure 5. Design space for a sandwich lay-up graphite/epoxy cylinder $\left[90^{\circ} / \pm 20^{\circ} / 90^{\circ}\right]$.

constraint

$$
\sum_{k=1}^{4} \hat{h}_{k}=1
$$

is always satisfied. Figure 5 shows the developed $\hat{p}_{c r}$-isomerits in the $\hat{h}_{1}-\hat{h}_{2}$ design space. The contours inside the feasible domain, which is bounded by the three lines $\hat{h}_{1}=0, \hat{h}_{2}=0$ and $\hat{h}_{1}+2 \hat{h}_{2}=1$ (that is, $\hat{h}_{4}=0$ ), are obliged to turn sharply to be asymptotes to the line $\hat{h}_{4}=0$, in order not to violate the thickness equality constraint. This is why they appear in the figure as zigzagged lines.

It is clear now that all tabulated results given by Rasheed and Yousif [2001] can be directly obtained from just one design point in Figure 5, namely $\left(\hat{h}_{1}, \hat{h}_{2}\right)=(0.25,0.25)$ at which $\hat{p}_{c r}=16.43$ (see Table 6). As a general observation, as the thickness of the hoop layers increase, a substantial increase in the critical

\begin{tabular}{|rrrc|}
\hline & Baseline $\left[0_{3}^{\circ}\right]$ & {$\left[0^{\circ} / 90^{\circ} / 0^{\circ}\right]$} & {$\left[90^{\circ} / 0^{\circ} / 90^{\circ}\right]$} \\
$h / R$ & $\hat{p}_{c r}=1.00$ & 1.651 & 17.92 \\
\hline $1 / 15$ & 520.59 & 859.57 & 9331.19 \\
$1 / 50$ & 14.06 & 23.21 & 251.94 \\
$1 / 120$ & 1.02 & 1.68 & 18.23 \\
\hline
\end{tabular}

Table 5. Critical buckling pressure for graphite/epoxy cylinders $\left[\theta_{1} / \theta_{2} / \theta_{1}\right]$ $\left(p_{c r}=\hat{p}_{c r} \times 1.757(h / R)^{3} \mathrm{GPa}\right)$. 


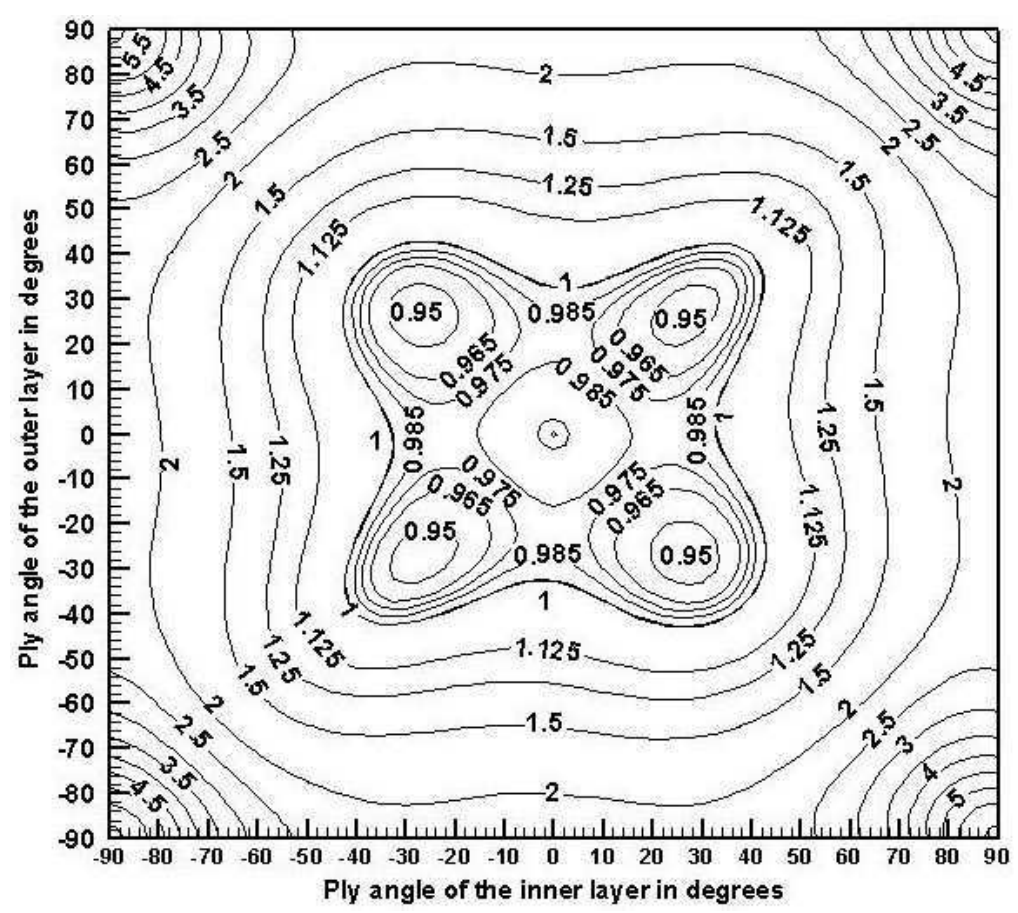

Figure 6. Butterfly-like zone containing the local minimal stability limits in $\left[\theta_{1} / \theta_{2}\right]$ design space for two-layer, $E$-glass/vinyl-easter ring $\left(\hat{h}_{1}=\hat{h}_{2}=0.5\right)$.

buckling pressure will be achieved; for example, at $\left(\hat{h}_{1}, \hat{h}_{2}\right)=(0.33,0.17), \hat{p}_{c r}=17.92$ representing a percentage increase of $(17.92-16.43) / 16.43=9.1 \%$.

5D. Anisotropic thin rings. In this section, some study cases of thin rings will be examined to see the effect of anisotropy for different angle-ply stacking sequences. The first example considers a ring fabricated from $E$-glass/vinyl-ester having two equal-thickness layers with stacking $\left[\theta_{1} / \theta_{2}\right]$. Figure 6 shows the developed $\hat{p}_{c r}$-isomerits, which are symmetrical about the two lines $\theta_{1}=0$ and $\theta_{2}=0$ corresponding to the baseline value of unity. The region in the middle resembles a butterfly containing four local minima $\left[ \pm 30^{\circ} / \pm 30^{\circ}\right.$, where $\hat{p}_{c r}=0.94$ representing about $6 \%$ degradation in the stability level. The butterfly bounding contour determines stacking sequences having buckling pressure equals to that of the baseline design (namely, $\hat{p}_{c r}=1$ ). The unconstrained global maximum value of the critical buckling pressure occurs at any one of the four design points $\left[\theta_{1} / \theta_{2}\right]=\left[ \pm 90^{\circ} / \pm 90^{\circ}\right]$ where $\hat{p}_{c r}=6.1$. The constrained

\begin{tabular}{|ccccc|}
\hline$h / R$ & $1 / 15$ & $1 / 20$ & $1 / 25$ & $1 / 50$ \\
\hline$p_{c r}(\mathrm{GPa})$ & 8553.0 & 3609.3 & 1847.5 & 231.0 \\
\hline
\end{tabular}

Table 6. Critical buckling pressure for graphite/epoxy cylinders $\left[90^{\circ} / \pm 20^{\circ} / 90^{\circ}\right]\left(\hat{p}_{c r}=\right.$ $\left.16.43, p_{\text {cro }}=1.757(h / R)^{3} \mathrm{GPa}, p_{c r}=\hat{p}_{c r} \cdot p_{\text {cro }}\right)$. 


\begin{tabular}{|lcccc|}
\hline & {$\left[+63^{\circ}\right]$} & {$\left[+63^{\circ} /-63^{\circ}\right]$} & {$\left[+63^{\circ} /-63^{\circ}\right]_{3}$} & {$\left[+63^{\circ} /-63^{\circ}\right]_{\infty}$} \\
$h / R$ & $\hat{p}_{c r}=1.754$ & 2.166 & 3.134 & 3.234 \\
\hline $1 / 20$ & 369.00 & 455.67 & 659.32 & 680.35 \\
$1 / 50$ & 23.62 & 29.16 & 42.20 & 43.54 \\
$1 / 100$ & 2.95 & 3.65 & 5.27 & 5.44 \\
\hline
\end{tabular}

Table 7. Critical buckling pressure for $E$-glass/vinyl-easter thin rings $\left(p_{c r}=\hat{p}_{c r} \times 1.683(h / R)^{3} \mathrm{GPa}\right)$.

solutions for the special case of $\pm 63^{\circ}$ angle-ply, which was considered by Rasheed and Yousif [2005], are summarized in Table 7, including also the extreme cases of full anisotropy represented by the lay-up with only $\left[+63^{\circ}\right]$ plies for the entire thickness and the fully orthotropic lamination consisting of many too thin alternating balanced plies $\left[+63^{\circ} /-63^{\circ}\right]$, which produce the highest possible buckling capacity.

A last example considers a thin ring fabricated from $S$-glass/epoxy with the mechanical properties given in Table 2. The lay-up consists of three $\left[+45^{\circ} /-45^{\circ}\right]$ balanced plies, each with equal thickness, that is, $\left[h_{k} / h_{k}\right]_{k=1,2,3}$ where $h_{k}$ is the thickness of a single lamina. Figure 7 depicts the developed $\hat{p}_{c r^{-}}$ isomerits in the $\hat{h}_{1}-\hat{h}_{2}$ design space. As seen, the feasible domain is bounded by the three straight lines $\hat{h}_{1}=0, \hat{h}_{2}=0$ and $\hat{h}_{1}+\hat{h}_{2}=0.5$, where an infinite number of level curves are obliged to turn to be tangent to the latter one in order not to violate the thickness equality constraint. The global optimal solution has shown to be of equal ply thickness: $\hat{h}_{k}=0.167, k=1,2,3$, where $\hat{p}_{c r}=1.2593$. The calculated dimensional value of the maximum buckling pressure is given in Table 8 for different thickness to radius ratios.

\section{Conclusions}

In this paper, a practical approach for enhancing the buckling stability limits of thin-walled anisotropic rings/long cylinders has been developed. The formulation of an optimal lamination design against buckling has been thoroughly investigated, where useful design charts are given for several types of anisotropic rings/long cylinders showing the functional dependence of the critical buckling pressure on the stacking sequence and ply thickness as well. An analytical buckling model has been implemented, which provides good sensitivity to lamination parameters, allowing the search for the needed optimal stacking sequences in an acceptable computational time. The proposed model deals with dimensionless quantities in order to be applicable for handling thin shells having arbitrary thickness-to-radius ratios, which is a major contribution of this work. Results have indicated that the optimized laminations induce significant increases, always exceeding several tens of percent, of the buckling pressures with respect to the reference

\begin{tabular}{|cccccc|}
\hline$h / R$ & $1 / 15$ & $1 / 20$ & $1 / 25$ & $1 / 50$ & $1 / 100$ \\
\hline$p_{c r}(\mathrm{KPa})$ & 1305.94 & 550.94 & 282.1 & 35.26 & 4.41 \\
\hline
\end{tabular}

Table 8. Maximum buckling pressure for $S$-glass/epoxy rings $\left[+45^{\circ} /-45^{\circ}\right]_{3}\left(\hat{p}_{c r}=\right.$ $\left.1.2593, p_{\text {cro }}=3.5(h / R)^{3} \mathrm{GPa}, p_{c r}=\hat{p}_{c r} \cdot p_{\text {cro }}\right)$. 


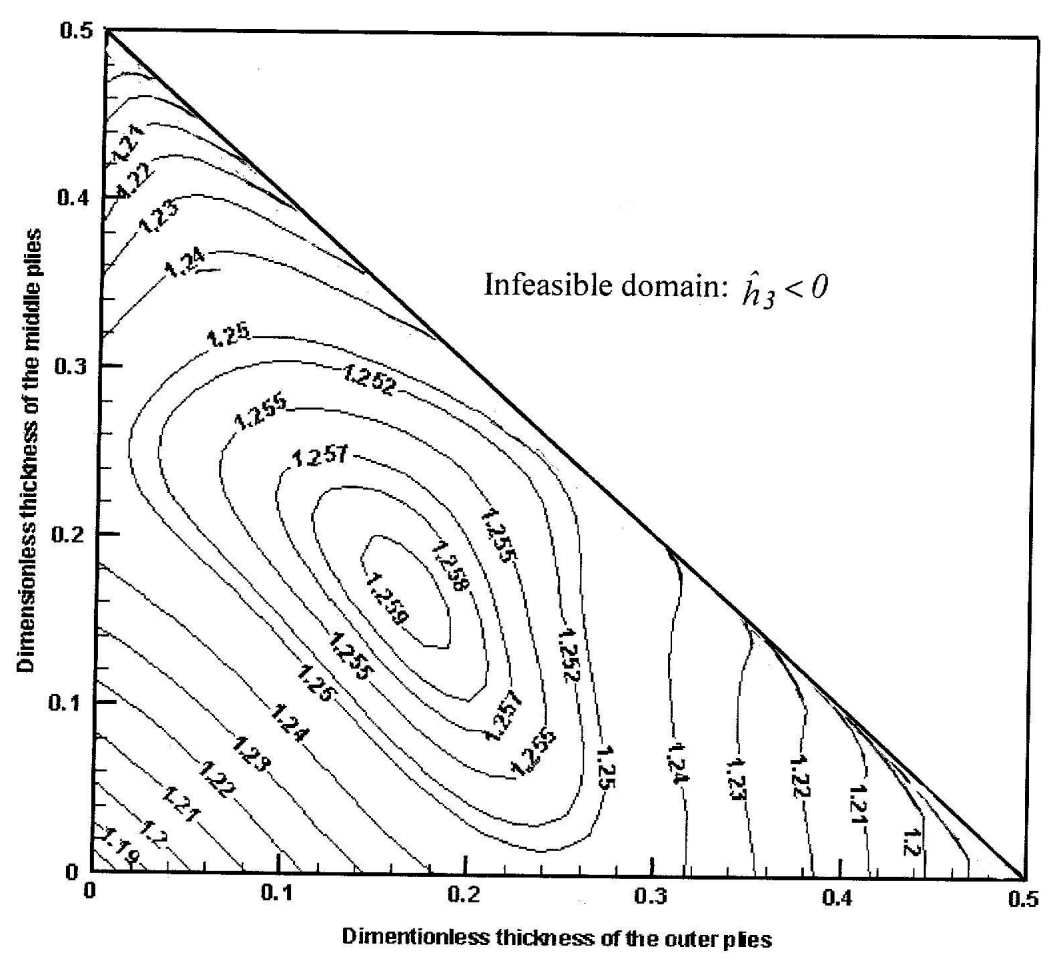

Figure 7. Global optimal stability limit in $\hat{h}_{1}-\hat{h}_{2}$ design space for a six-layer, $S$ glass/epoxy ring with a stacking sequence $\left[+45^{\circ} /-45^{\circ}\right]_{3}$.

or baseline design. It is assumed that the volume fractions of the composite material constituents do not significantly change during optimization, so that the total structural mass remains constant. Three types of composites were considered: $E$-glass/vinyl-ester, graphite/epoxy and $S$-glass/epoxy. It has been shown that the overall stability level of the laminated composite shell structures under considerations can be substantially improved by finding the optimal stacking sequence without violating any imposed side constraints. The stability limits of the optimized shells have been substantially enhanced as compared with those of the reference or baseline designs. The case of cylinders of finite length as well as the use of material grading concept for maximizing buckling stability boundaries under equality mass constraint shall be considered in the future.

\section{Appendix A}

Based upon the classical lamination theory [Reddy 2004], this appendix includes a brief derivation of the laminate stiffness parameters, which allows for a general stacking sequence optimization.

Constitutive relations. One difference between laminated composites and traditional engineering materials is that a composite response to loads is direction dependent. In order to analyze the response of a composite, we must be able to predict the behavior of individual unidirectional lamina, which is characterized by having all fibers oriented in the same direction. This model allows one to treat the lamina as an orthotropic material. In reality fibers are not perfectly straight or uniformly oriented within 
the lamina. There are generally several layers of fibers nested within a single lamina. The structural model used to represent the composite laminate is schematically shown in Figure A-1.

1, 2 and 3 are denoted the principal directions of an orthotropic lamina, defined as follows:

- Direction 1: principal fiber direction, also called fiber longitudinal direction,

- Direction 2: in-plane direction perpendicular to fibers, transversal direction, and

- Direction 3: out-of-plane direction perpendicular to fibers, normal direction.

The reduced form of Hooke's law for an orthotropic homogeneous lamina in a plane stress state may be written as

$$
\left(\begin{array}{l}
\sigma_{11} \\
\sigma_{22} \\
\tau_{12}
\end{array}\right)=\left(\begin{array}{ccc}
Q_{11} & Q_{12} & 0 \\
Q_{12} & Q_{22} & 0 \\
0 & 0 & Q_{66}
\end{array}\right)\left(\begin{array}{l}
\varepsilon_{11} \\
\varepsilon_{22} \\
\gamma_{12}
\end{array}\right)
$$

where $Q$ is referred to as the reduced stiffeners matrix of the $k$-th lamina, defined in terms of material properties:

$$
Q_{11}=\frac{E_{11}}{1-v_{12} v_{21}}, \quad Q_{22}=\frac{E_{22}}{1-v_{12} v_{21}}, \quad Q_{12}=\frac{v_{12} E_{22}}{1-v_{12} v_{21}}, \quad Q_{66}=G_{12},
$$

where $v_{12} E_{22}=v_{21} E_{11}$.

As seen from the above equations, there are four independent elastic constants: the Young's moduli in the 1 and 2 directions, $E_{11}$ and $E_{22}$, the shear modulus, $G_{12}$, and the major Poisson's ratio, $v_{12}$, upon which the stiffness matrix of a homogeneous orthotropic composite material is calculated.
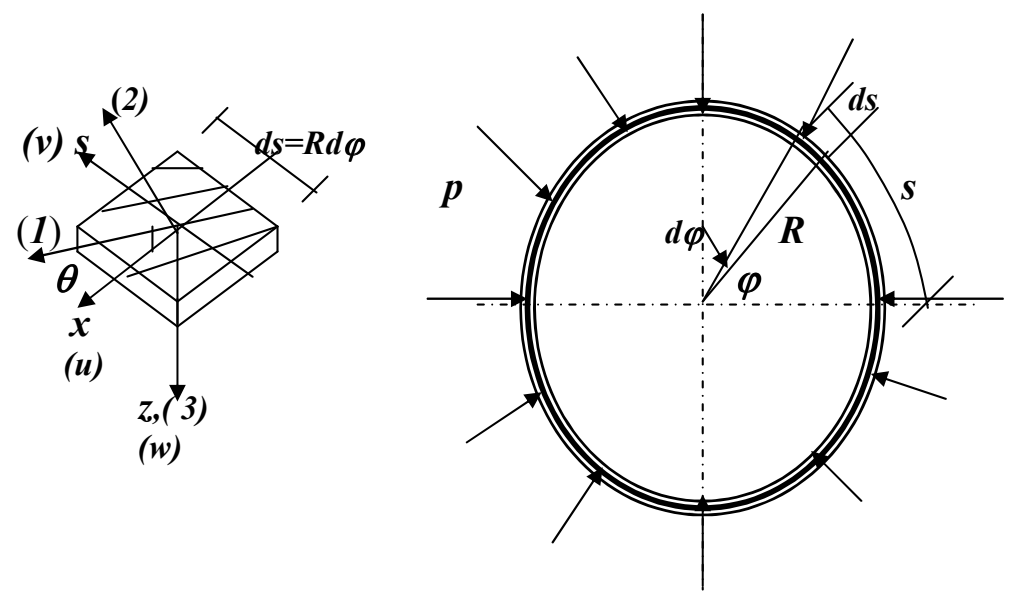

Figure A-1. Laminated composite ring/cylindrical shell under external pressure ( $u$ displacement in the axial direction $x, v$ in the tangential direction $s, w$ in the radial direction $z$ ). 
For a generally orthotropic material, (A.1) must be transformed to reflect rotated fiber orientation angles. The following matrix relation reflects this transformation [Daniel and Ishai 2006]:

$$
\left(\begin{array}{l}
\sigma_{x x} \\
\sigma_{s s} \\
\tau_{x s}
\end{array}\right)=\left(\begin{array}{lll}
\bar{Q}_{11} & \bar{Q}_{12} & \bar{Q}_{16} \\
\bar{Q}_{12} & \bar{Q}_{22} & \bar{Q}_{26} \\
\bar{Q}_{16} & \bar{Q}_{26} & \bar{Q}_{66}
\end{array}\right)\left(\begin{array}{c}
\varepsilon_{x x} \\
\varepsilon_{s s} \\
\gamma_{x s}
\end{array}\right) .
$$

The elements of the $k$-th lamina stiffness matrix $\bar{Q}$, which is now referred to the reference axes of the cylindrical shell $(x, s, z)$, are given by

$$
\begin{array}{ll}
\bar{Q}_{11}=U_{1}+U_{2} \cos 2 \theta+U_{3} \cos 4 \theta, & \bar{Q}_{22}=U_{1}-U_{2} \cos 2 \theta+U_{3} \cos 4 \theta, \\
\bar{Q}_{12}=U_{4}-U_{3} \cos 4 \theta, & \bar{Q}_{16}=0.5 U_{2} \sin 2 \theta+U_{3} \sin 4 \theta, \\
\bar{Q}_{26}=0.5 U_{2} \sin 2 \theta-U_{3} \sin 4 \theta, & \bar{Q}_{66}=0.5\left(U_{1}-U_{4}\right)-U_{3} \cos 4 \theta,
\end{array}
$$

where the invariant terms $U_{i}$ are solely function of the material properties. They are defined by the following expressions [Reddy 2004]:

$$
\begin{array}{ll}
U_{1}=0.125\left(3 Q_{11}+3 Q_{22}+2 Q_{12}+4 Q_{66}\right), & U_{2}=0.5\left(Q_{11}-Q_{22}\right), \\
U_{3}=0.125\left(Q_{11}+Q_{22}-2 Q_{12}-4 Q_{66}\right), & U_{4}=0.125\left(Q_{11}+Q_{22}+6 Q_{12}-4 Q_{66}\right) .
\end{array}
$$

For classical lamination theory, it is assumed that $n$ layers of material are perfectly bonded together, with infinitely thin, nonshear deformable boundaries. Using Kirchoff plate theory [Simitses 1976], which assumes that the in-plane displacements vary linearly through the thickness of the laminate, the displacements of a material point distance $z$ from the middle surface are

$$
\begin{aligned}
& u(x, s, z)=u_{0}(x, s)-z \frac{\partial w_{0}}{\partial x}, \\
& v(x, s, z)=v_{0}(x, s)-z\left(\frac{\partial w_{0}}{\partial s}-\frac{v_{0}}{R}\right), \quad\left(s \cong R \varphi, \frac{z}{R} \ll 1\right), \\
& w(x, s, z)=w_{0}(x, s),
\end{aligned}
$$

where $u_{0}(x, s), v_{0}(x, s)$ and $w_{0}(x, s)$ are the displacements of a generic point $(x, s)$ on the shell middle surface $(z=0)$ in $x, s$ and $z$ directions, respectively.

The strain-displacement relations in terms of the middle surface strains and shell curvatures are given in the following:

$$
\begin{aligned}
& \varepsilon_{x x}=\varepsilon_{x x}^{0}+z \kappa_{x x}, \\
& \varepsilon_{s s}=\varepsilon_{s s}^{0}+z \kappa_{s s}, \quad \text { or } \quad \quad\left(\begin{array}{c}
\varepsilon_{x x} \\
\varepsilon_{s s} \\
\gamma_{x s}
\end{array}\right)=\gamma_{x s}^{0}+z \kappa_{x s},
\end{aligned}=\left(\begin{array}{c}
\varepsilon_{x x}^{0} \\
\varepsilon_{s s}^{0} \\
\gamma_{x s}^{0}
\end{array}\right)+z\left(\begin{array}{c}
\kappa_{x x} \\
\kappa_{s s} \\
\kappa_{x s}
\end{array}\right),
$$


where the middle surface strains and curvatures are [Brush and Almroth 1975]

$$
\begin{array}{rlrl}
\varepsilon_{x x}^{0} & =\frac{\partial u_{0}}{\partial x}, & \kappa_{x x} & =-\frac{\partial^{2} w_{0}}{\partial x^{2}}, \\
\varepsilon_{s s}^{0} & =\frac{\partial v_{0}}{\partial s}+\frac{w_{0}}{R}+\frac{1}{2}\left(\frac{\partial w_{0}}{\partial s}-\frac{v_{0}}{R}\right)^{2}, & \kappa_{s s} & =-\frac{\partial}{\partial s}\left(\frac{\partial w_{0}}{\partial s}-\frac{v_{0}}{R}\right), \\
\gamma_{x s}^{0}=\frac{\partial u_{0}}{\partial s}+\frac{\partial v_{0}}{\partial x}, & \kappa_{x s} & =2 \frac{\partial}{\partial x}\left(\frac{\partial w_{0}}{\partial s}-\frac{v_{0}}{R}\right) .
\end{array}
$$

Substituting for the total strains from (A.3) into (A.2) we have

$$
\left(\begin{array}{c}
\sigma_{x x} \\
\sigma_{s s} \\
\tau_{x s}
\end{array}\right)_{k}=\left(\begin{array}{lll}
\bar{Q}_{11} & \bar{Q}_{12} & \bar{Q}_{16} \\
\bar{Q}_{12} & \bar{Q}_{22} & \bar{Q}_{26} \\
\bar{Q}_{16} & \bar{Q}_{26} & \bar{Q}_{66}
\end{array}\right)_{k}\left(\left(\begin{array}{c}
\varepsilon_{x x}^{0} \\
\varepsilon_{s s}^{0} \\
\gamma_{x s}^{0}
\end{array}\right)+z\left(\begin{array}{c}
\kappa_{x x} \\
\kappa_{s s} \\
\kappa_{x s}
\end{array}\right)\right) .
$$

The resultant forces and moments per unit length applied at the middle surface are defined by the integrals

$$
\text { Forces: } \quad \begin{aligned}
& \quad\left(\begin{array}{c}
N_{x x} \\
N_{s s} \\
N_{x s}
\end{array}\right)=\int_{-h / 2}^{h / 2}\left(\begin{array}{c}
\sigma_{x x} \\
\sigma_{s s} \\
\tau_{x s}
\end{array}\right) d z=\sum_{k=1}^{n} \int_{z_{k-1}}^{z_{k}}\left(\begin{array}{c}
\sigma_{x x} \\
\sigma_{s s} \\
\tau_{x s}
\end{array}\right) d z, \\
& \text { Moments: } \quad\left(\begin{array}{c}
M_{x x} \\
M_{s s} \\
M_{x s}
\end{array}\right)=\int_{-h / 2}^{h / 2}\left(\begin{array}{c}
\sigma_{x x} \\
\sigma_{s s} \\
\tau_{x s}
\end{array}\right) z d z=\sum_{k=1}^{n} \int_{z_{k-1}}^{z_{k}}\left(\begin{array}{c}
\sigma_{x x} \\
\sigma_{s s} \\
\tau_{x s}
\end{array}\right) z d z .
\end{aligned}
$$

Substituting for the stress-strain relationships of (A.4) into (A.5) and (A.6), we get

$$
\left(\begin{array}{l}
N_{x x} \\
N_{s s} \\
N_{x s} \\
M_{x x} \\
M_{s s} \\
M_{x s}
\end{array}\right)=\left(\begin{array}{llllll}
A_{11} & A_{12} & A_{16} & B_{11} & B_{12} & B_{16} \\
A_{12} & A_{22} & A_{26} & B_{12} & B_{22} & B_{26} \\
A_{16} & A_{26} & A_{66} & B_{16} & B_{26} & B_{66} \\
B_{11} & B_{12} & B_{16} & D_{11} & D_{12} & D_{16} \\
B_{12} & B_{22} & B_{26} & D_{12} & D_{22} & D_{26} \\
B_{16} & B_{26} & B_{66} & D_{16} & D_{26} & D_{66}
\end{array}\right)\left(\begin{array}{c}
\varepsilon_{x x}^{0} \\
\varepsilon_{s s}^{0} \\
\gamma_{x s}^{0} \\
\kappa_{x x} \\
\kappa_{s s} \\
\kappa_{x s}
\end{array}\right),
$$
where $A_{i j}$ are called the extensional stiffnesses given by $A_{i j}=\sum_{k=1}^{n}\left(\bar{Q}_{i j}\right)_{k}\left(z_{k}-z_{k-1}\right) . B_{i j}$ are called the
bending-extensional stiffnesses given by

$$
B_{i j}=\frac{1}{2} \sum_{k=1}^{n}\left(\bar{Q}_{i j}\right)_{k}\left(z_{k}^{2}-z_{k-1}^{2}\right) .
$$

$D_{i j}$ are called the bending stiffnesses

$$
D_{i j}=\frac{1}{3} \sum_{k=1}^{n}\left(\bar{Q}_{i j}\right)_{k}\left(z_{k}^{3}-z_{k-1}^{3}\right),
$$

where $n$ is the number of different plies in the stacking sequence. 


\section{Appendix B: The interior penalty function technique}

In this method the original objective function $F(\vec{x})$ is augmented with terms, called penalty terms, such that as $\vec{x}$ approaches a constraint surface one term increases indefinitely. Since the algorithm seeks to minimize the value of the objective function, it will try not to penetrate any constraint surface. Thus all constraints are taken into consideration by representing them by penalty terms in the objective function expression. The most commonly used interior penalty function [Vanderplaats 1994] is cast in the form

$$
\Phi(\vec{x}, r)=F(\vec{x})-r \sum_{j=1}^{M} \frac{1}{G_{j}(\vec{x})},
$$

where $\Phi(\vec{x}, r)$ is the modified objective function, $G_{j}(\vec{x})$ is the $j$-th constraint function and $r$ is a multiplier. A sequence of unconstrained minimization problems is solved with successively decreasing values of $r$. The MATLAB optimization toolbox [Venkataraman 2002] offers routines that implement the interior penalty function method via a built-in function named fminsearch.

\section{Acknowledgment}

The author wishes to thank Professor Hayder Rasheed of the Department of Civil Engineering, Kansas State University, for his valuable scientific discussions and help.

\section{References}

[Anastasiadis and Simitses 1993] J. S. Anastasiadis and G. J. Simitses, "Buckling of pressure-loaded, long, shear deformable cylindrical laminated shells", Compos. Struct. 23:3 (1993), 221-231.

[Brush and Almroth 1975] D. O. Brush and B. O. Almroth, Buckling of bars, plates and shells, McGraw-Hill, New York, 1975. [Chattopadhyay and Ferreira 1993] A. Chattopadhyay and J. Ferreira, "Design sensitivity and optimization of composite cylinders", Compos. Eng. 3 (1993), 169-179.

[Daniel and Ishai 2006] I. M. Daniel and O. Ishai, Engineering mechanics of composite materials, 2nd ed., Oxford University, New York, 2006.

[Davies and Chauchot 1999] P. Davies and P. Chauchot, Composites for marine applications -Part 2: underwater structures, Kluwer Academic, Dordrecht, 1999.

[Graham 1995] D. Graham, "Composite pressure hulls for deep ocean submersibles", Compos. Struct. 32:1-4 (1995), 331-343.

[Hodges 1999] D. H. Hodges, "Non-linear inplane deformation and buckling of rings and high arches", Int. J. Nonlinear Mech. 34:4 (1999), 723-737.

[Hodges and Harursampath 2002] D. H. Hodges and D. Harursampath, "Inplane buckling of anisotropic rings", in Proceedings of the 15th ASCE Engineering Mechanics Division Conference (New York), Columbia University, New York, June 2-5 2002.

[Librescu and Maalawi 2007] L. Librescu and K. Y. Maalawi, "Material grading for improved aeroelastic stability in composite wings", J. Mech. Mater. Struct. 2:7 (2007), 1381-1394.

[Maalawi 2002] K. Y. Maalawi, "Buckling optimization of flexible columns", Int. J. Solids Struct. 39:23 (2002), 5865-5876.

[Maalawi and El Chazly 2002] K. Y. Maalawi and N. M. El Chazly, "Global optimization of multi-element beam-type structures", in The 2nd international conference on advances in structural engineering and mechanics, ASEMO2 (Busan, South Korea), August 21-23 2002.

[Rasheed and Yousif 2001] H. A. Rasheed and O. H. Yousif, "Buckling of thin laminated orthotropic composite rings/long cylinders under external pressure", Int. J. Struct. Stab. Dyn. 1:4 (2001), 485-507. 
[Rasheed and Yousif 2005] H. A. Rasheed and O. H. Yousif, "Stability of anisotropic laminated rings and long cylinders subjected to external hydrostatic pressure", J. Aerospace Eng. 18:3 (2005), 129-138.

[Reddy 2004] J. N. Reddy, Mechanics of laminated composite plates and shells: theory and analysis, 2nd ed., CRC Press, Boca Raton, 2004.

[Simitses 1976] G. J. Simitses, An introduction to the elastic stability of structures, Prentice-Hall, Englewood Cliffs, N.J., 1976.

[Simitses 1996] G. J. Simitses, "Buckling of moderately thick laminated cylindrical shells: a review", Compos. Part B Eng. 27:6 (1996), 581-587.

[Soden et al. 1998] P. D. Soden, M. J. Hinton, and A. S. Kaddour, "Lamina properties, lay-up configurations and loading conditions for a range of fibre-reinforced composite laminates”, Compos. Sci. Technol. 58:7 (1998), 1011-1022.

[Sridharan and Kasagi 1997] S. Sridharan and A. Kasagi, "On the buckling and collapse of moderately thick composite cylinders under hydrostatic pressure", Compos. Part B Eng. 28:5-6 (1997), 583-596.

[Tanguy et al. 2002] M. Tanguy, P. Mariusz, G. Bernard, and C. Pierre, "Optimal laminations of thin underwater composite cylindrical vessels", Compos. Struct. 58:4 (2002), 529-537.

[Vanderplaats 1994] G. Vanderplaats, Numerical optimization techniques for engineering design: with applications, McGrawHill, New York, 1994.

[Venkataraman 2002] P. Venkataraman, Applied optimization with MATLAB programming, Wiley, New York, 2002.

[Vinson 1992] J. R. Vinson, The behavior of shells composed of isotropic and composite materials, Kluwer Academic, Dordrecht, 1992.

[ZitzEvancih 1985] L. D. ZitzEvancih, "Designing graphite cylinders to resist buckling", AIAA J. 85 (1985), 1101. Also, AIAA/SAE/ASME/ASEE 21st Joint Propulsion Conference, Monterey, CA: July 8-10, 1985.

Received 7 Oct 2007. Revised 21 Jan 2008. Accepted 22 Jan 2008.

KARAM Y. MAALAWI: maalawi@netscape.net

Department of Mechanical Engineering, National Research Center, 12622 Dokki, Cairo, Egypt

www.nrc.sci.eg 


\title{
A PLANE STRESS PERFECTLY PLASTIC MODE I CRACK PROBLEM FOR A YIELD CONDITION BASED ON THE SECOND AND THIRD INVARIANTS OF THE DEVIATORIC STRESS TENSOR
}

\author{
DAVID J. UNGER
}

\begin{abstract}
A statically admissible solution for the opening mode of fracture under plane stress loading conditions is obtained for a yield condition containing both the second and third invariants of the deviatoric stress tensor. This yield locus lies approximately midway between the Mises and Tresca yield loci in the principal stress plane. The crack problem addressed is analogous to an earlier one investigated by John W. Hutchinson for the Mises yield condition. A stress function approach to the present problem results in a differential algebraic equation rather than an ordinary differential equation as in the former case. It is found that a reduction of order is possible for this second order differential equation of the sixth degree through a simple transformation which generates a Clairaut equation. This equation can be integrated analytically to obtain the general solution of the governing second order differential equation for uniform states of stress. This general solution is applicable to two of three distinct sectors of the plane crack problem. The remaining sector in the plane is governed by the singular solution of this Clairaut equation. The first integral of the singular solution, which is the envelope of general solution, is found through the use of a contact transformation. This transformation aids in reduction of this equation to that of a first order differential equation of the thirtieth degree. The primitive of this first order differential algebraic equation is obtained by numerical solution. An approximate analytical solution to the problem is also provided. These results are compared to those obtained previously for the analogous crack problem under the Mises yield condition.
\end{abstract}

\section{Introduction}

Drucker [1949; 1962] illustrated the "truly remarkable correlation" of experimental data of an aluminum alloy [Osgood 1947] with a yield condition based upon the second and third invariants of the deviatoric stress tensor. Clearly what appeared to be a sizable band of experimental scatter in a plot of equivalent stress versus octahedral strain, under both the Tresca and Mises yield criteria, became a very narrow locus under this alternative yield condition. Despite the excellent agreement with experimental data, this particular yield condition has been used only rarely in the literature. The probable cause of this avoidance is the yield condition's mathematical complexity over the Mises yield condition, which is based on the second deviatoric stress invariant alone. However, with today's widespread availability of both symbolic mathematical and numerical computer software, this particular concern need not be the only deciding factor.

Admittedly, the results obtained here may in fact turn out to be a rather fine point with limited practical significance, as the predictions are very close to those found using the Mises yield condition, while the

Keywords: plane stress, mode I crack, perfectly plastic yield condition, second third invariants deviatoric stress tensor, differential algebraic equation, DAE. 


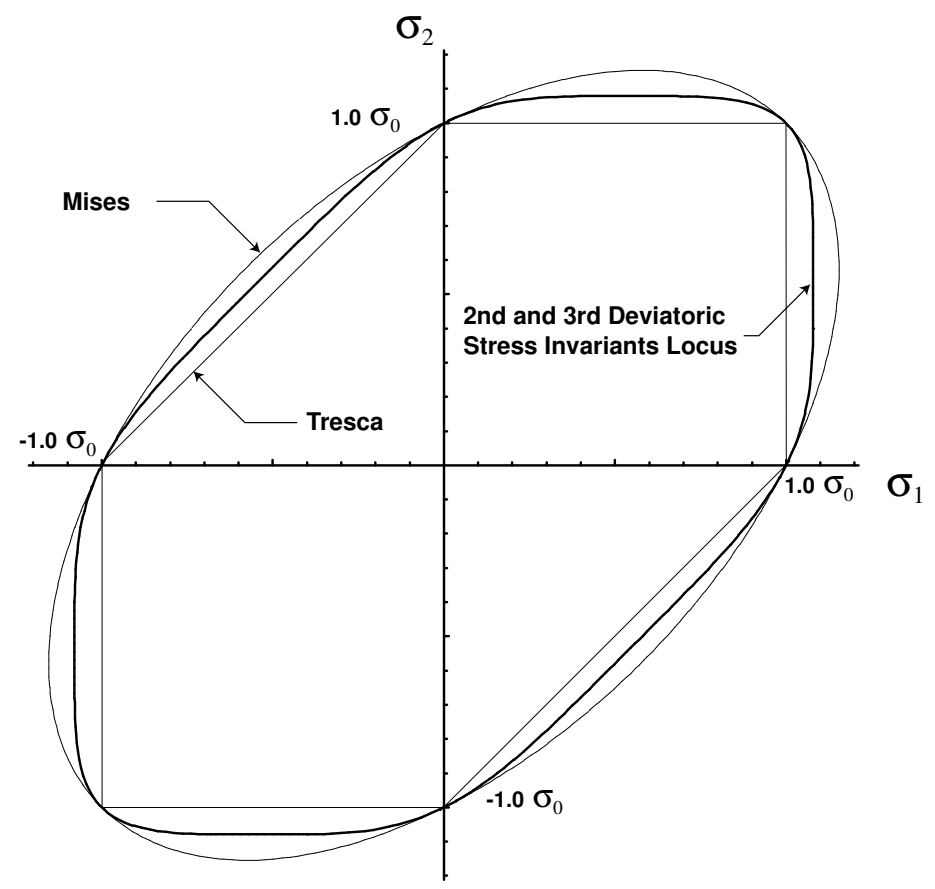

Figure 1. Three different yield loci in the principal stress plane.

increase in mathematical difficulty in obtaining solution is enormous. Nevertheless, this analysis contains some interesting and novel mathematical aspects that may aid others in their own research on either yield criteria or on differential algebraic equations (DAE). These include the use of a contact transformation in determining the singular solution of a DAE and the use of a phase plane analysis in determining an approximate singular solution in the form of a Jacobian elliptic function. In this respect there is merit to reporting the results.

The yield condition proposed by Drucker is shown in Figure 1 in the principal stress plane for a state of plane stress loading conditions, where $\sigma_{1}$ is the first principal stress and $\sigma_{2}$ is the second principal stress $\left(\sigma_{3}=0\right)$. In terms of the deviatoric stress invariants it assumes the algebraic form

$$
\left(J_{2}\right)^{3}-\left(3 J_{3} / 2\right)^{2}=\left(a \sigma_{0}\right)^{6},
$$

where $J_{2}$ and $J_{3}$ are the second and third invariants of the deviatoric stress tensor, respectively [Chakrabarty 1987], and $a$ is the proportionality constant ${ }^{1}$ between the yield stress in pure shear $\tau_{0}$ and the yield stress in tension $\sigma_{0}$

$$
\tau_{0}=a \sigma_{0}, \quad a=\sqrt[6]{2 / 81} \approx 0.540
$$

\footnotetext{
${ }^{1}$ This value $a$ is approximately midway between analogous relationships for the Tresca yield condition 0.500 and the Mises $\approx 0.577$.
} 
In polar coordinates, these invariants become explicitly

$$
\begin{aligned}
& J_{2}=\frac{1}{3}\left(\sigma_{r}^{2}-\sigma_{r} \sigma_{\theta}+\sigma_{\theta}^{2}\right)+\tau_{r \theta}^{2}, \\
& J_{3}=\frac{\sigma_{r}+\sigma_{\theta}}{3}\left[\tau_{r \theta}^{2}-(1 / 9)\left(2 \sigma_{r}-\sigma_{\theta}\right)\left(2 \sigma_{\theta}-\sigma_{r}\right)\right],
\end{aligned}
$$

where $\sigma_{r}$ and $\sigma_{\theta}$ are the normal stresses in the radial and transverse directions respectively and $\tau_{r \theta}$ is the shear stress.

Drucker [1949] and Freudenthal and Geiringer [1958] introduce this yield condition Equation (1) as being the simplest possible form containing both the second and third deviatoric stress invariants, although neither reference indicates its priority.

\section{General and singular solutions}

By introducing a plastic stress function $\phi(r, \theta)$ in polar form

$$
\phi(r, \theta)=r^{2} f(\theta)
$$

the stresses derived from it [Unger 2005; 2007]

$$
\begin{aligned}
\sigma_{\theta} & =2 f(\theta), \\
\tau_{r \theta} & =-f^{\prime}(\theta)=-p, \\
\sigma_{r} & =f^{\prime \prime}(\theta)+2 f(\theta)=p \frac{d p}{d f}+2 f,
\end{aligned}
$$

will automatically satisfy the equilibrium equations in the plane, where the number of prime symbols applied to the function $f(\theta)$ indicate the order of differentiation with respect to $\theta$. By substituting Equations (5)-(7) into Equation (3) and subsequently substituting those results into Equation (1), the governing differential equation for the stress function becomes

$$
\left(q^{2}-6 f q+6 Q\right)^{3}-(1 / 12) q^{2}\left(2 q^{2}-18 f q+18 Q\right)^{2}=(2 / 3) \sigma_{0}^{6},
$$

where for brevity the following notation has been adopted in Equation (8)

$$
Q=p^{2} / 2+2 f^{2}, \quad q=d Q / d f .
$$

Equation (8) has the form of a Clairaut differential equation [Zwillinger 1989, pp. 150-160]. As such, a Clairaut operator $U(f)$ may be defined as

$$
U=f d Q / d f-Q(f)=f q-Q,
$$

which brings Equation (8) into the form

$$
3\left(q^{2}-6 U\right)^{3}-q^{2}\left(q^{2}-9 U\right)^{2}=2 \sigma_{0}^{6} .
$$

The operational procedure to solve a Clairaut equation is to substitute a constant in place of the first derivative of the dependent variable with respect to the independent variable and to solve for the dependent variable. The dependent variable constitutes the solution of the problem. 
In this case $q$ will be set equal to $c$ with $Q$ being identified temporarily as the dependent variable, which is a function of $f$. Solving Equation (11) for $U(f)$ and substituting Equation (10) into the result, one finds that

$$
Q(f)=c f-\frac{c^{2}}{8}+\frac{5 c^{4}}{24 \cdot 3^{1 / 3}} \frac{1}{M\left(c, \sigma_{0}\right)}-\frac{M\left(c, \sigma_{0}\right)}{24 \cdot 3^{2 / 3}},
$$

where the constant $M\left(c, \sigma_{0}\right)$ is defined in terms of the two other parameters as

$$
M\left(c, \sigma_{0}\right)=\left[3 c^{6}-192 \sigma_{0}^{6}+8 \sqrt{6} \sqrt{c^{12}-3 c^{6} \sigma_{0}^{6}+96 \sigma_{0}^{12}}\right]^{1 / 3} .
$$

Reintroducing the fundamental definition of $Q$ from Equation (9), one may now separate variables and integrate to obtain the following branch of the general solution of Equation (8)

$$
f(\theta)=\frac{c}{4}-\frac{1}{4 \cdot 3^{2 / 3}} \sqrt{\frac{5 c^{4}}{M\left(c, \sigma_{0}\right)}-\frac{M\left(c, \sigma_{0}\right)}{3^{1 / 3}}} \sin (2 \theta+\alpha),
$$

where $\alpha$ represents the second constant of integration.

An additional solution to that of Equation (14) exists for Equation (8) in the form of a singular solution. The singular solution of Equation (8) represents the envelope of the family of ellipses that Equation (14) will generate in the phase space $(f, p)$ upon varying the parameter $c$ (the choice of parameter $\alpha$ is immaterial). It cannot be obtained directly from Equation (14) by simply selecting particular values of the constants $c$ or $\alpha$.

The conventional approach to obtaining a singular solution of a Clairaut equation is to first separate the governing equation into a function of the Clairaut operator, represented here by $U$, and a function of the first derivative $q$. By differentiating this expression with respect to the independent variable $f$, a pair of simultaneous equations results from which the first derivative can be eliminated to obtain a solution [Zwillinger 1989].

However, in the present situation obtaining the function of $U$ requires a solution of a cubic algebraic equation which is cumbersome.

As an alternative, a solution technique described in [Ince 1956], as the principal of duality, will be used here. To achieve solution, expand the parenthetic expressions in Equation (11) and rewrite the expansion as follows, using the fundamental definition of $U$

$$
2 q^{6}-36 q^{4}(f q-Q)+243 q^{2}(f q-Q)^{2}-648(f q-Q)^{3}=2 \sigma_{0}^{6} .
$$

Next, make the following substitutions for the variables appearing in Equation (15)

$$
f=P, \quad Q=X P-Y, \quad q=X,
$$

where $P$ is defined as the first derivative of the new dependent variable $Y$ with respect to the new independent variable $X$

$$
P=d Y / d X \text {. }
$$

Upon substitution of the variables defined by Equations (16) into Equation (15), one converts the differential equation into the following algebraic equation

$$
2 X^{6}-36 X^{4} Y+243 X^{2} Y^{2}-648 Y^{3}=2 \sigma_{0}^{6} .
$$


By differentiating Equation (18) with respect to $X$ and by employing the definition of $P$, one finds upon solving the expression for $P$ that

$$
P=X \frac{24 X^{2} Y-2 X^{4}-81 Y^{2}}{81 X^{2} Y-6 X^{4}-324 Y^{2}} .
$$

Now the inverse of the contact transformation, Equation (16), is given by

$$
X=q, \quad Y=f q-Q, \quad P=f .
$$

By transforming Equation (19) back to the original variables one finds that

$$
f=q \frac{24 q^{2}(f q-Q)-2 q^{4}-81(f q-Q)^{2}}{81 q^{2}(f q-Q)-6 q^{4}-324(f q-Q)^{2}} .
$$

Eliminating the common variable $q$ between Equations (15) and (21), and then substituting the definition for $Q$ from Equation (9) into the result, one reduces the order of the governing equation for the singular solution by one to:

$$
\begin{aligned}
& 387420489\left(4 f^{2}+p^{2}\right)^{12}\left[8 f^{6}+51 f^{4} p^{2}+24 f^{2} p^{4}+8 p^{6}\right] \\
& =28697814\left(4 f^{2}+p^{2}\right)^{6}\left[33280 f^{12}+163680 f^{10} p^{2}+16800 f^{8} p^{4}-17536 f^{6} p^{6}\right. \\
& \left.-16239 f^{4} p^{8}-2136 f^{2} p^{10}+88 p^{12}\right] \sigma_{0}^{6} \\
& -11337408\left[8783360 f^{18}+30758400 f^{16} p^{2}+2053440 f^{14} p^{4}\right. \\
& +4665120 f^{12} p^{6}-2198394 f^{10} p^{8}+352476 f^{8} p^{10} \\
& \left.-147783 f^{6} p^{12}+127980 f^{4} p^{14}-4986 f^{2} p^{16}+80 p^{18}\right] \sigma_{0}^{12} \\
& +1492992\left[598880 f^{12}+436800 f^{10} p^{2}+53400 f^{8} p^{4}-25322 f^{6} p^{6}\right. \\
& \left.+29955 f^{4} p^{8}-6153 f^{2} p^{10}+110 p^{12}\right] \sigma_{0}^{18}
\end{aligned}
$$

where $p$ is the first derivative of the function $f$ with respect to $\theta$, as in Equation (6).

This result, which is tedious to do by hand, was determined using the command function Eliminate of Mathematica ${ }^{\circ}$.

A plot of Equation (22) is shown in Figure 2 for the first quadrant of the phase plane. The insert in figure reveals that the complete locus of Equation (22) is barrel shaped; nearly flat on the top and bottom, with curved lateral sides. The complete locus is shown inscribed within a circle representing the locus of the Mises singular solution [Unger 2005].

Note that Equation (22) provides an exact relationship between stresses $\tau_{r \theta}$ and $\sigma_{\theta}$ of the singular solution once the associated relationships between the stress function $f$ and its first derivative $p$, such that Equations (5) and (6), are substituted into that equation. In Figure 3, this relationship is plotted as $\tau_{r \theta}$ versus $\sigma_{\theta}$ which generates an oval shape. An implicit plotting routine is required to find and draw the locus of this thirtieth order algebraic relationship. 


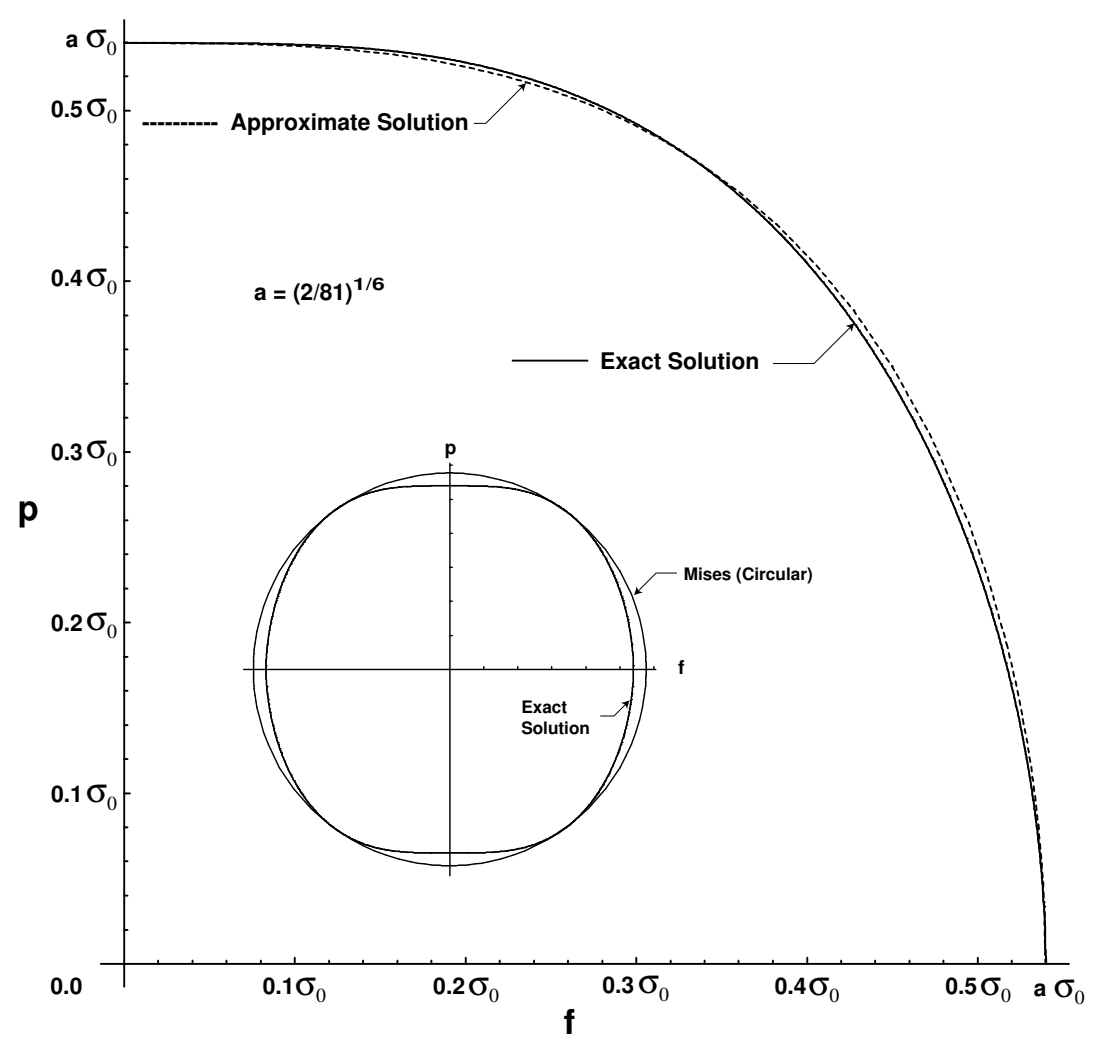

Figure 2. Phase plane analysis of the singular solution Equation (22), its approximation Equation (25), and the analogous Mises solution.

Now, by eliminating the common terms in parentheses $f q-Q$ from Equations (15) and (21), one obtains an expression that relates the remaining functions $f$ and $q$. The function $f$ is one half the value of $\sigma_{\theta}$ by Equation (5). In addition, one can relate $q$ to the sum of the normal stresses as

$$
q=p \frac{d p}{d f}+4 f=\sigma_{r}+2 f=\sigma_{r}+\sigma_{\theta},
$$

by using Equations (5), (7) and (9). Thus an exact relationship can determined between the stresses $\sigma_{r}$ and $\sigma_{\theta}$ of the singular solution as

$$
\begin{array}{r}
32 \sigma_{r}^{15}+336 \sigma_{r}^{14} \sigma_{\theta}+1587 \sigma_{r}^{13} \sigma_{\theta}^{2}+4453 \sigma_{r}^{12} \sigma_{\theta}^{3}+8274 \sigma_{r}^{11} \sigma_{\theta}^{4}+10758 \sigma_{r}^{10} \sigma_{\theta}^{5} \\
+9977 \sigma_{r}^{9} \sigma_{\theta}^{6}+6039 \sigma_{r}^{8} \sigma_{\theta}^{7}+396 \sigma_{r}^{7} \sigma_{\theta}^{8}-4972 \sigma_{r}^{6} \sigma_{\theta}^{9}-7755 \sigma_{r}^{5} \sigma_{\theta}^{10}-6909 \sigma_{r}^{4} \sigma_{\theta}^{11} \\
\quad-3998 \sigma_{r}^{3} \sigma_{\theta}^{12}-1482 \sigma_{r}^{2} \sigma_{\theta}^{13}-321 \sigma_{r} \sigma_{\theta}^{14}-31 \sigma_{\theta}^{15}-32 \sigma_{r}^{9} \sigma_{0}^{6}+144 \sigma_{r}^{8} \sigma_{\theta} \sigma_{0}^{6} \\
+1575 \sigma_{r}^{7} \sigma_{\theta}^{2} \sigma_{0}^{6}+4791 \sigma_{r}^{6} \sigma_{\theta}^{3} \sigma_{0}^{6}+7767 \sigma_{r}^{5} \sigma_{\theta}^{4} \sigma_{0}^{6}+7983 \sigma_{r}^{4} \sigma_{\theta}^{5} \sigma_{0}^{6}+5709 \sigma_{r}^{3} \sigma_{\theta}^{6} \sigma_{0}^{6} \\
+2925 \sigma_{r}^{2} \sigma_{\theta}^{7} \sigma_{0}^{6}+981 \sigma_{r} \sigma_{\theta}^{8} \sigma_{0}^{6}+157 \sigma_{\theta}^{9} \sigma_{0}^{6}+1944 \sigma_{r}^{3} \sigma_{0}^{12}-5832 \sigma_{r}^{2} \sigma_{\theta} \sigma_{0}^{12} \\
+5832 \sigma_{r} \sigma_{\theta}^{2} \sigma_{0}^{12}-1944 \sigma_{\theta}^{3} \sigma_{0}^{12}=0
\end{array}
$$




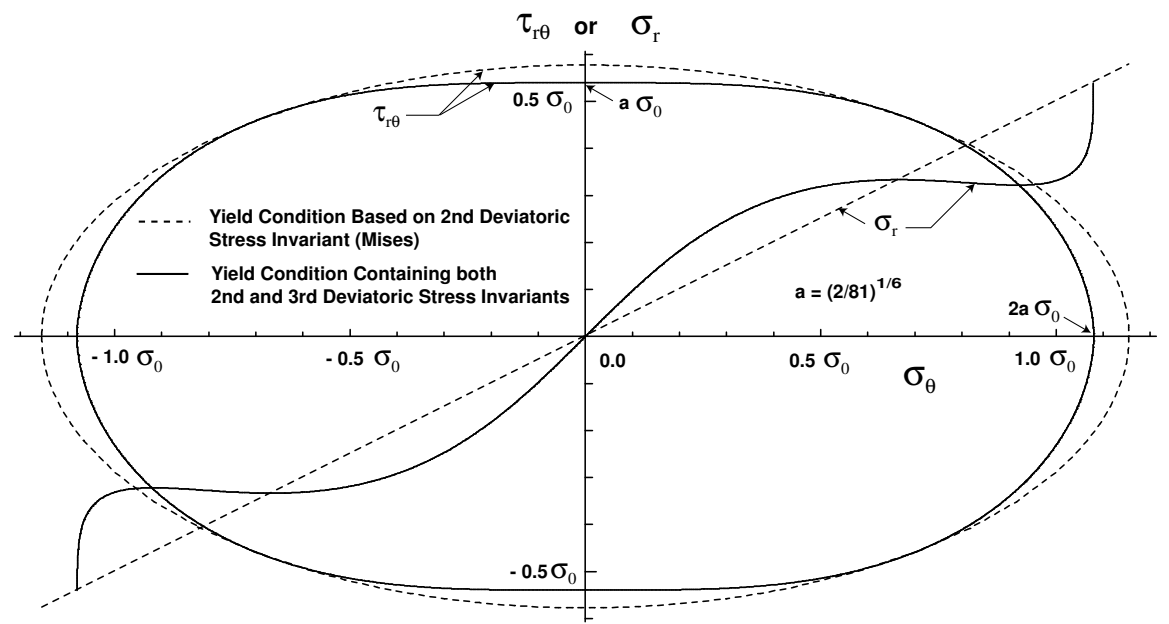

Figure 3. Exact relationships among stresses for the singular solutions of two different yield conditions.

This relationship appears as an open curve when plotted in Figure 3.

The continuous curves in Figure 3 constitute exact relationships among the stresses for the singular solution of Equation (8), and consequently satisfy yield condition, Equation (1). For comparison, the analogous stresses for the Mises yield condition are also plotted using dashed line segments. The greatest difference between the two different yield criteria is in $\sigma_{r}$ which is a straight line in the case of the Mises yield condition and a curve in case of yield condition, Equation (1). The plots of $\sigma_{r}$ as a function of $\sigma_{\theta}$ reveal two distinct ovals for the two different yield criteria. In the case of the Mises yield condition, this oval is also an ellipse.

\section{Analytical approximation to the singular solution}

The form of the differential algebraic equation, Equation (22), for the singular solution appears to be insoluble by exact techniques. Consequently, approximate analytical and numerical methods will be used for evaluation.

One approximation of the exact locus Equation (22), but much simpler in form, is given by

$$
\left(\frac{p_{\text {approx }}}{a \sigma_{0}}\right)^{2}+\left(\frac{f_{\text {approx }}}{a \sigma_{0}}\right)^{3}=1 .
$$

The closeness of fit between the approximate and exact loci in the phase plane is shown in Figure 2. The simplicity of Equation (25) also allows its exact primitive $f_{\text {approx }}$ to be found as a solution of an ordinary differential equation. This solution will serve as a useful analytical approximation to the solution of Equation (22). 


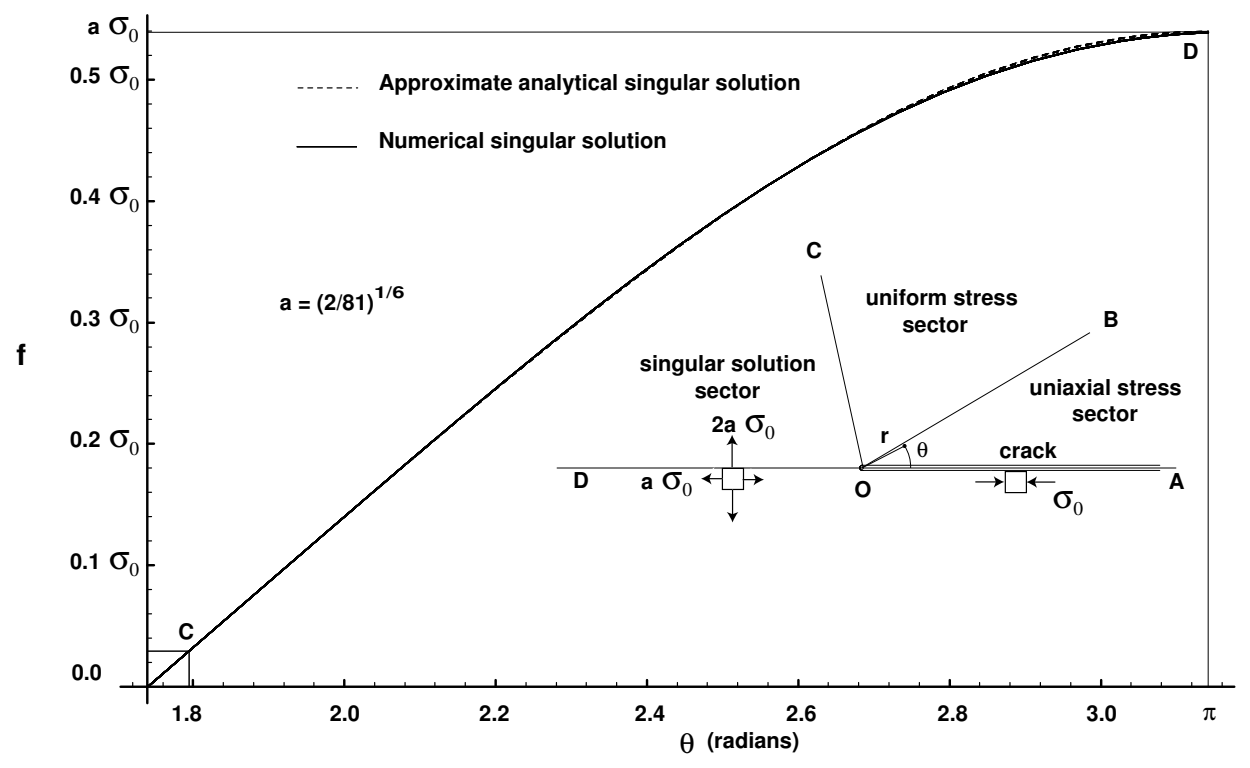

Figure 4. Comparison of the numerical singular solution to the analytical approximation, Equation (29).

The variables of the approximate relationship Equation (25) can be separated and integrated to generate the following relationship

$$
\theta+\beta=\int_{g}^{1} \frac{d u}{\sqrt{1-u^{3}}}, \quad g=\frac{f_{\text {approx }}}{a \sigma_{0}},
$$

where $\beta$ is a constant of integration.

The remaining integral in Equation (26) can be evaluated using [Gradshteyn and Ryzhik 1980, table entry 3.139 2]. It is represented below as an incomplete elliptic integral of the first kind $F($, , , as in

$$
\theta+\beta=\frac{1}{\sqrt[4]{3}} F(\psi, k), \quad \psi=\cos ^{-1} \frac{\sqrt{3}-1+g}{\sqrt{3}+1-g}, \quad k=\sin 75^{\circ},
$$

where the incomplete elliptic integral $F($,$) is defined below as [Gradshteyn and Ryzhik 1980, relation-$ ship 8.111 2]

$$
F(\psi, k)=\int_{0}^{\psi} \frac{d x}{\sqrt{1-k^{2} \sin ^{2} x}} .
$$

Upon inversion of the elliptic integral appearing in Equation (27), one obtains after simplification that

$$
f_{\text {approx }}=a \sigma_{0}-\sqrt{3} a \sigma_{0} \frac{1-c n \sqrt[4]{3}(\theta+\beta)}{1+c n \sqrt[4]{3}(\theta+\beta)},
$$

where $c n$ ( ) is a Jacobian cosine-amplitude elliptic function of modulus $k$. The particular value of $k$ for use in this approximate solution is given in Equation (27). 
The function $f_{\text {approx }}$ provides a close approximation to the exact relationship between $f$ and $\theta$, when compared to results from a numerical solution, which are plotted in Figure 4. However, the approximate analytical solution should not be used for the direct determination of $\sigma_{r}$ by Equation (7), as this would require taking the second derivative of the function with respect to the angle $\theta$. Accuracy cannot be guaranteed by this procedure, nor will the yield condition be satisfied in general by the derived stresses.

Instead, an alternative method of stress evaluation is proposed which will ensure that the yield condition is satisfied.

A good approximation to the singular solution can be obtained by using Equation (29) to obtain an approximate relationship between $f$ and $\theta$ from which one uses Equation (5) to obtain $\sigma_{\theta}$ as a function of $\theta$. The same relationships used previously to generate stresses $\tau_{r \theta}$ and $\sigma_{r}$ as functions of $\sigma_{\theta}$ in Figure 3 can then be used to generate a stress field that satisfies yield condition Equation (1) for any given angle $\theta$.

\section{Crack problem}

A schematic diagram of the basic crack geometry is shown in the insert of Figure 4. The domain is composed of three distinct sectors which cover the upper half plane $0 \leq \theta \leq \pi$. The semiinfinite crack is positioned along the coordinate $\theta=0$. Two angles, $\theta_{A O B}$ and $\theta_{A O C}$, mark the divisions between the three sectors. These angles are measured counterclockwise from the crack line $O A$ to rays $O B$ and $O C$, respectively. Using symmetry, the solution for the lower half plane can be inferred from the solution of the upper half plane.

A dedicated computer program was written to solve the mode I perfectly plastic crack problem for the upper half plane under yield condition, Equation (1).

The code assumes a uniaxial state of compression in sector $A O B$. The stress function applicable to region $O A B$ is obtained by substituting the following parameters into Equation (14)

$$
c=-\sigma_{0}, \quad \alpha=\frac{3 \pi}{2} .
$$

The associated stress function assumes the simple form

$$
f_{A O B}=-\frac{\sigma_{0}}{2} \sin ^{2} \theta, \quad 0 \leq \theta<\theta_{A O B},
$$

after an elementary trigonometric identity has been introduced. This stress function generates a state of compression of magnitude $\sigma_{0}$ in region $A O B$ and meets the traction-free boundary condition along the crack surface $O A$ as depicted in Figure 4.

Equilibrium requires that both the stress functions and their normal derivatives be continuous across sector boundaries. An iterative process in the software determines the angles from crack line $O A$ to rays $O B$ and $O C$ as

$$
\theta_{A O B}=0.53360=30.573^{\circ}, \quad \theta_{A O C}=1.79229=102.69^{\circ} .
$$

The values of the parameters of Equation (14) for the middle sector $B O C$ are found simultaneously as

$$
c=0.11383 \sigma_{0}, \quad \alpha=-0.44270 .
$$




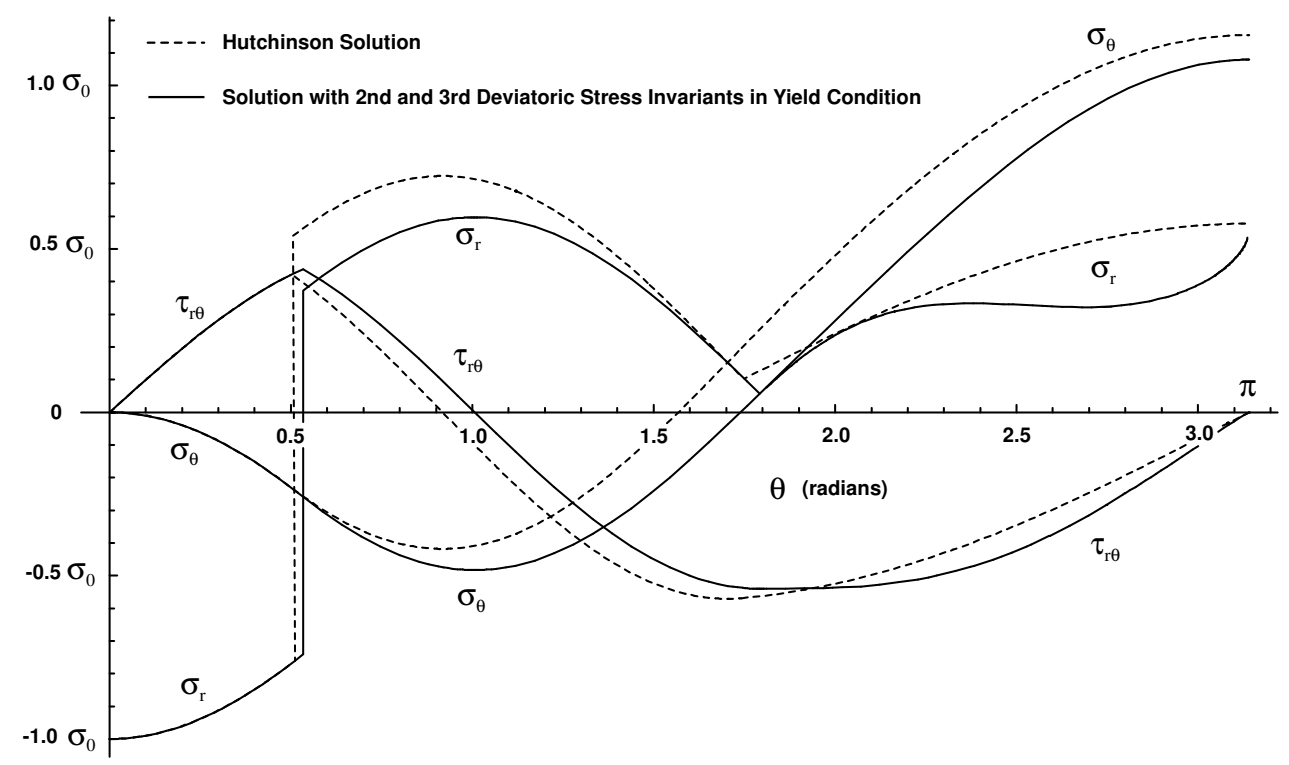

Figure 5. Comparison of perfectly plastic stress fields of the mode I fracture problem.

With these constants and Equation (14), one determines the state of stress in sector $B O C$ using Equations (5)-(7).

The approximate singular solution Equation (29) with $\beta=-\pi$ is used to relate the angle $\theta$ to the function $f$ in the leading sector $C O D$. The exact stresses, which were plotted previously in Figure 3, can be parametrized in terms of the stress function $f$. As these stresses satisfy the yield condition exactly, the error introduced by this procedure is limited to the approximate correlation of $f$ to $\theta$. The numerical data of Figure 4 suggest that the differences are small. See the Appendix for details on the numerical singular solution.

The stresses obtained from this analysis are plotted in Figure 5 as solid curves. Note that a stress discontinuity exists in $\sigma_{r}$ at the boundary between regions $A O B$ and $B O C$, as with ray $O B$ of Figure 4 .

The use of the exact relationships among singular solution stresses, as plotted in Figure 3, are necessary in order to duplicate the results shown in Figure 5. In this respect, the author found it computationally expedient to solve the problem with a continuous exact stress $\sigma_{r}$ and stress function $f$ at the second interface $\theta_{A O C}$, rather than relying on the continuity of the stress function and its normal derivative $p$. The original computational scheme, which did not employ this logic, seemed relatively insensitive to small changes in the interface angle $\theta_{A O C}$. This response was related to the nearly constant slope $p$ of the stress function in this region. As a consequence, a small stress discontinuity in $\sigma_{r}$ formed at the interface $\theta_{A O C}$, which was unexpected. However, upon implementation of the alternative computational scheme, the stress discontinuity was eliminated leaving the stress function $f$, its normal derivative $p$, and the normal stress $\sigma_{r}$ continuous at the second interface $\theta_{A O C}$.

In [Hutchinson 1968] a statically-admissible solution for a perfectly plastic material under the Mises yield condition was obtained for a traction-free semiinfinite mode I crack under plane stress loading conditions. The stress field of Hutchinson for the Mises yield condition is plotted in Figure 5 as dashed 
lines. A stress discontinuity exists in normal stress $\sigma_{r}$ on the boundary between the trailing sectors as previously noted for yield condition, Equation (1). In the region adjacent to the crack, the stresses are identical for the two different yield criteria. Consequently the dashed line representation of the Hutchinson solution is indiscernible in the portion of the figure where it has been overdrawn by the solid curve. The analogous value of the parameter $a$ multiplying normal stresses ahead of the crack in Figure 4 is $1 / \sqrt{3}$ for the Mises yield condition.

In Hutchinson [1968], the crack was oriented to the left of the crack tip rather than to the right as illustrated in Figure 4. This reflection of the semiinfinite crack about the vertical axis through the origin $\mathrm{O}$ allows for a less congested plot of the three stresses $\sigma_{r}, \sigma_{\theta}$, and $\tau_{r \theta}$ as functions of the angle $\theta$. This is particularly true when two different solutions are superposed on the same graph as they are in Figure 5.

\section{Conclusions}

There are relatively few perfectly plastic solutions for the mode I crack problem using yield criteria other than the traditional Mises (see Unger [2007] for one example). In fact, the second most commonly used yield criterion for metals, the Tresca, does not even admit a concentrated fan of mathematical characteristics under plane stress loading conditions from which one could determine an analogous mode I solution to Hutchinson's [Unger 2005]. Thus, while the predictions of the Mises and Tresca yield conditions are often similar in nature, this conclusion cannot be universally drawn. In a similar light, the behavior of yield condition Equation (1), as it pertains to crack problems, was unknown before the present analysis.

As noted in the previous section, a number of similarities exist between the statically admissible stress fields of the mode I fracture problem under the Mises yield condition and under the alternative yield condition, Equation (1). In the case of Equation (1), the smoothness of this yield surface in the principal stress plane is the likely source of its good correlation with the Mises. In contrast, the Tresca is only piecewise smooth.

What stands out between Hutchinson's mode I solution and the present analysis is the rapid rise in the slope of $\sigma_{r}(\theta)$ as $\theta \rightarrow \pi$ for the solution based on yield condition, Equation (1). Just the opposite behavior is exhibited in Figure 5 for the solution based on the Mises yield condition where the slope of $\sigma_{r}(\theta)$ tends to zero as $\theta \rightarrow \pi$. One might expect for microstructural defects driven by hydrostatic stress gradients, that this difference might have a significant effect on any mathematical model describing their distribution near the crack tip.

Nevertheless, it is anticipated that in general a slightly better fit of experimental data by Equation (1) will not compensate for its increased complexity over the conventional Mises yield condition.

\section{Appendix A: Numerical evaluation of the singular solution}

The validity of the approximate solution $f_{\text {approx }}$ is supported by the data shown in Figure 4, where a numerical solution of Equation (22) is compared to the approximate solution Equation (29). In Equation (29) the phase angle $\beta$ of the function $c n$ ( ) was chosen as

$$
\beta=-\pi
$$


in order to meet boundary conditions on traction for a crack oriented as shown in the insert of Figure 4. With this particular value of $\beta$, the function $c n \sqrt[4]{3}(\theta+\beta)$ evaluates to one at $\theta=\pi$, and consequently by Equation (29) the function $f_{\text {approx }}=a \sigma_{0}$. By substituting this particular value for $f_{\text {approx }}$ into Equation (25), one finds the corresponding value of $p_{\text {approx }}$ is zero. Collectively these values of $f_{\text {approx }}$ and $p_{\text {approx }}$ evaluated at $\theta=\pi$ fulfill the boundary conditions on traction directly ahead of the crack tip. Correspondingly, along ray $O D$ of Figure 4 the normal stress $\sigma_{\theta}$ has a value of $2 a \sigma_{0}$ and the shear stress $\tau_{r \theta}$ has a value of zero.

The initial condition for the numerical evaluation of Equation (22) is

$$
\left.f(\theta)\right|_{\theta_{0}}=f_{0}(1.7394)=0,
$$

where the value of the initial angle 1.7394 is near to the location where the function $f_{\text {approx }}=0$ with phase angle $\beta$ given by Equation (A.1). The range on the independent variable for the numerical solution of $f$ will be $1.7394 \leq \theta \leq \pi$, which spans approximately one quarter of the function's period.

At first glance it may seem more logical to start the numerical marching scheme at $\theta=\pi$, taking the initial value of the function $f_{0}$ as $a \sigma_{0}$ (to fulfill the boundary condition) and using negative increments in the angle $\theta$ over the desired range. However, one can prove by direct substitution that $f=a \sigma_{0}=$ const is an exact solution to Equation (22). Consequently any numerical scheme initiated with this particular value of $f_{0}$ will generate this solution regardless of the starting position $\theta_{0}$. Other solutions of Equation (22) having initial data near to the value $a \sigma_{0}$, but differing slightly, will prove computationally unstable. Because of these difficulties the alternative numerical scheme cited was adopted.

The solutions $f= \pm a \sigma_{0}$ to Equation (22) are also singular solutions, but of a different classification than the periodic singular solution. They are extraneous solutions because they violate the yield condition. They represent mathematically the envelope (upper and lower bounds respectively) of the periodic singular solution.

The command function NDSolve in Mathematica 5.2 was used to solve the associated differential algebraic Equation (22), subject to the initial condition, Equation (A.2). The difference between the numerical solution of Equation (22), subject to the initial condition (A.2), and the approximate analytical solution, Equation (29), with $\beta$ given in Equation (A.1), is nearly indistinguishable in the plot of these two functions in Figure 4.

See Zwillinger [1989] for a short discussion and additional references on differential algebraic equations and their solution.

\section{Acknowledgements}

The author thanks Dr. Robert A. Clark, Director of the Institute for Global Enterprises in Indiana, and the other members of the Deans' Council of the University of Evansville for his appointment as a U. E. Global Scholar for 2007-2008. The software purchased under this program's professional development budget was essential to the successful completion of this project.

\section{References}

[Chakrabarty 1987] J. Chakrabarty, Theory of plasticity, McGraw-Hill, New York, 1987. 
[Drucker 1949] D. C. Drucker, "Relationship of experiments to mathematical theories of plasticity", J. Appl. Mech. (Trans. ASME) 16 (1949), 349-357.

[Drucker 1962] D. C. Drucker, "Basic concepts", Chapter 46, pp. 46-1-46-15 in Plasticity and viscoelasticity, edited by W. Flügge, Handbook of Engineering Mechanics, McGraw-Hill, New York, 1962.

[Freudenthal and Geiringer 1958] A. M. Freudenthal and H. Geiringer, "The mathematical theories of the inelastic continuum, elasticity and plasticity", pp. 282-283 in Handbuch der physik, vol. VI, edited by S. Flügge, Springer-Verlag, Berlin, 1958.

[Gradshteyn and Ryzhik 1980] I. S. Gradshteyn and I. M. Ryzhik, Table of integrals, series, and products, Academic Press, New York, 1980.

[Hutchinson 1968] J. W. Hutchinson, "Plastic stress and strain fields at a crack tip", J. Mech. Phys. Solids 16:5 (1968), 337-342. [Ince 1956] E. L. Ince, Ordinary differential equations, Dover, New York, 1956.

[Osgood 1947] W. R. Osgood, "Combined-stress tests on 24S-T aluminum alloy tubes", J. Appl. Mech. (Trans. ASME) 14 (1947), 147-153.

[Unger 2005] D. J. Unger, "A plane stress perfectly plastic mode I crack solution with continuous stress field”, J. Appl. Mech. (Trans. ASME) 72:1 (2005), 62-67.

[Unger 2007] D. J. Unger, “A complete perfectly plastic solution for the mode I crack problem under plane stress loading conditions", J. Appl. Mech. (Trans. ASME) 74:3 (2007), 586-589.

[Zwillinger 1989] D. Zwillinger, Handbook of differential equations, Academic Press, Boston, 1989.

Received 11 Nov 2007. Revised 29 Feb 2008. Accepted 6 Mar 2008.

DAVID J. UNGER: du2@evansville.edu

Department of Mechanical and Civil Engineering, University of Evansville, 1800 Lincoln Avenue, Evansville, IN 47722, United States 


\title{
SUBMISSION GUIDELINES
}

\section{ORIGINALITY}

Authors may submit manuscripts in PDF format on-line. Submission of a manuscript acknowledges that the manuscript is original and has neither previously, nor simultaneously, in whole or in part, been submitted elsewhere. Information regarding the preparation of manuscripts is provided below. Correspondence by email is requested for convenience and speed. For further information, write to:

\author{
Marie-Louise Steele \\ Division of Mechanics and Computation \\ Durand Building, Room 262 \\ Stanford University \\ Stanford CA 94305
}

\section{LANGUAGE}

Manuscripts must be in English. A brief abstract of about 150 words or less must be included. The abstract should be selfcontained and not make any reference to the bibliography. Also required are keywords and subject classification for the article, and, for each author, postal address, affiliation (if appropriate), and email address if available. A home-page URL is optional.

\section{FORMAT}

Authors are encouraged to use $\mathrm{LAT}_{\mathrm{E}} \mathrm{X}$ and the standard article class, but submissions in other varieties of $\mathrm{TEX}$, and, exceptionally in other formats, are acceptable. Electronic submissions are strongly encouraged in PDF format only; after the refereeing process we will ask you to submit all source material.

\section{REFERENCES}

Bibliographical references should be listed alphabetically at the end of the paper and include the title of the article. All references in the bibliography should be cited in the text. The use of $\mathrm{BIBT}_{\mathrm{E}} \mathrm{X}$ is preferred but not required. Tags will be converted to the house format (see a current issue for examples), however, in the manuscript, the citation should be by first author's last name and year of publication, e.g. "as shown by Kramer, et al. (1994)". Links will be provided to all literature with known web locations and authors are encoraged to provide their own links on top of the ones provided by the editorial process.

\section{FigURES}

Figures prepared electronically should be submitted in Encapsulated PostScript (EPS) or in a form that can be converted to EPS, such as GnuPlot, Maple, or Mathematica. Many drawing tools such as Adobe Illustrator and Aldus FreeHand can produce EPS output. Figures containing bitmaps should be generated at the highest possible resolution. If there is doubt whether a particular figure is in an acceptable format, the authors should check with production by sending an email to:

$$
\text { production@mathscipub.org }
$$

Each figure should be captioned and numbered so that it can float. Small figures occupying no more than three lines of vertical space can be kept in the text ("the curve looks like this:"). It is acceptable to submit a manuscript with all figures at the end, if their placement is specified in the text by means of comments such as "Place Figure 1 here". The same considerations apply to tables.

\section{White SPACE}

Forced line breaks or page breaks should not be inserted in the document. There is no point in your trying to optimize line and page breaks in the original manuscript. The manuscript will be reformatted to use the journal's preferred fonts and layout.

\section{Proofs}

Page proofs will be made available to authors (or to the designated corresponding author) at a web site in PDF format. Failure to acknowledge the receipt of proofs or to return corrections within the requested deadline may cause publication to be postponed. 


\title{
Journal of Mechanics of Materials and Structures
}

\author{
Volume 3, № $4 \quad$ April 2008
}

Interfacial crack kinking subjected to contact effects

BaOxiang X. Shan, Assimina A. Pelegri And Yi Pan 591

A variational deduction of second gradient poroelasticity II: an application to the

consolidation problem - ANGELA MADEO, FRANCESCO DELL'ISOLA, NicoletTA

IANIRO AND GIULIO SCIARRA 607

Effects of layer stacking order on the $V_{50}$ velocity of a two-layered hybrid armor system

Pankaj Kumar Porwal and Stuart Leigh Phoenix

A finite element for dynamic analysis of a cylindrical isotropic helical spring _ MOHAMED

TAKTAK, FAKHREDDINE DAMMAK, SAID A BID AND MOHAMED HADDAR 641

Perturbation technique for wave propagation analysis in a notched beam using wavelet spectral element modeling

Mira Mitra, S. Gopalakrishnan, Massimo

RuZZene, Nicole A PETRE AND S. HANAGUd 659

Integration of measures and admissible stress fields for masonry bodies

Massimiliano LucCHesi, Miroslav Šilhavý AND Nicola ZaNi

Concentrated force acting on a power law creep half-plane

JEFFREY JORDAN, IWONA JASIUK AND A LEKSANDER ZUBELEWICZ

Optimization of penetration into geological and concrete shields by impactor with jet thruster

GABI BEN-DOR, ANATOLY DUBINSKY AND TOV ELPERIN

A new tailoring optimization approach for improving structural response and energy

absorption capability of laminated and sandwich composites

UGO ICARDI AND LAURA FERRERO

Interaction between a screw dislocation and a piezoelectric circular inclusion with viscous interface

Xu Wang, ERnian Pan AND A. K ROY

Optimal buckling design of anisotropic rings/long cylinders under external pressure

KARAM Y. MAALAWI

A plane stress perfectly plastic mode I crack problem for a yield condition based on the second and third invariants of the deviatoric stress tensor

DAVID J. UNGER 